\title{
Design for Support in the Initial Design of Naval Combatants
}

\author{
by \\ Syavash Esbati \\ A thesis submitted in fulfilment of the requirements for the degree of \\ Doctor of Philosophy \\ (Naval Architecture and Marine Engineering)
}

Department of Mechanical Engineering

University College London

2018 
To my family. 


\section{Declaration}

I, Syavash Esbati, declare that except where explicit reference is made to other sources, this thesis is the result of my own work. I confirm that this thesis has not been submitted for any other degree at University College London or any other institution.

Print Name:

Signature: 


\section{Abstract}

The decline of defence budgets coupled with the escalation of warship procurement costs have significantly contributed to fleet downsizing in most major western navies despite little reduction in overall commitments, resulting in extra capability and reliability required per ship. Moreover, the tendency of governments to focus on shortterm strategies and expenditure has meant that those aspects of naval ship design that may be difficult to quantify, such as supportability, are often treated as secondary issues and allocated insufficient attention in Early Stage Design. To tackle this, innovation in both the design process and the development of individual ship designs is necessary, especially at the crucial early design stages. Novelty can be achieved thanks to major developments in computer technology and in adopting an architecturally-orientated approach to early stage ship design. The existing technical solutions aimed at addressing supportability largely depend on highly detailed ship design information, thus fail to enable rational supportability assessments in the Concept Phase. This research therefore aimed at addressing the lack of a quantitative supportability evaluation approach applicable to early stage naval ship design.

Utilising Decision Analysis, Effectiveness Analysis, and Analytic Hierarchy Process, the proposed approach tackled the difficulty of quantifying certain aspects of supportability in initial ship design and provided a framework to address the issue of inconsistent and often conflicting preferences of decision makers. Since the ship's supportability is considered to be significantly affected by its configuration, the proposed approach utilised the advantages of an architecturally-orientated early stage ship design approach and a new concept design tool developed at University College London. The new tool was used to develop concept level designs of a frigate-sized combatant and a number of variations of it, namely configurational rearrangement with enhancement of certain supportably features, and an alternative ship design style. The design cases were then used to demonstrate the proposed evaluation approach.

The overall aim of proposing a quantitative supportability evaluation approach applicable to concept naval ship design was achieved, although several issues and limitations emerged during both the development as well as the implementation of the approach. Through identification of the research limitations, areas for future work aimed at improving the proposal have been proposed. 


\section{Impact Statement}

Despite the considerable number of issues that need further investigation, it is considered that this research has contributed to advancing the extent of what can be assessed in the early, formative stages of the design process of what Graham (US Navy Commander, quoted by Gates and Rusling (1982)) described as 'the most the most complex, diverse and highly integrated of any engineering systems' that is regularly manufactured today. The existing body of knowledge regarding the Early Stage Design of naval ships could benefit from this research in a number of ways, both outside and inside academia.

This project was partly sponsored by BAE Systems; the largest defence and shipbuilding company in the UK and one of the world's largest producers of complex warships. During three and half years of research work at UCL, regular project meetings with BAE Systems representatives helped gain valuable practical insights. Additionally, the ship designs developed as part of this research were based on the broad specifications of the Type 26 Global Combat Ship programme that recently entered construction at BAE Systems' Govan and Scotstoun shipyards on the River Clyde in Glasgow, UK. It is hoped that the outcome of this research will support BAE Systems involvement with the UK MoD in better addressing the UK MoD's stated intentions to give equal emphasis to through-life (support) issues as to initial (purchasing) affordability. By stating how Design for Support (DfS) can start to be addressed at the earliest design stages in complex naval vessels, this research will encourage BAE Systems to take such approaches as are demonstrated in this thesis into actual ship design programmes of the future.

The insights generated could be used to justify further research into a number of areas, namely naval ship life cycle costing and application of the proposal in this thesis to those aspects of naval ship supportability that were not considered in this research and to a wider range of ship types. In addition, the feedback from this research on the application of academic concept ship design tools could help improve these softwares' robustness and capability. 


\section{Acknowledgments}

Whilst I as sole author bear full responsibility for the research presented in this thesis, it would not have been possible without the support and encouragement of a number of individuals both from within the UCL community and beyond.

First and foremost, I would like to express my gratitude to my supervisors; Prof. David Andrews, for giving me the opportunity to work in his research groups and his exemplary support and guidance throughout my PhD; and Dr. Rachel Pawling for her generous assistance, insightful comments, and the many casual, yet productive discussions.

I would also like to acknowledge the help readily provided to me by the staff of the Naval Architecture and Marine Engineering Office and the wider UCL Department of Mechanical Engineering, in particular Dr. Alexander Piperakis and Dr. Nick Bradbeer.

Funding for this project was provided by EPSRC (CASE studentship) and sponsorship by BAE Systems, for which I am grateful to both institutes. Special thanks go to BAE Systems representatives - Dr. Malcolm Robb; Dr. Richard Trumper; and Mr David Lewis, all of whom belong to the Research \& Technology Group at BAE Systems Maritime Naval Ships - for the valuable practical insights gained during regular project meetings.

Finally, I am especially grateful to my family for their infinite support, understanding, and continued encouragement throughout my $\mathrm{PhD}$. 


\section{Table of Contents}

Table of Contents

Table of Figures ___ 11

List of Tables _ 18

Nomenclature___ 21

List of Symbols _ 27

Chapter 1: Introduction _ 29

1.1 Preamble _ 29

1.2 Research Scope and Aim __ 31

1.3 Thesis Structure___ 33

Chapter 2: A Review of the State of the Art in Design for Support for Naval Ships _ 35

2.1 Introduction __ 35

2.2 Naval Ship Design __ 37

2.2.1 Background to the Process of Naval Ship Design and Procurement

2.2.2 The Cost of Owning a Warship __ 39

2.2.3 Introduction to Naval Ship Design ___ 47

2.2.4 Traditional Early Stage Naval Ship Design ___ 51

2.2.5 Architectural Modelling in Early Stage Naval Ship Design___ 54

2.2.6 The Design Building Block Approach __ 57

2.3 Naval Ship Supportability ___ 66

2.3.1 Introduction to Naval Ship Supportability ___ 66

2.3.2 Architecturally Discernible Aspects of Naval Ship Through-Life Support _ 69

2.3.3 Technical Solutions Addressing the Architecturally Discernible Aspects of Naval Ship Through-Life Support __ 72

2.4 Top-down Structuring of Multi-Criteria Decision Problems ___ 84

2.4.1 Decision Analysis___ 84

2.4.2 Effectiveness Analysis ___ 85

2.4.3 Multi-Criteria Decision Making Techniques ___ 88

2.5 Conclusion from the State of the Art Review ___ 92

Chapter 3: Development of the Design for Support Evaluation Approach _96 
3.1 Introduction 96

3.2 Initial Proposals for the Design for Support Evaluation Approach 97

3.2.1 Critical Path Method and Programme Evaluation and Review Technique 97

3.2.2 Prospect Theory-Based Real Option Analysis 99

3.3 The Proposed Design for Support Evaluation Approach 102

3.3.1 Top-Down Structuring of the Evaluation of Naval Ship TLS _ 102

3.3.2 Developing Rational and Indicative Supportability Metrics 104

3.3.3 A Proposed Framework to Capture, Incorporate, Apply, and Modify Expert Knowledge 105

3.4 Customisation to a Specific Naval Ship Supportability Case 114

3.4.1 Overall Measure of Effectiveness (oMoE) 114

3.4.2 Objectives 114

3.4.3 Measures of Effectiveness (MoE) 115

3.4.4 Measures of Performance (MoP) 115

3.4.5 Dimensional Parameters (DP) 117

3.4.6 Maintainability Assessment Hierarchy 119

Chapter 4: $\quad$ Ship Design Case Studies 121

4.1 Introduction _ 121

4.2 The New UCL Ship Concept Design Software___ 122

4.3 Baseline Frigate Design __ 128

4.3.1 Ship Performance Requirements and Payload Equipment ___ 129

4.3.2 Ship Complement and Accommodation___ 131

4.3.3 Major Ship Characteristics and Dimensional Ratios __ 131

4.3.4 Resistance Estimation and Power Plant Selection ___ 133

4.3.5 Stability Assessments ___ 136

4.3.6 Internal Arrangement ___ 140

4.3.7 Ship Costing ___ 142

4.3.8 Supportability Features ___ 143

4.3.9 Ship Design Aspects not Considered in the Baseline Frigate Design Study___ 144

4.4 Ship Design Variants ___ 146

4.4.1 Configurational Variants of the Baseline Frigate Design ___ 146

4.4.2 Enhancement of Certain Supportability Features ____ 148

4.4.3 Alternative Ship Design Style___ 151 
Chapter 5: Application of the Proposed Design for Support Evaluation Approach and Presentation of the Results 156

5.1 Introduction 156

5.2 Maintainability of Ship Machinery Spaces ___ 157

5.2.1 Individual Hierarchy Levels ___ 159

5.2.2 Additional Aspects Taken into Account___ 169

5.2.3 Application of the Maintainability Evaluation Hierarchy___ 173

5.3 Maintainability Assessment Results ___ 175

5.3.1 Baseline Frigate Design and Configurational Variants___ 175

5.3.2 Enhanced Supportability Features ___ 178

5.3.3 Alternative Ship Design Style___ 180

Chapter 6: Discussion of Design for Support in the Initial Design of Naval Combatants

6.1 Introduction ___ 183

6.2 Analysis of Maintainability Assessment Results ___ 184

6.2.1 Baseline Frigate Design and Configurational Variants____ 184

6.2.2 Enhanced Supportability Features ___ 185

6.2.3 Alternative Ship Design Style___ 187

6.2.4 Ship Costing and Maintainability Assessments ___ 190

6.2.5 Maintenance Scenarios and Maintainability Assessments ___ 192

6.3 Research Summary ___ 194

6.3.1 The Importance of Design for Support in Naval Ship Design _ 194

6.3.2 Design for Support in Naval Ship Early Stage Design___ 195

6.3.3 Design for Support in Architecturally-Orientated Design ___ 196

6.3.4 The Proposed Design for Support Evaluation Approach ___ 197

6.3.5 Research Limitations ___ 200

6.3.6 Future Work ___ 202

Chapter 7: Conclusions and Further Work

References

Appendices

Appendix 1 A Review of the State of the Art in Design for Support for Naval Ships __ 221

Appendix 2 Development of the Design for Support Evaluation Approach__ 230

Appendix 3 RINA Warship Conference 2015 __ 231 
Appendix 4 RINA ICCAS 2015 Conference 252

Appendix 5 Ship Design Case Studies 274 


\section{Table of Figures}

Figure 2.1: UK MoD design and procurement process (CADMID) (UK MoD, 2002)

Figure 2.2: Breakdown of warship WLC and typical example of the percentage breakdown in WLC for a warship (Brown and Andrews, 1980) 41

Figure 2.3: Undiscounted WLC of a typical 3,000 tonne frigate (Rawson, 1973) _ 41

Figure 2.4: Contribution of different factors to shipbuilding annual cost escalation for US Navy surface combatants: DDG-2 (FY 1961) and DDG-51 (FY 2002) (Arena et al., 2006)

Figure 2.5: The early stages of a programme and the opportunity to reduce WLC (NATO, 2009) 44

Figure 2.6: The Whole Life Costs Iceberg (NATO, 2012) 45

Figure 2.7: Temporal Ship's Whole Life Costs (Page, 2011) 46

Figure 2.8: The relationships between the three areas of human knowledge; humanities, science and design (Archer, 1979) 47

Figure 2.9: UK Naval ship design process (Andrews, 1992) 50

Figure 2.10: A summary representation of pre architecturally-orientated sequential synthesis in ship design, showing the feedback loops as dotted lines (Andrews, 2003b)

Figure 2.11: Combining two sets of circles of influence from preferences in compartment juxtapositions (Andrews, 1985) 58

Figure 2.12: A diagrammatic indication of a network representation of the compartment relationships for a full synthesis (Andrews, 1985) 58

Figure 2.13: A diagrammatic suggestion as to how a full synthesis suggested by Figure 2.11 and Figure 2.12 might be accomplished (Andrews, 1985) 59

Figure 2.14: The Design Building Block approach to Early Stage Ship Design applied to surface ships (Andrews and Dicks, 1997) (Andrews and Pawling, 2003) 62

Figure 2.15: Design phases of the UCL DBB approach showing major design choices (Andrews and Pawling, 2008) 63

Figure 2.16: ILS elements (UK MoD, 2013a) 66

Figure 2.17: Relationship of ILS objectives to mission area and needs (DSMC, 1986)

Figure 2.18: Adaptability leads to affordability over the lifecycle (Sturtevant et al., 2014) 71

Figure 2.19: VPS destroyer (Boerum and Birindelli, 1985) 73

Figure 2.20: Lockheed Martin monohull LCS design (Gouré, 2006) 74 
Figure 2.21: General Dynamics Trimaran LCS variant (Gouré, 2006) 75

Figure 2.22: The Stanflex 300 series of the Flyvefisken Class OPVs (Depetro, 2015)

Figure 2.23: Bolhm \& Voss MEKO concept (Glanville, 2010) 76

Figure 2.24: BMT Venetor mission bay (top) and arrangement of major compartments (bottom) (Kimber et al., 2008) 77

Figure 2.25: Proposed Through Life Business Model (TLBM) (John et al., 2003) _79 Figure 2.26: Current (boxed area) and future scope of SUPPORT Sim (Hill and Jarman, 2014)

Figure 2.27: The maintenance and modernisation framework captures the key dynamics of material condition and service life (Malone et al., 2014) 82

Figure 2.28: Schematic representation of the steps in Decision Analysis (Keeney, 1982) 84

Figure 2.29: The steps involved in the process of Effectiveness Analysis (Leite and Mensh, 1999) 87

Figure 2.30: Example oMoE hierarchy for assessing a set of military performance characteristics (Brown and Salcedo, 2002)

Figure 2.31: AHP applied to determine the best job after obtaining PhD degree (Saaty, 2008) 90

Figure 2.32: Variations of marginal utility for linear and non-linear utility functions with risk-acceptance and risk-averse regions (DeNeufville, 1990) 91

Figure 3.1: Simplified work breakdown network for a series of spaces commonly located amidships in a typical frigate (Esbati et al., 2015a) 98

Figure 3.2: Variation of risk-adjusted utility with mission bay conversation probability, showing a probability decision threshold of approximately $70 \%$ and negative utility for probability values of less than $40 \%$ (Esbati et al., 2015b) 101

Figure 3.3: Schematic representation of the steps in Decision Analysis (Keeney, 1982)

Figure 3.4: Generic top-down structure showing certain components of Decision Analysis (shown in red) and Effectiveness Analysis (shown in blue) being used in parallel 104

Figure 3.5: The weightings distribution produced by Integer Scale (top left), Balanced Scale (top right), and Power Scale (bottom) when comparing two and three attributes (Elliott, 2010) 109

Figure 3.6: Top-down structuring of a partial (architecturally dependent) evaluation of maintainability as a subset of naval ship supportability to arrive at an overall Measure of Effectiveness 120 
Figure 4.1: Network of required relationships for a selected set of DBBs from a database of layout guidance

Figure 4.2: Example of a whole ship adjacency network, showing the profile view and highlighting links to the mission bay for a UCL Offshore Support Vessel (OPV) design study (Pawling and Andrews, 2013), outputted by the UCL layout tool (Pawling et al., 2015) 124

Figure 4.3: Screenshots of the developmental JavaScript-based implementation of the UCL DBB approach, ship internal arrangement and interactive GUI and controls (top), close-up of the cellular representation (bottom) 126

Figure 4.4: Baseline Frigate Design Deep displacement weight breakdown 133

Figure 4.5: Baseline Frigate Design power speed curve developed using Holtrop and Mennen (1982) power prediction method

Figure 4.6: The CODLOG machinery configuration adopted for the Baseline Frigate Design 135

Figure 4.7: Baseline Frigate Design curve of statical stability for the Lightship case 137

Figure 4.8: The method proposed by Rawson and Tupper (2001) to correct the GZ curve for free surface effects 139

Figure 4.9: Baseline Frigate Design curve of statical stability for the Deep case with free surface effects correction 139

Figure 4.10: Baseline Frigate Design internal arrangement produced by the UCL JavaScript ship design tool 141

Figure 4.11: Pie chart showing UPC breakdown based on the warship seven weight groups system for Baseline Frigate Design (WG 7 not included since not relevant to UPC) 142

Figure 4.12: Baseline Frigate Design deck plan highlighting supportability features, produced by the UCL JavaScript ship design tool 144

Figure 4.13: Deck plan highlighting supportability features of the Baseline Frigate (top left), Variant 1 (top right), Variant 2 (bottom left), and Variant 3 (bottom right), produced by the UCL JavaScript ship design tool

Figure 4.14: Ship design Variant 1.2.1 internal arrangement produced by the UCL JavaScript ship design tool 155

Figure 5.1: Bottom-up application of the Design for Support evaluation approach for a partial (architecturally dependent) assessment of maintainability of machinery spaces 158

Figure 5.2: The linear regression method (left) and the segmented regression method with given breakpoint (right) considered in evaluating the sub-MoP actual values against the sub-MoP rejection and preference values 160 
Figure 5.3: Partially populated comparison matrix produced from pairwise comparisons of Maintenance Type 1 (transfer of Naval Stores and Spare Gear Stores to machinery spaces) sub-MoPs 162

Figure 5.4: Reciprocal comparison matrix produced from pairwise comparisons of Maintenance Type 1 (transfer of Naval Stores and Spare Gear Stores to machinery spaces) sub-MoPs 162

Figure 5.5: Priority vector for Maintenance Type 1 (transfer of Naval Stores and Spare Gear Stores to machinery spaces) sub-MoPs 163

Figure 5.6: Variation of relative importance of different types of maintenance with respect to the two maintenance scenarios at the MoE level 169

Figure 5.7: Sequential description of the ship design variants 175

Figure 5.8: Maintainability absolute oMoE results, measured for the Baseline Frigate Design and its three configurational variants (Variants 1, 2, and 3) 176

Figure 5.9: Absolute MoE for different types of maintenance (UM/AM), measured for the Baseline Frigate Design and its three configurational variants 176

Figure 5.10: Comparison of maintainability oMoE results with major ship design characteristics, produced for the Baseline Frigate Design and its three configurational variants, and normalised against the Baseline Frigate Design 177

Figure 5.11: Maintainability absolute oMoE results, showing the effects of increasing the overall capacity of certain supportability features 178

Figure 5.12: Comparison of maintainability oMoE results with major ship design characteristics, produced for the Baseline Frigate and ship design variants with enhanced provisions of supportability features, and normalised against the Baseline Frigate Design 179

Figure 5.13: Maintainability absolute oMoE results, showing the effects of the alternative ship design style 180

Figure 5.14: Absolute MoE of different types of maintenance (UM/AM), showing the effects of the alternative ship design style 181

Figure 5.15: Comparison of maintainability oMoE results with major ship design characteristics, produced for the ship design variants with original and alternative ship design styles and baseline and enhanced provisions of supportability features, and normalised against the Baseline Frigate Design 182

\section{Appendix 1}

Figure A1. 1: General design diagram (Evans, 1959) 221

Figure A1.2: Overall model of the ship design process and section through the model showing typical steps in the spiral (Andrews, 1981) 221

Figure A1. 3: Design spiral (Gale, 2003) 222 
Figure A1. 4: A simplified model of the initial ship sizing illustrating assumptions and typical sourced needed at the concept stages (Andrews, 1985) 224

Figure A1. 5: A summary representation of a more 'holistic' approach to a fully integrated ship synthesis (Andrews, 1985) 225

Figure A1. 6: A schematic modification of the initial ship synthesis of Figure A1. 4 with spatial totals which are laid out graphically before reiterating (Andrews, 1985)

Figure A1. 7: SURFCON representation showing the three panes for tree structure, graphics (Design Building Blocks) and tabular interfaces, with the results of stability and resistance analyses also visible (Andrews and Pawling, 2008) 227

Figure A1. 8: Phases and major milestones in the TLBM process (John et al., 2003)

\section{Appendix 2}

Figure A2. 1: The AHP utilised by Gregor (2003) for measuring oMoE as part of developing a method for valuing flexibility under (acquisition) uncertainty 230

\section{Appendix 5}

Figure A5. 1: Holtrop and Mennen applicability limitations (Holtrop and Mennen, 1982) 278

Figure A5. 2: The process of estimating the required ship propulsion power (Molland et al., 2011) 279

Figure A5. 3: Various cost estimation methods and their applicability at each stage of a defence programme (NATO, 2009) 280

Figure A5. 4: Ship design Variant 1 (two workshops and two Naval and Spare Gear Stores) internal arrangement, produced by the UCL JavaScript ship design tool _282

Figure A5. 5: Ship design Variant 2 (one workshop and three Naval and Spare Gear Stores) internal arrangement, produced by the UCL JavaScript ship design tool _283

Figure A5. 6: Ship design Variant 3 (two workshops and three Naval and Spare Gear Stores) internal arrangement, produced by the UCL JavaScript ship design tool _284

Figure A5. 7: Pie chart showing UPC breakdown based on the warship seven weight groups system for ship design Variant 1 (WG 7 not included since not relevant to UPC)

Figure A5. 8: Pie chart showing UPC breakdown based on the warship seven weight groups system for ship design Variant 2 (WG 7 not included since not relevant to UPC)

Figure A5. 9: Pie chart showing UPC breakdown based on the warship seven weight groups system for ship design Variant 3 (WG 7 not included since not relevant to UPC) 
Figure A5. 10: Ship design Variant 1.1 (two workshops (50\% extra overall capacity) and two Naval and Spare Gear Stores (overall capacity same as Baseline Frigate)) internal arrangement, produced by the UCL JavaScript ship design tool 286

Figure A5. 11: Ship design Variant 1.2 (two workshops (100\% extra overall capacity) and two Naval and Spare Gear Stores (overall capacity same as Baseline Frigate)) internal arrangement, produced by the UCL JavaScript ship design tool 287

Figure A5. 12: Pie chart showing UPC breakdown based on the warship seven weight groups system for ship design Variant 1.1 (WG 7 not included since not relevant to UPC) 288

Figure A5. 13: Pie chart showing UPC breakdown based on the warship seven weight groups system for ship design Variant 1.2 (WG 7 not included since not relevant to UPC) 288

Figure A5. 14: Ship design Variant 2.1 (one workshop (overall capacity same as Baseline Frigate) and three Naval and Spare Gear Stores (50\% extra overall capacity)) internal arrangement, produced by the UCL JavaScript ship design tool 289

Figure A5. 15: Ship design Variant 2.2 (one workshop (overall capacity same as Baseline Frigate) and three Naval and Spare Gear Stores (100\% extra overall capacity)) internal arrangement, produced by the UCL JavaScript ship design tool

Figure A5. 16: Pie chart showing UPC breakdown based on the warship seven weight groups system for ship design Variant 2.1 (WG 7 not included since not relevant to UPC) 291

Figure A5. 17: Pie chart showing UPC breakdown based on the warship seven weight groups system for ship design Variant 2.2 (WG 7 not included since not relevant to UPC) 291

Figure A5. 18: Ship design Variant 3.1 (two workshops (50\% extra overall capacity) and three Naval and Spare Gear Stores (50\% extra overall capacity)) internal arrangement, produced by the UCL JavaScript ship design tool 292

Figure A5. 19: Ship design Variant 3.2 (two workshops (100\% extra overall capacity) and three Naval and Spare Gear Stores (100\% extra overall capacity)) internal arrangement, produced by the UCL JavaScript ship design tool 293

Figure A5. 20: Pie chart showing UPC breakdown based on the warship seven weight groups system for ship design Variant 3.1 (WG 7 not included since not relevant to UPC) 294

Figure A5. 21: Pie chart showing UPC breakdown based on the warship seven weight groups system for ship design Variant 3.2 (WG 7 not included since not relevant to UPC) 294

Figure A5. 22: Ship design Variant 2.2.1 (two workshops (one workshop (overall capacity same as Baseline Frigate), three Naval and Spare Gear Stores (100\% extra 
overall capacity), and alternative ship design style) internal arrangement, produced by the UCL JavaScript ship design tool 295

Figure A5. 23: Ship design Variant 3.2.1 (two workshops (100\% extra overall capacity), three Naval and Spare Gear Stores (100\% extra overall capacity), and alternative ship design style) internal arrangement, produced by the UCL JavaScript ship design tool 296

Figure A5. 24: Pie chart showing UPC breakdown based on the warship seven weight groups system for ship design Variant 1.2.1 (WG 7 not included since not relevant to UPC) 297

Figure A5. 25: Pie chart showing UPC breakdown based on the warship seven weight groups system for ship design Variant 2.2.1 (WG 7 not included since not relevant to UPC) 297

Figure A5. 26: Pie chart showing UPC breakdown based on the warship seven weight groups system for ship design Variant 3.2.1 (WG 7 not included since not relevant to UPC) 297 


\section{List of Tables}

Table 3.1: A summary of the corresponding components of Decision Analysis and Effectiveness Analysis 104

Table 3.2: The numerical values used by the three common scale types and corresponding linguistic expressions of preference (Elliott, 2010) 108

Table 3.3: Random Consistency Index values for different comparison matrix dimensions (Saaty, 1987) 110

Table 3.4: $\lambda_{\max }$ values for various comparison matrix dimensions and consistency acceptance thresholds (Alonso and Lamata, 2006)

Table 3.5: The features required for the proper description of the objective to improve the effectiveness of three distinct Maintenance Types on-board a vessel 115

Table 4.1: Baseline Frigate Design main performance requirements based on the broad specifications of the General Purpose variant of the Type 26 Global Combat Ship (Saunders and Philpott, 2013; BAE Systems, 2015) and UCL (2013b) warship database

Table 4.2: Baseline Frigate Design payload equipment based on the broad specifications of the General Purpose variant of the Type 26 Global Combat Ship (Saunders and Philpott, 2013; BAE Systems, 2015) and UCL (2013b) warship database 130

Table 4.3: Baseline Frigate Design complement and total accommodation breakdown 131

Table 4.4: Baseline Frigate Design major ship characteristics 132

Table 4.5: Baseline Frigate Design major dimensional ratios 132

Table 4.6: Shape criteria for the GZ curve (UK MoD, 2000b) 136

Table 4.7: Evaluation of the Baseline Frigate Design Lightship intact stability assessment results against Defence Standards 02-109 (UK MoD, 2000b) 137

Table 4.8: The variable weights taken into account for Deep intact stability assessment 138

Table 4.9: Evaluation of the Baseline Frigate Design Deep intact stability assessment results against Defence Standards 02-109 (UK MoD, 2000b) 140

Table 4.10: Disposition of the Baseline Frigate Design supportability features 143

Table 4.11: Disposition of the supportability features in the Baseline Frigate Design and its configurational variants 146

Table 4.12: Major ship characteristics and UPC of the Baseline Frigate Design and configurational variants 148

Table 4.13: Ship design variants with extra overall workshop or/and Naval and Spare

Gear Store capacities 149 
Table 4.14: Major ship characteristics and UPC of ship design variants with extra overall workshop capacity

Table 4.15: Major ship characteristics and UPC of ship design variants with extra overall Naval and Spare Gear Stores capacity

Table 4.16: Major ship characteristics and UPC of ship design variants with extra overall workshop and Naval and Spare Gear Stores capacities

Table 4.17: Stylistic ship design variants with extra overall workshop or/and Naval and Spare Gear Store capacities 152

Table 4.18: Major ship characteristics and UPC of stylistic ship design variants with extra overall workshop capacity 152

Table 4.19: Major ship characteristics and UPC of stylistic ship design variants with extra overall Naval and Spare Gear Stores capacity

Table 4.20: Major ship characteristics and UPC of stylistic ship design variants with extra overall Workshop and Naval and Spare Gear Stores capacities 153

Table 5.1: Rejection and preference values used for assessing the sub-MoPs of maintainability of machinery spaces

Table 5.2: Pairwise comparisons of Maintenance Type 1 (transfer of Naval Stores and Spare Gear Stores to machinery spaces) sub-MoPs 161

Table 5.3: Determination of the priority vector for Maintenance Type 2 (transfer of equipment from machinery spaces to ship workshop facilities) sub-MoPs 164

Table 5.4: Determination of the priority vector for Maintenance Type 2 (transfer of Naval Stores and Spare Gear Stores to ship workshop facilities) sub-MoPs 164

Table 5.5: Determination of the priority vector for Maintenance Type 3 (transfer of equipment from machinery spaces to ship Equipment Removal Points) sub-MoPs165

Table 5.6: Determination of the priority vector for Maintenance Type 2 (transfer of equipment from machinery spaces and Naval and Spare Gear Stores to ship workshop facilities) MoPs 166

Table 5.7: Determination of the priority vector for the three Maintenance Types MoEs, Underway Maintenance scenario 167

Table 5.8: Determination of the priority vector for the three Maintenance Types MoEs, Alongside Maintenance scenario 168

Table 5.9: Distribution of maintenance demands across the machinery spaces for all ship design cases 171

Table 5.10: Capability measures of workshop facilities and Naval and Spare Gear Stores for all ship design cases outlined in Sections 4.4.1 - 4.4.3 172

Table 5.11: Routeability measures for the Equipment Removal Points with respect to the selected ship design style 173 
Table 6.1: Variation of oMoE improvements and UPC escalations with respect to the adopted ship design style.

\section{Appendix 1}

Table A1. 1: Taxonomy of $S^{4}$ plus CS characteristics, relevant ship design aspects to consider and corresponding features 223

Table A1. 2: ILS elements and brief description (UK MoD, 2013a) 228

\section{Appendix 5}

Table A5. 1: Weight and space margins used in the development of the Baseline Frigate Design (UCL, 2013a) 275

Table A5. 2: Weight of equipment mountings used in the development of the Baseline Frigate Design for noise and vibration attenuation purposes (UCL, 2013a) 276

Table A5. 3: Typical frigate characteristics (UCL, 2013a) 277

Table A5. 4: Typical frigate characteristics (Griethuysen, 1992, 1993) 277 


\section{Nomenclature}

AHP - Analytic Hierarchy Process (decision making method) ................................ 90

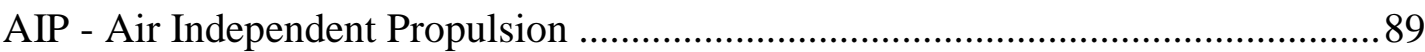

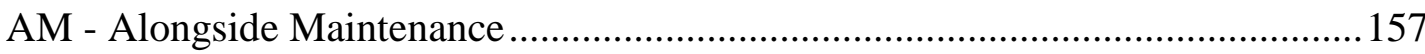

AMR - Auxiliary Machinery Room ..................................................................... 157

AoA - Analysis of Alternatives (US Department of Defence requirement for

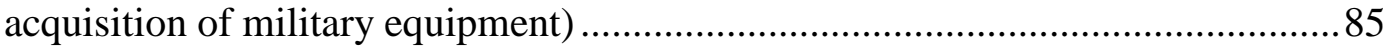

ARM - Availability, Reliability and Maintainability ….............................................. 30

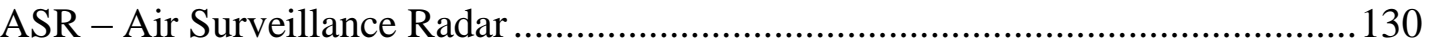

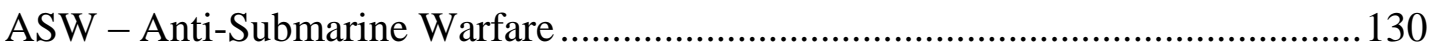

AVCAT - Aviation Carrier Air Turbine (aviation fuel) .......................................... 138

CAD - Computer-Aided Design..........................................................................56

CADMID - Concept, Assessment, Demonstration, Manufacture, In-Service and

Disposal (UK MoD stages in defence equipment acquisition) ............................66

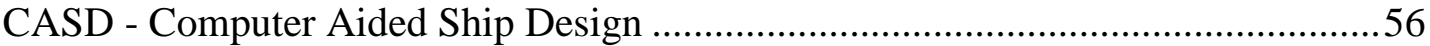

CBM - Condition Based Maintenance ….................................................................. 80

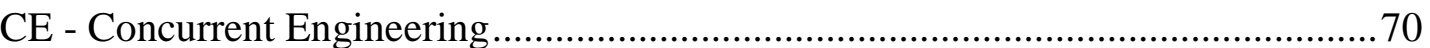

CER - Cost Estimating Relationships (causal relationships between system costs and

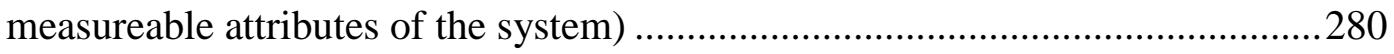

CfA - Contracting for Availability (contracting approach aimed to improve

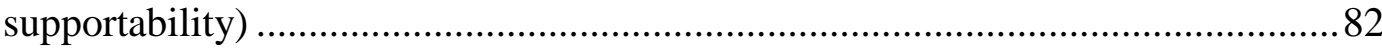

CI - Consistency Index (measure of consistency of a decision maker's judgments)

CIC - Combat Information Centre (US Navy term for the Operations Room)

CIWS - Close-in Weapon System (short range defence systems) ...........................130

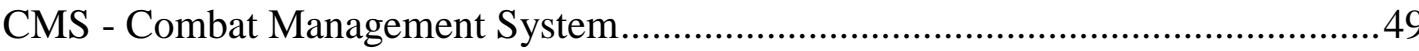

CODLOG - Combined Diesel-Electric or Gas (ship propulsion system) ............... 134

CODOG - Combined Diesel or Gas (ship propulsion system) ............................... 106

COTS - Commercial Off The Shelf …..................................................................... 192

CPM - Critical Path Method (technique for scheduling project activities)................97

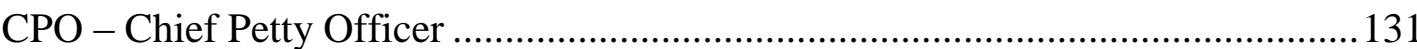


CR - Consistency Ratio (threshold for acceptable inconsistency of a decision maker's

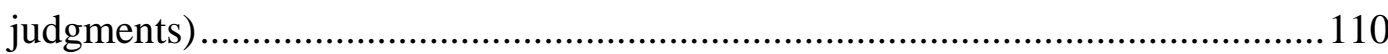

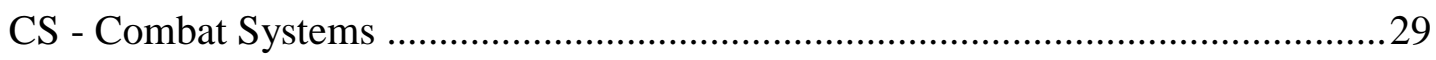

CSV - Comma Separated Value (file storage format in computing) ....................... 127

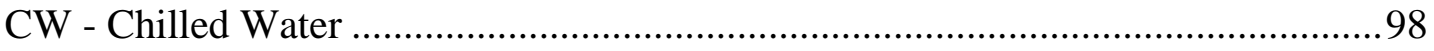

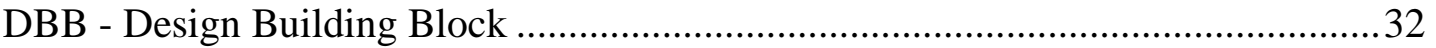

DCD - Damage Control Deck (the lowest deck in the ship where horizontal access is

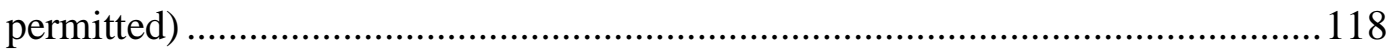

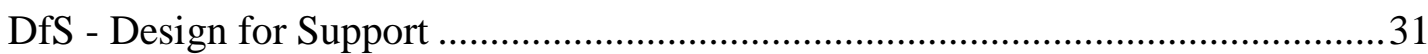

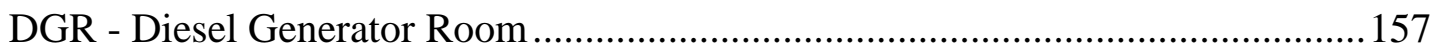

DP - Dimensional Parameter (term used in Effectiveness Analysis)........................ 86

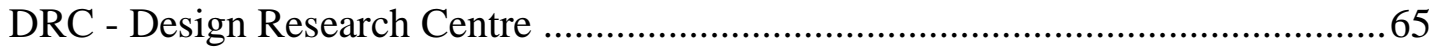

DSMC - Defence System Management College (a corporate university of the US

Department of Defence headquartered in Fort Belvoir, Virginia) .........................66

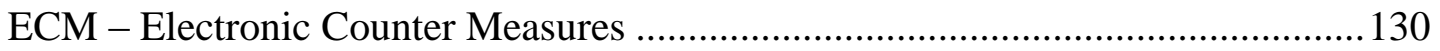

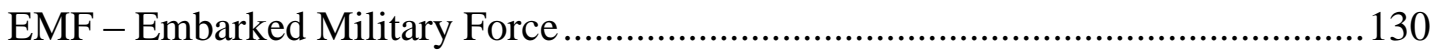

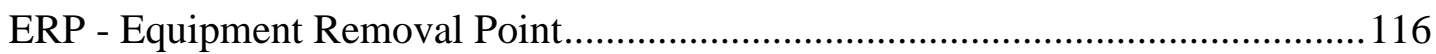

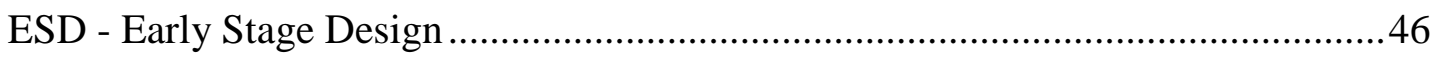

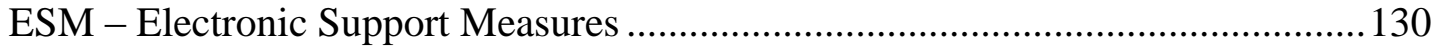

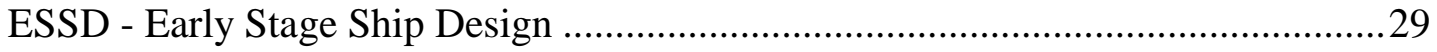

EVM - Eigenvector Method (method to estimate the principal eigenvector).......... 112

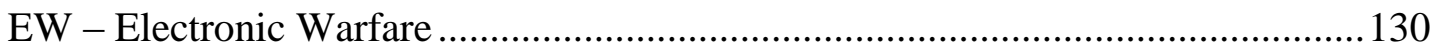

FICS - Fully Integrated Communications System .............................................. 130

FMECA - Failure Modes Effects and Criticality Analysis .....................................67

FOC - First of Class (lead ship of a class of ships) ..............................................40

FoM - Figure of Merit (numerical measure of system performance relative to the

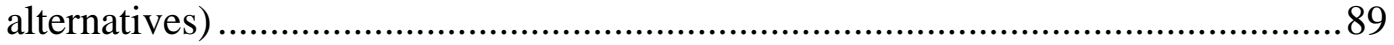

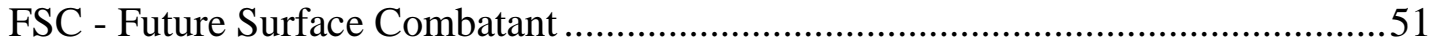

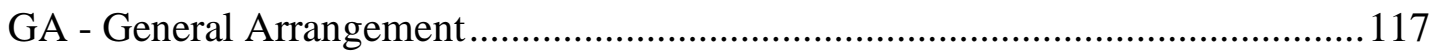

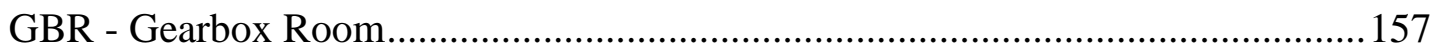

GCS - Global Combat Ship (Royal Navy Type 26 frigate) ................................... 82

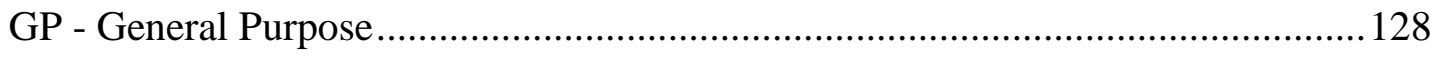

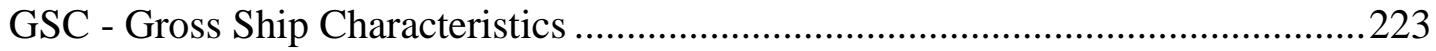

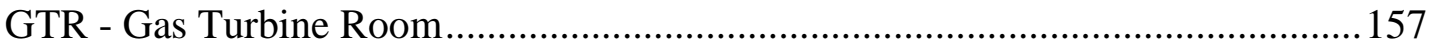




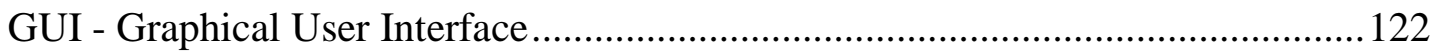

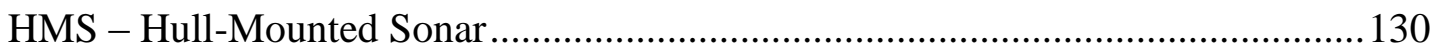

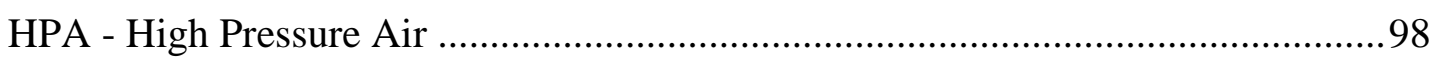

HVAC - Heat, Ventilation and Air Conditioning ................................................. 98

HWS - Hierarchical Weighted Sum (decision making method) ............................... 89

IEP - Integrated Electric Propulsion .......................................................201, 205

IFEP - Integrated Full Electric Propulsion (ship propulsion systems)......................64

ILS - Integrated Logistics Support (integrated and iterative approach to develop material and support strategy for military systems) ..........................................2. 29

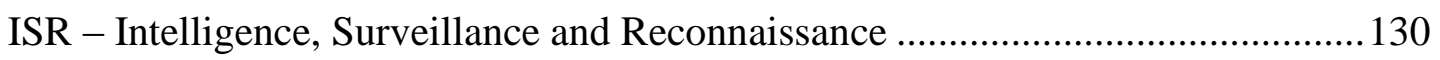

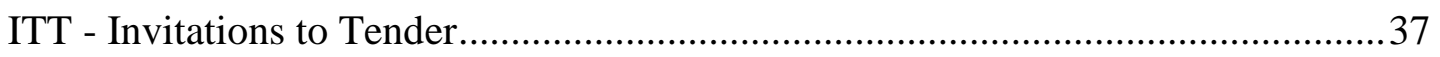

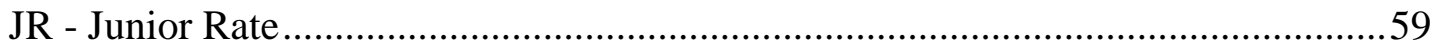

JSS - Joint Support Ship (multirole naval ship capable of launching and supporting

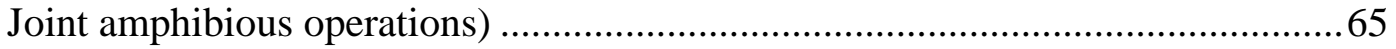

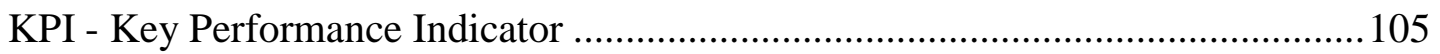

LCC - Life Cycle Cost (summation of initial and through-life costs) .......................40

LCS - Littoral Combat Ship (US Navy surface combatant) ...................................65

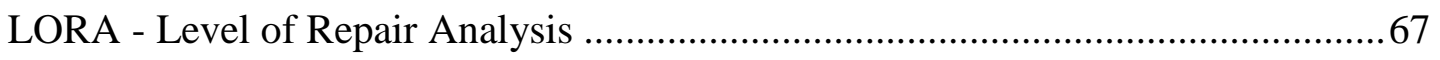

LSA - Logistic Support Analysis (method for analysing supportability and through-

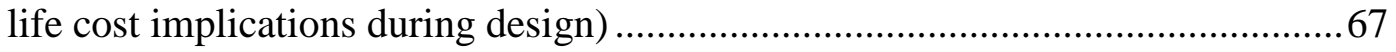

MAU - Multi-Attribute Utility (decision making method) ..................................... 91

MCDA - Multiple Criteria Decision Analysis ..................................................... 83

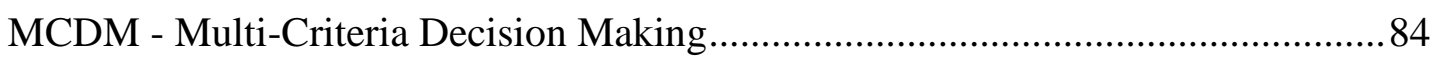

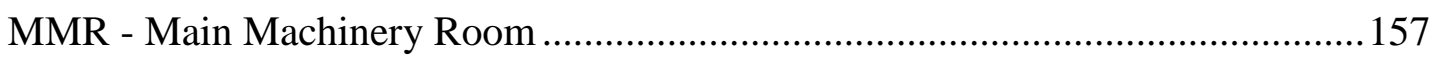

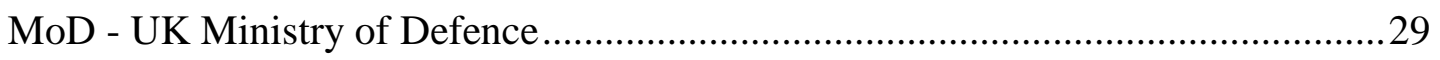

MoE - Measures of Effectiveness (term used in Effectiveness Analysis...................86

MoFE - Measure of Force Effectiveness (term used in Effectiveness Analysis) .......86

MoM - Measure of Merit (term used in Effectiveness Analysis) .............................. 86

MoP - Measures of Performance (term used in Effectiveness Analysis)...................86

MoSE - Measure of System Effectiveness (term used in Effectiveness Analysis)....86

MRG - Marine Research Group (UCL research group in Department of Mechanical

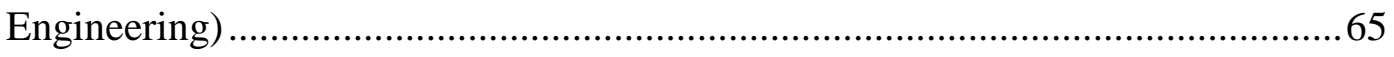

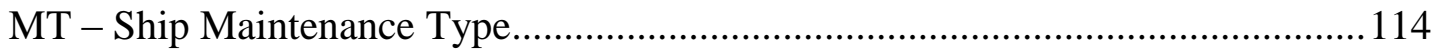

$\mathrm{MT}_{1}$ - Maintenance Type 1 (on-board in-space maintenance)............................... 114 
$\mathrm{MT}_{2}$ - Maintenance Type 2 (on-board workshop maintenance)

MT3 - Maintenance Type 3 (off-board maintenance carried out by naval dockyards or the OEM). .114

MTBF - Mean Time Between Failure ………..................................................... 71

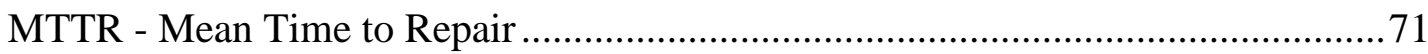

NAAFI - Navy, Army and Air Force Institutes (UK government organisation that runs recreational establishments used by the British Armed Forces) ........................ 138

NATO - North Atlantic Treaty Organization............................................................29

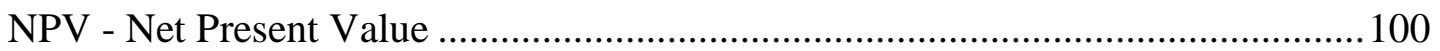

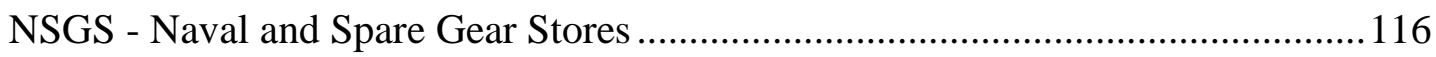

O\&S - Operations and Support (US Department of Defence term for through-life

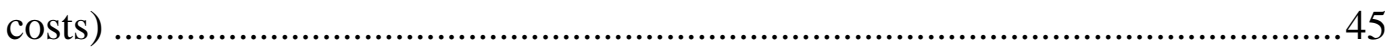

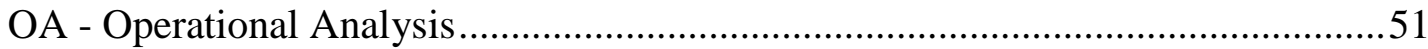

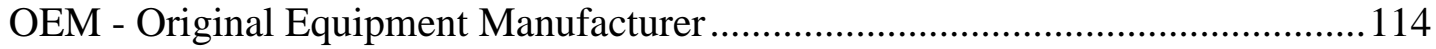

oMoE - overall Measure of Effectiveness (term used in Effectiveness Analysis).....86

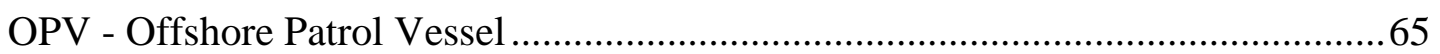

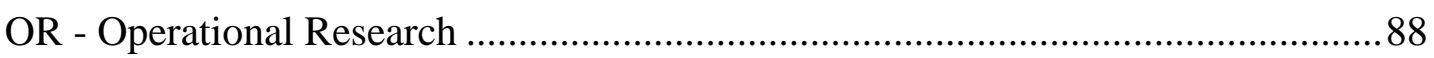

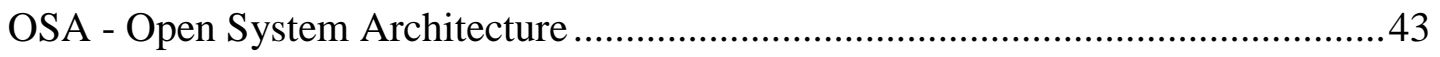

PAMMS - Principal Anti Air Missile System............................................................ 40

PB-ROA - Prospect Theory-Based Real Options Analysis (quantitative framework for evaluating adaptability in non-commercial engineering systems) ...................... 100

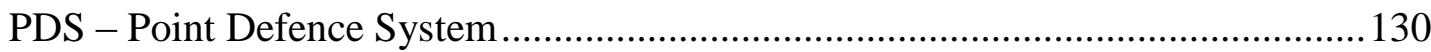

PERT - Programme Evaluation and Review Technique (statistical tool used in project

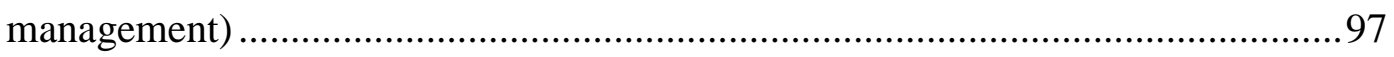

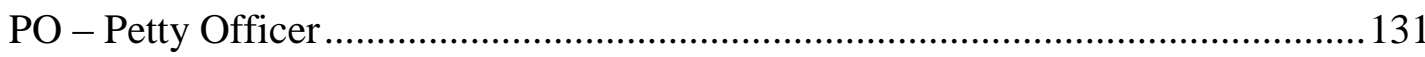

PSI - Proxy Supportability Indicator (indirect measures of supportability performance

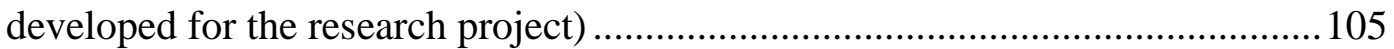

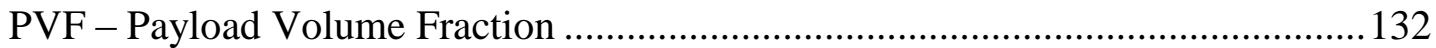

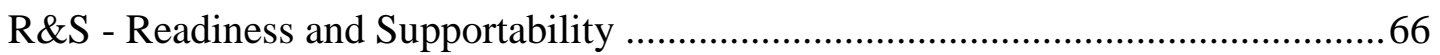

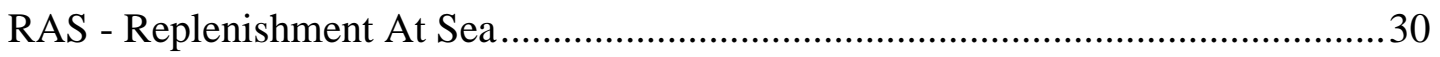

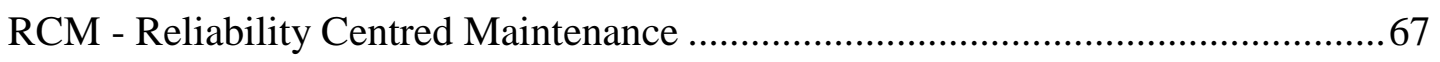

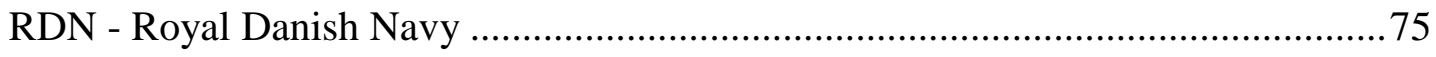

RGM - Row Geometric Mean (method to estimate the principal eigenvector)....... 113 
RI - Random Consistency Index (consistency index derived from a sample of randomly generated reciprocal matrices) .................................................... 110

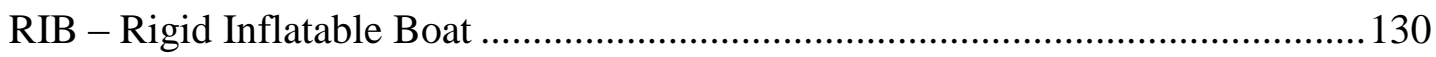

RMMV - Remote Multi-Mission Vehicle (unmanned, autonomous, semi-submersible

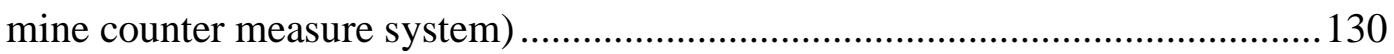

ROA - Real Options Analysis (options evaluation technique used in investment

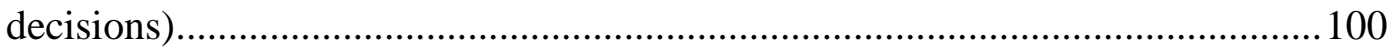

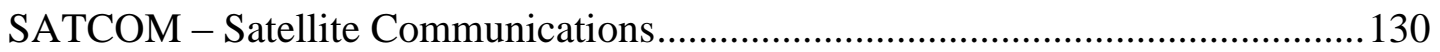

SCOT - Satellite Communications On-board Terminal ......................................... 130

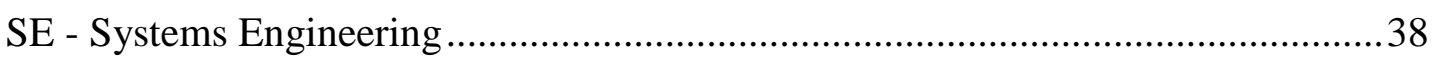

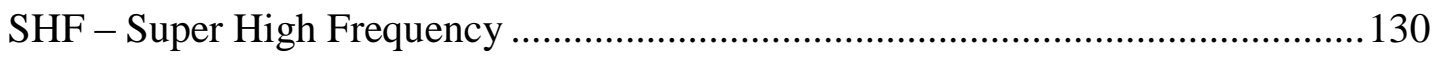

SIGMA - Stabilised Integrated Gun Missile Array (medium calibre gun for short

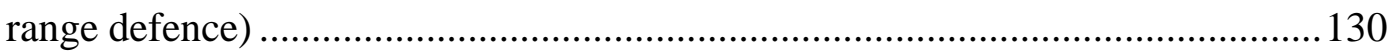

SRD - Systems Requirements Document (UK MoD term for features required to meet

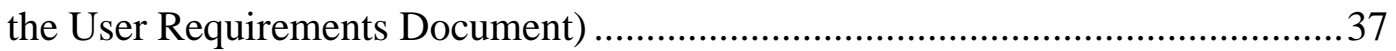

SSTDS - Surface Ship Torpedo Defence System............................................... 130

SVL - Soft Vertical Launch (method to reduce missile launch signature)............... 130

SWATH - Small Water Plane Area Twin Hull .......................................................50

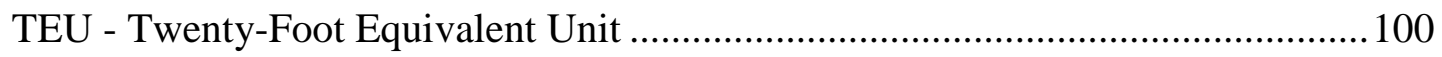

TLBM - Through-Life Business Model (maintenance data management approach) 78

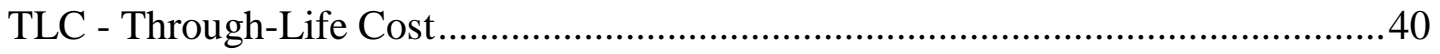

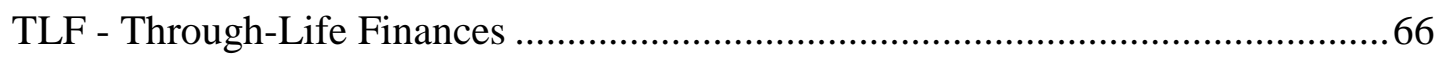

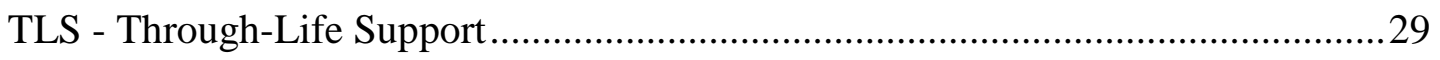

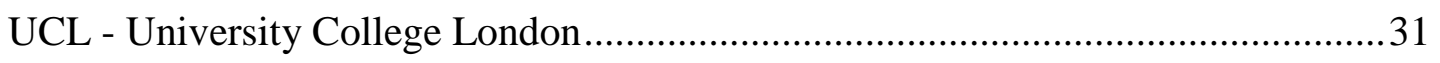

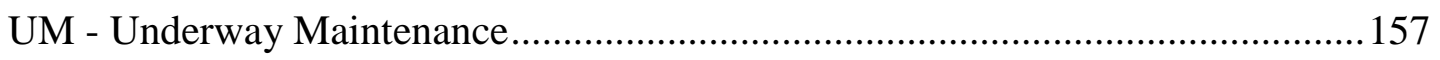

UPC - Unit Procurement Cost (UK MoD term for initial equipment costs)..............40

URD - User Requirements Document (UK MoD term for equipment requirements 37

UxV - Unmanned Vehicle.

VBA - Visual Basic for Applications (Microsoft event-driven programming language)

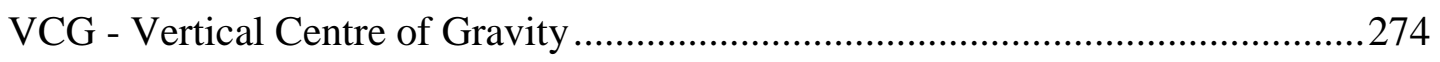

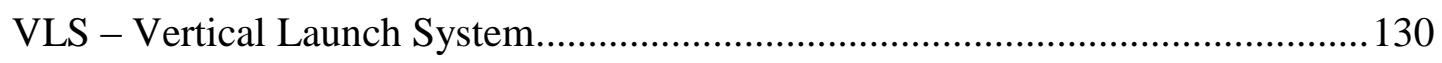

VPS - Variable Payload Ship (naval ship with modularised combat systems).......... 72

VTUAV - Vertical Take-off and Landing Unmanned Aerial Vehicle ..................... 130 
WBS - Weight Breakdown Structure (used to describe ships and associated

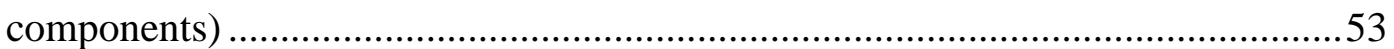

WLC - Whole Life Costs (summation of initial and through-life costs) ....................39

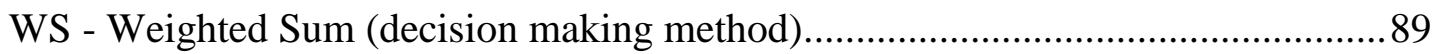

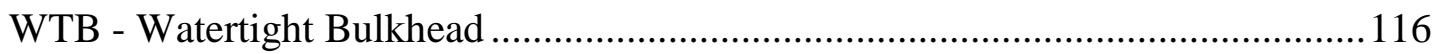




\section{List of Symbols}

$\Delta_{\mathrm{D}}$ - Ship Deep displacement

$\Delta_{\mathrm{L}}$ - Ship Light displacement

$\lambda_{\max }$ - Principal eigenvalue of the reciprocal pairwise comparison matrix

$\nabla$ - Ship volume of displacement

$1+\mathrm{k}_{1}-$ Ship hull form factor

a - Comparison matrix consistency threshold

$\mathrm{B}_{\mathrm{MD}}$ - Ship amidships main deck beam

$\mathrm{B}_{\mathrm{wl}}$ - Ship waterline beam

$\mathrm{C}_{\mathrm{B}}$ - Ship block coefficient

$\mathrm{C}_{\mathrm{M}}$ - Ship midship section coefficient

$\mathrm{C}_{\mathrm{P}}$ - Ship prismatic coefficient

CwP - Ship waterplane area coefficient

D - Ship amidships hull depth

F - Ship forward freeboard

$\mathrm{GM}_{\mathrm{F}}$ - Ship transverse metacentric height with free surface effects

$\mathrm{GM}_{\mathrm{S}}$ - Ship solid transverse metacentric height

GZ - Ship transverse righting lever arm

kts - Knots (unit of speed)

$\mathrm{L}_{\mathrm{oa}}$ - Ship overall length

$\mathrm{L}_{\mathrm{wl}}$ - Ship waterline length

$m$ - Number of machinery spaces on a given ship design

$\mathrm{n}$ - Pairwise comparison matrix dimension

$\mathrm{Nm}$ - Nautical miles

$\mathrm{R}_{\mathrm{A}}$ - Model-ship correlation resistance

$\mathrm{R}_{\mathrm{APP}}$ - Ship appendage resistance

$\mathrm{R}_{\mathrm{B}}$ - Ship additional pressure resistance of the bulbous bow near the water surface

$\mathrm{R}_{\mathrm{F}}$ - Ship frictional resistance according to ITTC-1957 correlation line

$\mathrm{R}_{\text {Total }}$ - Ship total ship resistance

$\mathrm{R}_{\mathrm{TR}}$ - Ship additional pressure resistance of the immersed transform stern

$\mathrm{R}_{\mathrm{W}}$ - Ship wave-making resistance

T - Ship amidships Deep draught 
$V_{G}$ - Ship total enclosed volume

$\mathrm{V}_{\mathrm{s}}$ - Ship superstructure proportion

$\rho$ - Ship overall density 


\section{Chapter 1: Introduction}

\subsection{Preamble}

Supportability is defined by NATO as 'a measure of the degree to which all the resources required to operate and maintain the system/equipment can be provided in sufficient quantity and time' (NATO, 2011). It is a significant aspect of design that is generally facilitated through Integrated Logistics Support (ILS) management and the associated technical procedures and processes. Given that the design decisions made early in the lifecycle have a major influence on design outcomes, then as many of the ILS activities (i.e. the enablers of system/equipment supportability) as possible should be considered early in the design process, albeit at top level. They also need to be considered in parallel with the system's acquisition and engineering design process in order to 'maximise availability, effectiveness, and capability of the system' (NATO, 2011). Failure to consider supportability at the early, formative stages of the design process can lead to longer and more expensive ship overhauls, less availability and reduced scope for adaptability and upgradeability (Coles et al., 2014).

The UK Ministry of Defence (MoD) has endorsed the application of ILS to all product acquisition as official policy (UK MoD, 2013a). However, its genuine implementation in the domain of naval ship design has been impeded by the process of naval ship concept design being historically carried out in the middle of an intense debate to reduce acquisition costs, while attempting to maximise mission capability. This approach has led to a focus on the numerically amenable characteristics, largely addressed in the first four items of the classic ' $\mathrm{S}^{51}$ (Brown and Andrews, 1980) (i.e. Speed, Seakeeping, Stability, (structural) Strength, the fifth being Style (Andrews, 2018)), and in the ship's Combat System's (CS) capabilities, in the application of the governments' budgetary criteria in the early stages of naval ship design. Thus insufficient attention has been given to the full range of capability aspects, particularly Through-Life Support (TLS) features. Consequently, TLS is addressed much later than the initial design synthesis by which time the design has effectively been constrained. In addition, the traditional numerical approach to Early Stage Ship Design (ESSD) (not to be confused with US Navy's definition of Preliminary Design 
described by Gale (2003)) has delayed the early investigation of Style aspects (being the $5^{\text {th }}$ of the $S^{5}$ categories mentioned above) and any architectural modelling (Andrews, 1984) which has only been properly considered in the subsequent design phases and within the confines of a defined hullform produced from a limited numerical balance. This further hinders early considerations of naval ship supportability as many of its constituent aspects, such as operational and through-life Adaptability, Availability, Reliability and Maintainability (ARM), Replenishment At Sea (RAS), and access policy are strongly influenced by the ship's design Style and its overall ship architecture and arrangement.

The shortcomings of both acquisition and design processes are likely to be exacerbated by a) rising warship ownership costs, b) the lack of a numerical and structured TLS evaluation approach that can be utilised during the early stages of design, and c) the loss of the naval ship design expert knowledge base. The rising costs of naval ship programmes (Arena et al., 2006) combined with the lack of a rational supportability assessment method, compatible with the high level design definitions produced in the Concept Phase, are likely to lead to cost cutting of specific programmes by the government. Additionally, given the political significance of defence procurement processes and the duration of such major projects exceeding single governments, they are vulnerable to changes in political direction. These changes could further hinder those aspects conventionally addressed during the later detailed design stages, such as TLS. Considering the issue of expert knowledge; the demand for engineers currently exceeds supply across all UK engineering sectors (Fidler and Harrison, 2013), including naval ship design (UK NEST, 2013), resulting in a reduction in the number of available experienced individuals. This is a major cause for concern given that TLS and other 'softer' aspects of naval ship design have usually been tackled by experienced ship designers and practitioners and then been addressed through detailed engineering applying lessons learnt from operational and maintenance practice. 


\subsection{Research Scope and Aim}

This research undertaken on supportability assessment has been focused on the creative and fluid early stages of the naval ship design process. During ESSD, a large variety of ship design options are investigated with reasonably low design efforts and resources yet having significant impact on the choice of the final design solution. The research did not tackle those aspects affecting supportability that are usually addressed downstream right into the detailed design stages, where most Design for Support (DfS) analyses require high levels of design information and the constrained nature of the design makes corrective design alterations lengthy and expensive.

The financial challenges of defence acquisition and the political tendencies of governments were considered to be outside the scope of this project. Instead the research focused on addressing the drawbacks caused by the traditional naval ESSD process and the lack of numerical and structured TLS evaluation approaches appropriate to the Concept Phase. The importance of ESSD and the advantages of architectural modelling during the Concept Phase were first investigated. The architecturally discernible aspects of naval ship TLS and some of the technical solutions aimed at addressing such issues and their applicability to this research were then explored. This led to identification of the main obstacles to a proper consideration of naval ship TLS in ESSD and the gaps in the current supportability assessment approaches. This was followed by the proposed approach to evaluate supportability in the early stages of naval ship design. The proposed evaluation approach was then demonstrated on a range of concept level ship design cases, namely frigate-sized naval combatants. However, it is considered to have applicability to other types and configurations of surface warships and possible relevance to naval auxiliaries.

The supportability evaluation approach described in this thesis is a combination of an architecturally-orientated ESSD approach, a new ship concept design software developed at University College London (UCL), and the application of some widely established decision making and appraisal techniques. The approach developed possesses four significant features. Firstly, certain supportability aspects amenable to being influenced by high level architecturally-driven early design choices were investigated. Secondly, without relying on overly detailed ship design information it 
addressed TLS in the Concept Phase. Thirdly, the difficulty of quantifying many aspects of naval ship TLS in ESSD was tackled. Fourthly, a framework was presented that captured, incorporated, and applied the accumulated naval ship design and TLS knowledge (e.g. through rules of thumb) in a rational manner.

The validity and applicability of the proposed approach was illustrated through a number of ship design case studies. These focused on: configurational rearrangement of certain supportably features; enhancement of those supportability features; and, finally, an alternative ship design style. The ship designs were developed using the UCL originated architecturally-orientated Design Building Block (DBB) approach to ESSD (Andrews, 1984; Andrews and Dicks, 1997) and detailed sufficiently for the application of the proposed evaluation approach. Due to the sensitive nature of naval ship design, the unclassified UCL naval ship design procedure (UCL, 2013a) and database (UCL, 2013b), that are considered to be representative of the UK MoD design practice, were used to develop the ship designs. Thus classified data for actual ship designs and naval weapon systems as well as classified tools were not utilised.

In summary, the overall aim of the research undertaken and presented in this thesis was to propose a new, quantitative supportability evaluation approach and demonstrate it on a range of ship design alternatives in investigating a limited number of supportability aspects. Crucially, the proposed approach was required to be applicable to the high level design definition of the Concept Phase of naval ship design. It was the intention of this research to utilise the enhanced emphasis on ship architecture and configuration introduced by the UCL architecturally-orientated DBB approach to ESSD. This enables a more effective and earlier consideration of TLS (during the Concept Phase) of naval ship design. 


\subsection{Thesis Structure}

The thesis is composed of seven main chapters accompanied by separate appendices providing additional material relevant to the main text.

Chapter 1 provides a brief introduction to naval ship TLS and the issues surrounding it as well as the scope, aim, and structure of the research project.

The second chapter, consisting of four main sections, presents a state of art review of the relevant background and provides a justification for the proposed supportability evaluation approach. Section 2.2 covers the background to the process of modern naval ship design and procurement, the cost of owning a warship, the traditional approach to early stage naval ship design, and how the UCL originated alternative architecturally-based synthesis approach, made available by the developments in computer graphics, means aspects like supportability can be considered in ESSD. Section 2.3, on naval ship supportability, provides a summary of the wider scope of naval ship supportability and then emphasises the importance of its early consideration in the initial design phase. The architecturally discernible aspects of naval ship TLS are then highlighted as being both feasible and appropriate to be addressed. Some of the technical solutions aimed at addressing the architecturally discernible aspects of naval ship TLS early in design are also covered. Section 2.4 discusses how the feasibility of supportability investigations in ESSD could be improved by reducing the need for overly detailed ship design information. The three sub-sections describe the three different topics that were explored in searching for how this could be achieved, discussing the associated advantages and shortcomings. Section 2.5 highlights the issues identified as the main obstacles to a proper consideration of naval ship TLS in ESSD and describes the gaps in current supportability assessment approaches. This confirms the consequent need for a method to evaluate supportability early in that design process. The chapter is concluded with an outline of the proposed evaluation approach.

Chapter 3 includes three main sections and focuses on the development of the supportability evaluation approach that was proposed as part of the research. Section 3.2 briefly covers the two methods initially investigated for the development of the DfS evaluation approach. Section 3.3 outlines the main proposal and provides 
a general description of the principal constituents of the proposed DfS evaluation approach and the associated issues. Section 3.4 gives a detailed account of how the principal features described in the second section were customised in order to investigate a specific naval ship supportability example.

The fourth chapter, consisting of three main sections, describes the development of the ship design cases that were used to demonstrate the application of the DfS evaluation approach outlined in Chapter 3. Section 4.2 covers the development of a new ship concept design software and the current status of that work at UCL. Second 4.3 describes in detail the Baseline Frigate Design developed as part of this research. Section 4.4 discusses the choice of ship design variations and outlines the resultant designs.

Chapter 5 has two main sections which outline the application of the proposed DfS evaluation approach to the ship design cases described in Chapter 4 and presents the assessment results. Section 5.2 provides a detailed account of the application of the proposed DfS evaluation approach to the assessment of the specific naval ship supportability example outlined at the end of Chapter 3. Section 5.3 presents the assessment results for all the ship design variants. A limited discussion of each set of results is also provided.

The sixth chapter consists of two main sections that provide a comprehensive discussion of DfS in naval ship ESSD. In Section 6.2, the results presented in Chapter 5 are discussed in detail and wider ship design implications are analysed. Section 6.3 lays the foundation for addressing whether the DfS evaluation approach has achieved the overall research aim outlined in Chapter 1. It links the proposed approach to the gaps identified in the research background outlined in Chapter 2, followed by discussing its implementation, before reviewing the limitations in the demonstrations. The chapter is concluded by a section that discusses the areas that require further investigation.

Based on the issues discussed in Chapter 6, the seventh and final chapter addresses whether and to what extent the overall research aim has been achieved. The major issues and limitations identified from the research work as well as the areas of potential future research are also summarised in a suggested order of significance. 


\section{Chapter 2: A Review of the State of the Art in Design for Support for Naval Ships}

\subsection{Introduction}

This chapter, consisting of four main sections, provides an overview of the investigations carried out to identify where there is a knowledge gap in Design for Support (DfS) for naval combatants. It delves into the body of knowledge in the areas of naval ship design and Through-Life Support (TLS) with the aim of identifying questions that are both relevant and feasible in investigating naval ship support early in design.

The next section is split into six sub-sections. The background to the process of naval ship design and procurement through the latter part of the $20^{\text {th }}$ century to date is first outlined, followed by considering the nature of the cost of owning a warship and how it has changed over the same time period. The third and fourth sub-sections further examine the process of naval ship design and cover the traditional approach to early stage naval ship design. The last two sub-sections investigate the advantages of architectural modelling in initial ship design and describe an established architecturally-based design approach as an alternative to both the traditional and other current approaches to Early Stage Ship Design (ESSD).

Section 2.3 is split into three sub-sections. The first is a summary of the scope of naval ship supportability, where the importance of its consideration in the initial design phase is emphasised. The architecturally discernible aspects of naval ship TLS are then highlighted since they are considered both feasible and appropriate to be addressed. The third and final sub-section covers some of the technical solutions aimed at addressing the architecturally discernible aspects of naval ship TLS early in design.

Section 2.4 addresses the need to reduce overly detailed ship design information in improving the feasibility of supportability investigations in ESSD. The three subsections summarise the three different topics that were explored in searching for how this could be achieved, discussing the associated advantages and shortcomings. 
The last section highlights the issues identified as the main obstacles to a proper consideration of naval ship TLS in ESSD and the need for a method to evaluate supportability early in that design process. The chapter concludes with an outline of the proposed approach, explaining why this approach to the problem was selected. 


\subsection{Naval Ship Design}

\subsubsection{Background to the Process of Naval Ship Design and Procurement}

The design and construction of a new naval vessel has usually been initiated in response to either the need to get a new military capability to sea to face new threats, or the need to renew existing capabilities (Andrews, 1987; Brown, 1993). The UK MoD mandates the use of the Smart Acquisition process, illustrated in Figure 2.1, for the design and procurement of defence capabilities (UK MoD, 2002).

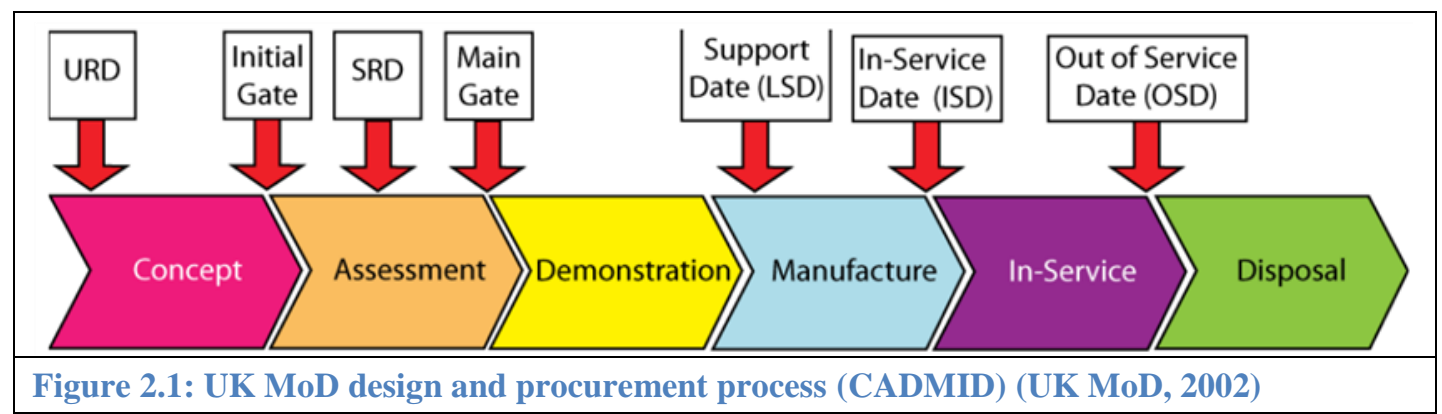

The UK MoD (2002) stated the process to be a whole life approach that begins with the Concept Phase at the end of which the operational requirements organisation issues a User Requirements Document (URD) outlining the need for particular capabilities to be met by a future equipment programme. The aims of the Concept Phase are to identify technology and procurement options that warrant further investigations, obtain funding, and identify the performance, cost and time parameters for the programme. At the end of the Concept Phase, the 'Initial Gate' review is carried out to assess the feasibility of the programme and if approved, release the funds for the Assessment Phase that follows. In the Assessment Phase, after considering each of the primary and secondary tasks separately, a Systems Requirements Document (SRD) is produced to spell out what the system(s) must do to achieve what was specified in the URD. Invitations to Tender (ITT) are issued to the industry and Assessment Phase contracts are subsequently awarded to a short-list of companies or consortia. Feasibility studies and trade-offs between cost, time, performance and risks should occur during this phase to identify the technological solution within the 'Initial Gate' boundaries. The next stage is the main investment decision of the programme called the 'Main Gate' Review, the point at which a preferred procurement option/supplier is generally chosen. The following Demonstration Phase works towards eliminating 
development risks in order to meet the performance targets for manufacture. The process is continued in the Manufacture Phase when production work is carried out to deliver the military capability within the time and cost parameters previously set and as according to the contract that includes build specifications. The In-Service Phase confirms that the military capability provided by the system is available for operational use, to the extent defined at 'Main Gate' and on In-Service Acceptance Date. The process is finalised by the Out of Service Date and Disposal Phase when plans are drawn for an efficient, effective and safe disposal of the system.

Although Smart Acquisition is the official UK MoD policy for the design and procurement of defence capabilities (UK MoD, 2002), the realities of naval ship design and procurement are very different. Andrews (2003a) criticised the Smart Acquisition 'practice of first investigating, in considerable depth and importantly in non-material specific terms, the requirements for a major naval programme,' as a) inappropriate for major warships due to the assumption that such large and physically complex system of systems can be designed in a manner akin to other military vehicles and software-led systems (e.g. military aircrafts, air traffic control systems, and warship Combat Systems (CS)), and b) bad Systems Engineering (SE) practice owing to falsely depicting SE as an appropriate design discipline for complex systems such as warships that don't have prototypes, rather than a broad approach that can be adopted by project teams to achieve best practice project management.

The naval ship design process and final design solution are largely based on the designers' interpretation of the often immature requirements (Andrews, 1985), hence informed discussion and dialogue between the requirements owner (UK MoD as the operational requirements organisation for naval vessels) and the procurement community are essential during the Concept Phase. The dialogue is vital in order to determine what is realistically achievable before the requirements are fixed (Andrews, 1992). In order to strike a balance between affordability and capability and achieve a realistic design solution (Heather, 1990; Crow, 2001), the requirements owner (i.e. the operational requirements organisation) needs, during the Concept Phase, to explore and with the nascent procurement project make several top level principal choices as part of the joint requirements elucidation process (Andrews, 2003a, 2013). Hawke (1988) listed some of these strategic choices for a warship, concerning different types of policies and requirements: 
- Specialised or general role and mission orientation;

- Short or long planned lifetime with subsequent implications on modernisation requirements;

- Cash limited or flexible cost policy;

- Endurance and deployment length;

- Degree of self-sufficiency with implications on ship's manning and demand for spare parts.

However, 'Requirements Engineering' such as Smart Acquisition is unable to facilitate proper dialogues between the requirements owner and the 'procurement project' to reach a balanced and affordable set of requirements for complex systems such as modern naval combatants (Andrews, 2003a). The consequence of this failure to carry out an effective requirements elucidation is a mismatch between design requirements and solutions (Andrews, 2003a). The Smart Acquisition process shown in Figure 2.1 is considered to be inappropriate to naval ship design because:

- It fails to ensure that top level requirements are fixed without full material options exploration by the end of the Concept Phase before continuing to the Assessment Phase where feasibility studies on a balanced preferred option are undertaken;

- It disregards the fact that trade-off analyses should be carried out as part of the Concept Phase and feasibility studies are essentially about working up the design to show that is technically affordable and achievable;

- Classic Demonstration of the overall ship before design to build and commencing Manufacture is not possible due to the lack of prototypes.

\subsubsection{The Cost of Owning a Warship}

Ship costing is a critical part of the concept design process for both naval and commercial ships (UK MoD, 2002) as it clearly determines the feasibility and costeffectiveness of a ship programme. It should also determine whether there are sufficient funds available to take the programme forward (NATO, 2009; Caprace, 2010), and ought to avoid budget overruns and the associated consequences (Gerdemann et al., 2012; Rudius, 2012). NATO (2009) described the total cost of ownership over the life of a warship, the Whole Life Costs (WLC) (also termed Life 
Cycle Cost (LCC) in some sources), as one of the key criteria (others being operational need and government constraints) that could assist the decision makers in deciding between alternative procurement options. WLC represents all the costs that will be expended during the life of a system (including the main system and support systems) to acquire, operate, support it and eventually dispose of it (NATO, 2003).

The two principal constituents of WLC are Unit Procurement Cost (UPC) and Through-Life Cost (TLC). UPC includes initial cost elements, such as equipment and material acquisition; shipbuilder labour costs; outfit and assembly; allowances for design changes; and test and trials costs. TLC consists of the costs incurred during the warship's in-service period, such as personnel (basic pay, national income contributions, pensions and gratuities, additional special service pay), training, consumables (e.g. fuel, stores and spares), maintenance (further divisible into; general maintenance, docking, minor refit and major modernisation) and disposal costs. There is an additional cost element, termed as the First of Class (FOC) cost that can be significant and is paid for separately to the lead shipbuilder or prime contractor and not proportioned out amongst ships in the class subsequent to the FOC. FOC costs include shipbuilder's office setup; design and project resources; drawings; recruitment; model productions; shore test facilities; and first ship trials (Brown and Andrews, 1980; Page, 2011; Piperakis, 2014). Figure 2.2 shows the general breakdown of warship WLC for a typical example of 1980s. Figure 2.3 illustrates an undiscounted WLC of a typical 3,000 tonne frigate built at a 1971 UPC of £12M. Inflation would probably lead to a value of approximately $£ 150 \mathrm{M}$ in 2018 but the WLC breakdown is considered to be still broadly applicable. The noticeably low weapons design cost in Figure 2.3 could be due to the less sophisticated and evolutionary nature of CS developments in the 1970s and clearly excludes any major new weapon development, such as the Principal Anti Air Missile System (PAMMS) for the Royal Navy's Type 45 Destroyers (Gates, 2014). 

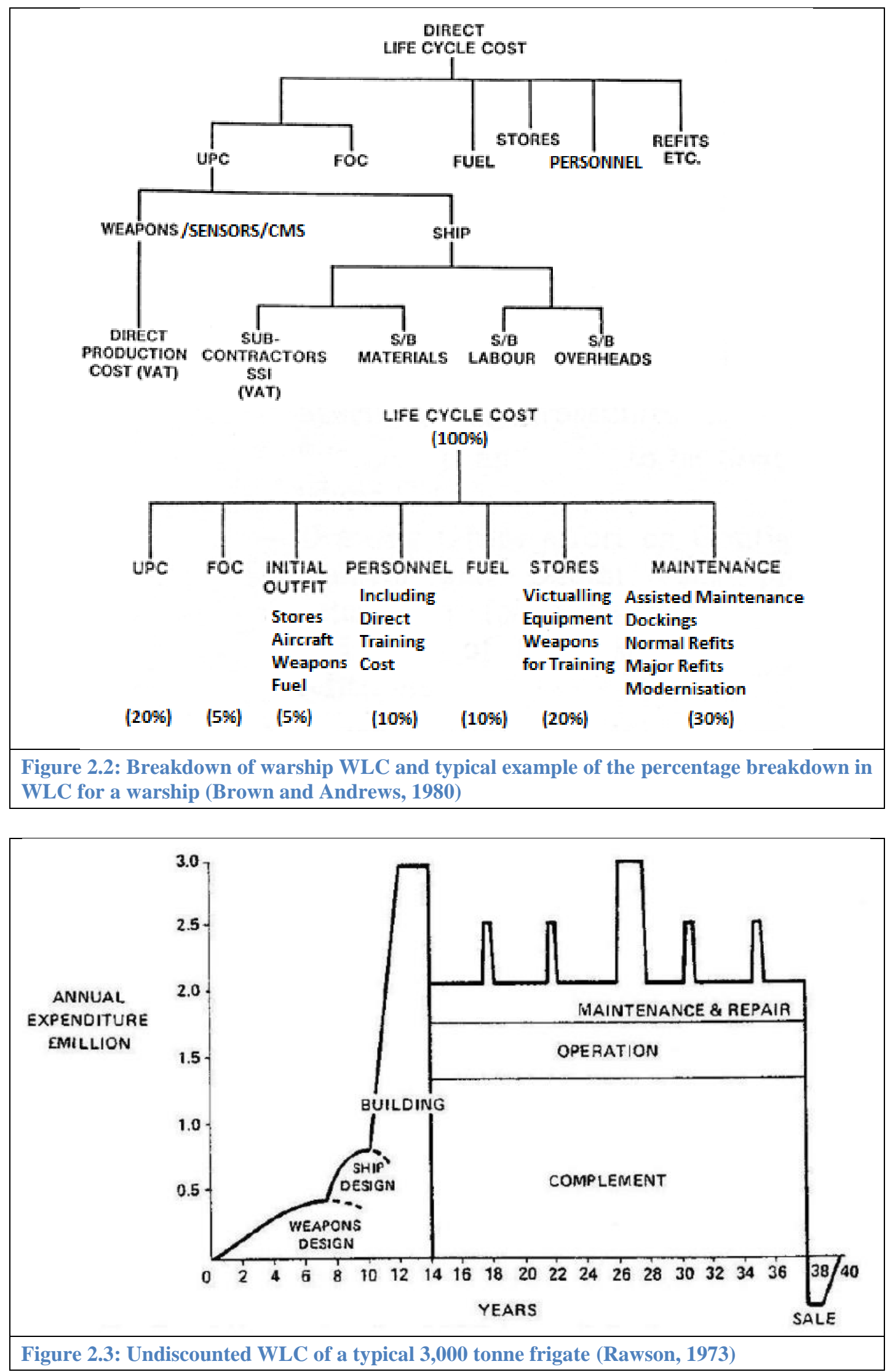
Considering the balance of affordability and capability that was mentioned in Section 2.2.1, Brown (1986) explained how the policy of military equipment procurement transformed the objectives in naval ship design during the $20^{\text {th }}$ century. At about the middle of the Cold War, the aim to obtain the technically best ship was first abandoned in favour of purchasing the most cost-effective ship; that was itself replaced by the current cash limited warship procurement policy. This has been caused by both the reduction in defence expenditure (as percentage of GDP) and the growth above inflation in the UPC of warships identified as approximately $9 \%$ per annum (Gates, 2005; Arena et al., 2006). Warship cost escalation has been attributed to two principal factors; economy-driven and customer driven. The economy-driven factors (e.g. material, labour, and equipment) have generally been comparable to the general inflation rate and amounted to around half the overall escalation. The customer-driven factors (e.g. complexity, standards and requirements, and declining procurement rates over which overheads are amortised) make up the other half (Arena et al., 2006). Figure 2.4 illustrates the breakdown of annual cost escalation factors resulting from a pairwise comparison between the US Navy guided missile destroyers DDG-2 (FY 1961) and DDG-51 (FY 2002).

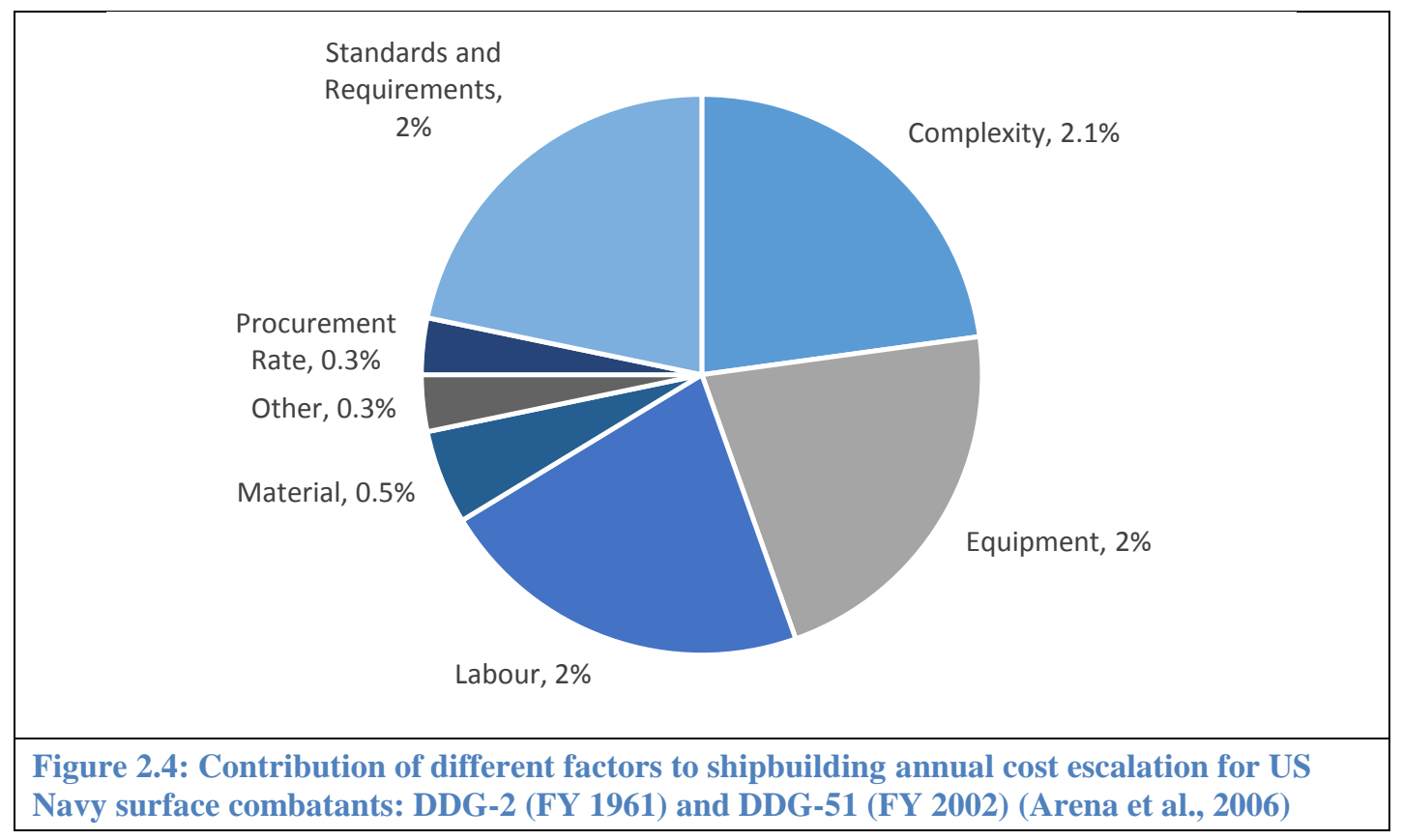

It can be seen that the most significant contributions in Figure 2.4 are due to the rising costs of equipment, labour, complexity and standards. These have been confirmed by Brown and Andrews (1980) and Brown and Tupper (1988) for UK naval acquisitions. 
In addition to the better reliability and availability of individual items of equipment and vessels (Alexander, 1988), the decline of defence budgets that begun at the end of the Cold War (Andrews and Hall, 1995), coupled with the above inflation escalation in UPC for comparable naval combatants have resulted in an overall reduction in fleet size for most major western navies post-Cold War (Taylor, 2010) despite little reduction in overall commitments (Collins et al., 2012). The consequence has been greater capability (Jones and Kimber, 2012) and reliability (Manley, 2012) required per ship. Although there are no easy or simple solutions to tackle this, a number of approaches have been suggested, most of which involve some level of compromise. These proposals cover the areas of naval ship design, programme management and acquisition strategy, and manufacturing and shipbuilding technologies. The examples given below are proposed strategies aimed at tackling the rising cost of naval ship acquisition.

- Build a mix of multirole ships with a) limited capability in each role and b) mission-focused ships capable of only one, or very few, roles but of first class quality in the selected role (Brown and Andrews, 1980; Arena et al., 2006);

- Implement the concepts of Open System Architecture (OSA) (Vasilakos et al., 2000), modularity and the separation of the mission and weapons systems from the rest of the ship (Drewry and Jons, 1975) and modular approaches, such as the late 1970s UK proposal of cellularity, that proposed to improve access and interchangeability for CS equipment during build and refit and physical compatibility through common transport and installation envelopes (Gates, 1985);

- Adopt a larger proportion commercial equipment and design standards (Brown and Andrews, 1980). This was seen to be especially attractive for noncombatants, such as auxiliary ships (Cooper et al., 2007) and amphibious warfare vessels (e.g. HMS Ocean (Allison, 2015)). However this can result in different and potentially unacceptable and risky survivability capabilities but be easier to build and less expensive initially (Arena et al., 2006) if questionable value for money (Andrews, 2018);

- Technical innovation (Andrews and Brown, 1982) and complement reduction (Brown and Andrews, 1980). 
It follows from all the above examples, that could be adopted due to economic reasons, that they have significant implications on the resulting ship design and can compromise the ship's, and in turn the fleet's, capability.

NATO considers the early stages of a programme (i.e. Concept Phase) as the best opportunity to achieve potential WLC reductions (NATO, 2009). This is supported by Rawson (1973) and Andrews (1987) since by the time the design is finalised, most of a ship design's upkeep characteristics and hence that element of WLC/LCC have been committed and are not recoverable, but the expenditure of design resources in terms of time and finance is relatively small, as illustrated in Figure 2.5.

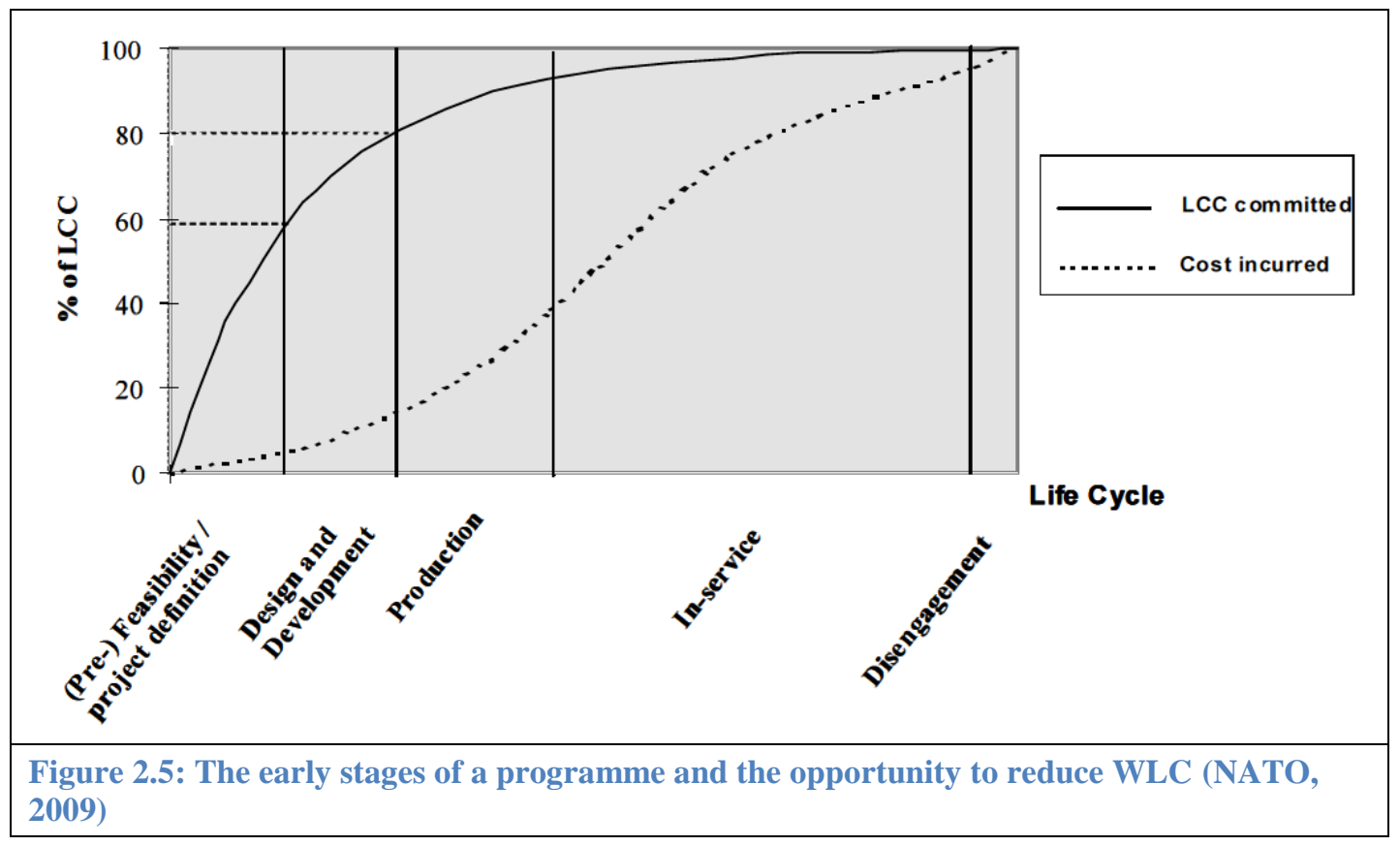

Estimating WLC during concept design allows the cost effects of varying the principal characteristics to be investigated while the design is still flexible (Carreyette, 1978). It also enables alternative solutions, cost reduction opportunities, and aspects of financial risk and uncertainty to be evaluated (NATO, 2009), as well as trade-off studies to be carried out (Rawson, 1973).

Despite the need to investigate cost-effectiveness in its entirety, cost reduction approaches have historically been aimed at reducing the UPC rather than the TLC of the warship (Brown and Andrews, 1980). Although the initial costs can be reduced, these approaches are unable to address the fact that the majority of a warship's WLC is incurred during the in-service period (i.e. the TLC component) (Depetro and Hoey, 
2011; Page, 2011). Brown and Andrews (1980) provided a typical example of a warship's WLC breakdown that shows a 1:3 ratio between UPC and TLC. The large difference in UPC and TLC is also illustrated in Figure 2.6 (TLC referred to as Operations and Support (O\&S) costs in this case).

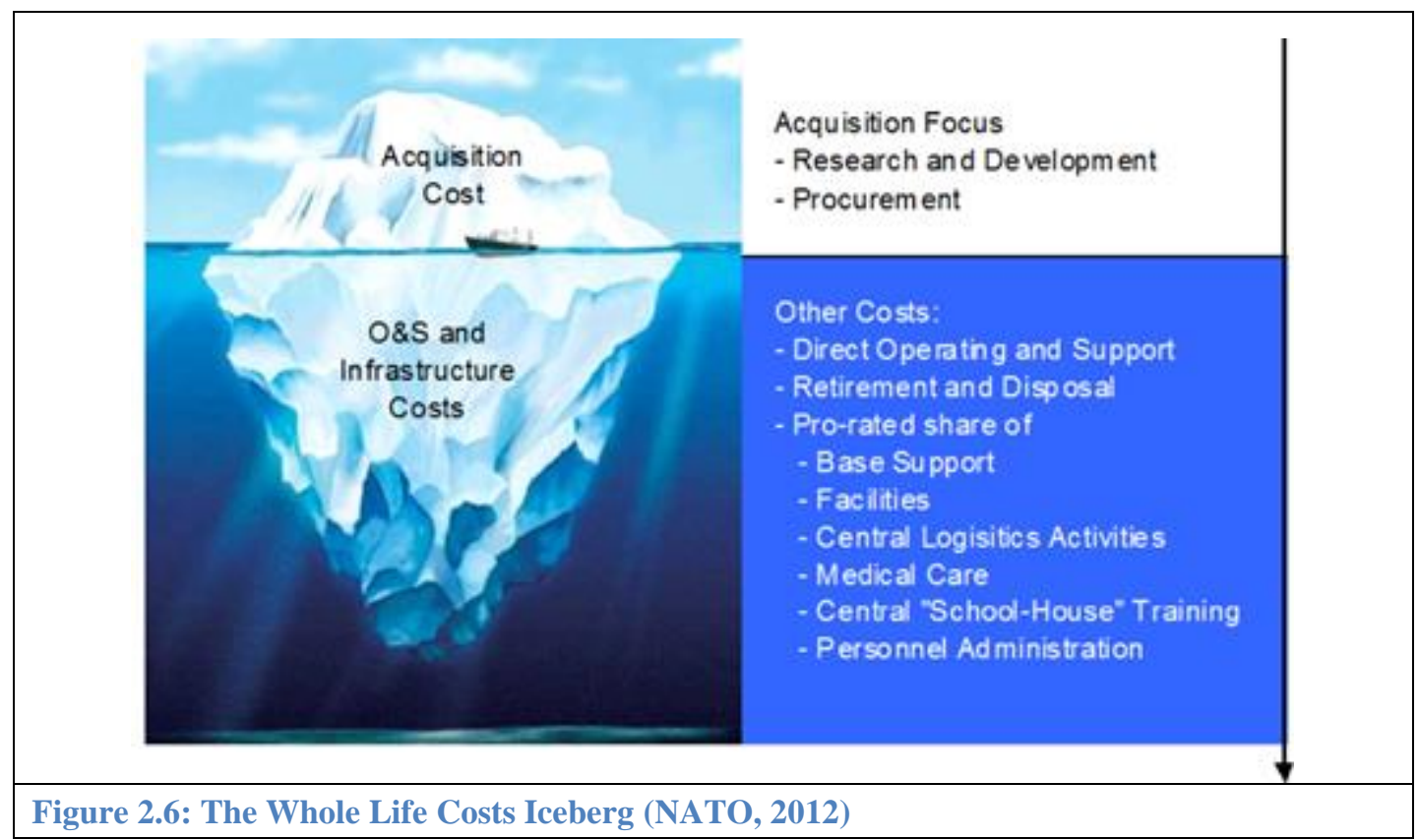

The WLC iceberg (Figure 2.6) illustrates the historical propensity of governments to focus on aspects that they consider to be more important to how their term in office will be judged, as well as the tendency of treasuries to massively discount early years' expenditure. Rizzo (2011) attributes this conduct to the intense political, media and leadership attention on acquisition that shifts the focus towards short-term schedule, budget, and specifications, thus leaving the designer with limited options should complications arise. Consequently, a lower priority is given to through-life aspects that leads to inadequate through-life sustainability and supportability. Historically, procurement decision making has attached higher real values to money spent in the near term, compared with money to be spent in the distant future, referred to as the time preference concept (Andrews and Brown, 1982). Figure 2.7 is a sample temporal illustration of a warship's WLC, showing how navies spend much more through-life than in procurement during a programme's lifespan of more than 40 years (Page, 2011). Figure 2.7 emphasises the cost-effectiveness of incorporating DfS features during ESSD in comparison to at build or even in-service. 


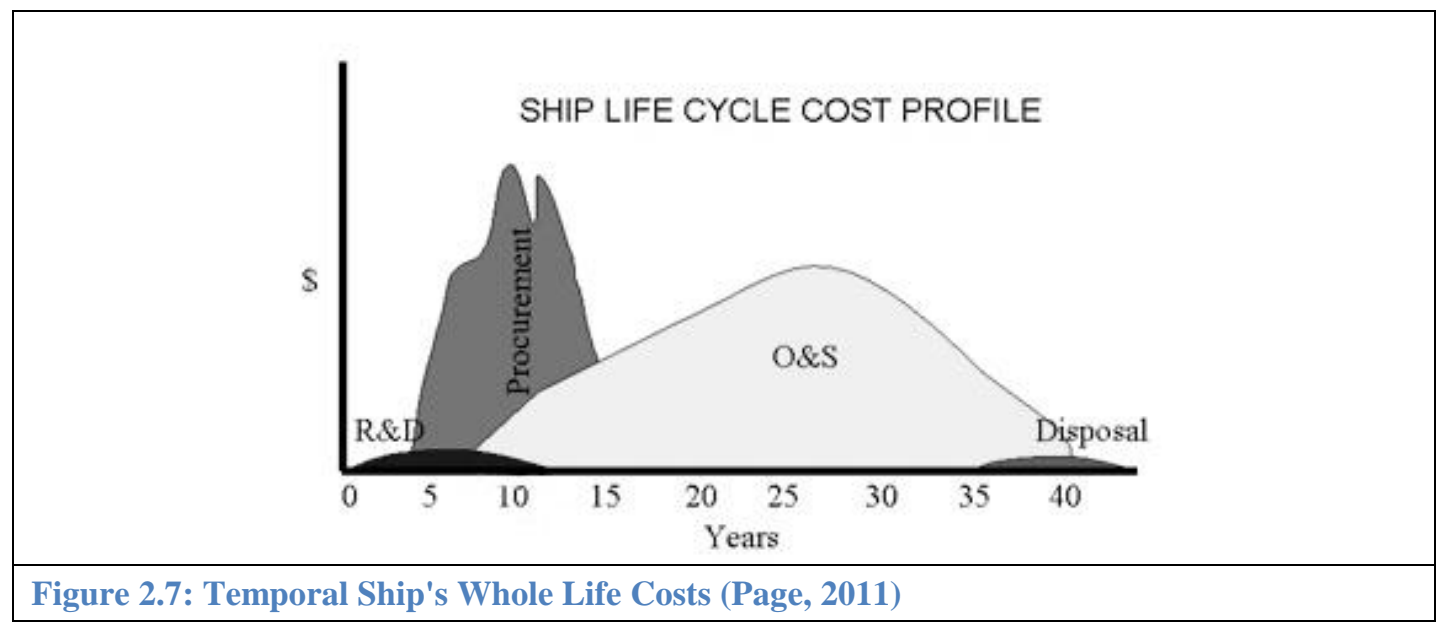

However, this time preference has three major flaws. First, it will distort Figure 2.7 into a 'perceived' (i.e. the customer's perception of cost relative to income) WLC. Second is that it overlooks the fact that the majority of crucial decisions are made during Early Stage Design (ESD) when most of the cost is incorporated in the design despite little of the design effort having been expended, especially for large complex systems of systems like warships (Andrews, 2013). Consequently, should design mistakes and shortcomings be revealed as the design moves into the more detailed phases and more specific design information becomes available, it becomes very expensive to rectify in these later design phases (Page, 2011; Andrews, 2013). The third flaw is the inapplicability of the time preference concept to defence investments, given that most defence benefits cannot be easily quantified (Andrews and Brown, 1982). Unlike commercial freight shipping, where the benefits of possessing a container ship or an oil tanker are easily quantifiable in cash terms, it is not so in the naval domain where certain capabilities are intrinsically difficult to quantify, whether this be a supportability feature, an element of the ship's survivability, or smaller capability subsets, such as the sea boats. In addition, although the full range of the ship's capabilities are crucial to its overall military value, in reality not all capabilities are continuously utilised, or possibly ever in the case of all out warfare, hence further complicating any application of cost-effectiveness decision making.

In summary, given the escalating cost of warships and the socio-technical factors involved in naval ship design and its procurement process, aspects such as naval ship supportability can be seen as attractive areas for cost reductions. However, there is a danger of short-sighted cuts in long-term vital investment of defence capability that are then imposed rather than arising from a well-engineered cost saving. This short- 
termism can then result in either increasing the overall WLC or at best failing to produce any real reductions. For example, the Royal Navy Type 42 Destroyers were designed and built for minimum UPC and as a result were difficult and expensive to refit and update (Friedman, 2006).

\subsubsection{Introduction to Naval Ship Design}

Archer (1979) argued that the process of design should be treated as distinctive but on a par with the other two areas of human knowledge; science and humanities. He showed the interrelationships between these three areas by means of the diagram shown in Figure 2.8.

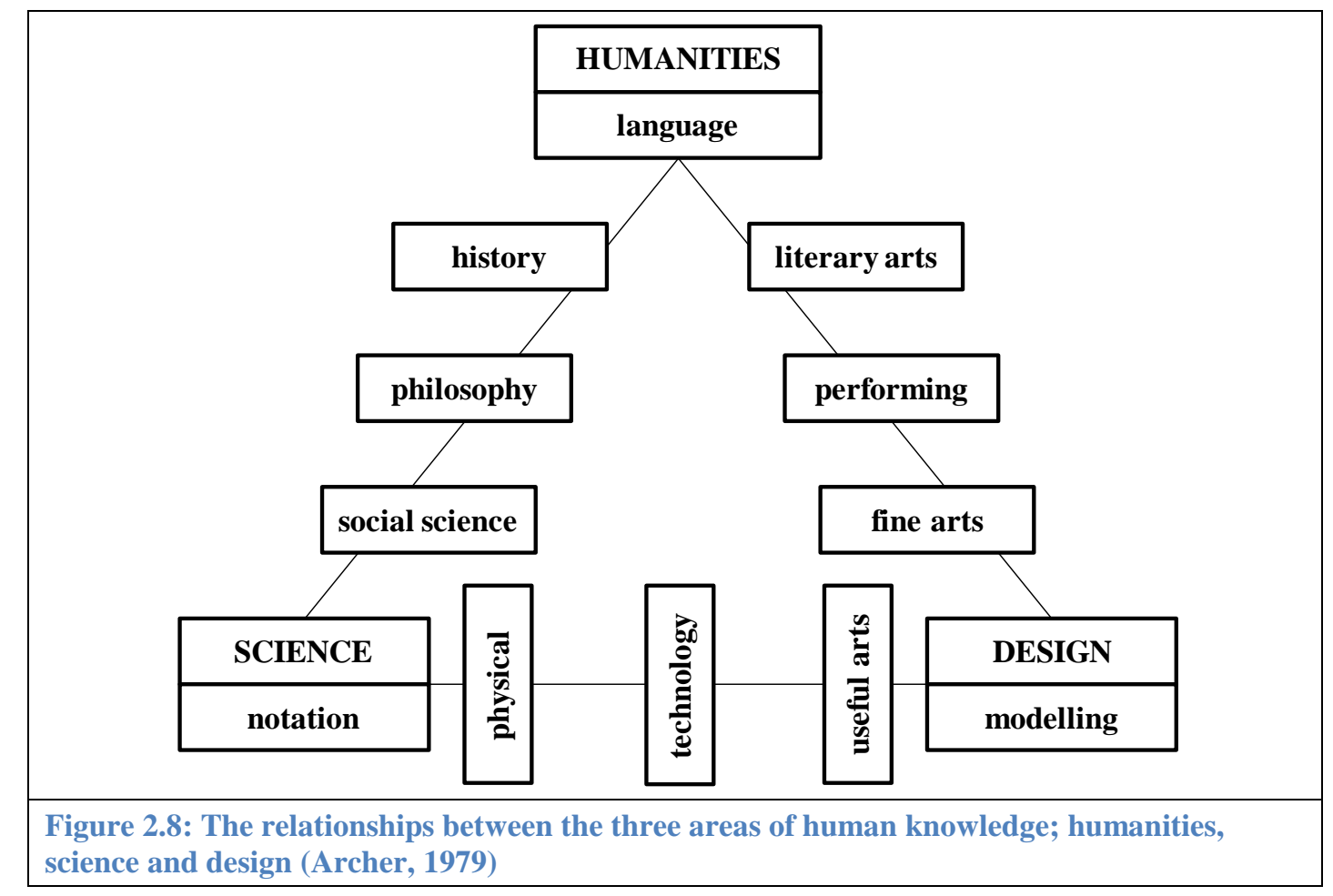

Andrews (1981) highlighted synthesis (i.e. the act of putting the pieces together in a new way (Jones, 1970)) as the distinctive element that distinguishes design from other human activities. As a part of synthesis, Andrews $(1981,1984,1985)$ emphasised the architectural element of ship design. He proposed an architectural approach to ESSD as a way of dealing with the fact that as a process, it is impossible to describe ship design as a purely scientific or engineering science endeavour, given the many disparate design style issues involved (Andrews, 2018). Similarly, Gale (2003) emphasised the nature of the ship design process, both scientifically and artistically, by describing it as the activity involved in producing the drawings (or 3-D computer 
models), specifications and other data needed to construct an object, in this case a ship. The ship design process can be considered to be affected by not just the type of ship being designed, but also the personal preferences of those in the design team (Andrews, 1985, 2012; Gale, 2003).

The act of 'putting it all together' or synthesis of the inputs from all specialist disciplines to create the total fighting unit, as well as the identification of the overall design style to be adopted; essentially constitute the role of the naval architect in the ship design process (Brown and Tupper, 1988). The naval ship design process is characterised, especially at the early phases, by the 'wicked' nature of the process of setting up and constraining the solution space to find the achievable and affordable requirement. First coined by Rittel and Webber (1973) for urban planning and large scale architecture, the term 'wicked problem' was then suggested as appropriate to complex ship design, since 'identifying what is the nature of the problem is the main problem' (Andrews, 2011, 2013). The iterative nature of the process has been attributed to the complex nature of ship design that has so far proven impossible to be described by a set of directly solvable equations (Gale, 2003). To illustrate how the naval architect deals with the various aspect of ship design sequentially and iteratively, the first design spiral (Figure A1. 1, Appendix 1) was produced by Evans (1959) for ship structural design and many other versions have since been developed (e.g. Figure A1. 2 and Figure A1. 3, Appendix 1). Andrews et al. (2009) provided a comprehensive set of different versions of the ship design spiral and Andrews et al. (2012) a critique of the ship design spiral. The spiral as a way representing the ship design process has received a number of criticisms, such as:

- It depicts the ship design process as a closed, sequential series of steps while the process is in fact neither closed nor sequential. The ship design process consists of externally imposed constraints and the inputs from outside the ship design team are numerous, diverse, and unpredictable. It consists of interacting closed loops and intuitive leaps by the naval architect from one spot to another in the spiral as new knowledge is gained, problems are encountered and design drivers are identified (Brown, 1986; Watson, 1998; Gale, 2003; Andrews et al., 2012);

- Performance functions are nonlinear and often discontinuous, and inequalities are more common than equations (Brown, 1986); 
- The design spiral describes individual action steps rather than the fundamental decision making in the ship design process itself (Andrews et al., 2012; Andrews, 2013);

- The design spiral is insensitive to ship type and programme drivers (Watson, 1998; Pawling et al., 2017b).

Among all ship types, warships, for various reasons, have been described as the most complex, diverse and highly integrated of any engineering systems (Graham (US Navy Commander) quoted by Gates and Rusling (1982)). Described as being distinctively large and complex (Andrews and Dicks, 1997), many aspects of a warship's performance are difficult to quantify (Brown and Tupper, 1988). They are designed to be effective in a variety of roles (Brown and Tupper, 1988) and operate globally at various levels of hostilities (Gates, 2005) while providing an appealing environment for the crew and as Graham put it, 'serve two hundred breakfasts' (Brown and Tupper, 1988). The design of warships also involves certain areas of high risks, the main areas of which were outlined by Heather (1990) in his study of fast (small) naval ships:

- Bottom-up estimation of weight in novel designs where a decent first prediction requires enormous amounts of effort;

- Auxiliary power generation requirements that are difficult to predict;

- Internal arrangement of key spaces, such as the bridge and Operations Room;

- The concurrent development of complex weapons and software items that are often delayed or don't perform as required, that particularly applies to the ship's Combat Information Centre (CIC) (i.e. Operations Room) and Combat Management System (CMS), pulling the elements of the CS together.

Gale (2003) considers both design complexity and risks to be directly proportional to design novelty, and there has been suggestions that as a rule of thumb, a new design should have $25 \%$ novelty and $75 \%$ well-tried practice (Baker, 1958), though Brown (1986) argues that since this would imply a total change only every four classes, there may be a case for greater novelty despite the risk implied. Andrews (1985) outlined the range of design processes in ascending order of novelty of the design solution: stretch of an existing ship; type-ship design (proven data base but restrictive); evolutionary (marginally less restrictive than type-ship design); historical (a compromise between good and bad, relevant and inappropriate previous designs); 
simple numerically based synthesis (restricted to reasonably conventional configuration solutions); broader synthesis (an extension of the simple synthesis by integrating an architectural synthesis); radical configuration (current technology adopted in totally new configuration, e.g. Small Water Plane Area Twin Hull (SWATH)); and radical technology (necessary to employ full scale research and development prototypes like the aerospace industry). In reality, the ship designer's task is a very complicated one, due to the variety of often conflicting objectives to be met with limited budgets (Brown and Tupper, 1988; Gale, 2003). Similar to civil engineering, the task is only made more difficult by the absence of complete prototypes due to time, cost and size factors (Brown and Tupper, 1988), the small product numbers in comparison to other industries like the aerospace and automotive (Andrews, 1998), and the lack of new production lines specific to a new design in the shipbuilding industry (Andrews, 2012).

Figure 2.9 is the illustration published by Andrews (1992) to describe the various phases of the naval ship design process prior to the Smart Acquisition process.

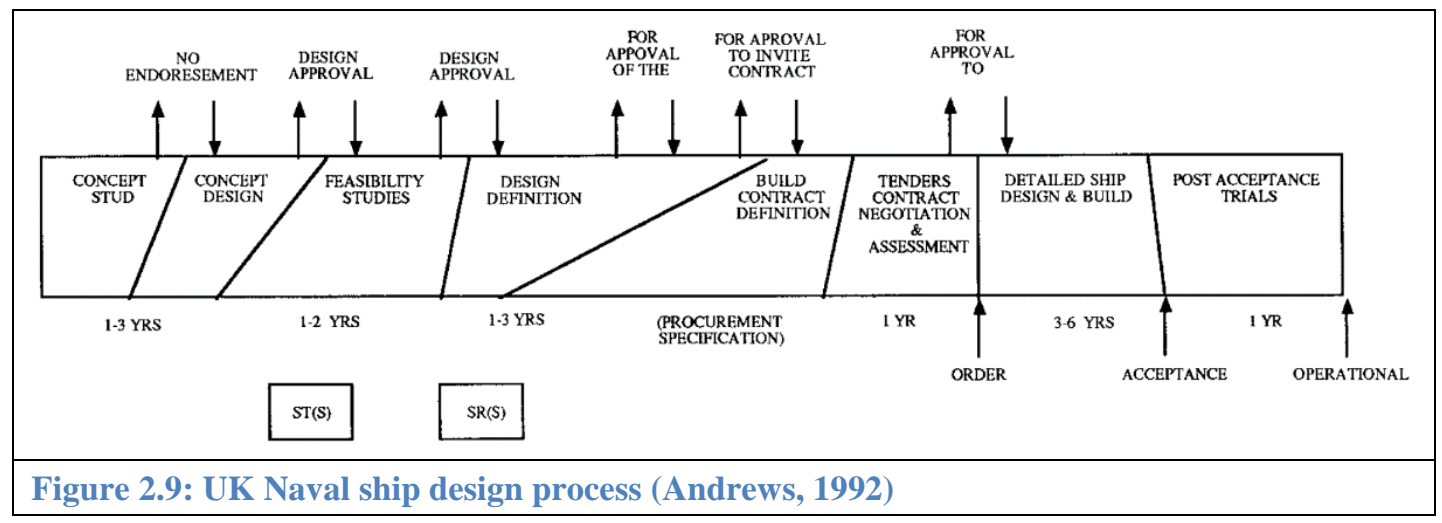

Brown (1986) described the Concept Phase as the only time to introduce genuine design novelty. During the Concept Phase, many ideas are explored by the designers in an attempt to understand the customer's needs through dialogue and convert them into a technical solution (Brown, 1986; Gale, 2003; Andrews, 2011). Andrews (1993) loosely divided the Concept Phase into three overlapping stages; Concept Exploration, Concept Studies and Concept Design, through which alternatives are explored and promising ideas are developed in sufficient details to enable realistic estimates to be made of size, cost and capability (Brown, 1986; Andrews and Pawling, 2007). Although the Concept Phase is when major decisions are made and trade-off studies carried out on requirements and affordability (Brown and Andrews, 1980; Andrews 
and Pawling, 2009), thus effectively committing the majority of future costs, the cost of this design effort in terms of time and resources is only a tiny fraction in comparison to that of the entire project (Andrews, 1987). Brown and Andrews (1980) described the Concept Phase of a typical naval combatant to require six people for six months as opposed to hundreds of people for the much longer later phases of the project (although in recent naval programmes getting through Initial Gate can be tortuous, e.g. nearly two decades in case of the Future Surface Combatant (FSC)/Type 26).

Such a large contrast makes it much more sensible to identify and tackle problematic issues and change the design, if required, during the Concept Phase (Heather, 1990) (Gale, 2003) as the cost of rectifying problems during the later design phases is disproportionately higher (Goossens, 1992). This means that should cost reductions become necessary in the detailed phases of the design, they are more likely to be implemented through omission of equipment rather than radical design rework (Andrews and Brown, 1982).

In conclusion, it can be said that the early phases of design, concept in particular, are the most important as the adopted design decisions effectively 'lock up' the corresponding design and cost aspects (Page, 2011). This was encapsulated by Andrews et al. (2006) as 'the often quoted truism that $90 \%$ of the major design decisions have been made when less than $10 \%$ of the design effort has been expended.'

\subsubsection{Traditional Early Stage Naval Ship Design}

Brown and Andrews (1980) listed the traditional subjects of naval architecture as the ' $\mathrm{S}$ ' - Speed, Seakeeping, Stability, (structural) Strength and Style. These were described as being 'like the sea, in essence unchanging.' Naval architects have focused on the first four characteristics $\left(\mathrm{S}^{4}\right)$ that although diverse, are considered as essentially definable in numerical terms and described as direct performance requirements. On the other hand, Style related issues, defined as cross-cutting ship design information (Pawling et al., 2013) have at best been examined at later stages (Andrews, 1984). Speed and Seakeeping requirements are largely determined through conducting an Operational Analysis (OA) in pre-defined operational scenarios. The results from these trade-off analyses also lead to the selection of CS characteristics. On the other hand, Stability and (structural) Strength are assessed separately from the OA by 
adopting the appropriate naval standards that have to be met and are unlikely to be subjected to trade-offs or negotiable. Table A1. 1 (Appendix 1) is a taxonomy of $\mathrm{S}^{4}$ plus CS characteristics, illustrating relevant ship design aspects to consider and corresponding features.

Similar to the design spirals shown in Appendix 1, the traditional design process is considered to consist of discrete sequential steps and feedbacks (Andrews, 1985; Andrews and Dicks, 1997), as shown in Figure 2.10.

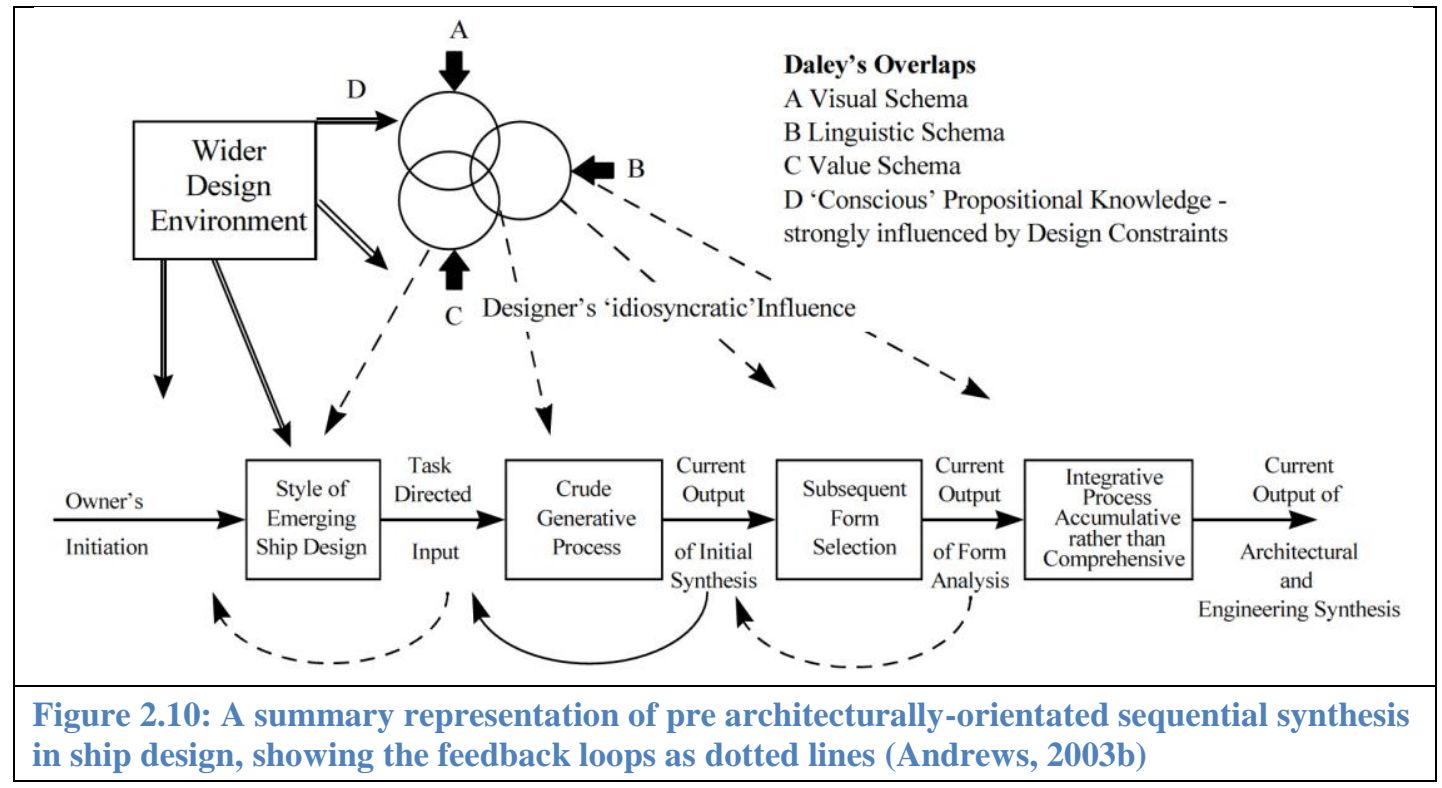

In the first iteration of the design process, the initial sizing has classically relied heavily on extensive amounts of existing ship data and scaling ratios to produce the first estimates of weight and space requirements and costs (Brown and Andrews, 1980). Andrews (1985) produced Figure A1. 4 (Appendix 1) to illustrate a simplified model of initial ship sizing purely as a mechanistic process and emphasise the assumptions implicit in such a design routine and the implied specific sources of data. This crude initial sizing can then be followed by the parametric survey to refine the main hull dimensions and underwater coefficients which, traditionally, has been mostly concerned with balancing stability and powering. Such a process only moves into the development of ship layouts once parametric survey is finalised (Andrews, 1985, 1987).

Several disadvantages have been attributed to this traditional ship design process. It does not represent a fully integrated design process, limits design creativity and innovation and restricts the application of computers to the numerically-based design 
steps only (i.e. initial sizing and parametric survey) (Andrews, 1985, 2003b). The sequential nature of the process results in having to develop the ship architecture and layout within the confines of a hullform already defined and fixed through initial sizing and parametric survey (Andrews, 1981), thus limiting the scope for design creativity and innovation or even ensuring that vital, architecturally dependent capabilities can be met within the envelope (Andrews, 1985). Architectural development is also constrained by naval standards that must be met regardless of other issues (e.g. transverse bulkhead locations determined by damage stability and zoning requirements) (Brown, 1987; Andrews, 2003b). The development of issues related to crewing, ship operations and personnel face the same restrictions and, through such a sequence as Figure 2.10, can only be investigated with the design already constrained. This can lead to operational inefficiencies and potentially hazardous environments onboard (Andrews, 2006), with corrective design alterations being lengthy and expensive at such design stages and likely to be compromised (Andrews et al., 2009).

Another weakness of the non-architectural approach is the purely numerical nature of initial sizing and parametric survey (Andrews and Dicks, 1997), leading to extensive reliance in the process on existing ship data (Gates, 2005) and the designer's ability to develop suitable scaling ratios (Cooper et al., 2007). Also, any numerically-based parametric survey will fail to take into account the architectural element of ship design (Andrews, 1985). Further shortcomings are caused by the group Weight Breakdown Structure (WBS) traditionally used by the process to describe ships and associated components (Garzke and Kerr, 1985). The UK MoD (2010) standard has the following eight weight groups: 1) hull and superstructure; 2) propulsion; 3) electrical; 4) control and communication; 5) ancillary systems; 6) outfit and furnishing; 7) armament; and 8) variable load. While this classification allows comparisons between a new ship design and existing ships to check design completeness (Andrews, 2003b), it is aimed towards how the ship manufacturing industry undertakes shipbuilding (Garzke and Kerr, 1985; Andrews and Pawling, 2009) and its particular use for ship costing (UK $\mathrm{MoD}, 2010)$ can be seen as an inhibitor with regards to design creativity and novel solutions (Andrews, 2003b). As a completeness check, it can result in avoiding innovative options and the subsequent adoption of design solutions excessively based on previous designs that are possibly no longer appropriate (Andrews, 2003b). Also, the weight breakdown description is not suitable for examining the overall effects on 
the ship from adding or removing certain capabilities that require a more architecturally-based definition (Andrews and Dicks, 1997).

In conclusion, Andrews (1985) argued that the traditional ship synthesis is neither comprehensive nor integrative but at best progressive, underlining the scalar description of the initial design as contributory to the failure of this process to be able to comprehend many of the important design Style issues (e.g. a gross measure of space is unable to address aspects such as accessibility). He stated that, since forward momentum and the pressure to maintain an agreed schedule is inevitable in development of an actual design project, the feedback loops in Figure 2.10 are somewhat optimistic. Thus there is a reluctance with a traditional ship synthesis to exploit this desirable iterative characteristic. While innovations in design development and design approach are seen to be necessary to respond to the pressures of diminishing budgets and loss of expert knowledge (Brown, 1993), the traditional approach to ESSD would seem to inhibit creativity and the full exploration of alternatives, resulting in most new designs being based on existing vessels for risk reduction reasons (Andrews, 2003b). However, the rapid development of computer graphics since the late 1990s has enabled a fully integrative ship design synthesis where style and architectural factors can be incorporated into the design decision making process from the very start (Andrews, 2003b).

\subsubsection{Architectural Modelling in Early Stage Naval Ship Design}

Brown (1987) stated that in naval ship design, the architecture often does not receive the attention that it deserves since aspects like ship hydrodynamics and internal partitioning (for damage stability) are prioritised. He emphasised the architectural element by defining a modern warship as 'an assembly of multipurpose spaces interacting in a complex manner and located within an envelope, the hullform, the overall shape of which is governed by hydrodynamic performance requirements and internal partitioning determined by structural continuity and damage containment requirements to a far greater extent than an onshore building.' Andrews (1985) had already suggested that the ship design process of Figure 2.10 should be integrated, such that a first step could be a preliminary internal arrangement focused on the ship's primary role, and followed by enveloping the layout within a stable and hydrodynamically efficient hullform. This suggestion is in line with the fact that 
structural, stability, and hydrodynamic considerations are no longer the only warship design drivers. It is instead the overall architecture and deck layout that significantly affect the build and running costs, fighting capability and crew efficiency and comfort, and so can be the design starting point while being readily adjustable to meet further emergent engineering requirements (Brown, 1987). However, due to the complex and interactive nature of a warship's architecture, as well as the multiple and often conflicting requirements it has to meet, evaluating the quality of a particular layout is difficult. For example, the link between machinery spaces and the upper deck through uptakes and downtakes will interact with the ship architecture in many ways (e.g. separation and duplication of vital spaces and zoning) (Andrews and Brown, 1982; Brown, 1993). Brown (1993) described this 'problematic web of interactions' as being related to architecture, thus regarding ships as architecturally-driven rather than by weight or even gross space arguments.

Given that warships can be seen to be primarily architecture driven, architectural modelling in early stage naval ship design is considered to better identify many design drivers and risk areas (Andrews, 2003b). Adding a new, configurational angle to the initial design synthesis process allows the naval architect to be innovative and better contribute to the initial divergent phases (Brown, 1986) and to deal more efficiently with design complexities (Andrews, 2003b). It is also possible to systematically develop and investigate a much larger number of design alternatives and improve trade-off studies and cost estimations, thus assist early design decision making and mitigate the risk of errors and prolonged expensive design reworks (Andrews, 1985; Andrews et al., 2006; Andrews and Pawling, 2007). Integrating the ship architecture and configuration with weight, space and form parameters enables the ship's overall dimensions to be readily adjustable throughout the evolutionary process of a concept study to achieve a more efficient layout (Andrews, 1985, 2003b). This, in essence, contradicts the traditional approach where the ship designer would appear to always strive to avoid any increase in ship size, especially in length and displacement (Andrews, 1981). A fully integrated design process enables the ship designer to explore design aspects that have traditionally been difficult or impossible to explore through a numerically-based ESSD. These include: design style aspects, such as zoning, vulnerability, cellularity, modularity, accessibility, choice of margins and habitability (Andrews, 1985) (see (Pawling et al., 2013; Andrews, 2018) for a more 
comprehensive study of design style aspects), and more informative early integration of the ship CS (Andrews, 1992). Despite the fact that questions have been raised as to how to evaluate certain aspects (e.g. vulnerability, habitability) (Garzke and Kerr, 1985), the ability to consider them during ESSD allows more believable design solutions to be produced through better cost estimates and for design comparisons to be based on operational performance as well as improved cost estimates (Andrews, 1985; Garzke and Kerr, 1985). Finally, since requirements elucidation can only be properly undertaken by concurrently producing design options (Andrews, 2003b), the essential dialogue between the parties involved, including the requirements owner, is likely to be more productive through early architectural and configurational modelling (Gale, 2003).

The dimensions of a space on-board a ship are not limited to length, area or gross volume, but rather include its location and shape (Brown, 1987). Therefore employing the advantages of modern Computer-Aided Design (CAD) tools enables the implementation of an architecturally-based, fully integrated, more innovative and adaptable ship design process (Andrews, 1981, 2003b). The rapid developments in computer graphics, since the early 2000s, have opened up the way for sophisticated architectural modelling (Andrews and Pawling, 2009), enabling ship designers to rapidly produce large numbers of design alternatives with less mistakes, thus reducing design reworks and costs (Gale, 2003), explore significantly different layout options and assess whether ship features are affected (Andrews, 2003b). Computer Aided Ship Design (CASD) allows the ship designer to carry out investigations that were traditionally done in the later design stages (Andrews, 1998; Cooper et al., 2007), for example the weight and cost estimations of alternative structural options (Andrews and Brown, 1982), the assessment of vulnerability and structural continuity (Andrews, 1993; Crow, 2001), circulation of personnel and logistic of freight movement are all addressable through simulation techniques (Andrews and Pawling, 2009), with investigation of highly congested areas facilitated by 3-D-modelling (Gale, 2003). These new capabilities could, to a certain extent, tackle the observations made by Garzke and Kerr (1985) regarding the evaluation of certain ship characteristics (e.g. mobility (hull, mechanical, electrical), survivability, habitability, multipurpose CSs). Also, the improvements in computer tools and 3-D models incorporating simulation and walk-through capabilities could potentially reduce the extent of physical 
modelling (i.e. mock-ups of certain parts of the ship) that are expensive and time consuming to both construct and modify (Gale, 2003; Gates, 2005).

Thanks to such major developments in computer graphics, it is evident that a more integrated and innovative ESSD is achievable (Andrews and Pawling, 2003) while the design is still being developed (Andrews et al., 2009). This then increases the designer's ability to broaden the scope of the divergent phases of the design (Brown, 1986), while enabling a more descriptive, comprehensive design to emerge. The resulting design should then better reveal what are the issues critical to major design and requirement decision making (Andrews, 1993).

\subsubsection{The Design Building Block Approach}

The proposal by Andrews (1981) to integrate ship architecture with the traditional numerical sizing was followed by the demonstration of 'creative synthesis' (Andrews, 1984) that was presented in the paper entitled 'An Integrated Approach to Ship Synthesis' (Andrews, 1985). In this work, a simple hullform generator was employed to produce the hull and deck outlines of a frigate, followed by placement of compartments. A graphical facility was used for layout and spatial auditing and proceeded by extraction of space and weight data and reiteration. Based on the desirability of relative juxtapositions of specific compartments, the concept of producing 'circles of influence' was proposed by Andrews (1985) as one way of developing an initial layout of a representative frigate before a hullform was sized and balanced. The combination of circles of influence of two groups of compartments is shown in Figure 2.11. Using this, Andrews (1985) produced the network representation shown in Figure 2.12 to illustrate the compartment relationships for a full synthesis. From this network, Andrews (1985) proposed positioning a disposition of compartment blocks on a graphical display as shown in Figure 2.13, at least for the major compartment groups in the main regions of arrangement conflict (e.g. superstructure and main through decks). Figure 2.13 demonstrates how a fully integrated ship synthesis might be carried out to produce a 3-D block layout of a frigate, around which a hullform could be 'wrapped' (Andrews, 1985, 2003b). 

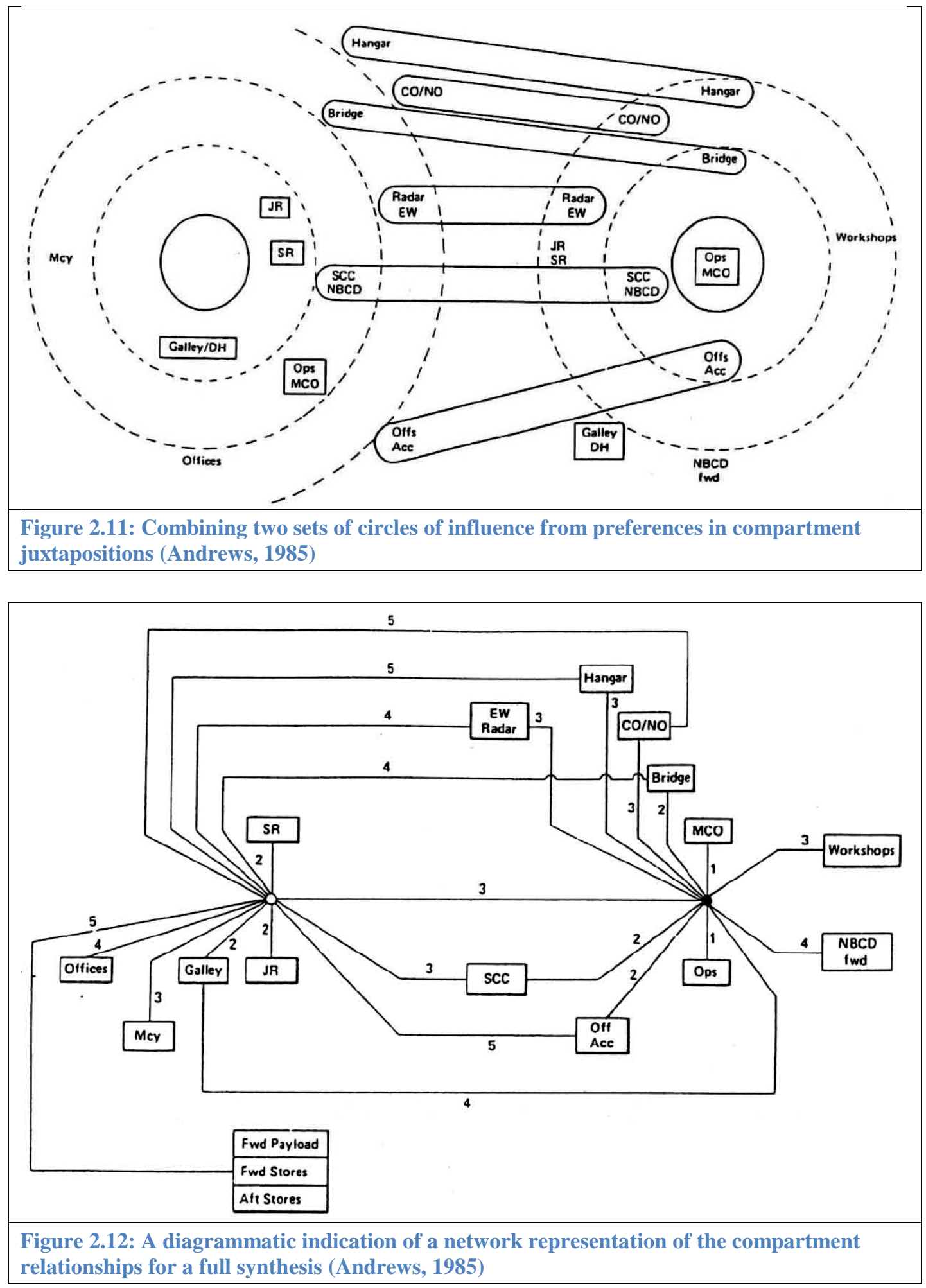


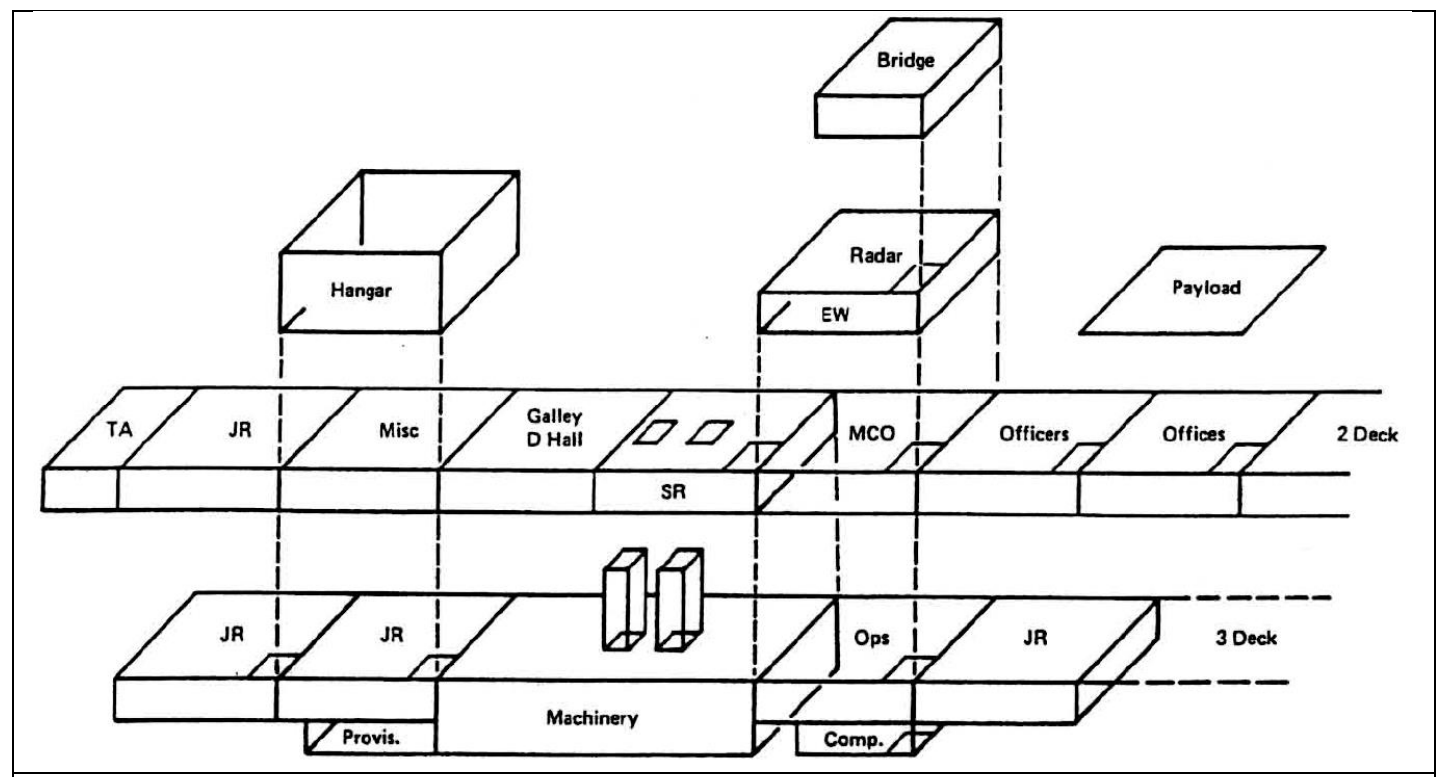

Figure 2.13: A diagrammatic suggestion as to how a full synthesis suggested by Figure 2.11 and Figure 2.12 might be accomplished (Andrews, 1985)

Brown (1987) denoted the Junior Rates (JR) Dining Halls as an obvious example with many layout relations that can be used to form 'circles of influence' (e.g. adjacent to and have good access to the galley complex and various types of store rooms, sited in areas of low vertical acceleration). Andrews (1985) also regarded the location of operational spaces like the Operations Room, machinery spaces, as well as accommodation spaces (given their preponderance in the space disposition) as the most important dispositions that should be central to the creation of 'circles of influence.' The study was however restricted due to the limited capabilities of 1980s' computers. The initial hullform, and thus deck plans, were developed using hull coefficients typical to frigates, that would not be easily available for exploring unconventional shapes and more general hullforms (Andrews, 1998). Nevertheless, the approach ensured that the disposition of principal spaces could be used to determine both the initial sizing and selection of hull dimensions, thus avoiding a purely numerical sizing and parametric survey process. Andrews (1985) demonstrated a more 'holistic' approach to a fully integrated ship synthesis (Figure A1. 5, Appendix 1) and revised the initial sizing process (Figure A1. 4, Appendix 1) to produce a process (Figure A1. 6, Appendix 1) that integrates the preliminary layout into the numerical synthesis process. 
As discussed in Section 2.2.4, the application of the group WBS is seen as inhibiting the traditional ESSD that was reconsidered in the approach to achieving a fully integrated ship synthesis. The motto, 'to float, to move, to fight' was drawn on by Brown and Tupper (1988) as the summary of the ship designer's objectives, representing a ship in terms of materials and configuration (Andrews, 1987). By adding an infrastructure group, it was seen to be appropriate to use this functional breakdown as an alternative to the group WBS to describe a vessel at the top level, as was used for the SUBCON demonstration of the architecturally-driven approach to submarine synthesis in the 1990s (Andrews et al., 1996). Some advantages of applying this more functional breakdown to ship descriptions were seen to be:

- Enabling the designer to readily assess the whole ship impact of a certain subsystem or a capability (Andrews, 2003b), thus improving the ability to engage in a dialogue with the requirements owner and better explore the costeffectiveness of potential solutions (Andrews, 2011);

- Producing a more informative breakdown of the cost of the ship (Andrews and Pawling, 2003). For example, Andrews and Brown (1982) determined the initial cost devoted to 'fighting the ship' to be about $70 \%$ of the total UPC following the classic float, move, fight functional subdivision, i.e. without an infrastructure group (Andrews et al., 1996; Andrews and Dicks, 1997);

- Enhancing creativity by reducing the extensive reliance of new ship designs on subtly input information from existing ships (i.e. the weight groups) and implied solution choices (Andrews and Pawling, 2009).

From the above studies, a new approach, namely the Design Building Block (DBB) approach to ESSD was developed, initially for submarines (Andrews et al., 1996), and later for surface vessels (Andrews and Dicks, 1997; Andrews and Pawling, 2003), and has since reached acceptance as a standard ESSD approach (Tupper, 2013). The basic idea behind the UCL DBB approach is for the designer to separate the ship's functions and sub-functions into discrete physically realisable elements (Design Building Blocks) and position them appropriately in a putative ship configuration. This, in contrast to the traditional sequential design process, puts architectural features at the centre of the ship synthesis process, in conjunction with the traditional numerically based sequential design process (Andrews and Dicks, 1997). The DBB approach 
enables the designer to avoid a type-based ship design or one limited to variations within the conventional configurations (Andrews and Dicks, 1997). By allowing a more thorough exploration of alternative designs and encouraging the investigation of novel solutions (Andrews and Pawling, 2009), the DBB approach significantly facilitates the iterative dialogue process of requirements elucidation, described by (Andrews, 2011, 2013) as the primary task of the Concept Phase. It is an 'open/glass box' that allows the designers to contribute and incorporate their experience and judgment to the design process and avoids having to rely on a 'Black Box' system that is usually based on someone else's work, and a 'soft' approach permitting the structure and processes used to be readily updated, algorithms improved, or even different modelling features incorporated (Andrews and Dicks, 1997). The architectural nature of the approach allows many design aspects beyond $\mathrm{S}^{4}$ to be investigated in the earliest phases and to do so while the design is still readily alterable (Andrews and Pawling, 2003). It also allows potential conflicts to be addressed (Andrews and Dicks, 1997) and better links the different phases of the ship design process (Andrews, 1998). Design aspects that can be addressed include:

- Design margin philosophy and specifically the extent of access, that can be considered as part of the development process of a particular design, rather than having to use default values, often based on inappropriate historic data that is unrepresentative of the actual ship being designed (e.g. using an assumed access route philosophy based only on a previous design) (Andrews and Dicks, 1997);

- Distributed systems details, topside design and CS integration, ILS, human factors and 'ilities' issues (e.g. producibility, supportability, survivability) (Andrews, 1998);

- Through-life costing, health and safety issues, and environmental aspects (Andrews and Pawling, 2009). 
A summary of the UCL DBB approach, shown schematically in Figure 2.14, is as follows (Andrews and Dicks, 1997):

- A need for a new conceptual design is conceived and an idea of the likely design Style to meet that requirement suggested;

- Drawing on novel ideas and/or historical data, a series of initial super design building blocks are defined in a CAD system. Each design building block contains geometric and technical attributes regarding the functions of that block;

- A design space is generated and the design building blocks are configured as initially seen to be required or desired within the design space;

- Overall balance and performance of the ship design are investigated using simple and flexible algorithms and, if necessary, using analysis programs external to the main system (e.g. seakeeping should that be a critical driver);

- The configuration is then manipulated until the designer is satisfied;

- Decomposition of the building blocks to greater levels of detail is undertaken, as necessary to increase confidence in the design solution.

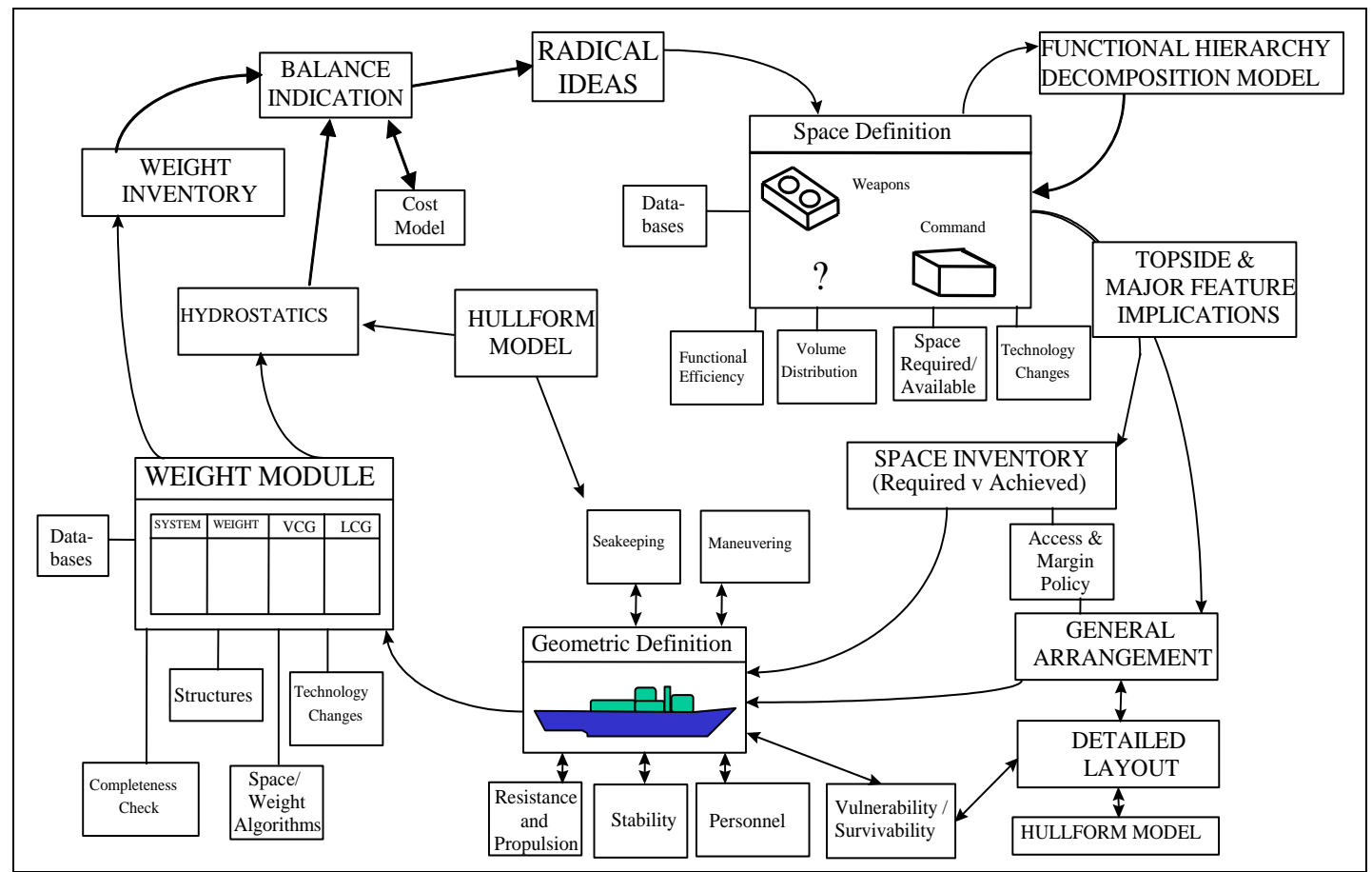

Figure 2.14: The Design Building Block approach to Early Stage Ship Design applied to surface ships (Andrews and Dicks, 1997) (Andrews and Pawling, 2003) 
Figure 2.14 shows the intent behind the DBB approach. It is not a formal process (like the representations of the 'design spiral'). Therefore 'inputs' like 'Hullform Model' are indicative rather than absolute. Figure 2.14 summarises a comprehensive set of analysis processes most of which are unlikely to be used in the initial setting up of the design and early iterations around the sequence of building blocks, geometric definition and size balance. In fact several of the inputs shown in Figure 2.14 are either specific to naval combatants, such as topside features, or omit aspects which could be dominant in specialist vessels, such as aircraft carriers, amphibious warfare vessels or cruise liners and large ferries where personnel and vehicle flow dominate the internal ship configuration. Figure 2.15 summarises the main activities in each design phase of the UCL DBB approach.

\begin{tabular}{|c|c|}
\hline & Design Preparation \\
\hline & Selection of Design Style \\
\hline & Topside and Major Feature Design Phase \\
\hline & Design Space Creation \\
\hline & Weapons and Sensor Placement \\
\hline & Engine and Machinery Compartment Placement \\
\hline & Aircraft Systems Sizing and Placement \\
\hline & Superstructure Sizing and Placement \\
\hline & Super Building Block Based Design Phase \\
\hline & Composition of Functional Super Building Blocks \\
\hline & Selection of Design Algorithms \\
\hline & Assessment of Margin Requirements \\
\hline & Placement of Super Building Blocks \\
\hline & Design Balance \& Audit \\
\hline & Initial Performance Analysis for Master B.B. \\
\hline & Building Block Based Design Phase \\
\hline & Decomposition of Super Building Blocks by function \\
\hline & Selection of Design Algorithms \\
\hline & Assessment of Margins and Access Policy \\
\hline & Placement of Building Blocks \\
\hline & Design Balance \& Audit \\
\hline & Further Performance Analysis for Master B.B. \\
\hline & General Arrangement Phase \\
\hline & Drawing Preparation \\
\hline
\end{tabular}

During each phase, the design is evolved and the level of detail increased and at the end of each step, the designer uses the feedback information from the design tool to assess the state of the design in terms of configuration, weight, space, stability, powering and any other aspects deemed critical to the given design study. The designer then decides as to what changes are necessary to achieve the required design balance (Andrews and Pawling, 2009). Andrews and Dicks (1997) demonstrated the advantages of the DBB approach by applying it to design a monohull frigate that was 
compared with one designed using the traditional numerical ESSD. The widened range of aspects considered by the DBB approach produced a design that, it was argued better satisfied the emergent requirements, with a more considered and feasible configuration that, in the case in question led, to an increased ship size. As not all these more holistic requirements (e.g. survivability, producibility, and supportability) could be assessed by the traditional (numerical) synthesis, such emergent insights would then require further downstream design iterations or significantly compromised designs being developed.

Following the rapid developments in computer capabilities and graphics, the DBB was further developed and in 2001 implemented to surface ships as the SURFCON module in QinetiQ GRC's Paramarine CASD suite (Munoz and Forrest, 2002). This ensured that the UCL DBB approach was incorporated in a commercially established ESSD software package and ship designers could draw on all the naval architectural analytical tools available within Paramarine (e.g. stability, powering, seakeeping, vulnerability, manoeuvring, structural analysis) (Andrews and Pawling, 2003). SURFCON, with its three dimensional nature, has been shown to be capable of achieving an architecturally-centred fully integrated ESSD process, applicable to a wide range of conventional and unconventional ships, and able to be used to investigate a broader range of issues directly related to the ship architecture and configuration, as well as other aspects (Andrews and Pawling, 2003; Andrews, 2018). The implementation of the SURFCON module was comprehensively explained by Andrews (2006) and Andrews and Pawling (2003, 2009). Figure A1. 7 (Appendix 1) shows an example of Paramarine's SURFCON module application to ESSD.

In line with one of the major elements of the DBB approach, SURFCON describes the ship by means of a functional breakdown, namely FLOAT, MOVE, FIGHT, and INFRASTRUCTURE (Andrews and Pawling, 2003). These four fundamental groups are broken down into more detailed DBB subgroups (Andrews and Pawling, 2009). Andrews and Dicks (1997) stated that FIGHT and MOVE groups tend to constrain a design more extensively due to having directly defined requirements and generally tightly controlled configuration choices, whereas, FLOAT and INFRASTRUCTURE are largely seen as dependent groups rather than main drivers. However, technologies such as Integrated Full Electric Propulsion (IFEP) and vertical launch missile silos can provide the ship designer with more architectural choices with regards to MOVE and 
FIGHT groups (Andrews and Dicks, 1997) as can the adoption of mission bays (Pawling and Andrews, 2010).

Since the inception and realisation of the DBB approach through SURFCON, the UCL Design Research Centre (DRC), established in 2000 as part of the UCL Marine Research Group (MRG), has utilised it in a wide range of investigative ship design studies. These include how an all-electric ship approach affects the configuration of a warship (Andrews et al., 2004), more efficient configurations for ship producibility (Andrews et al., 2005), integrating personnel movement simulation into ESSD (Andrews et al., 2008), and naval ship survivability (Piperakis and Andrews, 2014). Other examples include a high speed adaptable Littoral Combat Ship (LCS) (Andrews and Pawling, 2006), a Joint Support Ship (JSS) (Andrews and Pawling, 2007), innovative Offshore Patrol Vessel (OPV) designs incorporating a modular payload for Unmanned Vehicles (UxV), and the effect of large UxVs on minor war 'second-rate' vessels (Pawling and Andrews, 2013). More examples can be found in (Andrews and Pawling, 2009). In all these examples, the applicability of the DBB approach was proven by sizing the individual DBBs using the guidelines provided for the UCL MSc course ship design exercise (UCL, 2013a, 2013b). This helped realise the advantages of the approach, namely producing believable and coherent solutions through open, revelatory and creative methods (Andrews, 2003b).

In addition to the UCL DBB approach, other novel approaches to the architecture and arrangement of complex ships in ESSD are the University of Michigan Intelligent Ship Arrangement approach (Nick, 2008) and the Technical University of Delft Packing Approach (Oers, 2011). While the Intelligent Ship Arrangement approach works within the fixed envelope of the hull and topside and only solves the space arrangement part of the total ship design problem, the Packing Approach uses a highly flexible packing genetic algorithm to position the systems that are represented as building blocks. Both methods are driven by evolutionary algorithms and use automated layout generation processes, unlike the DBB Approach manual procedure (Pawling et al., 2013). It was concluded that the DBB approach, with its manual exploration and decision making process and high interactivity, would better facilitate the investigation of DfS early in naval ship design. The issue of naval ship supportability is now outlined before presenting the proposal as to how this might be done. 


\subsection{Naval Ship Supportability}

\subsubsection{Introduction to Naval Ship Supportability}

Supportability is defined by NATO as 'a measure of the degree to which all the resources required to operate and maintain the system/equipment can be provided in sufficient quantity and time' (NATO, 2011). As a disciplined approach applicable throughout the whole life of an engineering system project, Integrated Logistics Support (ILS) is the official UK MoD policy to facilitate the associated supportability aspects (UK MoD, 2013a). Despite its focus changing as the project progresses through the CADMID phases (see Figure 2.1), the principal aim of the ILS process is maximising system availability while improving Through-Life Finances (TLF). It should continually influence the product design process to ensure that the final product is supportable throughout its lifetime at an acceptable cost (UK MoD, 2013a). Figure 2.16 illustrates the several distinct yet integrated processes, termed as ILS elements. The UK MoD (2011, 2013a) and NATO (2011) have produced generic descriptions of ILS elements, shown in Table A1. 2 (Appendix 1).

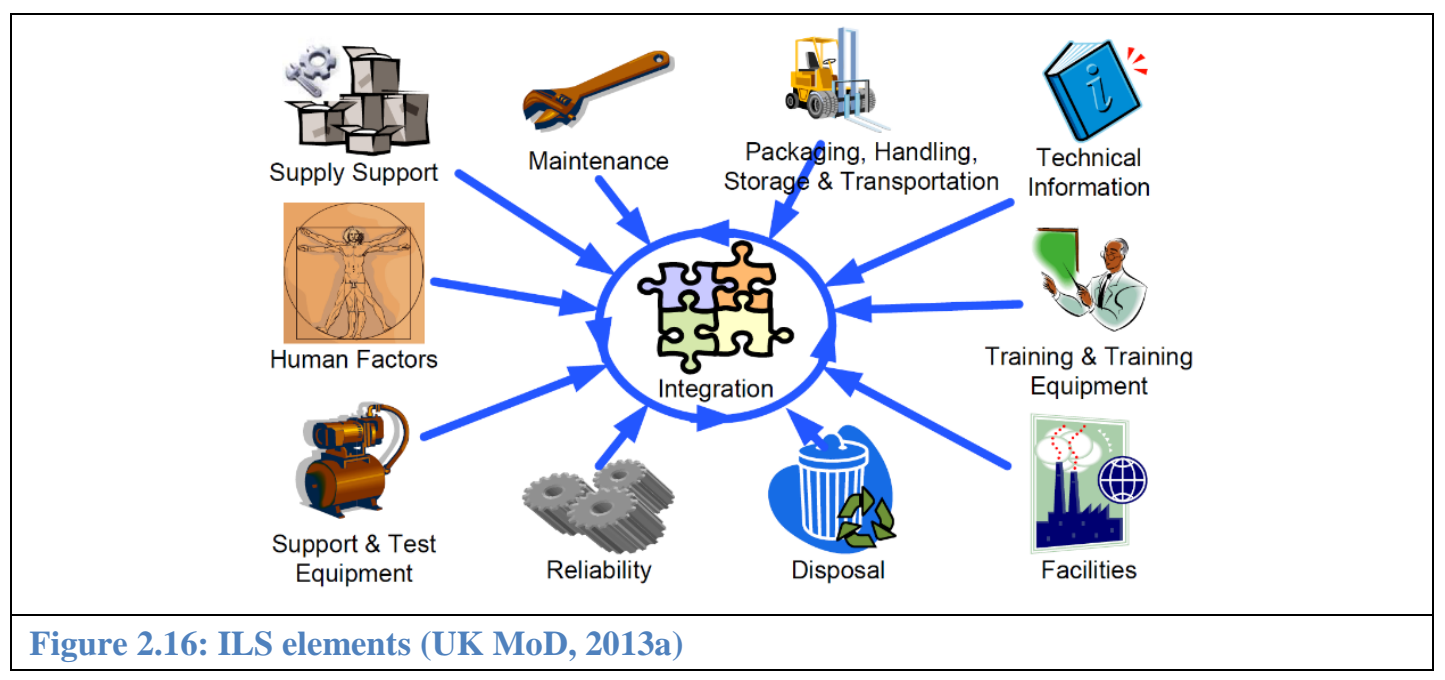

The development of military products demands that those responsible for the overall ILS process must carefully consider the relationship between the mission area and needs, peacetime readiness and wartime utilisation requirements, and the appropriate support solution. Termed as Readiness and Supportability (R\&S) objectives by the US Defence System Management College (DSMC), these determine or influence the manner in which the system will be designed and supported in its operational role, that 
in turn leads to supportability parameters being established for use in the system design process (DSMC, 1986). Figure 2.17 illustrates how the R\&S objectives help link mission area and needs, to the support solution.

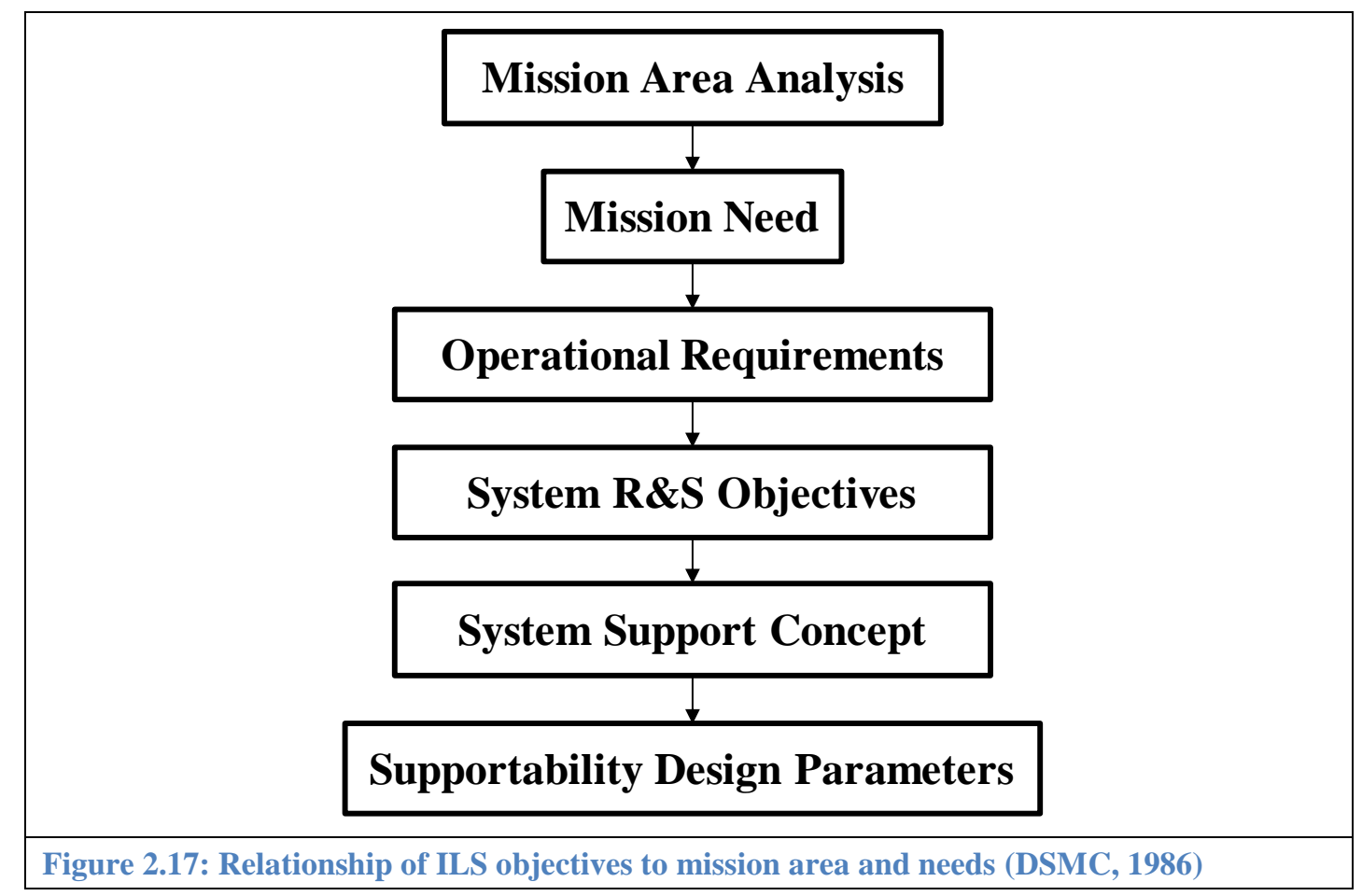

The UK MoD (2013a) describes ILS as using four main tools and techniques to develop the support solution:

- Logistic Support Analysis (LSA): being the most wide-ranging in scope, LSA is a structured method to help analyse the supportability implications of individual items of a product while they are being developed with the aim of identifying features of the design that could result in excessive TLC;

- Failure Modes Effects and Criticality Analysis (FMECA): performed in time to influence the product design with the aim of maximising availability and minimising maintenance requirments, thus reducing TLC;

- Reliability Centred Maintenance (RCM): performed in service to identify the most cost-effective maintenance methods;

- Level of Repair Analysis (LORA): largely done in service to determine the most suitable maintenance level (i.e. repair vs. discard and replace) for repairing the individual items of a product. 
An efficient, cost-effective ILS process requires the establishment of overall maintenance concepts during the Concept Phase of the design rather than later. Green (1993) considers this to be dependent on adequate raw data generated by the timely execution of the tools and techniques mentioned above. He therefore regards delays to ILS assessments as sources of logistical 'nightmare.' By carrying out the assessment during the Concept Phase when the development of design alternatives is still feasible without severe implications on schedule and cost, it is possible to ensure that the support solution is specifically tailored to suit the customer's requirements that will still be fluid and undergoing elucidation (Andrews, 2011), and avoid the extra labour, time and cost required for corrective actions later. Other benefits were listed by Green (1993) and UK MoD (2013b) as:

- Ensuring an adequate planning period to allow sufficient time to define and plan all approaches, tasks and funds required;

- Enabling up-front market research to investigate both military and commercial products already in existence before deciding if a new product or equipment needs to be developed;

- Ensuring realistic and timely budget estimates are approved to guarantee the availability of funding when required.

These advantages need to be incorporated in new ship programmes given that major navies currently face ever increasing challenges to affordably procure, keep available and sustain their ships into the future, often far beyond their initially intended service life (e.g. the Royal Navy's short life Type 23 Frigates, some likely to be in service almost double their intended life due to the failure of successive governments to hold on to the short life policy originally intended). The benefits have to be achieved against the backdrop of evolving threats, rapidly changing technology, rising procurement and through-life costs, and declining budgets (Sturtevant et al., 2014). Despite this, naval ship TLS is rarely modelled or assessed in ESSD, partly due to the socio-technical factors involved in the design and procurement process (see Section 2.2.2), but also due to its perceived 'soft' or uncertain nature compared to direct operational performance aspects (see Section 2.2.4). TLS requirements elucidation and the development of numerical design solutions are both regarded as highly uncertain, resulting in TLS only being considered once it is possible to undertake detailed 
modelling and investigation of all underlying aspects, including future operational and support scenarios. However, taking into account the unknown nature of future scenarios, some of which could be a few decades away, combined with the scope of ILS and the lack of detailed ship design information, means such comprehensive investigation is not currently considered to be a realistic task in the Concept Phase. Consequently, TLS has historically been assessed by experienced ship designers and practitioners in the design phases later than Concept when more information is available.

Rather than invariably associating TLS and other 'soft' design aspects with inherent ambiguities, it may well be true that many of the perceived uncertainties result from historical difficulties in establishing numerical and structured ways to address such design aspects at ESD. This is because such considerations rely on explicit knowledge, values or solutions that are not really possible with high level and incomplete design disclosure at ESD. While there have been some attempts to deliver innovative, systematic, sustainable and affordable TLS technical solutions (Cowper, 2014), the lack of such structured approach in the early phases of the design process has been largely accepted.

\subsubsection{Architecturally Discernible Aspects of Naval Ship Through-Life Support}

While it is appreciated that all the ILS elements (see Figure 2.16, Table A1. 2 (Appendix 1)) are pertinent to a comprehensive supportability analysis, those dealing with detailed equipment supportability analysis (i.e. LSA), strategic long-term planning and supply of maintenance and support, information and documentation, together with certain human factors aspects, and high level support management and contractual issues have been considered to not be readily suited to being addressed by the UCL DRC. Given the extensive knowledge and experience of the UCL DRC team on naval ship concept design, the implementation of the DBB approach, and analysis of ship configurations (see Section 2.2.6), this research was limited to those ILS elements that are relevant to and discernible through ship configuration and architecture, which essentially meant being amenable to the DBB approach.

Andrews and Pawling (2003) stated that the DBB approach enables the designer to bring several aspects of modern ship acquisition practice into the initial synthesis and 
importantly to break away from purely numerical sizing approaches that have encouraged the 'minimum sized ship to achieve a given role/performance' response. Amongst these aspects is Concurrent Engineering (CE), that rather than a technology is more of a philosophy in aiming to deliver a new product at the lowest cost and highest quality. It employs cross-functional design teams and uses parallel rather than sequential design approaches to enable a more holistic design process (Bennett and Lamb, 1996). One of the major elements of CE is DfS or the consideration of throughlife issues, namely adaptability (operationally and through-life) and sustainability, both described by Andrews and Pawling (2003) as being significantly affected by the ship's layout.

Carmel (2004) described a versatile design as one that allows altering the package of organic mission capabilities in responding to different threat environments and mission requirements, in an effective and focused way. Sturtevant et al. (2014) argued that 'flexibility leads to affordability over the lifecycle' (Figure 2.18) and listed four key attributes that characterise a 'flexible ship:'

- Adaptability: to reduce the time spent in refit and the modernisation costs to prevent the ship from becoming obsolete. Relevant features include rapidly reconfigurable spaces, modular payloads and payload stations, routes for equipment insertion and removal, and ship services and infrastructure with sufficient margins to support future upgrades;

- Modularity: to allow parallel development of payload and the ship itself, subsequent payload installation, and faster more efficient capability insertion and space reconfiguration. Its features include standardised interfaces and modular equipment components;

- Scalability: to allow systems of hardware/software combination (e.g. radar) to be increased or decreased in size to match the capability requirements of different-sized ships;

- Commonality: to reduce development costs through re-use of expensive payload systems and reduce acquisition costs through larger quantity buys and increased competition. Its features include using standardised design specifications that allow the same systems, at various scales, to be applied across multiple ship classes. 


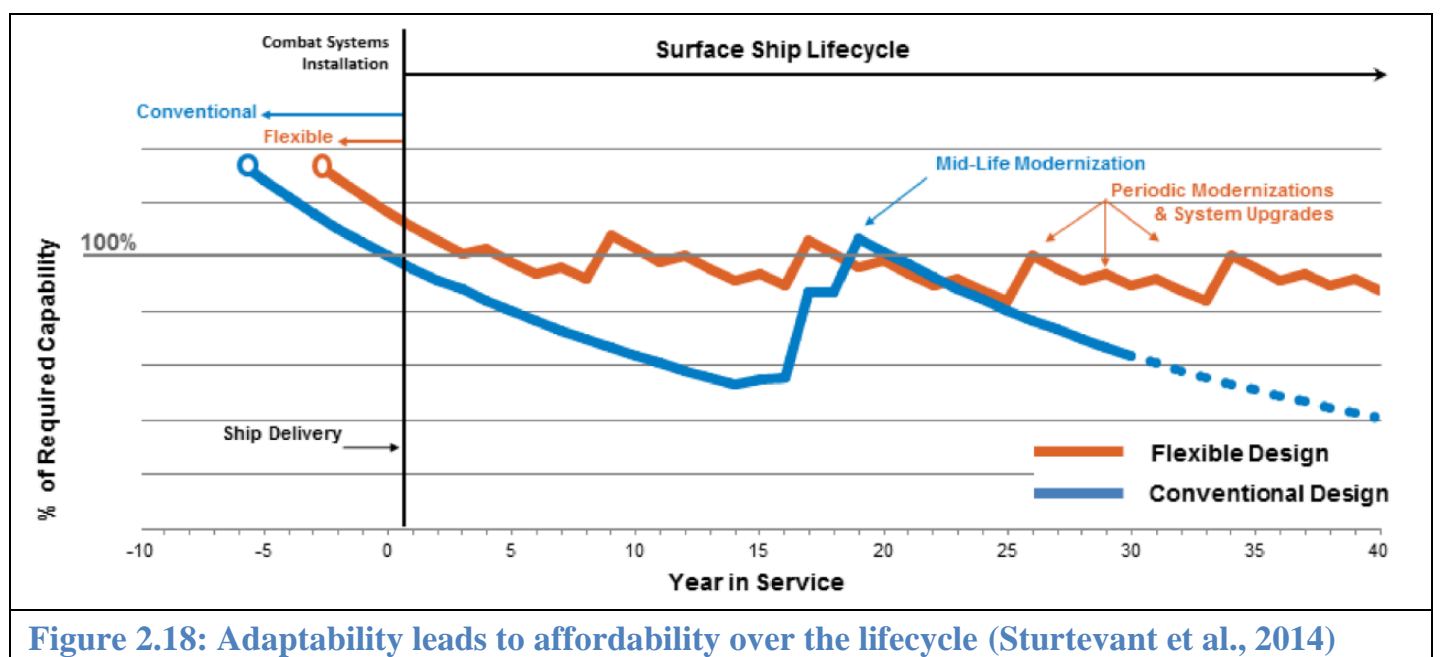

From the four attributes of a flexible ship listed, it is considered that the adaptability and modularity elements are far more relevant to the link between an early consideration of the ship's architecture and configuration and the supportability of the ship design. The other two characteristics (scalability and commonality) tend to be more appropriately dealt with in later detailed ship design phases, and so the design of individual systems and equipment and high level design and procurement policies across different ship classes were considered to be less relevant to the research that was undertaken. Both operational and through-life adaptability require recourse to the appropriate spatial margins (Andrews and Pawling, 2003) with direct implications on ship size and cost (Gale, 1975), and placement of sufficient, unobstructed, and if possible, completely vertical removal routes (Drewry and Jons, 1975) or other means of removing equipment (Boerum and Birindelli, 1985) that also interact with the ship's zoning philosophy (Doerry, 2006) and general layout style (Brown, 1987).

Another major supportability aspect with certain architecturally discernible features is ARM. Reliability and maintainability have been defined as inherent properties that must be designed and built into a system during its development and manufacture to ensure high in-service availability (UK MoD, 2013a). While reliability mostly concerns detailed component, equipment or system design to improve Mean Time Between Failure (MTBF), maintainability can readily be linked to the ship's configuration and architecture. The reduction of Mean Time to Repair (MTTR) with faster more efficient maintenance is achievable through considering the relationships between certain spaces and compartments (Andrews, 2003b) and better accessibility (Andrews and Pawling, 2003). This will affect aspects such as horizontal and vertical 
main access routes, dedicated removal and replacement routes, and placement of soft patches and compartments (i.e. areas designed to be made portable or easily cut away (UK MoD, 2000a)) with significant supportability implications.

\subsubsection{Technical Solutions Addressing the Architecturally Discernible Aspects of Naval Ship Through-Life Support}

It has been stated by many authors that central to the concept of a versatile ship is the decoupling of the ship design development from the parallel development of the CS, or, more generally, clearly isolating those parts of the ship, that need updating throughout its service life (Gates, 1985; Doerry, 2014; Sturtevant et al., 2014). This can be achieved by utilising modular blocks of proven design that can be assembled in different configurations (Carmel, 2004) and transferred from ship to ship, maximising utilisation and mission availability (Sturtevant et al., 2014). Since the 1970s, the concept of using modular blocks in the design of surface combatants has been proposed as a means of achieving adaptability, upgradeability, improved availability, and maximising a navy's return on investment (Drewry and Jons, 1975). Its objectives have been described as facilitating construction, integration, installation, removal, and interchangeability (Drewry and Jons, 1975). Often termed as the Variable Payload Ship (VPS), Broome et al. (1982) described a ship with a combat suite made up of modules of standard sizes with standard interfaces, thus easily removed, altered and upgraded. The concept of a VPS destroyer was proposed by Boerum and Birindelli (1985) and is shown in Figure 2.19. 


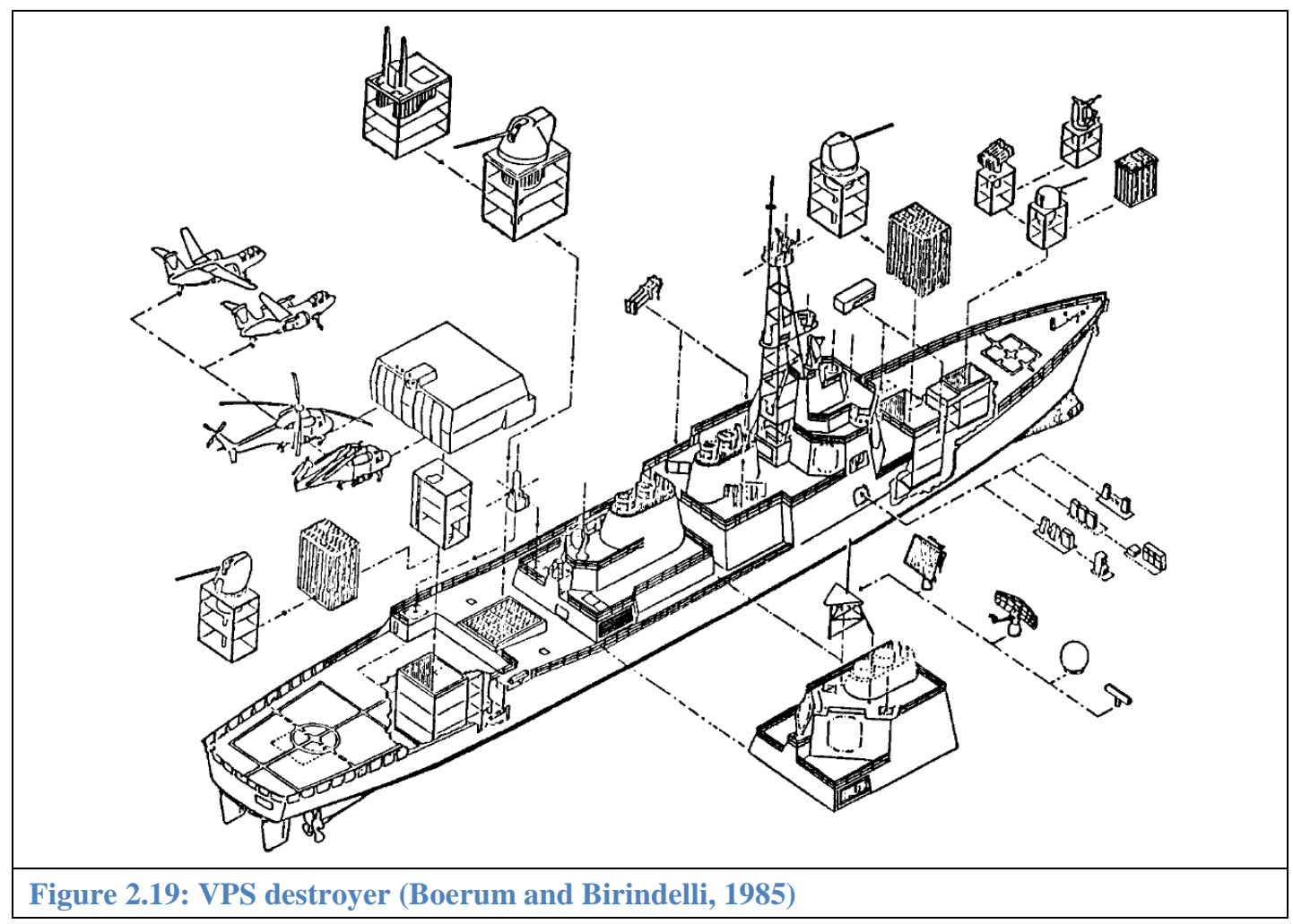

Drewry and Jons (1975) outlined numerous ways to achieve standard interfaces, including: interface buffering and use of adaptors, standardisation, consolidation, and complete interface elimination. Considering accessibility as a major ILS aspect, Boerum and Birindelli (1985) suggested that VPS equipment should be categorised on the basis of the expected frequency of maintenance, replacement or upgrade and thus the removal strategy being determined accordingly. This is similar to the approach for conventionally constructed ships. To determine the width of passageways and sizes of doors and hatchways, Broome et al. (1982) also suggested the use of standardised module sizes. They however pointed out that given the inflexible shape and dimensions inherent to any standardised unit, the choice on the fore and aft location of modules is severely limited due to the $\mathrm{V}$-shape of the hull forward and the after cut up.

The concept of modular blocks has been implemented in a number of well-known examples (Glanville, 2010): the US Navy LCS ships, the Danish Stanflex ships; the Blohm and Voss MEKO modular design; and the BMT Venator concept. The US Navy LCS vessels consist of a very large connected mission bay-hangar complex and are equipped with modular 'plug-and-play' mission packages, including UxVs. Rather than being a multi-mission ship, like the US Navy's larger surface combatants, the 
LCS is a mission-focused ship, meaning it is a ship equipped to perform one primary mission at any given time. The ship's mission orientation can be changed by changing out its mission packages (O'Rourke, 2016). Two LCS variants have been developed and a number of ships manufactured; the Lockheed Martin monohull variant (Figure 2.20), and the General Dynamics Trimaran variant (Figure 2.21) (Gouré, 2006).

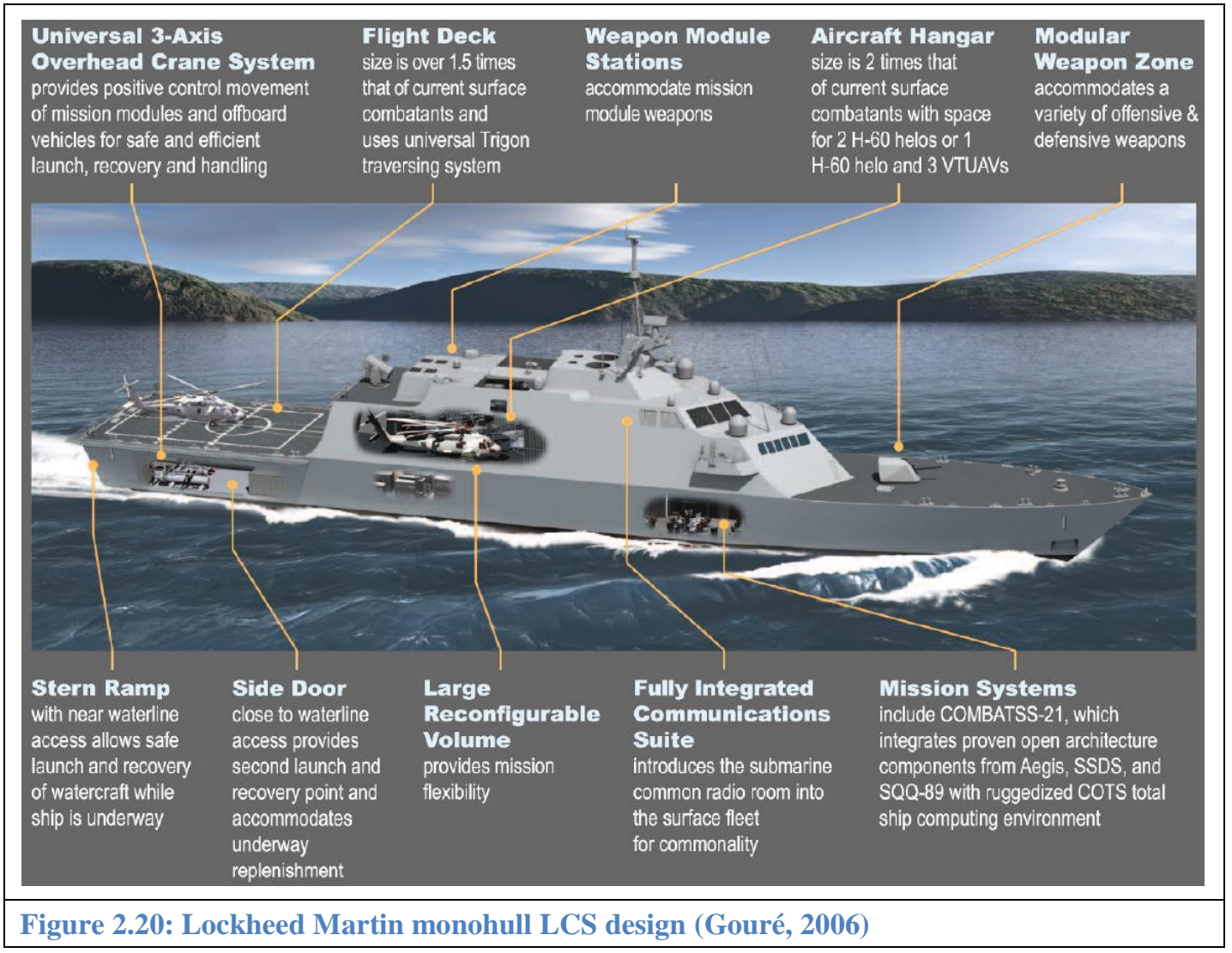




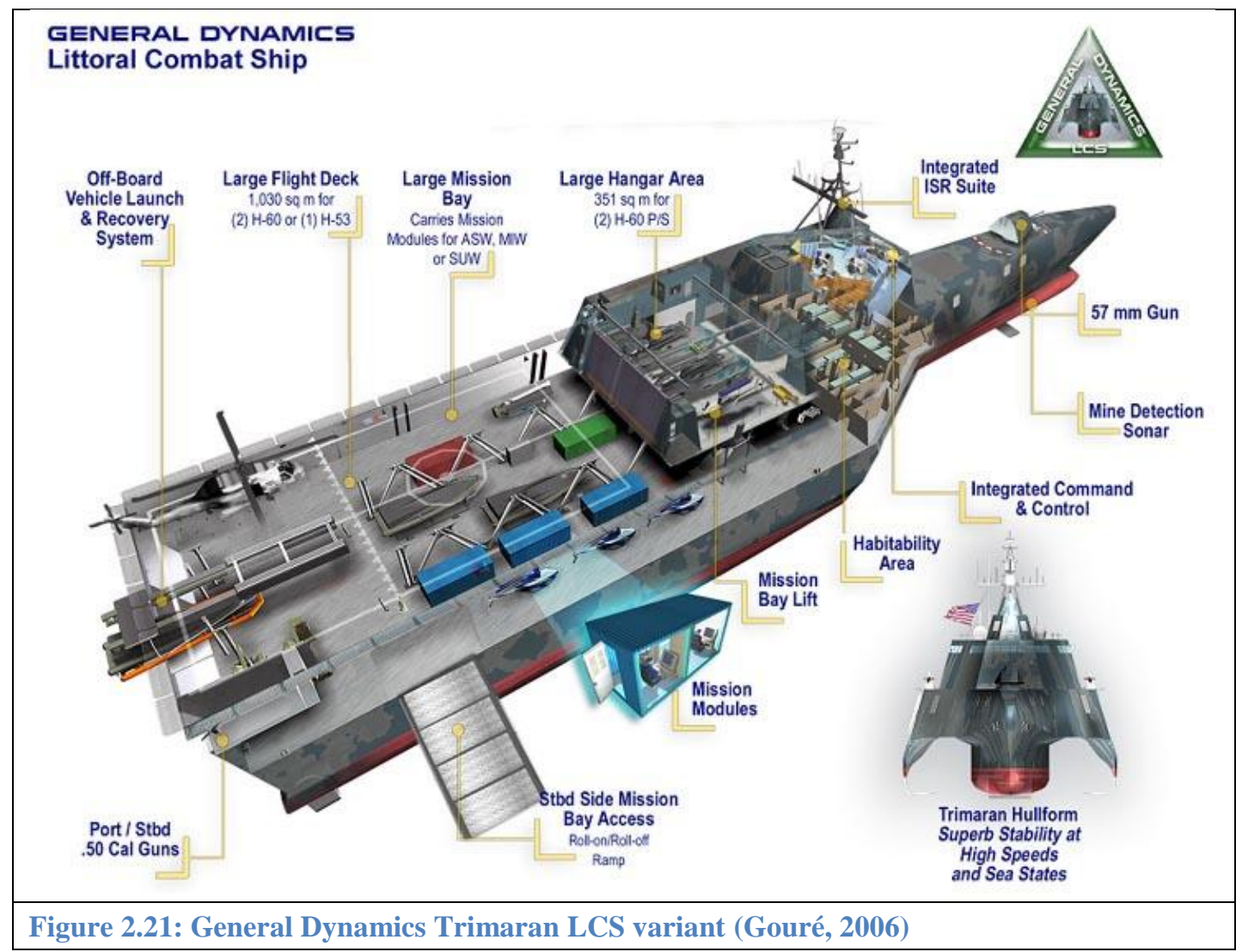

The Stanflex concept aims to improve the level of fleet capability with a reduced number of ships and weapons. With the Stanflex 300 series of the Flyvefisken Class of OPVs, Royal Danish Navy (RDN) benefits from a wide portfolio of modularised capability specific systems and equipment that are said to be readily interchangeable within the fleet without any special alongside facilities. Each Stanflex ship has onboard 'slots' to accommodate the required weapons and other equipment (Sorensen, 2015), as shown in Figure 2.22.

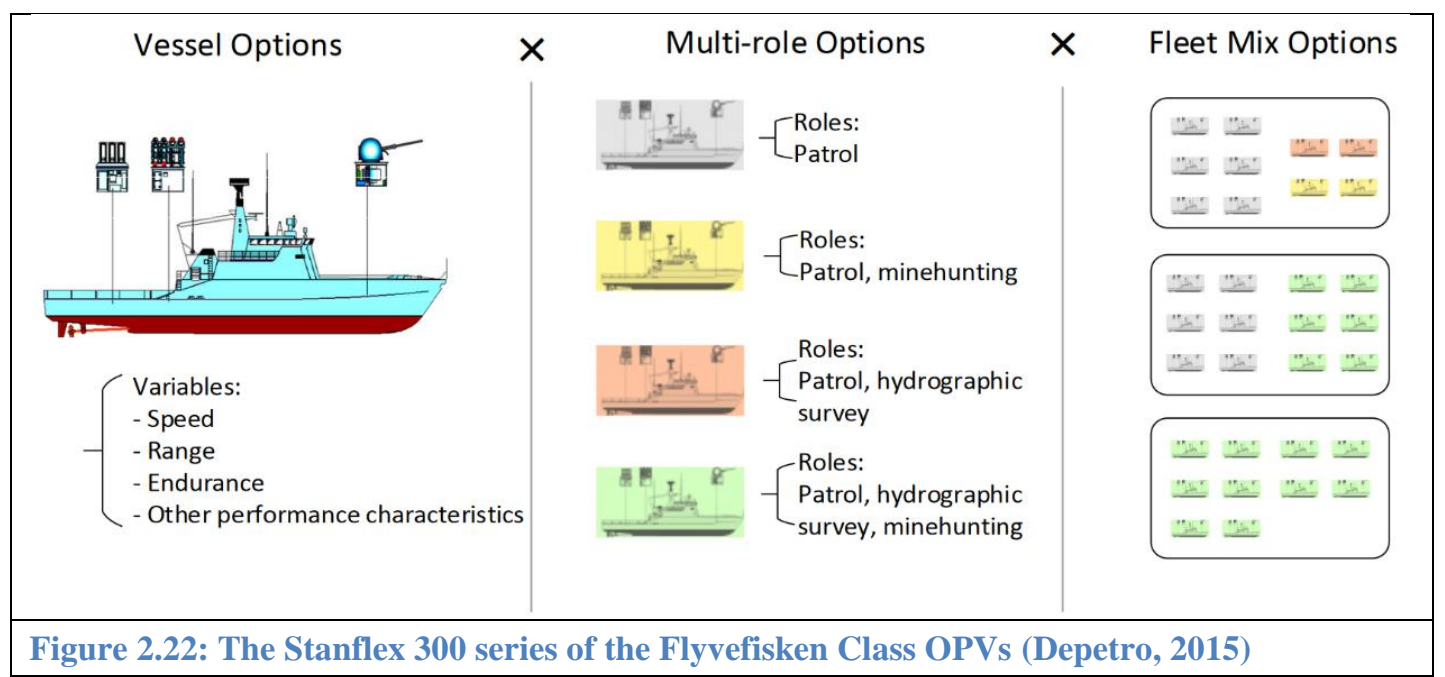


The MEKO concept does not consider the ship itself but focuses on modularisation of certain weapon and ship systems, accommodating all the components needed to run the system in a single module that can be incorporated into different ships (Glanville, 2010) as shown in Figure 2.23. MEKO has historically maximised design reuse and produced highly marketable warship solutions on the international market (Moss, 2007).

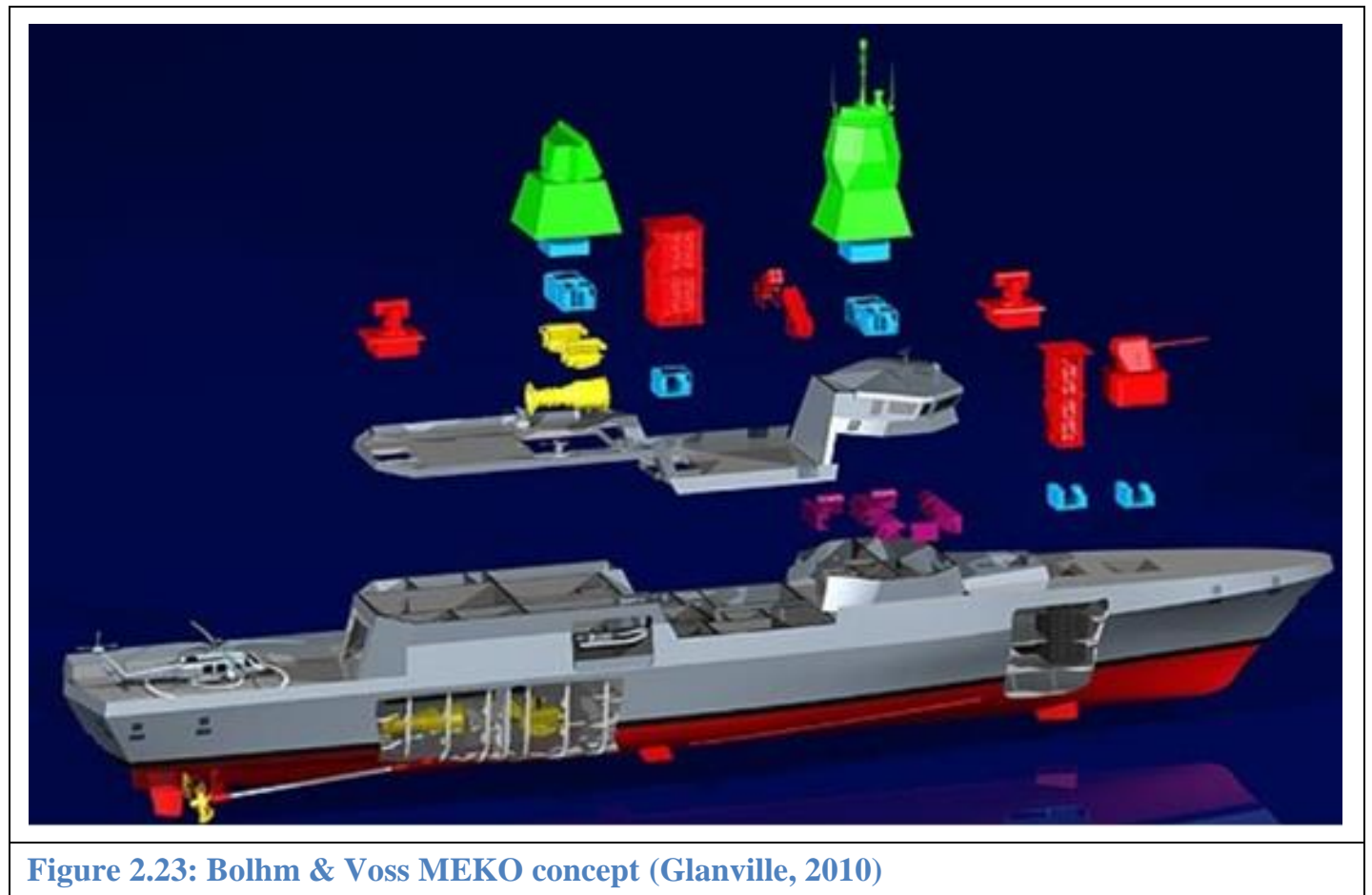

The BMT Venator concept design relies heavily on the use of reconfigurable internal mission spaces and modularised off-board vehicles, in contrast to the Stanflex concept of fast interchangeable standardised units. The BMT concept proposes a large reconfigurable mission bay, telescopic hangar arrangement, overhead gantry arrangement, and a permanent stern ramp for launching off-board vehicles (Glanville, 2010). The mission bay and the arrangement of major compartments of the BMT Venetor is shown in Figure 2.24. 


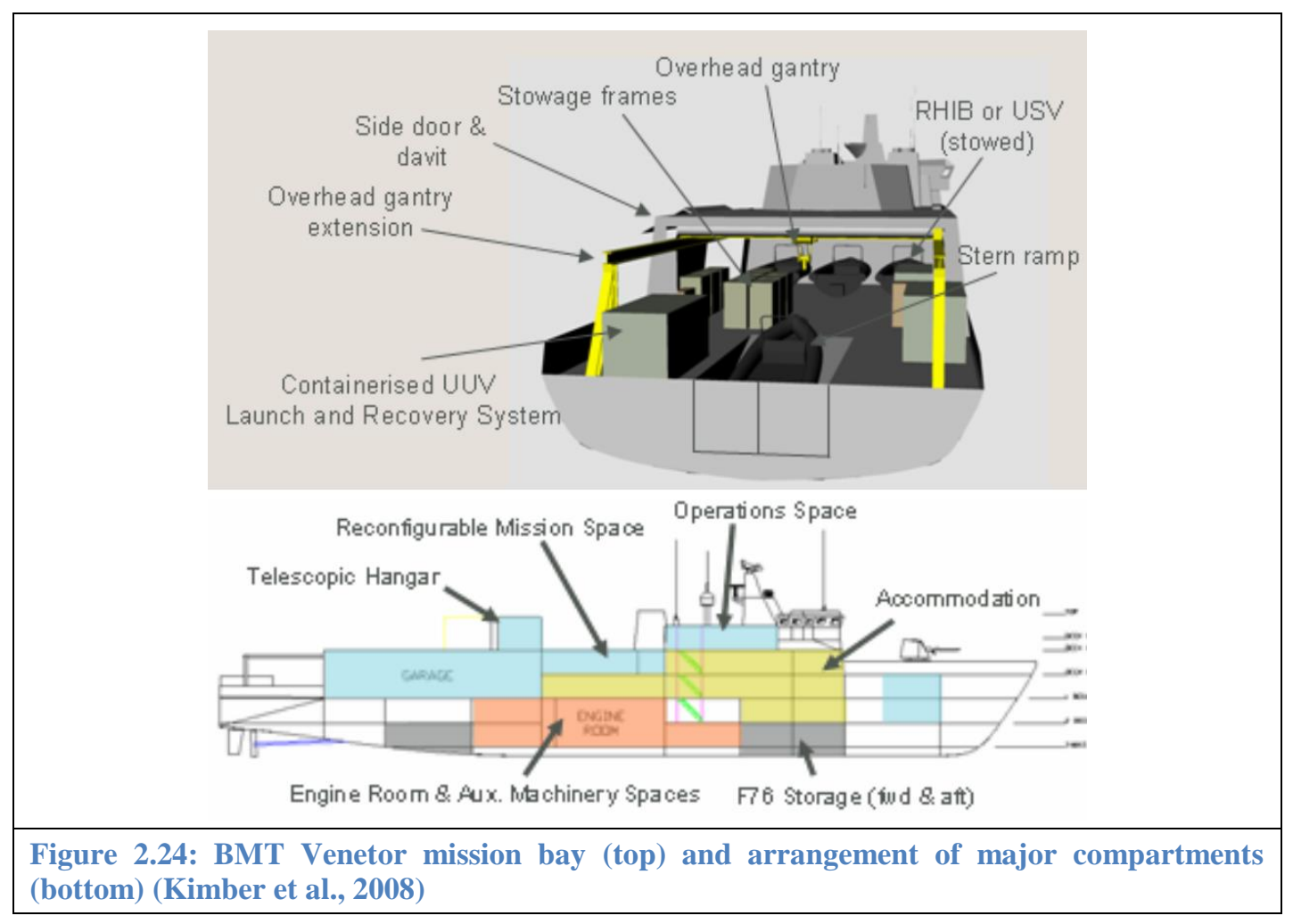

Despite the potential advantages of modular technology in areas of fleet adaptability and cost savings (Schank et al., 2016), it has been argued that it does not readily fit into the current and future UK procurement and operational contexts. Moss (2007) listed the following reasons:

- Widespread modularisation will certainly result in extra procurement costs;

- The space and weight constraints associated with the strict modularisation of certain systems may further restrict the achievable ship capabilities;

- A future UK surface combatant needs to evolve cost effectively alongside the Type 23 upgrades with some commonality of equipment, and sustain high-end, naval warfare capabilities at long range and far from other assets.

Moss (2007) therefore argued that, the requirements of the Royal Navy would perhaps be best satisfied by solutions found between the extremes of the traditional bespoke naval ship design and the fully containerised design approach.

Cellularity, as described by Gates (1985), is another concept that was intended to enhance the adaptability as well as producibility of naval ships with the core objectives of addressing installation difficulties. Traditionally most standard watertight doors and hatchways were sized according to the limits imposed by the human body, causing 
difficulties with equipment installation. Since cellularity concentrates on the areas of weapons electronics, Gates (1985) argued that the dimensions of electronic cabinets rarely exceed certain values for practical reasons, hence these limiting values could be used to define a transport envelope to improve access during build and through-life. The second standard, the installation envelope, was defined as an imaginary boundary to ensure that the space required to accommodate the cabinets, the necessary maintenance space, and the shock clearance have been properly considered. Gates (1985) also proposed that areas adjacent to the cellular weapons areas should be 'soft areas' (e.g. accommodation spaces, dining halls, stores and the like) to be 'consumed' for rapid conversion to spaces with additional combat equipment installed. Some of the design features that need to be considered to implement cellularity were seen to be constant frame spacing, routeing of through services, access and passageways, and standards for defining soft areas, as was comprehensively explained by Gates (1985). The benefits of cellularity were said to include: less outfit labour during build; improved through-life operability; more efficient maintenance and refitability; and reduced structural work during overhauls (Schank et al., 2016). Cellularity is considered to be far more flexible than the fully containerised design approach. However, these benefits and potential cost savings were found to be difficult to identify or appreciate within the narrow confines of highly initially cost constrained and UPC driven designs of the time (i.e. Type 23), and difficult to quantify, even if additional factors are included in the cost model (Gates, 1985). This instance also led Andrews (1987) to strongly argue for an architecturally-integrated synthesis as it could have revealed the advantages of cellularity early in the ship design process when the decision needed to be made.

Considering ARM as the other major supportability aspect identified as having certain architecturally discernible elements, improved and innovative concepts have become increasingly necessary given the ever higher warship sustainment costs and demands for improved availability. Hence there has been a lot of emphasis on developing tools for simulating maintenance tasks and detailed level analysis, as well as maintenance management strategies. John et al. (2003) tackled the need for a structured data-driven approach to maintenance by developing an integrated and structured systems approach in the construction industry, termed a Through-Life Business Model (TLBM), shown in Figure 2.25. This would manage the data required for maintenance support and 
decision making for building services systems, an area of engineering with sustainability issues that are very similar to the maritime world. Using the model, ILS was applied as part of a wider sequential (i.e. waterfall) process that consists of certain phases, stages, and milestones as shown in Figure A1. 8 (Appendix 1). However, in addition to the extensive requirement for reliable data, the sequential nature of the TLBM process was found to significantly inhibit genuine requirements elucidation (i.e. examining the requirements based on proper design feedback) as, according to Andrews (2011), is generally the case in sequential processes.

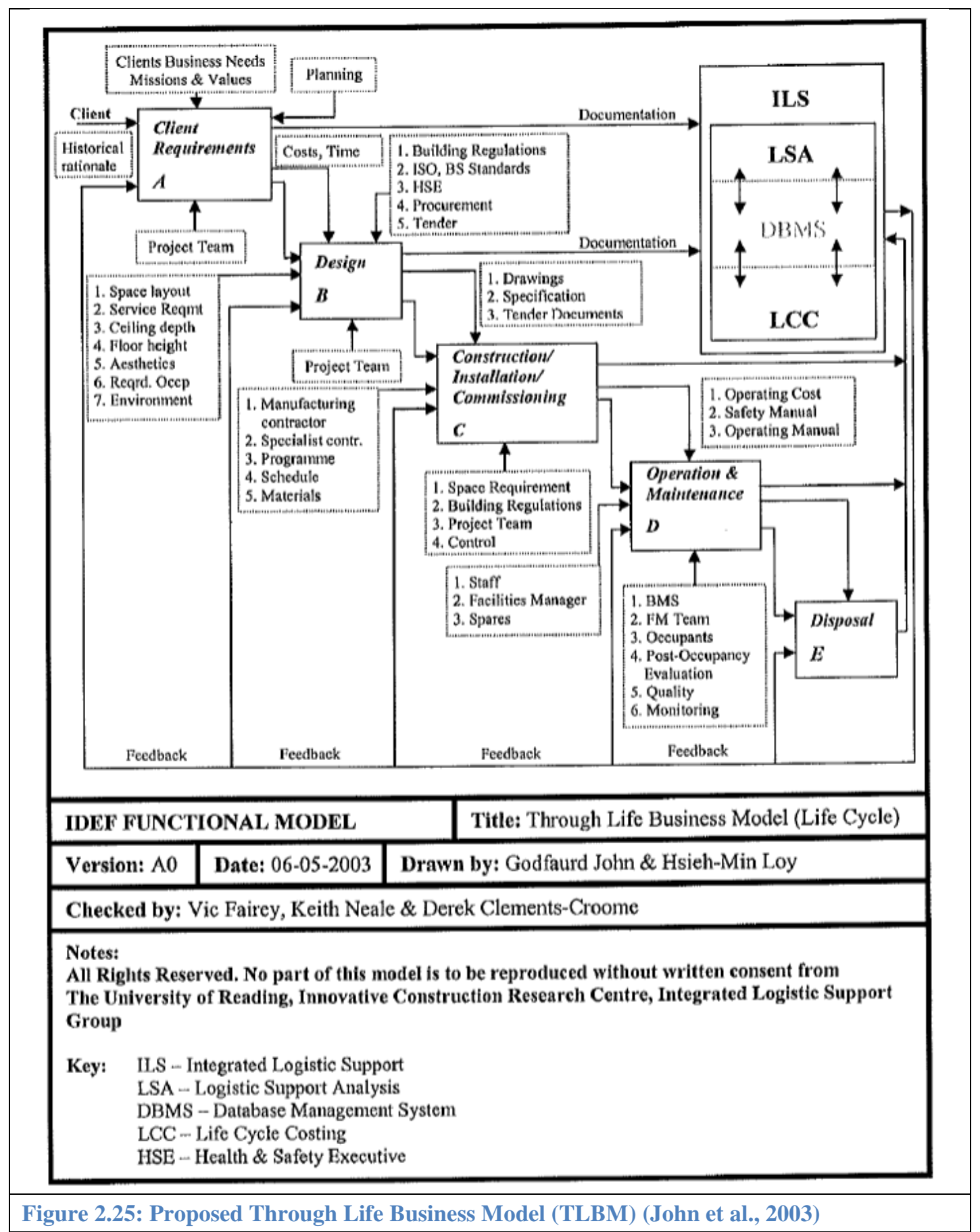


The lack of a structured data-driven approach was also highlighted by Drayton (2014) as the primary cause of failure to improve the cost and schedule performance of a naval ship maintenance programme. To counter this, a structured, multidimensional, and prioritised approach was developed by Drayton (2014), aimed at improving effectiveness of the maintenance workforce and to reduce the number of hours required to finish a given maintenance task. Despite delivering significant improvements across a range of maintenance, repair, and overhaul organisations, the approach was focused on tackling the organisational aspects of maintenance and did not address the design issues.

Tinga (2014) emphasised the importance of planning and execution of timely maintenance as a vital element in developing innovative predictive maintenance concepts to improve the life cycle management of naval systems. That work looked at developing:

- Preventive maintenance methods based on component failure mechanisms;

- Innovative sustainment based on advanced analysis of usage and failure data;

- Condition Based Maintenance $(\mathrm{CBM})$ in cases of unknown failure mechanisms;

- Optimisation of maintenance intervals at the whole ship level, based on the different usage patterns, failure mechanisms, and degradation rates of all subsystems.

Hill and Jarman (2014) of Babcock International Group have presented a modelling tool, 'SUPPORT Sim' (see Figure 2.26), which was developed to help ensure a continuous service can be maintained with a limited number of critical assets. The tool simulates failures and consequential repairs, and examines the $\operatorname{task}(\mathrm{s})$ the asset is assumed to be performing at the time of failure or is scheduled to perform during the expected duration of the repair activities. SUPPORT Sim has been used to assess both the overall design and the design of the support solution throughout the design phases of a key defence programme (Hill and Jarman, 2014). However, the tool was found to be heavily dependent on understanding how the asset behaves, interacts and responds when deviating from the planned maintenance and operating cycle. The required data includes MTBF, MTTR, whether or not failure can be repaired at different locations and, the probability of spares being available at different locations, and the probability 
of an item being beyond economic repair once failure has occurred. Considering the general lack of such information during ESSD, the SUPPORT Sim tool was found to be inapplicable to early considerations of TLS.

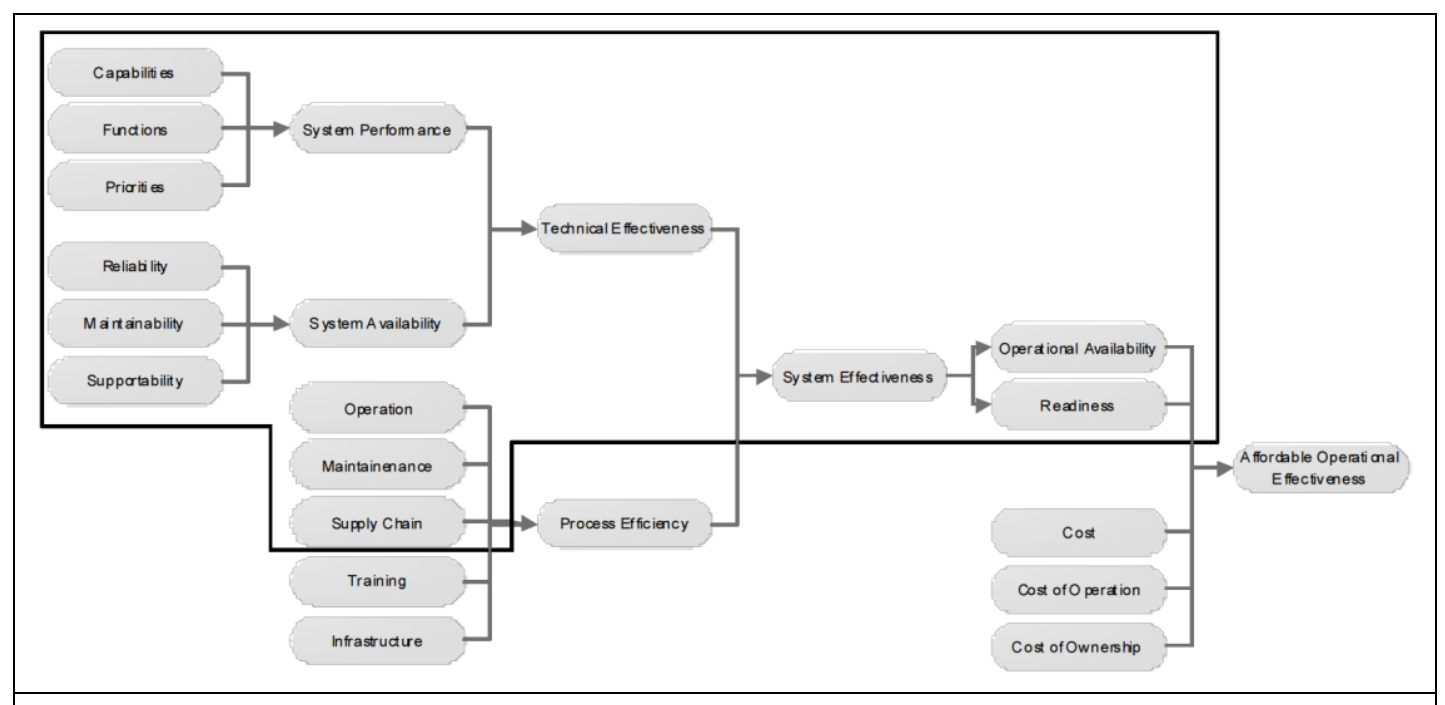

Figure 2.26: Current (boxed area) and future scope of SUPPORT Sim (Hill and Jarman, 2014)

Malone et al. (2014) developed a simulation model to investigate how long-term maintenance decisions could be made and how the issue of under-maintenance could contribute to a material-driven failure in a naval ship (e.g. loss of structural integrity due to corrosion caused by insufficient paint maintenance) to meet their mission requirements, particularly in the second half of the ship's lifetime. This work identified the interactions between maintenance backlog and modernisation, the effect of past and future asset usage on service life, how deferred maintenance could cause a capability gap (i.e. the difference between the desired and the actual capability of the system) that adds up over time and becomes more expensive to repair, and how limited maintenance budgets restrict maintenance activities to the most critical issues. This work strongly advocated the use of RCM and CBM as the two main analytical techniques used in the maintenance of complex systems to reduce the financial costs and impacts on availability. The key variables and interrelationships of the framework are shown in Figure 2.27. 


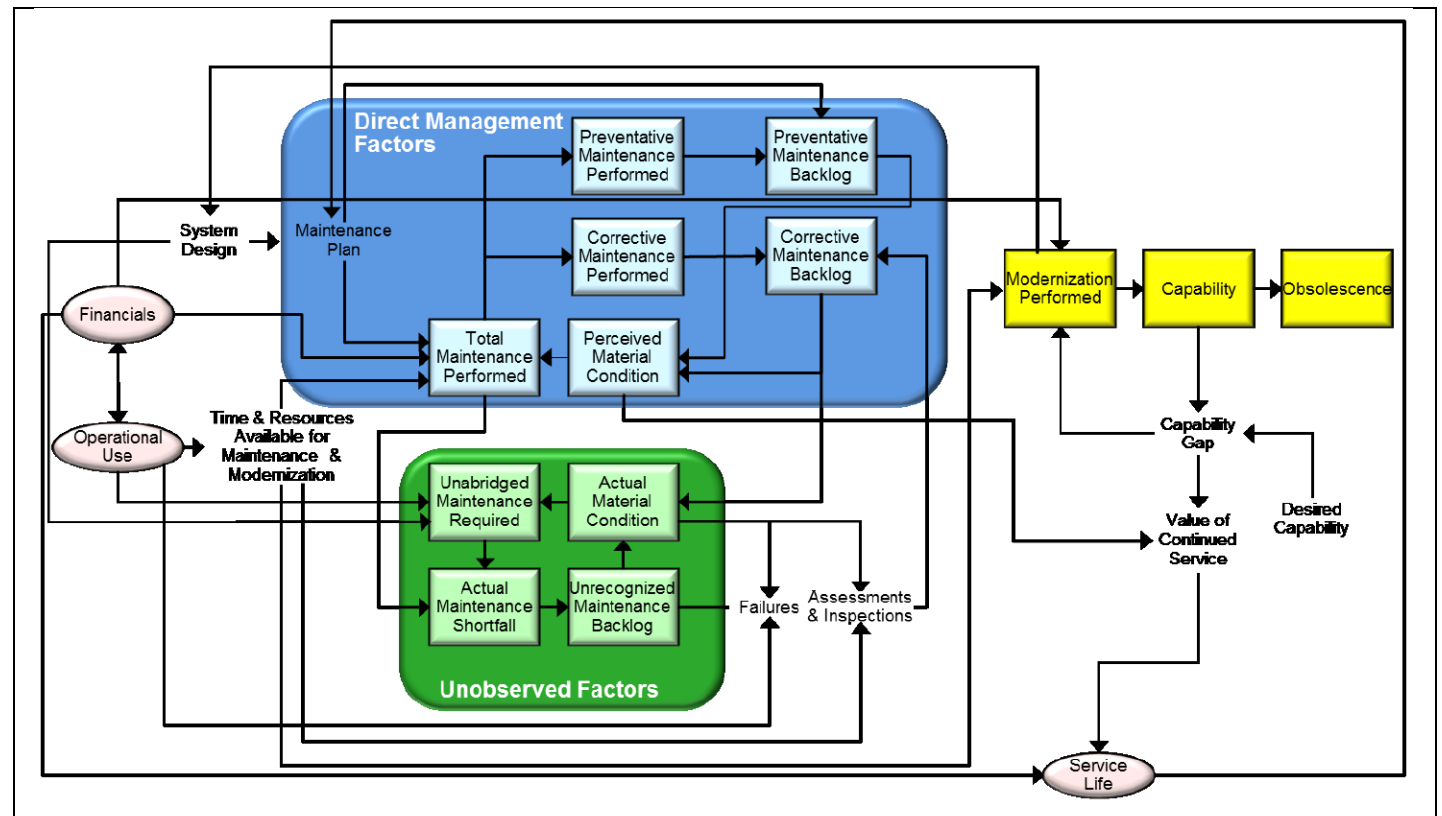

Figure 2.27: The maintenance and modernisation framework captures the key dynamics of material condition and service life (Malone et al., 2014)

Malone et al. (2014) stated that RCM and CBM are closely related, hence these techniques should be utilised in a complimentary manner instead of arguing in favour of one technique over the other. In RCM, the objective of maintenance is to preserve the function of an asset, that in turn justifies the need for CBM and provides an understanding of the applicability and effectiveness of the proposed CBM processes and technologies such as health monitoring and prognostics (Malone et al., 2014). According to the US Department of Defence (2011), RCM is a formally structured and highly documented process that includes the sequential identification of functions, functional failures, failure modes, failure effects, failure consequences, and maintenance tasks and intervals. Proper application of RCM relies on a great deal of supporting information and data that can only be produced from actual operational and test environments, and if unavailable, the analysis has to rely on assumptions based on expert knowledge (US Department of Defence, 2011), hence it is not applicable to ESSD of a new vessel.

Price et al. (2014) have explained how recent naval ship support arrangements set up by the UK MoD, including those for the latest programme (the Type 26 Global Combat Ship (GCS), have favoured Contracting for Availability (CfA). That approach aims to incentivise industry to be more innovative regarding TLS, to deliver improved availability and have more accountability for its delivery. However, the US Government Accountability Office (2010) reported that the increased reliance on the 
industry for deep maintenance, coupled with defence downsizing, has and will further deteriorate the experience and capabilities of navy maintainers in working on equipment, diagnosis and repair, thus reducing the cost-effectiveness of military support.

The CfA approach involves two fundamental techniques to analyse support options; bottom-up, or top-down modelling. The bottom-up approach, applied in programmes like the Type 45 Destroyers and Queen Elizabeth Class Carriers, produces robust answers but requires a large amount of data, including the reliability and maintainability characteristics of the equipment fitted, as well as the availability of a comprehensive spare parts list. The alternative is the top-down Multiple Criteria Decision Analysis (MCDA) approach that was implemented by Price et al. (2014) in the development of the Type 26 GCS support options to help argue for a more consistent approach to supportability from the initial design phases right into the inservice period. The key outcome of this work was that the Type 26 support solution should focus on supporting the on-board maintainers through the provision of better training and monitoring tools.

Despite not considering the configurational aspects of naval ship supportability, Price et al. (2014) demonstrated the advantages of top-down structuring of multi-criteria decision problems in comparison to bottom-up approaches. These benefits have been described as being able to provide an answer at a much shorter time span and consider much broader aspects, while reducing the need for overly detailed ship design information and being more resilient to changes in the data when compared to bottomup approaches (Price et al., 2014). These advantages are further highlighted considering the lack of highly detailed ship design information and robust data in the creative and fluid early stages of the naval ship design when the customer's requirements should still be undergoing elucidation (Andrews, 2011). The above justified further investigations into the top-down structuring of multi-criteria decision problems for DfS in ESSD, as described in the following section. 


\subsection{Top-down Structuring of Multi-Criteria Decision Problems}

To reduce the need for overly detailed ship design information and make supportability investigations feasible in ESSD, top-down structuring of multi-criteria decision problems was investigated. The three different topics explored were Decision Analysis; Effectiveness Analysis; and Multi-Criteria Decision Making (MCDM) techniques.

\subsubsection{Decision Analysis}

Keeney $(1982,1984)$ defined Decision Analysis as 'the formalisation of common sense for decision problems which are too complex for informal use of common sense' or more technically as 'a philosophy, articulated by a set of logical axioms, and a method and collection of systematic procedures, based upon those axioms, for responsibly analysing the complexities inherent in decision problems.' In the absence of a framework to articulate and integrate the values and professional judgments of decision makers and experts, the incorporation of judgments and preferences has been left to informal procedures, undefined assumptions, and the intuition of decision makers (Keeney, 1982). Decision Analysis, illustrated in Figure 2.28, is considered to provide such framework. A comprehensive explanation of Decision Analysis has been provided by Keeney and Raiffa (1993).

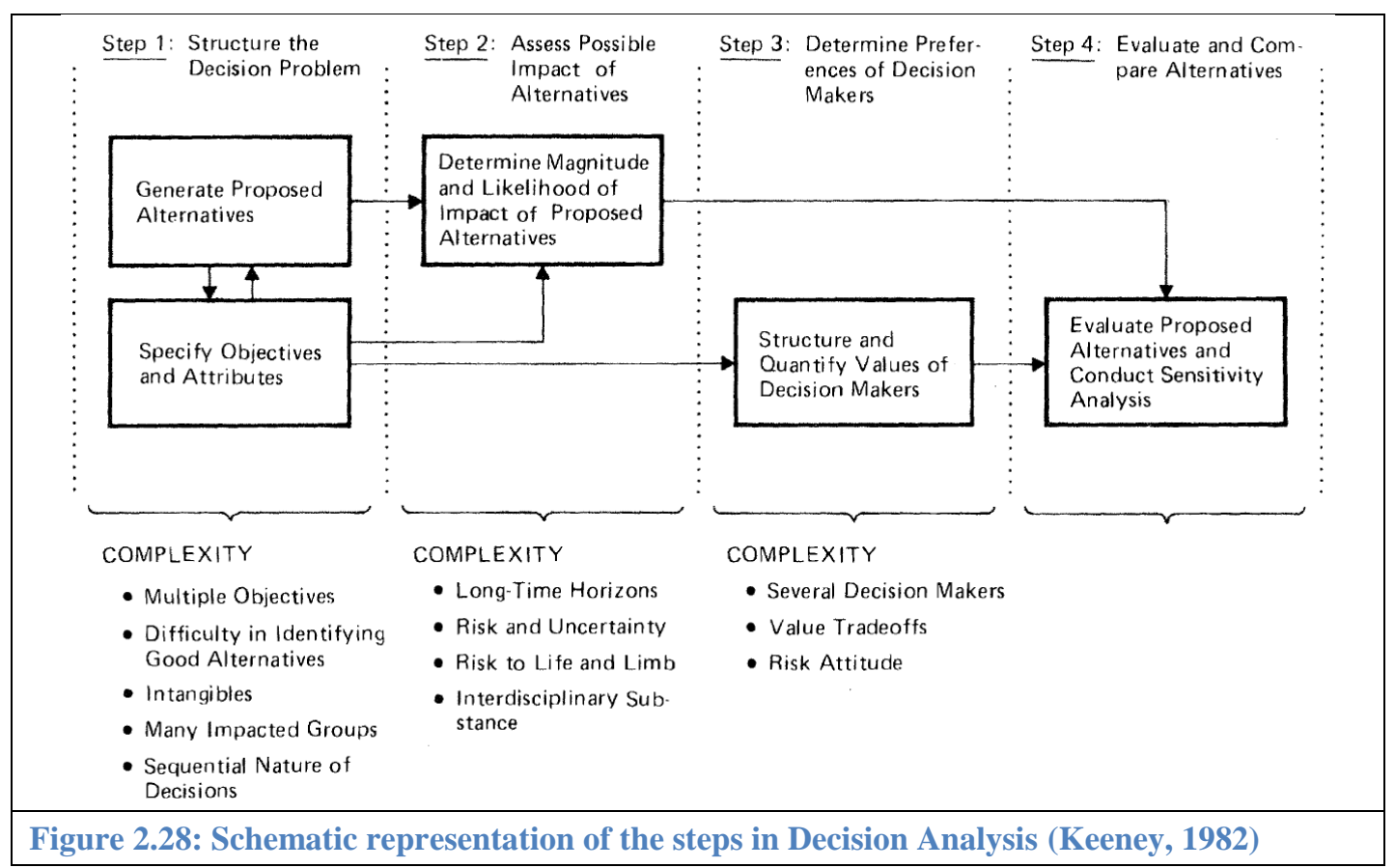


Keeney (1996) argued that the usual approach to decision making is alternativefocused. It is reactive rather than proactive and is focused on generating alternatives first and only considers the objectives afterwards. By just focusing on alternatives the decision makers will not be in control of the decision problem. Instead, Keeney (1996) proposed 'value-focused thinking' and defined it as 'a way to channel a critical resource - hard thinking - to lead to better decisions.' Unlike alternative-focused thinking that is designed to solve decision problems, value-focused thinking is designed to identify desirable decision opportunities and create alternatives. Valuefocused thinking particularly emphasises the need for a clear structure in developing the objectives in strategic decision contexts. In short, (Keeney, 1996) listed three features required for stating an objective explicitly; decision context; the object; and direction of preference; and used a simple example, that addressed the reduction of environmental impacts caused by the activities of a forest products company, to outline the procedure as:

1. Decision context: harvesting natural resources;

2. The object: environmental impact;

3. Direction of preference: less impact is preferred to more.

In addition, Keeney (1981) identified the lack of natural or direct attributes (e.g. cost in millions of dollars) as a major difficulty in many Decision Analysis studies. For example, in an investment decision scenario with the objective of maximising profits, a direct attribute could be defined in the form of net millions of pounds to measure the degree to which the objective is achieved. However, for the decision regarding a new nuclear power plant with the objective of minimising the socioeconomic and environmental impacts, the attributes are less clear and the impacts not as straightforward. To tackle this problem, Keeney (1981) proposed the use of proxy attributes that 'relate to the degree to which an objective is met, but do not directly measure it.'

\subsubsection{Effectiveness Analysis}

The US Office of Aerospace Studies (2013) described Effectiveness Analysis as the most complex element of the Analysis of Alternatives (AoA) that aims to determine the military value of the design alternatives in performing mission tasks. While there 
is no consensus on specific definitions, Hootman (2003) listed the terms broadly used in determining estimates of military performance:

- Effectiveness is 'the condition of achieving a requirement' (Hockberger, 1996);

- System effectiveness is 'the ability of a system to accomplish a mission, and achieve a favourable battle outcome' (Brown, 1995);

- Dimensional Parameters (DP) are 'properties or characteristics in physical entities the values of which determine the behaviour of the system under consideration' (Green and Johnson, 2002);

- Measures of Performance (MoP) are 'related to inherent parameters (physical and structural) and measure attributes of system behaviour' (Green and Johnson, 2002). MoPs are 'generally non-probabilistic performance measures and the consequence of specific configurations of physical elements' (Brown, 1995). MoPs are defined and measured with the system isolated and under standardised conditions (Leite and Mensh, 1999);

- Measures of Effectiveness (MoE) are 'measures of how individual systems perform their function within an operational environment' or 'metrics that measure the degree of effectiveness attained in achieving a requirement' (Green and Johnson, 2002). MoEs are defined according to the nature of the mission objectives and are external to the characteristics of the systems (Hockberger, 1996);

- Overall Measure of Effectiveness (oMoE), also known as the Measures of Force Effectiveness (MoFE) or Measures of System Effectiveness (MoSE) is the 'measure of how the overall system performs its mission' and is the collective result of all individual MoEs;

- Measures of Merit (MoM) are 'a general term for all measures that characterise a system under analysis' (Hootman, 2003) and 'and subsume all the measures that characterise a systems.' Depending on the context of the analysis, a MoM could be a MoP, MoE or oMoE (Green and Johnson, 2002). 
The steps involved in the process are shown in Figure 2.29.

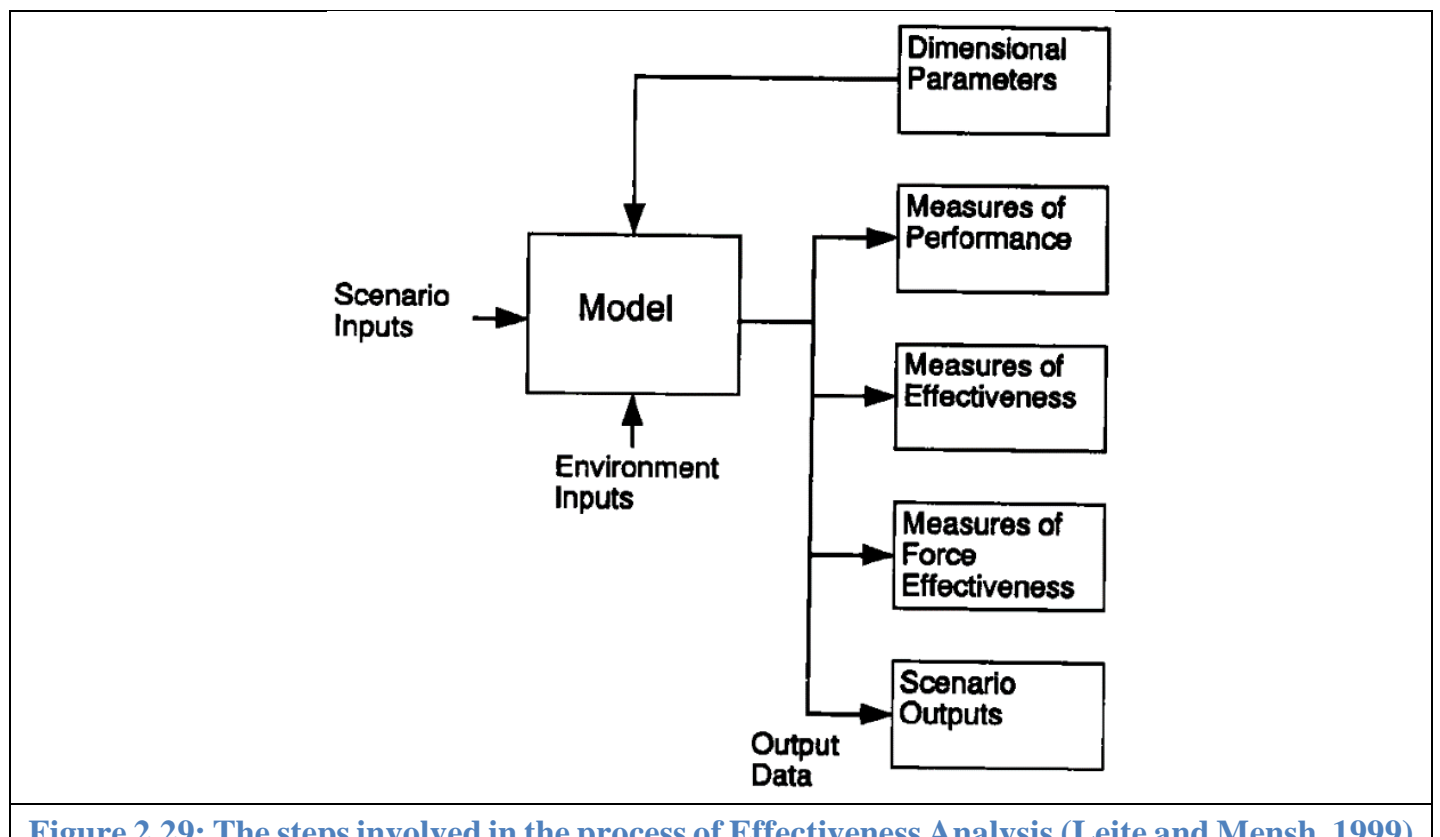

Figure 2.29: The steps involved in the process of Effectiveness Analysis (Leite and Mensh, 1999)

The process shown in Figure 2.29 is very much hierarchical as 'MoEs are often based on multiple MoPs and MoEs might support other MoEs at higher levels' (US Office of Aerospace Studies, 2013). An example oMoE hierarchy is shown in Figure 2.30.

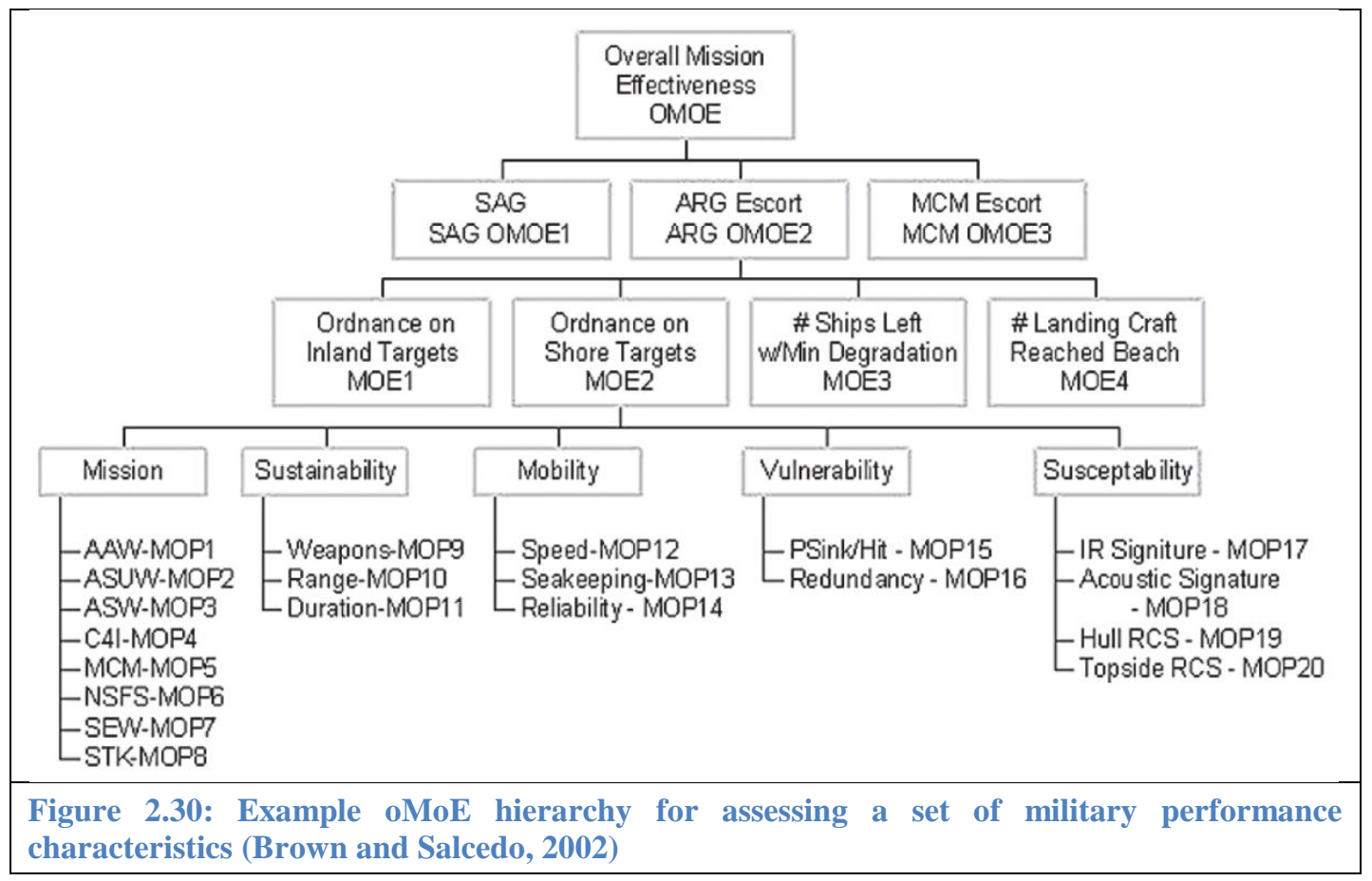

The instructions on how to define and determine each of the terms included in an Effectiveness Analysis hierarchy are mostly focused on military systems, their effectiveness in performing given military tasks, and a relatively small but frequently 
required and highly significant set of military performance characteristics (Hootman, 2003; US Office of Aerospace Studies, 2013). Therefore, Effectiveness Analysis might not immediately appear as entirely applicable to the analysis of non-military performance aspects of a given system, such as supportability. However, in a paper on the role of Operational Research (OR) in organisational decision making, Kerr (1983) advocated an alternative approach in which techniques like Effectiveness Analysis take on a 'facilitating' role. This provides the decision makers with improved methods to explore their own perceptions of the decision problems, either through closer integration with the process of producing solutions, or by providing the decision makers with a set of analytical tools to examine their own personal solution space. It was therefore considered that Effectiveness Analysis could be applicable to this DfS research specifically as a tool for structuring and combining various quantities to produce an overall measure that would help conduct comparative studies and identify design trends and drivers.

\subsubsection{Multi-Criteria Decision Making Techniques}

The investigation of a ship design should always take into consideration the fact that ships are composed of numerous different systems, many complicated in their own right and their interactions even more so (Hootman, 2003). Hence MCDM techniques might be useful to objectively model and evaluate what is essentially a system of systems. Similar to Effectiveness Analysis, MCDM was also considered to be potentially advantageous, but only to facilitate comparative investigations and generate insight, not to drive the engineering decision making process.

There are two approaches to modelling MCDM problems; multiplicative and additive. The multiplicative approach involves the multiplication of given attribute measures, leading to a single but unweighted overall measure. The additive method involves applying weightings to attributes that reflect their relative importance, followed by adding the weighted attributes to produce a single weighted overall measure (Brown, 1995). There are both advantages and disadvantages in the aggregation of attributes and preferences to form an overall effectiveness score or a single-attribute utility function, hence it should be approached with caution. Referred to as 'rolling up the results' by the US Office of Aerospace Studies (2013), aggregation can generally help simplify MCDM problems by enabling the comparison of alternatives using fewer 
effectiveness measures. This often involves mathematical transformations such as normalisation and will spare the decision makers from being overwhelmed by much more information that they can reasonably be expected to handle (Hootman, 2003). However, the disadvantage of such simplification is that information and potential insight are lost in the roll up process that could potentially lead to misleading results. Hence, the US Office of Aerospace Studies (2013) suggested that aggregation should be used only when the following conditions have been met:

- The aggregation arises naturally from relationships among the MoEs;

- The significance of the aggregates is clear;

- The aggregates tell a clearer story than the individual MoEs.

Given that the conflicting preferences of decision makers are an integral part of naval ship design (Hootman, 2003), additive MCDM methods were investigated for this research and the most prevalent approaches are as follows:

\section{Weighted Sum}

The simplest and most commonly used method is the Weighted Sum (WS), implemented by summing the product of individual attribute levels and their associated objective weights to achieve an overall Figure of Merit (FoM) (Whitcomb, 1998)

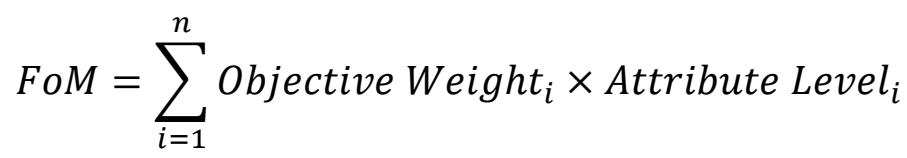

Whitcomb (1998) cautioned against using WS in decision problems because a) objectives and attributes are defined on a single level, potentially hiding the interrelationships, and b) risk and uncertainty are assessed through a single global weight and regardless of its nature.

\section{Hierarchical Weighted Sum}

The Hierarchical Weighted Sum (HWS) is a modification of WS that uses objective hierarchy instead of the single level objective sum of products. The method has been used in the design of a special warfare submarine (Price and Whitcomb, 1992) and for submarine Air Independent Propulsion (AIP) subsystem selection. HWS allows the 
inspection and modification of the weightings information prior to the evaluation of design alternatives.

\section{Analytic Hierarchy Process}

The Analytic Hierarchy Process (AHP) is a formalised evaluation method developed for solving multi-attribute decision problem. It involves structuring the decision problem in a top-down manner, consisting of top level objectives of the decision problem to the lowest level which is a set of system characteristics (Saaty, 2008). Crucially the method relies on constructing a set of pairwise comparison matrices to compare and weigh objectives and characteristics at different levels of the hierarchy. This creates a relative importance scale and allows the inspection and modification of the weightings information prior to the evaluation of design alternatives. The overall score of an alternative is obtained by aggregating the product of its individual objective scores and respective objective weights (Whitcomb, 1998). An example of the AHP applied to determine the most suitable job after a student obtains his/her $\mathrm{PhD}$ is shown in Figure 2.31.

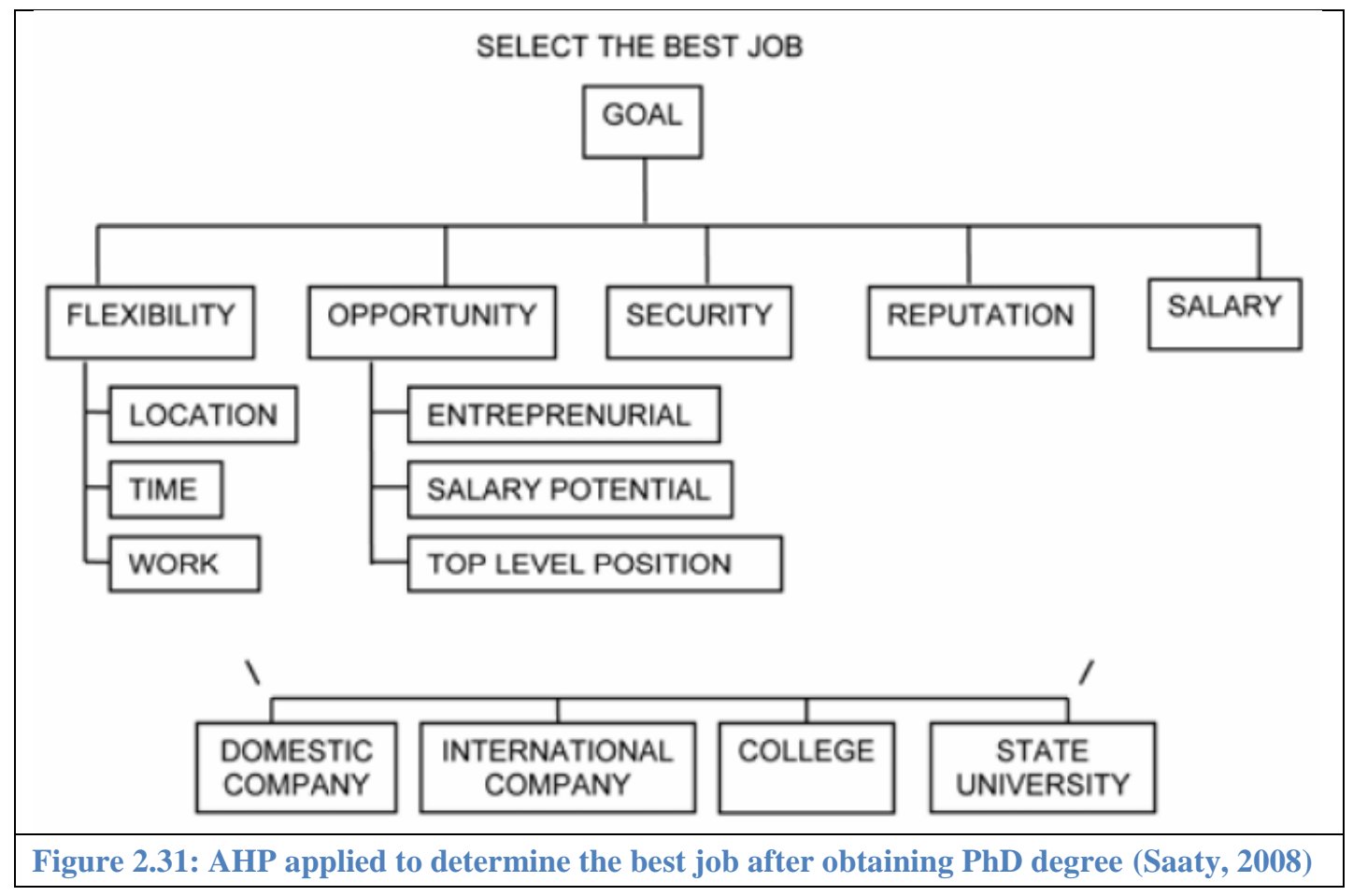




\section{Multi-Attribute Utility}

The Multi-Attribute Utility (MAU) analysis is solely based on the preferences and priorities of decision makers and includes characteristics such as uncertainty and risk in selecting design alternatives. The analysis relies on nonlinear single-attribute (i.e. independent) utility (i.e. measure of satisfaction) functions to capture the preferences of decision makers towards each of the individual objectives and attributes. Individual utilities are then combined into an MAU function. In utility theory, many decision makers demonstrate a combination of risk-acceptance and risk-aversion in their preference structure, depending on the level of attributes, as shown in Figure 2.32. Although a major benefit of MAU analysis is the ability to incorporate the decision makers' nonlinear preferences, it does not allow the single-attribute utility functions to be readily modified if the risk profile changes as the decision process unfolds and more information becomes available. Consequently, the entire utility function development process has to be performed again, with major implications on cost and time-effectiveness. Finally, while a hierarchy is not necessarily required for MAU analysis, it helps define independent attributes required for generating single-attribute utility functions (DeNeufville, 1990; Whitcomb, 1998; Hootman, 2003). However, the concept of truly independent attributes is considered to be unrealistic and unfeasible in the context of ship design, and even more so for warships.

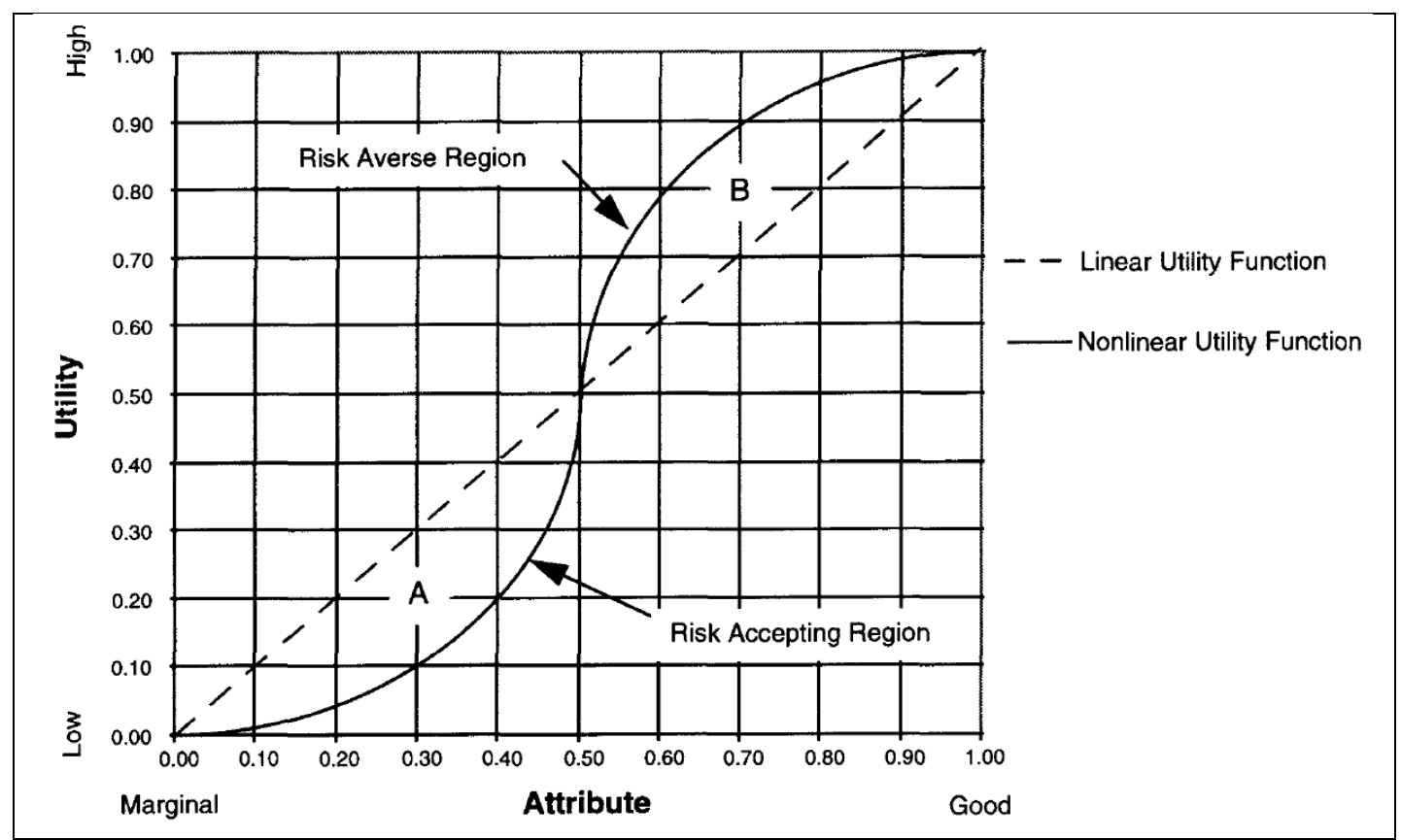

Figure 2.32: Variations of marginal utility for linear and non-linear utility functions with riskacceptance and risk-averse regions (DeNeufville, 1990) 


\subsection{Conclusion from the State of the Art Review}

Since the end of the Cold War, most major western navies have experienced reductions in fleet size, partly due to the much improved reliability and availability of individual items of equipment and vessels, but largely due to the decline of defence budgets, coupled with the above inflation escalation of warship procurement costs. To tackle these developments a number of wide-ranging and innovative approaches that potentially compromise the performance of the vessel have been proposed, notably in the area of naval ship design. However, efforts to develop well-engineered and innovative cost reduction approaches have been hindered by a series of issues.

The socio-technical nature of naval ship design and the tendency of governments to focus on short-term rather than long-term strategies, early years' expenditure, and requirements rather than solutions, coupled with the shrinkage in expert ship design knowledge of the last few decades, have meant that a lower priority is given to through-life aspects by the ship owner, the UK MoD in the case of the Royal Navy vessels. The UK MoD (2002) Smart Acquisition process includes a lot of ILS handwaving but fails to adequately 'engineer' ILS in the early phases of naval ship design. As a result, attempts to develop new cost reduction approaches have usually been aimed at UPC rather than the much larger TLC. Consequently, those aspects of naval ship design that have historically been difficult to quantify, like TLS, might imprudently seem like attractive areas for cost cuttings. This could result in ships that are too physically tight (e.g. The Royal Navy's Type 42 Destroyers and Type 23 Frigates) or poorly engineered (e.g. HMS Ocean and the warfighting inadequacy of commercial equipment), hence such ships are more likely to be unavailable at times of need, expensive to run, difficult or impossible to upgrade, and even more difficult to build in the first place.

TLS is rarely considered in ESSD, partly due to the socio-technical factors discussed above, but also due to its essentially 'soft' or uncertain nature compared to the more numerically quantifiable attributes. In naval ship design, capabilities like supportability features are inherently difficult to quantify and include intrinsic ambiguities, hence overall TLS has historically been assessed by experienced ship designers and ILS practitioners. However, it is considered that an early investigation 
of TLS aspects has also been traditionally limited by the lack of numerical and structured ways to address such design aspects in ESD, when relying on explicit knowledge, values or solutions (e.g. specific equipment selection, failure rates, and repair times) is not really possible. The lack of such approach compatible with the high level design definitions produced in the Concept Phase has not been questioned, leading to reliance on expert TLS knowledge in the subsequent ship design phases. Although innovative, the majority of developed TLS technical solutions, strategies, and tools investigated for this research were found to be either excessively solutionfocused or largely depend on highly detailed ship design information and a great deal of supporting information on specific equipment (see Section 2.3.3). Consequently the development of a rational Concept Phase evaluation scheme that gives ILS issues much greater consideration does not currently exist.

Additionally, innovation in design development and design approach is considered to be an essential element in tackling the challenges caused by diminishing budgets, escalating costs, and shrinking expert knowledge. However, the traditional naval ESSD might be considered to inhibit creativity. It postpones configurational modelling until after numerical synthesis, meaning that those aspects that affect the internal configuration are themselves unable to properly influence form selection and ship size. These shortcomings are further highlighted by the fact that novelty in design and assessment is best explored during ESSD, when most crucial decisions are made but the expenditure of design resources on technical investigations is relatively little.

In conclusion, the three main obstacles to a proper consideration of naval ship TLS in the early, crucial phase of design were considered to be:

- The socio-technical issues involved in the design and procurement process and the tendency of governments to focus on short-term rather than long-term strategies, early years' expenditure, and requirements rather than solutions;

- The lack of a numerical and structured TLS evaluation approach compatible with the high level design definitions of initial naval ship design;

- The traditional naval ESSD process that naturally inhibits creativity and innovation, caused by late configurational modelling, inadequate consideration of design Style issues, and producing simplistic and evolutionary-based numerical solutions. 
There was therefore seen to be a need for a novel approach to tackle these limitations that could demonstrate the potential benefits of a rational, if incomplete evaluation of supportability alternatives at the Concept Phase. While the financial challenges of defence acquisition and the political tendencies of governments were outside the scope of this project, the development of an approach to address the shortcomings caused by the traditional naval ESSD process and the lack of a numerical and structured TLS evaluation approach was considered possible. Realising that in modern warships, many of the underlying supportability issues are architecturally-constrained (Andrews, 2003b; Andrews and Pawling, 2003), it was sensible to take full advantage of alternative, architecturally-orientated ESSD approaches like the DBB approach and to draw on the UCL DRC's associated knowledge and experience. Other important features of the proposed approach were:

- Avoid requiring highly detailed ship design definitions to enable exploration of ILS issues in the Concept Phase;

- Tackling the difficulty of quantifying many aspects of naval ship TLS in ESSD;

- Enabling the structured representation and rational application of expert naval ship design and TLS knowledge (e.g. rules of thumb) in the ESSD supportability evaluation process.

Price et al. (2014) see reducing the need for highly detailed ship design information as one of the key advantages of top-down structuring of multi-criteria decision problems. As part of investigating a potential top-down multi-criteria approach, Decision Analysis, Effectiveness Analysis, and MCDM techniques were considered and the following potential advantages were identified:

- Decision Analysis and value-focused thinking could facilitate the development of objectives in strategic decision contexts and help structure the decision problem (Keeney, 1996);

- Effectiveness Analysis might provide the tools to help structure the decision problem (Kerr, 1983; Hootman, 2003);

- MCDM techniques might assist in structuring the decision problem, as well as incorporating the conflicting preferences of decision makers (Hootman, 2003). 
However, due to the multifaceted and non-heterogeneous nature of ILS in complex naval ship design, it is proposed that any numerical outputs from Effectiveness Analysis and MCDM techniques can only be applied to generate insight and help identify design trends and drivers, rather than used to directly make engineering decisions. Finally, the lack of natural or direct quantitative attributes identified by Keeney (1981) as a major problem in many decision problems, was seen to also be applicable to research on naval ship TLS. The proposed use of proxy or indirect measures was then explored as a means of addressing this issue to some extent.

Put together, the features described above were considered to provide a basis for providing ship designers with a framework for an early stage analysis of various ship internal arrangements with respect to a range of TLS aspects. Integration of consideration of ILS related aspects with traditional issues in naval ship design was considered to be necessary in order to avoid solely focusing on the proposal as a supportability indicator. This had a better likelihood of producing believable, informative, and implementable results. It is acknowledged that in just addressing the aspects of ILS that are influenced directly by ship architecture, meant the proposed approach would not be able to deal with the full scope of naval ship TLS. However, it should help tackle the problem and therefore contribute to producing a more balanced ship design in that some consideration of ILS would be included in a more holistic ESSD process. With the more nuanced architecturally-orientated UCL DBB approach to ship synthesis becoming acceptable in ESSD (at least for complex vessels) (Tupper, 2013), then those ILS aspects amenable to being influenced by high level architecturally-driven early design choices should also be addressed as part of the design options exploration in the early phases of design. This would at least mean that these supportability aspects are not treated as secondary issues for later consideration after major style and configurational decisions have already been made. This in turn results (at best) in supportability being shoehorned into an already highly constrained 'minimal' layout and hence results, concurrently, in both compromised and hard to adjust ship configurations. 


\section{Chapter 3: Development of the Design for Support Evaluation Approach}

\subsection{Introduction}

This chapter, consisting of three sections, focuses on the development of the supportability evaluation approach that was proposed in the research presented. The three main obstacles to an early, genuine consideration of naval ship Through-Life Support (TLS) have been outlined in Section 2.5. This concluded in bringing the consideration of TLS into Early Stage Ship Design (ESSD), the proposed evaluation approach should:

- Take full advantage of architecturally-orientated ESSD approaches, such as the UCL Design Building Block (DBB) approach, to identify Integrated Logistics Support (ILS) aspects that are configurationally driven;

- Avoid requiring highly detailed ship design definitions to enable exploration of ILS issues in the Concept Phase;

- Tackle the difficulty of quantifying many aspects of naval ship TLS in ESSD;

- Help capture, apply and modify the accumulated naval ship design and TLS knowledge (e.g. rules of thumb) for a more rational analysis of TLS.

In the next section, the two methods initially investigated for the development of the Design for Support (DfS) evaluation approach are briefly covered. Section 3.3 outlines the main proposal and provides a general description of the three principal constituents of the proposed DfS evaluation approach and the associated issues. Section 3.4 provides a detailed account of how the three principal features of the proposed DfS evaluation approach described in Section 3.3 were customised in order to carry out a specific naval ship supportability investigation. 


\subsection{Initial Proposals for the Design for Support Evaluation Approach}

This section gives a brief account of the two methods of dealing with DfS in ESSD that were presented at two conferences in 2015 prior to proposing the final approach. The outline of each paper and more importantly the comments the candidate received at each conference are covered in the following sub-sections. The complete papers can be found in Appendices 2 and 3.

\subsubsection{Critical Path Method and Programme Evaluation and Review Technique}

The paper presented by the candidate to the RINA Warship Conference 2015 (Esbati et al., 2015a) proposed an approach based on the Critical Path Method (CPM) and the Programme Evaluation and Review Technique (PERT) and applied it to naval ship TLS. Given notable applications, such as the US Navy's development of the Polaris ballistic missile system (Sapolsky, 1972) and the major midlife refit and update programme for the Royal Navy's Leander Class frigates (Whitwam and Watty, 1978), this work looked at how the CPM and PERT might help solve the general problem of reducing the time required to complete a project that consists of many interrelated tasks. The investigations were focused on the midlife refit and update of a naval combatant because: a) such projects satisfy the conditions for CPM applicability (e.g. clearly definable project objectives and start and finish points, interdependent activities with broadly estimable durations) (Esbati et al., 2015a), and b) the incorrect identification of the critical path can further increase the already high cost of dry docking and delay the ship's delivery (Whitwam and Watty, 1978).

The critical path of a project is the sequence of activities, within the overall activities network that has the least float (i.e. the amount of time an activity can be delayed without delaying the completion of the overall project), and thus determines the project's earliest possible completion time (Mercier and Nunnally, 1965). Accurate determination of the critical path of a project is crucial in ensuring that the project can run to programme and enables the examination of the effects of any slippage to the schedule of critical activities (Esbati et al., 2015a). 
Considering the midlife refit of a typical frigate, a qualitative simplified work breakdown network was developed for a series of spaces commonly located amidships in such a vessel (e.g. main and auxiliary machinery rooms, accommodation spaces, galley, provisions room, dining rooms) and is shown in Figure 3.1.

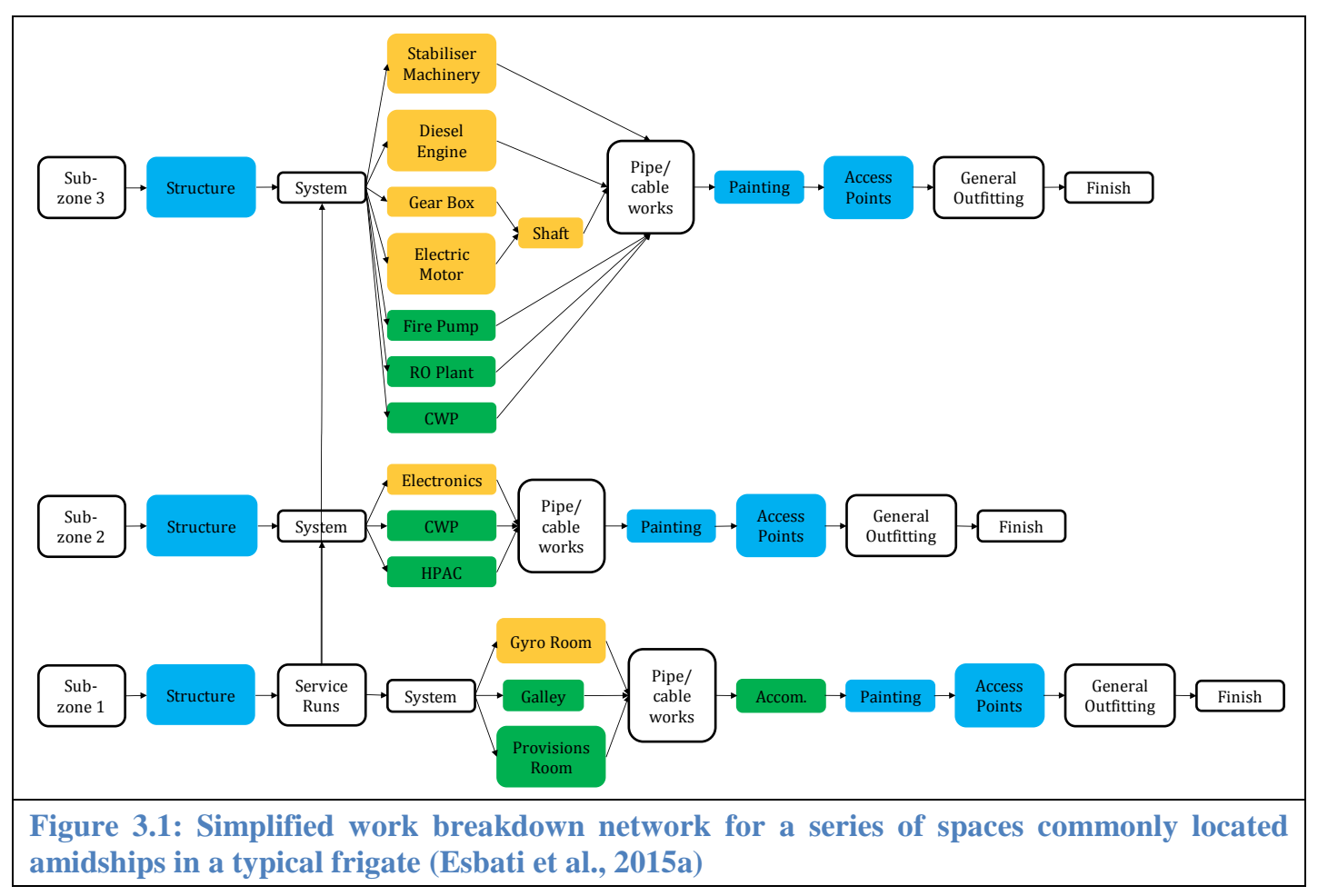

Similar networks were produced for a number of arrangement and architectural variations (e.g. double sided passageways, technical galleries on No. 2 or 3 Decks) (see page 244). Each configurational option was found to significantly affect the work breakdown network and revealed issues such as:

- Having to increase deck height to accommodate the routeing of distributed service systems (e.g. Chilled Water (CW), Heat, Ventilation and Air Conditioning (HVAC), High Pressure Air (HPA), and electrical power);

- Ballistic protection features required for double sided passageways;

- The difficulties of corrosion inspection of structural scantlings caused by the ballistic protection of vital ship services;

- Athwartship passageways that provide better personnel flow and facilitate cross deck access and escape with two sided passageways. 
If this approach was to have been taken forward, then the next approach would have been to populate the work breakdown networks with estimated durations of relevant support activities to enable design comparisons of different physical aspects. When in a refit a ship is opened up and inspected properly, emergent work (e.g. areas of corrosion) is often discovered. This will disrupt the work breakdown network and potentially affect the critical path. Hence incorporating the uncertainty caused by such emergent work was considered to be a significant element of the proposed approach. It was therefore decided to replace single time estimates with a PERT-based three point estimate system in approximating the activity durations. The creators of the traditional PERT (Malcolm et al., 1959) assumed that the activity durations in a three point estimate system would be subjectively determined and would fit a Beta distribution. This could then provide a rich family of distributional shapes when producing a distribution over some finite interval (Golenko-Ginzburg, 1988), say a few hundred workforce hours. In addition, it was also concluded that a four parameter Beta distribution with known bounds (Treat, 1984) was required to counter the limitations of the traditional PERT approach and enhance the flexibility of the distribution shape (Jensen, 2004).

However, when this approach to DfS in ESSD was presented at the 2015 RINA Warship Conference, this approach was generally treated with scepticism and concerns were raised with regards to:

- The traditional CPM/PERT model ignores the issue of emergent work as it assumes that everything would be known and planned for;

- Warships and their issues are not conventional, hence generalised distributions like the Beta distribution are questionable in dealing with problems such as emergent work;

- The proposed approach was generally considered not sufficiently multidisciplinary for a holistic investigation of warship TLS.

\subsubsection{Prospect Theory-Based Real Option Analysis}

To address the points raised at the earlier 2015 conference, an alternative approach was investigated, incorporating both operational and through-life adaptability, as subsets of TLS. This was presented at the RINA ICCAS Conference 2015 (Esbati et 
al., 2015b). The paper drew on recent work at University of Michigan, Department of Naval Architecture and Marine Engineering, which developed a novel quantitative framework for evaluating adaptability in non-commercial engineering systems (Knight and Singer, 2014). The framework, called Prospect Theory-Based Real Options Analysis (PB-ROA), takes the principles of the investment approach of Real Options Analysis (ROA) (Wang and Neufville, 2005) and applies them to noncommercial assets, such as those in the naval sector, that do not generate cash flows. PB-ROA corrects the underestimation of the value of adaptability that results from Net Present Value (NPV) and static budgetary techniques. It uses three techniques: Utility Theory (Read, 2007) to appraise assets in the absence of cash flows; Prospect Theory (Kahneman and Tversky, 1979) to help model the loss-averse attitude of organisations like navies and other military acquisition organisations; and Game Theory (Parsons and Wooldridge, 2002) to model the interdependencies of alternative design options for naval vessels and the resulting feedback to the operational environment.

In 2015 the candidate presented an example (Esbati et al., 2015b) in which PB-ROA was used to introduce some degree of quantification when comparing the extent of adaptability features that could be provided for certain ship design variants. Exploring the concept of a large reconfigurable mission bay, two design variants were investigated, a baseline variant with a fixed mission bay area, and a cellular (see Section 2.3.3) variant that had the same initial mission bay area but was able to be enlarged by 'consuming' the 'soft areas' located adjacent to the mission bay. The utility of each of the two design variants was estimated in terms of the number of Twenty-Foot Equivalent Unit (TEU) mission support containers that each mission bay could accommodate (i.e. the capability parameter). The utility values were also riskadjusted using Prospect Theory, to account for an anticipated risk-averse attitude by naval ship designers. The two design variants were then compared in terms of riskadjusted utility values plotted against the probability of the mission bay being converted to accommodate the TEUs. The probability decision threshold (i.e. the probability point where the design choice on the basis of utility would switch from one option to the other) and negative utility values were seen to be the more significant observations to be obtained by this approach. These are shown in Figure 3.2. This study demonstrated how the PB-ROA could be used to carry out a simplified yet 
quantitative comparison of the performance of two design variants in regard to different mission capabilities.

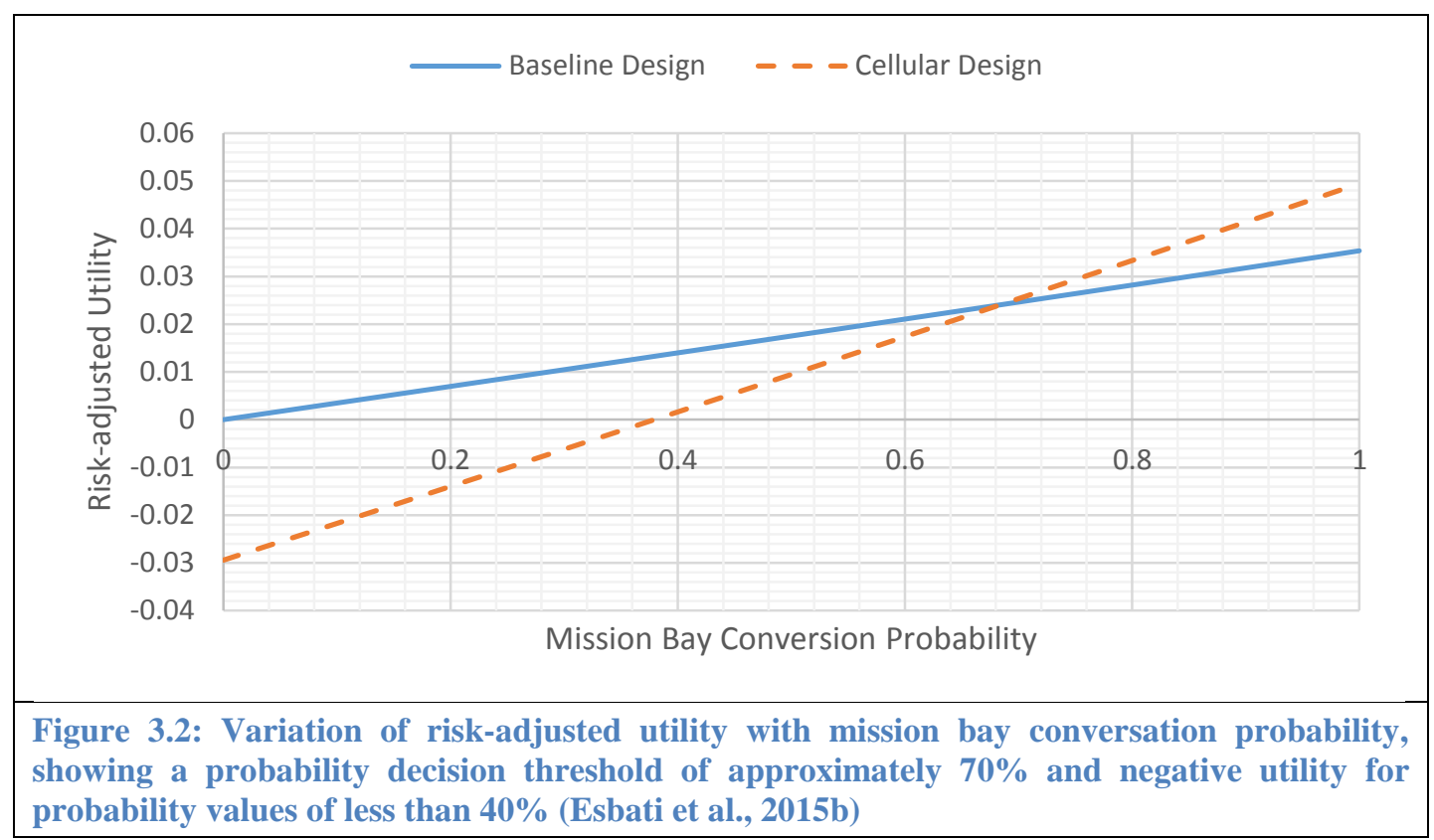

It was concluded that the next steps in such an approach could have been a more multidimensional assessment of the risk factors and capability parameters together with the incorporation of game theoretic interdependencies (Knight, 2014) that was not presented in the Esbati et al. (2015b) paper. Despite the improvements in comparison to the CPM/PERT proposal, especially in developing a more multidisciplinary and flexible approach that produced a numerical indicator of supportability, major concerns were also raised in the discussions at ICCAS 2015. The most important criticisms addressed the underlying assumptions, particularly the design complexity metric that was hard to define and had required an assumed figure. Hence the candidate concluded that this might make the study seem purely a numerical manipulation technique since any results would be based on educated guesswork.

Despite failing to meet the general characteristics considered essential to any TLS evaluation approach (see Section 3.1), a PB-ROA approach or indeed similar financial appraisal methods are considered suitable numerical techniques applicable to DfS, but only provided that numerical indicators, such as ship capability parameters and design complexity metrics, can be appropriately defined. 


\subsection{The Proposed Design for Support Evaluation Approach}

Having presented the two papers in 2015, it became apparent that in tackling the obstacles to an early and proper consideration of TLS (see Section 2.5), the required characteristics (see Section 3.1) were not totally satisfied by either approach. Major shortcomings included the applicability to naval ships and extensive reliance on information that is unavailable during traditional numerical ESSD processes. Hence the development of a proposed approach was focused on more established analysis tools and exploiting the high level ship design information available in ESSD and in particular in architecturally-orientated approaches such as the UCL DBB approach. Despite the inevitable simplicity due to the nature of the extractable data, this was considered likely to produce a more believable metrication and evaluation of supportability. While taking full advantage of the architecturally-orientated UCL DBB approach to ESSD, the proposed approach included the following principal features:

- Structuring the evaluation of naval ship TLS in a top-down manner to reduce the reliance on unavailable detailed ship design information;

- Developing a series of rational and indicative supportability metrics to tackle the difficulty in quantifying those aspects of naval ship TLS potentially available at ESSD;

- Constructing a framework to capture, incorporate, and apply the accumulated naval ship design and TLS knowledge in a rational manner.

Many overlapping issues were identified as part of tackling the above features, so they had to be addressed concurrently. The following three sub-sections provide a general description of each principal component.

\subsubsection{Top-Down Structuring of the Evaluation of Naval Ship TLS}

Both Decision Analysis and Effectiveness Analysis were found to correlate in a number of respects. Both methods require clearly specified objectives, both need to recognise the role of inconsistent prioritisation by decision makers in the design of real complex systems, and both have very similar component elements. Hence certain elements of the two methods can be used in parallel to form a more robust approach and obtain better insight into decision problems. 
Central to the application of both methods in structuring decision problems is explicit specification of objectives. In Decision Analysis, objectives need to be specified prior to assessing the possible decision impacts of different (design) alternatives (i.e. how well each alternative meets each objective), while in Effectiveness Analysis, Measures of Effectiveness (MoEs) are defined from consideration of the relevant missions or objectives. Explicit objectives are also required to structure and quantify the inconsistent preferences of decision makers. Dealing with the inconsistent priorities of decision makers is central to both methods. In Decision Analysis (illustrated again in Figure 3.3 below), the third step shown specifically deals with the relative preferences of a given decision maker, which then feed into the fourth and final step; the evaluation and comparison of alternatives.

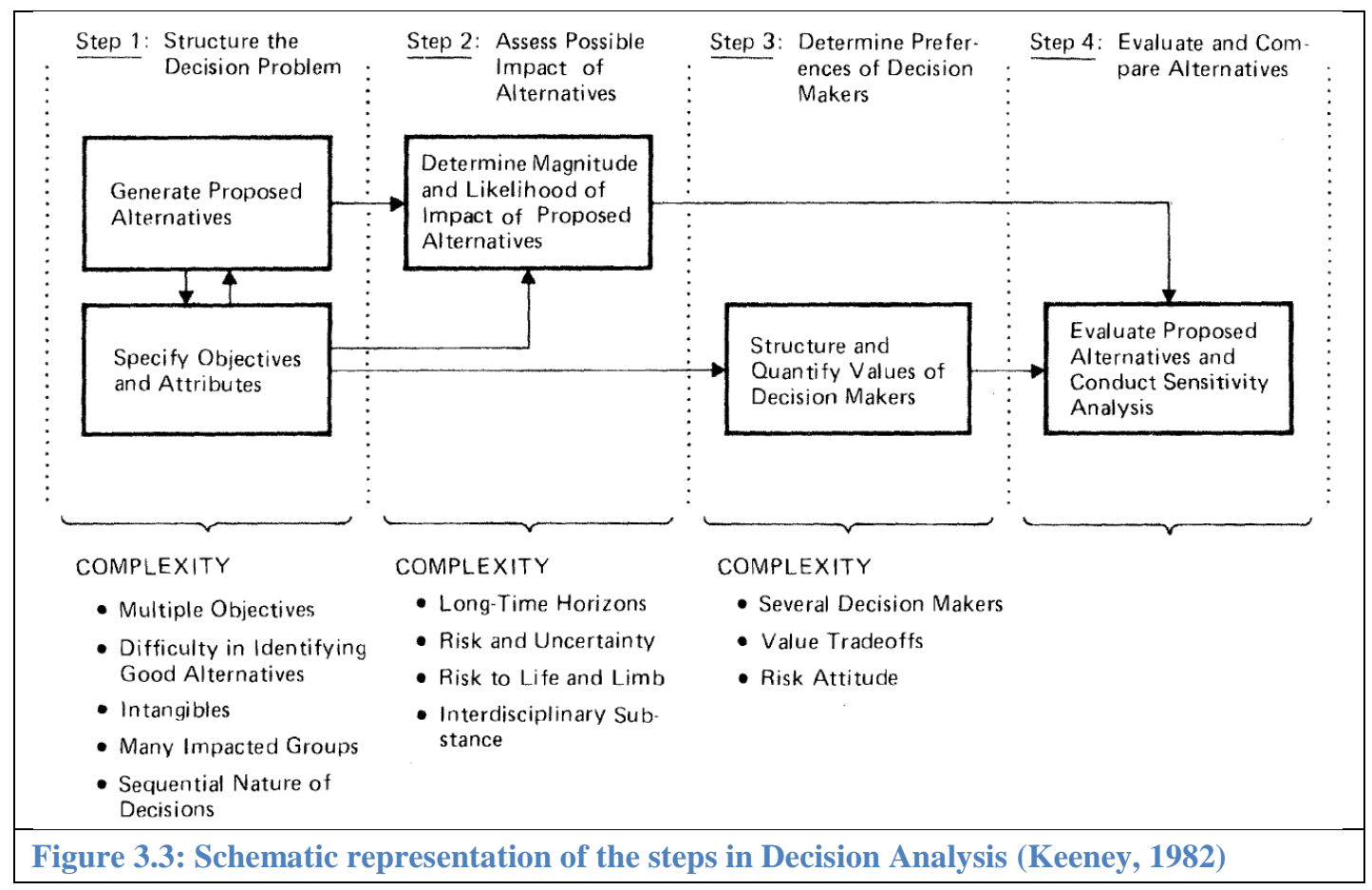

Similarly in Effectiveness Analysis, the MoEs are first weighted before being aggregated and used to assess the merit of each alternative. Finally, both methods have components that have similar definitions and roles in the assessment, as is summarised in Table 3.1. 


\begin{tabular}{|l|l|l|}
\hline \multicolumn{1}{|c|}{$\begin{array}{c}\text { General } \\
\text { Description }\end{array}$} & \multicolumn{1}{|c|}{$\begin{array}{c}\text { Decision } \\
\text { Analysis } \\
\text { Components }\end{array}$} & $\begin{array}{c}\text { Effectiveness } \\
\text { Analysis } \\
\text { Components }\end{array}$ \\
\hline $\begin{array}{l}\text { Degree of effectiveness attained in } \\
\text { performing a function based on system } \\
\text { performance }\end{array}$ & $\begin{array}{l}\text { Degree of } \\
\text { achieving } \\
\text { the objectives }\end{array}$ & $\begin{array}{l}\text { Measures of } \\
\text { Effectiveness } \\
\text { (MoEs) }\end{array}$ \\
\hline $\begin{array}{l}\text { Deterministic measures of system } \\
\text { performance based on system core } \\
\text { characteristics }\end{array}$ & $\begin{array}{l}\text { Direct or proxy } \\
\text { attributes }\end{array}$ & $\begin{array}{l}\text { Measures of } \\
\text { Performance } \\
\text { (MoPs) }\end{array}$ \\
\hline $\begin{array}{l}\text { Inherent physical characteristics that } \\
\text { determine system performance }\end{array}$ & $\begin{array}{l}\text { Core system } \\
\text { characteristics }\end{array}$ & $\begin{array}{l}\text { Dimensional } \\
\text { Parameters (DPs) }\end{array}$ \\
\hline
\end{tabular}

Table 3.1: A summary of the corresponding components of Decision Analysis and Effectiveness Analysis

Figure 3.4 presents a generic top-down structure, showing how certain components of Decision Analysis (shown in red) and Effectiveness Analysis (shown in blue) could be used in parallel to provide a better means of decision making with regard to complex issues such as DfS in the design of complex vessels.

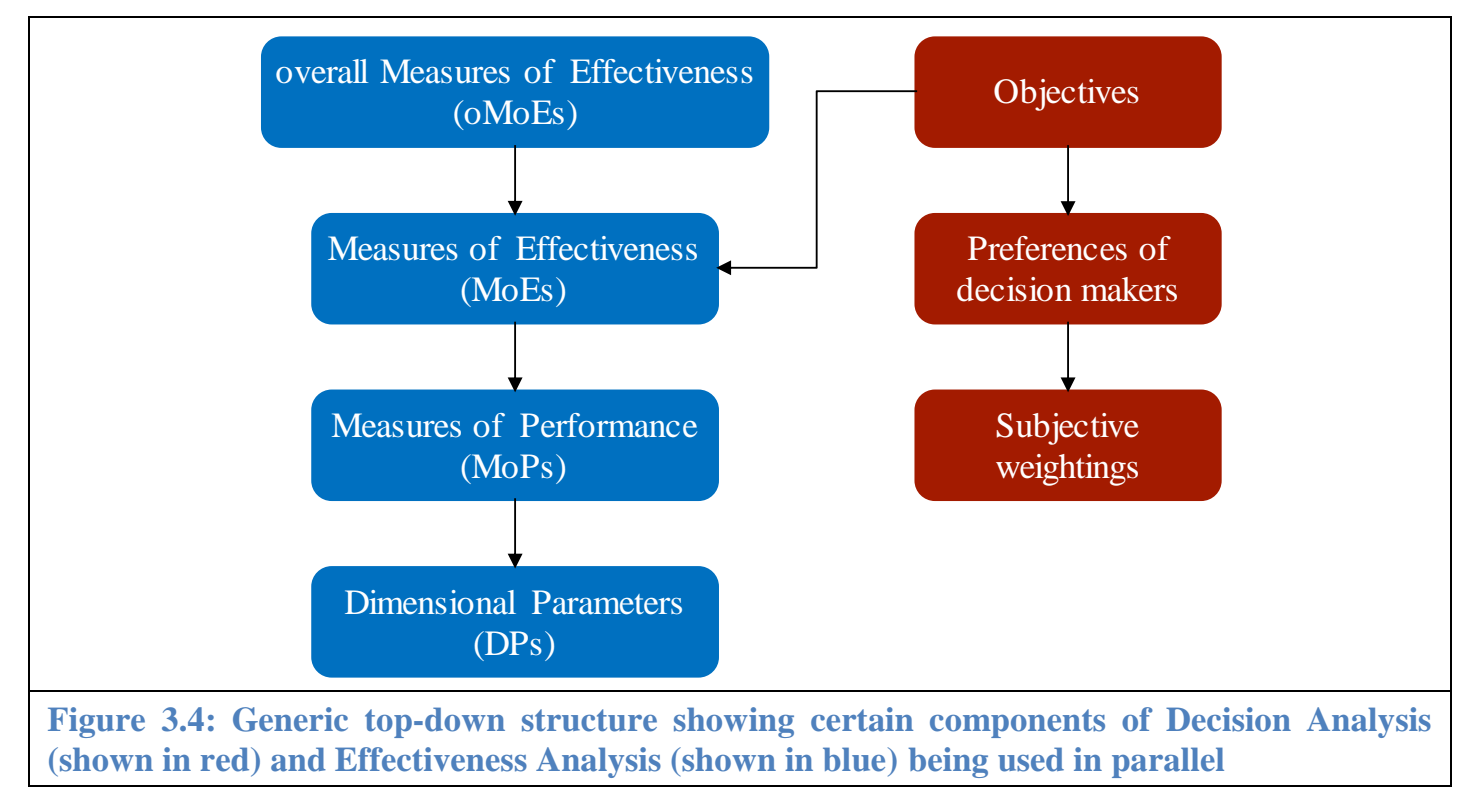

\subsubsection{Developing Rational and Indicative Supportability Metrics}

The work by Price et al. (2014) recognised that many aspects of TLS, particularly in the naval context, are not easily quantifiable and a 'currency' for measuring how effectively a system meets its assigned objectives could be difficult to establish. The proposal by Keeney (1981) to use proxy or indirect measures (see Section 2.4.1) was presented as a possible way to address this problem. Web Finance (2016) defined 
proxy indicators as 'approximate or representative measurements in the absence of direct values' (e.g. the use of ship fuel consumption as a proxy for shaft power (Aldous, 2015), the number of female members of a chamber of commerce is a proxy indicator of the percentage of female business owners or executives in society bounded by the scope of that chamber of commerce (Web Finance, 2016)). The proposal to use proxy measures was also considered by the candidate, as able to assist the use of Effectiveness Analysis in providing a 'facilitating' capability (see Section 2.4.2) when analysing the non-military performance aspects of naval ships, such as supportability.

Proxy Supportability Indicators (PSI) are essentially measures that, in the absence of direct DfS Key Performance Indicators (KPI), might enable the supportability impacts of concept design decisions to be captured. Despite being imperfect, these indicators could then potentially assist ship designers in making more informed design decisions with respect to DfS in ESSD. Given the context of this research, it was necessary to formulate proxy indicators to help analyse certain architecturally discernible aspects of TLS, such as adaptability and some aspects of maintainability.

\subsubsection{A Proposed Framework to Capture, Incorporate, Apply, and Modify Expert Knowledge}

Addressing the issue of inconsistent and, often, conflicting preferences of decision makers was a significant objective for the proposed approach. The multi-criteria nature of complex systems and the need for Multi-Criteria Decision Making (MCDM) methods to deal with aggregating performance measures have been highlighted in discussing both Decision Analysis and Effectiveness Analysis. Therefore, a framework was constructed to capture and quantify the relative priorities of decision makers and transform them into subjective numerical measures. From the four main additive MCDM methods covered in Section 2.4.3, one was selected as part of developing such a framework. The selection process was based on the advantages and shortcomings of each method explained below.

Weighted Sum (WS) (Whitcomb, 1998) was rejected because although the most commonly used, it lacks a hierarchy and limits the analysis to a single level and hence is likely to hide important interrelationships. This limitation could also cause the decision problem to become unmanageable in size since all weightings would have to 
be simultaneously determined. As for the remaining choices; Hierarchical Weighted Sum (HWS) (Price and Whitcomb, 1992), Analytic Hierarchy Process (AHP) (Saaty, 2008), and Multi-Attribute Utility (MAU) (DeNeufville, 1990); all three methods are or can be hierarchical, thus issues addressed in earlier discussion of Decision Analysis (see Section 2.4.1) and Effectiveness Analysis (see Section 2.4.2) are likely to be pertinent. By enabling a hierarchical analysis, these three remaining methods are able to ensure that important interrelationships are not neglected and the process of handling the preferences of decision makers should remain manageable. Based on work by Whitcomb (1998), the three remaining choices were compared as follows:

- The inspection and thus any necessary modification of the weightings information prior to the evaluation of design alternatives are enabled through HWS and AHP, but not MAU;

- AHP is inherently top-down, thus pertinent to the requirement for top-down structuring of evaluation of naval ship TLS in order to reduce the reliance on unavailable detailed ship design information;

- AHP uses pairwise comparisons that are considered to better facilitate the process of quantifying the decision makers' subjective preferences;

- Handling the decision makers' attitude towards risk and uncertainty is fundamental to MAU, and is possible to some extent using AHP;

- The standard procedure for developing the required single-attribute utility functions used in MAU analysis involves the construction of questionnaires and elaborate personal interviews. The questionnaires are constructed so that the personnel being interviewed are asked to compare various aspects of the decision making procedure, such as performance measures and uncertainties. The interview results are then collated to produce single-attribute utility functions (Keeney and Raiffa, 1993). However, it was concluded that the results of such interviews could be significantly biased and based on experience from outmoded vessels (e.g. heavily crewed) and old technologies (e.g. Combined Diesel or Gas (CODOG) frigates). Hence the single-attribute utility functions could be uncharacteristic of a real navy's current utility curves (Knight and Singer, 2014) and therefore may not be convincing. 
Considering the above pros and cons, AHP was selected as the preferred method to develop a framework to help capture, incorporate, apply, and modify expert knowledge. As part of applying AHP to quantify the relative preferences of a given decision maker, it was necessary to probe and select: a) the numerical scale used in pairwise comparisons, b) the method for assessing the consistency of pairwise comparison matrices, and c) the method for generating numerical preferences from pairwise comparison matrices. Each topic was thoroughly explored as described below.

\section{a) The Numerical Scale Used in Pairwise Comparisons}

In order to quantify the decision makers' subjective assessments using AHP, pairwise comparisons first need to be carried out at individual levels of the evaluation hierarchy (Cabala, 2010) to construct a set of positive reciprocal pairwise comparison matrices (Saaty, 2008). A square matrix of dimension $\mathrm{n}$ is said to be reciprocal about the leading diagonal provided that $a_{i j}=\frac{1}{a_{j i}}$ for all $i, j=1, \ldots, n$ (Shiraishi et al., 1998).

An important aspect of implementing pairwise comparisons is the underlying numerical scale used to convert the subjective linguistic expressions of personal preference into numerical values. The selection of the scale type can potentially affect the outcome of a decision problem and has important implications on the distribution and consistency of an individual's preferences. The three common numerical scale types are the traditional Integer Scale, Balanced Scale and Power Scale (Elliott, 2010). As shown in Table 3.2, for all three types, the number of linguistic phrases used is usually nine, or five if the relative preferences are less obvious (Saaty, 2008), but the numerical values used to represent the intensities of importance are different for each type. This difference is explained in the following paragraph. 


\begin{tabular}{|l|l|l|l|}
\hline $\begin{array}{c}\text { Traditional } \\
\text { Integer }\end{array}$ & Balanced & Power & \multicolumn{1}{c|}{ Linguistic Expressions of Preference } \\
\hline 1 & 1 & 1 & A is equally important to B \\
\hline 2 & 1.22 & 1.32 & A is weakly or slightly more important than B \\
\hline 3 & 1.33 & 1.73 & A is moderately more important than B \\
\hline 4 & 1.86 & 2.28 & A is moderately plus more important than B \\
\hline 5 & 2.33 & 3 & A is strongly more important than B \\
\hline 6 & 3 & 3.95 & A is strongly plus more important than B \\
\hline 7 & 4 & 5.2 & A is very strongly more important than B \\
\hline 8 & 5.67 & 6.84 & A is very, very strongly more important than B \\
\hline 9 & 9 & 9 & A is extremely more important than B \\
\hline
\end{tabular}

Table 3.2: The numerical values used by the three common scale types and corresponding linguistic expressions of preference (Elliott, 2010)

Several authors have pointed out that the traditional Integer Scale produces an uneven distribution of weightings that is highly skewed towards the extreme values, a behaviour that is not considered to be logically justifiable (Lootsma, 1993; Salo and Hamalainen, 1997; Elliott, 2010). This means that if the importance of A relative to B is increased from 8 (i.e. very, very strongly) to 9 (i.e. extremely), then the increase in the weighting of $\mathrm{A}$ is 15 times smaller than when the relative importance is increased from 1 (i.e. equally) to 2 (i.e. weakly or slightly) (Salo and Hamalainen, 1997). To tackle this issue, Salo and Hamalainen (1997) proposed an alternative scale, called Balanced Scale, where the increments in the weights are constant for all increments in judgment. However, the Balanced Scale produces evenly distributed weightings only when the number of attributes being compared is no more than two and fails to do so for larger numbers. Based on power law, Lootsma (1993) proposed a third method, called the Power Scale, that was further modified by Elliott (2010). The Power Scale addresses both the uneven distribution of weightings that result from the Integer Scale and the limited applicability of the Balanced Scale. The resulting scale ensures an almost uniform distribution for any number of attributes being compared. Elliott (2010) demonstrated the effect of scale type on the weightings distributions, as shown in Figure 3.5. 


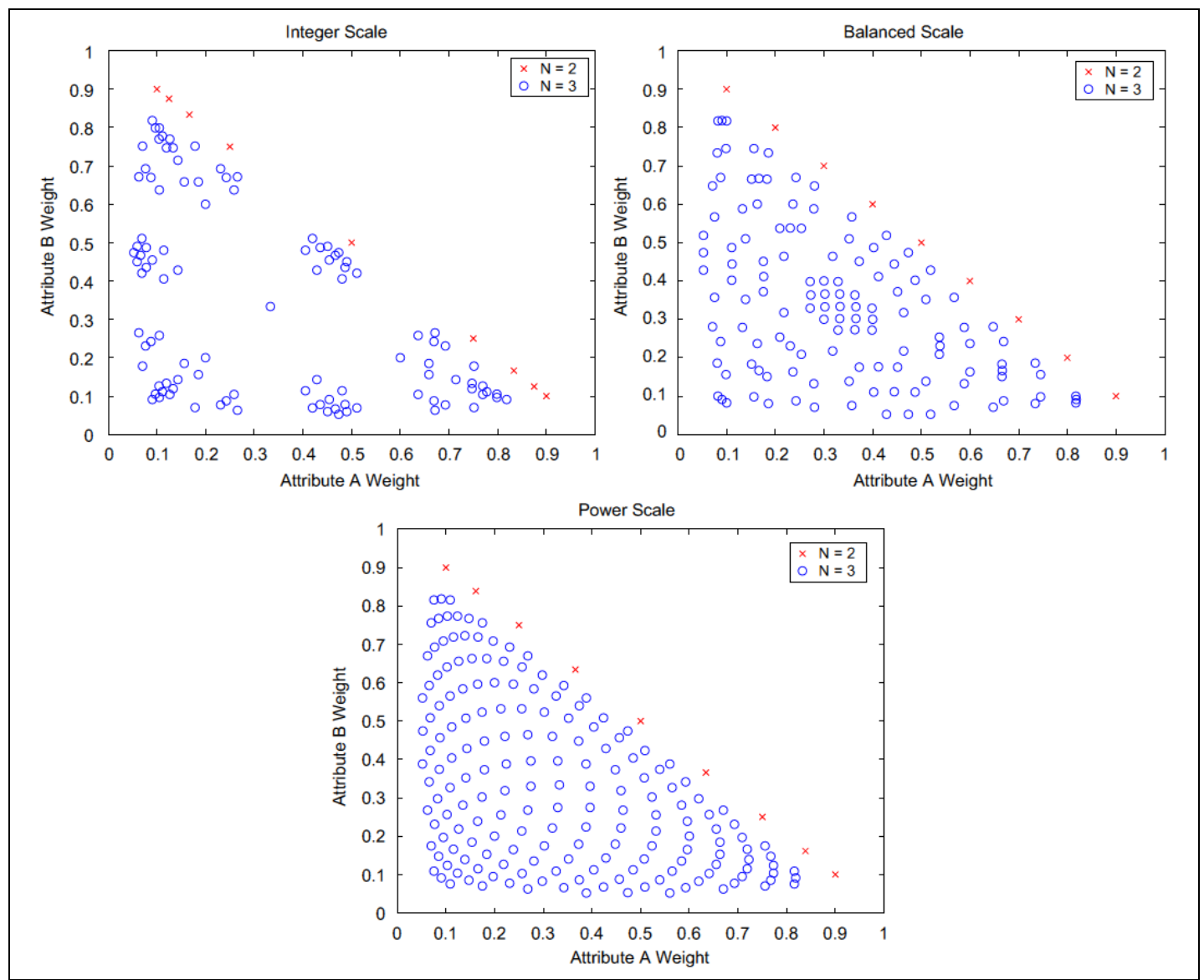

Figure 3.5: The weightings distribution produced by Integer Scale (top left), Balanced Scale (top right), and Power Scale (bottom) when comparing two and three attributes (Elliott, 2010)

The study by Elliott (2010) concluded that there doesn't seem to be universal agreement over the comparative performance of the different scale types and that it would be sensible to allow the decision makers to choose the type that best models their own particular problem. For this research, the Power Scale was adopted since it provides an almost even distribution of weightings while allowing the comparison of any number of attributes.

\section{b) Method for Assessing the Consistency of Pairwise Comparison Matrices}

According to Brunelli (2015), a perfectly rational decision maker is considered to be able to maintain total consistency when carrying out pairwise comparisons and avoid contradicting themselves. In mathematical terms, a comparison matrix produced through pairwise comparisons is only consistent if its elements meet the following condition, known as the transitivity requirement.

$$
a_{i j} \times a_{j k}=a_{i k} \quad \forall i, j, k=1, \ldots, n
$$


If this condition is met, the principal (i.e. maximum) eigenvalue of the pairwise comparison matrix $\left(\lambda_{\max }\right)$ will be equal to the dimension of the comparison matrix $(n)$ (i.e. $\lambda_{\max }=n$ ). However, Saaty (2003) argued that 'people are more likely to be cardinally inconsistent than cardinally consistent because they are unable to make precise measurements even from a known scale and worse when dealing with intangibles (i.e. linguistic expressions of preference).' He therefore argued that since a comparison matrix is supposed to be representative of realistic judgments, a degree of inconsistency might be better than forced consistency since the latter would limit the ability of the decision makers to respond appropriately to new evidence becoming available. Also mathematically speaking, the number of totally consistent comparison matrices (i.e. matrices that satisfy Equation 2) is very limited in practice. Alonso and Lamata (2006) demonstrated that using the traditional Integer Scale proposed by Saaty (1987), the number of perfectly consistent comparison matrices when comparing three attributes is either thirteen or just four, depending on whether or not indifference (i.e. equal importance) is allowed, thirteen and one for four attributes, fourteen and zero when comparing five attributes, and so on. Therefore, Saaty (1987) defined a threshold for acceptable inconsistency called Consistency Ratio (CR) by means of the Consistency Index (CI) and Random Consistency Index (RI).

$$
\begin{aligned}
& C I=\frac{\lambda_{\max }-n}{n-1} \\
& C R=\frac{C I}{R I}<0.1
\end{aligned}
$$

Saaty (1987) has stated that when a comparison matrix is inconsistent, its principal eigenvalue exceeds its dimension (i.e. $\lambda_{\max }>n$ ) and this departure from $\mathrm{n}$ (Equation 3) serves as a measure of inconsistency. RI is the average CI value measured for a large number of matrices of the same dimension and randomly chosen judgments. CR is then measured as the ratio between the CI of the matrix and RI. Saaty (1987) only considers a comparison matrix as consistent if $\mathrm{CR}$ is less than 0.1 , as indicated by Equation 4. Table 3.3 shows the RI values derived by Saaty (1987) for different matrix dimensions.

\begin{tabular}{|l|r|r|r|r|r|r|r|r|r|r|}
\hline$n$ & 1 & 2 & 3 & 4 & 5 & 6 & 7 & 8 & 9 & 10 \\
\hline$R I$ & 0 & 0 & 0.58 & 0.9 & 1.12 & 1.24 & 1.32 & 1.41 & 1.45 & 1.49 \\
\hline
\end{tabular}

Table 3.3: Random Consistency Index values for different comparison matrix dimensions (Saaty, 1987) 
Many authors have investigated the effect of the simulation method and the number of randomly generated matrices on the resulting RI values. The usual simulation method used to determine RI involves calculating the CI of a certain number of matrices of each dimension, and then estimating the RI as the arithmetic mean of those CI values. An extensive account of these studies by Alonso and Lamata (2006) showed that although the RI values produced by different authors are of the same order of magnitude, there is no universal agreement over the exact RI figures. Following these investigations, Alonso and Lamata (2006) proposed an alternative approach to assess consistency.

The approach replaces the CI value used in Equation 4 with an average principal eigenvalue $\left(\bar{\lambda}_{\max }\right)$ as the consistency indicator. Using a least squares adjustment straight line, Alonso and Lamata (2006) experimentally developed the function for measuring $\bar{\lambda}_{\text {max }}$ for matrices of dimension three to fifteen, that is shown in Equation 5.

$$
\bar{\lambda}_{\text {max }}(n)=2.7699 n-4.3513
$$

The function was found to be very closely correlated with experimentally calculated $\bar{\lambda}_{\text {max }}$ values in matrices of dimensions larger than fifteen (Alonso and Lamata, 2006). The other advantage of this alternative approach is that it enables a flexible consistency acceptance criterion by allowing the decision makers to specify the consistency threshold (a) that they consider applicable to their particular problem, as shown in Equation 6.

$$
\frac{\lambda_{\max }-n}{\bar{\lambda}_{\max }(n)-n} \leq a(\text { Consistency threshold })
$$

Combining Equations 5 and 6, Alonso and Lamata (2006) proposed Equation 7, enabling a flexible consistency check based on the dimension (n) of the comparison matrix in question and the consistency threshold (a) considered suitable by the decision makers.

$$
\lambda_{\max } \leq n+a(1.7699 n-4.3513)
$$


Table 3.4 shows various consistency thresholds and corresponding $\lambda_{\max }$ values for different comparison matrix dimensions. The fixed consistency check proposed by Saaty (1987) has been highlighted. Given the adaptability of the approach proposed by Alonso and Lamata (2006), it was adopted for this research. As for the consistency threshold and corresponding principal eigenvalues $\left(\lambda_{\max }\right)$ values, the candidate was unable to obtain figures that could be confidently related to naval ship applications. Therefore for the purpose of demonstrating the application of the framework, a consistency acceptance threshold of 0.1 was adopted and corresponding $\lambda_{\max }$ values were estimated using Equation 7.

\begin{tabular}{|l|c|c|c|}
\cline { 2 - 4 } \multicolumn{1}{c|}{} & \multicolumn{3}{c|}{ Comparison Matrix Dimension } \\
\cline { 2 - 4 } \multicolumn{1}{c|}{} & 3 & 5 & 10 \\
\hline Consistency Threshold (CR) & \multicolumn{3}{c|}{$\lambda_{\max }$} \\
\hline 0.01 & 3.0096 & 5.0450 & 10.1335 \\
\hline 0.05 & 3.0478 & 5.2248 & 10.6673 \\
\hline 0.08 & 3.0765 & 5.3597 & 11.0677 \\
\hline 0.1 & 3.0957 & 5.4497 & 11.3346 \\
\hline 0.2 & 3.1913 & 5.8993 & 12.6692 \\
\hline 0.5 & 3.4784 & 7.2483 & 16.6732 \\
\hline
\end{tabular}

Table 3.4: $\lambda_{\max }$ values for various comparison matrix dimensions and consistency acceptance thresholds (Alonso and Lamata, 2006)

\section{c) Method for Generating Numerical Preferences from Pairwise Comparison Matrices}

Based on the general Perron-Frobenius theorem (Xu and Wang, 2013), the classic approach for generating numerical preferences from a positive reciprocal consistent or near consistent pairwise comparison matrix is the Eigenvector Method (EVM) (Saaty, 2003). The output, commonly known as the priority vector, is the normalised principal eigenvector of the comparison matrix (i.e. the eigenvector that corresponds to the maximum eigenvalue) (Xu and Wang, 2013). 
Another widely used method to estimate the priority vector is the Row Geometric Mean (RGM). Using RGM, each component of the priority vector is obtained as the geometric mean of the elements on the respective row divided by a normalisation term so that the components add up to 1, as is the case in EVM (Williams, 1985). For a square matrix of dimension n, RGM is mathematically described in Equation 8.

$$
w_{i}=\frac{\left(\prod_{j=1}^{n} a_{i j}\right)^{\frac{1}{n}}}{\sum_{i=1}^{n}\left(\prod_{j=1}^{n} a_{i j}\right)^{\frac{1}{n}}}
$$

In addition to EVM and RGM, there are other methods to compute the priority vector, namely the normalised columns method and the least squares method, and there is ongoing debate as to which method should be used in AHP (Brunelli, 2015).

The mathematical approach adopted in this research to check that analysts are consistent in their decision making is only applicable if EVM is used to generate priority vectors (Alonso and Lamata, 2006). Additionally, investigating the relevant literature did not provide well-established consistency checks suitable for other prioritisation approaches such as RGM. Therefore EVM was adopted in this research as the method for quantifying the preferences of decision makers.

In summary, the three features for using an AHP-based framework to quantify the relative preferences of a given decision maker and produce priority vectors were:

- Using the Power Scale as the numerical scale for carrying out pairwise comparisons at individual levels of the assessment hierarchy;

- Checking the consistency of the pairwise comparison matrices against a consistency acceptance threshold of 0.1 and corresponding principal eigenvalues;

- Using the EVM for generating priority vectors from positive reciprocal consistent or near consistent pairwise comparison matrices. 


\subsection{Customisation to a Specific Naval Ship Supportability Case}

This section provides a detailed description of how the principal features of the proposed DfS evaluation approach described in Section 3.3 were customised in order to investigate a specific naval ship supportability example. The investigations were primarily centred on the issue of naval ship maintenance, sometimes considered to be a major subset of naval ship supportability and can be defined by considering the different types of activities aimed at preserving or returning equipment and spaces to their required operational status (Eriksen, 2001). Following the logic of the generic top-down structure developed previously (see Figure 3.4), the components of the hierarchy, including the Proxy Supportability Indicators (PSI) (see Section 3.3.2), were developed as described below.

\subsubsection{Overall Measure of Effectiveness (oMoE)}

The oMoE is the collective result of all the different effectiveness measures and is the metric used for the evaluation and comparison of design alternatives. Given that the primary focus of the investigations was on ship maintenance, the oMoE in this research represents a measure of ship maintainability.

\subsubsection{Objectives}

The top level objective was set to be improving naval ship maintainability, and to simplify the problem, this was further broken down to three sub-objectives; improving the effectiveness of three distinct Maintenance Types (MT). The MTs were:

- $\mathrm{MT}_{1}$ : on-board in-space maintenance;

- $\mathrm{MT}_{2}$ : on-board workshop maintenance;

- $\mathrm{MT}_{3}$ : off-board maintenance carried out by naval dockyards or the Original Equipment Manufacturers (OEM). 
Following the approach proposed by Keeney (1996), the three features required for a proper description of each sub-objective are shown in Table 3.5.

\begin{tabular}{|l|l|l|l|}
\hline $\begin{array}{l}\text { Maintenance } \\
\text { Type }\end{array}$ & \multicolumn{1}{|c|}{$\begin{array}{c}\text { Decision } \\
\text { Context }\end{array}$} & $\begin{array}{c}\text { The } \\
\text { Object }\end{array}$ & $\begin{array}{c}\text { Direction of } \\
\text { Preference }\end{array}$ \\
\hline$M T_{1}$ & $\begin{array}{l}\text { The transfer of spare parts from on- } \\
\text { board stores to certain shipboard } \\
\text { spaces where the equipment in } \\
\text { question is located }\end{array}$ & $\begin{array}{l}\text { The time } \\
\text { elapsed }\end{array}$ & $\begin{array}{l}\text { Shorter travel } \\
\text { times are } \\
\text { preferred }\end{array}$ \\
\hline$M T_{2}$ & $\begin{array}{l}\text { The transfer of spare parts from on- } \\
\text { board stores and certain ship } \\
\text { equipment from the relevant on- } \\
\text { board operational spaces to the ship } \\
\text { workshop facilities }\end{array}$ & $\begin{array}{l}\text { The time } \\
\text { elapsed }\end{array}$ & $\begin{array}{l}\text { Shorter travel } \\
\text { times are } \\
\text { preferred }\end{array}$ \\
\hline$M T_{3}$ & $\begin{array}{l}\text { The removal of certain ship } \\
\text { equipment from the ship }\end{array}$ & $\begin{array}{l}\text { The time } \\
\text { elapsed }\end{array}$ & $\begin{array}{l}\text { Shorter travel } \\
\text { times are } \\
\text { preferred }\end{array}$ \\
\hline
\end{tabular}

Table 3.5: The features required for the proper description of the objective to improve the effectiveness of three distinct Maintenance Types on-board a vessel

\subsubsection{Measures of Effectiveness (MoE)}

To help measure the degree of achieving the objective of improving each type of maintenance, the MoEs were simply defined as how effectively each type of maintenance can be carried out.

\subsubsection{Measures of Performance (MoP)}

In the absence of direct indicators, MoPs were defined to be proxy indicators and assign quantities to the MoEs. Having clearly stated the sub-objectives and resulting MoEs, the MoPs were defined in generic terms to be the time it takes to move ship equipment and spare parts between given shipboard spaces, assuming that shorter travel times would contribute to more effective maintenance. As each type of maintenance requires a different strategy, the design implications and associated MoPs would also be different for each type: 
- $\mathrm{MT}_{1}$ (on-board in-space maintenance): the travel time between Naval and Spare Gear Stores (NSGS) and the relevant shipboard equipment spaces;

- $\mathrm{MT}_{2}$ (on-board workshop maintenance): the travel time between relevant shipboard equipment spaces and ship workshop facilities, and between NSGSs and ship workshop facilities;

- $\mathrm{MT}_{3}$ (off-board maintenance by naval dockyards or OEMs): the travel time between relevant shipboard equipment spaces and ship Equipment Removal Points (ERP).

In the face of the difficulty of directly measuring the effectiveness of maintenance activities during ESSD, indirect or proxy measures like the above, could provide an indication, if not an actual measurement of effectiveness. Producing accurate travel time measurements was also considered to be too arduous a task to be undertaken during ESSD, hence the following indicative measures, termed sub-MoPs were instead adopted:

- The travel distance;

- The number of decks to lift or lower equipment or spare parts through;

- The number of Watertight Bulkheads (WTB) to pass equipment or spare parts through.

The Manhattan or Taxicab method was used to obtain a more practical measure of the travel distance, as opposed to the straight-line (Euclidean) method. The Manhattan distance between two points is defined as the sum of the absolute differences between their Cartesian coordinates (McCune et al., 2002):

$$
\Delta d=\left|x_{2}-x_{1}\right|+\left|y_{2}-y_{1}\right|+\left|z_{2}-z_{1}\right|
$$

It is appreciated that the Manhattan method is unable to accurately detect the actual travel route between two spaces of interest in a typical ship internal arrangement. However, the aim of this research was first and foremost, to reveal major indicators and provide estimated relative figures, not precise answers and bottom-up absolute values. Therefore the lack of absolute accuracy in the Manhattan method was not considered to be a serious shortcoming of the proposed comparative approach applied in ESSD to inform major design choices. 


\subsubsection{Dimensional Parameters (DP)}

Given the context of this research, the ship's architectural or configurational characteristics were seen to be central to defining the indicative measures of supportability (i.e. MoPs and sub-MoPs). Therefore, the ship's individual spaces and equipment, overall internal arrangement, and topology had to be associated with characteristics considered to be relevant to determining the Proxy Supportability Indicators (PSIs). A fundamental feature of the UCL DBB approach is the 'block object' that allows the designer to assign characteristics to ship spaces (Andrews, 2006). This is readily achievable through the design tools available at UCL. The description of each category of architectural or configurational characteristics and some example supportability-related features are provided in the following paragraphs.

\section{a) Individual ship spaces and equipment}

The features of individual spaces and equipment within them exist regardless of any General Arrangement (GA) adopted and so it is not necessary to first develop a GA in order to quantify them. For individual ship spaces and equipment (i.e. the Design Building Blocks) used as part or otherwise associated with any supportability investigations, the following features were seen to be relevant:

- Weight, gross (total) and net (unoccupied) plan area, volume, density;

- Criticality of functions and demand for ship services that may need disconnecting (e.g. CW, HVAC, HPA, electrical power, fuel, lube oil);

- The type of maintenance required;

- Susceptibility or immunity to various external effects (e.g. heat, noise, vibration, shock, ship motions, corrosion, fire);

- Equipment handleability (i.e. whether or not the crew requires special arrangements to be able to move the equipment), at least partially determined by the weight of the equipment;

- Level of human occupancy in the relevant spaces. 


\section{b) Overall internal arrangement}

These are characteristics applicable to the ship's overall internal arrangement that will only be quantified once a description of the ship's layout has been produced, however crudely, for Concept Studies. Some example supportability-related features are:

- The extent of access routes per relevant deck;

- Convexity (i.e. the straight line segment connecting two points in a vector space is fully contained by the same space (e.g. cube, circle)) or concavity (i.e. any part of the straight line segment that might lie outside the relevant space (e.g. crescent, L-shapes));

- Aspect ratio of essentially rectangular spaces of relevance;

- General position of certain spaces and equipment in the ship (e.g. hull or superstructure, amidships, aft or forward).

\section{c) Topology}

These characteristics concern the relative juxtapositions of spaces and, in a similar manner to the features associated with the ship's overall internal arrangement, may only be quantified once a sufficiently defined layout has been developed. Adjacency relationships can be modelled through network theory (Gillespie and Singer, 2012; Rigterink et al., 2014) as shown in the next chapter where specific ship descriptions are presented (see Figure 4.1 and Figure 4.2). Example supportability-related features are:

- Adjacency of certain spaces to one another (e.g. the juxtaposition of spaces and issues such as accessibility, fire safety, transmission of noise and vibration);

- Adjacency of certain spaces to a WTB or damage control zone boundary;

- Adjacency of certain spaces and systems of concern (e.g. fuel tanks and fuel systems);

- Relative position with respect to decks (e.g. below, on or above the Damage Control Deck (DCD);

- Adjacency of sources and sinks of the external effects (e.g. the degree of exposure of a susceptible space to the source of heat or corrosion). 


\subsubsection{Maintainability Assessment Hierarchy}

Having defined the oMoE, objectives, MoEs, MoPs/sub-MoPs, and DPs for a partial evaluation of maintainability as a subset of naval ship supportability, a top-down hierarchy was constructed and is shown in Figure 3.6. The approach used by Gregor (2003) as part of developing a method for assessing whole ship design flexibility under (acquisition) uncertainty was broadly applied by the candidate when considering the specific assessment of maintainability of naval vessels in ESSD. In particular, the AHP developed by Gregor (2003) (see Figure A2. 1, Appendix 2) was extensively used in producing the supportability evaluation hierarchy. Having outlined the proposed assessment of DfS in ESSD, it was necessary to produce a number of ship design case studies to which the assessment approach was applied. This is covered in the next chapter. 


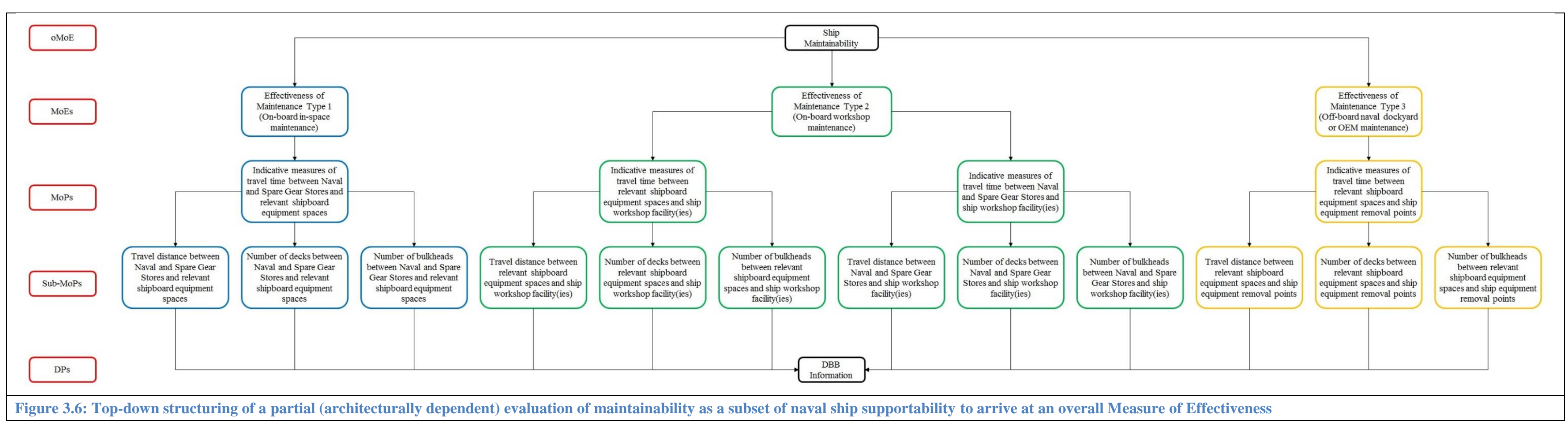




\section{Chapter 4: Ship Design Case Studies}

\subsection{Introduction}

This chapter, consisting of three main sections, describes the development of the ship designs that were used to demonstrate the application of the Design for Support (DfS) evaluation approach outlined in the previous chapter. In the next section, the justifications for developing a new ship concept design software and the current status of the work at UCL on this are covered. The Section 4.3 explains why the development of the baseline ship design for this research was focused on a frigate-sized combatant, followed by outlining the main ship performance requirements and main equipment selected. Major ship characteristics, certain ship design analyses performed and resultant ship features, together with an internal arrangement deck plan are also presented. Section 4.4 describes the ship design variants that were developed in order to investigate the effects of various changes to the internal arrangement from that of the baseline ship design, as well as investigating the impact of certain supportability features on the measure of supportability performance proposed in this research. 


\subsection{The New UCL Ship Concept Design Software}

Despite the advantages of implementing the UCL Design Building Block (DBB) approach through the SURFCON module in the Paramarine Computer-Aided Ship Design (CASD) suite (see Section 2.2.6), there were felt to be several drawbacks in using such a sophisticated (fully 3-D), high-fidelity, high-capability Computer-Aided Design (CAD) modelling tool in this Early Stage Ship Design (ESSD) investigation of DfS. These include: the apparent need to model to an unnecessarily high level of design detail; a degree of flexibility and precision felt to be inappropriate for, at least, the earliest (exploratory) stage of the Concept Phase; the very high software learning overhead; and the challenge of connecting the concept ship design tools in Paramarine with external analysis tools. The consequence is that although Paramarine offers great flexibility and extensive naval architectural analyses for ship design, it can be overwhelming for new users (Pawling et al., 2015, 2017a). In order to address these issues, an alternative implementation of the DBB approach (Andrews, 2003b) has been developed by the UCL Design Research Centre (DRC) for ESSD research applications. The key features sought in this simpler tool were noted by Pawling et al. (2015) as: wide availability; low learning and familiarisation overheads; fast operation; flexible levels of detail; task-focused and reliable; not based on ship type; not automated; appropriate levels of precision for ESSD; and integration of early stage ship concept models, datasets and evaluation approaches.

The new tool, described comprehensively by Pawling et al. (2015), was initially developed using commercial non-CAD software. Specifically at that stage, the tool consisted of a Microsoft Excel spreadsheet-based layout model, which used a cellular representation of the space on the ship with each square cell representing $0.5 \mathrm{~m} \times 0.5 \mathrm{~m}$ of ship dimensions. Excel was chosen because: it is widely available; has a preexisting Graphical User Interface (GUI); permits scripting and a limited degree of programming, via Visual Basic for Applications (VBA); and inherently provides an addressable cellular model - albeit only in two dimensions, rather than Paramarine's 3-D. 
The Excel-based ship design toolset was found to be capable of auditing areas and volumes of ship layouts, and providing data (e.g. centroids, hydrostatics, compartment and equipment adjacencies and proximities), either to other tools for further analysis or as feedback to designers, on the implications regarding ship performance (e.g. basic stability assessment and powering calculation). Using network analysis and 2-D modelling, it enabled a wide range of spatial properties to be incorporated as required relationships from a database of layout guidance (see Figure 4.1), or extracted for the subsequent analysis of the configuration (see Figure 4.2). Such spatial properties included compartment proximity, adjacency, perimeter, and deck area.

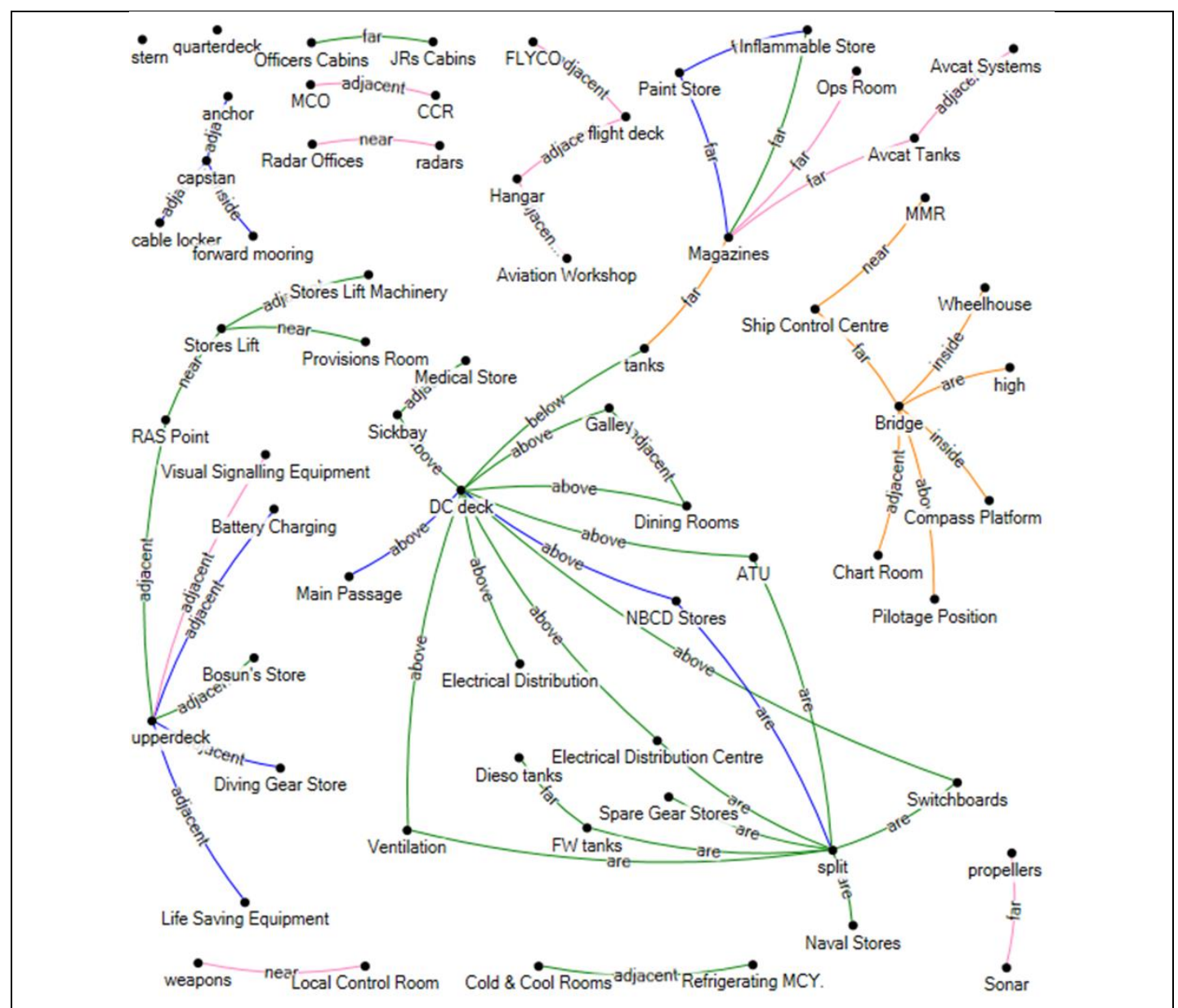

Figure 4.1: Network of required relationships for a selected set of DBBs from a database of layout guidance 


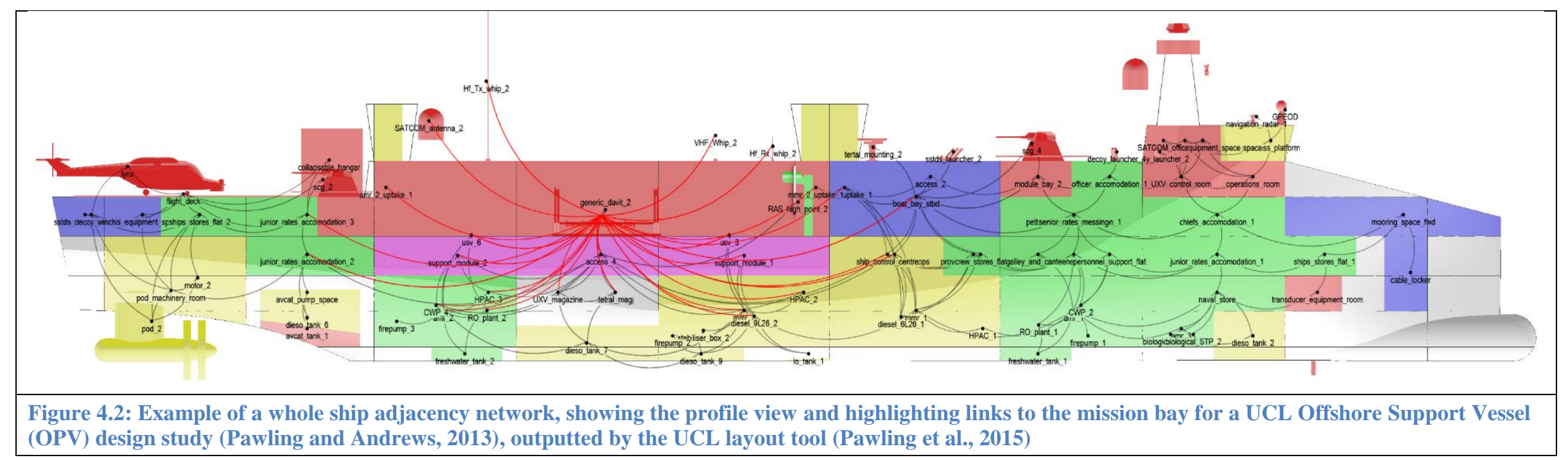


This simplified arrangement model included several features intended to satisfy the needs of specific investigations being undertaken at that time, in particular the ability to interface with separate, dedicated tools (e.g. Paramarine, MATLAB, Python) for more complex analyses. The generation of layout and connectivity information allows such analysis to be undertaken at earlier stages of the ship design process without adding a significant modelling burden. Given such capabilities, the toolset was considered to provide ESSD architectural models which could then enable the evaluation of supportability aspects in the early stages of naval ship design.

Despite the above capabilities, the VBA environment severely restricted the calculation speed and responsiveness of the Excel-based UCL tool and so the designer was unable to model sufficiently quickly, especially when sketching the internal arrangement and needing to rapidly generate and extract spatial properties. It took up to one hour of processing time to sketch each new internal arrangement and extract the required spatial properties. Therefore, inspired by the work presented by Gaspar et al. (2014), the tool was transferred from Excel to JavaScript, a remarkably versatile scripting language that is many times faster than VBA (Gaspar, 2016). The processing time was reduced from up to one hour to around five minutes, allowing the designer to be much more explorative without being worried that implementing a few changes to the internal arrangement might take up to an hour to process. Other advantages of JavaScript include wider availability, as it can run directly from any modern internet browser without the need to install any bespoke programs; easier sharing of results publically (online server) or privately (HTML file); and it can benefit from many ready-made graphical libraries, thus has a more customisable GUI than the Excelbased tool (Gaspar, 2016). Hence running inside a web browser on a typical consumer desktop PC, the online JavaScript-based tool includes an interactive GUI that enables the inspection and modification of a typical concept ship design level of hullform and internal arrangement description appropriate to ESSD. It provides scalability of hullform dimensions and the ability to audit and modify the dimensions, areas, volumes and centroids of the individual DBBs, and change the Watertight Bulkheads (WTB) locations along the hull, if required. The tool can also output matrices describing the proximity and adjacency relationships of all the DBBs in an ESSD (Pawling et al., 2017a). Considering the current focus at the UCL DRC on investigating the applications of online and open-source softwares to research into 
ESSD, the JavaScript-based tool, despite being currently limited to monohull ship designs, represents a major improvement of the original Excel-based implementation.

Figure 4.3 shows two different illustrations of the graphical output of the tool for the ESSD of a typical naval combatant.

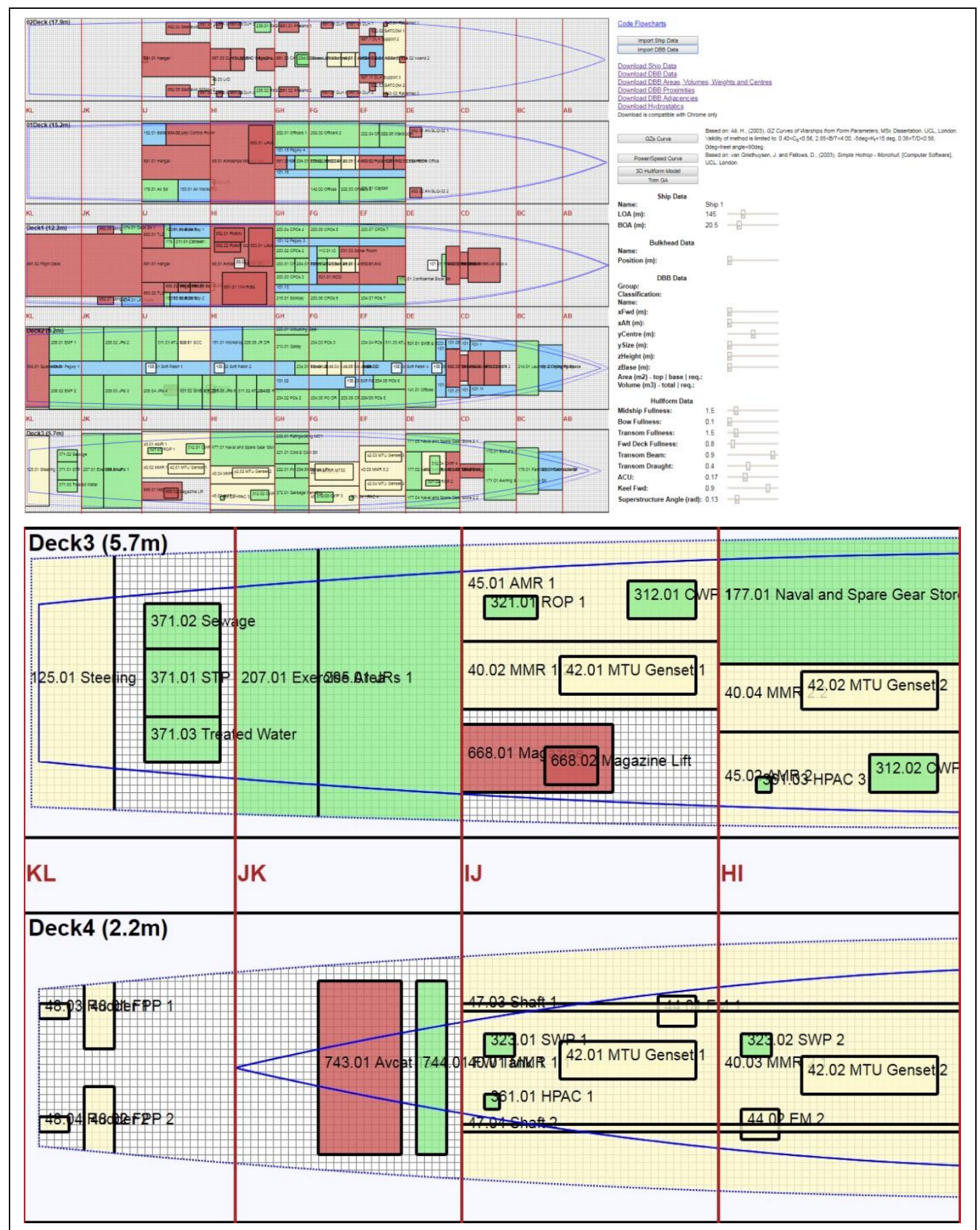

Figure 4.3: Screenshots of the developmental JavaScript-based implementation of the UCL DBB approach, ship internal arrangement and interactive GUI and controls (top), close-up of the cellular representation (bottom) 
The JavaScript-based tool relies on two principal components as input data, namely the 'Ship Data' and the 'DBB Data', formatted as Comma Separated Value (CSV) files. The 'Ship Data' contains information such as major ship dimensions, position of WTBs, number and position of decks in the hull and superstructure, and certain hullform geometrical constraints (covered in Appendix 5, Section Hullform Geometry Coefficients). The 'DBB Data' component requires a tabular description of the DBBs, giving the locations, dimensions, weight, area and volume characteristics, and any other parameters that may be provided by the designer simply by adding additional columns to the spreadsheet. The tabular format has the advantage that all appropriate aspects of a DBB can be contained in a single row in the table. The 'DBB Data' is structured using the UCL defined ship functional groups of FLOAT, MOVE, FIGHT and INFRASTRUCTURE for naval vessels (Andrews and Pawling, 2003). The tool then reads the information from the 'Ship Data' and 'DBB Data' files and generates a display of the ship design's internal arrangement. Since the tool allows design changes to be applied visually on the computer screen, it saves the designer the trouble of having to go back to the spreadsheets for every design modification. Once the designer is satisfied with the amended design, the data describing the new design can be obtained through the output 'Ship Data' and 'DBB Data' files produced by the JavaScript tool. A much more comprehensive description of the tool is intended to be published following this thesis' completion. 


\subsection{Baseline Frigate Design}

The basis behind developing the Baseline Frigate Design was to produce a 'balanced' (i.e. balance of weight, space, stability, speed, range, and power) concept level ship design so that subsequent investigations of (architecturally-driven) DfS aspects could be conducted (using the proposed DfS evaluation approach) through variants of this design study. Frigates are the most common warship for deep-ocean medium-sized navies, such as the Royal Navy, hence provide a point of reference for most new developments in naval ship design. The baseline design for this research was therefore representative of a blue-water navy frigate-sized combatant. The Type 26 programme was seen to be a sensible starting point since it is currently one of the two major ongoing UK surface fleet projects (the other being the Queen Elizabeth class aircraft carrier). The Type 26 programme entered the Demonstration Phase in April 2015 (Fallon, 2015), moved further forward with the first long lead items ordered in August 2015 (Larrinaga, 2015), the steel cut began in summer 2017 (Holland, 2017), and the ships are expected to enter service from the mid-2020s to replace the ageing Type $23 \mathrm{~s}$ and essentially form the backbone of the Royal Navy for the next 40 years (UK MoD, 2015a).

Without using any past or current official detailed design solutions, the broad specifications for the General Purpose (GP) variant of the Type 26 Global Combat Ship (GCS) (Saunders and Philpott, 2013; BAE Systems, 2015) as well as the UCL (2013b) warship database were drawn on to form the broad performance requirements and the appropriate payload equipment listing. The development of the baseline design for this research was carried out in accordance with the procedure, data, and parametric relationships available for the ship design module in the UCL MSc Naval Architecture course (UCL, 2013a, 2013b), and other published data sources, such as BAE Systems (2011, 2012), Lockheed Martin (2014), Northrop Grumman (2012), SEA (2015), Ultra Electronics (2014a, 2014b), (Rolls Royce, 2016a, 2017), and MTU (2013). 
What follows is a description of the main ship performance requirements, payload equipment, complement and accommodation breakdown, major ship characteristics and dimensional ratios, resistance estimation and power plant selection, stability assessments, internal arrangement, ship costing, the supportability features relevant to the hierarchy shown in Figure 3.6, and finally the ship design aspects that were not considered primarily due to limiting the design definition to that appropriate to ESSD (Andrews, 2018).

\subsubsection{Ship Performance Requirements and Payload Equipment}

The main ship performance requirements and payload equipment incorporated into the Baseline Frigate Design are shown in Table 4.1 and Table 4.2 respectively.

\begin{tabular}{|l|r|}
\hline Maximum speed (kts) & 28 \\
\hline Cruise speed (task group escort) (kts) & 18 \\
\hline Cruise speed (maximum efficiency) (kts) & 15 \\
\hline Range (Nm/kts) & 60 \\
\hline Stores endurance (days) & 7000Nm at $15 \mathrm{kts}$ \\
\hline
\end{tabular}

Table 4.1: Baseline Frigate Design main performance requirements based on the broad specifications of the General Purpose variant of the Type 26 Global Combat Ship (Saunders and Philpott, 2013; BAE Systems, 2015) and UCL (2013b) warship database 


\begin{tabular}{|c|c|c|}
\hline Payload Equipment & \multicolumn{2}{|c|}{ Description } \\
\hline $1 \times$ BAE Systems MK 45 Mod 4 & \multicolumn{2}{|c|}{ Lightweight medium calibre naval artillery } \\
\hline $\begin{array}{l}3 \times 8 \text { cell strike length MK } 41 \\
\text { VLS }\end{array}$ & \multicolumn{2}{|l|}{ Modular long range VLS } \\
\hline $\begin{array}{l}48 \text { Quad packed MBDA Sea } \\
\text { Ceptor SAM SVL technology }\end{array}$ & \multirow{2}{*}{\multicolumn{2}{|c|}{ PDS against missiles and aircraft }} \\
\hline $2 \times$ Phalanx CIWS & & \\
\hline $\begin{array}{l}2 \times \text { triple barrel deck mounted } \\
\text { torpedo launch system }\end{array}$ & \multicolumn{2}{|c|}{$\begin{array}{l}\text { Anti-submarine acoustic homing torpedo } \\
\text { launch system }\end{array}$} \\
\hline $2 \times$ Seahawk SIGMA & \multirow{2}{*}{\multicolumn{2}{|c|}{ General ship protection systems }} \\
\hline $2 \times$ Mk 25 Mod 0 Minigun & & \\
\hline $2 \times 7 \mathrm{~m}$ standard navy RIB & Armed transport vehicle & Ship's boats \\
\hline $1 \times$ Hangered Merlin HM.2 & $\begin{array}{l}\text { Medium-lift helicopter for } \\
\text { ASW or transportation }\end{array}$ & \multirow{4}{*}{$\begin{array}{l}\text { Modular mission } \\
\text { payload }\end{array}$} \\
\hline $\begin{array}{l}2 \times \text { Hangered Northrop } \\
\text { Grumman MQ-8C Fire Scout }\end{array}$ & VTUAV for ISR & \\
\hline $2 \times 11 \mathrm{~m}$ standard navy RIB & $\begin{array}{l}\text { Armed transport vehicle } \\
\text { specifically for the } \\
\text { Embarked Military Force } \\
(\text { EMF) }\end{array}$ & \\
\hline $1 \times$ Lockheed Martin RMMV & Mine Counter Measures & \\
\hline $\begin{array}{l}8 \times 6 \text { tubed Outfit Thales SIREN } \\
(\mathrm{DLH}) \text { launchers }\end{array}$ & $\begin{array}{l}\text { Active parachute-retarded } \\
\text { aerial jammer for defence } \\
\text { against incoming missiles }\end{array}$ & \multirow{3}{*}{$\begin{array}{l}\text { Electronic } \\
\text { Warfare }\end{array}$} \\
\hline $\begin{array}{l}1 \times \text { SSTDS (Ultra Electronics } \\
\text { type } 2170 \text { ) }\end{array}$ & $\begin{array}{l}\text { Active and passive torpedo } \\
\text { decoy system }\end{array}$ & \\
\hline $\begin{array}{l}2 \times \text { Raytheon AN/SLQ-32 (V.3) } \\
\text { shipboard ESM \& ECM }\end{array}$ & $\begin{array}{l}\text { EW system with radar } \\
\text { jamming capability }\end{array}$ & \\
\hline $1 \times$ Raytheon I-band type 1047 & Ship navigation & \multirow{3}{*}{ Radars } \\
\hline $1 \times$ Raytheon I-band type 1047 & Helicopter navigation & \\
\hline $1 \times$ BAE Systems 997 Artisan & Medium range 3-D ASR & \\
\hline $1 \times$ Thales type $2050 \mathrm{HMS}$ & Medium range bow sonar & Sonars \\
\hline $1 \times$ Astrium SCOT 5 SATCOM & $\begin{array}{l}\text { SHF Naval satellite } \\
\text { communications }\end{array}$ & \multirow[b]{2}{*}{ Communications } \\
\hline $\begin{array}{l}1 \times \text { Thales FICS, } \\
1 \times \text { outfit ALK aerial } \\
(\text { Communications II) }\end{array}$ & $\begin{array}{l}\text { Ship communications } \\
\text { system }\end{array}$ & \\
\hline Active/passive sonobuoys & \multicolumn{2}{|c|}{ Launched from Merlin HM.2 to conduct ASW } \\
\hline $2 \times$ Radamec 2500 & \multicolumn{2}{|c|}{ Electro optical tracking system } \\
\hline $30 \times$ Royal Marines & \multicolumn{2}{|l|}{ Platoon size EMF } \\
\hline
\end{tabular}

Table 4.2: Baseline Frigate Design payload equipment based on the broad specifications of the General Purpose variant of the Type 26 Global Combat Ship (Saunders and Philpott, 2013; BAE Systems, 2015) and UCL (2013b) warship database 


\subsubsection{Ship Complement and Accommodation}

An in-depth study of frigate complementing was not considered to be necessary for this supportability-focused research. The breakdowns of ship complement and accommodation was roughly estimated using empirical ratios and the information available on the Type 26 GCS and Type 45 Destroyers. At the time of developing the Baseline Frigate Design, the information available on the Type 26 GCS suggested a complement of 118 and a total accommodation of 208 (BAE Systems, 2015). The breakdown of the complement and total accommodation was estimated using UCL guidelines on complementing (UCL, 2013a) and the information available on the Type 45 Destroyers (Chambers et al., 2009; Gates, 2014), the Royal Navy's latest operational combatant. Table 4.3 shows the breakdown adopted in the Baseline Frigate Design.

\begin{tabular}{|l|r|r|}
\hline \multicolumn{1}{|c|}{ Rank } & Complement & Total Accommodation Provision \\
\hline Captain & 1 & 1 \\
\hline Officers & 13 & 14 \\
\hline Chief Petty Officers (CPO) & 14 & 26 \\
\hline Petty Officers (PO) & 26 & 48 \\
\hline Junior Rates (JR) & 64 & 119 \\
\hline Total & 118 & 208 \\
\hline
\end{tabular}

Table 4.3: Baseline Frigate Design complement and total accommodation breakdown

Having broken down the complement of 118 , it was assumed that the remaining ninety accommodation spaces account for training and advancement of CPOs, POs and JRs (Officers advance through different schemes), the flight crew, and the platoon size EMF. The EMF platoon commander is usually accommodated with the other Officers, hence one extra Officer cabin was provided. The remaining 89 accommodation spaces were then distributed between CPOs, POs and JRs according to the complement breakdown ratios. Finally, the accommodation spaces were sized based on the Future Surface Combatant (FSC) accommodation standards provided by UCL (2013b).

\subsubsection{Major Ship Characteristics and Dimensional Ratios}

Major ship characteristics and dimensional ratios of the balanced Baseline Frigate Design are shown in Table 4.4 and Table 4.5 respectively. These values were compared with the principal characteristics of the Type 26 GCS released by BAE 
Systems (2015) and typical frigate characteristics provided in Table A5. 3 and Table A5. 4 (Appendix 5), and were found to be comparable.

\begin{tabular}{|l|r|}
\hline Total enclosed volume $\left(\mathrm{V}_{\mathrm{G}}\right)$ & $23873 \mathrm{~m}^{3}$ \\
\hline Deep displacement $\left(\Delta_{\mathrm{D}}\right)$ & $6632 \mathrm{te}$ \\
\hline Light displacement $\left(\Delta_{\mathrm{L}}\right)$ & $4570 \mathrm{te}$ \\
\hline Overall density $(\rho)$ & $0.278 \mathrm{te} / \mathrm{m}^{3}$ \\
\hline Superstructure Proportion $\left(\mathrm{V}_{\mathrm{s}}\right)$ & 0.310 \\
\hline Payload Volume Fraction $(\mathrm{PVF})$ & 0.218 \\
\hline Waterline length $\left(\mathrm{L}_{\mathrm{wl}}\right)$ & $137.0 \mathrm{~m}$ \\
\hline Overall length $\left(\mathrm{L}_{\mathrm{oa}}\right)$ & $145.0 \mathrm{~m}$ \\
\hline Waterline beam $\left(\mathrm{B}_{\mathrm{wl}}\right)$ & $18.3 \mathrm{~m}$ \\
\hline Amidships Main Deck beam $\left(\mathrm{B}_{\mathrm{MD}}\right)$ & $20.6 \mathrm{~m}$ \\
\hline Amidships Deep draught $(\mathrm{T})$ & $5.7 \mathrm{~m}$ \\
\hline Amidships hull depth $(\mathrm{D})$ & $12.2 \mathrm{~m}$ \\
\hline Forward freeboard $(\mathrm{F})$ & $7.9 \mathrm{~m}$ \\
\hline
\end{tabular}

Table 4.4: Baseline Frigate Design major ship characteristics

\begin{tabular}{|l|r|}
\hline Waterline length to waterline beam ratio $\left(\mathrm{L}_{\mathrm{wl}} / \mathrm{B}_{\mathrm{wl}}\right)$ & 7.5 \\
\hline Overall length to amidships hull depth ratio $\left(\mathrm{L}_{\mathrm{oa}} / \mathrm{D}\right)$ & 11.9 \\
\hline Waterline beam to amidships hull depth ratio $\left(\mathrm{B}_{\mathrm{wl}} / \mathrm{D}\right)$ & 1.5 \\
\hline Waterline beam to amidships Deep draught ratio $\left(\mathrm{B}_{\mathrm{w}} / \mathrm{T}\right)$ & 3.2 \\
\hline Circular M & 7.35 \\
\hline Block coefficient $\left(\mathrm{C}_{\mathrm{B}}\right)$ & 0.45 \\
\hline Prismatic coefficient $\left(\mathrm{C}_{\mathrm{P}}\right)$ & 0.57 \\
\hline Midship section coefficient $\left(\mathrm{C}_{\mathrm{M}}\right)$ & 0.79 \\
\hline Waterplane area coefficient $\left(\mathrm{C}_{\mathrm{WP}}\right)$ & 0.73 \\
\hline
\end{tabular}

Table 4.5: Baseline Frigate Design major dimensional ratios

The top level weight breakdown for the Deep Condition, including all margins and an allowance for items, such as mountings appropriate for noise and vibration attenuation purposes, is shown in Figure 4.4. Additional information regarding the adopted margin policy and equipment mountings can be found in Appendix 5, Section Margins and Equipment Mountings. 


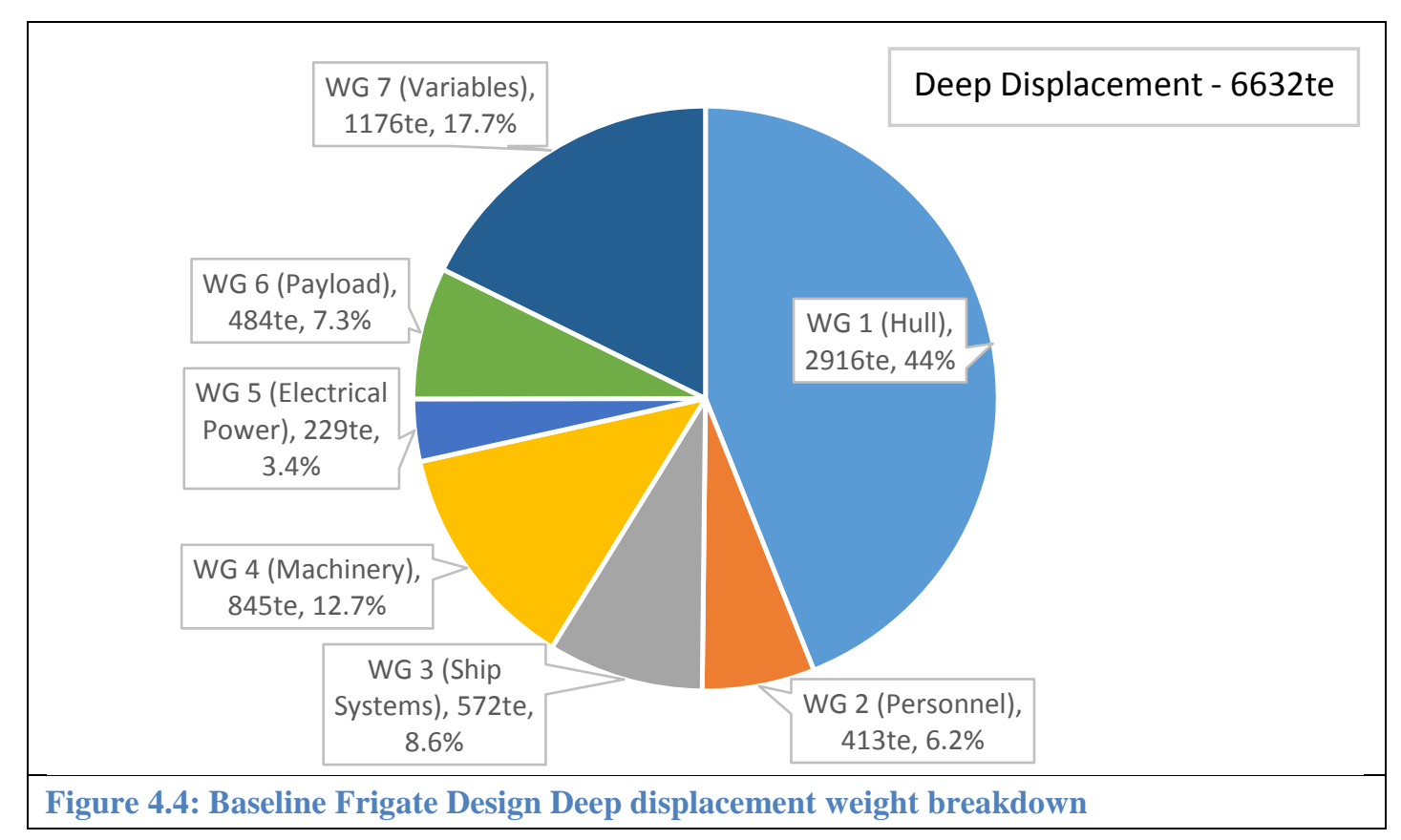

\subsubsection{Resistance Estimation and Power Plant Selection}

Total ship resistance and the required propulsion power were estimated using the widely applied Holtrop and Mennen power prediction method (Holtrop and Mennen, 1978, 1982; Holtrop, 1984). A short description of the method has been provided in Appendix 5, Section Ship Resistance and Propulsion. Figure 4.5 shows the Baseline Frigate Design power speed curve developed for a ship speed range of $0-28 \mathrm{kts}$, indicating a shaft power requirement of $35.5 \mathrm{MW}$ at $28 \mathrm{kts}$, including a sea margin of $20 \%$. The required hotel load was based on similar frigate designs developed by Piperakis (2014) and assumed to be approximately 2.5MW, that was then doubled to $5 \mathrm{MW}$ to meet duplication requirements. 


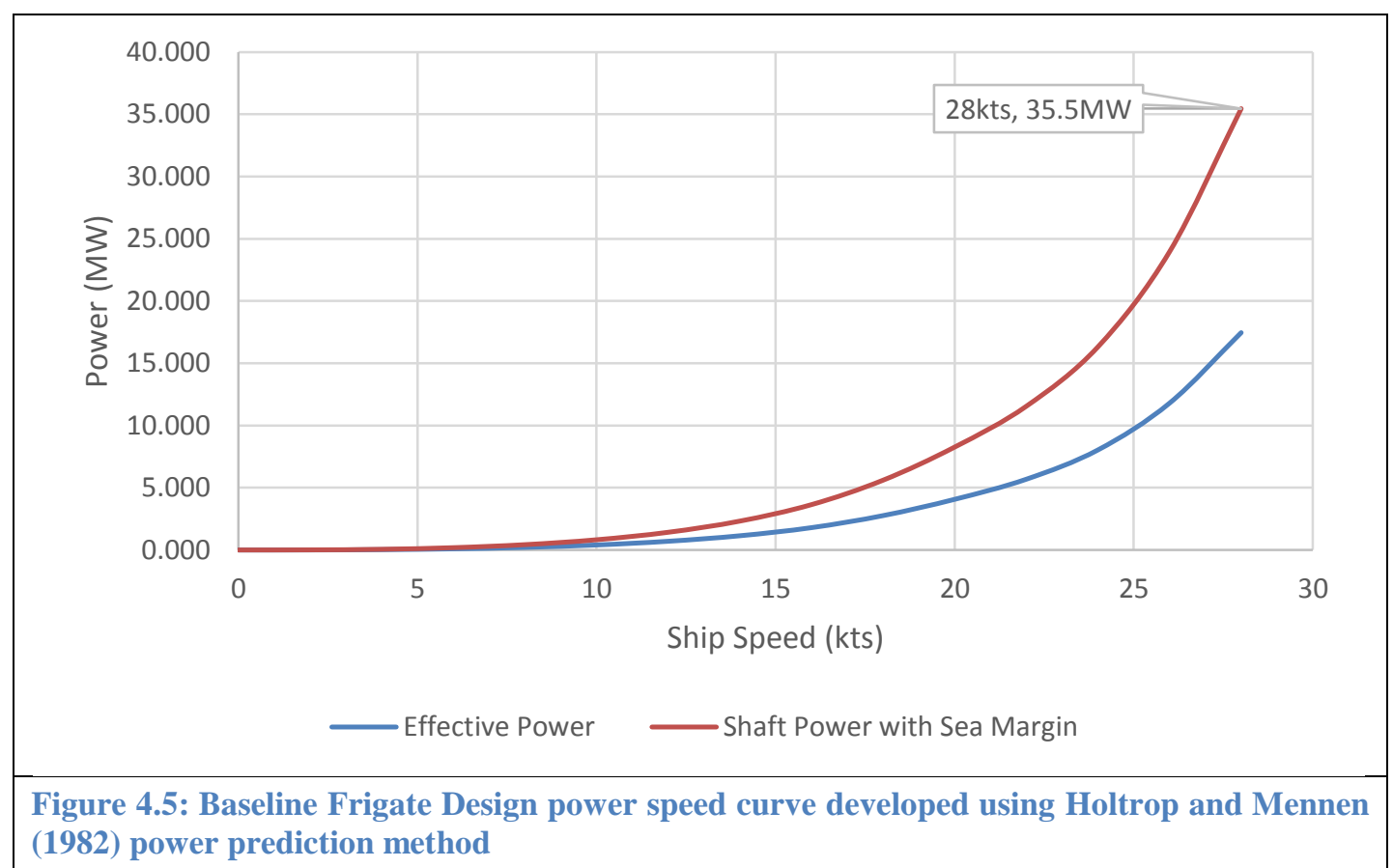

The selection of the main machinery systems for the Baseline Frigate Design was carried out according to the information available on the Type 26 GCS. The new Royal Navy frigates will have a Combined Diesel-Electric or Gas (CODLOG) system (BAE Systems, 2015). Such a hybrid machinery configuration is now considered mature after more than 30 years of service on-board the Type 23 Frigates (Buckingham, 2016). The chosen CODLOG fit consists of a mix of diesel generating set(s) and gas turbine(s). The acoustically isolated diesel generating set(s) provide low noise signatures and economical fuel consumption during transit while the gas turbine(s) enable fast sprinting (Bhatt and Arsenie, 2017). The Type 26 GCS will consist of a single Rolls Royce MT30 gas turbine (40MW) and four 20V 4000 M53B diesel generating sets (about 3MW each), providing a total installed power of approximately 52MW (Rolls Royce, 2015, 2016b). That machinery configuration was also adopted for the Baseline Frigate Design because: 
- The principal ship characteristics were very similar to the information available on the Type 26 GCS (less than $5 \%$ different);

- The total installed power of approximately 52MW was considered to meet the estimated hotel load as well as propulsion power requirements for both cruise and maximum speeds;

- The information on the Type 26 GCS machinery configuration was obtained from the supplier (Rolls Royce, 2016b) and therefore considered reliable enough for ESSD.

The machinery configuration adopted for the Baseline Frigate Design is shown in Figure 4.6.

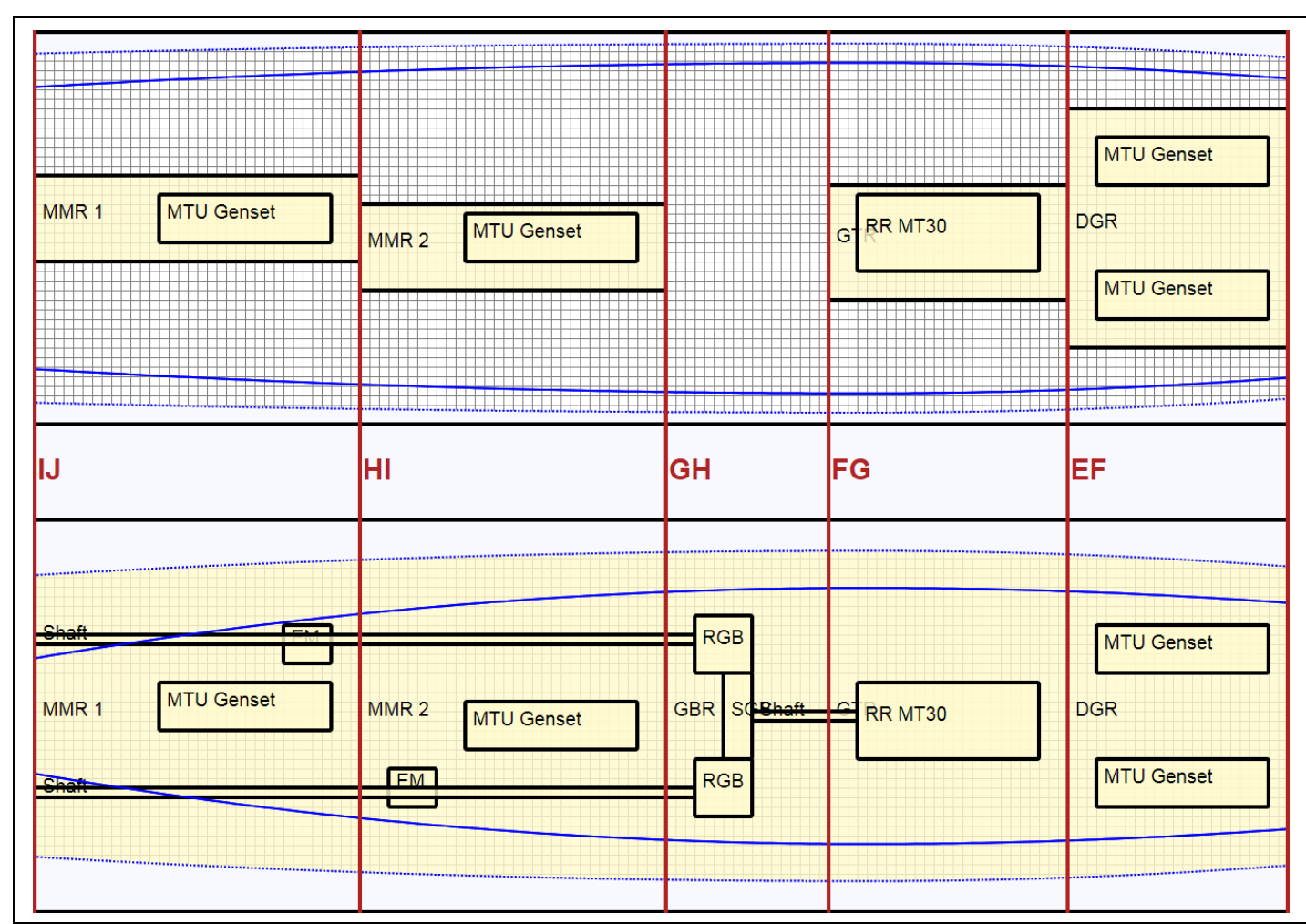

Figure 4.6: The CODLOG machinery configuration adopted for the Baseline Frigate Design 


\subsubsection{Stability Assessments}

Transverse intact stability analysis was carried out for two loading conditions, Light (i.e. the summation of all fixed weights) and Deep (i.e. the summation of all fixed and variable weights). The assessments were carried out using a frigate stability analysis tool developed by Ali (2003) as part of an MSc dissertation project at UCL and based on the regression analysis of 98 frigate hullforms. The results were evaluated against the Defence Standard 02-109 (NES 109) (UK MoD, 2000b), summarised in Table 4.6.

\begin{tabular}{|l|l|}
\hline Area under the GZ curve up to $30^{\circ}$ & Not less than $0.08 \mathrm{~m} . \mathrm{rad}$ \\
\hline Area under the GZ curve up to $40^{\circ}$ & Not less than $0.133 \mathrm{~m} \cdot \mathrm{rad}$ \\
\hline Area under the GZ curve between $30^{\circ}$ and $40^{\circ}$ & Not less than $0.048 \mathrm{~m} \cdot \mathrm{rad}$ \\
\hline Maximum GZ value & Not less than $0.3 \mathrm{~m}$ \\
\hline Angle of the maximum GZ value & Not less than $30^{\circ}$ \\
\hline $\mathrm{GM}_{\mathrm{S}}$ or GM $\mathrm{F}$ depending on the loading condition & Not less than $0.3 \mathrm{~m}$ \\
\hline Angle of vanishing stability & Not less than $70^{\circ}$ \\
\hline
\end{tabular}

Table 4.6: Shape criteria for the GZ curve (UK MoD, 2000b)

Input information to the tool included $\mathrm{L}_{\mathrm{wl}}, \mathrm{B}_{\mathrm{wl}}, \mathrm{T}, \mathrm{C}_{\mathrm{B}}, \mathrm{C}_{\mathrm{WP}}, \mathrm{D}$, and the vertical distance between the centre of gravity and keel. This distance was calculated by taking moments of all available individual weights for the Lightship condition. The Lightship GZ values were obtained using the procedure developed by Ali (2003) for angles of heel ranging between $0-90^{\circ}$. However, the tool does not perform all the checks required by Defence Standard 02-109 (UK MoD, 2000b), hence some basic naval architecture approaches were employed to complete the calculations. GMs was estimated by finding the intersection between the tangent going through the GZ curve at small angles (i.e. $0-10^{\circ}$ ) and the 1 radian ordinate. The areas under the GZ curve were approximated using the Simpson's rule for numerical integration, as well as by constructing a number of triangular and trapezoidal shapes, with both methods producing very close results. Figure 4.7 provides a graphical demonstration of this procedure. 


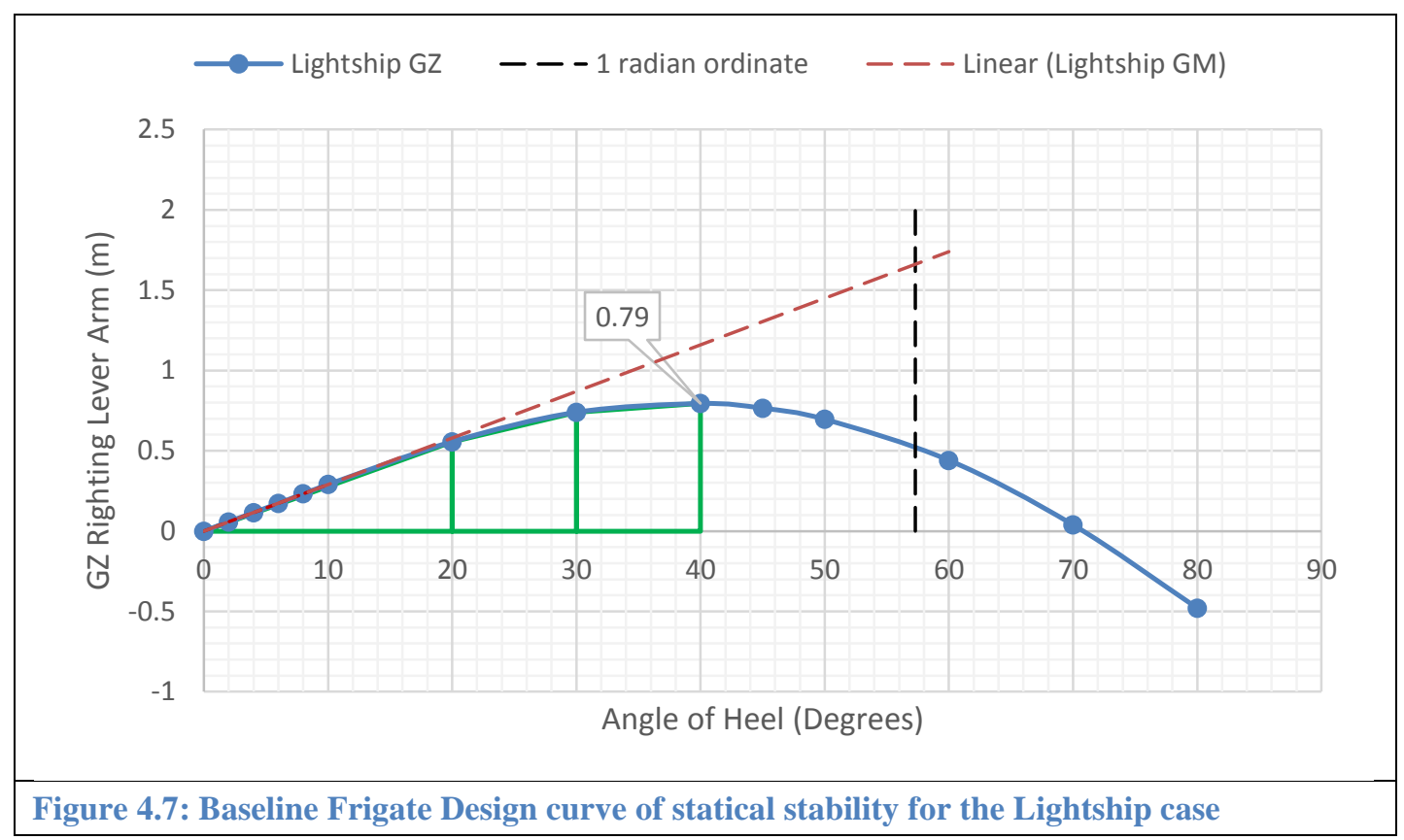

The evaluation of the Lightship transverse intact stability assessment results against the Defence Standard 02-109 (UK MoD, 2000b) is shown in Table 4.7, indicating a pass in all categories.

\begin{tabular}{|l|l|l|}
\cline { 2 - 3 } \multicolumn{1}{c|}{} & $\begin{array}{l}\text { Intact Stability } \\
\text { Assessment Results }\end{array}$ & $\begin{array}{l}\text { Defence Standard } \\
02-109\end{array}$ \\
\hline Area under the GZ curve up to $30^{\circ}$ & $0.21 \mathrm{~m} . \mathrm{rad}$ & $>0.08 \mathrm{~m} . \mathrm{rad}$ \\
\hline Area under the GZ curve up to $40^{\circ}$ & $0.34 \mathrm{~m} . \mathrm{rad}$ & $>0.133 \mathrm{~m} . \mathrm{rad}$ \\
\hline Area under the GZ curve $30^{\circ}$ to $40^{\circ}$ & $0.13 \mathrm{~m} . \mathrm{rad}$ & $>0.048 \mathrm{~m} . \mathrm{rad}$ \\
\hline Maximum GZ value & $0.79 \mathrm{~m}$ & $>0.3 \mathrm{~m}$ \\
\hline Angle of the maximum GZ value & $40^{\circ}$ & $>30^{\circ}$ \\
\hline $\mathrm{GM}_{\mathrm{S}}$ & 1.6 & $>0.3 \mathrm{~m}$ \\
\hline Angle of vanishing stability & $71^{\circ}$ & $>70^{\circ}$ \\
\hline
\end{tabular}

Table 4.7: Evaluation of the Baseline Frigate Design Lightship intact stability assessment results against Defence Standards 02-109 (UK MoD, 2000b) 
The same procedure was followed to analyse transverse intact stability for the Deep Condition, taking into account the variable weights summarised in Table 4.8.

\begin{tabular}{|l|l|}
\hline \multicolumn{1}{|c|}{ Variable Weights } & \multicolumn{1}{|c|}{ Description } \\
\hline $\begin{array}{l}\text { Liquid tanks (trim tanks, fuel oil, lube } \\
\text { oil, Aviation Carrier Air Turbine } \\
\text { (AVCAT) fuel, fresh water, sewage and } \\
\text { treated water }\end{array}$ & $\begin{array}{l}\text { As instructed by UCL (2013b), all } \\
\text { liquid tanks were assumed to be 85\% } \\
\text { full to allow for lack of pumpability, } \\
\text { tanks not being fully pressed, and the } \\
\text { volume consumed by the structure }\end{array}$ \\
\hline $\begin{array}{l}\text { Magazines (Anti-air, surface, } \\
\text { submarine, and electronic warfare) }\end{array}$ & $\begin{array}{l}\text { Full capacity was assumed } \\
\text { provisions, Navy, Army and Air Force } \\
\text { Institutes (NAAFI), naval stores, spare } \\
\text { parts, clothing and mess gear) }\end{array}$ \\
\hline $\begin{array}{l}\text { Modular mission payload (helicopter } \\
\text { and other mission specific vehicles) }\end{array}$ & $\begin{array}{l}\text { Interchangeable items of mission- } \\
\text { specific equipment }\end{array}$ \\
\hline
\end{tabular}

Table 4.8: The variable weights taken into account for Deep intact stability assessment

Given that the approach by Ali (2003) does not account for the free surface effects caused by the liquid tanks, the GZ curve had to be corrected. Ship tanks are usually not simple geometric shapes, hence determining the corrected GZ curve for multiple angles of inclination is not easily achievable without the use of computerised integrators. It is therefore common to employ simplifying assumptions as proposed by Rawson and Tupper (2001). The proposal involves computing the GZ value for $45^{\circ}$ angle of heel allowing for free surface. The corrected GZ curve up to $45^{\circ}$ is then constructed by drawing the curve through the corrected GZ value at $45^{\circ}$, following the general character of the uncorrected GZ curve. For angles of inclination larger than $45^{\circ}$ the reduction of $\mathrm{GZ}$ at $45^{\circ}$ is applied as a constant correction, as shown in Figure 4.8. 


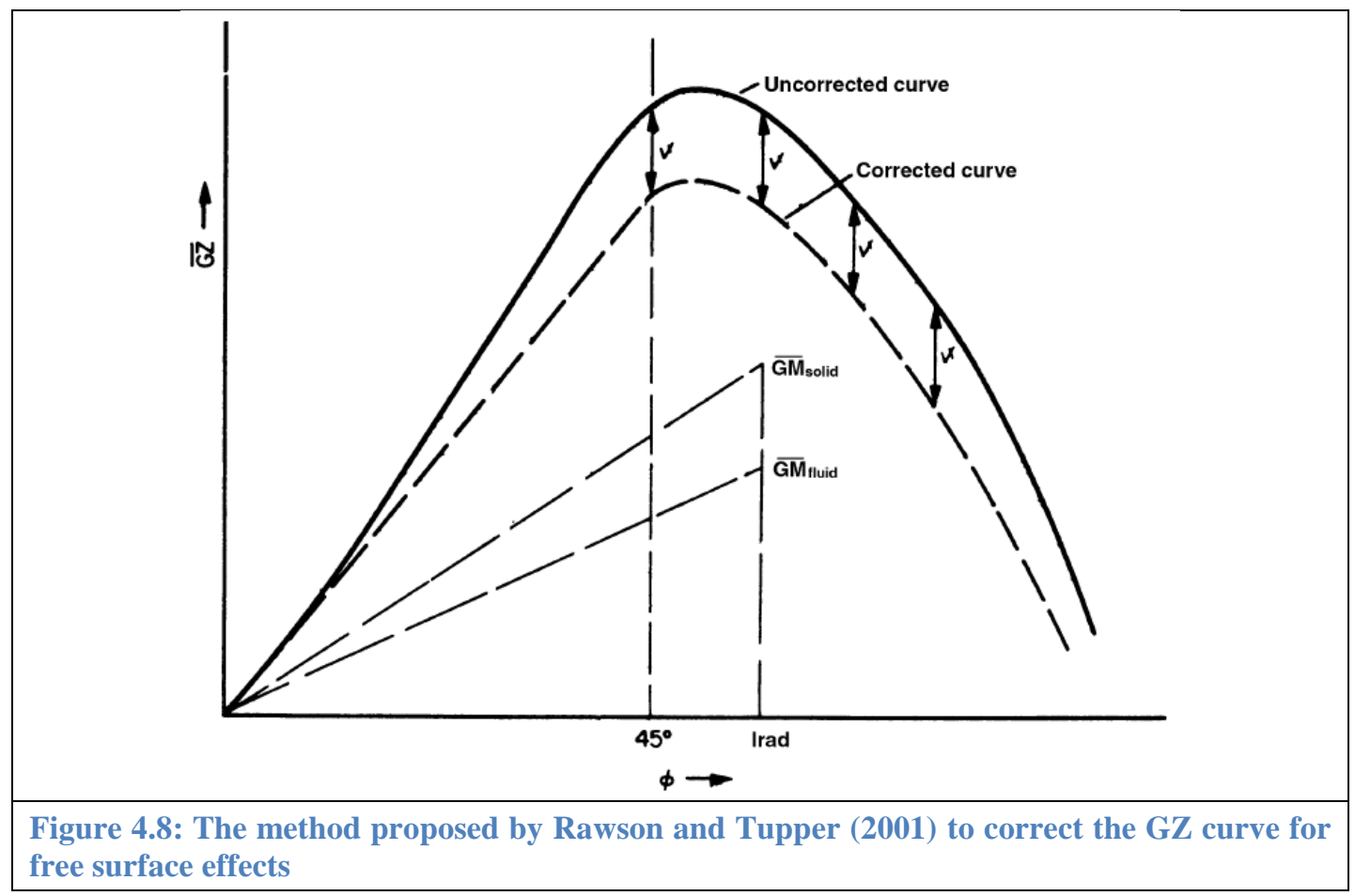

The Deep uncorrected GZ curve was produced using the procedure developed by Ali (2003) for angles of heel ranging between $0-90^{\circ}$, and was corrected following the method shown in Figure 4.8. $\mathrm{GM}_{\mathrm{F}}$ was then estimated by finding the intersection between the tangent going through the corrected GZ curve at small angles (i.e. $0-10^{\circ}$ ) and the 1 radian ordinate. The areas under the corrected GZ curve were estimated using the same methods applied for the Lightship case. This procedure is shown graphically in Figure 4.9.

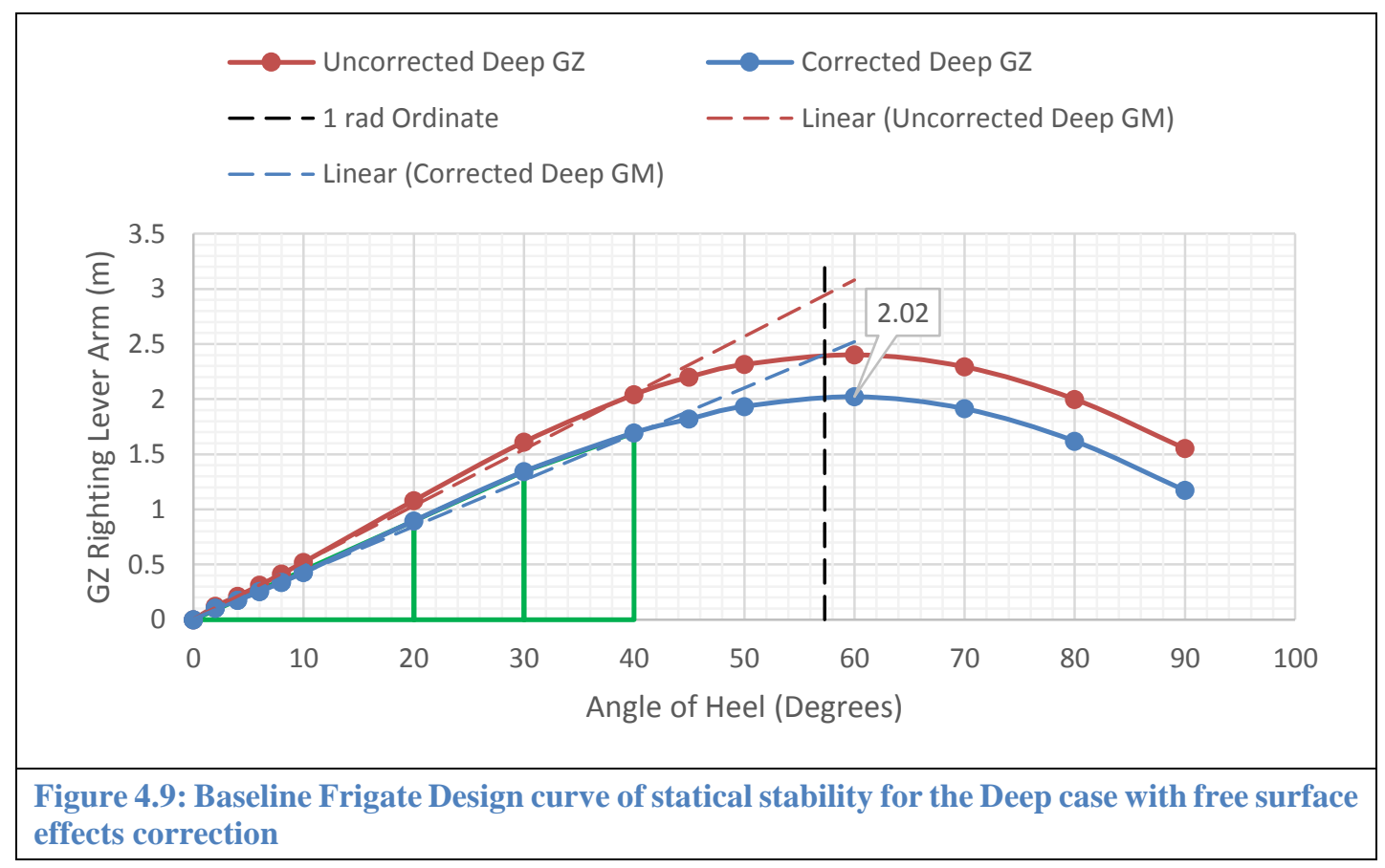


The evaluation of the Deep transverse intact stability assessment results against the Defence Standard 02-109 (UK MoD, 2000b) is shown in Table 4.9, indicating a pass in all categories.

\begin{tabular}{|l|l|l|}
\cline { 2 - 3 } \multicolumn{1}{c|}{} & $\begin{array}{l}\text { Intact Stability } \\
\text { Assessment Results }\end{array}$ & $\begin{array}{l}\text { Defence Standard } \\
02-109\end{array}$ \\
\hline Area under the GZ curve up to $30^{\circ}$ & $0.35 \mathrm{~m} \cdot \mathrm{rad}$ & $>0.08 \mathrm{~m} \cdot \mathrm{rad}$ \\
\hline Area under the GZ curve up to $40^{\circ}$ & $0.61 \mathrm{~m} . \mathrm{rad}$ & $>0.133 \mathrm{~m} \cdot \mathrm{rad}$ \\
\hline Area under the GZ curve $30^{\circ}$ to $40^{\circ}$ & $0.26 \mathrm{~m} . \mathrm{rad}$ & $>0.048 \mathrm{~m} \cdot \mathrm{rad}$ \\
\hline Maximum GZ value & $2.02 \mathrm{~m}$ & $>0.3 \mathrm{~m}$ \\
\hline Angle of the maximum GZ value & $60^{\circ}$ & $>30^{\circ}$ \\
\hline GM & 2.40 & $>0.3 \mathrm{~m}$ \\
\hline Angle of vanishing stability & $107^{\circ}$ & $>70^{\circ}$ \\
\hline
\end{tabular}

Table 4.9: Evaluation of the Baseline Frigate Design Deep intact stability assessment results against Defence Standards 02-109 (UK MoD, 2000b)

\subsubsection{Internal Arrangement}

The Baseline Frigate Design was intended to represent a conventional modern frigate type ship. The hull consisted of four internal decks, including the double bottom. No. 2 Deck or Damage Control Deck (DCD) was the only passing deck and served as the main passageway deck, containing a single central passageway. It was argued in Section 2.2.6 that in describing a vessel at the top level, functional breakdown is a sensible alternative to the current practice of using group weight breakdowns. The development of the Baseline Frigate Design and in particular the internal arrangement were carried out according to the UCL functional groups of FLOAT, MOVE, FIGHT and INFRASTRUCTURE for naval vessels (Andrews and Pawling, 2003). Figure 4.10 illustrates the Baseline Frigate Design internal arrangement produced using the UCL JavaScript ship design tool. The DBBs are assigned a numerical identifier, a visible outline and nametag, and a colour to identify them as belonging to a specific functional group in the GA using the existing DBB functional breakdown colour code, namely FLOAT - Blue, MOVE - Yellow, FIGHT - Red, INFRASTRUCTURE - Green (Andrews and Pawling, 2003). Figure 4.10 also displays the deck outlines (blue lines), deck vertical positions measured from the keel, and disposition of the main WTBs (red lines). 


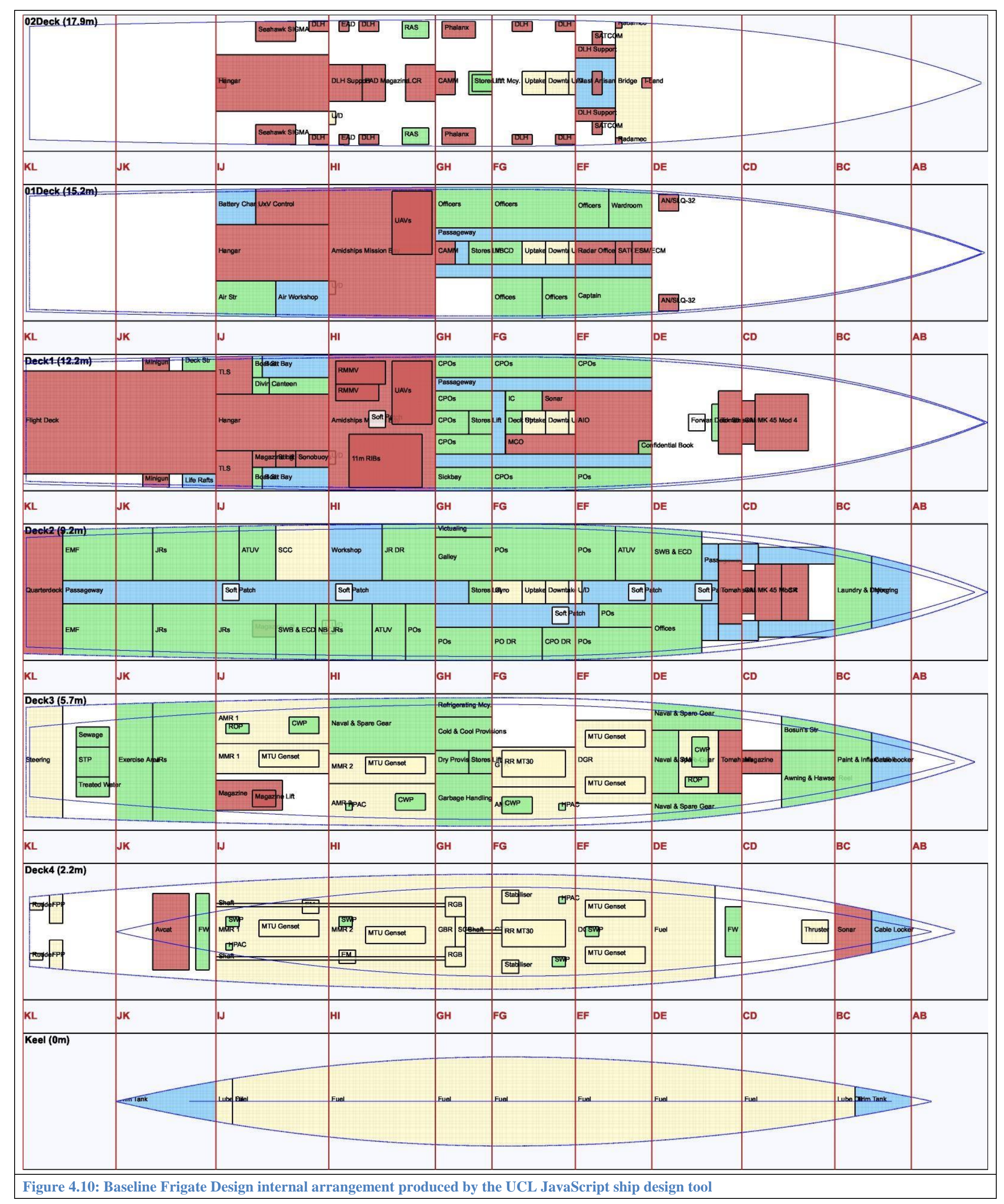




\subsubsection{Ship Costing}

The Unit Procurement Cost (UPC) of the Baseline Frigate Design was estimated by applying the top-down (parametric) approach to ship costing (see Appendix 5, Section Ship Costing). The analysis was carried out for the Lightship displacement (i.e. no variable weights) with no Board and Growth Margins included. The UPC data was based on cost returns for a 4000te Royal Navy frigate constructed in the UK during the 1990s (UCL, 2013b). At the time of developing the cost model, the latest complete annual inflation data that could be obtained from the Office for National Statistics (2017) was for the year 2016, thus the figures were inflated to equivalent 2016 prices. The figures were representative of the fourth vessel of a class of twelve ships and did not include First of Class (FOC) costs. Figure 4.11 shows the Baseline Frigate Design total UPC and breakdown based on the warship seven weight groups. The cost figures include minor equipment (8\%) and industrial contingency margins for lack of positive data $(10 \%)$ and the displacement correction factor $(0.5 \%)$ to account for the fact that the parametric relationships were related to a 4000te frigate (Dirksen, 1996).

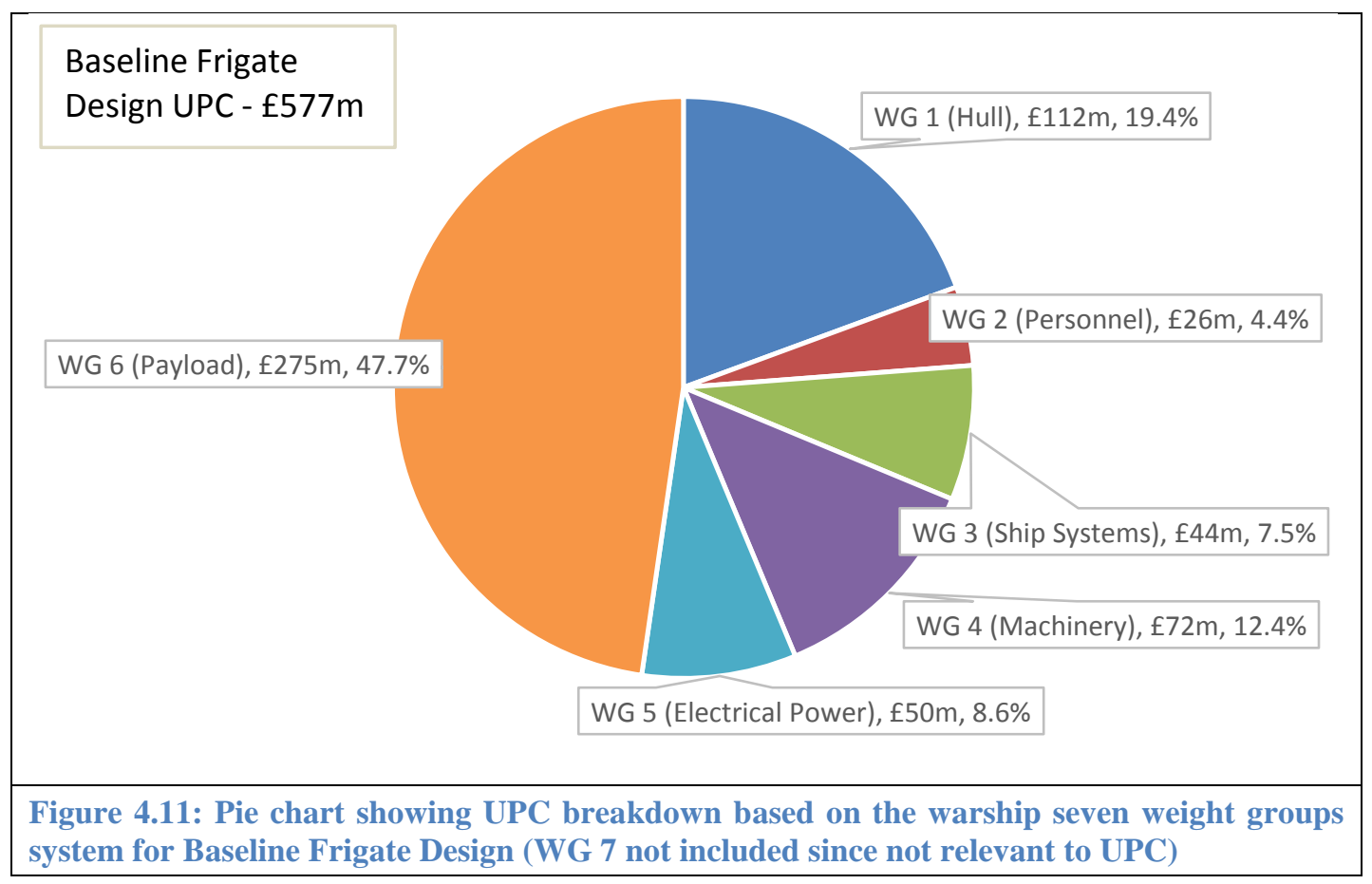


The cost analysis did not include the estimation of Through-Life Cost (TLC) because a) personnel, training, fuel, stores, and disposal cost elements were not considered to be directly relevant to an architectural investigation of supportability, and b) the UCL (2013b) parametric procedure for costing the supportability issues (e.g. general maintenance, minor refit and major modernisation) is very rudimentary. The procedure estimates the costs associated with ship maintenance, refit, and upgrade as proportional to the UPC, based on fixed intervals and person-hour values, and regardless of how the adopted ship internal arrangement might affect some of these issues. Given that many aspects of naval ship supportability are architecturally-driven (see Section 2.3.2) as well as the configurational emphasis of this research, it was concluded that such crude TLC estimations were unlikely to produce worthwhile supportability costing information.

\subsubsection{Supportability Features}

The supportability features considered in this research for a partial evaluation of ship maintainability were a) the on-board workshop, b) Naval and Spare Gear Stores (NSGS), and c) the Equipment Removal Points (ERP). The Baseline Frigate Design was intended to represent the minimum sensible levels of these features given the ship's capability, as described in Table 4.10 and shown in Figure 4.12.

\begin{tabular}{|l|l|}
\hline \multicolumn{1}{|c|}{$\begin{array}{c}\text { Supportability } \\
\text { Feature }\end{array}$} & \multicolumn{1}{c|}{ Description } \\
\hline On-board workshop & $\begin{array}{l}\text { Single space, astern of amidships on No. 2 Deck, weight } \\
\text { and volume information were based on the UCL (2013b) }\end{array}$ \\
\hline $\begin{array}{l}\text { Naval and Spare } \\
\text { Gear Stores }\end{array}$ & $\begin{array}{l}\text { Split in two spaces, forward and aft on No. 3 Deck, weight } \\
\text { and volume information were based on the UCL (2013b) }\end{array}$ \\
\hline $\begin{array}{l}\text { Equipment Removal } \\
\text { Points }\end{array}$ & $\begin{array}{l}\text { Two locations, amidships mission bay and forward of the } \\
\text { superstructure on No. 1 Deck }\end{array}$ \\
\hline
\end{tabular}

Table 4.10: Disposition of the Baseline Frigate Design supportability features 


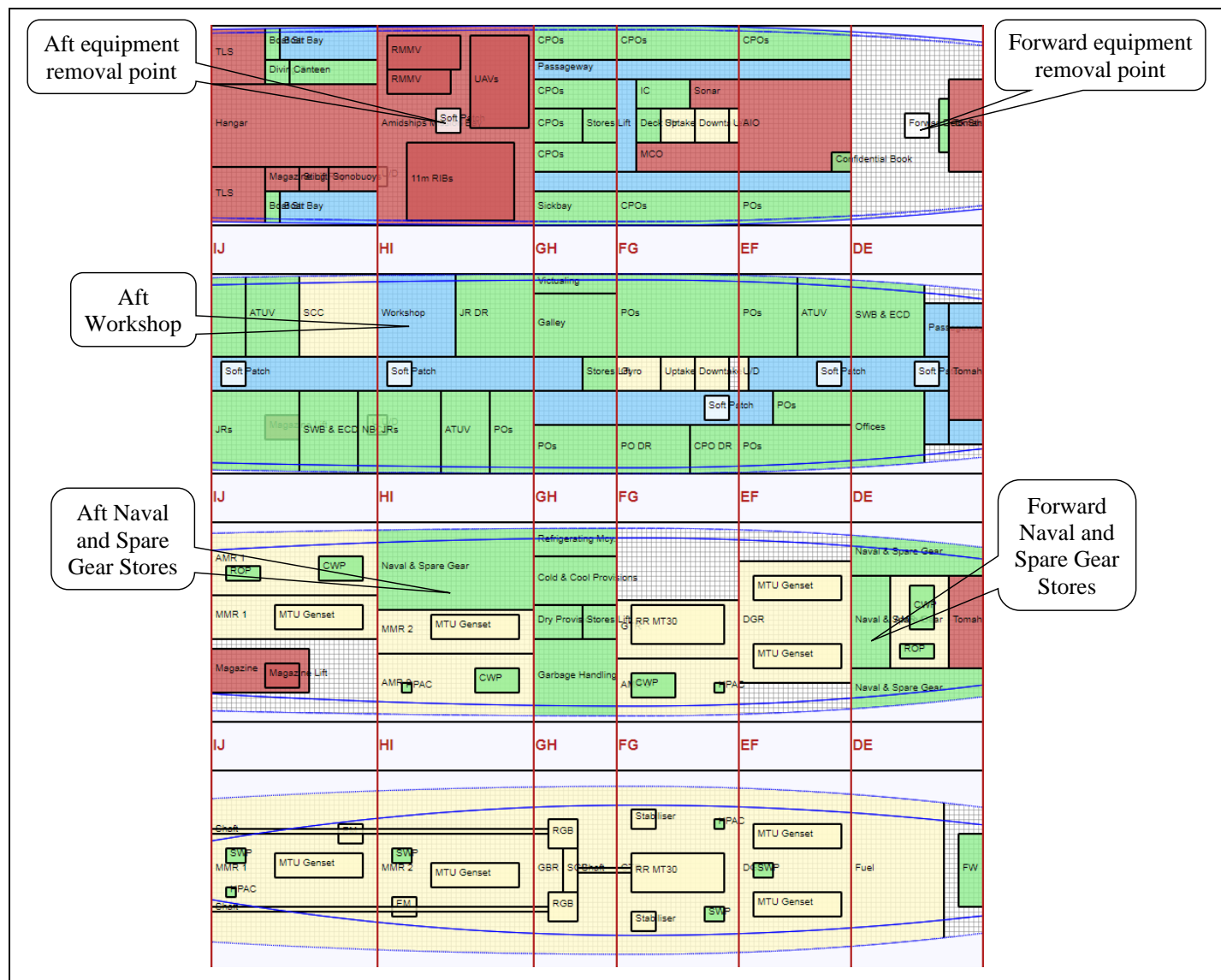

Figure 4.12: Baseline Frigate Design deck plan highlighting supportability features, produced by the UCL JavaScript ship design tool

\subsubsection{Ship Design Aspects not Considered in the Baseline Frigate Design Study}

A number of ship design aspects were not considered due to the limited number of analytical tools associated with the JavaScript tool comparing to Paramarine (Munoz and Forrest, 2002). The UCL attempt to reduce the reliance on fully sophisticated 3D CAD softwares (e.g. Paramarine) for ESSD research and the subsequent lack of computerised integration for this research, meant that damage stability could not be investigated. A proper consideration of structural design aspects, involving the extraction of wave-induced bending moments, amidships section structural analysis, and grillage design were considered beyond normal ESSD and not carried out. Structural considerations were limited to meeting the restriction on overall length to amidships hull depth ratio (i.e. should be less than 12 at concept design (Chalmers, 1993)), locating bulkheads at the forward and aft ends of the superstructure and after cut-up, and ensuring that structural continuity was maintained throughout the ship as best as possible. Survivability considerations were restricted to good naval ship design practice (i.e. duplicating and separating major ship systems (e.g. power generation, 
chilled water, high-pressure air, and sea and fresh water generation) and locating certain important ship spaces (e.g. Operations Room, bridge, and Ship Machinery Control Centre) far from each other). Seakeeping and manoeuvring were not investigated given the early stage nature of the ship design and as they were not considered crucial to the nascent concept.

While fully acknowledging that the proper development of a naval ship design requires that all design aspects are thoroughly investigated, issues considered to be more relevant to the evaluation of naval ship supportability were prioritised given the focus of this research project. 


\subsection{Ship Design Variants}

Ship design variants were chosen to explore the effects of various internal arrangements and implications of altering the on-board workshop facilities and NSGS capacities using the proposed measure of supportability performance. Therefore the main ship performance requirements, payload equipment, and complement and accommodation breakdown adopted in all variants were identical to those of the Baseline Frigate Design.

\subsubsection{Configurational Variants of the Baseline Frigate Design}

Departing from the original disposition of the Baseline Frigate Design supportability features shown in Table 4.10 and Figure 4.12, three layout variants were initially produced with the aim of exploring the effects of better accessibility for support. While the number and location of the ERPs were left unchanged for all the ship design variants, the on-board workshop and NSGSs were broken up into a greater number of but individually smaller spaces (i.e. less weight and volume), in order to reduce the indicative travel time between them and given shipboard spaces. Crucially, this was carried out without altering the overall combined capacity (volume) of these particular spaces. The Baseline Frigate Design and its configurational variations are described in Table 4.11. The supportability features are highlighted in Figure 4.13. Complete internal arrangements can be found in Figure A5. 4 - Figure A5. 6 (Appendix 5).

\begin{tabular}{|l|l|}
\hline \multicolumn{1}{|c|}{ Ship Design } & \multicolumn{1}{c|}{ Description of the Supportability Features } \\
\hline $\begin{array}{l}\text { Baseline } \\
\text { Frigate }\end{array}$ & $\begin{array}{l}\text { One workshop, astern of amidships on No. 2 Deck } \\
\text { Two NSGSs, forward and aft on No. 3 Deck }\end{array}$ \\
\hline Variant 1 & $\begin{array}{l}\text { Two workshops, forward and aft on No. 2 Deck } \\
\text { Two NSGSs, forward and aft on No. 3 Deck }\end{array}$ \\
\hline Variant 2 & $\begin{array}{l}\text { One workshop, astern of amidships on No. 2 Deck } \\
\text { Three NSGSs, forward, amidships and aft on No. 3 Deck }\end{array}$ \\
\hline Variant 3 & $\begin{array}{l}\text { Two workshops, forward and aft on No. 2 Deck } \\
\text { Three NSGSs, forward, amidships and aft on No. 3 Deck }\end{array}$ \\
\hline
\end{tabular}

Table 4.11: Disposition of the supportability features in the Baseline Frigate Design and its configurational variants 


\begin{tabular}{|c|c|c|c|c|c|c|c|c|c|c|c|c|c|c|c|}
\hline \multirow[t]{4}{*}{$\begin{array}{l}\text { Aft equipment } \\
\text { removal point }\end{array}$} & 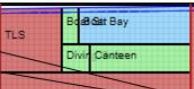 & enever & 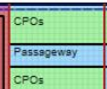 & $\frac{100}{100}$ & enter & $=$ & \multirow[t]{18}{*}{$\begin{array}{c}\text { Forward } \\
\text { equipment removal }\end{array}$} & \multirow[t]{4}{*}{$\begin{array}{l}\text { Aft equipment } \\
\text { removal point }\end{array}$} & \multirow{3}{*}{ 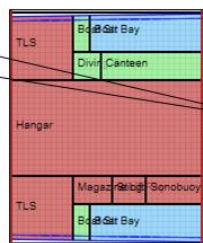 } & \multirow{2}{*}{ rave } & \multicolumn{2}{|l|}{ 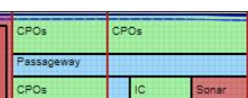 } & 100, & \multirow[t]{2}{*}{ wam } & \multirow[t]{2}{*}{$\begin{array}{c}\text { Forward } \\
\text { equipment removal }\end{array}$} \\
\hline & 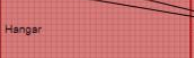 & $x$ & \begin{tabular}{|c|c|}
$\infty$ & $=0-0$ \\
\end{tabular} & 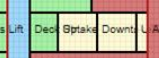 & & & & & & & \begin{tabular}{l|l}
$\cos ^{\cos }$ & \\
\end{tabular} & 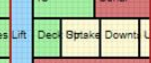 & H.o & & \\
\hline & 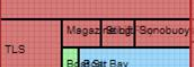 & 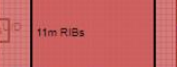 & sess & 100 & & & & & & \multirow{2}{*}{ 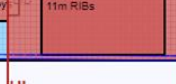 } & coss & & & & \\
\hline & w & HI & GH & FG & EF & OE & & & IN & & \multirow{3}{*}{\multicolumn{2}{|c|}{$\frac{\mathrm{GH}}{\mathrm{Oang}}$}} & EF & \multirow{2}{*}{ DE } & \\
\hline \multirow{4}{*}{$\begin{array}{c}\text { Aft } \\
\text { Workshop } \\
\end{array}$} & tave & wateros & oner & pos & $\infty$ & & & \multirow{4}{*}{$\begin{array}{c}\text { Aft } \\
\text { Workshop }\end{array}$} & \multirow[b]{2}{*}{ 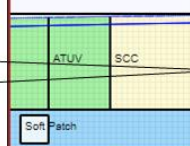 } & 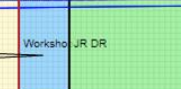 & & & \multirow{2}{*}{ atov } & & \multirow{4}{*}{ 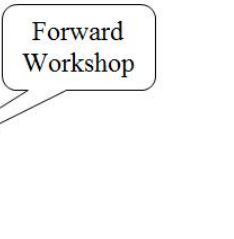 } \\
\hline & $\frac{1}{80 \times n+\infty}$ & 䂩我 & $1 \mathrm{sen}$ & 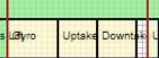 & 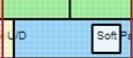 & ten & & & & & & & & 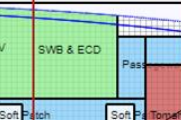 & \\
\hline & & 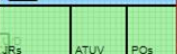 & 工 & 50 & $P^{\infty}$ & areacen & & & & 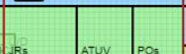 & - & 㑭 & $\int^{-1}$ & & \\
\hline & 8 & \begin{tabular}{l|l|l} 
& \\
\end{tabular} & Pos & \begin{tabular}{|l|l|}
0008 & 00008 \\
\end{tabular} & & & & & & 8 & $\infty$ & \begin{tabular}{|c|c|} 
Poor & Coo 08 \\
\end{tabular} & pos & & \\
\hline Aft Naval & s & HI & GH & $=6$ & EF & DE & & $\begin{array}{l}\text { Aft Naval } \\
\text { and Spare }\end{array}$ & s & HI & oH & & & $\mathrm{DE}$ & $\begin{array}{l}\text { Forward } \\
\text { Naval and }\end{array}$ \\
\hline $\begin{array}{l}\text { and Spare } \\
\text { Gear Stores }\end{array}$ & 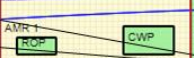 & 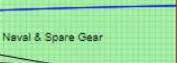 & $\cos a \cos \theta=$ & 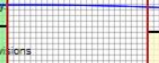 & (5) & & & Gear Stores & 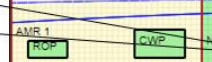 & trasencour & costcosent & & 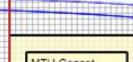 & 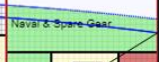 & $\begin{array}{l}\text { Naval Gear } \\
\text { Spare Gear } \\
\text { Stores }\end{array}$ \\
\hline & & & & & 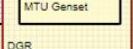 & 网 & & & & & . & & nurvenes & & \\
\hline & $=$ & wame $=$ & 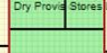 & Aerurso & (s) & 年 & & & $=$ & 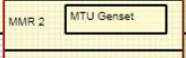 & or bects & Aneranos & (1) & - & \\
\hline & - & 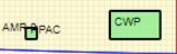 & aromonto & tere & 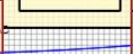 & 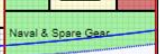 & & & 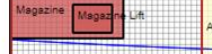 & W & anoment & $\sqrt{m}$ & 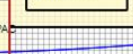 & 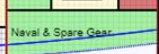 & \\
\hline & s & HI & कн & FG & $E F$ & DE & & & so & H1 & कн & Fo & EF & DE & \\
\hline & & & 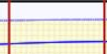 & & ( & E & & & & & 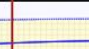 & & +2 & t & \\
\hline & 西 & & $=$ =网 & 四 & Erocosenter & 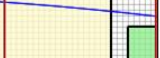 & & & 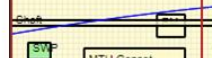 & & $=$ & sas & 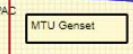 & & \\
\hline & 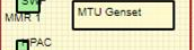 & 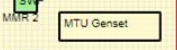 & $\cos 5=$ & 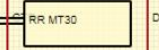 & & Fene & & & Wunt & 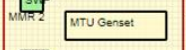 & $\cos 13=5$ & 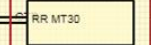 & 四 & fow & \\
\hline $\begin{array}{l}\text { Frigate } \\
\text { Design }\end{array}$ & & 䍙 & $=$ =006 & 国必 & Ervocmen & & & Variant 1 & & & =成 & 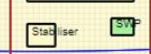 & rrovestan & & \\
\hline Aft equipment & 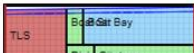 & 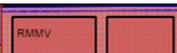 & coon & +oon & कon & एक्य & Forward & Aft equipment & ns & $\overline{\text { pauv }}$ & coos & & (cose & प्w? & Forward \\
\hline & $1 \mathrm{plom}$ & \begin{tabular}{|l|l|} 
Emom & \\
\end{tabular} & cons & & - & & equipment removal & removal point & 17 & $\begin{aligned} \operatorname{mov} \\
\end{aligned}$ & cosos & & & & equipment removal \\
\hline & Hown & $-\infty$ & 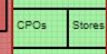 & 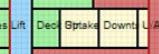 & & & & & tom & 政 & cos 15 & & & & \\
\hline & 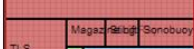 & at & 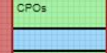 & 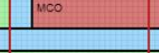 & & & & & is & 1nasas & sos & & & & \\
\hline & 年 & & scoen & (con & $+\infty$ & Pan & & & Fisten & & scosen & 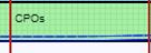 & $=$ & 国皮 & \\
\hline & su & H1 & он & $F=$ & EF & DE & & & is & Hi & GH & FG & EF & DE & \\
\hline $\begin{array}{c}\text { Aft } \\
\text { Workshop }\end{array}$ & \begin{tabular}{|l|l|} 
& anv \\
asc
\end{tabular} & $=$ & onest & $+\infty$ & tow & suseceo & & $\begin{array}{c}\text { Aft } \\
\text { Workshop }\end{array}$ & TE & & onem & Pow & ano & sorsecos & $\begin{array}{l}\text { Forward } \\
\text { Workshop }\end{array}$ \\
\hline & 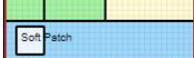 & 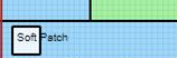 & $1 \mathrm{sen}$ & 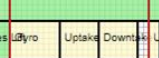 & 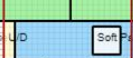 & & & & 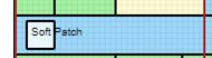 & ⿶as & $\mathrm{T}_{\mathrm{s}}$ & 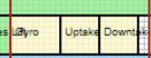 & & & \\
\hline & \begin{tabular}{|l|l|} 
& \\
\end{tabular} & \begin{tabular}{|l|l|l|} 
& & \\
\end{tabular} & 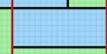 & 国保 & $P^{+\infty}$ & & & & \begin{tabular}{|l|l}
$-a$ & \\
\end{tabular} & 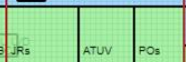 & 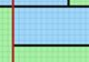 & 囦 & $P^{\text {pos }}$ & & \\
\hline & 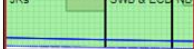 & $0^{10}$ & pos & 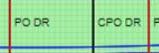 & Pos & & & & & 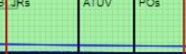 & pos & coovo & & 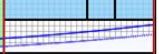 & \\
\hline Aft Naval & $\omega$ & HI & $\mathrm{GH}$ & $F=$ & EF & OE & Forward & Aft Naval & is & H1 & он & $=6$ & EF & DE & Forward \\
\hline $\begin{array}{l}\text { and Spare } \\
\text { Gear Stores }\end{array}$ & Nave & & $+\infty$ & . & 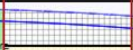 & 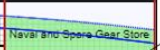 & $\begin{array}{l}\text { Naval and } \\
\text { Spare Gear }\end{array}$ & $\begin{array}{l}\text { and Spare } \\
\text { Gear Stores }\end{array}$ & & & + & & 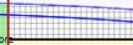 & 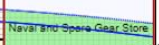 & 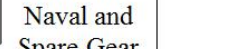 \\
\hline & 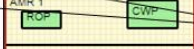 & 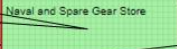 & 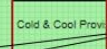 & 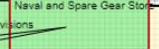 & 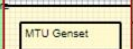 & & $\begin{array}{l}\text { Spare Gear } \\
\text { Stores }\end{array}$ & Gear stores & $\infty$ & 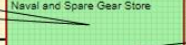 & costeces & 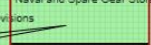 & 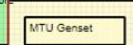 & & $\begin{array}{l}\text { Spare Gear } \\
\text { Stores }\end{array}$ \\
\hline & 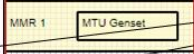 & 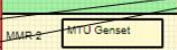 & 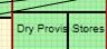 & Serentron & & $17-$ & & Amidshins & 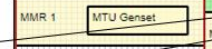 & 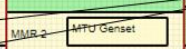 & Doproupls: & fiperarion & & at. & \\
\hline $\begin{array}{l}\text { Amidssips } \\
\text { Naval and }\end{array}$ & 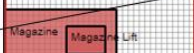 & (10me & & & 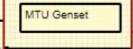 & 国 & & Naval and & 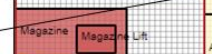 & 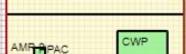 & onsone. & & 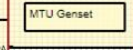 & 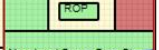 & \\
\hline $\begin{array}{l}\text { Spare Gear } \\
\text { Stores }\end{array}$ & ـ & 西 & 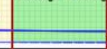 & 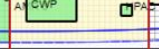 & 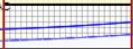 & & & $\begin{array}{l}\text { Spare Gear } \\
\text { Stores }\end{array}$ & 年 & $\square$ & & & 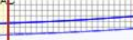 & ( & \\
\hline & su & Hi & $\mathrm{GH}$ & $F G$ & EF & OE & & & L & H1 & GH & $=6$ & EF & DE & \\
\hline & & $D^{2}$ & 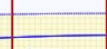 & & 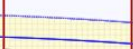 & 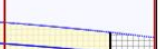 & & & & & & & & & \\
\hline & & & =网 & $-\quad 0^{\circ}$ & wruoserser & & & & & & =䎟 & 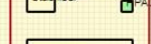 & 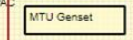 & П & \\
\hline & wind & 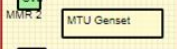 & $\cos 25=0$ & enerson & 반 & & & & $a_{0 \rightarrow 0}$ & Wrut conset & osens $5=$ & 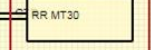 & 0 河 & Fout & \\
\hline Variant 2 & & 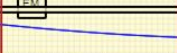 & $=$ & 㖥 & tone & 15 & & Variant 3 & & & 4 & 囦 & 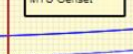 & 1 & \\
\hline
\end{tabular}


Table 4.12 shows the major ship characteristics and estimated UPC of the Baseline Frigate and the three configurational variants described in Table 4.11 (see Figure A5. 7 - Figure A5. 9 (Appendix 5) for UPC breakdowns). It can be seen that since the variants were essentially configurational rearrangements of the Baseline Frigate Design, the overall design impacts were commensurately very small.

\begin{tabular}{|l|r|r|r|r|}
\hline $\begin{array}{c}\text { Major Ship } \\
\text { Characteristics }\end{array}$ & \multicolumn{1}{c|}{$\begin{array}{c}\text { Baseline } \\
\text { Frigate }\end{array}$} & $\begin{array}{c}\text { Ship Design } \\
\text { Variant 1 }\end{array}$ & \multicolumn{1}{c|}{$\begin{array}{c}\text { Ship Design } \\
\text { Variant 2 }\end{array}$} & \multicolumn{1}{c|}{$\begin{array}{c}\text { Ship Design } \\
\text { Variant 3 }\end{array}$} \\
\hline$V_{G}$ & $23873 \mathrm{~m}^{3}$ & $23850 \mathrm{~m}^{3}$ & $23863 \mathrm{~m}^{3}$ & $23850 \mathrm{~m}^{3}$ \\
\hline$\Delta_{D}$ & $6632 \mathrm{te}$ & $6637 \mathrm{te}$ & $6639 \mathrm{te}$ & $6637 \mathrm{te}$ \\
\hline$\Delta_{L}$ & $4570 \mathrm{te}$ & $4584 \mathrm{te}$ & $4585 \mathrm{te}$ & $4584 \mathrm{te}$ \\
\hline$\rho$ & $0.278 \mathrm{te} / \mathrm{m}^{3}$ & $0.278 \mathrm{te} / \mathrm{m}^{3}$ & $0.278 \mathrm{te} / \mathrm{m}^{3}$ & $0.278 \mathrm{te} / \mathrm{m}^{3}$ \\
\hline$v_{s}$ & 0.310 & 0.319 & 0.315 & 0.319 \\
\hline$L_{w l}$ & $137.0 \mathrm{~m}$ & $137.0 \mathrm{~m}$ & $137.0 \mathrm{~m}$ & $137.0 \mathrm{~m}$ \\
\hline$L_{o a}$ & $145.0 \mathrm{~m}$ & $145.0 \mathrm{~m}$ & $145.0 \mathrm{~m}$ & $145.0 \mathrm{~m}$ \\
\hline$B_{w l}$ & $18.3 \mathrm{~m}$ & $18.3 \mathrm{~m}$ & $18.3 \mathrm{~m}$ & $18.3 \mathrm{~m}$ \\
\hline$B_{M D}$ & $20.6 \mathrm{~m}$ & $20.6 \mathrm{~m}$ & $20.6 \mathrm{~m}$ & $20.6 \mathrm{~m}$ \\
\hline$T$ & $5.7 \mathrm{~m}$ & $5.7 \mathrm{~m}$ & $5.7 \mathrm{~m}$ & $5.7 \mathrm{~m}$ \\
\hline$D$ & $12.2 \mathrm{~m}$ & $12.2 \mathrm{~m}$ & $12.2 \mathrm{~m}$ & $12.2 \mathrm{~m}$ \\
\hline$F$ & $7.8 \mathrm{~m}$ & $7.8 \mathrm{~m}$ & $7.9 \mathrm{~m}$ & $7.8 \mathrm{~m}$ \\
\hline$U P C$ & $£ 577 \mathrm{~m}$ & $£ 578 \mathrm{~m}$ & $£ 578 \mathrm{~m}$ & $£ 578 \mathrm{~m}$ \\
\hline
\end{tabular}

Table 4.12: Major ship characteristics and UPC of the Baseline Frigate Design and configurational variants

\subsubsection{Enhancement of Certain Supportability Features}

Given that the overall workshop and NSGS capacities remained unchanged in the above ship design cases, the next series of ship designs were developed with the aim of investigating how increasing the overall capacity (volume) of these spaces might affect the proposed measure of supportability. Outlined in Section 4.3, the Baseline Frigate was treated as the benchmark for these investigations. For ship design Variants 1,2 , and 3 , the overall workshop or NSGS capacities were increased while maintaining the same dispositions shown in Figure 4.13. As outlined in Table 4.13, overall workshop capacity was first increased, followed by the NSGSs, and finally both spaces were enlarged simultaneously in order to identify any major implications. 


\begin{tabular}{|l|l|}
\hline Ship Design & \multicolumn{1}{c|}{ Description of the Supportability Features } \\
\hline Variant 1.1 & $\begin{array}{l}\text { Two workshops, overall capacity increased by } \mathbf{5 0 \%} \\
\text { Two NSGSs, overall capacity unchanged from Baseline Frigate }\end{array}$ \\
\hline Variant 1.2 & $\begin{array}{l}\text { Two workshops, overall capacity increased by 100\% } \\
\text { Two NSGSs, overall capacity unchanged from Baseline Frigate }\end{array}$ \\
\hline Variant 2.1 & $\begin{array}{l}\text { One workshop, overall capacity unchanged from Baseline Frigate } \\
\text { Three NSGSs, overall capacity increased by } \mathbf{5 0 \%}\end{array}$ \\
\hline Variant 2.2 & $\begin{array}{l}\text { One workshop, overall capacity unchanged from Baseline Frigate } \\
\text { Three NSGSs, overall capacity increased by } \mathbf{1 0 0 \%}\end{array}$ \\
\hline Variant 3.1 & $\begin{array}{l}\text { Two workshops, overall capacity increased by } \mathbf{5 0 \%} \\
\text { Three NSGSs, overall capacity increased by } \mathbf{5 0 \%}\end{array}$ \\
\hline Variant 3.2 & $\begin{array}{l}\text { Two workshops, overall capacity increased by } \mathbf{1 0 0 \%} \\
\text { Three NSGSs, overall capacity increased by } \mathbf{1 0 0 \%}\end{array}$ \\
\hline
\end{tabular}

Table 4.13: Ship design variants with extra overall workshop or/and Naval and Spare Gear Store capacities

Ship design Variants 1.1 and 1.2 were developed by increasing the overall workshop capacity (volume) of Variant 1, first by $50 \%$ and then $100 \%$. Major ship characteristics and estimated UPC of the resulting ship designs as well as those of Variant 1 are shown in Table 4.14. Complete internal arrangements and UPC breakdowns can be found in Figure A5. 10 - Figure A5. 13 (Appendix 5).

\begin{tabular}{|c|c|c|c|}
\hline $\begin{array}{c}\text { Major Ship } \\
\text { Characteristics }\end{array}$ & $\begin{array}{c}\text { Ship } \\
\text { Design } \\
\text { Variant 1 }\end{array}$ & $\begin{array}{c}\text { Ship Design Variant } \\
1.1 \text { (50\% extra overall } \\
\text { workshop capacity) }\end{array}$ & $\begin{array}{c}\text { Ship Design Variant } 1.2 \\
\text { (100\% extra overall } \\
\text { workshop capacity) }\end{array}$ \\
\hline$V_{G}$ & $23850 \mathrm{~m}^{3}$ & $23950 \mathrm{~m}^{3}$ & $23995 \mathrm{~m}^{3}$ \\
\hline$\Delta_{D}$ & 6637 te & 6655 te & 6668te \\
\hline$\Delta_{L}$ & 4584te & 4600te & 4611te \\
\hline$\rho$ & $0.278 \mathrm{te} / \mathrm{m}^{3}$ & $0.278 \mathrm{te} / \mathrm{m}^{3}$ & $0.278 \mathrm{te} / \mathrm{m}^{3}$ \\
\hline$v_{s}$ & 0.319 & 0.318 & 0.318 \\
\hline$L_{w l}$ & $137.0 \mathrm{~m}$ & $137.1 \mathrm{~m}$ & $137.2 \mathrm{~m}$ \\
\hline$L_{o a}$ & $145.0 \mathrm{~m}$ & $145.1 \mathrm{~m}$ & $145.2 \mathrm{~m}$ \\
\hline$B_{w l}$ & $18.3 \mathrm{~m}$ & $18.3 \mathrm{~m}$ & $18.4 \mathrm{~m}$ \\
\hline$B_{M D}$ & $20.6 \mathrm{~m}$ & $20.6 \mathrm{~m}$ & $20.7 \mathrm{~m}$ \\
\hline$T$ & $5.7 \mathrm{~m}$ & $5.7 \mathrm{~m}$ & $5.7 \mathrm{~m}$ \\
\hline$D$ & $12.2 \mathrm{~m}$ & $12.2 \mathrm{~m}$ & $12.2 \mathrm{~m}$ \\
\hline$F$ & $7.8 \mathrm{~m}$ & $7.9 \mathrm{~m}$ & $7.9 \mathrm{~m}$ \\
\hline$U P C$ & $£ 578 \mathrm{~m}$ & $£ 579 \mathrm{~m}$ & $£ 580 \mathrm{~m}$ \\
\hline
\end{tabular}

Table 4.14: Major ship characteristics and UPC of ship design variants with extra overall workshop capacity 
Ship design Variants 2.1 and 2.2 were developed by increasing the overall NSGS capacity (volume) of Variant 2, first by $50 \%$ and then $100 \%$. Major ship characteristics and estimated UPC of the resulting ship designs as well as those of Variant 2 are shown in Table 4.15. Complete internal arrangements and UPC breakdowns can be found in Figure A5. 14 - Figure A5. 17 (Appendix 5).

\begin{tabular}{|l|r|r|r|}
\hline $\begin{array}{c}\text { Major Ship } \\
\text { Characteristics }\end{array}$ & $\begin{array}{c}\text { Ship } \\
\text { Design } \\
\text { Variant } 2\end{array}$ & $\begin{array}{c}\text { Ship Design Variant 2.1 } \\
\text { (50\% extra overall } \\
\text { NSGS capacity) }\end{array}$ & $\begin{array}{c}\text { Ship Design Variant 2.2 } \\
\text { (100\% extra overall } \\
\text { NSGS capacity) }\end{array}$ \\
\hline$V_{G}$ & $23863 \mathrm{~m}^{3}$ & $24147 \mathrm{~m}^{3}$ & $2466 \mathrm{~m}^{3}$ \\
\hline$\Delta_{D}$ & $6639 \mathrm{te}$ & $6708 \mathrm{te}$ & $6808 \mathrm{te}$ \\
\hline$\Delta_{L}$ & $4585 \mathrm{te}$ & $4644 \mathrm{te}$ & $4732 \mathrm{te}$ \\
\hline$\rho$ & $0.278 \mathrm{te} / \mathrm{m}^{3}$ & $0.278 \mathrm{te} / \mathrm{m}^{3}$ & $0.276 \mathrm{te} / \mathrm{m}^{3}$ \\
\hline$v_{s}$ & 0.315 & 0.311 & 0.305 \\
\hline$L_{w l}$ & $137.0 \mathrm{~m}$ & $137.5 \mathrm{~m}$ & $138.2 \mathrm{~m}$ \\
\hline$L_{o a}$ & $145.0 \mathrm{~m}$ & $145.5 \mathrm{~m}$ & $146.2 \mathrm{~m}$ \\
\hline$B_{w l}$ & $18.3 \mathrm{~m}$ & $18.4 \mathrm{~m}$ & $20.7 \mathrm{~m}$ \\
\hline$B_{M D}$ & $20.6 \mathrm{~m}$ & $20.7 \mathrm{~m}$ & $5.8 \mathrm{~m}$ \\
\hline$T$ & $5.7 \mathrm{~m}$ & $5.7 \mathrm{~m}$ & $12.3 \mathrm{~m}$ \\
\hline$D$ & $12.2 \mathrm{~m}$ & $12.2 \mathrm{~m}$ & $8.0 \mathrm{~m}$ \\
\hline$F$ & $7.9 \mathrm{~m}$ & $7.9 \mathrm{~m}$ & $£ 588 \mathrm{~m}$ \\
\hline$U P C$ & $£ 578 \mathrm{~m}$ & $£ 582 \mathrm{~m}$ & $\mathrm{~N}$ \\
\hline
\end{tabular}

Table 4.15: Major ship characteristics and UPC of ship design variants with extra overall Naval and Spare Gear Stores capacity

Ship design Variants 3.1 and 3.2 were developed by increasing both the overall workshop and NSGS capacities (volume) of Variant 3, first by $50 \%$ and then $100 \%$. Major ship characteristics and estimated UPC of the resulting ship designs as well as those of Variant 3 are shown in Table 4.16. Complete internal arrangements and UPC breakdowns can be found in Figure A5. 18 - Figure A5. 21 (Appendix 5). 


\begin{tabular}{|l|r|r|r|}
\hline $\begin{array}{c}\text { Major Ship } \\
\text { Characteristics }\end{array}$ & $\begin{array}{c}\text { Ship } \\
\text { Design } \\
\text { Variant 3 }\end{array}$ & $\begin{array}{c}\text { Ship Design Variant 3.1 } \\
\text { (50\% extra overall } \\
\text { workshop and NSGS } \\
\text { capacity) }\end{array}$ & $\begin{array}{c}\text { Ship Design Variant 3.2 } \\
\text { (100\% extra overall } \\
\text { workshop and NSGS } \\
\text { capacity) }\end{array}$ \\
\hline$V_{G}$ & $23850 \mathrm{~m}^{3}$ & $24232 \mathrm{~m}^{3}$ & $24873 \mathrm{~m}^{3}$ \\
\hline$\Delta_{D}$ & $6637 \mathrm{te}$ & $6725 \mathrm{te}$ & $6843 \mathrm{te}$ \\
\hline$\Delta_{L}$ & $4584 \mathrm{te}$ & $4659 \mathrm{te}$ & $4768 \mathrm{te}$ \\
\hline$\rho$ & $0.278 \mathrm{te} / \mathrm{m}^{3}$ & $0.278 \mathrm{te} / \mathrm{m}^{3}$ & $0.275 \mathrm{te} / \mathrm{m}^{3}$ \\
\hline$v_{s}$ & 0.319 & 0.315 & 0.31 \\
\hline$L_{w l}$ & $137.0 \mathrm{~m}$ & $137.6 \mathrm{~m}$ & $138.4 \mathrm{~m}$ \\
\hline$L_{o a}$ & $145.0 \mathrm{~m}$ & $145.6 \mathrm{~m}$ & $18.4 \mathrm{~m}$ \\
\hline$B_{w l}$ & $18.3 \mathrm{~m}$ & $20.7 \mathrm{~m}$ & $20.7 \mathrm{~m}$ \\
\hline$B_{M D}$ & $20.6 \mathrm{~m}$ & $5.8 \mathrm{~m}$ & $5.8 \mathrm{~m}$ \\
\hline$T$ & $5.7 \mathrm{~m}$ & $12.2 \mathrm{~m}$ & $12.3 \mathrm{~m}$ \\
\hline$D$ & $12.2 \mathrm{~m}$ & $7.9 \mathrm{~m}$ & $8.1 \mathrm{~m}$ \\
\hline$F$ & $7.8 \mathrm{~m}$ & $£ 583 \mathrm{~m}$ & $£ 590 \mathrm{~m}$ \\
\hline$U P C$ & $£ 578 \mathrm{~m}$ & & $0 v 6$ \\
\hline
\end{tabular}

Table 4.16: Major ship characteristics and UPC of ship design variants with extra overall workshop and Naval and Spare Gear Stores capacities

\subsubsection{Alternative Ship Design Style}

The last series of ship design variants were developed by applying major stylistic changes to ship design Variants 1.2, 2.2, and 3.2 (i.e. those with 100\% extra workshop or/and NSGS capacities (volume) relative to the Baseline Frigate Design). The style originally adopted for all three variants included one passing deck, single central passageway, and conventionally sized superstructure. The alternative style consisted of a deeper, two passing deck hull, double sided and athwartships passageway arrangement, and a correspondingly reduced superstructure. The resulting ship design variants, labelled Variants 1.2.1, 2.2.1, and 3.2.1, are described in Table 4.17. Major ship characteristics and estimated UPC are shown in the tables further below. 


\begin{tabular}{|c|c|c|}
\hline $\begin{array}{c}\text { Ship } \\
\text { Design }\end{array}$ & $\begin{array}{l}\text { Ship Design } \\
\text { Style }\end{array}$ & Description of the Supportability Features \\
\hline $\begin{array}{l}\text { Variant } \\
1.2\end{array}$ & Original & \multirow{2}{*}{$\begin{array}{l}\text { Two workshops, overall capacity increased by } \mathbf{1 0 0 \%} \\
\text { Two NSGSs, overall capacity unchanged from } \\
\text { Baseline Frigate }\end{array}$} \\
\hline $\begin{array}{l}\text { Variant } \\
1.2 .1\end{array}$ & Alternative & \\
\hline $\begin{array}{l}\text { Variant } \\
2.2\end{array}$ & Original & \multirow{2}{*}{$\begin{array}{l}\text { One workshop, overall capacity unchanged from } \\
\text { Baseline Frigate Three NSGSs, overall capacity } \\
\text { increased by } \mathbf{1 0 0 \%}\end{array}$} \\
\hline $\begin{array}{l}\text { Variant } \\
2.2 .1\end{array}$ & Alternative & \\
\hline $\begin{array}{l}\text { Variant } \\
3.2\end{array}$ & Original & \multirow{2}{*}{$\begin{array}{l}\text { Two workshops, overall capacity increased by } \mathbf{1 0 0 \%} \\
\text { Three NSGSs, overall capacity increased by } \mathbf{1 0 0 \%}\end{array}$} \\
\hline $\begin{array}{l}\text { Variant } \\
3.2 .1\end{array}$ & Alternative & \\
\hline
\end{tabular}

Table 4.17: Stylistic ship design variants with extra overall workshop or/and Naval and Spare Gear Store capacities

\begin{tabular}{|l|r|r|}
\hline Major Ship Characteristics & $\begin{array}{r}\text { Ship Design Variant 1.2 } \\
\text { (Original Style) }\end{array}$ & $\begin{array}{c}\text { Ship Design Variant } 1.2 .1 \\
\text { (Alternative Style) }\end{array}$ \\
\hline$V_{G}$ & $23995 \mathrm{~m}^{3}$ & $25370 \mathrm{~m}^{3}$ \\
\hline$\Delta_{D}$ & $6668 \mathrm{te}$ & $6850 \mathrm{te}$ \\
\hline$\Delta_{L}$ & $4611 \mathrm{te}$ & $4780 \mathrm{te}$ \\
\hline$\rho$ & $0.278 \mathrm{te} / \mathrm{m}^{3}$ & $0.270 \mathrm{te} / \mathrm{m}^{3}$ \\
\hline$v_{s}$ & 0.318 & 0.121 \\
\hline$L_{w l}$ & $137.2 \mathrm{~m}$ & $138.4 \mathrm{~m}$ \\
\hline$L_{o a}$ & $145.2 \mathrm{~m}$ & $146.4 \mathrm{~m}$ \\
\hline$B_{w l}$ & $18.4 \mathrm{~m}$ & $18.5 \mathrm{~m}$ \\
\hline$B_{M D}$ & $20.7 \mathrm{~m}$ & $20.8 \mathrm{~m}$ \\
\hline$T$ & $5.7 \mathrm{~m}$ & $5.8 \mathrm{~m}$ \\
\hline$D$ & $12.2 \mathrm{~m}$ & $12.3 \mathrm{~m}$ \\
\hline$F$ & $7.9 \mathrm{~m}$ & $8.0 \mathrm{~m}$ \\
\hline$U P C$ & $£ 580 \mathrm{~m}$ & $£ 590 \mathrm{~m}$ \\
\hline
\end{tabular}

Table 4.18: Major ship characteristics and UPC of stylistic ship design variants with extra overall workshop capacity 


\begin{tabular}{|l|r|r|}
\hline Major Ship Characteristics & $\begin{array}{c}\text { Ship Design } \\
\text { Variant } 2.2 \\
\text { (Original Style) }\end{array}$ & $\begin{array}{c}\text { Ship Design Variant } \\
2.2 .1 \text { (Alternative } \\
\text { Style) }\end{array}$ \\
\hline$V_{G}$ & $24662 \mathrm{~m}^{3}$ & $25784 \mathrm{~m}^{3}$ \\
\hline$\Delta_{D}$ & $6808 \mathrm{te}$ & $6958 \mathrm{te}$ \\
\hline$\Delta_{L}$ & $4732 \mathrm{te}$ & $4871 \mathrm{te}$ \\
\hline$\rho$ & $0.276 \mathrm{te} / \mathrm{m}^{3}$ & $0.270 \mathrm{te} / \mathrm{m}^{3}$ \\
\hline$v_{s}$ & 0.305 & 0.119 \\
\hline$L_{w l}$ & $138.2 \mathrm{~m}$ & $139.2 \mathrm{~m}$ \\
\hline$L_{o a}$ & $146.2 \mathrm{~m}$ & $147.2 \mathrm{~m}$ \\
\hline$B_{w l}$ & $18.4 \mathrm{~m}$ & $18.6 \mathrm{~m}$ \\
\hline$B_{M D}$ & $20.7 \mathrm{~m}$ & $21.0 \mathrm{~m}$ \\
\hline$T$ & $5.8 \mathrm{~m}$ & $5.8 \mathrm{~m}$ \\
\hline$D$ & $12.3 \mathrm{~m}$ & $12.3 \mathrm{~m}$ \\
\hline$F$ & $8.0 \mathrm{~m}$ & $8.1 \mathrm{~m}$ \\
\hline$U P C$ & $£ 588 \mathrm{~m}$ & $£ 596 \mathrm{~m}$ \\
\hline
\end{tabular}

Table 4.19: Major ship characteristics and UPC of stylistic ship design variants with extra overall Naval and Spare Gear Stores capacity

\begin{tabular}{|l|r|r|}
\hline Major Ship Characteristics & $\begin{array}{c}\text { Ship Design } \\
\text { Variant } 3.2 \\
\text { (Original Style) }\end{array}$ & $\begin{array}{c}\text { Ship Design Variant } \\
3.2 .1 \text { (Alternative } \\
\text { Style) }\end{array}$ \\
\hline$V_{G}$ & $24873 \mathrm{~m}^{3}$ & $26027 \mathrm{~m}^{3}$ \\
\hline$\Delta_{D}$ & $6843 \mathrm{te}$ & $7005 \mathrm{te}$ \\
\hline$\Delta_{L}$ & $4768 \mathrm{te}$ & $4911 \mathrm{te}$ \\
\hline$\rho$ & $0.275 \mathrm{te} / \mathrm{m}^{3}$ & $0.269 \mathrm{te} / \mathrm{m}^{3}$ \\
\hline$v_{s}$ & 0.31 & 0.118 \\
\hline$L_{w l}$ & $138.4 \mathrm{~m}$ & $139.5 \mathrm{~m}$ \\
\hline$L_{o a}$ & $146.4 \mathrm{~m}$ & $147.5 \mathrm{~m}$ \\
\hline$B_{w l}$ & $18.4 \mathrm{~m}$ & $18.7 \mathrm{~m}$ \\
\hline$B_{M D}$ & $20.7 \mathrm{~m}$ & $21.0 \mathrm{~m}$ \\
\hline$T$ & $5.8 \mathrm{~m}$ & $5.8 \mathrm{~m}$ \\
\hline$D$ & $12.3 \mathrm{~m}$ & $12.3 \mathrm{~m}$ \\
\hline$F$ & $8.1 \mathrm{~m}$ & $8.1 \mathrm{~m}$ \\
\hline$U P C$ & $£ 590 \mathrm{~m}$ & $£ 599 \mathrm{~m}$ \\
\hline
\end{tabular}

Table 4.20: Major ship characteristics and UPC of stylistic ship design variants with extra overall Workshop and Naval and Spare Gear Stores capacities 
Figure 4.14 illustrates the internal arrangement of ship design Variant 1.2.1, showing the deeper two passing deck (No. 1 and 2 Decks) hull, double sided passageways on No. 1 and 2 Decks, athwartship passageways on No. 2 Deck, and a correspondingly much reduced superstructure. The complete internal arrangement of the ship design Variants 2.2.1, 3.2.1 and UPC breakdowns of all three ship design variants incorporating the alternative style are provided in Figure A5. 22 - Figure A5. 26 (Appendix 5). 


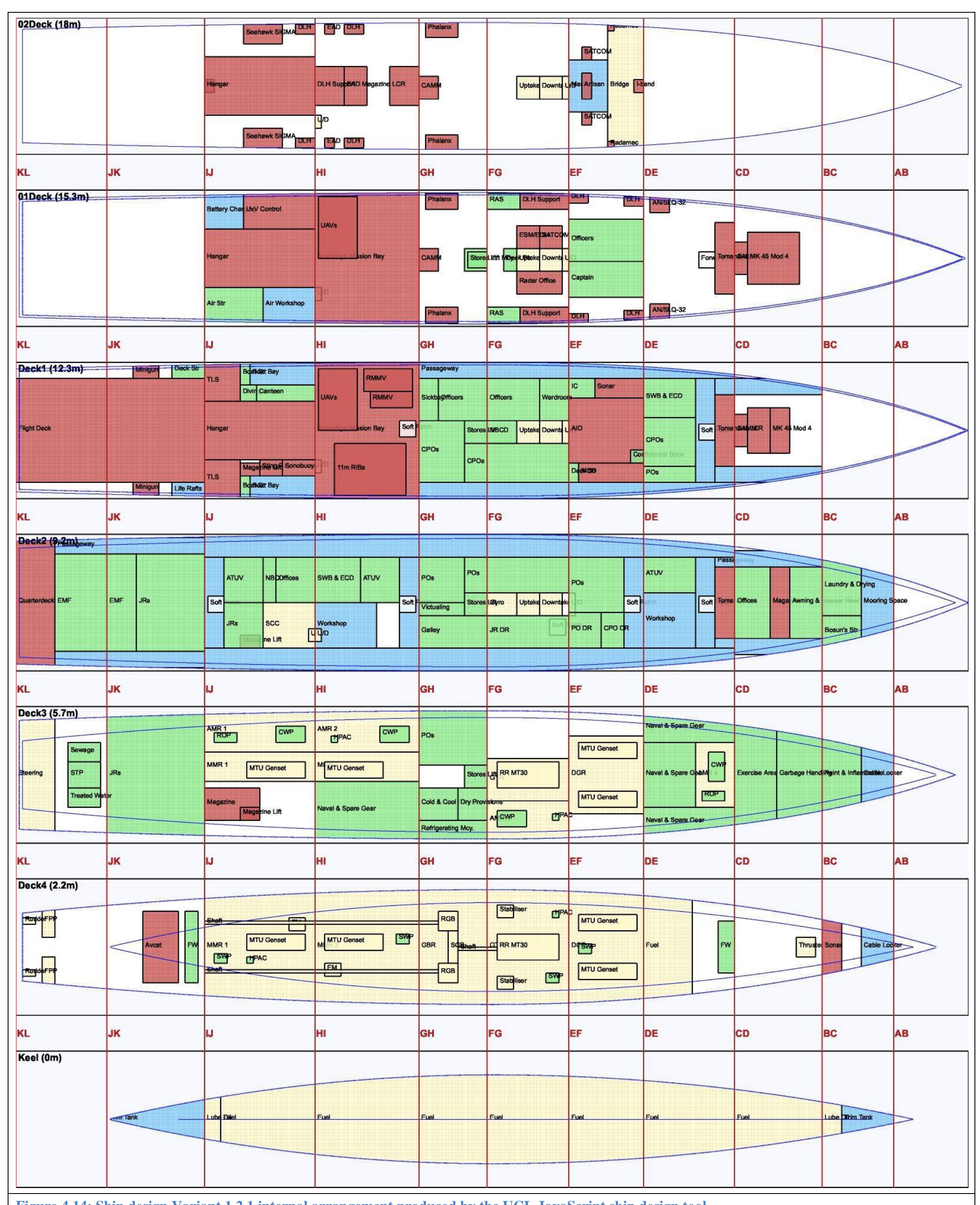

Figure 4.14: Ship design Variant 1.2.1 internal arrangement produced by the UCL JavaScript ship design tool 


\section{Chapter 5: Application of the Proposed Design for Support Evaluation Approach and Presentation of the Results}

\subsection{Introduction}

This chapter has two main sections, describing the application of the proposed Design for Support (DfS) evaluation approach to the ship design cases, which are outlined in Chapter 4 and presenting the assessment results. Focusing on the investigation of maintainability of ship machinery spaces, a detailed explanation of the bottom-up application of the proposed evaluation approach is provided in Section 5.2, covering individual hierarchy levels, the additional aspects that were taken into account to further rationalise the results, and the process through which these various elements were utilised together. Section 5.3 presents the maintainability assessment results, first for the Baseline Frigate Design and its configurational variants, followed by the ship designs in which certain supportability features were enhanced, and finally the ship designs that incorporated an alternative design style. A limited discussion of each set of results is also provided. A comprehensive discussion of the research, including wider ship design implications is presented in Chapter 6. 


\subsection{Maintainability of Ship Machinery Spaces}

As stated in Section 3.3.3, the supportability investigations undertaken as part of this research were primarily centred on the issue of naval ship maintenance. Considering the broad range of vital items of equipment usually located inside Main Machinery Rooms (MMR), Auxiliary Machinery Rooms (AMR), Gearbox Rooms (GBR), Diesel Generator Rooms (DGR), and Gas Turbine Rooms (GTR) of a typical naval combatant, the proposed DfS evaluation approach was applied to partially assess the maintainability of these spaces. Applying the hierarchy shown in Figure 3.6 (shown at the end of Chapter 3) to the ship design case studies described in Chapter 4, an overall Measure of Effectiveness (oMoE) for the maintainability of equipment in the machinery spaces of the ship design studies produced was calculated for two distinct ship maintenance scenarios; Underway Maintenance (UM) and Alongside Maintenance (AM). Although the hierarchy shown in Figure 3.6 was constructed in a top-down manner, its application, shown in Figure 5.1, followed a bottom-up procedure. 
Chapter 5: Application of the Proposed Design for Support Evaluation Approach and Presentation of the Results

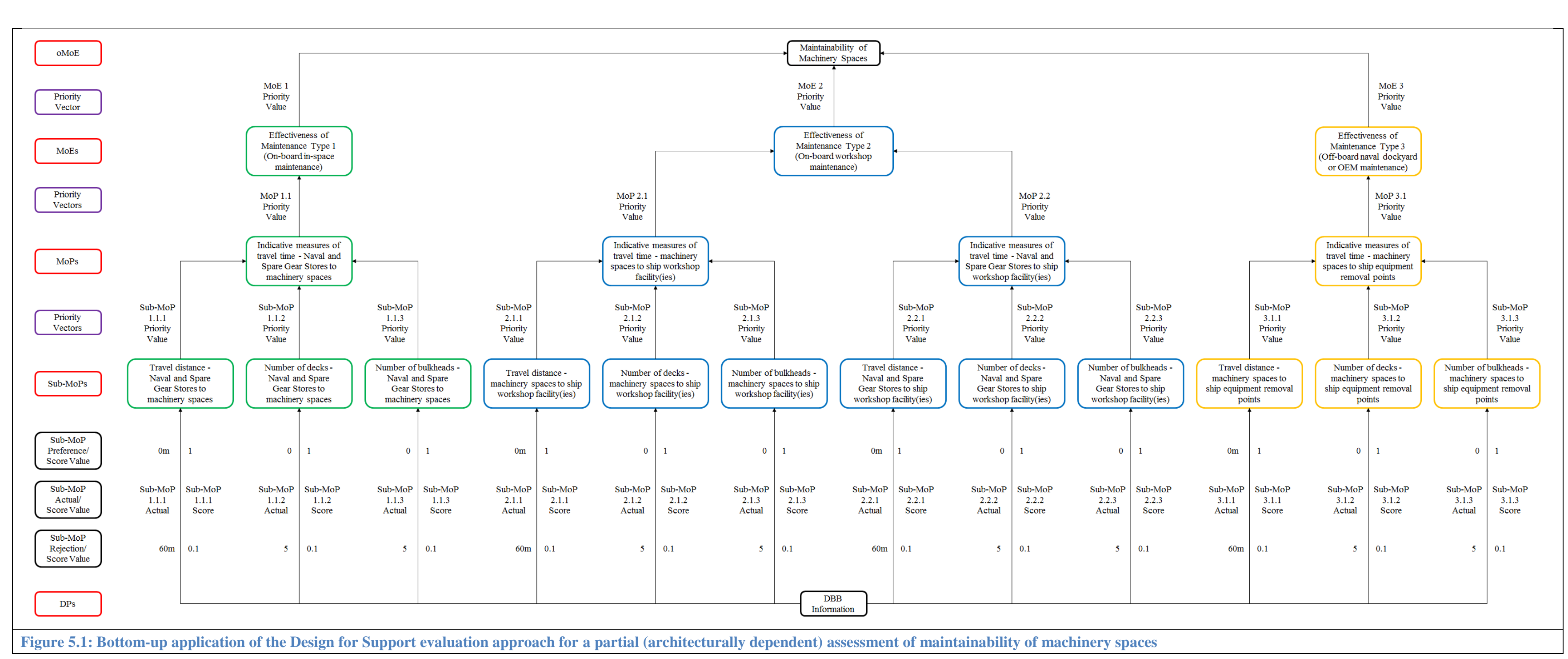




\subsubsection{Individual Hierarchy Levels}

In utilising the maintainability evaluation hierarchy shown in Figure 5.1, different hierarchy levels were first separately assessed, as described below.

\section{a) Dimensional Parameters (DP)}

The Design Building Block (DBB) information considered as relevant to the calculation of the sub-MoPs was first extracted from the ship designs. This DBB information included the exact Cartesian coordinates of MMRs, AMRs, Naval and Spare Gear Stores (NSGS), ship workshop facilities, and Equipment Removal Points (ERP), their areas and adjacency relationships of these spaces, and the number of equipment items present in each of the machinery spaces considered.

\section{b) Sub-Measures of Performance (sub-MoP)}

Next up the hierarchy, the extracted DPs were used to calculate the sub-MoPs. The sub-MoPs comprised the Manhattan travel distance and the number of decks and bulkheads to lift, lower or pass equipment or spare parts through. In order to aggregate the measured sub-MoPs to produce MoPs, they were first assessed against certain criteria and normalised (i.e. range $0-1$ ). Simple minimum and maximum values of travel distance and numbers of decks and bulkheads were used as the evaluation criteria. These limits were termed rejection and preference values, so they were meant to be quantitative representations of the accumulated naval ship design and supportability knowledge (e.g. rules of thumb), hence they should not be considered as irrational or arbitrary values. Table 5.1 shows the rejection and preference values used to evaluate the actual sub-MoP values. These values have already been presented in Figure 5.1.

\begin{tabular}{|c|c|c|}
\hline $\begin{array}{c}\text { Sub-MoP Rejection } \\
\text { Value }\end{array}$ & $\begin{array}{c}\text { Sub-MoP Actual } \\
\text { Value }\end{array}$ & $\begin{array}{c}\text { Sub-MoP Preference } \\
\text { Value }\end{array}$ \\
\hline $60 \mathrm{~m}$ & Manhattan Travel Distance & 0 \\
\hline 5 & Number of Decks & 0 \\
\hline 5 & Number of Bulkheads & 0 \\
\hline
\end{tabular}

Table 5.1: Rejection and preference values used for assessing the sub-MoPs of maintainability of machinery spaces 
Chapter 5: Application of the Proposed Design for Support Evaluation Approach and Presentation of the Results

Figure 5.2 shows the two approaches that were considered in evaluating the actual sub-MoP values; a linear regression method (left) and a segmented regression method with a given breakpoint (right).

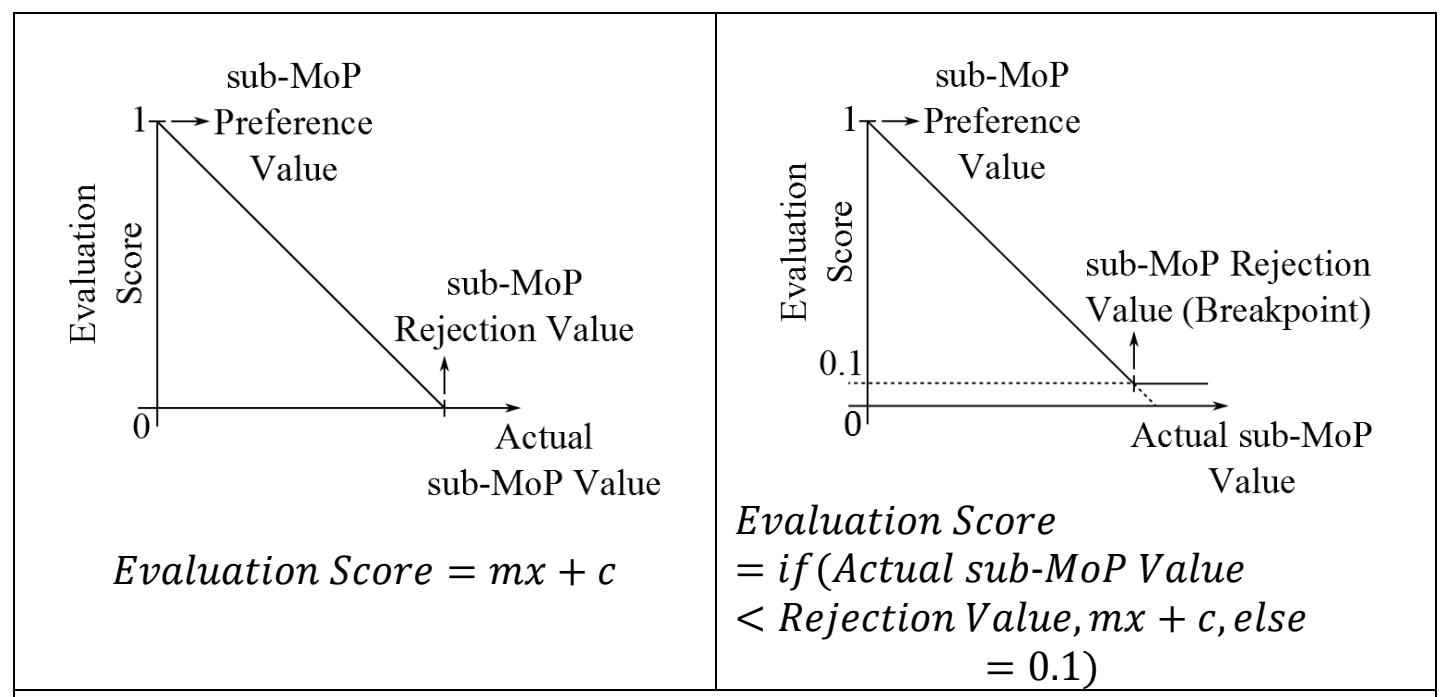

Figure 5.2: The linear regression method (left) and the segmented regression method with given breakpoint (right) considered in evaluating the sub-MoP actual values against the subMoP rejection and preference values

The linear regression implies that if the actual value of a particular sub-MoP is outside the allowable range set by the rejection and preference values (see Table 5.1), the contributions of the associated supportability feature towards overall ship supportability would be zero. However, this was considered to be unrealistic since spaces like NSGSs, workshop facilities, and equipment removal routes would still be provided in a ship design and then utilised, even if the associated sub-MoPs were found to be outside the range considered to be acceptable by the decision makers. Hence an alternative segmented regression was used to capture the very small contributions of supportability features towards overall ship supportability. The two segments were separated by the breakpoint that essentially replaced the zero score of the linear regression with a low value (e.g. 0.1 in this case). Therefore, if the actual value of a particular sub-MoP was outside the allowable range, it was given an evaluation score of 0.1 .

Having established a method for assessing the sub-MoPs, the associated priority vectors (i.e. the numerical preferences of decision makers in matrix form) were determined to enable weighted aggregations. Considering the different nature of the maintenance work associated with each of the three types of maintenance, different 
degrees of preference were assigned to the sub-MoPs of each type. The Analytic Hierarchy Process (AHP)-based framework described in Section 3.3.3 was used to quantify the relative preferences of a given decision maker and produce priority vectors. The three features of this process were:

- Using the Power Scale (see Section 3.3.3 Part a) to numerically carry out the pairwise comparisons at each of the three levels of the assessment hierarchy;

- Checking the consistency of the pairwise comparison matrices against a consistency acceptance threshold of 0.1 (see Section 3.3.3 Part b) and corresponding principal eigenvalues (see Table 3.4);

- Using the Eigenvector Method (EVM) (see Section 3.3.3 Part c) for generating priority vectors from positive reciprocal consistent or near consistent pairwise comparison matrices.

The pairwise comparisons carried out on the sub-MoP and the other two hierarchy levels (Measures of Performance (MoPs) and Measures of Effectiveness (MoEs) described further below) were based on the candidate's engineering judgment and so the results cannot be treated as representing expert knowledge. Below is a worked example showing the process to determine the priority vector for $\mathrm{MT}_{1}$ (on-board inspace maintenance) sub-MoPs (see Figure 5.1).

For this particular example of ship supportability, the sub-MoPs associated with $\mathrm{MT}_{1}$ concern the transfer of spare parts from the on-board NSGSs to the ship's machinery spaces (see Table 3.5). The pairwise comparisons of the three sub-MoPs were carried out using the Power Scale (see Section 3.3.3 Part a) as described in Table 5.2.

\begin{tabular}{|l|r|}
\hline \multicolumn{1}{|c|}{$\begin{array}{c}\text { Pairwise } \\
\text { Comparison }\end{array}$} & $\begin{array}{c}\text { Corresponding } \\
\text { Numerical Value }\end{array}$ \\
\hline $\begin{array}{l}\text { Travel distance (sub-MoP 1.1.1) is strongly more } \\
\text { important than No. of decks (sub-MoP 1.1.2) }\end{array}$ & \\
\hline $\begin{array}{l}\text { No. of decks (sub-MoP 1.1.2) is moderately more } \\
\text { important than No. of bulkheads (sub-MoP 1.1.3) }\end{array}$ & 1.73 \\
\hline $\begin{array}{l}\text { Travel distance (sub-MoP 1.1.1) is very strongly more } \\
\text { important than No. of bulkheads (sub-MoP 1.1.3) }\end{array}$ & \\
\hline
\end{tabular}

Table 5.2: Pairwise comparisons of Maintenance Type 1 (transfer of Naval Stores and Spare Gear Stores to machinery spaces) sub-MoPs 
Chapter 5: Application of the Proposed Design for Support Evaluation Approach and Presentation of the Results

The numerical values that correspond to the pairwise comparisons were then used to produce what is known as the reciprocal comparison matrix. First, the elements to the right side of the matrix leading diagonal were populated by the following procedure: if the judgment in the pairwise comparison is towards the left-hand side, the matrix is populated with the actual value (i.e. 3), but if it is to the right-hand side, then the matrix is populated with the reciprocal value (i.e. 1/3). The judgments in all the pairwise comparisons shown in Table 5.2 were towards the left-hand side, hence the elements to the right side of the matrix leading diagonal were populated with the actual values as shown in Figure 5.3.

\begin{tabular}{c|c|c|c} 
Comparison Matrix & $\begin{array}{c}\text { Sub-MoP } \\
1.1 .1\end{array}$ & $\begin{array}{c}\text { Sub-MoP } \\
1.1 .2\end{array}$ & $\begin{array}{c}\text { Sub-MoP } \\
1.1 .3\end{array}$ \\
\hline Sub-MoP 1.1.1 & 1 & 3 & 5.2 \\
\hline Sub-MoP 1.1.2 & & 1 & 1.73 \\
\hline Sub-MoP 1.1.3 & & & 1
\end{tabular}

Figure 5.3: Partially populated comparison matrix produced from pairwise comparisons of Maintenance Type 1 (transfer of Naval Stores and Spare Gear Stores to machinery spaces) subMoPs

Moreover, the comparison matrix needs to be reciprocal about the leading diagonal for the pairwise comparison to be correct both ways. A matrix is said to be reciprocal about the leading diagonal provided that $a_{i j}=\frac{1}{a_{j i}}$ for all $i, j=1, \ldots, n$ (Shiraishi et al., 1998). Figure 5.4 illustrates the fully populated reciprocal comparison matrix developed based on the pairwise comparisons shown in Table 5.2.

\begin{tabular}{c|c|c|c} 
Reciprocal Comparison & Sub-MoP & Sub-MoP & Sub-MoP \\
Matrix & 1.1 .1 & 1.1 .2 & 1.1 .3 \\
\hline Sub-MoP 1.1.1 & 1 & 3 & 5.2 \\
\hline Sub-MoP 1.1.2 & $1 / 3$ & 1 & 1.73 \\
\hline Sub-MoP 1.1.3 & $1 / 5.2$ & $1 / 1.73$ & 1
\end{tabular}

Figure 5.4: Reciprocal comparison matrix produced from pairwise comparisons of Maintenance Type 1 (transfer of Naval Stores and Spare Gear Stores to machinery spaces) sub-MoPs

Next, the consistency of the comparison matrix was checked. Using the consistency check method (see Section 3.3.3 Part b), a matrix is considered consistent provided that its principal eigenvalue is less than or equal to the maximum allowable principal eigenvalue, given the matrix dimension and the selected consistency threshold. For a $3 \times 3$ matrix, such as shown in Figure 5.4, the maximum allowable principal eigenvalue that corresponds to a consistency threshold of 0.1 is 3.096 (see Table 3.4). Using the 
built-in functions of Microsoft Excel, the principal eigenvalue of the comparison matrix was found to be 3 . This value suggests a perfectly consistent matrix.

Once the consistency requirement was satisfied, the EVM was used to generate the priority vector (i.e. the numerical preferences of decision makers in matrix form). Using Microsoft Excel built-in functions, the principal eigenvector of the comparison matrix was first calculated. This is the eigenvector that corresponds to the principal eigenvalue. The principal eigenvector was then normalised to produce the priority vector as shown in Figure 5.5.

\begin{tabular}{c|c|c} 
Matrix Element & Principal Eigenvector & $\begin{array}{c}\text { Priority Vector } \\
\text { (Normalised Principal Eigenvector) }\end{array}$ \\
\hline Sub-MoP 1.1.1 & 0.933 & 0.655 \\
\hline Sub-MoP 1.1.2 & 0.311 & 0.219 \\
\hline Sub-MoP 1.1.3 & 0.180 & 0.126
\end{tabular}

Figure 5.5: Priority vector for Maintenance Type 1 (transfer of Naval Stores and Spare Gear Stores to machinery spaces) sub-MoPs

Having determined the priority vector for $\mathrm{MT}_{1}$ (on-board in-space maintenance) subMoPs, the same procedure was followed to calculate the priority vectors for the subMoPs associated with $\mathrm{MT}_{2}$ (on-board workshop maintenance) and $\mathrm{MT}_{3}$ (off-board naval dockyard or Original Equipment Manufacturer (OEM) maintenance). Table 5.3 to Table 5.5 show the pairwise comparisons (highlighted in red, see Table 3.2 for corresponding linguistic expressions), the reciprocal comparison matrices, the consistency checks, and the resultant priority vectors for the sub-MoPs of $\mathrm{MT}_{2}$ and $\mathrm{MT}_{3}$. 
Chapter 5: Application of the Proposed Design for Support Evaluation Approach and Presentation of the Results

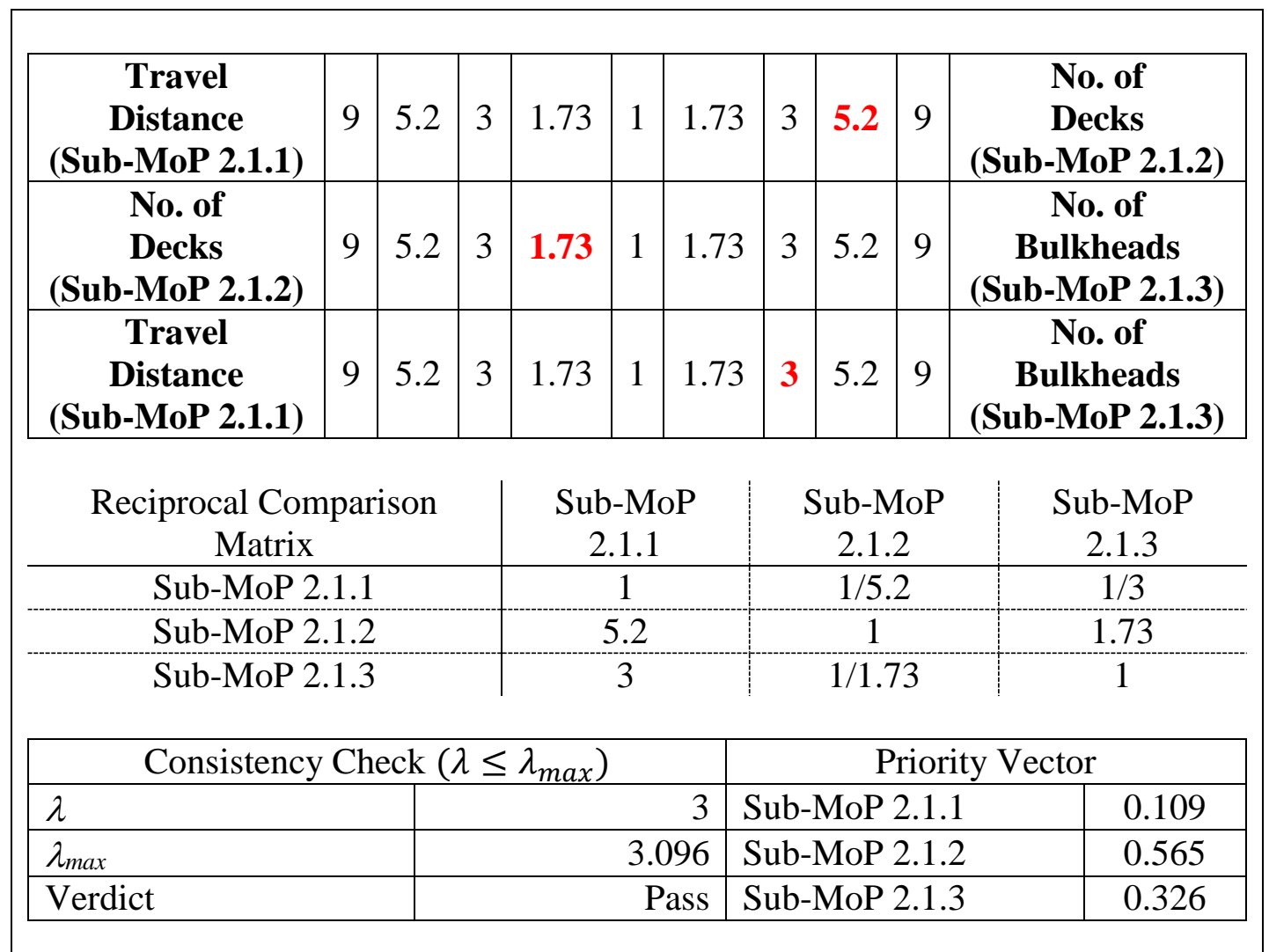

Table 5.3: Determination of the priority vector for Maintenance Type 2 (transfer of equipment from machinery spaces to ship workshop facilities) sub-MoPs

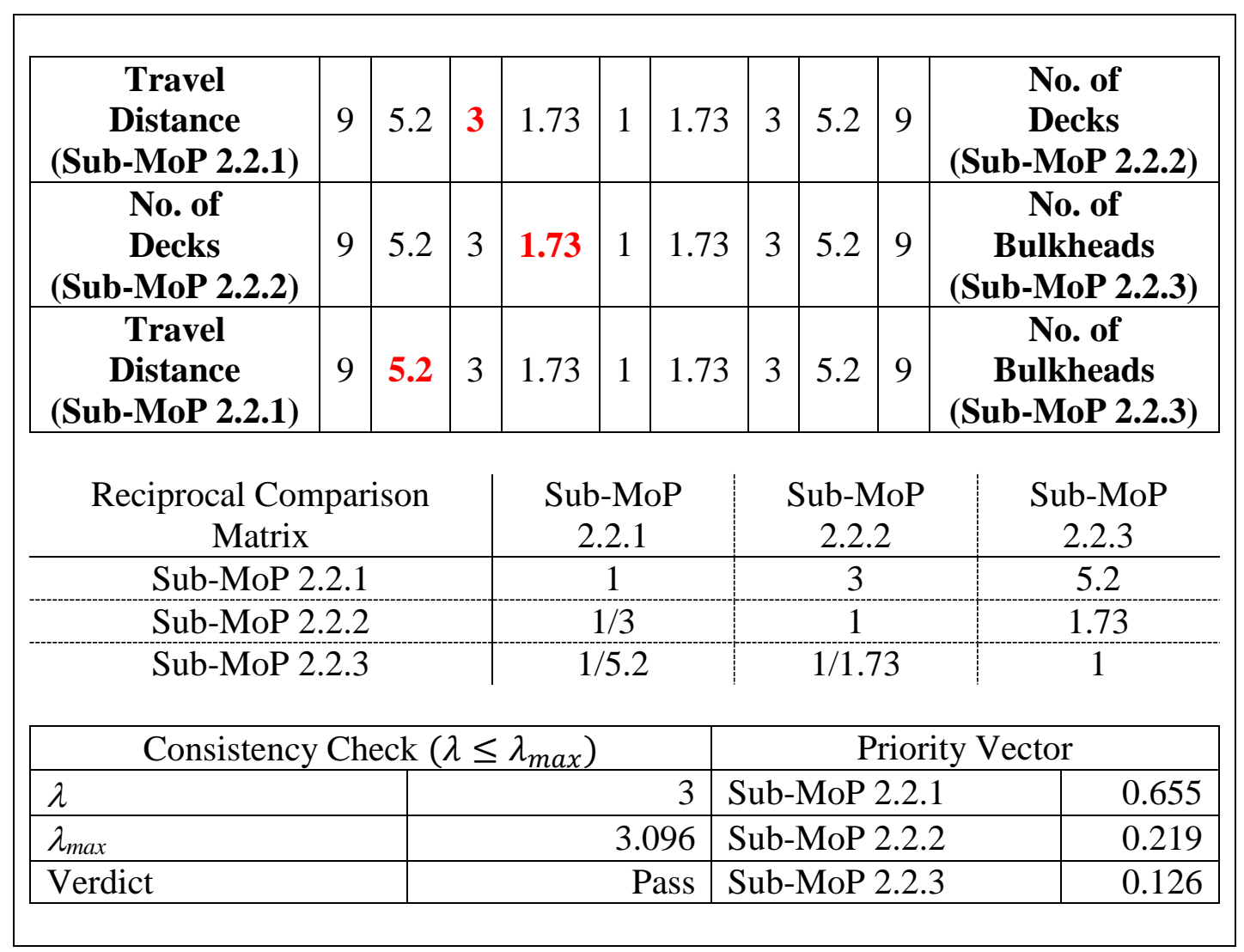

Table 5.4: Determination of the priority vector for Maintenance Type 2 (transfer of Naval Stores and Spare Gear Stores to ship workshop facilities) sub-MoPs 


\begin{tabular}{|c|c|c|c|c|c|c|c|c|c|c|}
\hline $\begin{array}{c}\text { Travel } \\
\text { Distance } \\
\text { (Sub-MoP 3.1) }\end{array}$ & 9 & 5.2 & 3 & 1.73 & 1 & 1.73 & 3 & 5.2 & 9 & $\begin{array}{c}\text { No. of } \\
\text { Decks } \\
\text { (Sub-MoP 3.2) }\end{array}$ \\
\hline $\begin{array}{c}\text { No. of } \\
\text { Decks } \\
\text { (Sub-MoP 3.2) }\end{array}$ & 9 & 5.2 & 3 & 1.73 & 1 & 1.73 & 3 & 5.2 & 9 & $\begin{array}{c}\text { No. of } \\
\text { Bulkheads } \\
\text { (Sub-MoP 3.3) }\end{array}$ \\
\hline $\begin{array}{c}\text { Travel } \\
\text { Distance } \\
\text { (Sub-MoP 3.1) } \\
\end{array}$ & 9 & 5.2 & 3 & 1.73 & 1 & 1.73 & 3 & 5.2 & 9 & $\begin{array}{c}\text { No. of } \\
\text { Bulkheads } \\
\text { (Sub-MoP 3.3) } \\
\end{array}$ \\
\hline \multicolumn{4}{|c|}{$\begin{array}{l}\text { Reciprocal Comparison } \\
\text { Matrix }\end{array}$} & \multicolumn{3}{|c|}{$\begin{array}{c}\text { Sub-MoP } \\
3.1 .1\end{array}$} & \multicolumn{3}{|c|}{$\begin{array}{l}\text { Sub-MoP } \\
3.1 .2\end{array}$} & $\begin{array}{c}\text { Sub-MoP } \\
3.1 .3\end{array}$ \\
\hline \multicolumn{4}{|c|}{ Sub-MoP 3.1.1 } & \multicolumn{3}{|c|}{1} & \multicolumn{3}{|c|}{$1 / 5.2$} & $1 / 3$ \\
\hline \multicolumn{4}{|c|}{ Sub-MoP 3.1.2 } & \multicolumn{3}{|c|}{5.2} & \multicolumn{3}{|c|}{1} & 1.73 \\
\hline \multicolumn{4}{|c|}{ Sub-MoP 3.1 .3} & \multicolumn{3}{|c|}{3} & \multicolumn{3}{|c|}{$1 / 1.73$} & 1 \\
\hline \multicolumn{7}{|c|}{ Consistency Check $\left(\lambda \leq \lambda_{\max }\right)$} & \multicolumn{4}{|c|}{ Priority Vector } \\
\hline \multicolumn{3}{|l|}{$\lambda$} & \multicolumn{4}{|c|}{ (med } & \multicolumn{3}{|c|}{ Sub-MoP 3.1.1 } & 0.109 \\
\hline \multirow{2}{*}{\multicolumn{3}{|c|}{$\lambda_{\max }$}} & \multirow{2}{*}{\multicolumn{4}{|c|}{3.096}} & \multicolumn{3}{|c|}{ Sub-MoP 3.1.2 } & 0.565 \\
\hline & & & \multicolumn{2}{|c|}{ Pass } & & & \multicolumn{3}{|c|}{ Sub-MoP 3.1.3 } & 0.326 \\
\hline
\end{tabular}

Table 5.5: Determination of the priority vector for Maintenance Type 3 (transfer of equipment from machinery spaces to ship Equipment Removal Points) sub-MoPs 
Chapter 5: Application of the Proposed Design for Support Evaluation Approach and Presentation of the Results

\section{c) Measures of Performance (MoP)}

Next up the hierarchy, priority vectors associated with the MoPs (the indicative measures of travel time to and from the various machinery spaces) were quantified. From Figure 5.1, it can be seen that $\mathrm{MT}_{2}$ (i.e. on-board workshop maintenance) consisted of two MoPs while the other two types of maintenance, $\mathrm{MT}_{1}$ and $\mathrm{MT}_{3}$, consisted of a single MoP each, thus did not require pairwise comparisons. Using the same procedure described in the worked example earlier (see Section 5.2.1 Part b), pairwise comparisons were only carried out for $\mathrm{MT}_{2}$. For $\mathrm{MoP}_{2}$, Table 5.6 shows the pairwise comparisons (highlighted in red, see Table 3.2 for corresponding linguistic expressions), the corresponding reciprocal comparison matrix, the consistency check, and the resultant priority vector are shown in Table 5.6.

\begin{tabular}{|c|c|c|c|c|c|c|c|c|c|c|}
\hline $\begin{array}{c}\text { Indicative } \\
\text { measures of } \\
\text { travel time - } \\
\text { machinery } \\
\text { spaces to ship } \\
\text { workshop } \\
\text { facilities } \\
\text { (MoP 2.1) } \\
\end{array}$ & 9 & 5.2 & 3 & 1.73 & 1 & 1.73 & 3 & 5.2 & I & $\begin{array}{c}\text { Indicative } \\
\text { measures of } \\
\text { travel time - } \\
\text { Naval and Spare } \\
\text { Gear Stores to } \\
\text { ship workshop } \\
\text { facilities } \\
\text { (MoP 2.2) } \\
\end{array}$ \\
\hline \multicolumn{7}{|c|}{ Reciprocal Comparison Matrix } & \multicolumn{3}{|c|}{ MoP 2.1} & MoP 2.2 \\
\hline \multicolumn{7}{|c|}{ MoP 2.1} & \multicolumn{3}{|c|}{1} & 1.73 \\
\hline \multicolumn{7}{|c|}{ MoP 2.2} & \multicolumn{3}{|c|}{$1 / 1.73$} & 1 \\
\hline \multicolumn{8}{|c|}{ Consistency Check $\left(\lambda \leq \lambda_{\max }\right)$} & \multicolumn{3}{|c|}{ Priority Vector } \\
\hline$\lambda$ & & & & & & & 2 & Mo & 2.1 & \begin{tabular}{l|l} 
& 0.634 \\
\end{tabular} \\
\hline$\lambda_{\max }$ & & & & & & & 2 & Mo & 2.2 & 0.366 \\
\hline \multicolumn{3}{|c|}{ Verdict } & & \multicolumn{4}{|c|}{ Pass } & & & \\
\hline
\end{tabular}

Table 5.6: Determination of the priority vector for Maintenance Type 2 (transfer of equipment from machinery spaces and Naval and Spare Gear Stores to ship workshop facilities) MoPs 


\section{d) Measures of Effectiveness (MoE)}

Maintenance MoEs were seen to be particularly significant since the implications from the two distinct maintenance scenarios mentioned at the start of Section 5.2 were captured at this level. Different degrees of preference were assigned to the MoE for the three types of maintenance depending on the two maintenance scenarios. Following the same procedure described in the worked example earlier (see Section 5.2.1 Part b), the associated priority vectors were determined. Table 5.7 and Table 5.8 show the MoE pairwise comparisons (highlighted in red, see Table 3.2 for corresponding linguistic expressions), reciprocal comparison matrices, consistency checks, and resultant priority vectors.

\begin{tabular}{|c|c|c|c|c|c|c|c|c|c|c|}
\hline $\begin{array}{c}\text { Maintenance } \\
\text { Type 1 } \\
\text { Effectivenes } \\
\text { (MoE 1) }\end{array}$ & 9 & 5.2 & 3 & 1.73 & 1 & 1.73 & 3 & 5.2 & \multicolumn{2}{|c|}{$\begin{array}{c}\text { Maintenance } \\
\text { Type } 2 \\
\text { Effectiveness } \\
\text { (MoE 2) } \\
\end{array}$} \\
\hline $\begin{array}{l}\text { Maintenance } \\
\text { Type } 2 \\
\text { Effectiveness } \\
\text { (MoE 2) }\end{array}$ & 9 & 5.2 & 3 & 1.73 & 1 & 1.73 & 3 & 5.2 & \multicolumn{2}{|c|}{$\begin{array}{l}\text { Maintenance } \\
\text { Type 3 } \\
\text { Effectiveness } \\
\text { (MoE 3) }\end{array}$} \\
\hline $\begin{array}{c}\text { Maintenance } \\
\text { Type 1 } \\
\text { Effectiveness } \\
\text { (MoE 1) } \\
\end{array}$ & 9 & 5.2 & 3 & 1.73 & 1 & 1.73 & 3 & 5.2 & \multicolumn{2}{|c|}{$\begin{array}{c}\text { Maintenance } \\
\text { Type 3 } \\
\text { Effectiveness } \\
\text { (MoE 3) } \\
\end{array}$} \\
\hline \multicolumn{6}{|c|}{ Reciprocal Comparison Matrix } & \multicolumn{3}{|c|}{ MoE 1} & MoE 2 & MoE 3 \\
\hline \multicolumn{6}{|c|}{$\mathrm{MoE} 1$} & \multicolumn{3}{|c|}{1} & $1 / 1.73$ & 3 \\
\hline \multicolumn{6}{|c|}{ MoE 2} & \multicolumn{3}{|c|}{1.73} & 1 & 5.2 \\
\hline \multicolumn{6}{|c|}{ MoE 3} & \multicolumn{3}{|c|}{$1 / 3$} & $1 / 5.2$ & 1 \\
\hline \multicolumn{8}{|c|}{ Consistency Check $\left(\lambda \leq \lambda_{\max }\right)$} & \multicolumn{3}{|c|}{ Priority Vector } \\
\hline$\lambda$ & & & & & & & 3 & & $\mathrm{oE} 1$ & 0.326 \\
\hline$\lambda_{\max }$ & & & & & & & .096 & & $\mathrm{oE} 2$ & 0.565 \\
\hline Verdict & & & & & & & Pass & & $\mathrm{oE} 3$ & 0.109 \\
\hline
\end{tabular}

Table 5.7: Determination of the priority vector for the three Maintenance Types MoEs, Underway Maintenance scenario 
Chapter 5: Application of the Proposed Design for Support Evaluation Approach and Presentation of the Results

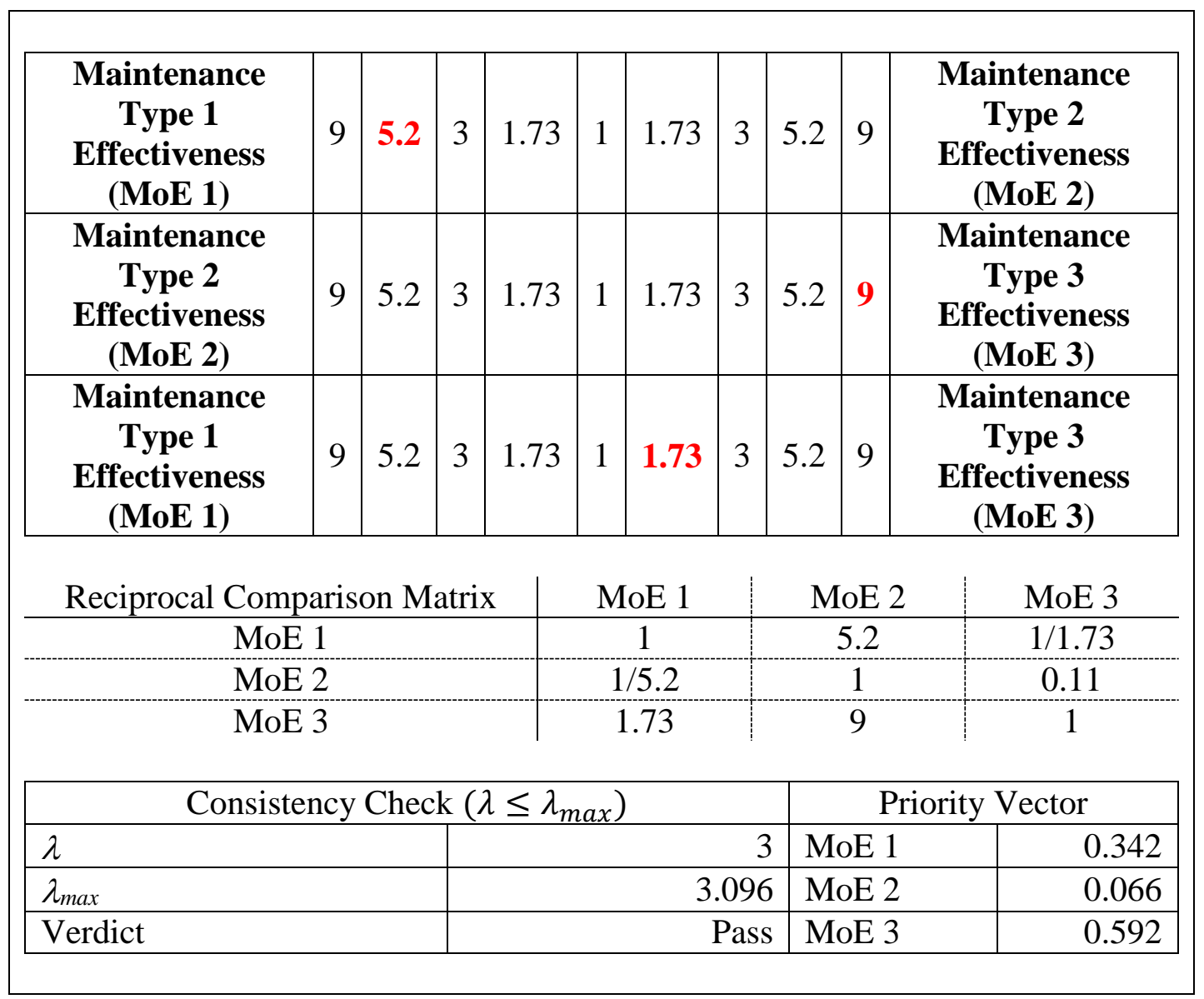

Table 5.8: Determination of the priority vector for the three Maintenance Types MoEs, Alongside Maintenance scenario

Figure 5.6 summarises the priority vectors from Table 5.7 and Table 5.8 and shows the variation of importance of different types of maintenance with respect to each of the two maintenance scenarios. It can be seen that for the three types of maintenance considered; $\mathrm{MT}_{1}$ (on-board in-space maintenance); $\mathrm{MT}_{2}$ (on-board workshop maintenance); and $\mathrm{MT}_{3}$ (off-board naval dockyard or OEM maintenance), the order of MT importance for Underway Maintenance (UM) was $\mathrm{MT}_{2}>\mathrm{MT}_{1}>\mathrm{MT}_{3}$, for Alongside Maintenance $(\mathrm{AM})$ it was $\mathrm{MT}_{3}>\mathrm{MT}_{1}>\mathrm{MT}_{2}$. 


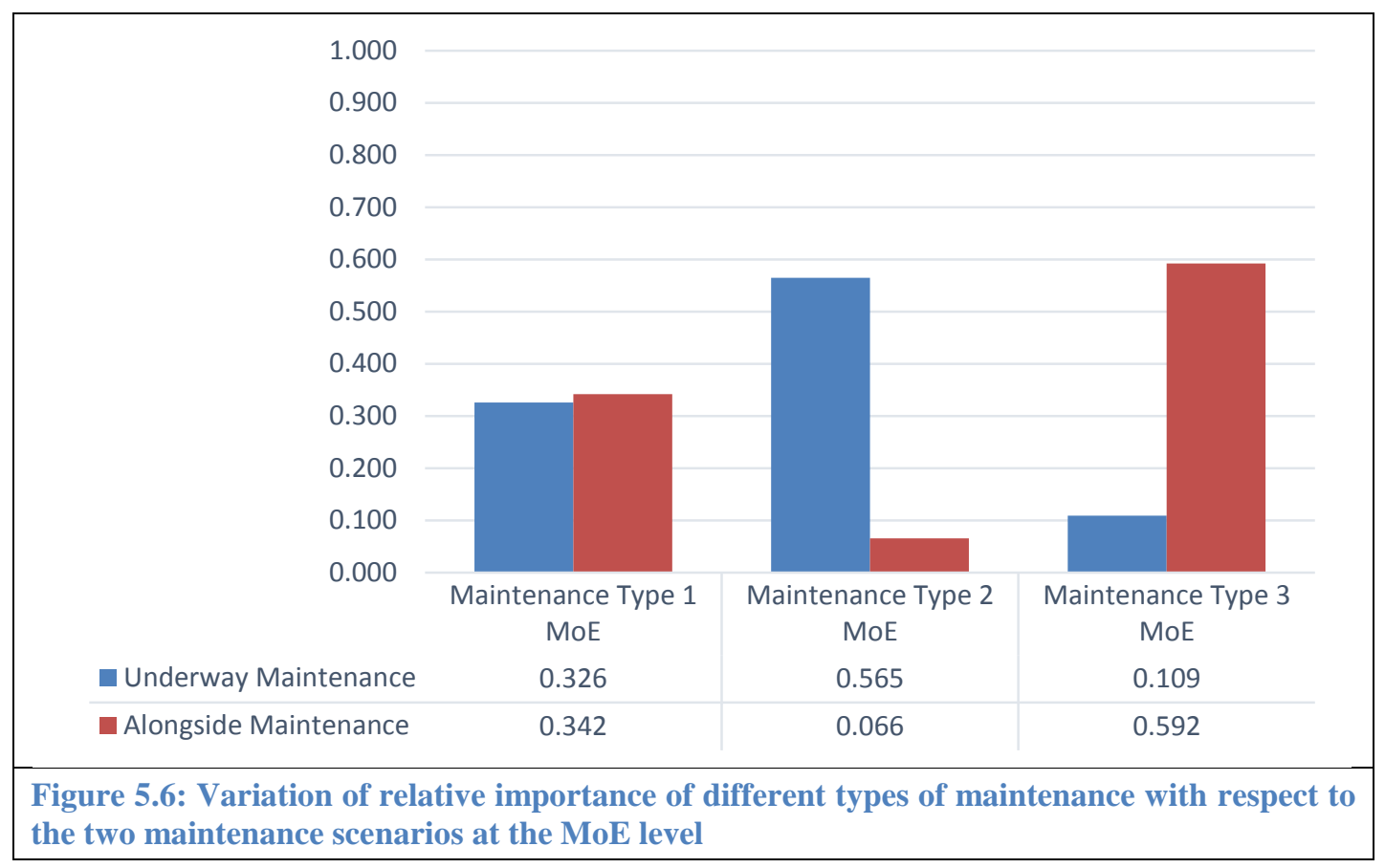

\subsubsection{Additional Aspects Taken into Account}

In order to further rationalise the maintainability oMoE and the underlying maintenance MoE values adopted in this research, and to further improve their sensitivity to differences in configuration from the Baseline Frigate Design, four additional aspects were taken into consideration before applying the maintainability evaluation hierarchy shown in Figure 5.1. These are described in the following four sub-sections.

\section{a) Maintenance Planning}

For ships that consist of several workshop facilities, NSGSs, ERPs, and machinery spaces, there is usually a logic behind the way in which these spaces are located with respect to each other. For a ship that usually includes several machinery spaces and NSGSs, it was assumed in carrying out maintenance on a particular machinery space (e.g. $\mathrm{MT}_{1}$, on-board in-space maintenance) the spare parts required would be located in the NSGS that is closest and most accessible (i.e. travelling the shortest Manhattan distance, and moving through smallest number of decks and bulkheads), that would lead to the lowest indicative travel time. Hence the sub-MoPs and MoPs were calculated for all the relevant spatial combinations but only the results associated with the closest and most accessible options were used to calculate the MoE of all three types of maintenance. 
Chapter 5: Application of the Proposed Design for Support Evaluation Approach and Presentation of the Results

\section{b) Machinery Space Measures of Maintenance Demand}

Given the broad range of items of equipment located inside the machinery spaces of naval vessels, it was concluded that it was unrealistic to assume that the maintenance demand would be evenly split across all spaces. Hence a measure was incorporated into the evaluation hierarchy to capture the effects of varying maintenance demands across the machinery spaces. Scaling maintenance demand with machinery space size was considered inappropriate, since maintenance complexity varies between different types of equipment and the number of items of equipment per unit of volume would be unlikely to be the same for all machinery spaces on a naval ship due to redundancy and survivability considerations. Instead, an indicative measure, based on equipment MTBF values, was adopted. The measure was defined as the ratio between the sum of MTBF values of items of equipment located in a particular machinery space to the sum of MTBF values of items of equipment located in all of the ship's machinery spaces. For a ship consisting of ' $m$ ' machinery spaces, the maintenance demand of a given machinery space was calculated using Equation 10.

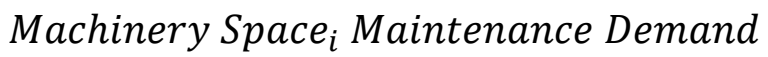

$$
\begin{aligned}
& =\frac{\sum \text { MTBF Machinery Space }}{i} \quad \text { for } i: 1 \ldots m
\end{aligned}
$$

In the absence of real data, the MTBF of all items of machinery equipment was assumed to be unity. For example, considering MMR 1 in the Baseline Frigate Design (see Figure 4.10), it consisted of three items of machinery equipment out of a total number of twenty six, hence its maintenance demand was 3/26. All ship design cases described in Chapter 4 were assumed to have the same disposition of machinery spaces and equipment, hence they would have the same distribution of maintenance demands, which is summarised in Table 5.9. Indicative maintenance demand values were applied when calculating the MoE of all the three types of maintenance addressed in this research. 


\begin{tabular}{|c|c|c|c|}
\hline $\begin{array}{l}\text { Machinery } \\
\text { Space }\end{array}$ & $\begin{array}{r}\text { Item } \\
\text { Type }\end{array}$ & $\begin{array}{c}\text { Item } \\
\text { Quantity }\end{array}$ & $\begin{array}{c}\text { Maintenance } \\
\text { Demand }\end{array}$ \\
\hline \multirow{4}{*}{ MMR 1} & Diesel generating set & 1 & \multirow{4}{*}{$\frac{4}{26}$} \\
\hline & Electric motor & 1 & \\
\hline & High Pressure Air Compressor & 1 & \\
\hline & Sea water pump & 1 & \\
\hline \multirow{3}{*}{ MMR 2} & Diesel generating set & 1 & \multirow{3}{*}{$\frac{3}{26}$} \\
\hline & Electric motor & 1 & \\
\hline & Sea water pump & 1 & \\
\hline \multirow{2}{*}{ GBR } & Reduction gearbox & 2 & \multirow{2}{*}{$\frac{3}{26}$} \\
\hline & Splitter gearbox & 1 & \\
\hline \multirow{4}{*}{ GTR } & Gas turbine & 1 & \multirow{4}{*}{$\frac{5}{26}$} \\
\hline & High Pressure Air Compressor & 1 & \\
\hline & Sea water pump & 1 & \\
\hline & Stabiliser box & 2 & \\
\hline \multirow{2}{*}{ DGR } & Diesel generating set & 2 & 3 \\
\hline & Sea water pump & 1 & $\overline{26}$ \\
\hline \multirow{2}{*}{ AMR 1} & Chilled water plant & 1 & 2 \\
\hline & Reverse osmosis plant & 1 & $\overline{26}$ \\
\hline \multirow{2}{*}{ AMR 2} & Chilled water plant & 1 & 2 \\
\hline & High Pressure Air Compressor & 1 & $\overline{26}$ \\
\hline \multirow{2}{*}{ AMR 3} & Chilled water plant & 1 & 2 \\
\hline & High Pressure Air Compressor & 1 & $\overline{26}$ \\
\hline \multirow{2}{*}{ AMR 4} & Chilled water plant & 1 & 2 \\
\hline & Reverse osmosis plant & 1 & $\overline{26}$ \\
\hline
\end{tabular}

Table 5.9: Distribution of maintenance demands across the machinery spaces for all ship design cases

\section{c) Measures of Capability of Supportability Features}

The indicative measures of travel time, based on Manhattan travel distance and the number of decks and bulkheads to lift, lower or pass equipment or spare parts through, did not in any way account for the capability of supportability features such as any ship workshop facilities and NSGSs on the proposed measure of ship supportability. Assuming the capability of these spaces scales with their size, indicative size-based measures were adopted. For a given ship design, the capability of an individual workshop facility or NSGS was defined as the ratio between its actual size and the values recommended for the overall ship by the UCL (2013b) database. In other words, if the size of an individual workshop facility or NSGS met the recommended UCL values, its capability was chosen to be 1.0, and if smaller than the recommended UCL value, its capability was reduced proportionally. Table 5.10 shows the capability 
Chapter 5: Application of the Proposed Design for Support Evaluation Approach and Presentation of the Results

measures for all ship design cases investigated. Indicative capability values were applied when calculating the MoE of $\mathrm{MT}_{1}$ (on-board in-space maintenance) and $\mathrm{MT}_{2}$ (on-board workshop maintenance).

\begin{tabular}{|l|r|r|r|r|r|}
\cline { 2 - 7 } \multicolumn{1}{l|}{} & \multicolumn{6}{c|}{ Supportability Feature } \\
\hline Ship Design & $\begin{array}{c}\text { Aft } \\
\text { Workshop }\end{array}$ & $\begin{array}{c}\text { Forward } \\
\text { Workshop }\end{array}$ & $\begin{array}{c}\text { Aft Naval } \\
\text { and Spare } \\
\text { Gear } \\
\text { Stores }\end{array}$ & $\begin{array}{c}\text { Amidships } \\
\text { Naval and } \\
\text { Spare Gear } \\
\text { Stores }\end{array}$ & $\begin{array}{c}\text { Forward } \\
\text { Naval and } \\
\text { Spare Gear } \\
\text { Stores }\end{array}$ \\
\hline $\begin{array}{l}\text { Baseline } \\
\text { Frigate }\end{array}$ & 1 & N/A & 0.5 & N/A & 0.5 \\
\hline Variant 1 & 0.5 & 0.5 & 0.5 & N/A & 0.5 \\
\hline Variant 1.1 & 0.75 & 0.75 & 0.5 & N/A & 0.5 \\
\hline Variant 1.2 & 1 & 1 & 0.5 & N/A & 0.5 \\
\hline Variant 1.2.1 & 1 & 1 & 0.5 & N/A & 0.5 \\
\hline Variant 2 & 1 & N/A & 0.33 & 0.33 & 0.33 \\
\hline Variant 2.1 & 1 & N/A & 0.5 & 0.5 & 0.5 \\
\hline Variant 2.2 & 1 & N/A & 0.73 & 0.52 & 0.75 \\
\hline Variant 2.2.1 & 0.5 & 0.5 & 0.33 & 0.49 & 0.77 \\
\hline Variant 3 & 0.75 & 0.75 & 0.5 & 0.5 & 0.33 \\
\hline Variant 3.1 & 1 & 1 & 0.73 & 0.52 & 0.75 \\
\hline Variant 3.2 & 1 & 1 & 0.74 & 0.49 & 0.77 \\
\hline Variant 3.2.1 & 1 & 1 & & 0.5 \\
\hline
\end{tabular}

Table 5.10: Capability measures of workshop facilities and Naval and Spare Gear Stores for all ship design cases outlined in Sections 4.4.1 - 4.4.3

\section{d) Measures of Routeability}

Routeability was defined as the ease of routeing a removal route between machinery spaces and certain locations in the ship where equipment can be removed from the ship (i.e. Equipment Removal Points or ERPs). The implemented Routeability measures used purely representative values, ranging from 0.0 to 1.0 , with higher values indicating better routeability, meaning faster transits. All ship design cases included two ERPs, one situated in the amidships mission bay and the other forward of the superstructure on No. 1 and No. 01 Decks for the original and alternative ship design styles respectively. 
It was assumed that due to the functional nature of the amidships mission bay, removing equipment through it would require more preparatory work than the forward removal point. Hence representative routeability measure of 0.5 and 0.8 were assumed for the amidships mission bay and forward removal point respectively. Assuming that double sided and athwartships passageway arrangements provide a degree of passageway redundancy and so generally improve routeability, the routeability measures of the alternative ship design style were assumed to be $25 \%$ larger than the original configuration. Table 5.11 shows the routeability measures for ERPs with respect to the selected ship design style. Indicative routeability measures were applied when calculating the MoE of $\mathrm{MT}_{3}$ (off-board maintenance by naval dockyards or OEMs).

\begin{tabular}{|l|l|r|}
\hline \multicolumn{1}{|c|}{ Adopted Style } & \multicolumn{1}{|c|}{ Equipment Removal Point } & Routeability Measure \\
\hline \multirow{2}{*}{ Original style } & Amidships mission bay & 0.5 \\
\cline { 2 - 3 } & Forward removal point & 0.8 \\
\hline \multirow{2}{*}{ Alternative style } & Amidships mission bay & 0.625 \\
\cline { 2 - 3 } & Forward removal point & 1.0 \\
\hline
\end{tabular}

Table 5.11: Routeability measures for the Equipment Removal Points with respect to the selected ship design style

\subsubsection{Application of the Maintainability Evaluation Hierarchy}

Having separately assessed the individual levels of the maintainability evaluation hierarchy shown in Figure 5.1 and taken into consideration the four additional aspects discussed above, maintainability of machinery spaces was assessed for all the ship design cases described in Chapter 4, Sections 4.4.1 - 4.4.3. The various elements of the assessment are summarised below.

1) Nodal priority values of every sub-MoP, MoP, and MoE were obtained as part assessing the individual levels of the maintainability evaluation hierarchy for both of the two maintenance scenarios;

2) The sub-MoPs associated with the three types of maintenance were measured for every machinery space using the Cartesian coordinates and adjacency 
Chapter 5: Application of the Proposed Design for Support Evaluation Approach and Presentation of the Results

relationships of the relevant spaces previously extracted from the DBB information;

3) The resulting sub-MoP values were then assessed against sub-MoP rejection and preference values using the segmented method shown in Figure 5.2 and transformed into sub-MoP score values;

4) The sub-MoP score value of each branch of the hierarchy was then multiplied by a) the sub-MoP, MoP, and MoE nodal priority values along that same hierarchy branch, and b) the additional measures incorporated to improve the rationality of the calculations. As a result, weighted sub-MoP score values were produced;

5) The weighted sub-MoP score value of all branches of the hierarchy were then aggregated to produce maintainability oMoE values of machinery spaces for both maintenance scenarios.

The assessment results are described in the following section. 


\subsection{Maintainability Assessment Results}

The assessment results are presented in the following three sub-sections. First the results from assessing the Baseline Frigate Design and its configurational variants (Variants 1, 2, and 3) are provided, followed by the results from investigating ship designs in which certain supportability features were enhanced (Variants 1.1, 1.2, 2.1, $2.2,3.1,3.2)$, and finally the results from evaluating the effects a different ship design style (Variants 1.2.1, 2.2.1, 3.2.1). Figure 5.7 provides a sequential description of the ship design variants. The term 'maintainability oMoE' used in all sub-sections refers to the combined maintainability scores of machinery spaces in each ship design case.

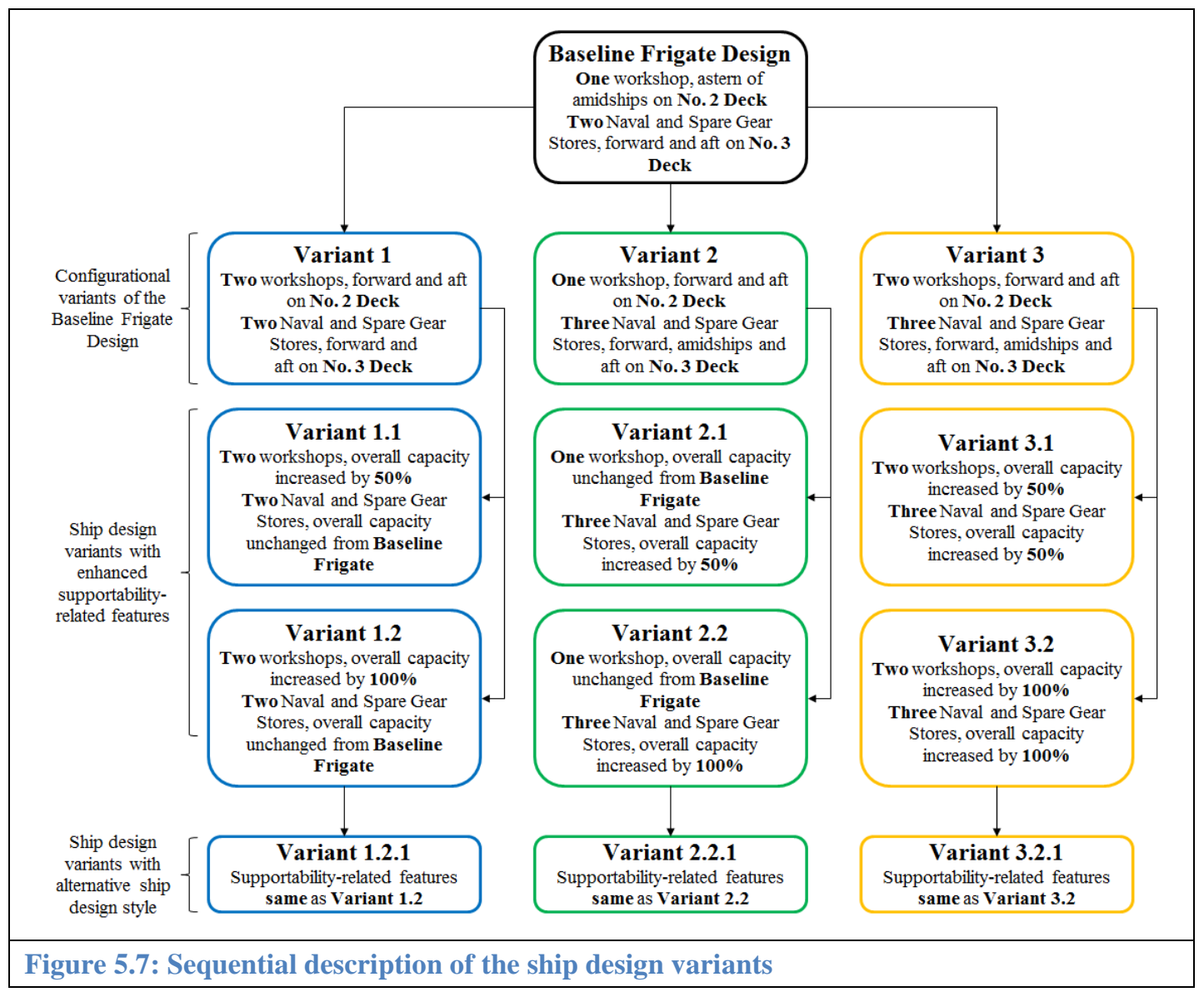

\subsubsection{Baseline Frigate Design and Configurational Variants}

Following the procedure described in Section 5.2.3, the Baseline Frigate Design and its three configurational variants were first assessed with respect to Underway Maintenance (UM) and Alongside Maintenance (AM) scenarios. The maintainability oMoE results are shown in Figure 5.8. 
Chapter 5: Application of the Proposed Design for Support Evaluation Approach and Presentation of the Results

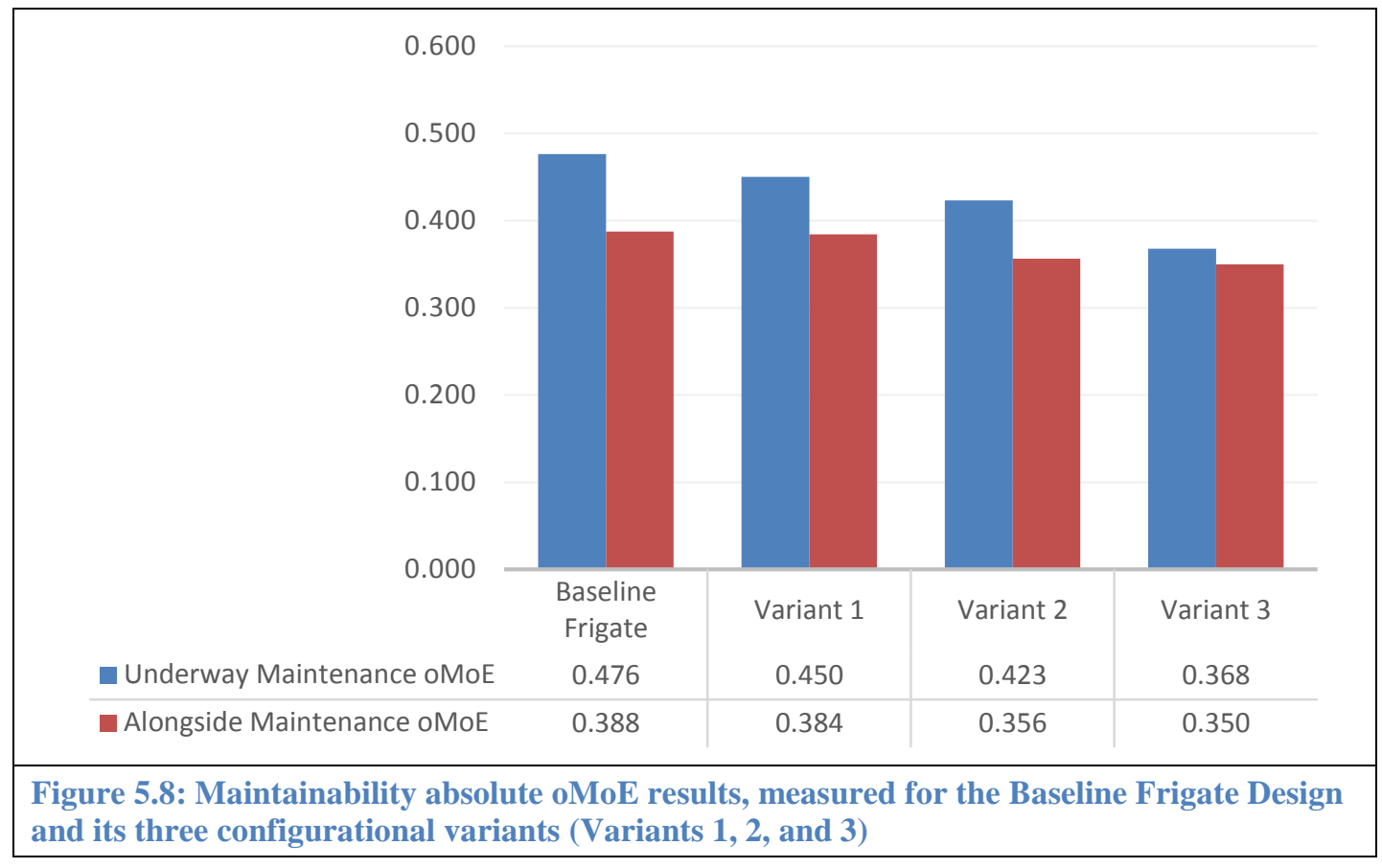

Figure 5.9 provides a more detailed set of results, showing the variation of MoEs of different types of maintenance with maintenance scenario.

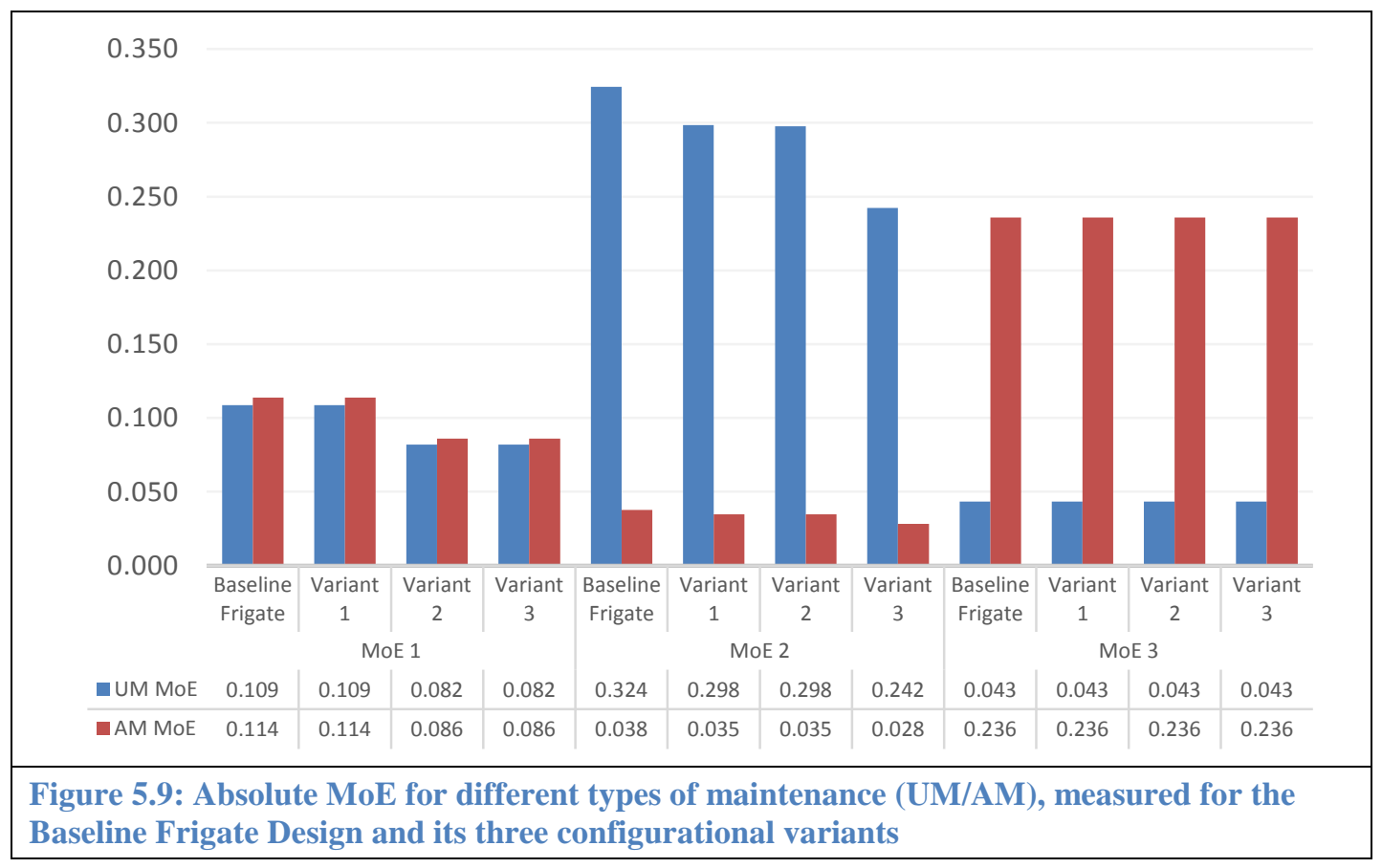

From Figure 5.8 and Figure 5.9, it can be seen that dividing the ship's workshop facilities and its NSGSs into a greater number of but individually smaller spaces without increasing the overall capacity might degrade certain MoEs (i.e. MoE 1 and $\mathrm{MoE}_{2}$ in this case), thus reducing maintainability oMoE. This result is entirely the 
consequence of incorporating capability measures (see Section 5.2.2) into the calculations and assuming there is a direct 1:1 relationship between the weight and volume of a supportability feature and its postulate of capability to contribute to ship maintenance. $\mathrm{MoE}_{3}$ remained unchanged since the Baseline Frigate Design and its configurational variants included the same number and disposition of ERPs.

To enable a more holistic comparison, the maintainability oMoE results shown in Figure 5.8 were compared with a number of major ship design characteristics, namely displacement, gross volume, density, and Unit Procurement Cost (UPC). All these values were normalised to the Baseline Frigate Design for comparison and the results are shown in Figure 5.10.

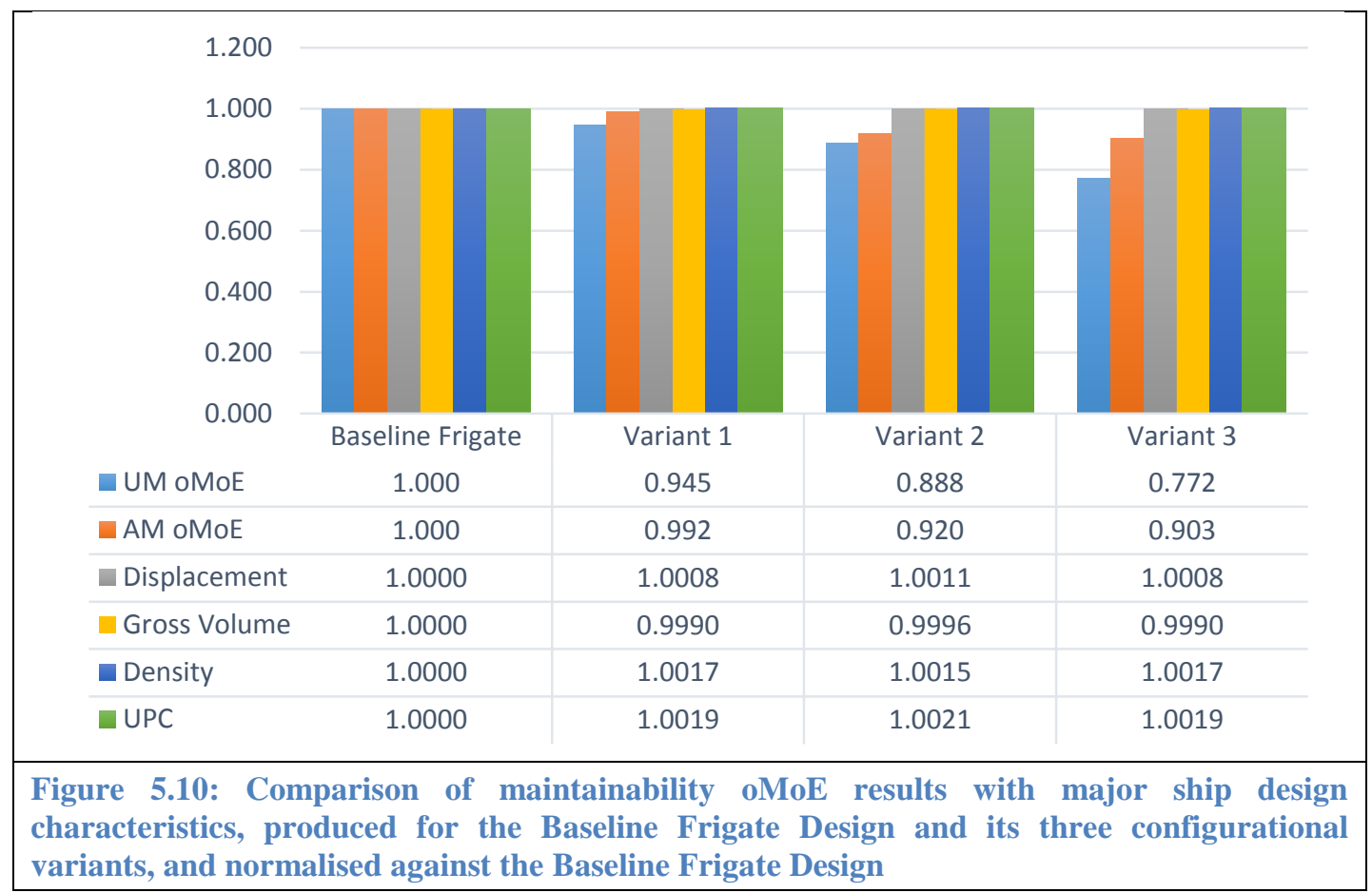

As also illustrated in Table 4.12, the variants were essentially configurational rearrangements of the Baseline Frigate Design, hence the impacts on displacement, gross volume, density, and UPC were expectably very small (less than $0.5 \%$, see Table 4.12). However, it can be seen that for ship designs of similar overall characteristics, the proposed measure of supportability performance was significantly affected by the various configurations implemented (Variant $3 \mathrm{UM}$ and AM oMoEs were reduced by approximately $26 \%$ and $11 \%$ respectively). A more in-depth discussion of the implications of such small departures from the Baseline Frigate Design on the maintainability oMoE is provided in Section 6.2.1. 
Chapter 5: Application of the Proposed Design for Support Evaluation Approach and Presentation of the Results

\subsubsection{Enhanced Supportability Features}

While maintaining the same disposition of supportability features (see Figure 4.13), the effects of increasing the overall capacity of workshop facilities and NSGSs on the proposed measure of supportability was investigated. First, the overall workshop capacity of ship design Variant 1 was increased to produce Variants 1.1 and 1.2, followed by increasing the overall NSGS capacity of ship design Variant 2 to produce Variant 2.1 and 2.2, and finally the overall capacities of both spaces were enhanced in ship design Variant 3 to produce Variant 3.1 and 3.2. Figure 5.11 compares the maintainability oMoE results of the Baseline Frigate and ship design variants with enhanced supportability features for both maintenance scenarios.

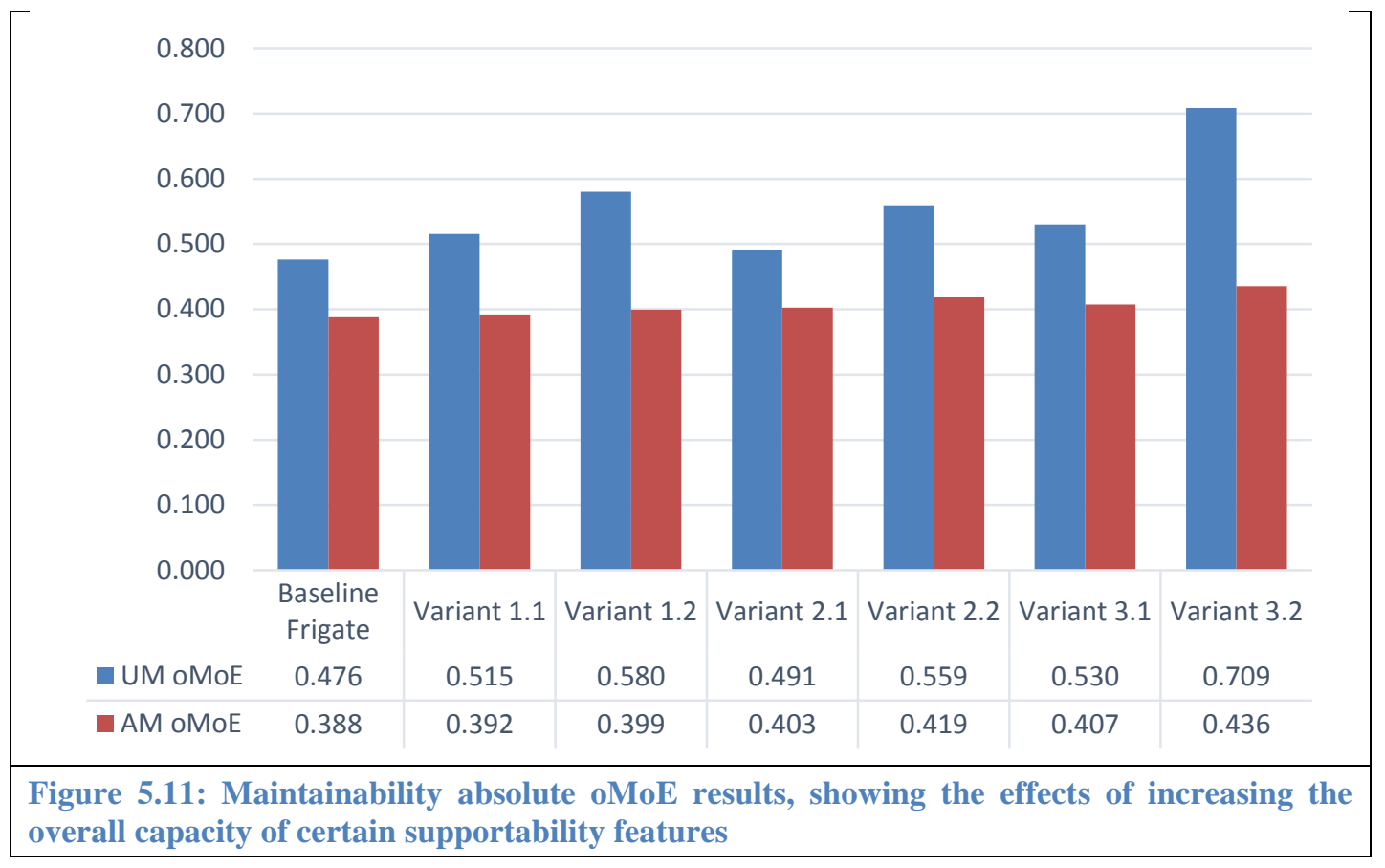

Figure 5.11 suggests that compared to the Baseline Frigate Design, the proposed measure of supportability (maintainability oMoE) improved, albeit at different rates, by expanding certain supportability features. As expected, doubling the capacity of workshop facilities or/and NSGSs (i.e. ship design Variants 1.2, 2.2, and 3.2) resulted in the highest maintainability oMoE values. The largest oMoE improvements were produced by Variant 3.2 in which workshop and NSGS capacities were both doubled. In this case, the maintainability oMoE was increased by approximately $39 \%$ for UM and $12 \%$ for AM, an average improvement of approximately $25 \%$ over the two 
maintenance scenarios. The ship design variants all consisted of the same number of ERPs, hence the improvements to AM oMoE were less than a third of UM.

These results can also be used to assist a ship designer who faces the dilemma of having to choose between only increasing the overall workshop or NSGS capacities. Increasing the overall workshop capacity (Variants 1.1 and 1.2) produced higher UM oMoE values than increasing the overall capacity of NSGSs (Variants 2.1 and 2.2) (e.g. approximately $+19.6 \%$ for Variant 1.2 and $+16 \%$ for Variant 2.2 with respect to the Baseline Frigate). However the opposite effect was observed in case of AM (e.g. approximately $+3 \%$ for Variant 1.2 and $+7.7 \%$ for Variant 2.2 ). Both choices produced practically the same mean oMoE improvements (e.g. about $+11.3 \%$ for Variant 1.2 and $+11.8 \%$ for Variant 2.2 with respect to the Baseline Frigate).

In an assessment similar to the Baseline Frigate Design, the maintainability oMoE results shown in Figure 5.11 were then compared to the same major ship design characteristics as in Figure 5.10. To help comparisons, the characteristics of ship design variants with enhanced supportability features were normalised to the Baseline Frigate. The results are shown in Figure 5.12.

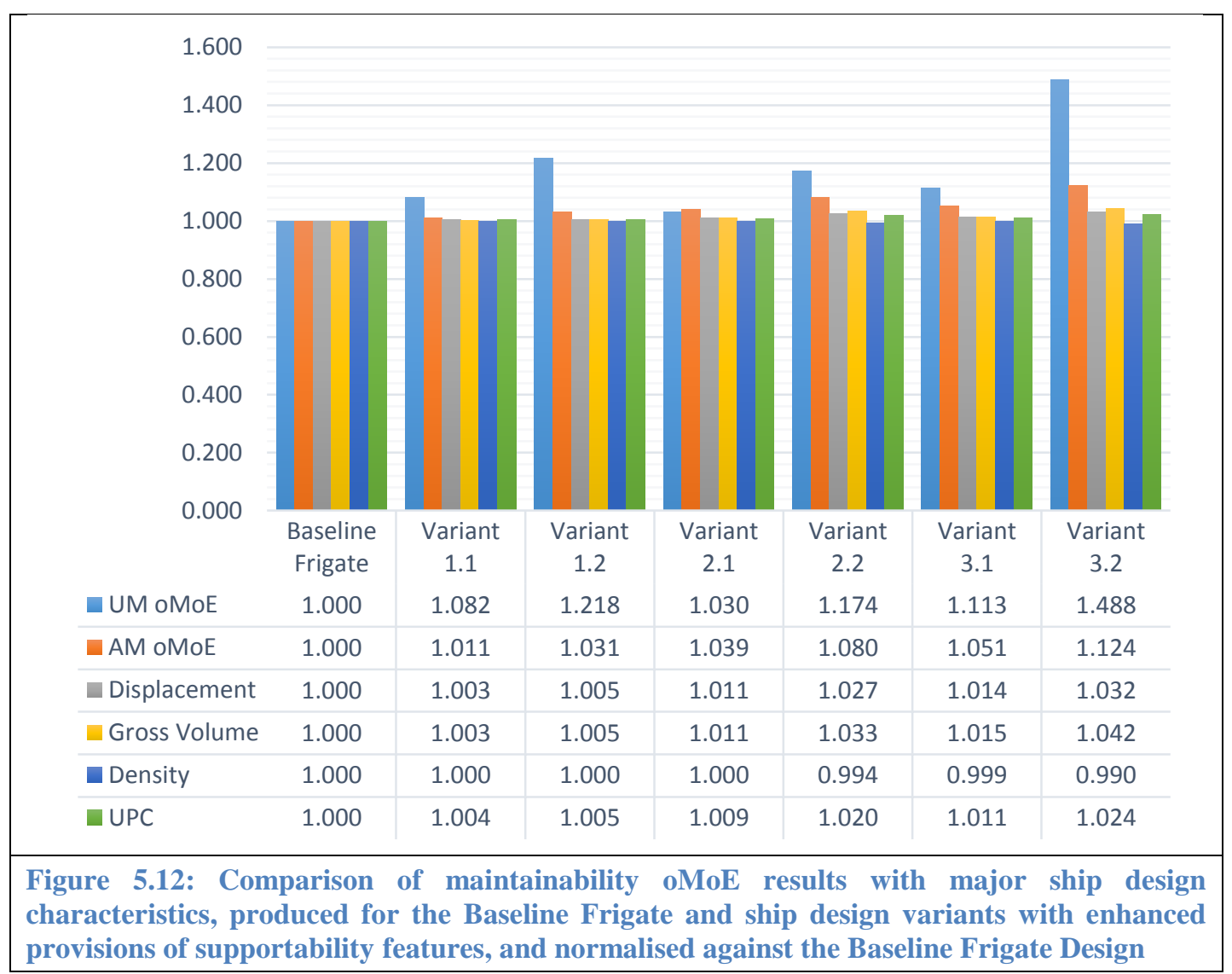


Chapter 5: Application of the Proposed Design for Support Evaluation Approach and Presentation of the Results

The overall conclusion from the data shown in Figure 5.12 is that considerable supportability improvements might be achievable for much smaller increases in major ship design characteristics, most importantly the ship UPC. For example, ship design Variant 3.2 improved the maintainability oMoE of the Baseline Frigate by an average of approximately $25 \%$ over the two maintenance scenarios, but its UPC was estimated to be only $2.5 \%$ or about $£ 14 \mathrm{~m}$ larger. Ship displacement and gross volume grew by about $3 \%$ and $4 \%$ respectively, reducing the overall ship density by approximately 1\%. A more in-depth discussion of these results is provided in Section 6.2.2.

\subsubsection{Alternative Ship Design Style}

The third and last set of studies involved assessing the effects of applying major stylistic design changes on the proposed measure of supportability. Ship design Variants 1.2, 2.2, and 3.2 had the highest maintainability oMoE values, hence formed the basis for adopting the alternative ship design style. Ship design Variants 1.2.1, 2.2.1, and 3.2.1 had the same supportability features as Variants 1.2, 2.2, and 3.2 respectively, but incorporated a deeper, two passing deck hull, double sided and athwartships passageway arrangement, and a correspondingly reduced superstructure. Figure 5.13 shows the maintainability oMoE results of the Baseline Frigate and ship design variants with the same supportability features but distinctly different ship design styles.

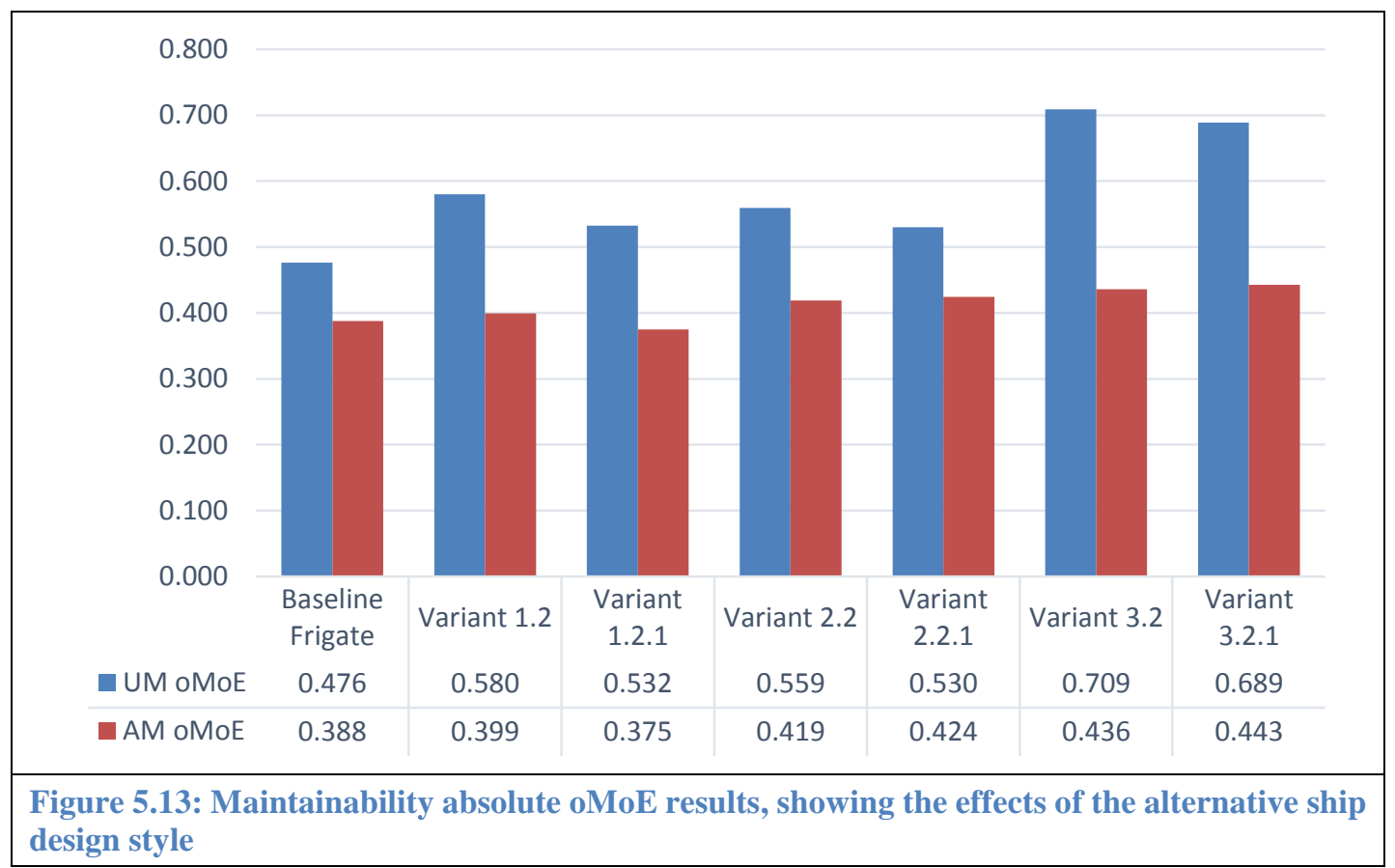


From Figure 5.13, it can be seen that in all the ship design variants incorporating the original style (Variants 1.2, 2.2, and 3.2), improvements to UM oMoE were larger in comparison to those integrating the alternative style (Variants 1.2.1, 2.2.1, and 3.2.1). Incorporating the alternative style, ship design Variants 2.2.1 and 3.2.1 produced slightly better AM oMoE results while Variant 1.2.1 performed worse than not just its original style equivalent but also the Baseline Frigate. Averaged over the two maintenance scenarios, the original ship design style returned higher maintainability oMoE values than the alternative option considered, though the gap appears to be shrinking as can be seen in Figure 5.13.

Figure 5.14 provides a more detailed set of results, showing the effects of the alternative ship design style on MoEs of different types of maintenance for the two maintenance scenarios. It can be seen that while incorporating the alternative ship design style generally degraded $\mathrm{MoE}_{1}$ and $\mathrm{MoE}_{2}$ of both maintenance scenarios, though at a decreasing rate in case of the latter, it resulted in $\mathrm{MoE}_{3}$ improvements. For example, Variant 3.2.1 outperformed Variant 3.2 in case of $\mathrm{MoE}_{3}$ by up to approximately $12 \%$.

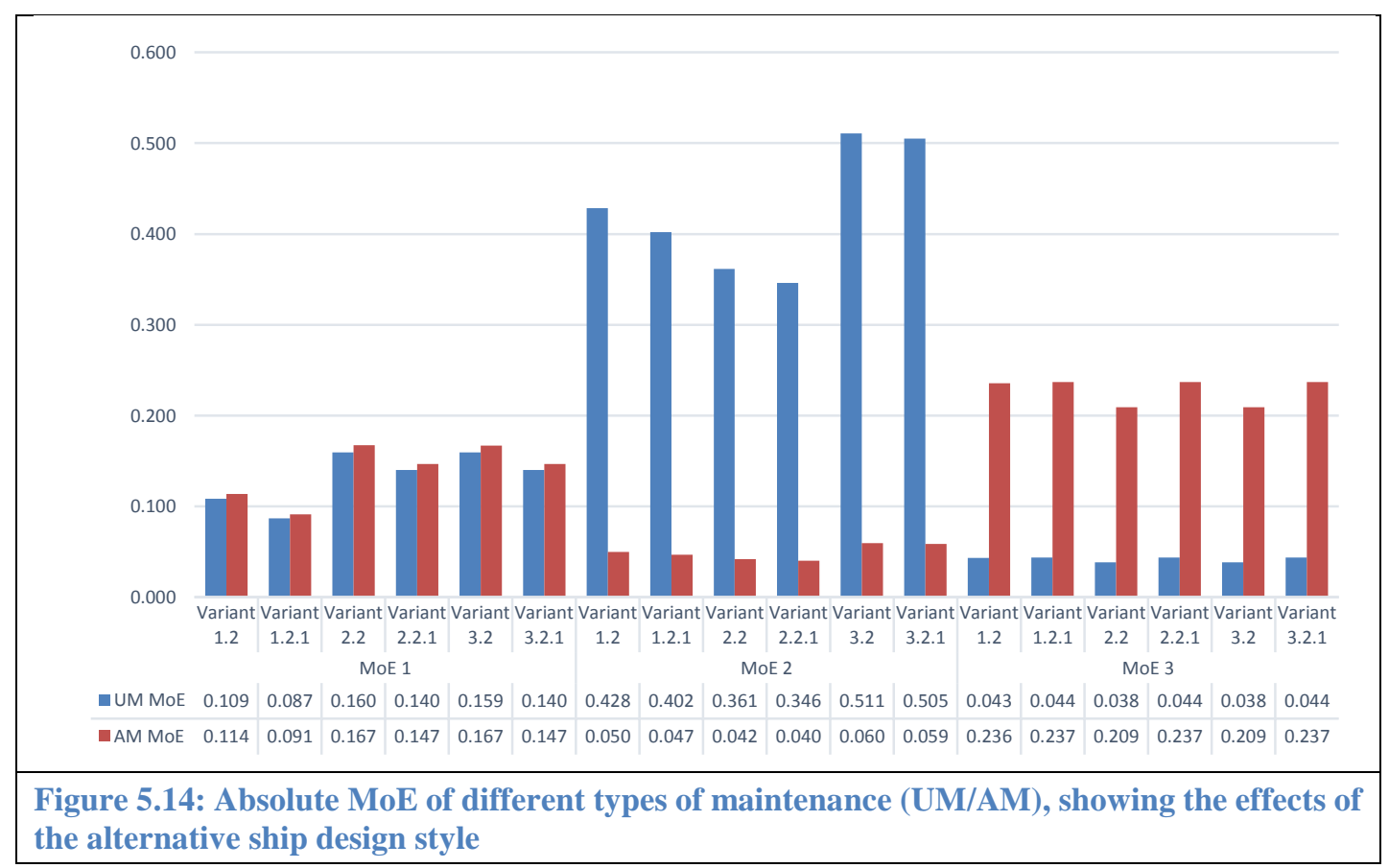


Chapter 5: Application of the Proposed Design for Support Evaluation Approach and Presentation of the Results

As previously, the maintainability oMoE results shown in Figure 5.13 were then compared with a number of major ship design characteristics. The results, normalised to the Baseline Frigate, are shown in Figure 5.15.

\begin{tabular}{|c|c|c|c|c|c|c|c|}
\hline \multicolumn{8}{|l|}{1.600} \\
\hline \multicolumn{8}{|l|}{1.200} \\
\hline \multicolumn{8}{|l|}{1.000} \\
\hline \multicolumn{8}{|l|}{0.800} \\
\hline \multicolumn{8}{|l|}{0.600} \\
\hline \multicolumn{8}{|l|}{0.400} \\
\hline \multicolumn{8}{|l|}{0.200} \\
\hline 0.000 & $\begin{array}{l}\text { Baseline } \\
\text { Frigate }\end{array}$ & $\begin{array}{c}\text { Variant } \\
1.2\end{array}$ & $\begin{array}{c}\text { Variant } \\
1.2 .1\end{array}$ & $\begin{array}{c}\text { Variant } \\
2.2\end{array}$ & $\begin{array}{c}\text { Variant } \\
2.2 .1\end{array}$ & $\begin{array}{c}\text { Variant } \\
3.2\end{array}$ & $\begin{array}{c}\text { Variant } \\
3.2 .1\end{array}$ \\
\hline - UM oMoE & 1.000 & 1.218 & 1.118 & 1.174 & 1.112 & 1.488 & 1.445 \\
\hline - AM oMoE & 1.000 & 1.031 & 0.968 & 1.080 & 1.095 & 1.124 & 1.143 \\
\hline Displacement & 1.000 & 1.005 & 1.033 & 1.027 & 1.049 & 1.032 & 1.056 \\
\hline Gross Volume & 1.000 & 1.005 & 1.063 & 1.033 & 1.080 & 1.042 & 1.090 \\
\hline Density & 1.000 & 1.000 & 0.972 & 0.994 & 0.971 & 0.990 & 0.969 \\
\hline UPC & 1.000 & 1.005 & 1.023 & 1.020 & 1.034 & 1.024 & 1.039 \\
\hline $\begin{array}{l}\text { Figure 5.15: Con } \\
\text { characteristics, pro } \\
\text { styles and baseline } \\
\text { the Baseline Frigat }\end{array}$ & $\begin{array}{l}\text { arison } \\
\text { dced for } \\
\text { d enhan } \\
\text { Design }\end{array}$ & $\begin{array}{l}\text { main } \\
\text { e ship d } \\
\text { d provi }\end{array}$ & $\begin{array}{l}\text { Iability } \\
\text { gn vari: } \\
\text { as of su] }\end{array}$ & $\begin{array}{l}\text { MoE r } \\
\text { S with o } \\
\text { ortabilit }\end{array}$ & $\begin{array}{l}\text { alts wi } \\
\text { sinal an } \\
\text { eatures }\end{array}$ & $\begin{array}{l}\text { major } \\
\text { alternati } \\
\text { nd norn }\end{array}$ & $\begin{array}{l}\text { ship design } \\
\text { ship design } \\
\text { lised against }\end{array}$ \\
\hline
\end{tabular}

From Figure 5.15, it can be seen that the alternative ship design style resulted in ships that were heavier, more voluminous, less dense, more expensive to build, and crucially less supportable compared to the original style according to the proposed supportability measure. For example, despite consisting of identical supportability features, ship design Variant 2.2 (original style) improved the average maintainability oMoE by an extra 2\% comparing to Variant 2.2.1 (alternative style), but it was calculated to be approximately $1.5 \%$ or $£ 8.5 \mathrm{~m}$ less expensive to procure. A more comprehensive discussion of these results is provided in Section 6.2.3. 


\section{Chapter 6: Discussion of Design for Support in the Initial Design of Naval Combatants}

\subsection{Introduction}

This chapter consists of two main sections. The next section takes up the maintainability assessment results presented in Chapter 5 and provides a more indepth discussion of those detailed results and considers the wider ship design implications. Section 6.3 provides the basis for addressing whether the proposed Design for Support (DfS) evaluation approach has achieved the overall research aim described in Chapter 1. First, the proposed approach is linked to the research background covered in Chapter 2, followed by discussing its implementation, before reviewing the limitations in the demonstrations. The chapter concludes by discussing the areas that require further investigation. On this basis, Chapter 7 discusses whether and to what extent the overall research aim has been achieved. 


\subsection{Analysis of Maintainability Assessment Results}

This section provides a more in-depth analysis of the maintainability assessment results presented in Chapter 5. Divided into three sub-sections, the results from assessing the Baseline Frigate Design and its configurational variants are first discussed, followed by an analysis of the results from investigating ship designs in which certain supportability features were enhanced, and finally the results from evaluating the effects of ship design style variants are examined.

\subsubsection{Baseline Frigate Design and Configurational Variants}

The results from assessing the maintainability of machinery spaces in the Baseline Frigate and its three configurational variants (Variant 1,2, and 3) with respect to two distinct maintenance scenarios, Underway Maintenance (UM) and Alongside Maintenance (AM), demonstrated that breaking up certain supportability features like ship workshop facilities and Naval and Spare Gear Stores (NSGS) might result in less supportable ships (see Figure 5.8). Although better accessibility to and from the machinery spaces was provided by splitting the workshop facilities and NSGSs into more locations, the new spaces had less individual capacity since the overall capacities were not altered. Considering the assumption that the capability of such supportability features scale with their size through a direct 1:1 relationship (see Section 5.2.2), the reduction in the defined maintainability overall Measure of Effectiveness (oMoE) observed in every configurational variant of the Baseline Frigate was considered to be reasonable. At the more detailed Measures of Effectiveness (MoE) level, the results showed that while measures of maintenance effectiveness for on-board, in-space and workshop maintenance (i.e. $\mathrm{MoE}_{1}$ and $\mathrm{MoE}_{2}$ respectively) were reduced for every configurational variant of the Baseline Frigate, the effectiveness of off-board, naval dockyard or even, Original Equipment Manufacturer (OEM) maintenance (i.e. $\mathrm{MoE}_{3}$ ) essentially remained unchanged (see Figure 5.9). This was considered to be a reasonable observation since the Baseline Frigate Design and its configurational variants, had the same number and disposition of Equipment Removal Points (ERP).

The maintainability oMoE results for both maintenance scenarios were also compared against a number of major ship design characteristics, namely displacement, gross 
volume, density, and Unit Procurement Cost (UPC). From these comparisons it was concluded that ship designs of very similar overall characteristics (i.e. less than $0.5 \%$ difference in any of these measures, see Figure 5.10) could have their supportability performance significantly affected by the configurational features investigated. For example ship design Variant 3, that incorporated the highest degree of fragmentation of workshop facilities and NSGSs without any overall growth in their capacity, experienced a reduction of approximately $26 \%$ in $\mathrm{UM}$ oMoE and $11 \%$ in $\mathrm{AM} \mathrm{oMoE}$ (see Figure 5.8). Therefore it might be suggested that supportability performance is more sensitive to the ship's internal configuration than certain major ship design characteristics have historically been assumed to be.

The percentage reductions in oMoEs as a result of purely configurational variations were very different due to the nature of the variants as well as the maintenance priorities, as is shown in Figure 5.6. While the Baseline Frigate workshop facilities and NSGSs were broken up into a greater number of but individually smaller spaces, the number and disposition of ERPs remained the same. Additionally, in case of UM, maintenance priorities were heavily skewed towards the types of maintenance heavily affected by the configurational changes. These were on-board in-space and on-board workshop maintenance works, each of which was considered to be directly affected by the changes to workshop facilities and NSGSs. On the other hand, AM was assumed to mostly depend on off-board maintenance, the main supportability feature of which (i.e. the number and disposition of ERPs) was not altered. This meant that the UM oMoE was more severely affected by the configurational variations than was AM oMoE.

\subsubsection{Enhanced Supportability Features}

Having concluded that splitting up certain supportability features, such as ship workshop facilities and NSGSs, without increasing the overall capacity provided might degrade ship supportability, the effect of expanding these spaces must be investigated. Extra overall workshop and NSGS capacities were incorporated into the configurational variants of the Baseline Frigate Design and the proposed measure of maintainability of machinery spaces was found to generally improve (see Figure 5.11). While the rates of improvement were different, depending on which feature was 
enhanced, simultaneously doubling the capacity of workshop facilities and NSGSs (i.e. ship design Variant 3.2) returned the largest improvements. In this case, the maintainability oMoE was increased by approximately $39 \%$ for UM and $12 \%$ for AM scenarios, which meant an average improvement of about $25 \%$ over the two maintenance scenarios.

The broad trends seen in the results presented in Figure 5.11 could assist ship designers who might be restricted to having to choose between increasing either the overall workshop or the NSGS capacities. Although options that change workshop or NSGS capacities essentially produced the same mean oMoE values, increasing the overall workshop capacity resulted in higher UM oMoE values, while expanding NSGSs produced higher AM oMoEs. Different capability measures of workshop facilities and NSGSs, and varying degrees of preference of different types of maintenance with respect to maintenance scenarios were identified as the reasons for the different patterns of oMoE improvement for UM and AM scenarios and are explained in the following paragraph.

All indicative measures of travel time between ship machinery spaces, workshop facilities, and NSGSs (i.e. MoEs) were weighted by corresponding capability measures. Although overall workshop and NSGSs capacities were doubled (compared to the Baseline Frigate Design) in Variants 1.2 and 2.2 respectively, Variant 1.2 incorporated two full capacity workshops (i.e. resulting in a capability measure of 1), which were located fore and aft, while Variant 2.2 incorporated three less than full capacity NSGSs placed fore, amidships, and aft (see Table 5.10). The large space requirements associated with increased overall capacities, combined with compartmentalisation and accessibility limitations, meant that it was not possible to locate spaces with the highest capability measures in places with maximum extent of accessibility, for example, amidships. Thus generally the most accessible NSGSs incorporated into ship design Variant 2.2, also had the smallest capacity and therefore the least measure of (storage) capability. This meant that when considering UM, increasing the capacity of workshop facilities appeared to be the preferable option. On the other hand, the lower capability measures of NSGSs compared to workshop facilities were to some extent counterbalanced by different maintenance priorities. At the Measures of Performance (MoP) (i.e. the indicative measures of travel time to and from the various machinery spaces) level, the importance of the single MoP directly 
related to the capability of ship workshop facilities (i.e. $\mathrm{MT}_{2} \mathrm{MoP}_{1}$ ) was 0.358 and 0.042 for UM and AM respectively. The two MoPs directly related to the capability of NSGSs (i.e. $\mathrm{MT}_{1} \mathrm{MoP}_{1}$ and $\mathrm{MT}_{2} \mathrm{MoP}_{2}$ ) had a total importance of 0.533 and 0.366 for UM and AM respectively. The larger importance values associated with NSGSrelated MoPs, meant that for the AM scenario, enhancing the capacity of NSGSs appeared to be preferable to increasing the capacity of workshop facilities.

In an assessment similar to the Baseline Frigate Design, the oMoE maintainability results of the ship design variants with enhanced supportability features were also compared against corresponding displacement, gross volume, density, and UPC figures (see Figure 5.12). The overall message from these comparisons was that a naval ship's supportability might be noticeably improved with much smaller increases in the major ship design characteristics that were considered, most importantly the ship UPC. For example, ship design Variant 3.2, that improved the maintainability oMoE of the Baseline Frigate by an average of about $25 \%$, was estimated to be only $2.5 \%$ or about $£ 14 \mathrm{~m}$ more expensive to procure than the Baseline Frigate.

Small fluctuations in overall ship density were also observed. The largest values were noted for ship design Variant 3.2 in which ship displacement and gross volume grew by about $3 \%$ and $4 \%$ respectively, reducing the overall ship density by approximately $1 \%$. Although very small, the increasing reduction in overall ship density might be seen as a trend that suggests less densely packed ships are likely to be more supportable.

\subsubsection{Alternative Ship Design Style}

Having found by simultaneously expanding and improving the accessibility of certain supportability features, such as ship workshop facilities and NSGSs, that the proposed measure of maintainability of machinery spaces was generally improved, the effects of applying major stylistic design differences were assessed. Ship design variants with enhanced supportability features that resulted in the highest maintainability oMoE values (Variant 1.2, 2.2, 3.2), formed the basis for adopting and comparing with the alternative ship design style. Averaged over the two maintenance scenarios, the resulting ship design variants (Variant 1.2.1, 2.2.1, and 3.2.1) returned lower maintainability oMoE values than their original style equivalent (see Figure 5.13). 
However, from Figure 5.13 the gap can be seen to be diminishing. While Variant 1.2 outperformed Variant 1.2 .1 by approximately $7.5 \%$ on the oMoE value, Variant 2.2 was approximately $2 \%$ better than Variant 2.2.1, and Variant 3.2 performed better than Variant 3.2 .1 by as little as $0.5 \%$. The reasons for the decreasing difference were identified as the routeability measures of the ERPs with respect to the adopted ship design style, and accessibility to and from workshop facilities and NSGSs and are explained in the following paragraphs.

The indicative measures of travel time between ship machinery spaces, workshop facilities, and NSGSs (i.e. MoEs) were increased as a result of incorporating the alternative ship design style. The double sided and athwartships passageway arrangement meant that for most cases of equipment and spare parts transfers, objects needed to be moved back and forth between the ship's centreline where hatches for vertical access were located and the sided passageways that were used for horizontal access, resulting in larger (Manhattan) travel distances. Very few cases of equipment and spare parts transfer were unaffected by the arrangement of the passageways. These were the cases in which the relevant spaces were vertically aligned and equipment and spare parts could be transferred without having to move to and fro the ship's centreline and sides (e.g. the direct transfer of equipment from the No. 2 Main Machinery Room (MMR 2) to the amidships missions bay for removal, see Figure 4.14).

Despite the negative impact of the alternative ship design style on the indicative measures of travel time, the effects were to some extent counterbalanced by the assumption that the double sided and athwartships passageway arrangement provided a better degree of passageway redundancy and hence improved routeability (i.e. the ease of routeing a removal route between machinery spaces and certain locations in the ship where equipment can be removed from the ship). The alternative ship design style was assumed to improve the routeability of the ERPs by $25 \%$. This resulted in higher $\mathrm{MoE}_{3}$ values comparing to the original style equivalent ship designs. For example, Variant 3.2.1 outperformed Variant 3.2 in case of $\mathrm{MoE}_{3}$ by up to approximately $12 \%$ (see Figure 5.14). Additionally, by having more workshop facilities and NSGSs on-board, direct access to these space was improved and the extent to which equipment and spare parts needed to be moved back and forth between the ship's centre and sides was reduced. This further improved the performance of ship design variants that adopted the alternative style. For example, while Variant 1.2 
outperformed its alternative style equivalent (Variant 1.2.1) in case of $\mathrm{MoE}_{2}$ by approximately 6\%, the comparison between Variants 3.2 and 3.2.1 showed a negligible difference in $\mathrm{MoE}_{2}$ values (see Figure 5.14). The consequence of the above is the decreasing difference in maintainability oMoE of the two stylistic variants shown in Figure 5.13.

In an assessment similar to the previous investigations, the oMoE maintainability results of the ship design variants with the original and alternative styles were also compared against corresponding displacement, gross volume, density, and UPC figures (see Figure 5.15). At first instance, the overall message from these comparisons was very similar to the investigations discussed in Section 6.2.2; that it might be possible to noticeably improve the supportability of a naval ship without substantially increasing the major ship design characteristics considered, most importantly the ship UPC. However, it can be seen from Table 6.1 that the oMoE improvements and corresponding UPC escalations, measured with respect to the Baseline Frigate Design, were different for the two ship design style variants.

\begin{tabular}{|l|l|r|r|}
\hline \multicolumn{1}{|c|}{$\begin{array}{c}\text { Ship } \\
\text { Design }\end{array}$} & \multicolumn{1}{c|}{$\begin{array}{c}\text { Ship Design } \\
\text { Style }\end{array}$} & $\begin{array}{c}\text { Maintainability oMoE } \\
\text { Improvement }\end{array}$ & $\begin{array}{c}\text { UPC } \\
\text { Escalation }\end{array}$ \\
\hline Variant 1.2 & Original & $11.5 \%$ & $0.5 \%$ \\
\hline Variant 1.2 .1 & Alternative & $4 \%$ & $2.5 \%$ \\
\hline Variant 2.2 & Original & $12 \%$ & $2 \%$ \\
\hline Variant 2.2 .1 & Alternative & $10 \%$ & $3.5 \%$ \\
\hline Variant 3.2 & Original & $25 \%$ & $2.5 \%$ \\
\hline Variant 3.2.1 & Alternative & $25 \%$ & $4 \%$ \\
\hline
\end{tabular}

Table 6.1: Variation of oMoE improvements and UPC escalations with respect to the adopted ship design style.

Despite including identical supportability features, ship design Variant 1.2 (original style) improved the average maintainability oMoE by an extra $7.5 \%$ comparing to Variant 1.2 .1 , but it was approximately $2 \%$ or $£ 10.5 \mathrm{~m}$ less expensive to procure. Variant 2.2 was approximately $1.5 \%$ or $£ 8.5 \mathrm{~m}$ less expensive to procure than its alternative style supportability equivalent Variant 2.2.1, but average maintainability oMoE was improved by an extra 2\%. Finally, ship design Variants 3.2 (original style) and 3.2.1 (alternative style) produced the same average maintainability improvements of approximately $25 \%$, but the latter was about $1.5 \%$ or $£ 9 \mathrm{~m}$ more expensive to procure. 
An observation made at the end of Section 6.2.2 was that less densely packed ships are likely to be more supportable. The results presented in Figure 5.15 do not support this conclusion, however. Ship design variants that incorporated the original style were slightly more densely packed and returned better average maintainability oMoE improvements than those that incorporated the alternative style. It might be suggested that although less densely packed ships could potentially be more supportable, the adopted ship design style and corresponding ship configuration could have significant effects on the extent of supportability improvements, for better or worse.

\subsubsection{Ship Costing and Maintainability Assessments}

From a ship design decision making perspective, the most important ship design characteristic that the maintainability assessment results were compared against was the estimated UPC values. The general argument is that early investments in carefully identified ship supportably features could result in disproportionately larger savings during the ship's lifetime (i.e. Through-Life Cost (TLC)). However, there were two obstacles in the way of being able to properly defend such an assertion.

First, there were significant uncertainties involved inside the parametric costing models used to estimate the UPC values. Certain assumptions regarding the cost of material and equipment, labour, shipyard hourly rates, purchasing overhead factors, were all based on historical monohull warship data and therefore considered to be unrepresentative of innovative design solutions and new technologies. The uncertainties are exacerbated due to the inaccuracies in the costs of specific items of equipment (e.g. gas turbine, diesel generators, electric motors, Combat System (CS) equipment). Piperakis (2014) comprehensively discussed the sources of uncertainties in UPC parametric estimations. Consequently, the estimated UPC values were only used in an indicative manner.

Second, the UPC incremental changes caused by enhanced supportability features could not be compared against quantified TLC values due to the restrictions of the UCL (2013b) TLC estimation method. The parametric procedure estimates the costs associated with ship maintenance, refit, and upgrade as proportional to the UPC, based on fixed upkeep intervals and person-hour values, and regardless of how the adopted ship internal arrangement and the supportability features provided might affect some 
of these issues. Considering the configurational emphasis of this research and the ship design cases developed, as well as the fact that supportability aspects, such as maintenance, refit, and upgrade, are strongly affected by the ship's configuration (see Section 2.3.2), it was concluded that such crude estimations were unlikely to capture the potential TLC reductions or demonstrate the value of supportability enhancements. For example, using fixed person-hour values fails to capture the effects of improved accessibility to certain supportability features of the ship. In fact, since the UCL (2013b) guidance estimates the costs associated with Through-Life Support (TLS) as proportional to the ship's UPC, the ship design cases with enhanced supportability features and seemingly better supportability performance (see Sections 5.3.2 and 5.3.3) would have resulted in larger TLC values, which would have been a contradictory conclusion.

Considering the 1:3 ratio between the UPC and TLC of a typical warship provided by (Brown and Andrews, 1980) and assuming that for every $£ 1$ of extra UPC the TLC is reduced by $£ 3$, it might be possible to produce representative figures of potential TLC savings to support the general argument of this research. For example, considering the ship design Variant 3.2 that resulted in the highest average maintainability oMoE improvements, it was also $£ 14 \mathrm{~m}$ more expensive than the Baseline Frigate Design to procure. Based on the assumed inverse relationship between UPC increases and TLC reductions, ship design Variant 3.2 would cost about $£ 40 \mathrm{~m}$ less to run over its lifetime comparing to the Baseline Frigate. However, if the same relationship was applied to ship design Variant 3.2.1 that was $£ 9$ m more expensive than Variant 3.2, that would suggest an extra $£ 27$ TLC savings. However, this would be contradictory since ship design Variant 3.2.1 was shown to produce the same degree of maintainability oMoE improvements as Variant 3.2.

While, based on the metrics proposed in this thesis, it can be generally asserted that more supportable ships would be expected to be more expensive to procure and that it is possible to roughly capture such cost escalations, a proper TLC estimation model appropriate to Early Stage Ship Design (ESSD) is evidently lacking. Such models should appreciate the fact that many aspects of naval ship supportability are configurationally-driven and better account for the impact of changes to the internal arrangement on TLC estimations. Failure to demonstrate the long-term financial 
benefits of enhanced ship supportability features against initial investments during ESSD, might mean that when the ship designer finally has access to more sophisticated costing models in the later more detailed design phases, many supportability enhancement features might well have been excluded.

\subsubsection{Maintenance Scenarios and Maintainability Assessments}

The maintainability oMoE results for the two distinct maintenance scenarios, UM and AM, were combined using simple averaging with no particular preferences being made between underway and alongside maintenance. In reality however, the decision makers might have very different preferences considering the development of maintenance concepts, such as those listed by Kettle and Figg (1998), Price et al. (2014), and Bergsma et al. (2016):

- Condition-Based Monitoring (CBM) in case of unknown failure mechanisms;

- Sponsored reserves to allow skilled personnel from OEMs to be deployed with the ship;

- Tele-engineering or video conferencing for maintenance and diagnostics;

- Forward support team and shore-based support or the capability to fly in staff and spares for maintenance support in order to improve maintenance response time and hence availability;

- On-board scaling or changing the size of on-board spare holdings based on better estimation of requirements (different scales of spare holdings were investigated as part of this research)

- On-board 3-D printing for rapid manufacture of spare parts and provision of increased flexibility without high inventory cost;

- The use of Commercial Off The Shelf (COTS) equipment to encourage replacement instead of repair, though there are question marks due to the equipment not being specifically designed for military applications.

Moreover, the world's geopolitical situation is also likely to affect the decision makers' attitude towards maintenance and supportability. During peacetime, a military system is always designed in accordance with the associated financial pressures that often results in the preferred design being the one that maximises assumed mission effectiveness per UPC. Naval ships like any other military system are usually designed 
and produced under the pressures of peacetime expenditure constraints. As hostilities rise however, the cost factor usually becomes much less critical than capability and the design that maximises mission effectiveness and early deployment is generally chosen. Cost as a constraint is effectively replaced by the availability of resources and delivery speed. However, the current geopolitical situation raises certain complications. Describable as 'semi-war', it involves peacekeeping, anti-piracy and minor actions, yet keeping down the overall defence expenditure is still the priority (UK MoD, 2015b). Given the significance of the issue and its long-term implications, the need to investigate DfS in ESSD is therefore evident. 


\subsection{Research Summary}

\subsubsection{The Importance of Design for Support in Naval Ship Design}

Most major western navies have experienced reductions in fleet size since the end of the Cold War, partly due to better reliability and availability of individual items of equipment and vessels (Alexander, 1988), but mostly caused by the decline of defence budgets, coupled with the above inflation escalation of warship procurement costs (Andrews and Hall, 1995; Taylor, 2010). This has happened despite little reduction in overall commitments (Collins et al., 2012), thus resulting in greater capability (Jones and Kimber, 2012) and reliability (Manley, 2012) required per ship.

The cash-limited warship procurement policy increasingly implemented in recent years to challenge the above financial realities (Brown, 1986) means investigation of cost-effectiveness is now a significant element of naval ship design practice. However, out of the two components of a warship's Whole Life Cost (WLC), UPC has historically been the focus of most cost reduction approaches and there has been a failure to address the fact that TLC or the costs incurred during the in-service period form the majority of a warship's WLC. This is illustrated by the WLC iceberg model (see Figure 2.6) and the 1:3 ratio between the UPC and TLC of a typical warship provided by Brown and Andrews (1980).

The lower priority given to through-life aspects by the ship owner, the UK MoD in the case of Royal Navy vessels, has been attributed to the historical tendency of governments to focus on aspects that they consider to be more important to how their term in office will be judged (Rizzo, 2011). Concentrating on short-term rather than long-term strategies, early years' expenditure, and requirements rather than solutions, has meant inadequate attention being given to the ship's through-life sustainability and supportability. The problem is exacerbated by the fact that in the naval domain, the benefit of certain capabilities (e.g. a supportability feature or survivability component) are intrinsically difficult to quantify and therefore hard to justify incorporating. The consequence is that TLS could be falsely presented as an attractive area for cost cutting UPC allocations. Such imprudent attitude could result in ships that are too physically tight (e.g. The Royal Navy's Type 42 Destroyers and Type 23 Frigates) or poorly 
engineered (e.g. HMS Ocean and the implications of the warfighting inadequacy of commercial equipment), hence future naval vessels could be unavailable at times of need, expensive to run, difficult or impossible to upgrade, and even more difficult to build in the first place.

\subsubsection{Design for Support in Naval Ship Early Stage Design}

The ship design process has so far proven impossible to be described by a set of directly solvable equations (Gale, 2003), hence in dealing with the various, often conflicting aspects, especially in the highly complex domain of naval ship design, naval architects use sequential and iterative approaches (e.g. the ship design spiral critiqued by Andrews et al. (2009)). The challenges faced are only made more difficult by the absence of prototypes due to time, cost and size factors (Brown and Tupper, 1988), meaning that unlike the aerospace industry, the final ship design solution does not benefit from fully demonstrating a prototype before the production run is ordered. Therefore, an extensive number of ship design options should be explored and evaluated during the Concept Phase before proceeding to the later design phases.

The Concept Phase is when major decisions are made and trade-off studies carried out on requirements and affordability, thus effectively committing the majority of future costs (Brown and Andrews, 1980; Andrews and Pawling, 2009). However, the expenditure of design resources in terms of time and finance are small comparing to the entire programme. Hence, complicated issues like TLS should be addressed during the Concept Phase instead of the later design phases where the cost of rectifying problems would be disproportionally higher (Goossens, 1992; Gale, 2003). In addition, introducing design novelty, whether that be to insert a new capability or to reduce costs, is best facilitated during the Concept Phase while the design is still flexible and the designers can explore many ideas (Brown, 1986). The advantages of a proper consideration of naval ship TLS in the early, crucial phase of design are therefore evident.

However, early considerations of TLS are restricted by the absence of numerical and structured ways to address such design aspects in ESSD. Although innovative, the majority of developed TLS technical solutions, strategies, and tools investigated for this research were found to be either excessively solution-focused or largely depend 
on highly detailed ship design information and a great deal of supporting information on specific equipment (see Section 2.3.3). The lack of a rational TLS evaluation scheme that is compatible with the high level design definitions produced in the Concept Phase has led to significant reliance on expert TLS knowledge in the later ship design phases where corrective design actions would be costly.

\subsubsection{Design for Support in Architecturally-Orientated Design}

Innovations in design development and design approach are seen to be necessary to respond to the pressures of diminishing budgets and escalating costs (Brown, 1993). However, given that warships are considered as architecturally-limited (Brown, 1993), the traditional naval ESSD would seem to inhibit creativity and the full exploration of alternatives (Andrews, 2003b) since it postpones configurational modelling until after numerical synthesis and fails to comprehend many of the important design Style issues (Andrews, 1985). These shortcomings are further highlighted by the fact that novelty in design and assessment is best facilitated during ESSD (Brown, 1986).

Therefore, incorporating alternative, fully integrated and configurationally-orientated approaches to ESSD is considered to enhance the contribution of ship designers and allow them to be more creative and innovative, enable the rapid exploration of large numbers of design alternatives, and help better identify design drivers and risk areas and avoid prolonged expensive design reworks (Andrews, 2003b). Amongst several aspects that can be brought into the initial synthesis by implementing configurationally-orientated approaches to ESSD is the consideration of through-life issues, namely adaptability (operationally and through-life) and sustainability, both considered to be significantly affected by the ship's layout (Andrews and Pawling, 2003).

The rapid developments in Computer-Aided Design (CAD) tools and computer graphics, since the late 1990s, have enabled the implementation of an architecturallybased, fully integrated, more innovative and adaptable ESSD approach known as the Design Building Block (DBB) approach that was originally developed at UCL (Andrews, 2003b). The basic idea behind the DBB approach to ESSD is to allow the ship designers to separate the ship's functions and sub-functions into discrete physically realisable elements (i.e. Design Building Blocks) and position them 
appropriately in a putative ship configuration, thus putting ship architecture at the centre of the process (Andrews and Dicks, 1997). The UCL DBB approach was implemented through the SURFCON module in the commercially established QinetiQ GRC's Paramarine (Munoz and Forrest, 2002), hence the naval architectural analytical tools available inside Paramarine were integrated with architectural modelling in ESSD.

Despite its advantages, the overwhelming nature of using a sophisticated (fully 3-D) CAD modelling tool like Paramarine, meant that it was not considered to be entirely appropriate in this ESSD investigation of DfS (see Section 4.2). Hence an alternative implementation of the UCL DBB approach that was initially developed as a Microsoft Excel-based spreadsheet (Pawling et al., 2015) and later transferred to the JavaScript environment for improvements (Pawling et al., 2017a) was used to develop the ship design cases investigated in this research.

\subsubsection{The Proposed Design for Support Evaluation Approach}

From the three main obstacles to a proper consideration of naval ship TLS in the early, crucial phase of ship design (see Section 2.5), the financial challenges of defence acquisition and the political tendencies of governments were considered to be outside the scope of this project. The development of the supportability evaluation approach proposed as part of this research was therefore carried out with the aim of addressing the shortcomings caused by the traditional naval ESSD process and the lack of a numerical and structured TLS evaluation approach compatible with the high level design definitions produced in the Concept Phase. Realising that modern warships are architecturally-constrained and considering the relevant experience, knowledge, and the tools available at UCL Design Research Centre (DRC), the development of the approach was focused on the architecturally identifiable aspects of Integrated Logistics Support (ILS). The proposed approach included the four principal features discussed below.

First, the shortcomings of the traditional naval ESSD process in handling the architecturally-driven aspects of ship design (e.g. DfS) were tackled by utilising the UCL DBB architecturally-orientated ESSD approach and the UCL JavaScript concept design tool. Though still under development, the JavaScript tool used to develop the 
ship design cases proved capable of fast modelling and generating the high level design definitions required by the maintainability evaluation hierarchy produced without the very high software learning overheads associated with Paramarine.

Second, to enable exploration of ILS issues in the Concept Phase, the reliance on overly detailed ship design information was reduced by structuring the evaluation of naval ship TLS in a top-down manner. Using certain components of Decision Analysis and Effectiveness Analysis (See Section 3.3.1) in parallel, as well as the Analytic Hierarchy Process (AHP), a top-down hierarchy for a partial (architecturally dependent) evaluation of maintainability (as a subset of naval ship supportability) was developed. Three distinct types of maintenance appropriate for investigation in ESSD were considered, namely on-board in-space maintenance, on-board workshop maintenance, and off-board maintenance by naval dockyards or equipment manufacturers (see Figure 3.6).

Third, the difficulty of quantifying many aspects of naval ship TLS in ESSD was tackled by employing a number of rational and indicative (i.e. proxy) supportability metrics. The time it takes to move ship equipment and spare parts between shipboard spaces was treated as an indication of effectiveness in achieving maintenance tasks, with shorter travel time generally taken to mean more effective maintenance. The travel time measurements were indicative and based on the Manhattan travel distance and the number of decks and bulkheads to lift, lower or pass equipment or spare parts through. Although such metrication might seem rather crude and simple, it was considered appropriate given the limited high level design information available during ESSD.

Fourth, a framework was developed to enable the structured representation and rational application of expert naval ship design and TLS knowledge (e.g. rules of thumb) in the supportability evaluation process. The framework was primarily centred on the use of pairwise comparisons (an important component of the AHP) of architecturally discernible attributes. This facilitated the process of capturing and quantifying the decision makers' inconsistent and often conflicting subjective preferences. The three major topics that were taken into account as part of developing the framework were: a) the numerical scale used in the pairwise comparisons and its effects on the distribution of the weightings; b) the method used to check the 
consistency of the pairwise comparison matrices and its implications on the resultant numerical preferences, and c) the method used for generating the numerical preferences from the pairwise comparison matrices and how it related to the other two topics.

To demonstrate the proposed approach, the evaluation hierarchy was first customised to the investigation of maintainability of ship machinery spaces and the maintenance of items of equipment usually located inside (see Figure 5.1). Before implementing the maintainability evaluation hierarchy, four additional aspects were taken into account to improve the rationality of the assessment results and enhance their sensitivity to configurational ship design variations. Firstly, since naval ships normally include several workshop facilities, NSGSs, ERPs, and machinery spaces, it was considered that there is usually a logic behind the way in which these spaces are located with respect to each other. For this research, it was assumed that the maintenance of the items of equipment located inside each machinery space would be conducted using the closest and most accessible relevant ship supportability features. Secondly, it was concluded that the maintenance demand would not be evenly split across various machinery spaces given the broad range of items of equipment located inside. Although the measures of maintenance demand were indicative and based on Mean Time Between Failure (MTBF) unity figures due to lack of real data (see Table 5.9), the supportability implications of the disposition of machinery spaces and relevant items of equipment were captured to some extent. Thirdly, assuming that the ability of relevant ship supportability features to contribute to ship maintenance scales with their size, capability measures were calculated proportionally to the values recommended by the UCL (2013b) database. Though rather crude, these measures helped capture the supportability consequences of reducing or enhancing the supportability features considered. Fourthly, the routeability measures, despite being essentially representative, helped take into account the fact that the difficulty of routeing a removal between machinery spaces and ERPs varies depending on the travel path of the removal route and the compartments it has to pass through.

With the assistance of the four additional aspects discussed above, the customised evaluation hierarchy was then applied to the ship design cases developed as part of 
this research and a partial (architecturally dependent) assessment of maintainability of machinery spaces, as a subset of ship supportability, was carried out.

\subsubsection{Research Limitations}

The development of the proposed supportability evaluation approach was entirely focused on the architecturally discernible aspects of ILS and addressed the lack of a numerical and structured assessment method appropriate for ESSD. Consequently, the full range of naval ship TLS, the financial challenges of defence acquisition, and the political tendencies of governments were not addressed in this research. However, it is considered that these issues will significantly impact comprehensive, fleet level considerations of naval ship supportability. The supportability assessments were further restricted due to being primarily centred on the issue of naval ship maintenance and more specifically the maintenance of equipment usually located inside the ship machinery spaces. The maintainability or adaptability of other significant features of the ship, such as the CS were not investigated.

There were certain limitations regarding the application of the proposed DfS evaluation approach. The indicative measures of travel time treated as Proxy Supportability Indicators (PSI) comprised of the Manhattan travel distance and the number of decks and bulkheads to lift, lower or pass equipment or spare parts through. Although the number of decks and bulkheads could be accurately counted, the Manhattan method was unable to accurately detect the actual travel route between two spaces of interest in a typical ship internal arrangement. In addition, the Consistency Threshold (CR) value selected for this research (0.1) was purely selected in order to be able to conduct the maintainability assessments without knowing how appropriate this number was to this particular problem and the wider domain of naval ship design. Moreover, to best utilise the experience, knowledge, and the tools available at the UCL DRC regarding architectural naval ship concept design, ship design-related investigations of supportability were prioritised. Consequently, no sensitivity analyses were carried out on the numerical preferences of the decision makers (priority vectors) used in the weighed aggregation of the proposed supportability metrics. Also, both maintenance scenarios investigated as part of this research (underway and alongside) were assumed to be of equal significance from a naval ship TLS perspective. This was probably a somewhat unrealistic assumption given the development of new 
maintenance concepts, the financial pressures of defence acquisition and ownership, and the unpredictable nature of international geopolitics. Finally, the additional aspects taken into consideration included the following limitations: the MTBF values of all items of machinery equipment were assumed to be unity due to lack of real data; a potentially questionable 1:1 relationship was assumed to exist between the capability of ship supportability features (e.g. workshop facilities and NSGSs) and their size; and the routeability measures were essentially representative and based on the assumption that the double sided and athwartships passageway arrangements provide a degree of passageway redundancy and so generally improve routeability.

The ship design cases developed as part of this research were based on a baseline monohull frigate-sized combatant with a number of relatively small variations of it, namely configurational rearrangement of certain supportably features (workshop facilities, NSGSs), enhancement of those supportability features, and finally an alternative ship design style (double sided and athwartships passageway arrangement). Other types of combatants (e.g. corvettes, destroyers), ship design styles (e.g. multihull), and different ship types (e.g. auxiliaries) or technologies (e.g. Integrated Electric Propulsion (IEP)) were not investigated. In addition, the Baseline Frigate Design and ship design variants were developed based on the UCL warship design procedure and database (UCL, 2013a, 2013b), thus considered to be only fully representative of Royal Navy ships and UK MoD practices and data. The database and ship design procedures used by other countries and navies are likely to be different. Moreover, despite the evident capabilities of the UCL JavaScript concept design tool, an important downside was that at the time of writing this thesis, the tool was yet to be capable of analysing certain aspects of naval ship ESSD, such as damage stability. Thus some relevant investigations could not be carried out and the design studies presented must be considered preliminary and not necessarily balanced to the extent usually appropriate to concept naval ship design. Also, the expansion of certain supportability features, such as ship workshop facilities, is likely to result in extra crew being required to operate the larger facilities. However, the whole ship implications on the ship's complement and accommodation requirements, and the resulting crew support facilities were not investigated. Finally, due to the limitations of the UCL (2013b) costing procedures, the cost analyses carried out as part of developing the ship design cases were limited to UPC and did not include proper TLC estimations. 
Therefore, potential TLC savings as a result of better considerations of TLS in ESSD could not be demonstrated.

\subsubsection{Future Work}

To improve the proposed ESSD supportability evaluation approach and tackle some of research limitations listed, the issues discussed below need to be addressed.

\section{a) The Proposed Design for Support Evaluation Approach}

\section{1) Proxy Supportability Indicators (PSI)}

The Manhattan travel distance, used as a component of the indicative measures of travel time between ship machinery spaces, workshop facilities, and NSGSs, should be replaced with more accurate measures. This might be addressed by defining the actual travel path between given spaces (e.g. machinery space to workshop facilities or ERPs) before measuring the distance along that path.

\section{2) The Numerical Scale Used in Pairwise Comparisons}

The numerical scale used to quantify the decision makers' subjective assessments through pairwise comparisons and its effects on the resulting numerical preferences and supportability evaluation results warrants further investigation. Numerical scales other than the Power Scale used in this research (e.g. Integer Scale) should be used to investigate the effects of uneven distribution of weightings on the supportability evaluation results.

\section{3) The Consistency of Pairwise Comparison Matrices}

The effects of various degrees of acceptable inconsistency on the supportability evaluation results should be further investigated. For this research, a standard CR value of 0.1 was used to check the consistency of the comparison matrices produced through pairwise comparisons (see Table 3.4). Departing from this value and allowing the decision makers to be cardinally more inconsistent in their preferences is likely to significantly affect the comparison matrices and consequently the supportability evaluation results, hence further investigation is required. In addition, CR values appropriate to the domain of naval ship design might be estimated through constructing elaborate questionnaires and conducting extensive personal interviews with supportability experts to determine how the 
various aspects of specific naval ship supportability decision making problems compare together.

\section{4) The Method Used for Generating Numerical Preferences}

Further investigation is required regarding the method used for generating the numerical preferences of the decision makers from the comparison matrices produced through pairwise comparisons. Mathematical methods to investigate as potential alternatives to the Eigenvector Method (EVM) used in this research include: the Row Geometric Method (RGM), the normalised columns method, and the least squares method (see Section 3.3.3 Part c).

\section{5) Sensitivity Analyses on the Numerical Preferences}

Sensitivity analyses should be conducted on the numerical preferences of the decision makers (i.e. priority vectors) to investigate how the supportability evaluation results might be affected. If the decision makers are certain about the overall rank order of their numerical preferences but uncertain about the precise numerical values, then sensitivity analyses could be carried out using the exponential method proposed by Hurley (2001). That method implodes or explodes the distribution of the numerical preferences (depending on the exponential input) while avoiding any reversals in the general rank order. However, this particular sensitivity analysis method raises another question regarding how certain are the decision makers regarding the ranking of their own preferences.

\section{6) Maintenance Scenarios}

Considering the development of new maintenance concepts in the face of financial pressures and unpredictable geopolitical situations, the attitude of decision makers towards different maintenance and upkeep policies (e.g. maintenance while underway vs. major refit programmes) need further investigation.

\section{b) Additional Aspects Taken into Account for Maintainability Assessments}

\section{1) Machinery Space Measures of Maintenance Demand}

The figure of unity assumed for the MTBF of all items of machinery equipment (see Table 5.9) should ideally be replaced with real MTBF data. Though this could prove problematic and perhaps even impossible, due to commercial sensitivity 
issues, if obtained, this would produce a more sensible measure of maintenance demand distribution.

\section{2) Measures of Capability of Supportability Features}

The assumed 1:1 link between the size and capability of ship supportability features (e.g. workshop facilities and NSGSs) needs further investigation. Such investigation will probably require a more clear definition of what is meant by the 'capability' of such ship supportability features.

\section{3) Measures of Routeability}

The purely representative routeability measures of ERPs and the assumption that routeability is generally improved by the double sided and athwartships passageway arrangement should be validated against systematically quantified values. This might be done by first defining the actual travel path between given shipboard spaces from where equipment needs to be removed and the ship ERPs while avoiding the 'hard' compartments (e.g. Operations Room, distributed system routeings). The routeability measure of each ERP would then be the sum of the routability measures of the individual spaces along the travel path. Routeability of individual spaces might be based on the following:

- Net area and density of the space;

- Handleability of individual items of equipment inside the space that might need to be displaced to facilitate removal of other equipment (at least partially determined by the weight of the equipment);

- The difficulty of displacing the equipment inside the space depending on the installation method (modularised vs. bespoke and built-in equipment);

- The amount of pipes, cables and material that need to be removed depending on the space's demand for ship distributed services (e.g. Chilled Water (CW), Heat, Ventilation and Air Conditioning (HVAC), High Pressure Air (HPA), electrical power, fuel, lube oil) and the extent of insulation and linings. 


\section{c) Ship Design Aspects}

\section{1) The Investigation of Different Types of Ships}

Other types of combatants (e.g. corvettes, destroyers), ship design styles (e.g. multihull), and ship types (e.g. auxiliaries) should be investigated to improve the validity of the proposed DfS evaluation approach. It may even be proposed to apply beyond naval vessels to cruise ships and large passenger ferries. In addition, the maintainability assessments should be expanded by investigating the effects of incorporating technologies such as Integrated Electric Propulsion (IEP) and corresponding machinery arrangement on the evaluation results.

\section{2) The UCL JavaScript Concept Design Tool}

In order for the supportability evaluation results to be more believable, informative, and implementable, the tool should be further expanded to include important ESSD analyses such as damage stability.

\section{3) Wider Ship Implications of Additional Supportability Enhancements}

A more in depth investigation of the effects of enhancing certain supportability features on the ship design should be carried out. Such effects involve, but are not limited to, extra complement and accommodation requirements and crew support facilities, and may have knock on implications leading to a different machinery fit, and increased fuel consumption for example.

\section{4) Cost Estimation Methods}

To properly demonstrate the value of TLS considerations in ESSD, better TLC estimation models are required. Such models should recognise the fact that many aspects of naval ship supportability are configurationally-driven and be capable of capturing the effects of changes in the ship's internal arrangement and enhanced supportability features on the TLC estimations. 


\section{Chapter 7: Conclusions and Further Work}

The main aim of this research was defined as: to propose and demonstrate a novel, quantitative, and integrated supportability evaluation approach that is compatible with the high level design definitions of the Concept Phase and tackles the issues identified as obstacles to a proper consideration of naval ship Through Life Support (TLS) in the early, crucial phase of design. In general, this aim was met and a number of fundamental conclusions were noted and are described in this final chapter. Despite meeting the overall aim, several issues and limitations emerged during both the development as well as the implementation of the proposed Design for Support (DfS) evaluation approach for Early Stage Ship Design (ESSD). Hence, a number of aspects are seen to need further investigation leading to improving the proposed evaluation approach. The limitations and areas of potential future work are also summarised in this chapter.

In developing the evaluation approach, it was realised that modern warships, including many of their underlying supportability issues (e.g. maintainability and adaptability) are architecturally-constrained. The experience, knowledge, and tools available in UCL Design Research Centre (DRC) regarding architectural naval ship concept design were also fully recognised. Therefore the development of the approach was focused on the architecturally identifiable aspects of Integrated Logistic Support (ILS) and how these might be affected by the choice of ship configuration and its configurationally discernible supportability features. The advantages of the architecturally-orientated UCL DBB approach in ESSD and its JavaScript implementation were seen in the development of the ship design cases to which the proposed approach was applied. For example, fast modelling and the ability to quickly implement major configurational and stylistic design changes, efficiently locate and audit areas and volumes of spaces of interest, and the ability to rapidly generate the high level design information required for the assessments, became clear. It was concluded that a new naval ship design could be described in terms of its weight breakdown and form, in order for the ship designers to be able to demonstrate design completeness and balance, as well as architecturally and following the ship's functional breakdown, in order to better deal with the more complex issues of naval 
ship design (e.g. supportability). Without a proper configurational model, a rational, if incomplete evaluation of naval ship supportability alternatives during ESSD would not be possible.

Structuring the evaluation of naval ship TLS in a top-down manner meant the assessments did not require the highly detailed ship design information that is generally unavailable during ESSD. It simplified the process of defining the Proxy Supportability Indicators (PSI) that were utilised in the absence of direct DfS Key Performance Indicators (KPI) of supportability. It also helped link the indicative supportability metrics to the ship design information that could be obtained through the UCL JavaScript concept design tool. It was therefore concluded that top-down structuring is crucial to enable exploration of ILS issues in the Concept Phase. Moreover, a framework was successfully developed to help quantify and incorporate the decision makers' inconsistent and often conflicting subjective preferences into the supportability evaluation hierarchy. The framework was primarily based on carrying out pairwise comparisons of the hierarchy components and accounted for important issues such as: the distribution of the resultant quantified preferences; the degree of consistency of the decision makers' judgements; and the different mathematical methods that could be used to quantify the decision makers' linguistic preferences. Although no sensitivity analyses were carried out on the numerical preferences used in this research in order to prioritise ship design-related investigations of supportability, the simple framework provided a process for a rational capture and incorporation of the accumulated naval ship design and TLS knowledge. Finally, the additional aspects that were taken into account in applying the DfS evaluation hierarchy to a partial (architecturally dependent) assessment of maintainability of ship machinery spaces, were considered to successfully improve the rationality and (crucially) the sensitivity of the assessment results to variations in ship configuration.

The proposed DfS evaluation approach was crucially integrated with the analysis of the traditional issues of naval ship design in order to produce believable, informative, and implementable results and avoid appearing solely as a supportability indicator. Though certain ship design aspects were not investigated, most importantly damage stability, this was due to the limited number of analytical tools currently associated with the UCL JavaScript tool and not a limitation caused by investigating supportability in ESSD. It was therefore concluded that certain supportability aspects 
could be investigated as part and parcel of the design options exploration in the early phases of design, without obstructing the analysis of the traditional, numerically definable aspects of naval ESSD.

The costing studies undertaken as part of developing the ship design cases were intended to help argue that although early investments in carefully identified ship supportably features are likely to cause the ship Unit Procurement Cost (UPC) to rise, the savings during the ship's lifetime (i.e. Through-Life Cost (TLC)) could be disproportionately larger. However, the UCL (2013b) parametric TLC estimation method was found to be unable to adequately capture how the adopted ship internal arrangement and the supportability features provided might affect supportability aspects, such as ship maintenance, refit, and upgrade. The need for a better understanding of the potential benefits of a rational, if partial (architecturally dependent) evaluation of supportability alternatives at the Concept Phase is therefore evident.

Despite the research limitations discussed in Section 6.3.5, particularly the inability to demonstrate potential TLC savings, the proposed DfS evaluation approach might help tackle the DfS problem to some extent. Thus it could contribute to producing more balanced ship designs by giving some consideration to ILS in a more holistic ESSD process. Being linked to the architecturally-orientated UCL DBB approach to ESSD, the proposed approach allows the effects of factors, such as the ship's overall configuration, the provision of supportability features, and ship design style on the proposed measure supportability performance, to be investigated in the early, formative stages of the design process. These are the stages when the expenditure of design resources are minimal compared to the whole of the programme and the ship design is still flexible and amenable to modifications at manageable costs, unlike the later more detailed stages when design adjustments would be very costly or even impossible. Moreover, rational quantification of those supportability aspects, which are amenable to being influenced by high level architecturally-driven early design choices, would help justify the enhancement of relevant supportability features. It is hoped this would at least mean that these supportability aspects are not treated as secondary issues and vulnerable to cost cutting in the mistaken belief that (generally) tighter designs are cheaper to acquire. 
Despite the considerable number of issues that need further investigation, it is considered that this research has contributed to advancing the extent of what can be usefully assessed in the early, formative stages of the design process of what Graham (US Navy Commander, quoted by Gates and Rusling (1982)) described as 'the most the most complex, diverse and highly integrated of any engineering systems' that is regularly manufactured today. Main areas of future work that were discussed in detail in Section 6.3.6 are summarised here in a suggested order of significance:

- Developing TLC estimation models that recognise the fact that many aspects of naval ship supportability are configurationally-driven and are amenable to variations of the ship's internal arrangement;

- Expanding the UCL JavaScript Concept Design tool to include important ESSD analyses such as damage stability;

- Investigating other types of combatants, ship design styles, ship types, and the effects of different technologies such as Integrated Electric Propulsion (IEP);

- Conducting sensitivity analyses on the numerical preferences of potential decision makers and investigating the effects on the assessment results;

- Exploring the attitude of potential decision makers towards different maintenance and upkeep policies (e.g. maintenance while underway vs. major refit programmes);

- Replacing the Manhattan travel distance with more accurate values based on actual travel path between given shipboard spaces;

- Investigating the numerical scale used in the pairwise comparisons and its effects on the distribution of the weightings and the assessment results;

- Examining the method used to check the consistency of the pairwise comparison matrices and its effects on the resultant numerical preferences;

- Investigating the method used for generating the numerical preferences from the pairwise comparison matrices and its links to the numerical scale and consistency check method;

- Improving the maintainability assessments by obtaining real Mean Time Between Failure (MTBF) figures, investigate the capability measure of the supportability features, and develop systematic ways to quantify the measures of routeability. 


\section{References}

Aldous, L.G., 2015. Ship Operational Efficiency Performance Models and Uncertainty Analysis (Naval Architecture PhD Thesis). University College London, London, UK.

Alexander, A.J., 1988. The Cost and Benefits of Reliability in Military Equipment. RAND Corporation, Santa Monica, California, USA.

Ali, H., 2003. GZ Curves of Warships from Form Parameters (Naval Architecture MSc Thesis). University College London, London, UK.

Allison, G., 2015. HMS Ocean not Decommissioning Early [WWW Document]. UK

Defence Journal. URL https://ukdefencejournal.org.uk/hms-ocean-not/ (accessed 25.8.2016).

Alonso, J.A., Lamata, M.T., 2006. Consistency in the Analytic Hierarchy Process A New Approach. International Journal of Uncertainty 14, 445-459.

Andrews, D.J., 1981. Creative Ship Design. Transactions of RINA 123, 447-471.

Andrews, D.J., 1984. Synthesis in Ship Design (Naval Architecture PhD Thesis). University College London, London, UK.

Andrews, D.J., 1985. An Integrated Approach to Ship Synthesis. Transactions of RINA 128, 73-91.

Andrews, D.J., 1987. Explorations in the Nature of Frigate Preliminary Design, in: Proceedings of the RINA Warship Conference May 1987. RINA, London, UK.

Andrews, D.J., 1992. The Management of Warship Design - The MoD Warship Project Manager's Perspective. Transactions of RINA 134, 1-24.

Andrews, D.J., 1993. Preliminary Warship Design. Transactions of RINA 135, 37-55.

Andrews, D.J., 1998. A Comprehensive Methodology for the Design of Ships (and Other Complex Systems). Proceedings of the Royal Society A 454, 187-211.

Andrews, D.J., 2003a. Marine Design - Requirement Elucidation rather than Requirement Engineering, in: Proceedings of the International Marine Design Conference (IMDC) May 2003. Athens, Greece.

Andrews, D.J., 2003b. A Creative Approach to Ship Architecture. International Journal of Maritime Engineering 145, 229-252.

Andrews, D.J., 2006. Simulation and the Design Building Block Approach in the Design of Ships and Other Complex Systems. Proceedings of the Royal Society A 462, 3407-3433.

Andrews, D.J., 2011. Marine Requirements Elucidation and the Nature of Preliminary Ship Design. International Journal of Maritime Engineering 153, 23-39.

Andrews, D.J., 2012. Art and Science in the Design of Physically Large and Complex Systems. Proceedings of the Royal Society A 468, 891-912.

Andrews, D.J., 2013. The True Nature of Ship Concept Design - And What it Means for the Future Development of CASD, in: Proceedings of the International Conference on Computer and IT Applications in the Maritime Industries (COMPIT) May 2013. COMPIT, Cortona, Italy, pp. 33-50.

Andrews, D.J., 2018. Choosing the Style of a New Design - The Key Ship Design Decision. International Journal of Maritime Engineering 160, 71-90.

Andrews, D.J., Brown, D.K., 1982. Cheap Warships are not Simple, in: Proceedings of the SNAME Symposium 1982. SNAME, New York, USA. 
Andrews, D.J., Burger, D., Zhang, J., 2005. Design for Production Using the Design Building Block Approach. International Journal of Maritime Engineering 147, 18-24.

Andrews, D.J., Casarosa, L., Pawling, R.J., Galea, E., Deere, S., Lawrence, P., 2008. Integrating Personnel Movement Simulation into Preliminary Ship Design. International Journal of Maritime Engineering 150, 19-34.

Andrews, D.J., Cudmore, A.C., Humble, P., Wilson, D., 1996. SUBCON - A New Approach to Submarine Concept Design, in: Proceedings of the RINA Warship Conference June 1996. RINA, London, UK.

Andrews, D.J., Dicks, C.A., 1997. The Building Block Design Methodology Applied to Advanced Naval Ship Design, in: Proceedings of the International Marine Design Conference (IMDC) June 1997. Newcastle, UK.

Andrews, D.J., Greig, A.R., Pawling, R.J., 2004. The Implications of an All Electric Ship Approach on the Configuration of a Warship, in: Proceedings of the International Naval Engineering Conference (INEC) April 2004. IMarEST, Amsterdam, Netherlands.

Andrews, D.J., Hall, H.J., 1995. The Trimaran Frigate - Recent Research and Potential for the Next Generation, in: Proceedings of the International Maritime Defence Expo March 1995. London, UK.

Andrews, D.J., Keane, R.G., Lamb, T., Sen, P., Vassalos, D., 2006. State of the Art Report on Design Methodology, in: Proceedings of the International Marine Design Conference (IMDC) May 2006. Michigan, USA.

Andrews, D.J., Papanikolaou, A., Erichsen, S., Vasudevan, S., 2009. State of the Art Report on Design Methodology, in: Proceedings of the International Marine Design Conference (IMDC) May 2009. Trondheim, Norway.

Andrews, D.J., Pawling, R.J., 2003. SURFCON - A 21st Century Ship Design Tool, in: Proceedings of the International Marine Design Conference (IMDC) May 2003. Athens, Greece.

Andrews, D.J., Pawling, R.J., 2006. Innovative Ship Design for High Speed Adaptable Littoral Warfare, in: Proceedings of the RINA Warship Conference June 2006. RINA, London, UK.

Andrews, D.J., Pawling, R.J., 2007. Concept Studies for a Joint Support Ship, in: Proceedings of the RINA Warship Conference June 2007. RINA, Bath, UK.

Andrews, D.J., Pawling, R.J., 2008. A Case Study in Preliminary Ship Design. International Journal of Maritime Engineering 150.

Andrews, D.J., Pawling, R.J., 2009. The Impact of Simulation on Preliminary Ship Design, in: Proceedings of the International Marine Design Conference (IMDC) May 2009. Trondheim, Norway.

Andrews, D.J., Percival, V., Pawling, R.J., 2012. Just how Valid is the Ship Design Spiral Given the Existence of Cliffs and Plateaux, in: Proceedings of the International Marine Design Conference (IMDC) May 2012. Glasgow, Scotland, UK, pp. 317-338.

Archer, B., 1979. Design as a Discipline - Whatever Became of Design Methodology. Design Studies 1, 17-20.

Arena, M.V., Blickstein, I., Younossi, O., Grammich, C.A., 2006. Why Has the Cost of Navy Ships Risen? RAND Corporation, Santa Monica, California, USA.

BAE Systems, 2011. Artisan 3-D. BAE Systems, Surrey, UK.

BAE Systems, 2012. Mk 45 Naval Gun System. BAE Systems, Portsmouth, UK. 
BAE Systems, 2015. Type 26 Global Combat Ship [WWW Document]. BAE Systems. URL https://www.baesystems.com/en-uk/product/global-combatship (accessed 31.1.2018).

Baker, R., 1958. Some Problems in the Construction of Warships Today. RCNC, London, UK.

Bennett, J.G., Lamb, T., 1996. Concurrent Engineering Application and Implementation for U.S. Shipbuilding. Journal of Ship Production and Design $12,107-125$.

Bergsma, J., Zalm, M. van der, Pruyn, J., 2016. 3D-Printing and the Maritime Construction Sector, in: Proceedings of High-Performance Marine Vehicles Conference October 2016. HIPER, Cortona, Italy.

Bhatt, A., Arsenie, N.A., 2017. Hybrid Car Technology Adopted in Next Generation M-Frigates. Militaire Spectator 186, 114-124.

Boerum, R.E., Birindelli, J.B., 1985. How Modular Combat Systems Will Enhance Support of Surface Combatants. Naval Engineers Journal 97, 53-66.

Broome, G.W., Nelson, D.W., Tootle, W.D., 1982. The Design of Variable Payload Ships. Naval Engineers Journal 94, 147-178.

Brown, A., Salcedo, J., 2002. Multiple-Objective Optimization in Naval Ship Design, in: Proceedings of ASNE Day April 2002. ASNE, Alexandria, Virginia, USA.

Brown, D.K., 1986. Defining a Warship. Naval Engineers Journal 98, 31-40.

Brown, D.K., 1987. The Architecture of Frigates, in: Proceedings of the RINA Warship Conference May 1987. RINA, London, UK.

Brown, D.K., 1993. Naval Architecture. Naval Engineers Journal 105, 42-50.

Brown, D.K., Andrews, D.J., 1980. The Design of Cheap Warships, in: Proceedings of the International Naval Technology Expo June 1980. Interavia S.A., Rotterdam, Netherlands.

Brown, D.K., Tupper, E.C., 1988. The Naval Architecture of Surface Warships. Transactions of RINA 131, 29-56.

Brown, K.W., 1995. Measuring the Effectiveness of Weapons Systems in Terms of System Attributes (Management MSc Thesis). United States Naval Postgraduate School, Monterey, California, USA.

Brunelli, M., 2015. Priority Vector and Consistency, in: Introduction to the Analytic Hierarchy Process. Springer, Berlin, Germany, pp. 17-31.

Buckingham, J., 2016. Geared Electric Propulsion. BMT Defence Services Ltd, Bath, UK.

Cabala, P., 2010. Using the Analytic Hierarchy Process in Evaluating Decision Alternatives. Operations Research and Decisions 1, 5-23.

Caprace, J.D., 2010. Cost Effectiveness and Complexity Assessment in Ship Design within Concurrent Engineering and Design for X Framework (Engineering Sciences PhD Thesis). University of Liège, Liège, Wallonia, Belgium.

Carmel, S.M., 2004. Adaptability in Sea-Base Platform Design. RUSI, London, UK.

Carreyette, J., 1978. Preliminary Ship Cost Estimation. Transactions of RINA 119.

Chalmers, D.W., 1993. Chapter 6: Structural Synthesis, in: Design of Ship's Structures. London HMSO, London, UK, p. 101.

Chambers, M., Makin, A., Mills, P., Ralph, M., Richmond, D., Wheatley, M., Banfield, T., 2009. Providing Anti-Air Warfare Capability: the Type 45 Destroyer. National Audit Office, London, UK.

Coles, J., Greenfield, P., Fisher, A., Davies, S., 2014. Study into the Business of Sustaining Australia's Strategic Collins Class Submarine Capability. Commonwealth of Australia, Canberra, New South Wales, Australia. 
Collins, L., Ward, P., Davidson, G., Fonseca, P., 2012. The Affordable Modular Frigate, in: Proceedings of the RINA Warship Conference June 2012. RINA, Bath, UK.

Cooper, S.L., Burger, D.P.G., McDonald, T.P., 2007. Concepts for a Fleet Tanker: An Exploration into Options and Pricing, in: Proceedings of the RINA International Conference: Military Support Ships 2007. RINA, London, UK.

Cowper, I., 2014. Warship Through Life Support The Future Challenge, in: Proceedings of the International Naval Engineering Conference (INEC) May 2014. IMarEST, Amsterdam, Netherlands.

Crow, A.C.D., 2001. Influences in Surface Naval Ship Design, in: Proceedings of the RINA Warship Conference June 2001. RINA, London, UK.

DeNeufville, R., 1990. Applied System Analysis: Engineering Planning and Technology Management. McGraw-Hill, New York, USA.

Depetro, A., 2015. Rapid Ship Design Evolution Using Computer Algorithms - A Scientific Approach to SEA 1180. BMT Design \& Technology, Melbourne, Victoria, Australia.

Depetro, A., Hoey, R., 2011. Rapid Generation and Optimisation of Ship Compartment Configuration Based on Life Cycle Cost and Operational Effectiveness. BMT Design \& Technology, Melbourne, Victoria, Australia.

Dirksen, G.C., 1996. Consideration of Warship Cost (Naval Architecture and Marine Engineering MSc Thesis). University College London, London, UK.

Doerry, N., 2006. Zonal Ship Design. Naval Engineers Journal 118, 39-51.

Doerry, N.H., 2014. Institutionalizing Modular Adaptable Ship Technologies. Journal of Ship Production and Design 30, 126-141.

Drayton, P., 2014. Improving Cost and Schedule Performance in Naval Marine Maintenance Programmes, in: Proceedings of the International Naval Engineering Conference (INEC) May 2014. IMarEST, Amsterdam, Netherlands.

Drewry, J.T., Jons, O.P., 1975. Modularity - Maximising the Return on the Navy's Investment. Naval Engineers Journal 87, 198-214.

DSMC, 1986. Integrated Logistics Support Guide. Defence Systems Management College, Department of Defence, Fort Belvoir, Virginia, USA.

Elliott, M.A., 2010. Selecting Numerical Scales for Pairwise Comparisons. Reliability Engineering and System Safety 95, 750-763.

Eriksen, J.H., 2001. NATO R\&M Terminology Applicable to ARMPs. NATO, Brussels, Belgium.

Esbati, S., Piperakis, A.S., Pawling, R.J., Andrews, D.J., 2015a. Evaluation of Supportability in the Preliminary Design of Naval Ships, in: Proceedings of the RINA Warship Conference June 2015. RINA, Bath, UK.

Esbati, S., Piperakis, A.S., Pawling, R.J., Andrews, D.J., 2015b. Design for Support in the Initial Design of Naval Combatants, in: Proceedings of the RINA International Conference on Computer Applications in Shipbuilding (ICCAS) September 2015. RINA, Bremen, Germany.

Evans, J.H., 1959. Basic Design Concepts. Naval Engineers Journal 71, 671-678.

Fallon, M., 2015. House of Commons Written Statement - Type 26 Global Combat Ship. UK House of Commons, London, UK.

Fidler, K., Harrison, M., 2013. Skills for the Nation Engineering Undergraduates in the UK. Royal Academy of Engineering, London, UK.

Friedman, N., 2006. The Post-Carrier Generation, in: British Destroyers \& Frigates. Chatham Publishing, London, UK, pp. 285-289. 
Gale, P.A., 1975. Margins in Naval Surface Ship Design. Naval Engineers Journal 87, 174-188.

Gale, P.A., 2003. Chapter 5: The Ship Design Process, in: Lamb, T. (Ed.), Ship Design and Construction. SNAME, Jersey City, New Jersey, USA.

Garzke, W.H., Kerr, G., 1985. A New Warship Design Strategy - A Perspective. SNAME Transactions 93, 129-146.

Gaspar, H.M., 2016. Introduction to JavaScript Applied to Design and Engineering. Norwegian University of Science and Technology, Trondheim, Norway.

Gaspar, H.M., Brett, P.O., Ebrahimi, A., Keane, A., 2014. Data-Driven Documents (D3) applied to Conceptual Ship Design Knowledge, in: Proceedings of the International Conference on Computer and IT Applications in the Maritime Industries (COMPIT) May 2014. Redworth. UK, pp. 569-583.

Gates, P.J., 1985. Cellularity: An Advanced Weapon Electronics Integration Technique. Transactions of RINA 105-120.

Gates, P.J., 2005. Surface Warships: An Introduction to Design Principles. Brassey's (UK) Ltd., London, UK.

Gates, P.J., 2014. Type 45 Destroyer Owner's Workshop Manual. Haynes Publishing, UK.

Gates, P.J., Rusling, S.C., 1982. The Impact of Weapons Electronics on Surface Warship Design. Transactions of RINA 124, 341-355.

Gerdemann, G., Kuramochi, N.F., Mooshage, O., Puppe, D., Rudius, M., 2012. A Comprehensive Approach for the Planning of Future Naval Vessels, in: Proceedings of the RINA Warship Conference June 2012. RINA, Bath, UK.

Gillespie, J., Singer, D.J., 2012. Helping Bridge the Gap Between Space-Only Arrangements and Distributed System Layouts, in: Proceedings of ASNE Day February 2002. ASNE, Arlington, Virginia, USA.

Glanville, J., 2010. Capability Analysis of Modular Multi-Role Warships for Australia, in: Proceedings of the Pacific January 2010. IEEE, Sydney, Australia.

Golenko-Ginzburg, D., 1988. On the Distribution of Activity Time in PERT. Journal of the Operational Research Society 39, 767-771.

Goossens, I.L., 1992. What's Driving the Hydrodynamic Ship Design?, in: Proceedings of MARIN Workshops on Advanced Vessels, Station Keeping, Propulsor-Hull Interaction, and Nautical Simulators August 1992. Elsevier Ltd., Wageningen, Netherlands.

Gouré, D., 2006. Modularity, the Littoral Combat Ship and the Future of the United States Navy. Lexington Institute, Arlington, Virginia, USA.

Green, J.P., Johnson, B.W., 2002. Towards a Theory of Measures of Effectiveness, in: Proceedings of Command and Control Research and Technology Symposium June 2002. ICCI, Monterey, California, USA.

Green, M.W., 1993. Logistic Support Analysis - Lessons to be Learned. Naval Engineers Journal 105, 137-145.

Gregor, J.A., 2003. Real Options for Naval Ship Design and Acquisition A Method for Valuing Flexibility under Uncertainty (Naval Architecture and Marine Engineering MSc Thesis). Massachusetts Institute of Technology, Cambridge, Massachusetts, USA.

Griethuysen, W.J., 1992. On the Variety of Monohull War Ship Geometry. Transactions of RINA 134, 267-289. 
Griethuysen, W.J., 1993. On the Choice of Monohull Warship Geometry - A Technique for Optimizing Hull Forms for Seakeeping, High Speed Resistance and Fuel Consumption Performance. Transactions of RINA 136, 57-78.

Hawke, M.B., 1988. Warship Design for Economic Procurement. Naval Engineers Journal 31, 1-12.

Heather, R.G., 1990. Some Preliminary Design Considerations for Fast Displacement Ships and Planing Craft, in: Proceedings of the RINA Warship Conference June 1990. RINA, London, UK.

Hill, C., Jarman, R.S., 2014. Maintaining a Continuous Service with Limited Critical Assets, in: Proceedings of the International Naval Engineering Conference (INEC) May 2014. IMarEST, Amsterdam, Netherlands.

Hockberger, W.A., 1996. Total System Ship Design in a Supersystem Framework. Naval Engineers Journal 108, 147-169.

Holland, L.B., 2017. The Royal Navy's new Frigates and the National Shipbuilding Strategy. UK House of Commons Library, London, UK.

Holtrop, J., 1984. A Statistical Re-Analy sis of Resistance and Propulsion Data. Journal of International Shipbuilding Progress 31, 272-276.

Holtrop, J., Mennen, G.G.J., 1978. A Statistical Power Prediction Method. Journal of International Shipbuilding Progress 25, 253-256.

Holtrop, J., Mennen, G.G.J., 1982. An Approximate Power Prediction Method. Journal of International Shipbuilding Progress 29, 166-170.

Hootman, J.C., 2003. A Military Analysis and Decision Making Framework for Naval Ship Design and Acquisition (Ocean Systems Management MSc Thesis). Massachusetts Institute of Technology, Cambridge, Massachusetts, USA.

Hurley, W.J., 2001. The Analytic Hierarchy Process A Note on an Approach to Sensitivity Which Preserves Rank Order. Computers \& Operations Research 28, 185-188.

Jensen, P.A., 2004. Project Management - Beta Distribution [WWW Document]. Operations Management/Industrial Engineering. URL http://www.me.utexas.edu/ jensen/ORMM/omie/computation/unit/project/be ta.html (accessed 15.5.2015).

John, G.A., Loy, H.M., Clements-Croome, D.J., Fairey, V., Neale, K., 2003. A System's Model for Maintenance Support and Decision Making in Building Services Systems, in: Proceedings of the 2nd International Conference on Innovation in Architecture, Engineering and Construction (AEC) June 2003. Millpress Science Publishers, Loughborough, UK, pp. 705-716.

Jones, A., Kimber, A., 2012. Balancing Survivability, Operability and Cost for a Corvette Design, in: Proceedings of the RINA Warship Conference June 2012. RINA, Bath, UK.

Jones, J.C., 1970. Design Methods: Seeds of Human Futures. John Wiley \& Sons, New York, USA.

Kahneman, D., Tversky, A., 1979. Prospect Theory: An Analysis of Decision under Risk. Econometrica 47, 263-291.

Kålås, S., 2015. Small-Scale Automation in Shipbuilding (Marine Systems Design MSc Thesis). Norwegian University of Science and Technology, Trondheim, Norway.

Keeney, R.L., 1981. Measurement Scales for Quantifying Attributes. Behavioral Science 26, 29-36.

Keeney, R.L., 1982. Decision Analysis: An Overview. Operations Research 30, 803838. 
Keeney, R.L., 1984. Ethics, Decision Analysis, and Public Risk. Risk Analysis 4, $117-$ 129.

Keeney, R.L., 1996. Value-focused Thinking Identifying Decision Opportunities and Creating Alternatives. Operations Research 92, 537-549.

Keeney, R.L., Raiffa, H., 1993. Decision Analysis with Multiple Conflicting Objectives, Preferences and Value Tradeoffs. New York, USA.

Kerr, R.M., 1983. The Role of Operational Research in Organizational Decision Making. European Journal of Operational Research 14, 270-278.

Kettle, P.H.V., Figg, K.A., 1998. Commercial off the Shelf Equipment, Will it Survive. Naval Engineers Journal 38, 74-85.

Kimber, A., Giles, W., Dinham-Peren, T., 2008. The Globally Deployable Minor Warship - A Conceptualisation of Future Solutions, in: Proceedings of the International Naval Engineering Conference (INEC) April 2008. IMAREST, Hamburg, Germany.

Knight, J.T., 2014. A Prospect Theory-Based Real Option Analogy for Evaluating Flexible Systems and Architectures in Naval Ship Design (Naval Architecture and Marine Engineering PhD Thesis). University of Michigan, Ann Arbor, Michigan, USA.

Knight, J.T., Singer, D.J., 2014. Applying Real Options Analysis to Naval Ship Design, in: Proceedings of ASNE Day April 2014. ASNE, Arlington, Virginia, USA.

Larrinaga, N. de, 2015. First Parts Ordered for UK's New Type 26 Frigates [WWW Document]. IHS Jane's 360. URL http://www.janes.com/article/53476/firstparts-ordered-for-uk-s-new-type-26-frigates (accessed 30.5.2016).

Leite, M.J., Mensh, D.R., 1999. Definition of Evaluation Criteria for System Development Acquisition Modeling and Simulation. Naval Engineers Journal 111, 55-64.

Lockheed Martin, 2014. MK41 VLS. Lockheed Martin, Bethesda, Maryland, USA.

Lootsma, F.A., 1993. Scale Sensitivity in the Multiplicative AHP and SMART. Journal of Multi-Criteria Decision Analysis 2, 87-110.

Malcolm, D.G., Roseboom, J.H., Clark, C.E., Fazar, W., 1959. Application of a Technique for Research and Development Program Evaluation. Journal of the Operational Research Society 7, 646-669.

Malone, M.L., Trost, C.S., Firestone, C., Mullen, T., Krebs, W.K., 2014. Maximising Value Across the Lifecycle of Long Lived Capital Intensive Assets. Naval Engineers Journal 126, 67-79.

Manley, D., 2012. The Falklands Conflict 30 Years On - Lessons on Ship Design for the Modern Era, in: Proceedings of DE\&S 2012 Marine Engineering Conference (MEC). DE\&S, Abbey Wood, Bristol, UK.

McCune, B., Urban, D.L., Grace, J.B., 2002. Distance Measures, in: Analysis of Ecological Communities. Mjm Software Design, Edinburgh, UK.

Mercier, A.G., Nunnally, R.S., 1965. The Critical Path Method: Its Fundamentals (Naval Management MSc Thesis). United States Naval Postgraduate School, Monterey, California, USA.

Molland, A.F., Turnock, S.R., Hudson, D.A., 2011. Ship Resistance and Propulsion: Practical Estimation of Ship Propulsive Power, Second. ed. Cambridge University Press, New York, USA.

Moss, N., 2007. Small But Able Frigate Fleets, The Shape of the Future Surface Combatant. RUSI, London, UK.

MTU, 2013. MTU Diesel Engines. MTU, Friedrichshafen, Germany. 
Munoz, J.A., Forrest, C.J.M., 2002. Advantages of Software Integration from Initial Design Through to Production Design, in: Proceedings of the RINA International Conference on Computer Applications in Shipbuilding (ICCAS) September 2002. RINA, Malmö, Sweden.

NATO, 2003. Cost Structure and Life Cycle Costs for Military Systems. NATO, Paris, France.

NATO, 2007. Methods and Models for Life Cycle Costing. NATO, Paris, France.

NATO, 2009. Code of Practice for Life Cycle Costing. NATO, Paris, France.

NATO, 2011. NATO Guidance on Integrated Logistics Support for Multinational Armament Programmes. NATO, Brussels, Belgium.

NATO, 2012. NATO Independent Cost Estimating and the Role of Life Cycle Cost Analysis in Managing the Defence Enterprise. NATO, Paris, France.

Nick, E.K., 2008. Fuzzy Optimal Allocation and Arrangement of Spaces in Naval Surface Ship Design (Naval Architecture and Marine Engineering $\mathrm{PhD}$ Thesis). University of Michigan, Ann Arbor, Michigan, USA.

Northrop Grumman, 2012. MQ-8C Fire Scout. Northrop Grumman, Falls Church, Virginia, United States.

Oers, B.J.V., 2011. A Packing Approach for the Early Stage Design of Service Vessels (Naval Architecture PhD Thesis). Delft University of Technology, Delft, Netherlands.

Office for National Statistics, 2017. Inflation and Price Indices [WWW Document]. URL https://www.ons.gov.uk/economy/inflationandpriceindices (accessed 5.3.2018).

O’Rourke, R., 2016. Navy Littoral Combat Ship (LCS)/Frigate Program Background and Issues for Congress. Congressional Research Service, Washington, D.C., USA.

Page, J., 2011. Flexibility in Early Stage Design of US Navy Ships (Engineering and Management MSc Thesis). Massachusetts Institute of Technology, Cambridge, Massachusetts, USA.

Parsons, S., Wooldridge, M., 2002. Game Theory and Decision Theory in Multi-Agent Systems. Autonomous Agents and Multi-Agent Systems 5, 243-254.

Pawling, R.J., Andrews, D.J., 2010. Three Innovative OPV Designs Incorporating a Modular Payload for UXVs, in: Proceedings of the RINA Warship Conference June 2010. RINA, London, UK.

Pawling, R.J., Andrews, D.J., 2013. Large Unmanned Vehicles and the Minor War Vessel, in: Proceedings of the RINA Warship Conference June 2013. RINA, Bath, UK.

Pawling, R.J., Andrews, D.J., Piks, R., Singer, D., Duchateau, E., Hopman, H., 2013. An Integrated Approach to Style Definition in Early Stage Design, in: Proceedings of International Conference on Computer and IT Applications in the Maritime Industries (COMPIT) May 2013. COMPIT, Cortona, Italy, pp. $248-263$.

Pawling, R.J., Kouriampalis, N., Esbati, S., Bradbeer, N., Andrews, D.J., 2017a. Expanding the Scope of Early Stage Computer Aided Ship Design, in: Proceedings of the International Conference on Computer and IT Applications in the Maritime Industries (COMPIT) May 2017. Cardiff, UK, pp. 362-376.

Pawling, R.J., Percival, V., Andrews, D.J., 2017b. A Study into the Validity of the Ship Design Spiral in Early Stage Ship Design. Journal of Ship Production and Design 33, 81-100. 
Pawling, R.J., Piperakis, A.S., Andrews, D.J., 2015. Developing Architecturally Oriented Concept Ship Design Tools For Research and Education, in: Proceedings of the International Marine Design Conference (IMDC) May 2015. Tokyo, Japan.

Piperakis, A.S., 2014. An Integrated Approach to Naval Ship Survivability in Preliminary Ship Design (Naval Architecture PhD Thesis). University College London, London, UK.

Piperakis, A.S., Andrews, D.J., 2014. A Comprehensive Approach to Survivability Assessment in Naval Ship Concept Design. International Journal of Maritime Engineering 156, 333-352.

Price, G., Wilson, D., Rottier, P., Kyte, P., 2014. UK Type 26 Global Combat Ship Support Solution Decision Point Project, in: Proceedings of the International Naval Engineering Conference (INEC) May 2014. IMarEST, Amsterdam, Netherlands.

Price, S., Whitcomb, C.A., 1992. Special Warfare Submarine. Massachusetts Institute of Technology, Cambridge, Massachusetts, USA.

Rawson, K.J., 1973. Towards Economic Warship Acquisition and Ownership. Transactions of RINA 115.

Rawson, K.J., Tupper, E.C., 2001. Stability, in: Basic Ship Theory. ButterworthHeinemann, Oxford, UK.

Read, D., 2007. Experienced Utility - Utility Theory from Jeremy Bentham to Daniel Kahneman. Psychology Press 13, 45-61.

Rigterink, D., Piks, R., Singer, D.J., 2014. The Use of Network Theory to Model Disparate Ship Design Information. International Journal of Naval Architecture and Ocean Engineering 6, 484-495.

Rittel, W.J., Webber, M.M., 1973. Dilemmas in a General Theory of Planning - 1973. Journal of Policy Sciences 4, 155-169.

Rizzo, P.J., 2011. Plan to Reform Support Ship Repair and Management Practices. Commonwealth of Australia, Canberra, New South Wales, Australia.

Rolls Royce, 2015. Rolls-Royce Signs Gas Turbine Supply Contract for UK Royal Navy’s Type 26 Global Combat Ship. Rolls Royce, Dunfermline, Fife, UK.

Rolls Royce, 2016a. MT30 Gas Turbine. Rolls Royce, Dunfermline, Fife, UK.

Rolls Royce, 2016b. Rolls-Royce Signs MTU Diesel Genset Supply Contract for UK Royal Navy's Type 26 Global Combat Ship. Rolls Royce, Dunfermline, Fife, UK.

Rolls Royce, 2017. Rolls Royce Propulsors. Rolls Royce, Dunfermline, Fife, UK.

Rudius, M., 2012. Approaches to Cost Risk Analysis in Naval Shipbuilding, in: Proceedings of the RINA Warship Conference June 2012. RINA, Bath, UK.

Saaty, T.L., 1987. The Analytic Hierarchy Process - What It is and How It is Used. Mathematical Modelling 9, 161-176.

Saaty, T.L., 2003. Decision-making with the AHP Why is the Principal Eigenvector Necessary. European Journal of Operational Research 145, 85-91.

Saaty, T.L., 2008. Decision Making with the Analytic Hierarchy Process. International Journal of Services Sciences 1, 83-98.

Salo, A.A., Hamalainen, R.P., 1997. On the Measurement of Preferences in the Analytic Hierarchy Process. Journal of Multi-Criteria Decision Analysis 6, 309-319.

Sapolsky, H.M., 1972. The Polaris System Development. Harvard University Press.

Saunders, S., Philpott, T., 2013. Jane's Fighting Ships 2013-2014. Jane's Information Group, London, UK. 
Schank, J.F., Savitz, S., Munson, K., Perkinson, B., McGee, J., Sollinger, J.M., 2016. Designing Adaptable Ships - Modularity and Flexibility in Future Ship Designs. RAND Corporation, Santa Monica, California, USA.

SEA, 2015. Torpedo Launch System. SEA, Beckington, Frome, UK.

Shiraishi, S., Obata, T., Daigo, M., 1998. Properties of a Positive Reciprocal Matrix and their Application to AHP. Journal of Operations Research Society of Japan 41, 404-414.

Sorensen, K., 2015. The Danish Experience with Flexible Warships. Odense Maritime Technology, Odense, Denmark.

Sturtevant, G.H., Flitter, L.A., Marshall, H.L., Voth, J.M., 2014. Flexible Ships: Affordable Relevance Over the Lifecycle. Naval Engineers Journal 126, 5366.

Taylor, C., 2010. A Brief Guide to Previous British Defence Reviews. UK House of Commons Library, London, UK.

Tinga, T., 2014. Innovative Predictive Maintenance Concepts to Improve Life Cycle Management, in: Proceedings of the International Naval Engineering Conference (INEC) May 2014. IMarEST, Amsterdam, Netherlands.

Treat, B.R., 1984. Parameter Estimation for the Four Parameter Beta Distribution (Operations Research MSc Thesis). Air Force Institute of Technology, Dayton, Ohio, USA.

Tupper, E.C., 2013. Introduction to Naval Architecture, 5th ed. Butterworth Heinemann, Oxford, UK.

UCL, 2013a. UCL Department of Mechanical Engineering, Naval Architecture and Marine Engineering MSc, Ship Design Procedure.

UCL, 2013b. UCL Department of Mechanical Engineering, Naval Architecture and Marine Engineering MSc, Ship Design Data Book.

UK MoD, 2000a. Requirements for Maintenance Envelopes and Removal Routes. UK MoD, London, UK.

UK MoD, 2000b. Defence Standard 02-109 - Stability Standards For Surface Ships. UK MoD, London, UK.

UK MoD, 2002. The Acquisition Handbook. UK MoD, London, UK.

UK MoD, 2010. Defence Standard 02-163 - Classification for Weight Groups for Surface Ships. UK MoD, London, UK.

UK MoD, 2011. DEFSTAN 00-600 Integrated Logistic Support. UK MoD, London, UK.

UK MoD, 2013a. JSP 886 DLS Chain Manual - Integrated Logistic Support Integrated Logistic Support Policy (Short Version). UK MoD, London, UK.

UK MoD, 2013b. JSP 886 DLS Chain Manual Supportability Engineering - Integrated Logistic Support Management (Master Version). UK MoD, London, UK.

UK MoD, 2015a. SDSR 2015 Defence Fact Sheets. UK MoD, London, UK.

UK MoD, 2015b. Future Navy Vision - The Royal Navy Today, Tomorrow and Towards 2025. UK MoD, London, UK.

UK NEST, 2013. The Naval Engineering Workforce. UK NEST, London, UK.

Ultra Electronics, 2014a. ALFEA Sonobuoy. Ultra Electronics, London, UK.

Ultra Electronics, 2014b. HIDAR Sonobuoy. Ultra Electronics, London, UK.

US Department of Defence, 2011. Department of Defense Reliability Centred Maintenance Manual. US Department of Defence, Fort Belvoir, Virginia, USA.

US Government Accountability Office, 2010. Depot Maintenance - Improved Strategic Planning Needed to Ensure that Navy Depots can Meet Future 
Maintenance Requirements (No. GAO-10-585). US GAO, Washington, D.C., USA.

Usher, P.J., Dorey, A.L., 1982. A Family of Warships. Transactions of RINA 124, 85112.

US Office of Aerospace Studies, 2013. Analysis of Alternatives (AOA) Handbook A Practical Guide to the Analysis of Alternatives. US Office of Aerospace Studies, Albuquerque, New Mexico, USA.

Vasilakos, J., Devries, R., Tompkins, K.T., 2000. Total Ship Open Systems Architecture. Naval Engineers Journal 112, 59-77.

Wang, T., Neufville, R., 2005. Real Options “in" Projects, in: Proceedings of the Real Options Annual International Conference June 2005. Paris, France.

Watson, D.G.M., 1998. Practical Ship Design (Elsevier Ocean Engineering Book Series). Elsevier Ltd, Oxford, UK.

Web Finance, 2016. Proxy Indicator [WWW Document]. Business Dictionary. URL http://www.businessdictionary.com/definition/proxy-indicator.html (accessed 19.6.2016).

Whitcomb, C.A., 1998. Naval Ship Design Philosophy Implementation. Naval Engineers Journal 110, 49-63.

Whitwam, D.F., Watty, A.J., 1978. Modernising the Leander Class Frigates. Transactions of RINA 37-52.

Williams, C.G., 1985. A Note on the Analysis of Subjective Judgment Matrices. Journal of Mathematical Psychology 29, 387-405.

Xu, Y., Wang, H., 2013. Eigenvector Method, Consistency Test and Inconsistency Repairing for an Incomplete Fuzzy Preference. Applied Mathematical Modelling 37, 5171-5183. 


\section{Appendices}

\section{Appendix 1 A Review of the State of the Art in Design for Support for Naval Ships}
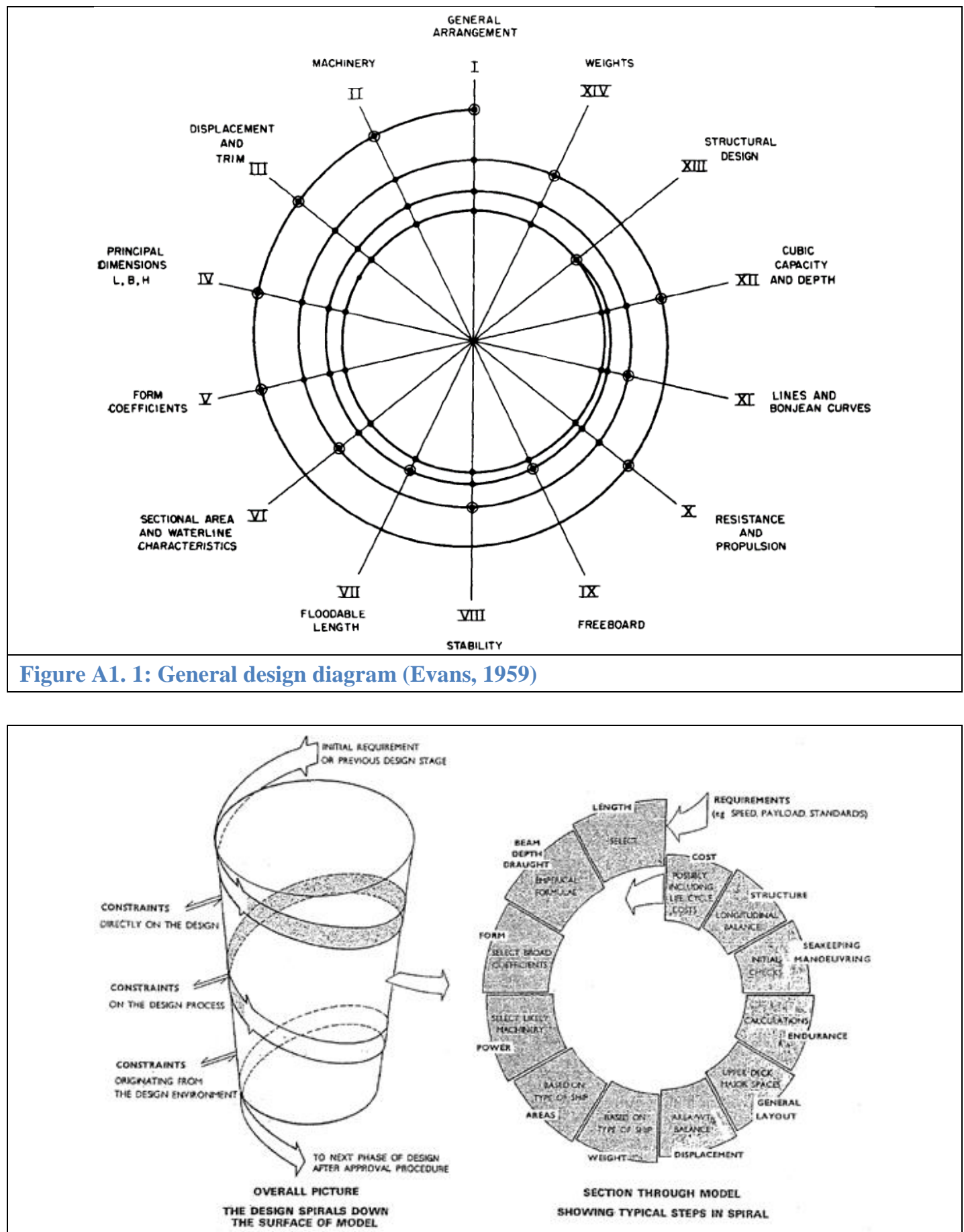

Figure A1. 2: Overall model of the ship design process and section through the model showing typical steps in the spiral (Andrews, 1981) 


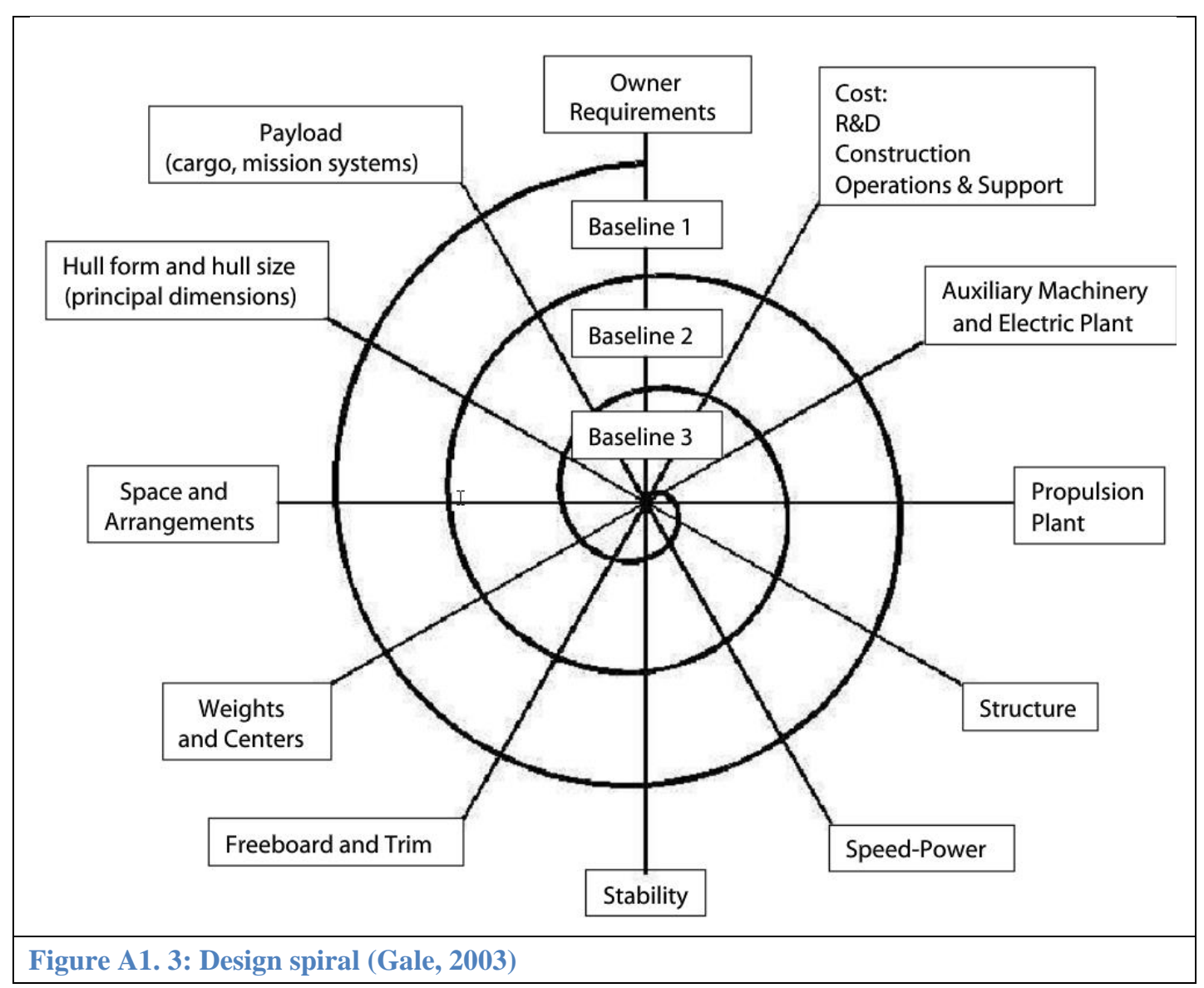




\begin{tabular}{|c|c|c|c|c|c|c|c|c|c|c|}
\hline \multicolumn{11}{|c|}{$\mathrm{S}^{4}$ plus Combat System Characteristics } \\
\hline \multicolumn{5}{|c|}{ Gross Ship Characteristics (GSC) to select } & \multicolumn{2}{|c|}{ Ship's payload to select } & \multicolumn{4}{|c|}{ Naval standards to meet } \\
\hline \multirow{2}{*}{\multicolumn{2}{|c|}{ Speed \& endurance }} & \multirow{2}{*}{\multicolumn{3}{|c|}{ Seakeeping }} & \multirow{2}{*}{\multicolumn{2}{|c|}{$\begin{array}{l}\text { Combat System } \\
\text { capabilities and } \\
\text { relevant systems }\end{array}$}} & \multicolumn{2}{|c|}{ Stability } & \multicolumn{2}{|c|}{ (structural) Strength } \\
\hline & & & & & & & \multicolumn{2}{|c|}{ NES 109} & \multicolumn{2}{|c|}{$\begin{array}{l}\text { Lloyd's rules and } \\
\text { regulations for the } \\
\text { classification of naval } \\
\text { ships }\end{array}$} \\
\hline \multicolumn{11}{|c|}{ Relevant ship design aspects to consider } \\
\hline $\begin{array}{c}\text { Max } \\
\text { speed }\end{array}$ & $\begin{array}{c}\text { Cruise } \\
\text { speed and } \\
\text { endurance }\end{array}$ & $\begin{array}{l}\text { Mission } \\
\text { (naval \& } \\
\text { support) }\end{array}$ & $\begin{array}{c}\text { Environment } \\
\text { (wind \& } \\
\text { wave) }\end{array}$ & $\begin{array}{c}\text { Performance } \\
\text { criteria }\end{array}$ & $\begin{array}{c}\text { Primary } \\
\text { roles }\end{array}$ & $\begin{array}{c}\text { Secondary } \\
\text { roles }\end{array}$ & Intact & Damage & $\begin{array}{l}\text { Extreme } \\
\text { loading }\end{array}$ & $\begin{array}{l}\text { Fatigue } \\
\text { loading }\end{array}$ \\
\hline \multicolumn{11}{|c|}{ Corresponding ship design features } \\
\hline \multicolumn{2}{|c|}{$\begin{array}{ll}\text { - } & \text { Propulsion plant } \\
\text { - } & \text { size } \\
\text { - } & \text { Pullfopulsor type } \\
\text { - } & \text { Appendages } \\
\text { - } & \text { Fuel tankage }\end{array}$} & \multicolumn{3}{|c|}{$\begin{array}{ll}\text { - } & \text { Hullform } \\
\text { - } & \text { Appendages } \\
\text { - Sheer line rise } \\
\text { - } & \text { Weight distribution } \\
\text { Equipment \& compartment location }\end{array}$} & \multicolumn{2}{|c|}{ 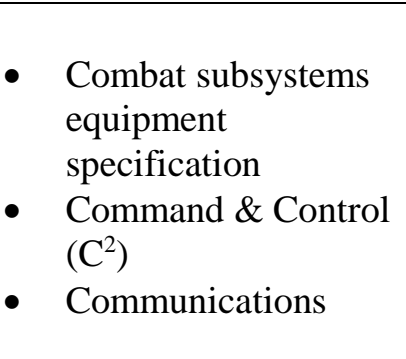 } & \multicolumn{2}{|c|}{$\begin{array}{ll}\text { - } & \text { Hullform } \\
\text { - } & \text { WTB } \\
\text { - } & \text { Zoning } \\
\text { - Hull flare }\end{array}$} & \multicolumn{2}{|c|}{$\begin{array}{ll}\text { - } & \text { Hullform } \\
\text { - } & \text { Weight distribution } \\
& \text { Amidships section } \\
\text { - } & \text { Superstructure } \\
& \text { effectiveness } \\
\text { - } & \text { Structural style \& } \\
\text { scantlings }\end{array}$} \\
\hline
\end{tabular}

Table A1. 1: Taxonomy of $S^{4}$ plus CS characteristics, relevant ship design aspects to consider and corresponding features 


\begin{tabular}{|c|c|c|}
\hline ASSUMPTIONS & STEPS IN PROCESS & SOURCES \\
\hline $\begin{array}{l}\text { Final ship design solution will } \\
\text { not demand major equipment } \\
\text { changes }\end{array}$ & Payload & $\begin{array}{l}\text { Historical data on equipment or } \\
\text { estimates by equipment } \\
\text { designers (weight, space, } \\
\text { services, complement) }\end{array}$ \\
\hline $\begin{array}{l}\text { The level of complement } \\
\text { machinery, services, structure, } \\
\text { etc. will be in line with current } \\
\text { solutions }\end{array}$ & Total internal volume & $\begin{array}{l}\text { Based on past practice from } \\
\text { payload volume }\end{array}$ \\
\hline $\begin{array}{l}\text { The degree of complexity, } \\
\text { structural philosophy, standards } \\
\text { of accommodation, upkeep, } \\
\text { margin philosophy implied }\end{array}$ & $\begin{array}{l}\text { First shot at } \\
\text { displacement }\end{array}$ & $\begin{array}{l}\text { Measures of the density of the } \\
\text { current ships of that category }\end{array}$ \\
\hline $\begin{array}{l}\text { Conventional wisdom on form } \\
\text { parameters, shafting, machinery } \\
\text { redundancy, etc. }\end{array}$ & $\begin{array}{l}\text { Selection of } \\
\text { machinery }\end{array}$ & $\begin{array}{l}\text { Power/speed/displacement plots } \\
\text { Machinery data (volume, mass, } \\
\text { auxiliary power, complement) }\end{array}$ \\
\hline $\begin{array}{l}\text { Level of operation (endurance), } \\
\text { maintenance philosophy, navy } \\
\text { or company manning } \\
\text { philosophy and organizational } \\
\text { structure }\end{array}$ & Complement & $\begin{array}{l}\text { Either overall figure displacement } \\
\text { dependant or given by the sum } \\
\text { of complement for machinery, } \\
\text { payload and remainder }\end{array}$ \\
\hline $\begin{array}{l}\text { Systems operating and upkeep } \\
\text { philosophies, redundancy, etc. }\end{array}$ & $\begin{array}{c}\text { Auxiliary power and } \\
\text { services }\end{array}$ & $\begin{array}{l}\text { Equipment data } \\
\text { Data book of historical demand } \\
\text { related to ship size complement }\end{array}$ \\
\hline $\begin{array}{l}\text { Applicability of hydrodynamics } \\
\text { data (triplet, methodical } \\
\text { series), usage of auxiliaries, } \\
\text { philosophy on tank allocation } \\
\text { (Stability, longitudinal balance, } \\
\text { proximity) }\end{array}$ & Tank volume & $\begin{array}{l}\text { Endurance calculation (hydro- } \\
\text { dynamics data). } \\
\text { Hotel fuel consumption } \\
\text { Fresh water, lub oil, voids }\end{array}$ \\
\hline $\begin{array}{l}\text { Assumed structural design } \\
\text { philosophy and material choice, } \\
\text { implied configuration solution } \\
\text { (\% access), design point, } \\
\text { stability philosophy }\end{array}$ & $\begin{array}{l}\text { Overall displacement } \\
\text { and internal volume }\end{array}$ & $\begin{array}{l}\text { Sum of payload, mcy, services } \\
\text { outfit, structure } \\
\text { Margins on items, growth, 'Board' } \\
\text { Superstructure volume as } \\
\text { proportion }\end{array}$ \\
\hline $\begin{array}{l}\text { Judgement of 'Satisfactory } \\
\text { Balance' }\end{array}$ & $\begin{array}{c}\text { Reiterate until } \\
\text { displacement and volume } \\
\text { balance }\end{array}$ & \\
\hline
\end{tabular}




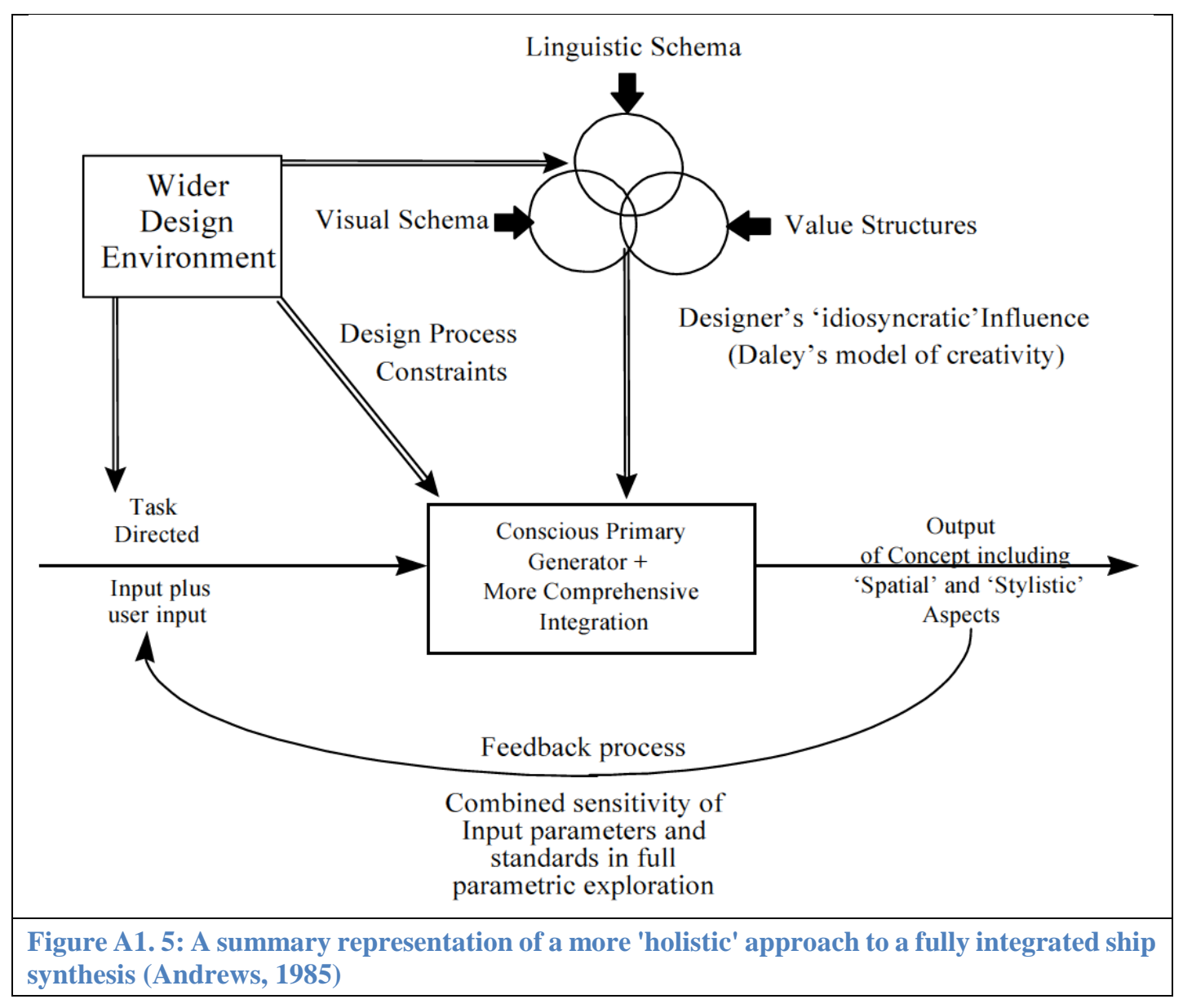




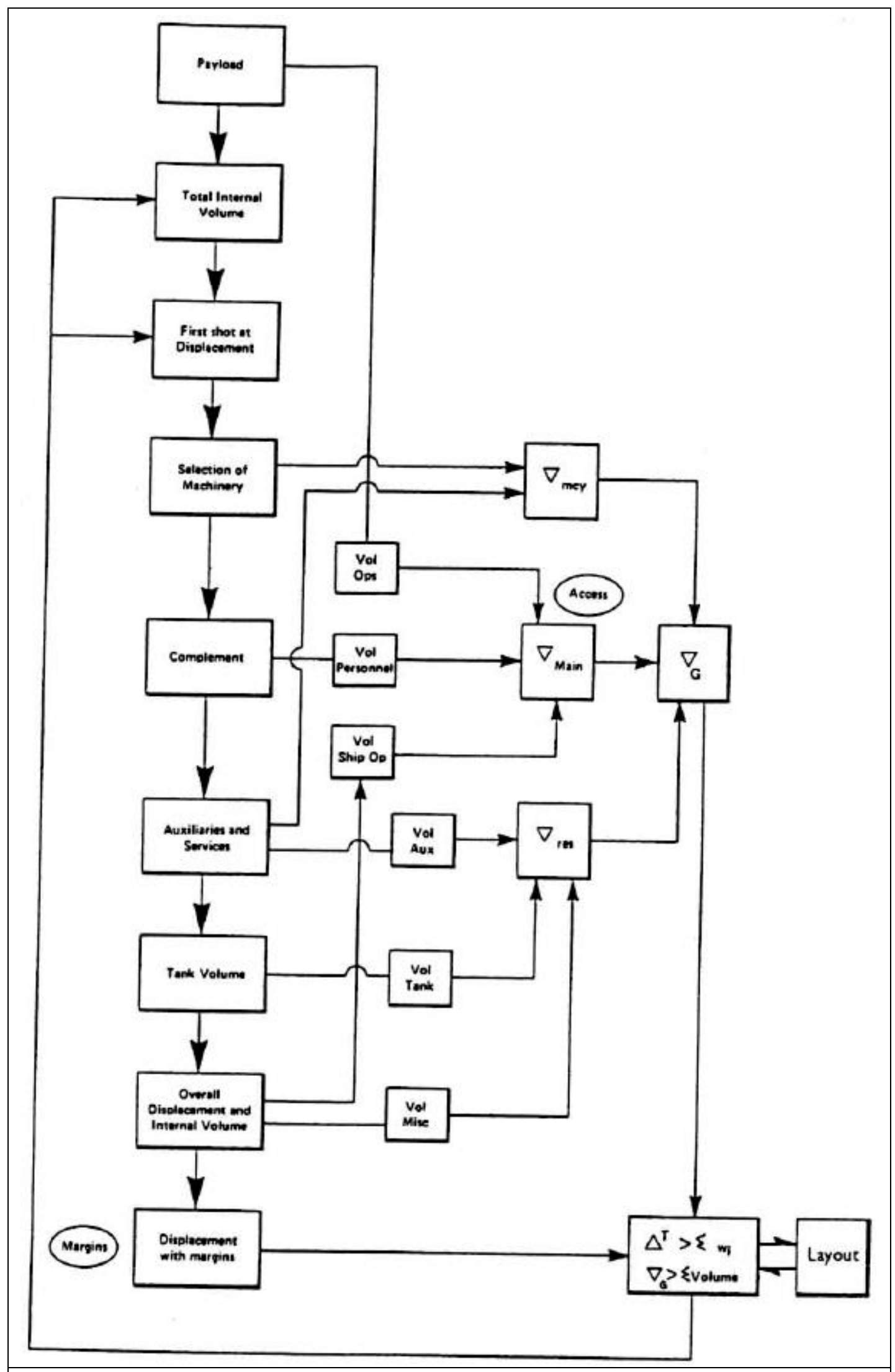

Figure A1. 6: A schematic modification of the initial ship synthesis of Figure A1. 4 with spatial totals which are laid out graphically before reiterating (Andrews, 1985) 


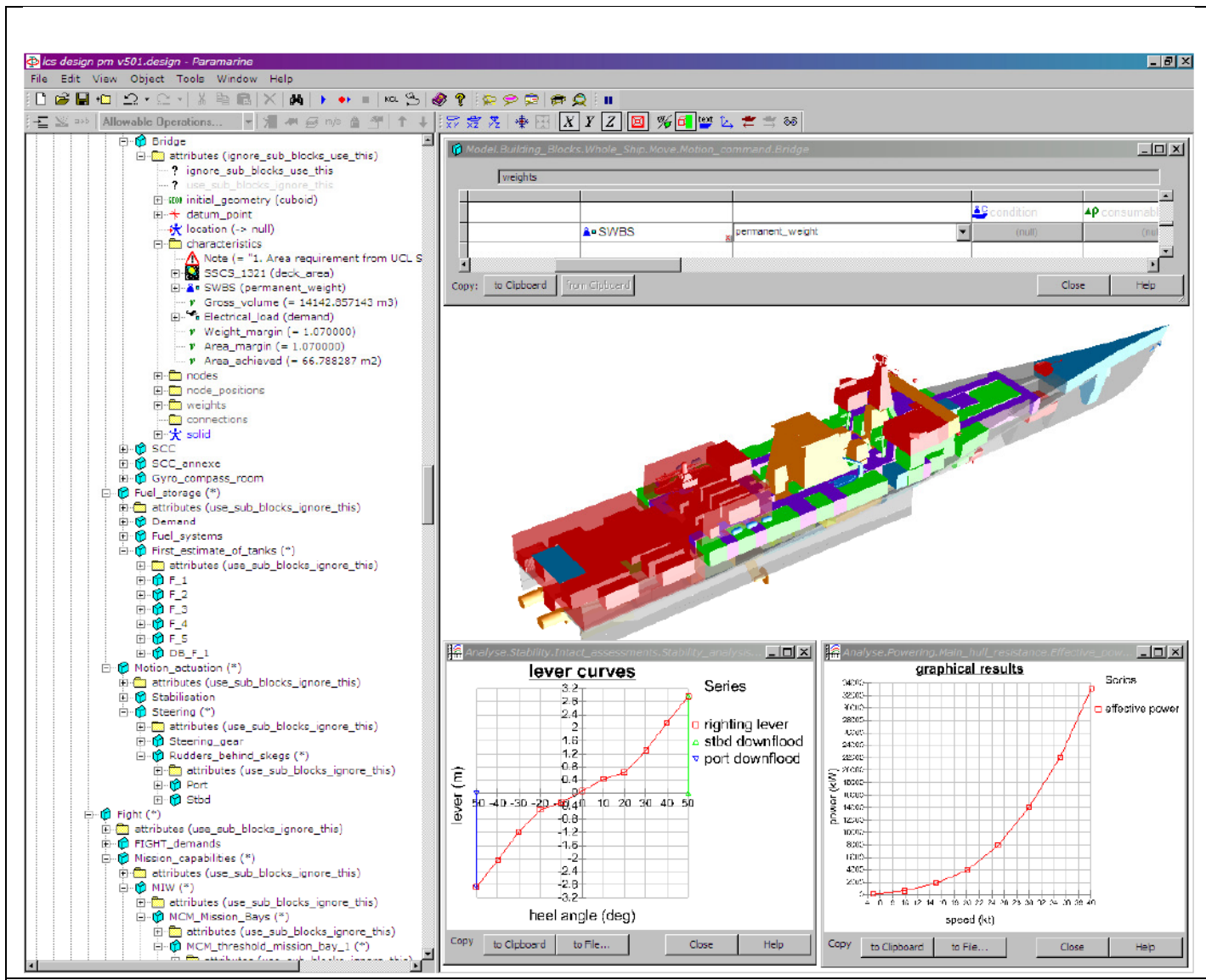

Figure A1. 7: SURFCON representation showing the three panes for tree structure, graphics (Design Building Blocks) and tabular interfaces, with the results of stability and resistance analyses also visible (Andrews and Pawling, 2008) 


\begin{tabular}{|c|c|}
\hline ILS Element & Element Description \\
\hline $\begin{array}{l}\text { Maintenance } \\
\text { Planning }\end{array}$ & $\begin{array}{l}\text { Establish maintenance concepts and requirements and draw a } \\
\text { detailed maintenance plan, involving the identification of } \\
\text { hardware, software, materiel, facilities, personnel, processes and } \\
\text { data needed for the efficient provision of product maintenance. }\end{array}$ \\
\hline $\begin{array}{l}\text { Supply } \\
\text { Support }\end{array}$ & $\begin{array}{l}\text { Process the data resulting from maintenance planning to } \\
\text { determine, for the initial period of years of the contract, the } \\
\text { requirements to acquire, catalogue, receive, implement, store, } \\
\text { transfer, issue and dispose of spares, repair parts, and updates. }\end{array}$ \\
\hline $\begin{array}{l}\text { Technical } \\
\text { Information }\end{array}$ & $\begin{array}{l}\text { Utilise the results of the supply support process to create a } \\
\text { database of the information necessary to operate, maintain, repair, } \\
\text { support and dispose of a product throughout its life, and identify } \\
\text { the standard(s) for the supply of information and data. }\end{array}$ \\
\hline $\begin{array}{l}\text { Support \& Test } \\
\text { Equipment }\end{array}$ & $\begin{array}{l}\text { The identification, planning and ensuring the availability of } \\
\text { equipment (fixed or mobile) required to support the operation and } \\
\text { maintenance of a system, including the associated multiuse end } \\
\text { items, maintenance equipment, tools, metrology and calibration } \\
\text { test equipment and automatic test equipment. }\end{array}$ \\
\hline $\begin{array}{l}\text { Facilities \& } \\
\text { Infrastructure }\end{array}$ & $\begin{array}{l}\text { The permanent and semi-permanent physical infrastructure } \\
\text { required to integrate, inspect, test, store, operate, train, maintain } \\
\text { and dispose of the equipment. }\end{array}$ \\
\hline $\begin{array}{l}\text { Training \& } \\
\text { Training } \\
\text { Equipment }\end{array}$ & $\begin{array}{l}\text { The processes, procedures, techniques, training devices and } \\
\text { equipment, used to train personnel to operate, maintain and } \\
\text { support a system, as determined by the training needs analysis. }\end{array}$ \\
\hline $\begin{array}{l}\text { Packaging, } \\
\text { Handling, } \\
\text { Storage \& } \\
\text { Transportation }\end{array}$ & $\begin{array}{l}\text { The resources, processes, procedures, and design considerations } \\
\text { to ensure that all systems, equipment, and support items are } \\
\text { preserved, packaged, handled, and transported properly, } \\
\text { considering the environmental limitations, product preservation } \\
\text { requirements for short and long term storage, the handling of } \\
\text { items during repair tasks and transport requirements. }\end{array}$ \\
\hline $\begin{array}{l}\text { Human Factors } \\
\text { Integration }\end{array}$ & $\begin{array}{l}\text { The evaluation of human factor considerations across both } \\
\text { operational and support areas of a product in order to ensure that } \\
\text { during product definition and acquisition, the capabilities and } \\
\text { limitations of the military and civilian personnel needed to operate } \\
\text { and maintain the product or facility are properly elucidated. }\end{array}$ \\
\hline $\begin{array}{l}\text { Reliability \& } \\
\text { Maintainability }\end{array}$ & $\begin{array}{l}\text { Properties that must be designed and built into a system during } \\
\text { development and build to ensure high in-service availability. } \\
\text { Mostly centred on the use of RCM to define the maintenance } \\
\text { regime and revise maintenance programmes if necessary. }\end{array}$ \\
\hline $\begin{array}{l}\text { Disposal \& } \\
\text { Termination }\end{array}$ & $\begin{array}{l}\text { Rundown plan and disposal strategy, confirming to safety and } \\
\text { environmental legislations, for the efficient, effective and safe } \\
\text { disposal of a product, together with its spares and consumables. }\end{array}$ \\
\hline
\end{tabular}

Table A1. 2: ILS elements and brief description (UK MoD, 2013a) 


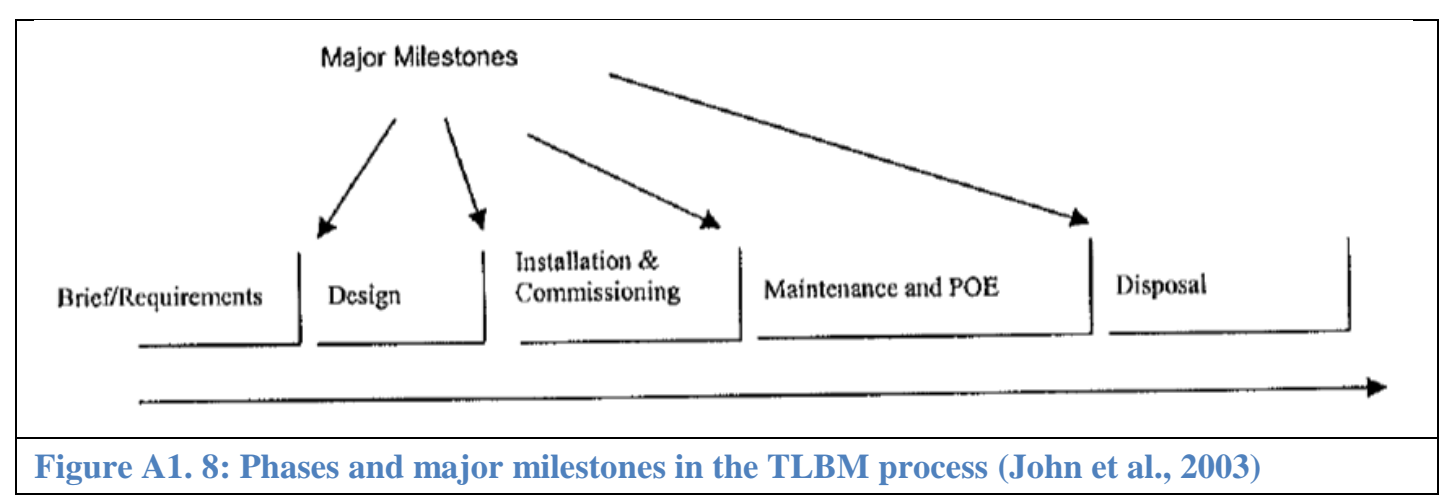


Appendix 2 Development of the Design for Support Evaluation Approach

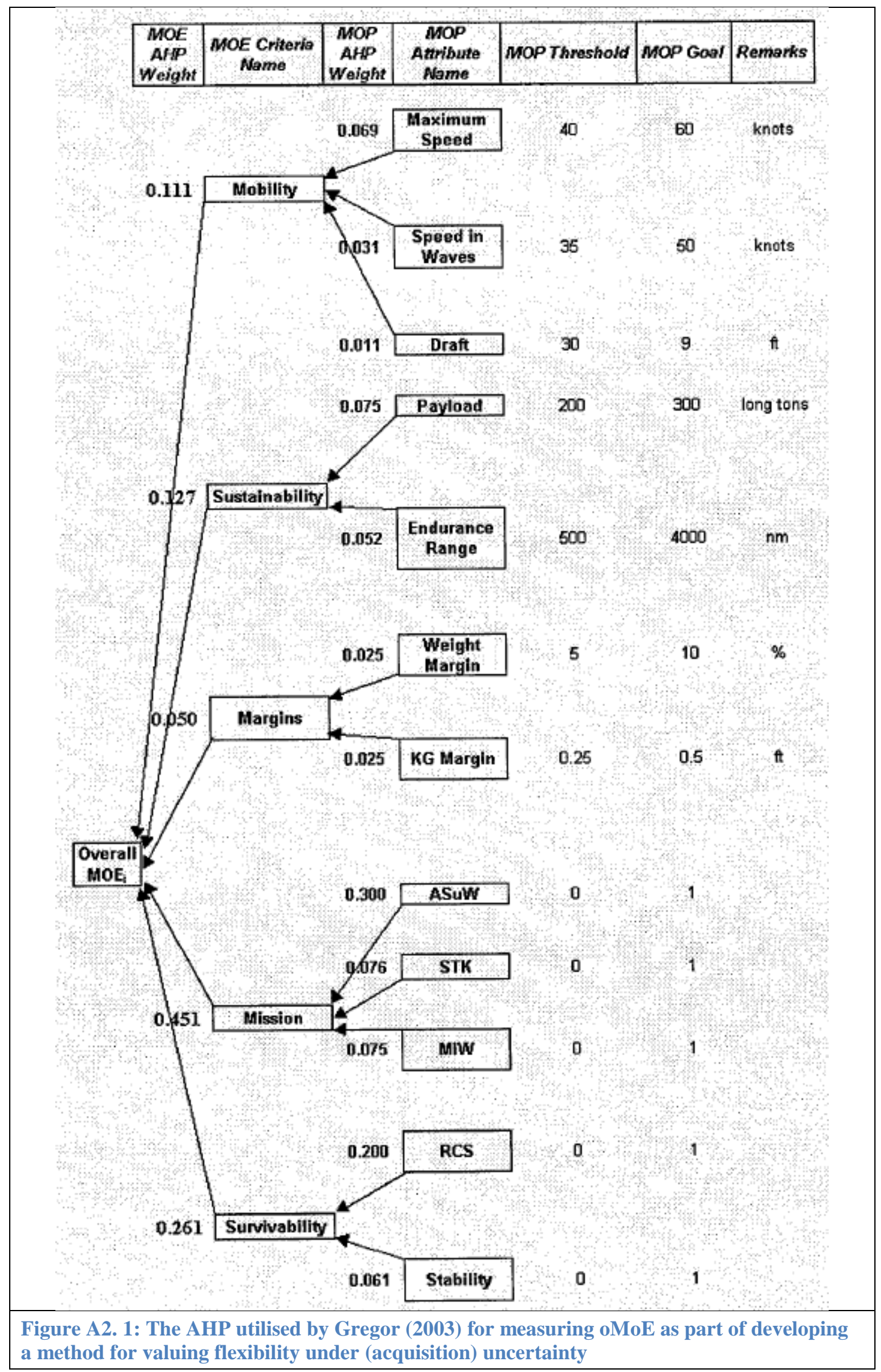




\section{Appendix 3 RINA Warship Conference 2015}

\section{EVALUATION OF SUPPORTABILITY IN THE PRELIMINARY DESIGN OF NAVAL SHIPS}

S Esbati, A S Piperakis, R J Pawling, D J Andrews, Design Research Centre, Marine Research Group, Department of Mechanical Engineering, University College London, UK

\section{SUMMARY}

Failing to include Design for Support features in the early stages of the design of naval vessels can result in certain disadvantages, most notably long and expensive overhauls, reduced through-life adaptability and upgradability, and diminished operability. A genuine implementation of Design for Support features in the early stages of the ship design could be made feasible by the early consideration of the vessel's internal arrangement. This paper describes ongoing work at University College London to develop an analysis tool which provides ship designers with a framework for early stage assessment of various internal arrangements from a through-life support perspective. The research project considers both method and tool development.

\section{NOMENCLATURE}

$\begin{array}{ll}\text { ARM } & \text { Availability, Reliability and Maintainability } \\ \text { ASW } & \text { Anti-Submarine Warfare } \\ \text { CAD } & \text { Computer Aided Design } \\ \text { CASD } & \text { Computer Aided Ship Design } \\ \text { CBM } & \text { Condition Based Monitoring } \\ \text { CPM } & \text { Critical Path Method } \\ \text { DBB } & \text { Design Building Block } \\ \text { DfS } & \text { Design for Support } \\ \text { DRC } & \text { Design Research Centre } \\ \text { ESD } & \text { Early Stage Design } \\ \text { GA } & \text { General Arrangement } \\ \text { GCS } & \text { Global Combat Ship } \\ \text { GUI } & \text { Graphical User Interface } \\ \text { ILS } & \text { Integrated Logistics Support } \\ \text { MoD } & \text { UK Ministry of Defence } \\ \text { OPV } & \text { Offshore Patrol Vessel } \\ \text { PERT } & \text { Programme Evaluation and Review Technique } \\ \text { PSD } & \text { Preliminary Ship Design } \\ \text { RAS } & \text { Replenishment At Sea } \\ \text { TLF } & \text { Through Life Finance } \\ \text { TLS } & \text { Through Life Support } \\ \text { UCL } & \text { University College London }\end{array}$




\section{INTRODUCTION}

Despite the fact that the principles of concurrent engineering were endorsed by the MoD over two decades ago, a genuine implementation of those principles has rarely been attempted in naval ship acquisition. One of the major aspects in meeting concurrent engineering objectives is to be able to incorporate Design for Support (DfS) features in the ship design from the very start of the ship synthesis. Currently the question of naval ship Through Life Support (TLS) is addressed much later than the initial synthesis when the design has been constrained by the need to achieve a set of diverse performance criteria. Failure to consider DfS features at the early, formative stages of the design process will degrade the supportability performance of the ship. This will result in longer and more expensive overhauls, diminished operability and reduced scope for through-life adaptability and upgradeability.

A genuine implementation of DfS features in naval ship Early Stage Design (ESD) can be facilitated by early consideration of the vessel's architecture. However, traditional naval ship design inhibits this as it too often only considers the ship's configuration in any depth after achieving an initial numerically balanced design (achieving speed, seakeeping, stability, strength and mission requirements). Hence there is a clear need to bring consideration of the architectural features to the centre of the initial ship synthesis process [1].

This paper provides a short background to naval ship TLS before describing ongoing work at University College London (UCL) Design Research Centre (DRC) on how the UCL originated Design Building Block (DBB) approach facilitates an architecturally focused investigation of through-life supportability during ESD, namely, while the ship design is still fluid.

\section{NAVAL SHIP THROUGH LIFE SUPPORT}

It is MoD policy that Integrated Logistics Support (ILS) will be applied to all product acquisition [2]. ILS, being a disciplined approach that influences the product design and develops the support solution to optimise supportability and Through Life Finance (TLF), is applicable throughout the whole life of a project. The focus however changes as the project progresses through the Concept, Assessment, Demonstration, Manufacture, In-Service and Disposal phases of the product life-cycle [2]. A summary of the totality of ILS is given in Figure 1. Note that some aspects of ILS, namely technical information, training and training equipment, and facilities, are not directly interrelated with ship architecture. 


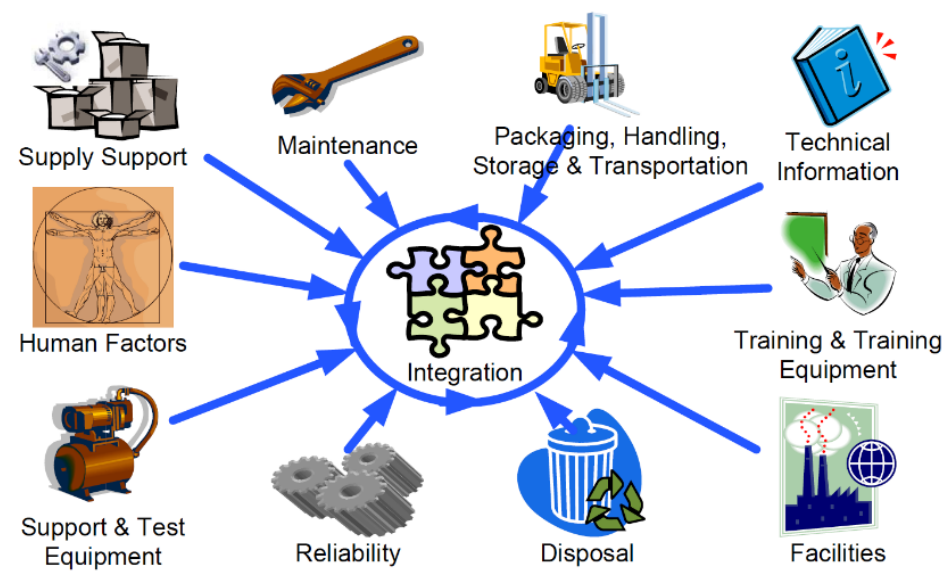

Figure 1: Integrated Logistics Support breakdown [2]

Despite the fact that ILS is official MoD policy, historically the process of naval ship design has been carried out in the middle of an intense debate to reduce costs while attempting to maximise mission capability, thus insufficient attention is given to the full range of capability aspects, namely TLS and availability. It is therefore considered essential to adopt design practices that make TLS more prominent during Preliminary Ship Design (PSD).

\section{PRELIMINARY NAVAL SHIP DESIGN}

\subsection{STYLE IN PRELIMINARY NAVAL SHIP DESIGN}

'Style' was explicitly incorporated as a characteristic of a ship design by Brown and Andrews [3] as the fifth ' $S$ ' in their "S5", the others being Speed, Seakeeping, Stability, and Strength (S4). Incorporating style enables the designer to accommodate uncertainty, in form of 'hard' knowledge (e.g. structural standards) and 'soft' knowledge (e.g. guidance on internal arrangement from a range of perspectives) that can be conceptually connected [4].

An example of a highly stylistic issue is the degree of through-life supportability of naval ships. Style is essentially a cross-cutting issue since one decision explicitly influences a wide range of solution areas [4]. A decision on the level of supportability to be adopted in a given ship design can influence a large range of both overall and detailed design features. Thus such aspects as the access policy, number and routing of removal routes, ship service runs, compartment adjacency, Replenishment At Sea (RAS) features, and Availability, Reliability and Maintainability (ARM) requirements, all strongly influence the resulting overall ship architecture. Hence an early evaluation of supportability requires the ship architecture to be at the centre of the initial synthesis of naval ship design.

\subsection{LIMITATIONS OF TRADITIONAL PRELIMINARY NAVAL SHIP DESIGN}

The traditional approach to naval ship design delays early architectural modelling as it focuses on the aspects of speed, seakeeping, stability, strength and the combat 
system to drive the initial ship sizing [5] and only considers internal arrangement once an initial numerically balanced design has been reached. It postpones the examination of style related issues (such as supportability) to later stages, despite the fact that warships are generally not weight or space limited, but architecturally driven [6]. The outcome is a design which is constrained by the issues traditionally seen to drive the design but with little or no emphasis on style, thus design developments necessitating from a later incorporation of style aspects result in disproportionate extra costs, which are exacerbated the later they are found to be necessary.

\subsection{DESIGN BUILDING BLOCK AS THE PREFERRED PSD APPROACH}

Andrews' [5] proposal to integrate ship architecture with the traditional numerical sizing was followed by the demonstration of 'creative synthesis', presented in a paper entitled 'An Integrated Approach to Ship Synthesis' [7]. From this work, a new approach, namely the Design Building Block approach to PSD [8] was developed and has reached acceptance as a standard PSD approach [9].

The basic idea behind the DBB approach is for the designer to separate the ship's functions and sub-functions into discrete physically realisable elements (Design Building Blocks) and position them appropriately in a putative ship configuration. This then puts architectural features at the centre of the ship synthesis process, alongside the traditional numerically based sequential design process [8]. The architectural approach allows a more thorough exploration of alternative designs in an interactive process of requirement elucidation [10]. It also encourages the investigation of novel solutions and human factors issues [11]. Figure 2 gives a summary of the DBB approach.

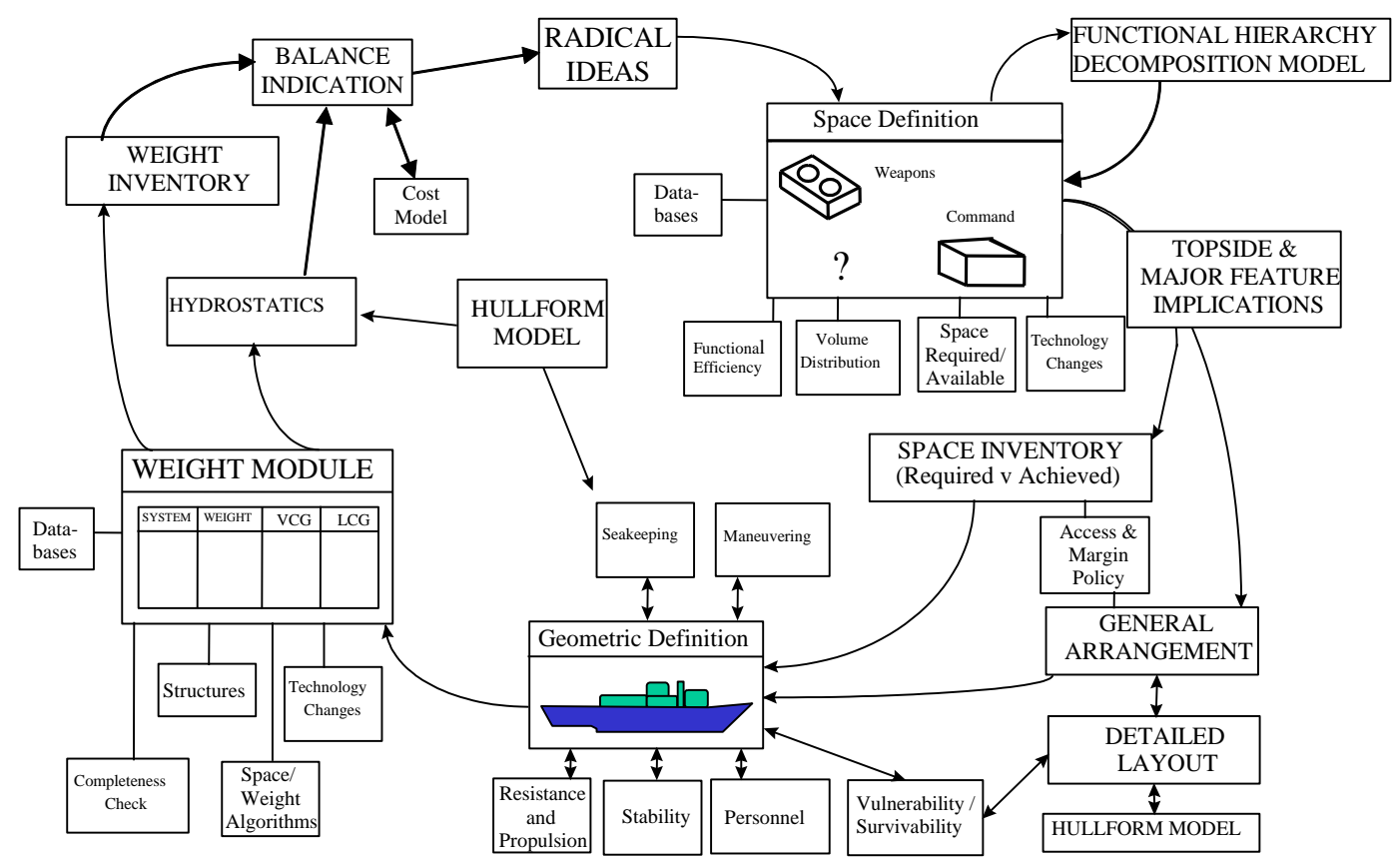

Figure 2: The Design Building Block approach to preliminary ship design applied to surface ships [8] 


\section{NEED FOR A NEW SHIP CONCEPT DESIGN SOFTWARE TOOL AND CURRENT PROGRESS}

The DBB approach to PSD (Section 3.3) was developed and implemented in an industry standard PSD toolset following the rapid developments in computer capabilities. The DBB approach was incorporated as the SURFCON module in QinetiQ GRC's PARAMARINE Computer Aided Ship Design (CASD) suite [12]. SURFCON's proof of concept was then described in [13]. By implementing the DBB approach through the SURFCON module in the PARAMARINE CASD suite, the DBB approach was linked to a commercially established PSD software package [12] and ship designers could then draw on all the naval architectural analytical tools available within PARAMARINE [13].

However, there are several disadvantages regarding the use of sophisticated (fully 3D) Computer Aided Design (CAD) models in early stage ship design research. These include: the need to resort to modelling to an unnecessarily high level of detail; excessive flexibility and precision for most early stage designs; the very high software learning overhead; and the difficulties in connecting design tools with external analysis tools. These issues are comprehensively discussed by Pawling et al [14].

In order to address these issues, a ship design toolset implementing the DBB approach and able to easily integrate new simulation and analysis tools into the design process is being developed at UCL for use in research into PSD [14]. This toolset is being developed using commercial non-CAD software. Specifically, the basic tool is a Microsoft Excel spreadsheet-based layout model using a cellular representation of the arrangeable space on the ship. Excel was chosen because it is widely available, has a pre-existing Graphical User Interface (GUI), permits scripting and a limited degree of programming (via Visual Basic for Applications, VBA) and inherently provides an addressable cellular model - albeit only in 2D.

\subsection{DESCRIPTION OF THE SOFTWARE TOOL}

The basic UCL tool is comprised of three principal components; namely the "Framework", the "DBB Model" and the "GA". This section gives a brief description of these components, including screenshots, with reference to a UCL designed Offshore Patrol Vessel (OPV) carrying unmanned vehicles [15].

\section{1 (a) Framework}

The "Framework", shown in Figure 3, contains common variables used by many DBBs, such as the number of crew and weight margins associated with a given DBB. The framework also contains variables describing the size of the vessel and the locations of decks and main watertight bulkheads. 

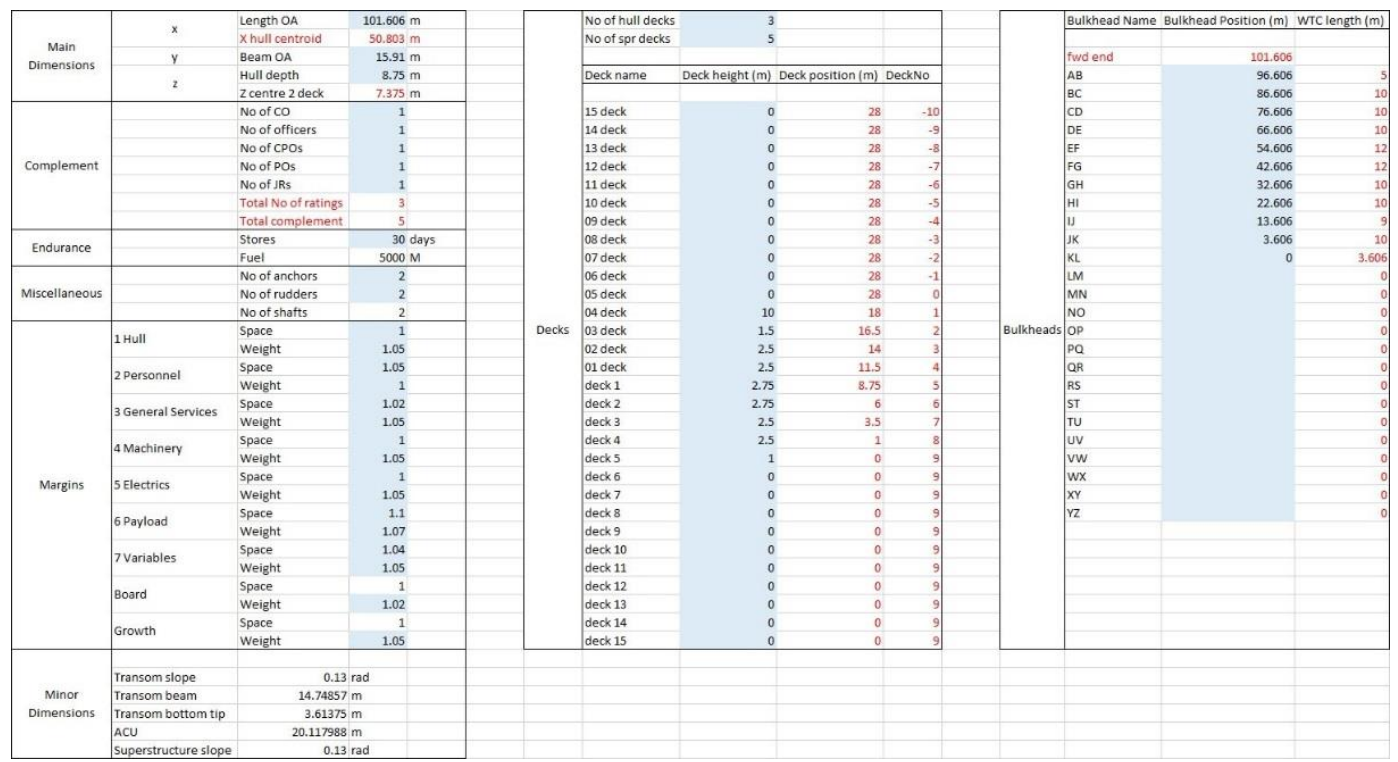

Figure 3: The UCL Ship Design tool's "Framework" component, showing tabular definition of ship dimensions, deck and main watertight bulkhead positions and other variables

\section{1 (b) DBB Model}

The DBBs are defined in a set of Excel sheets forming the "DBB Model", an example of which is shown in Figure 4. The "DBB Model" provides a tabular description of the DBBs, containing columns giving their location, dimensions, weights and any other parameters that may be added by the designer simply by adding additional columns to the spreadsheet. The DBBs may be connected to each other or to overall "Framework" variables. The tabular format has the advantage that all aspects of a DBB can be contained in a single row in the table. The "DBB Model" is structured using the UCL categories of FLOAT, MOVE, FIGHT and INFRASTRUCTURE functional breakdown for naval vessels [13].

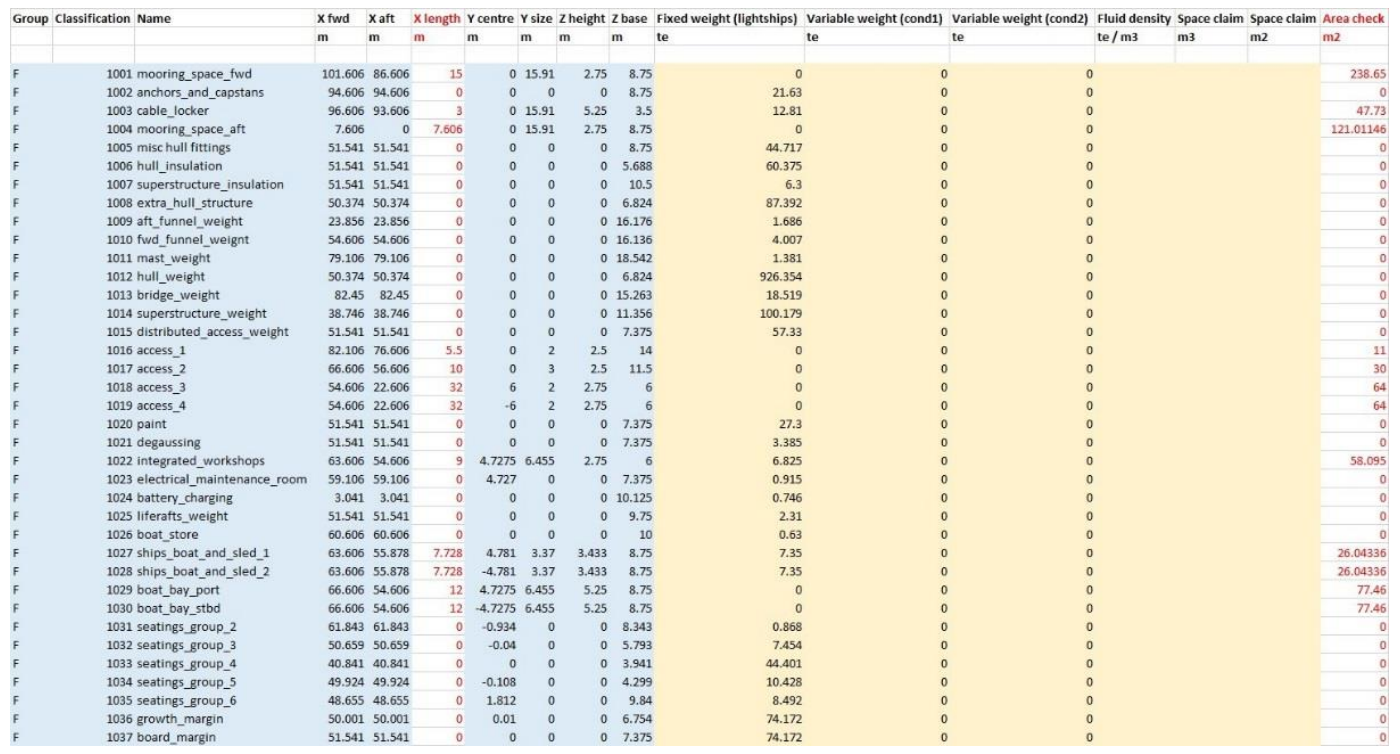

Figure 4: The UCL Ship Design tool's "DBB Model" component, showing tabular definition of the size and weight of the Design Building Blocks for the FLOAT category 


\section{1 (c) General Arrangement}

Finally, the design's General Arrangement (GA) can be visualised in the "GA" component. This is a separate workbook, which contains macros to read the "Framework" and "DBB Model" data and generate a display of the GA. An example GA for a UCL PSD study of a UXV OPV [15] (using the DBB functional breakdown colour code, namely FLOAT - Blue, MOVE - Yellow, FIGHT - Red, INFRASTRUCTURE - Green [13]) is illustrated in Figure 5.

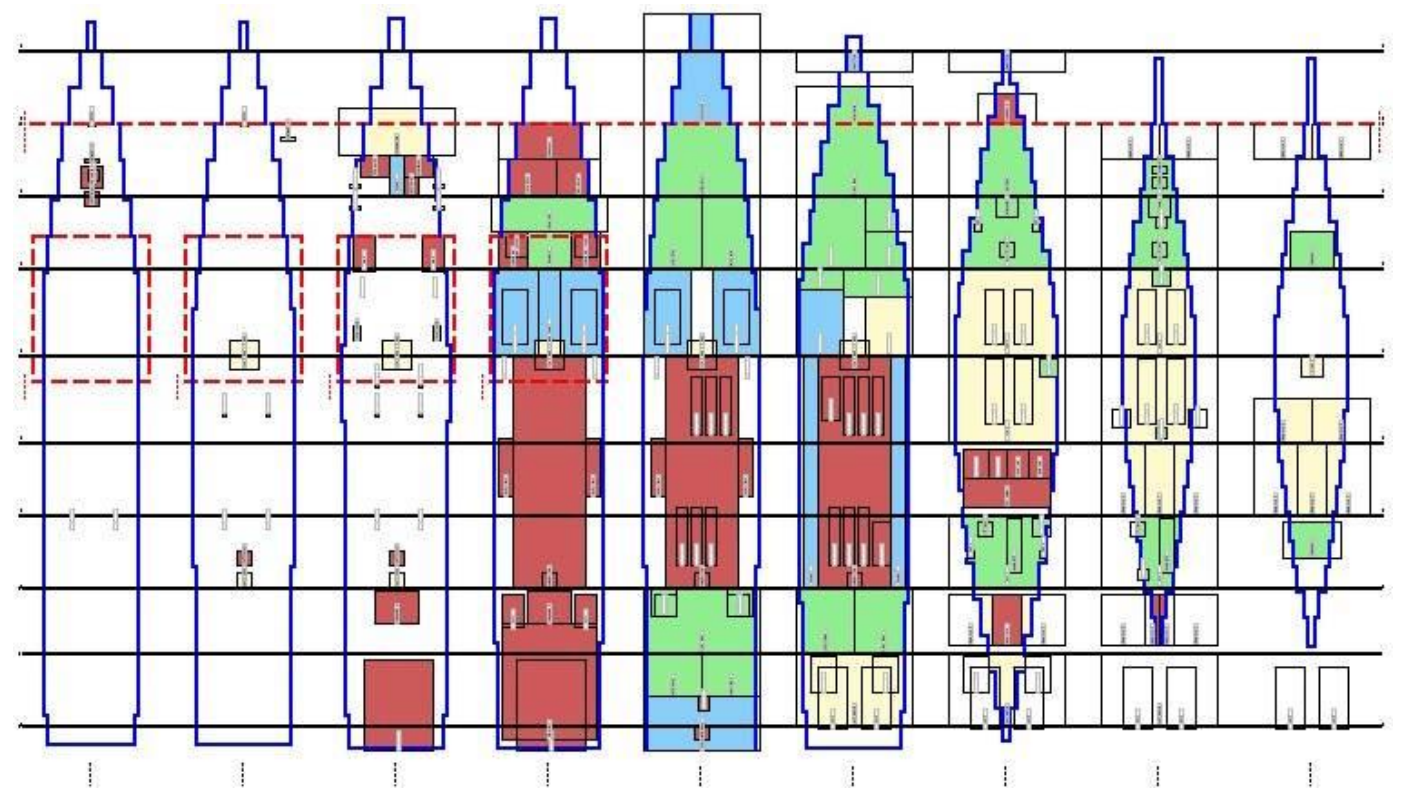

Figure 5: The UCL Ship Design Tool's "GA" component, showing the layout of a typical early stage design model for a UXV OPV study [15]

Figure 5 shows the simple method used to represent DBBs in the design. The cells in the spreadsheet represent a square grid; a group of cells is assigned a numerical identifier and the appropriate DBB colour to identify them as a specific functional group in the GA [13]. Each space also has a visible outline and nametag. Figure 5 also displays the deck outlines (blue line), location of the main watertight bulkheads (black line) and guidance provided to the designer for the location or limits for some spaces and equipment, based on design guidance (red dashed line). Specifically, limits on the location of upper deck weapons and sensors (e.g. due to green sea loading) and the bridge (for visibility and motions) are shown.

\subsection{SOFTWARE TOOL APPLICATIONS}

The UCL developed ship design toolset is being used to develop tools for analysing ship layout and providing this data, either to external tools, or for feedback to designers on the consequences to ship performance. Currently, using network analysis and 2D modelling, the toolset enables the quick extraction of a wide range of spatial properties to be used for the analysis of the configuration; such spatial properties include proximity, adjacency (via network diagrams, Figures 6 and 7), perimeter and surface area. In addition, this simplified arrangement model has several features applicable to 
more sophisticated analysis, which allows such analysis to be undertaken at an earlier stage of the design process without adding a significant modelling burden.

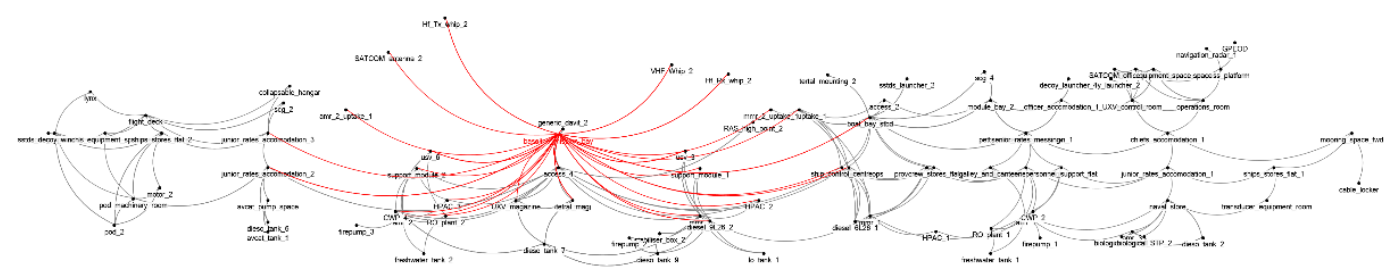

Figure 6: Example of a whole ship adjacency network, shown fixed to the profile view, outputted by the UCL layout tool [14]

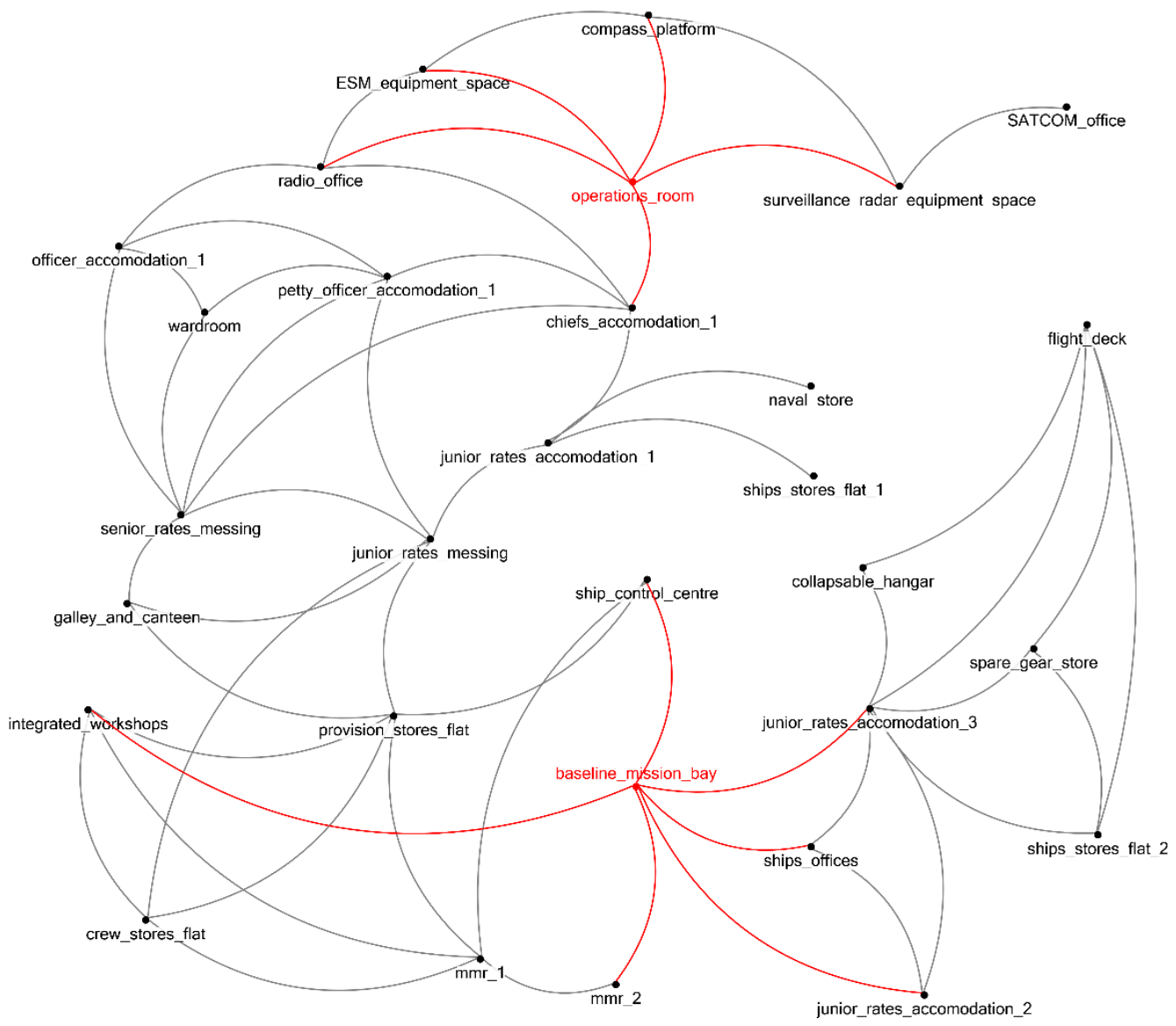

Figure 7: Example of an adjacency network for selected DBBs of the ship design from Figure 6, not to scale, outputted by the UCL layout tool

The generation of layout and connectivity information would allow personnel movement analysis [16] and fire safety assessment [17]. If this flexible and fast model of the general arrangement is combined with a tool that models damage effects from weapons and a network representation of ship systems, it could allow the findings of recent work on recoverability [18] to be incorporated into concept design studies. Similarly, the modelling of systems in concert with the arrangement is important for assessing the producability of designs [19] at the preliminary stages of ship design. 
Thus the UCL ship design toolset will facilitate the incorporation of additional design aspects early in design. This will satisfy the needs of specific projects and be able to interface with dedicated tools for more complex analysis. Given that the toolset satisfies the need for an architectural model necessary to capture DfS drivers, it can enable the evaluation of supportability in the preliminary design of naval ships.

\section{DESIGN FOR SUPPORT EVALUATION APPROACH}

Several discussions held between UCL and BAE Systems (the project's industry sponsor) have resulted in a consensus that the aim of the research project is to develop an approach to evaluate the supportability of a ship design in a rational manner and assist the designer in making more informed early, yet significant, design decisions. Essentially this could provide designers with a framework for an early stage analysis of various internal arrangements for a range of support aspects. The approach should:-

- Demonstrate real benefits in an early stage introduction of DfS features. Naval and government 'customers' are often not convinced by demonstrating that these benefits will only occur after 25 years of service life;

- Not act as an absolute indicator, but should instead indicate whether heading in a particular direction is a good idea or not from a DfS perspective;

- Not only concentrate on DfS features but enable a holistic analysis, thus also assess how S4 (Section 3.1) and combat system aspects might be affected by an early consideration of DfS.

\subsection{EXPLOITING THE RESEARCH AREAS ON DESIGN FOR SUPPORT}

Section 3.1 emphasised the need for an architectural approach in order to examine and evaluate supportability during the initial ship synthesis. Given the extensive experience at UCL on ship concept design and the implementation of the DBB method, the study is concentrating on developing an approach to assess the internal arrangement from a TLS perspective. It is aimed at elucidating how architectural decisions (e.g. access policy, service runs, routing of removal routes, zoning logic) affect the supportability performance of the ship (e.g. ease of refits, ARM, RAS) as well as the traditional ship design drivers (Section 3.2).

In the ship design research group at UCL, it appreciated that various other TLS aspects, such as high level support management and contractual issues, strategic longterm support planning, support documentation and numerous human factors, are also very pertinent to a comprehensive approach to DfS for naval vessels (Section 2). However, the UCL ship design research group is not deeply expert in these topics, where data and information is extensive but not necessarily ship configuration focused. Given the latter is where UCL DRC's expertise lies, the study has focused on the appropriate items in the previous paragraph. 


\subsection{PROPOSED WAY FORWARD}

Investigation of the Critical Path Method (CPM) [20] and its implementation in real projects suggest the requirements listed in Section 5 could be addressed by the approach. CPM has found wide application in solving the general problem of reducing the time required to complete a project that consists of many interrelated tasks. In the past, the technique has been used to reduce the time required for completing the midlife refit of the Royal Navy Leander Class frigates [21].

The critical path of a project is the sequence of activities, within the overall activities network that has the least float, thus determines the project's earliest possible completion time [20]. Accurate determination of the critical path of a project is crucial in ensuring the project is running on time and for examining the effects of any slippage in the critical activities' schedule.

The following sections outline ongoing work on how the CPM, concept naval ship design and architectural modelling could be brought together to address TLS aspects, such as midlife refit and repair programmes.

\section{2 (a) Why Address Midlife Refit and Repair Programmes}

The high cost of dry-docking a ship and the non-financial implications of ship unavailability are amongst the major consequences of a ship's refit and repair project not running to schedule. This could result from the wrong identification of the critical path, as it did during the midlife refit of the Leander Class Frigates. In that project, the critical path was initially identified as being the main cable reeving and the fitting out of the Operations Room. After one year of the refit project however, the critical path was found to be the work in way of the machinery spaces. This resulted in an 8 week delay to the delivery of HMS Cleopatra [21].

It is considered that a ship's refit and repair programme satisfies the conditions for CPM applicability to such projects. These conditions are [20]:-

- There must be a clearly definable start and finish to the project, (i.e. it is not suitable for continuing programmes);

- There must be a feasible and achievable objective to be accomplished;

- Each task or activity in the project must be definable;

- The duration or time for each activity must be estimable, at least within broad limits;

- There must be interdependencies among the activities;

- There must be more than one path through the network from start to finish.

However, it is appreciated that there is currently a major desire in both the MoD and industry, to avoid large refit and repair programmes, through methods such as Condition Based Monitoring (CBM) [22]. 


\section{2 (b) CPM Procedure}

Having an internal arrangement of the vessel is crucial in determining the critical path of a refit and repair project. Unlike the traditional naval ship concept design, the DBB method allows configurational modelling at the very start of the process. Having produced an internal arrangement, the procedure for determining the critical path is as follows [23]:-

- Subdivide the vessel. As the research project involves naval ships, zonal subdivision is preferable to functional subdivision adopted in merchant ship building. It would be more realistic if such subdivision in naval vessels corresponded to zoning from a survivability perspective based on vulnerability considerations;

- Divide each zone into sub-zones;

- Produce a sequential work breakdown based on a list of all maintenance, repair and refit activities in each zone and sub-zone, activity dependencies and precedence relationships;

- Associate each activity with an estimation of its duration;

- Determine the critical path by working out which path through the network has zero or the least float.

\section{2 (c) The Issue of Uncertainty}

It is very common for naval vessels to enter a midlife refit and repair programme, only to discover issues which were previously unknown and need to be undertaken on top of the pre-planned activities. These uncertainties could be incorporated into the estimation of the activity durations, when determining the critical path.

The activity duration in CPM is a single time estimate, usually based on experience with similar projects [24], thus it is difficult to accommodate uncertainty. To model uncertainty, an approach based on the Programme Evaluation and Review Technique (PERT) could be incorporated into CPM. Utilising probability theory, the approach essentially uses a three point estimate system to approximate the activity duration [24]:-

- Optimistic time (shortest possible duration);

- Most probable time;

- Pessimistic time (longest possible duration).

\subsection{DESIGN FOR SUPPORT CPM EXAMPLE}

As explained in Section 3.1, architectural decisions and through-life supportability performance are tightly linked together. The qualitative example below shows how architectural decisions on accessibility and distributed service runs might interact with the refit and repair critical path (in a zone which consists of three vertically placed sub-zones), and also the subsequent effects on other design aspects. 


\section{3 (a) Refit/Repair Work Breakdown in Zone and Sub-zones}

Figure 8 shows an example zone and its three sub-zones containing elements of FLOAT, MOVE and INFRASTRUCTURE DBBs. The zone is located amidships on a typical frigate consisting of three internal decks (excluding the double bottom), with two machinery decks and one passing deck.
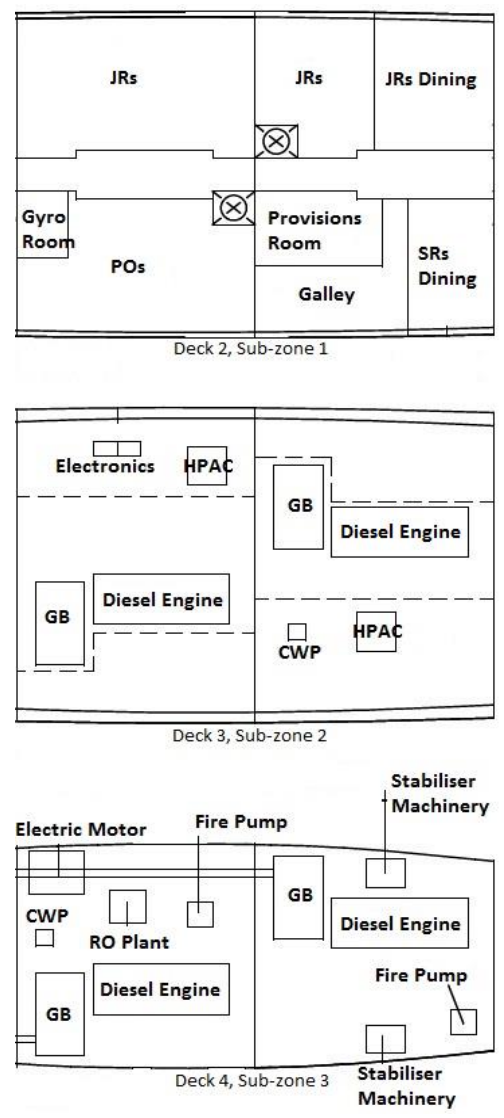

Figure 8: Amidships vertical zone for a typical frigate study consisting of three sub-zones

This configuration involves a single central passageway with main service runs passing through its deck head. Figure 9 is a simplified work breakdown network for the three sub-zones, showing activity dependencies and precedence relationships within and between the sub-zones. Note that the DBB functional breakdown colour code (Section 4.1 (c)) has been implemented in Figure 9. 


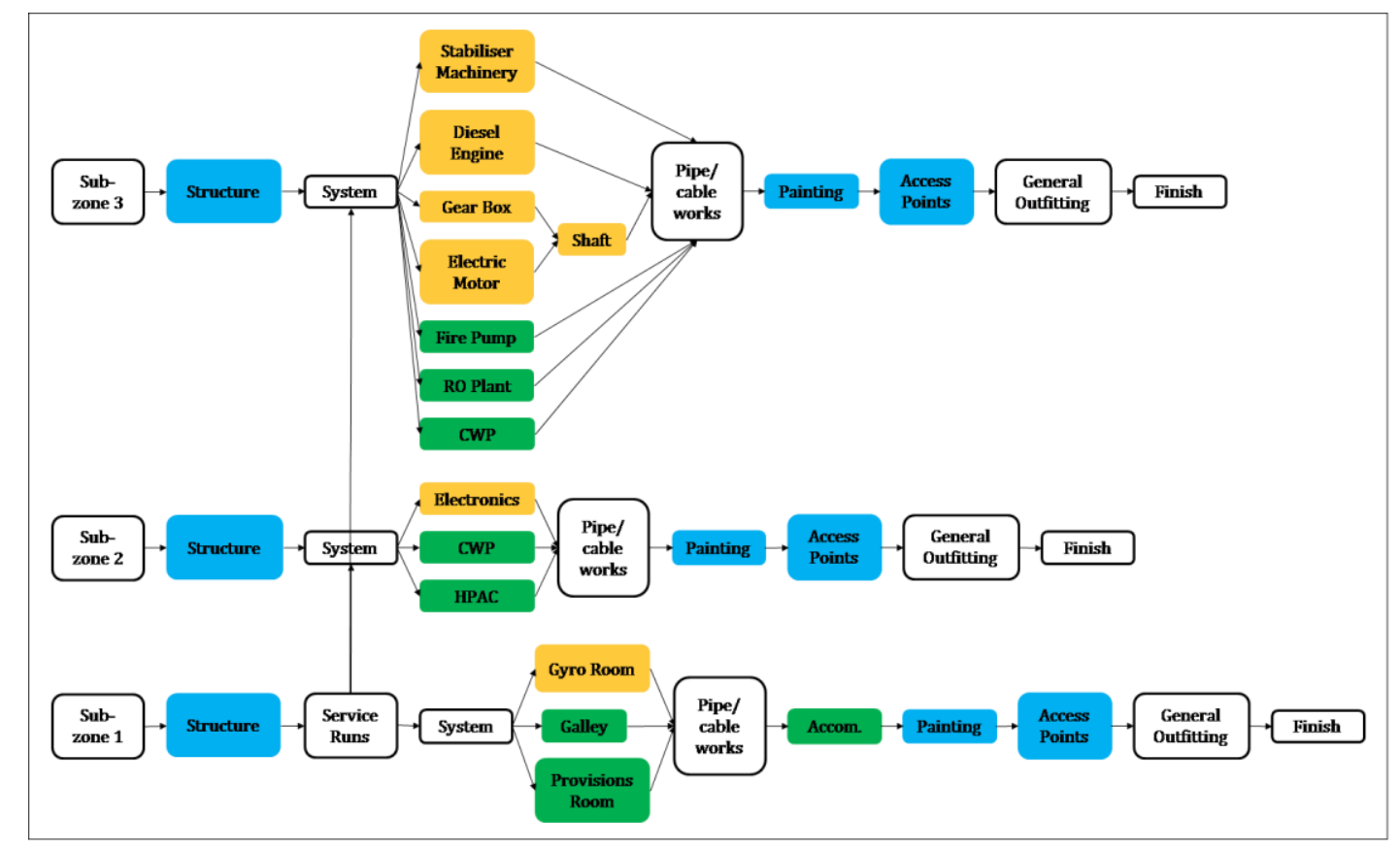

Figure 9: Simplified work breakdown network for three sub-zones from Figure 8

The designer's decisions on the arrangement of ship passageways and service runs plays a key role in determining the work breakdown in a zone or sub-zone. This particular configuration (Figure 8) means that if the passageway on No. 2 Deck is to be used for system removal and replacement activities for all three sub-zones, it should not be disturbed by refit work on the service runs, also located on the main passageway. The effect of this on the work breakdown is that service run activities have to be undertaken after any structural work and precede works on adjacent systems. Of relevance is the relationship between the completion of service run activities in sub-zone 1 and start of system activities in all three sub-zones. Lack of passageway redundancy means the overall duration is highly sensitive to unplanned disturbances in the passageway, especially if it is to form part of the routing for the relevant equipment removal routes.

\section{3 (b) Configurational Options for Main Access Routes and Service Runs}

Table 1 demonstrates various configurational options on accessibility and main run of services, as well as the issues resulting from each option. 


\begin{tabular}{|c|c|c|c|c|c|c|}
\hline \multirow[b]{2}{*}{ Option } & \multirow[b]{2}{*}{$\begin{array}{l}\text { Passage- } \\
\text { way }\end{array}$} & \multirow[b]{2}{*}{ Service Runs } & \multicolumn{2}{|c|}{ Redundancy } & \multirow[b]{2}{*}{$\begin{array}{l}\text { Ballistic } \\
\text { Protection }\end{array}$} & \multirow[b]{2}{*}{ Other Issues } \\
\hline & & & $\begin{array}{c}\text { Passagewa } \\
\mathrm{y}\end{array}$ & $\begin{array}{c}\text { Service } \\
\text { Runs }\end{array}$ & & \\
\hline 1 & $\begin{array}{c}\text { Single } \\
\text { central } \\
\text { on No. } 2 \\
\text { Deck } \\
\end{array}$ & $\begin{array}{c}\text { Through the } \\
\text { passageway deck } \\
\text { head }\end{array}$ & No & No & $\begin{array}{l}\text { Not } \\
\text { required }\end{array}$ & $\begin{array}{l}\text { Have to increase } \\
\text { No. } 2 \text { Deck height } \\
\text { to accommodate } \\
\text { service runs }\end{array}$ \\
\hline 2 & $\begin{array}{l}\text { Single } \\
\text { central } \\
\text { on No. } \\
2 \text { Deck }\end{array}$ & $\begin{array}{l}\text { Double technical } \\
\text { galleries on } \\
\text { ship's } \\
\text { sides on No. } 2 \\
\text { Deck } \\
\end{array}$ & No & Yes & Required & $\begin{array}{c}\text { Problematic } \\
\text { corrosion } \\
\text { inspection since } \\
\text { having to use } \\
\text { ballistic protection }\end{array}$ \\
\hline 3 & $\begin{array}{c}\text { Double } \\
\text { side } \\
\text { passage } \\
\text { ways } \\
\text { on No. } \\
2 \text { Deck }\end{array}$ & $\begin{array}{l}\text { Through the deck } \\
\text { head of both } \\
\text { passageways }\end{array}$ & Yes & Yes & Required & $\begin{array}{c}\text { Problematic } \\
\text { corrosion } \\
\text { inspection since } \\
\text { having to use } \\
\text { ballistic protection. } \\
\text { Also, have to } \\
\text { increase No. } 2 \\
\text { Deck height to } \\
\text { accommodate } \\
\text { service runs. } \\
\text { Athwartship } \\
\text { passageways are } \\
\text { required for access } \\
\text { and escape } \\
\text { between the two } \\
\text { passageways }\end{array}$ \\
\hline 4 & $\begin{array}{c}\text { Double } \\
\text { side } \\
\text { passage } \\
\text { ways } \\
\text { on No. } \\
2 \text { Deck }\end{array}$ & $\begin{array}{l}\text { Double technical } \\
\text { galleries on } \\
\text { ship's } \\
\text { sides on No. } 2 \\
\text { Deck }\end{array}$ & Yes & Yes & Required & $\begin{array}{l}\text { Problematic } \\
\text { corrosion } \\
\text { inspection since } \\
\text { having to use } \\
\text { ballistic protection. } \\
\text { May have to } \\
\text { increase beam to } \\
\text { accommodate } \\
\text { technical galleries. } \\
\text { Athwartship } \\
\text { passageways are } \\
\text { required for access } \\
\text { and escape } \\
\text { between the two } \\
\text { passageways }\end{array}$ \\
\hline 5 & $\begin{array}{l}\text { Single } \\
\text { central } \\
\text { on No. } \\
2 \text { Deck }\end{array}$ & $\begin{array}{l}\text { Double technical } \\
\text { galleries on } \\
\text { ship's } \\
\text { sides on No. } 3 \\
\text { Deck }\end{array}$ & No & Yes & Required & $\begin{array}{c}\text { Problematic } \\
\text { corrosion } \\
\text { inspection since } \\
\text { having to use } \\
\text { ballistic protection }\end{array}$ \\
\hline 6 & $\begin{array}{l}\text { Double } \\
\text { side } \\
\text { passage } \\
\text { ways } \\
\text { on No. } \\
2 \text { Deck }\end{array}$ & $\begin{array}{l}\text { Double technical } \\
\text { galleries on } \\
\text { ship's } \\
\text { sides on No. } 3 \\
\text { Deck }\end{array}$ & Yes & Yes & Required & $\begin{array}{c}\text { Problematic } \\
\text { corrosion } \\
\text { inspection since } \\
\text { having to use } \\
\text { ballistic protection. } \\
\text { Athwartship } \\
\text { passageways are } \\
\text { required for access } \\
\text { and escape } \\
\text { between the two } \\
\text { passageways } \\
\end{array}$ \\
\hline
\end{tabular}


In addition to the different issues arising from each configuration, the baseline work breakdown (Figure 9) will also be affected differently by each option:-

- Option 2 (Single central passageway with technical galleries on ship's sides on No. 2 Deck): This configuration separates service runs from the passageways, thus allowing system refit activities in all three sub-zones and service run work to follow structural activities in parallel. As with Option 1, lack of passageway redundancy means the overall duration is highly sensitive to unplanned disturbances in the passageway.

- Option 3 (Double passageways on No. 2 Deck with service runs through the deck head of both): This configuration allows service run activities in one of the passageways to follow structural activities while the other passageway is used to start system activities in parallel. Once work on service runs are completed in one passageway, work on the other could start without disturbing removal or replacement of systems equipment.

- Option 4 (Double passageways with technical galleries on ship's sides on No. 2 Deck): This configuration removes the dependency of system activities on service run work, thus allowing their parallel start following structural work. The overall duration is less sensitive to unplanned disturbances in the passageways.

- Option 5 (Single central passageway on No. 2 Deck with technical galleries on ship's sides on No. 3 Deck): By placing the main passageways and service runs on different decks, system activities in all three sub-zones and service run work could follow after structural work in parallel. However, lack of passageway redundancy means the overall duration is highly sensitive to unplanned disturbances in the passageway.

- Option 6 (Double passageways on No. 2 Deck with technical galleries on ship's sides on No. 3 Deck): This option has an identical work breakdown network to Option 5, but the overall duration is less sensitive to unplanned disturbances in the passageways.

Using the PERT-based three point estimate, the work breakdown networks of all options can be populated with estimations of activity duration to determine the critical path for the zone. Once some sensible metrics have been established for a single zone, zonal subdivision and the CPM would be implemented for a full internal arrangement.

It is believed by applying the CPM to certain TLS scenarios and determining the critical path for relevant compartments, the ship designer can be assisted in making major architectural decisions.

\section{3 (c) Removal and Replacement Routes}

In addition to passageways and service runs, the critical path in a zone or sub-zone is affected by the disposition of equipment removal and replacement routes. The designer has to make the following considerations when arranging these routes:- 
- Use of passageways, as part of the arrangement, and sizing them accordingly;

- Use of 'soft' compartments (i.e. those with minimal services or equipment, e.g. storerooms, dining rooms) in the arrangement;

- Most extreme and highly undesirable case of having to remove equipment through ad hoc openings in the ship's sides.

Both horizontal and vertical access routes (usually through hatches) are sized to allow equipment, up to certain dimensions and weight, to pass through. For example the Type 45 passageways and hatches are sized to allow equipment of maximum dimension $760 \mathrm{~mm}$ and maximum weight $50 \mathrm{~kg}$ to be transported by the crew without any special arrangements [25]. However, large and heavy equipment (e.g. gas turbine modules, diesel engine and alternator parts) would require pre-planned removal and replacement routes and special equipment. The number and location of these routes should be based on the number and location of major equipment items that require them. For example, a ship with three main and auxiliary machinery spaces will require three removal and replacement routes, at least up to the main passageway deck, if not to the Upper Deck (e.g. in way of the hangar).

The most efficient removal route is one which goes directly up and onto the Upper Deck. This will however be very much dependent on the overall internal and topside arrangements. For example if a removal route has to go through compartments which are not considered as 'soft' (e.g. Operations Room, bridge, weapons systems) to reach the Upper Deck, then this would be unacceptable. To avoid having to remove equipment through ad hoc openings in the ship's sides, the designer should consider using 'soft' compartments along the removal route, as well as including the main passageways as part of the route to move the equipment fore or aft before being lifted out through a more appropriate space. Having to change the arrangement for several compartments, or move equipment fore or aft between zones, will clearly influence the critical path in any affected zone and sub-zones.

The issue is, however, far more cross-cutting than just affecting a specific refit's critical path, and this would make the above decisions less straightforward. Displacing compartments could affect many aspects; for example, moving the bridge or Operations Room could affect the ship's survivability, while moving the bridge alone could result in greater impact of green seas and visibility problems, and weapons systems, if displaced, could suffer from insufficient firing arcs and green seas effects. Relocating compartments could also affect the location of main watertight bulkheads with major implications on issues such as survivability and structural continuity, with potential growth in the rebalanced ship design solution. In addition, using the passageways as part of the removal and replacement routes is likely to affect the passageway width, which in turn could have knock on effects on the whole ship design. 


\section{BASELINE SHIP DESIGN FOR THE RESEARCH PROJECT}

The main merit of this research project is seen to be the development of a supportability evaluation approach to assist naval ship designers during ESD. Thus the baseline design and other design variants can be used to demonstrate the robustness of the approach. Hence, ongoing work on the current baseline design is briefly outlined in this section.

Having entered the Demonstration Phase in February 2015 [26], the Type 26 Global Combat Ship (GCS) will form the backbone of the Royal Navy for the next 40 years. The programme consists of the design, plan for manufacture and support solution of eight Anti-Submarine (ASW) and five general purpose frigates [27]. While not using or planning to use any current official design solutions, the specification for the general purpose frigates has been drawn on to form the baseline design broad requirements set for these DfS studies, and to demonstrate the new UCL ship concept design toolset (Section 4).

Major ship characteristics of the research project baseline design at the time of writing this paper are given in Table 2 .

\begin{tabular}{|l|r|l|}
\hline Total Enclosed Volume & 21500 & $\mathrm{~m}^{3}$ \\
\hline Deep Displacement & 5565 & te \\
\hline Waterline Length & 126.5 & $\mathrm{~m}$ \\
\hline Overall Length & 134.5 & $\mathrm{~m}$ \\
\hline Waterline Beam & 18 & $\mathrm{~m}$ \\
\hline Amidships Upper Deck Beam & 20.5 & $\mathrm{~m}$ \\
\hline Amidships Draught & 5.5 & $\mathrm{~m}$ \\
\hline
\end{tabular}

Table 2: Major baseline ship design characteristics

Major payload equipment include:-

- $1 \times$ BAE Systems $127 \mathrm{~mm}$ naval gun;

- $2 \times$ Quad MK 141 Harpoon launchers;

- $6 \times 8$ cell MK 41 VLS;

- $2 \times$ Phalanx CIWS;

- $64 \times$ Quad packed MBDA Sea Ceptor SAMs;

- $1 \times$ hangared Merlin HM.2;

- $1 \times$ medium range 3-D BAE Systems 997 Artisan radar.

- $1 \times$ medium range Thales type 2050 bow sonar

\section{CONCLUSIONS AND FURTHER WORK}

Despite being entirely qualitative, the findings to date of the UCL DfS project indicate the highly style dependant and cross-cutting nature of both naval ship TLS and ship architecture. For a simplified refit and repair programme for a naval combatant, the developed work breakdown network is seen to be affected by major configurational decisions associated particularly with main passageways and ship service runs. In addition, the disposition of the removal and replacement routes, depending on whether 
or not they can be accommodated in a specific zone or sub-zone, seems to have a major influence on not just refit and repair activities, but various other design aspects which are not directly related to TLS concerns.

For a full implementation of the CPM applied to frigate refit activities, the work breakdown network in each zone and sub-zone will have to be populated with appropriate activity durations. A PERT-based three point estimate system will be used to incorporate uncertainty into the estimation of activity durations. Once a sensible link has been established between the results of the CPM in a single zone and the ship design's architectural consequences, the study will concentrate on the production of a complete internal arrangement appropriate to concept level investigations to further test the robustness of the proposed approach.

Parallel to developing the proposed approach, ongoing work on the baseline design will continue. To discern any distinction in applying DfS metrics across ship roles and types, it is intended that the investigation will extend from the baseline design to include studies of smaller, larger and multihull combatant designs meeting different specifications.

\section{ACKNOWLEDGEMENTS}

Financial support for the first author's PhD studentship has been provided through an EPSRC industrial CASE award with BAE Systems sponsorship and is greatly acknowledged, as is the latter's ongoing input to the research project.

\section{REFERENCES}

1. ANDREWS, D.J., 'A Creative Approach to Ship Architecture', Transactions of RINA, Vol. 145, 2003.

2. UK MOD., 'The Defence Logistic Support Chain Manual - Integrated Logistic Support', Integrated Logistic Support Policy JSP 886, 2013.

3. BROWN, D.K., and ANDREWS, D.J., 'The Design of Cheap Warships', Proceedings of the International Naval Technology Expo 1980, Rotterdam, Netherlands, February 1980.

4. PAWLING, R.J., ANDREWS, D.J., PIKS, R., SINGER, D., DUCHATEAU, E., HOPMAN, H., 'An Integrated Approach to Style Definition in Early Stage Design', Proceedings of COMPIT 2013, Cortona, Italy, May 2013.

5. ANDREWS, D.J., 'Creative Ship Design', Transactions of RINA, Vol. 123, 1981.

6. BROWN, D.K., 'Naval Architecture', US Naval Engineers Journal, Vol. 105 (1), 1993. 
7. ANDREWS, D.J., 'An Integrated Approach to Ship Synthesis', Transactions of RINA, Vol. 128, 1986.

8. ANDREWS, D.J., DICKS, C.A., 'The Building Block Design Methodology Applied to Advanced Naval Ship Design', Proceedings of IMDC 1997, Newcastle, UK, May 1997.

9. TUPPER, E.C., 'Introduction to Naval Architecture, Fifth Edition', Butterworth-Heinemann, UK, 2013.

10. ANDREWS, D.J., 'Marine Requirements Elucidation and the Nature of Preliminary Ship Design', IJME Vol. 153, Part A1, 2011.

11. ANDREWS, D.J., PAWLING, R.J., 'The Impact of Simulation on Preliminary Ship Design', Proceedings of IMDC 2009, Trondheim, Norway, May 2009.

12. MUNOZ, J.A., FORREST, C.J.M., 'Advantages of Software Integration from Initial Design Through to Production Design', Proceedings of ICCAS 2002, Malmö, Sweden, September 2002.

13. ANDREWS, D.J., PAWLING, R.J., 'SURFCON - A 21st Century Ship Design Tool', Proceedings of IMDC 2003, Athens, Greece, May 2003.

14. PAWLING, R.J., PIPERAKIS, A.S., ANDREWS, D.J., 'Developing Architecturally Oriented Concept Ship Design Tools For Research and Education', Proceedings of IMDC 2015, Tokyo, Japan, May 2015.

15. PAWLING, R.J., ANDREWS, D.J., 'Large Unmanned Vehicles and the Minor War Vessel', Proceedings of Warships 2013, Bath, UK, June 2013.

16. ANDREWS, D.J., CASAROSA, L., PAWLING, R.J., GALEA, E., DEERE, S., LAWRENCE, S., 'Integrating Personnel Movement Simulation into Preliminary Ship Design', IJME Vol. 150 Part A1, Discussion IJME, Vol. 150, Part A3, 2008.

17. PAWLING, R.J., GRANDISON, A., LOHRMANN, P., MERMIRIS, G., PEREIRA DIAS, C., 'Methods and Tools for Risk-Based Approach to Fire Safety in Ship Design', Ship Technology Research/Schiffstechnik, Vol. 59(3) pp. 38-49, 2012.

18. PIPERAKIS, A.S., ANDREWS, D.J., 'A Comprehensive Approach to Survivability Assessment in Naval Ship Concept Design', IJME Vol. 156, Part A4, 2014.

19. ANDREWS, D.J., BURGER, D., ZHANG, J., 'Design for Production Using the Design Building Block Approach', Transactions of RINA, 2005.

20. MERCIER A. G., NUNNALLY R. S., 'The Critical Path Method: Its Fundamentals', United States Naval Postgraduate School, Monterey, California, 1965. 
21. WHITMAN D. F., WATTY A. J., 'Modernising the Leander Class Frigates', Transactions of RINA, 1978.

22. COULL, G., 'Condition Monitoring in the Marine Sector', The Naval Architect, April 2015 pp. 20-22, UK, 2015.

23. SHARMA, A. V., 'Project Management Strategies for Shipyard', Proceedings of Offshore Inspection, Maintenance and Repair Conference, Singapore, November 2009

24. CHINNECK, J. W., 'PERT for Project Planning and Scheduling', Carleton Department of Systems and Computer Engineering, Ottawa, Canada, 2009.

25. GATES, J., 'Type 45 Destroyer Manual', Haynes Owners' Workshop Manuals, UK, 2014.

26. FALLON, M., 'Type 26 Global Combat Ship', House of Commons: Written Statement (HCWS289), 2015.

27. PRICE, G., WILSON D., ROTTIER, P., KYTE, P., 'UK Type 26 Global Combat Ship Support Solution Decision Point Project', Proceedings of INEC 2014, Amsterdam, Netherlands, May 2014.

\section{AUTHORS BIOGRAPHY}

Syavash Esbati completed his MEng in Naval Architecture and Marine Engineering at UCL in 2013. He is currently a PhD student at UCL DRC investigating naval ship through-life support in preliminary ship design.

Dr. Alexander S. Piperakis (MEng PhD AMRINA) completed the MEng in Mechanical Engineering at the University of Bath in 2008. He then joined the UCL DRC as a research student investigating naval ship survivability in preliminary ship design. Gaining his PhD in 2013, he has continued his research in the DRC in projects involving the incorporation of human factors into Risk-Based Design of ships and preliminary ship design methodology.

Dr. Rachel J. Pawling (MEng PhD AMRINA) is the BMT DSL Research Fellow for Naval Ship Design at UCL. She completed the MEng in Naval Architecture and Marine Engineering at University College London in 2001 and then joined the UCL $\mathrm{DRC}$ as a research student investigating the application of the Design Building Block approach to innovative ship design. Gaining her $\mathrm{PhD}$ in 2007, she has continued her research both in the DRC and via a secondment in industry. She is the recipient of the 2008 RINA Samuel Baxter Prize, 2009 RINA WHC Nicholas Prize, and 2012 COMPIT GL Award for papers describing her research.

Prof David J. Andrews (BSc(Eng), MSc, PhD, CEng, FRINA) was appointed Professor of Engineering Design at UCL in September 2000 following a career in ship design and acquisition management in the UK Defence Procurement Agency. He leads 
UCL's design research in computer aided ship design, design methodology and design practice. He is a Fellow of RINA, Fellow of IMechE and was elected to the Royal Academy of Engineering in 2000 and as a Vice President of RINA in 2006. 


\section{DESIGN FOR SUPPORT IN THE INITIAL DESIGN OF NAVAL COMBATANTS}

S Esbati, A S Piperakis, R J Pawling, D J Andrews, Design Research Centre, Marine Research Group, Department of Mechanical Engineering, University College London, UK

\section{SUMMARY}

Failure to address Design for Support aspects in the early, formative stages of the design of naval vessels will result in certain disadvantages, most notably reduced through-life adaptability and upgradability, long and expensive overhauls, and diminished operability. A genuine implementation of Design for Support in the early stages of the ship design could be made feasible through consideration of relevant Design for Support features, better addressed through integrating the vessel's architecture, into the concept design process. This paper describes ongoing work at University College London to develop an analysis tool which provides ship designers with a framework for early stage assessment of various internal arrangements from a through-life support perspective. The research project considers both method and tool development.

\section{NOMENCLATURE}

$\begin{array}{ll}\text { ARM } & \text { Availability, Reliability and Maintainability } \\ \text { ASW } & \text { Anti-Submarine Warfare } \\ \text { CAD } & \text { Computer Aided Design } \\ \text { CADMID } & \text { Concept, Assessment, Demonstration, Manufacture, In-Service and } \\ & \text { Disposal } \\ \text { CASD } & \text { Computer Aided Ship Design } \\ \text { DBB } & \text { Design Building Block } \\ \text { DfS } & \text { Design for Support } \\ \text { DRC } & \text { Design Research Centre } \\ \text { ESD } & \text { Early Stage Design } \\ \text { ESSD } & \text { Early Stage Ship Design } \\ \text { GA } & \text { General Arrangement } \\ \text { GCS } & \text { Global Combat Ship } \\ \text { GUI } & \text { Graphical User Interface } \\ \text { ILS } & \text { Integrated Logistics Support } \\ \text { JHSV } & \text { Joint High-Speed Vessel (US Navy) } \\ \text { LCS } & \text { Littoral Combat Ship (US Navy) } \\ \text { MoD } & \text { UK Ministry of Defence } \\ \text { NPV } & \text { Net Present Value } \\ \text { OPV } & \text { Offshore Patrol Vessel } \\ \text { PB-ROA } & \text { Prospect Theory-Based Real Options Analysis } \\ \text { PSD } & \text { Preliminary Ship Design }\end{array}$




$\begin{array}{ll}\text { RAS } & \text { Replenishment At Sea } \\ \text { ROA } & \text { Real Options Analysis } \\ \text { TEU } & \text { Twenty-Foot Equivalent Unit (ISO containers) } \\ \text { TLF } & \text { Through Life Finance } \\ \text { TLS } & \text { Through Life Support } \\ \text { UCL } & \text { University College London } \\ \text { USN } & \text { United States Navy } \\ \text { UXV } & \text { Unmanned (Air, Surface or Underwater) Vehicles } \\ \text { VCG } & \text { Vertical Centre of Gravity } \\ \text { VLS } & \text { Vertical Launch System (for ship launched missiles) }\end{array}$

\section{INTRODUCTION}

Despite the fact that the principles of concurrent engineering were endorsed by the MoD over two decades ago, a genuine implementation of those principles has proven difficult to satisfactorily implement in naval ship acquisition. One of the major reasons for this failure to meet concurrent engineering objectives is the inability to incorporate Design for Support (DfS) features in the ship design from the very start of the ship synthesis. Currently the question of naval ship Through Life Support (TLS) is addressed much later than the initial synthesis when the design has been constrained by the need to achieve a set of diverse performance and budgetary criteria. Failure to consider DfS features at the early, formative stages of the design process results in the supportability performance of the ship being poor. This leads to longer and more expensive overhauls, diminished availability and reduced scope for through-life adaptability and upgradeability.

For naval ships, a genuine implementation of DfS features in Early Stage Ship Design (ESSD) can be facilitated by early consideration of the vessel's architecture. However, traditional naval ship design does not incorporate this as too often, it only considers the ship's configuration in any depth after having achieved an initial numerically balanced design (i.e. achieving speed, seakeeping, stability, strength and mission requirements). Hence there is a clear need to bring consideration of the architectural features to the centre of the initial ship synthesis process [1].

This paper provides a short background to naval ship TLS before describing ongoing work at the University College London (UCL) Design Research Centre (DRC) on how the UCL originated Design Building Block (DBB) approach to preliminary ship design facilitates an architecturally focused investigation of through-life supportability during ESSD, namely, while the ship design is still fluid. This is presented through the use of a newly developed ship concept design tool and the application of a proposed method for evaluating the TLS performance of concept ship designs. 


\section{NAVAL SHIP THROUGH LIFE SUPPORT}

It is MoD policy that Integrated Logistics Support (ILS) will be applied to all product acquisition [2]. ILS, being a disciplined approach that influences the product design and develops the support solution to optimise supportability and Through Life Finance (TLF), is applicable throughout the whole life of a project. The focus, however, changes as the project progresses through the Concept, Assessment, Demonstration, Manufacture, In-Service and Disposal (CADMID) phases of the product life-cycle [2]. A summary of the totality of ILS is given in Figure 1.

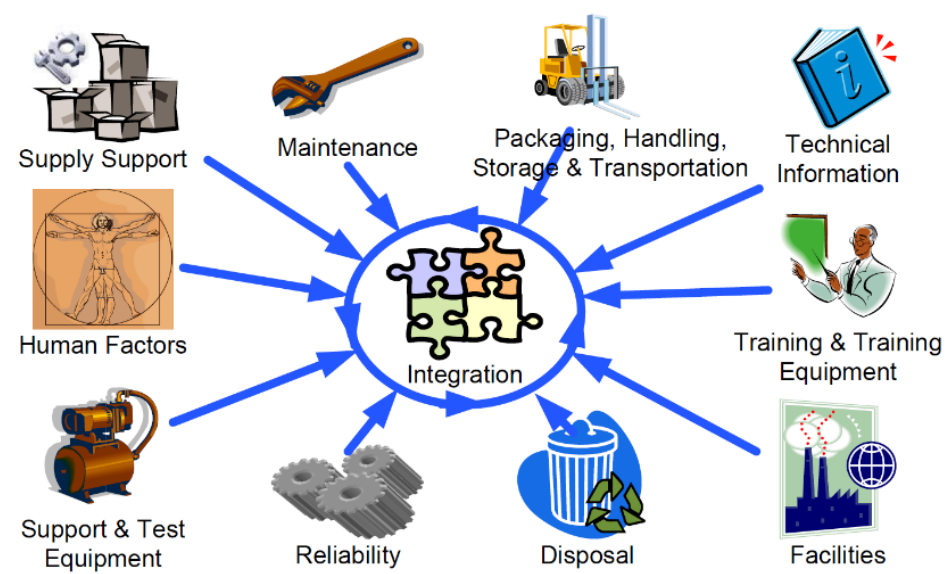

Figure 1: Integrated Logistics Support breakdown [2]

Note that some aspects of ILS, namely technical information and facilities, are not strongly interrelated with ship architecture.

Despite the fact that ILS is official MoD policy, historically the process of naval ship design has been carried out in the middle of an intense debate to reduce costs while attempting to maximise mission capability. This has meant that insufficient attention is given to the full range of capability aspects, namely TLS and availability. It is therefore considered necessary to adopt design practices that make TLS more prominent during Preliminary Ship Design (PSD).

\section{PRELIMINARY NAVAL SHIP DESIGN}

\subsection{STYLE IN PRELIMINARY NAVAL SHIP DESIGN}

'Style' was explicitly incorporated as a characteristic of a ship design by Brown and Andrews [3] as the fifth ' $\mathrm{S}$ ' in their "S5"; the others being Speed, Seakeeping, Stability, and Strength (S4). Style was later defined as a type of information in ship design that is cross-cutting, i.e. affects multiple design areas [4]. Incorporating style enables the designer to accommodate uncertainty and conceptually connect it, not just in the form of 'hard' knowledge (e.g. structural standards) but also 'soft' knowledge (e.g. guidance on internal arrangement from a range of perspectives) [4].

An example of a highly stylistic issue is the degree of through-life supportability of naval ships. As shown in Figure 1, TLS is inherently cross-cutting as a decision 
explicitly influences a wide range of solution areas. A decision on the level of supportability to be adopted in a given ship design can influence a large range of both overall and detailed design features. Thus aspects such as the access policy, number and routing of removal routes, ship service runs, compartment adjacency, Replenishment At Sea (RAS) features, and Availability, Reliability and Maintainability (ARM) requirements, and adaptability requirements, are all strongly influenced by the overall ship architecture. Hence an early evaluation of supportability requires the ship architecture to be at the centre of the initial synthesis of naval ship design.

\subsection{LIMITATIONS OF TRADITIONAL PRELIMINARY NAVAL SHIP DESIGN}

The traditional approach to naval ship design delays architectural modelling as it focuses on the numerical aspects of speed, seakeeping, stability, strength and the combat system to drive the initial ship sizing [5] and only considers internal arrangement properly once an initial numerically balanced design has been achieved. It postpones the examination of style related issues (such as supportability) to later stages, despite the fact that warships are generally not weight or space limited, but architecturally driven [6]. The outcome is a design which is constrained by the issues traditionally seen to drive the design but with little or no emphasis on those more architecturally driven aspects of style. Thus design developments that arise once these aspects are subsequently incorporated result in additional costs, which increase proportionately the later they are found to be necessary.

\subsection{THE DBB APPROACH AS THE PREFERRED PSD APPROACH}

Andrews' [5] proposal to integrate ship architecture with the traditional numerical sizing was followed by the demonstration of 'creative synthesis' [7]. From this work, a new approach, namely the Design Building Block approach to PSD [8] was developed and has reached acceptance as a standard PSD approach [9].

The basic idea behind the DBB approach is for the designer to separate the ship's functions and sub-functions into discrete physically realisable elements (Design Building Blocks) and position them appropriately in a putative ship configuration. This then puts architectural features at the centre of the ship synthesis process, alongside the traditional numerically based sequential design process [8]. The architectural approach allows a more thorough exploration of alternative designs in an interactive process of requirement elucidation [10]. It also encourages the investigation of novel solutions and "softer", user-centric and stylistic aspects such as human factors [11]. Figure 2 gives a summary of the DBB approach. 


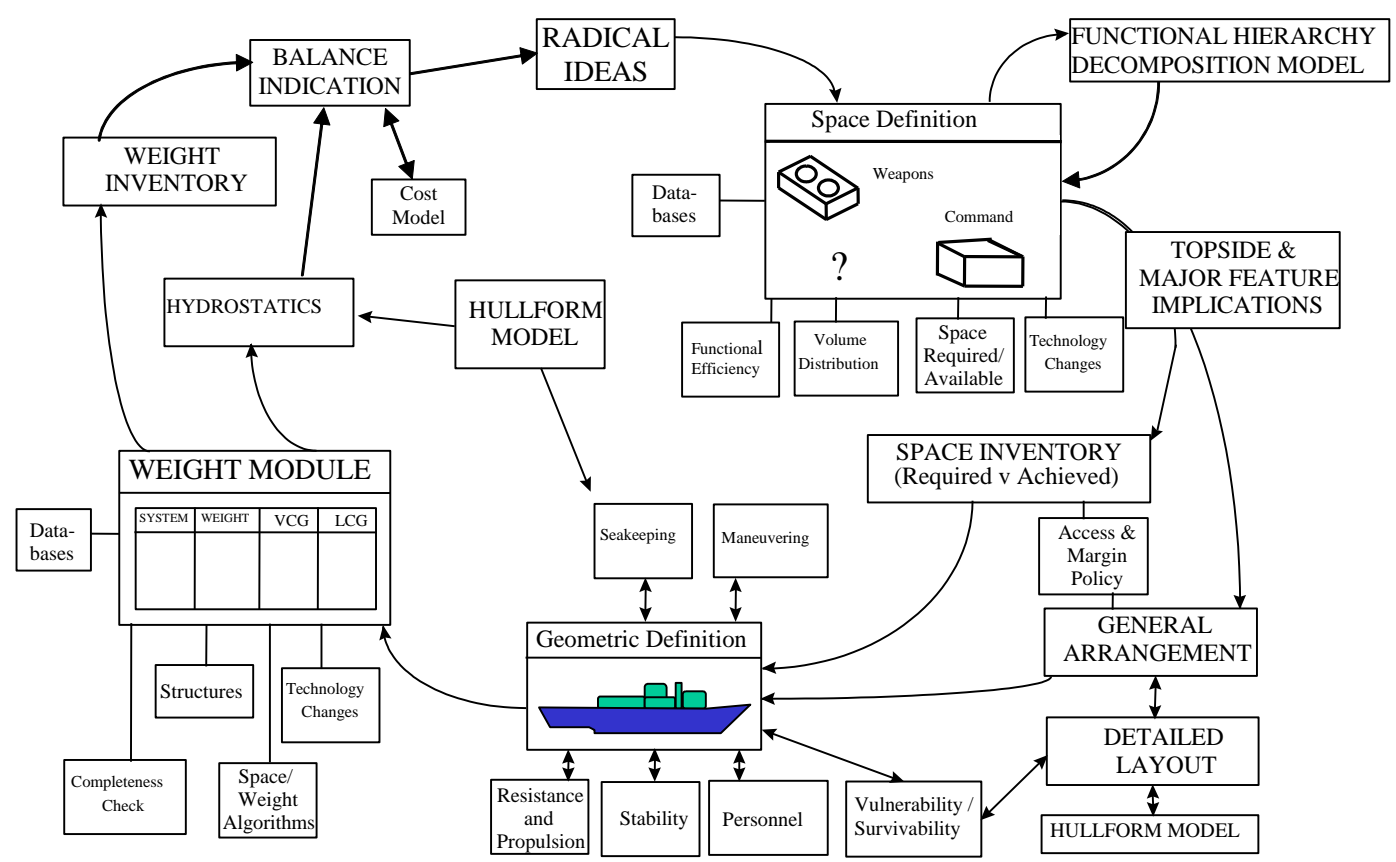

Figure 2: The Design Building Block approach to preliminary ship design applied to surface ships [8]

\section{THE NEED FOR A NEW SHIP CONCEP DESIGN SOFTWARE TOOL AND CURRENT PROGRESS}

The DBB approach to PSD (Section 3.3) was developed and implemented in an industry standard PSD tool following the rapid developments in computer graphics capabilities. The DBB approach was incorporated as the SURFCON module in QinetiQ GRC's Paramarine Computer Aided Ship Design (CASD) suite [12]. SURFCON's proof of concept was then described in [13]. By implementing the DBB approach through the SURFCON module in the Paramarine CASD suite, the DBB approach was linked to a commercially established PSD software package [12] and ship designers could then draw on all the naval architectural analytical tools available within Paramarine [13].

However, there are several disadvantages regarding the use of sophisticated (fully 3D) Computer Aided Design (CAD) models in Early Stage Ship Design (ESSD) research. These include: the need to resort to modelling to an unnecessarily high level of detail; excessive flexibility and precision inappropriate for most early stage designs; the very high software learning overhead; and the difficulties in connecting design tools with external analysis tools [14].

In order to address these issues, a ship design toolset implementing the DBB approach and able to easily integrate new simulation and analysis tools into the design process is being developed at UCL for use in research into PSD [14]. This toolset is being developed using commercial non-CAD software. Specifically, the basic tool is a Microsoft Excel spreadsheet-based layout model using a cellular representation of the arrangeable space on the ship. Excel was chosen because it is widely available, has a pre-existing Graphical User Interface (GUI), permits scripting and a limited degree of 
programming (via Visual Basic for Applications, VBA) and inherently provides an addressable cellular model - albeit only in 2D.

\subsection{DESCRIPTION OF THE SOFTWARE TOOL}

The basic UCL tool is comprised of three principal components; namely the "Framework", the "DBB Model" and the "GA". This section gives a brief description of these components, including screenshots, with reference to a UCL Offshore Patrol Vessel (OPV) design study carrying unmanned vehicles [15].

\section{1 (a) Framework}

The "Framework", shown in Figure 3, contains common variables used by many DBBs, such as the number of crew and the weight and space margins associated with a given DBB. The framework also contains variables describing the size of the vessel and the locations of decks and main watertight bulkheads.
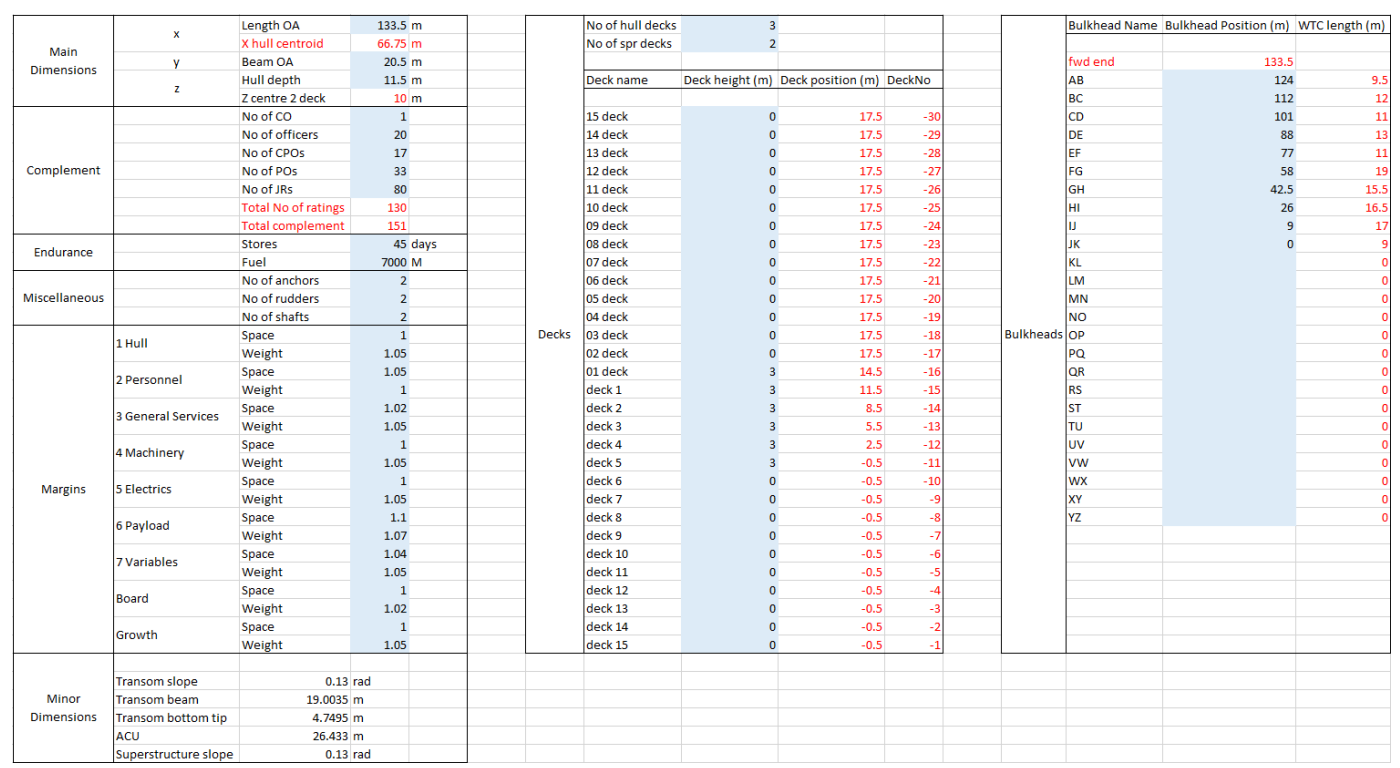

Figure 3: The UCL Ship Design tool's "Framework" component, showing tabular definition of ship dimensions, deck and main watertight bulkhead positions and other variables

\section{1 (b) DBB Model}

The DBBs are defined in a set of Excel sheets forming the "DBB Model", an example extract of which is shown in Figure 4. The "DBB Model" provides a tabular description of the DBBs, containing columns giving their location, dimensions, weights and any other parameters that may be added by the designer simply by adding additional columns to the spreadsheet. The DBBs may be connected to each other or to overall "Framework" variables. The tabular format has the advantage that all aspects of a DBB can be contained in a single row in the table. The "DBB Model" is structured using the UCL functional groups of FLOAT, MOVE, FIGHT and INFRASTRUCTURE for naval vessels [13]. 


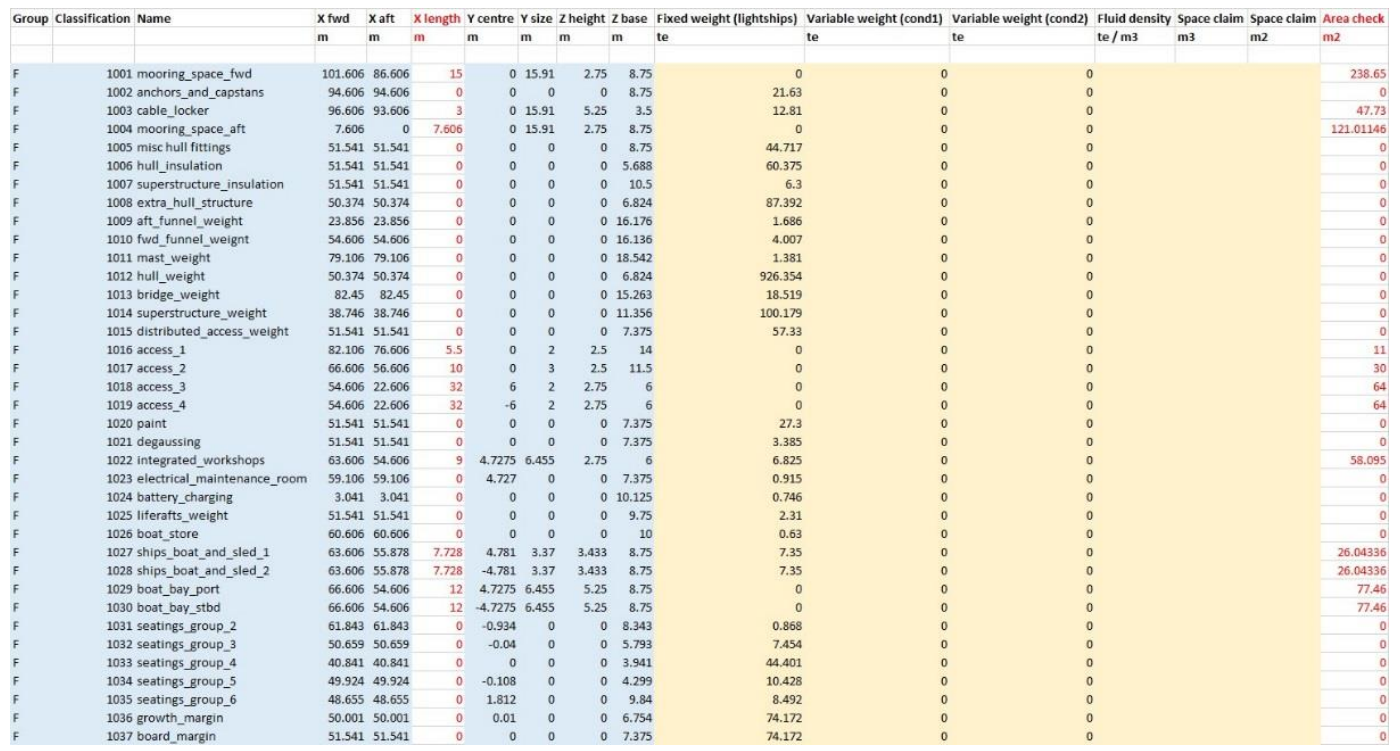

Figure 4: The UCL Ship Design tool's "DBB Model" component, showing tabular definition of the location, size and weight of the Design Building Blocks belonging to the FLOAT functional group

\section{1 (c) General Arrangement}

Finally, the design's General Arrangement (GA) can be visualised in the "GA" component. This is a separate workbook, which contains macros to read the "Framework" and "DBB Model" data and generate a display of the GA. An example GA for a UCL PSD study of a UXV OPV [15] is illustrated in Figure 5 (using the DBB functional breakdown colour code, namely FLOAT - Blue, MOVE - Yellow, FIGHT - Red, INFRASTRUCTURE - Green [13]).

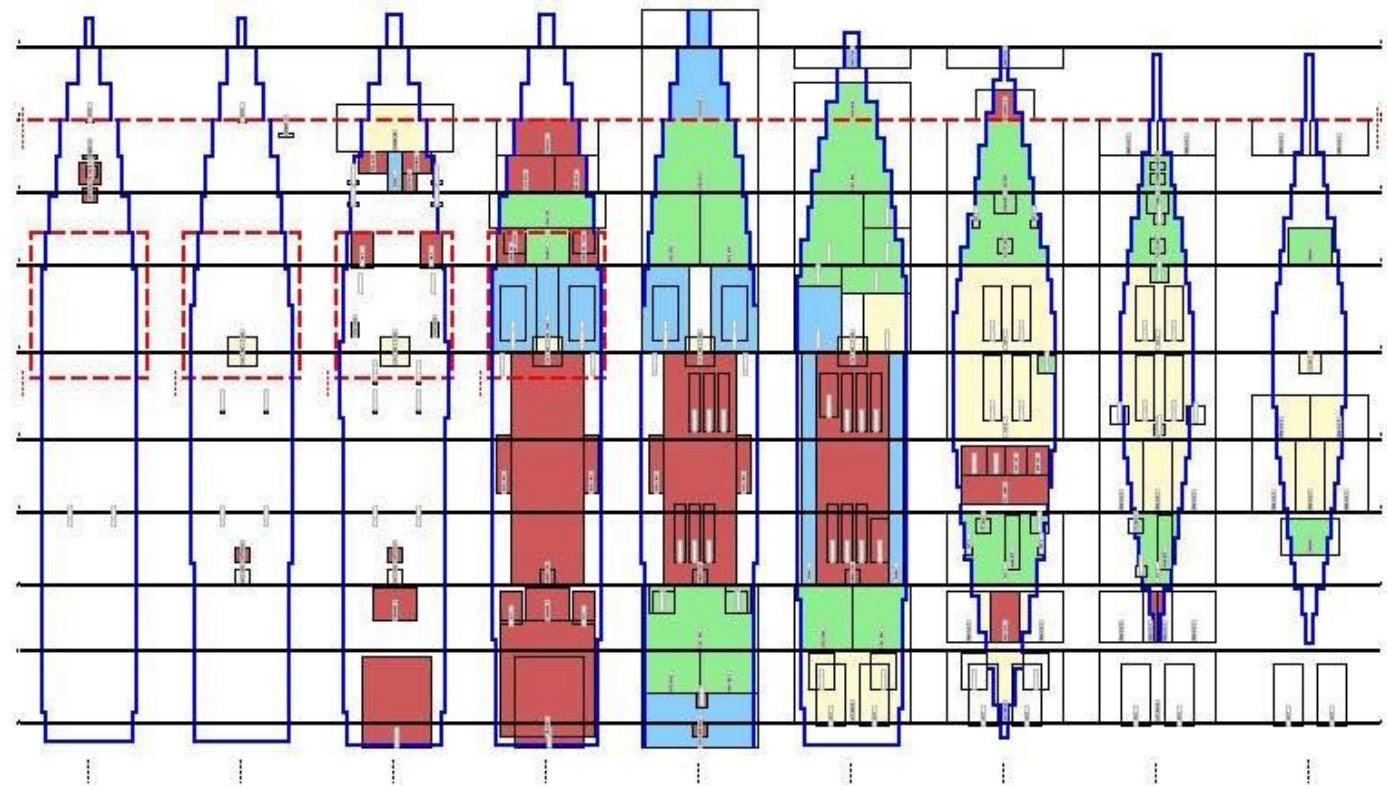

Figure 5: The UCL Ship Design Tool's "GA" component, showing the layout of a typical early stage design model for a UXV OPV study [15] 
Figure 5 shows the simple approach used to represent DBBs in the design. The cells in the spreadsheet represent a square grid; a group of cells is assigned a numerical identifier to identify them as part of a specific DBB and a colour to identify them as belonging to a specific functional group in the GA [13]. Each space also has a visible outline and nametag. Figure 5 also displays the deck outlines (blue lines), location of the main watertight bulkheads (black lines) and guidance provided to the designer for the location or limits for some spaces and equipment, based on design guidance (red dashed lines). Specifically, limits on the location of upper deck weapons and sensors (e.g. due to green sea loading) and the bridge (for visibility and motions) are shown.

\subsection{SOFTWARE TOOL APPLICATIONS}

The UCL developed ship design toolset is being used to develop tools for analysing ship layout and providing data, either to external tools or as feedback to designers, on the consequences to ship performance. Currently, using network analysis and 2D modelling, the toolset enables the quick extraction of a wide range of spatial properties for the analysis of the configuration. Such spatial properties include proximity, adjacency (via network diagrams, see Figures 6 and 7), perimeter and surface area. In addition, this simplified arrangement model has several features applicable to more sophisticated analysis. This allows such analysis to be undertaken at earlier stages of the design process without adding a significant modelling burden.

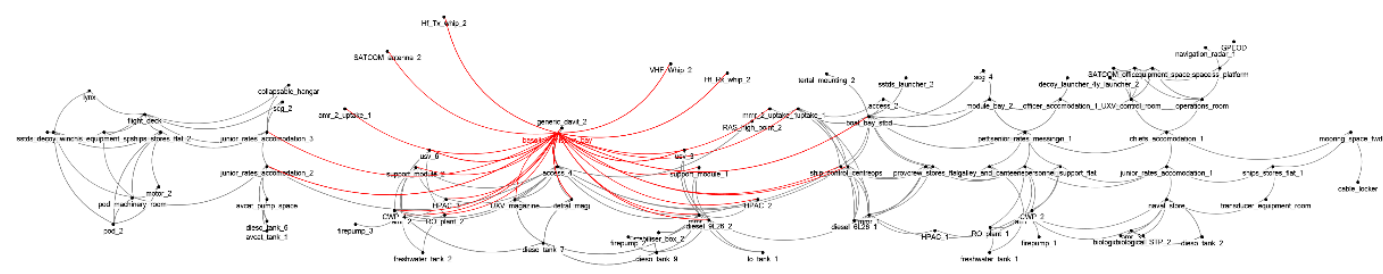

Figure 6: Example of a whole ship adjacency network, showing the profile view, outputted by the UCL layout tool [14] 


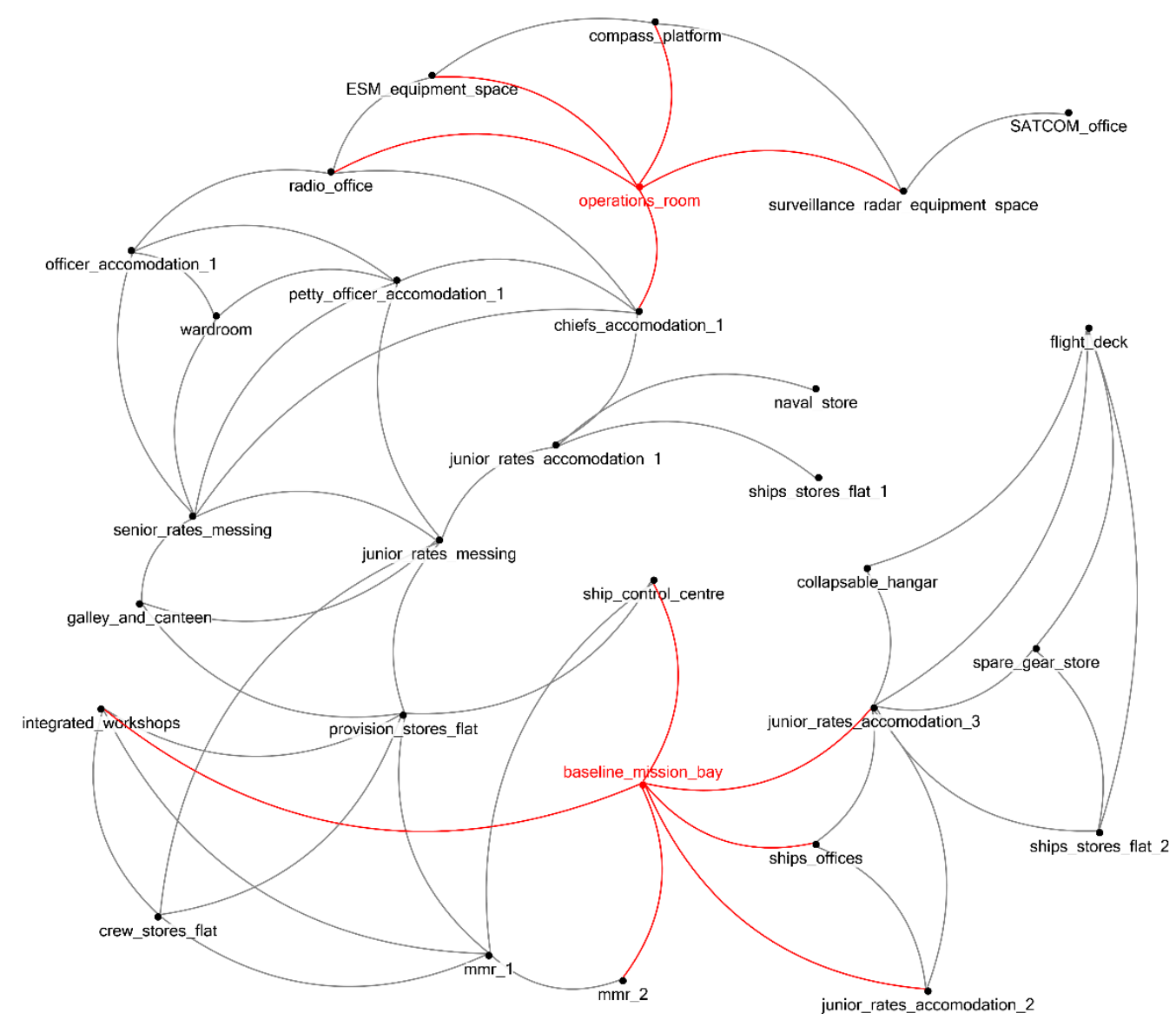

Figure 7: Example of an adjacency network for a selected set of DBBs of the specific ship design modelled in Figure 6. (This is not to scale and has been outputted from the UCL layout tool)

The generation of layout and connectivity information would facilitate early analysis of, for example, personnel movement [16] and fire safety assessment [17]. If this flexible and fast model of the general arrangement were to be combined with a tool that models damage effects from weapons and a network representation of ship systems, it could allow the findings of recent work on survivability [18] to be incorporated into concept design studies. Similarly, the modelling of systems in concert with the arrangement is important for assessing the producability of designs [19] at the preliminary stages of ship design.

Thus the UCL ship design toolset will facilitate the incorporation of additional design aspects early in design. This could satisfy the needs of specific projects and be able to interface with dedicated tools for more complex analysis. Given that the toolset satisfies the need for an ESD architectural model necessary to capture DfS drivers, it is believed that it can enable the evaluation of supportability in the preliminary design of naval ships. 


\section{DESIGN FOR SUPPORT EVALUATION APPROACH}

Several discussions held between UCL and BAE Systems (the project's industry sponsor) have resulted in a consensus that the aim of the research project is to develop an approach to evaluate the supportability of a ship design in a rational manner and assist the designer in making more informed early, yet significant, design decisions. This could essentially provide ship designers with a framework for an early stage analysis of various ship internal arrangements for a range of support aspects. The approach should:-

- Demonstrate real benefits in an early stage introduction of DfS features. Naval and government 'customers' are often not convinced by demonstrating that these benefits will only accrue financially after 25 years of service life;

- Not act as an absolute indicator, but should instead indicate whether or not, from a DfS perspective, it is a good idea to head in a particular direction;

- Not only concentrate on DfS features but enable a holistic analysis, thus also assess how S4 (Section 3.1) and combat system aspects might be affected by an early consideration of DfS.

\subsection{EXPLOITING THE RESEARCH AREAS ON DESIGN FOR SUPPORT}

Section 3.1 emphasised the need for an architectural approach in order to examine and evaluate supportability during the initial ship synthesis. Given the extensive experience at UCL on ship concept design and the implementation of the DBB method, the study is concentrating on developing an approach to assess the internal arrangement from a TLS perspective. It is aimed at elucidating how certain architectural decisions (e.g. access policy, service runs, routing of removal routes, zoning logic) could affect the supportability performance of the ship (e.g. ease of refits, ARM, RAS, adaptability) as well as the traditional ship design drivers (Section 3.2).

It is appreciated that various other TLS aspects, such as high level support management and contractual issues, strategic long-term support planning, support documentation and certain human factors issues, are also very pertinent to a comprehensive approach to DfS for naval vessels (Section 2). However, the UCL DRC's expertise is more focused on overall ship design and specifically the ship configuration. Therefore, this study has focused on the appropriate configuration related items identified in the previous paragraph.

\subsection{PROPOSED WAY FORWARD}

The need to incorporate both operational and through-life adaptability is considered to be an important aspect in improving the TLS performance of a naval ship. This is evident in the current trend in major navies towards highly adaptable and modular architectures, seen as attractive given squeezed budgets and rapid technology advancements. Significant relevant examples include the modular packages on the 
United States Navy's Littoral Combat Ship (LCS) [20], commercial standards on the Joint High-Speed Vessel (JHSV) and the missile Vertical Launch System (VLS) on the USS Arleigh Burke Class destroyers [21].

However, historically a rigorous mathematical framework for evaluating adaptability has been absent, forcing ship designers to employ insufficient metrics, such as cost and mission effectiveness, while relying on their own intuition, engineering experience or anecdotal evidence for decision making [22].

In tackling the above issue, the use of a novel quantitative framework for evaluating adaptability in non-commercial engineering systems has been proposed by Knight [23]. The framework, called Prospect Theory-Based Real Options Analysis (PBROA), takes the principles of Real Options Analysis (ROA) and applies them to noncommercial assets that do not generate cash flows. PB-ROA corrects the underestimation of the value of adaptability that results from Net Present Value (NPV) and static budgetary techniques by applying the ROA theory to the naval domain. However, in doing so Knight and Singer [21] outline the limitations of the three key assumptions of ROA theory:-

- The generation of cash flows by the asset, while modelling risk as the variance of the cash flows, both measured in some currency. However, naval ships don't generate cash flows measureable in any currency. So non-monetary measures of value and risk are necessary;

- The existence of a market to accurately price the assets using an "equivalent martingale measure", also known as the risk-neutral measure [24]. But no market exists for naval ships to incorporate the risk aversion of the decision makers into the price of the asset, so another mechanism is required;

- The market is tacitly assumed to be composed of many rivals and agents, each sufficiently small that no action taken by any agent will have a measureable effect on the market behaviour. However, this assumption is incorrect in many naval domains where the decision making process is interactive between a few major "agents", with various consequences on the operating environment.

Addressing the above shortcomings through PB-ROA and the mathematical procedure is comprehensively discussed by Knight [23]. In short it can be said that:

- Utility Theory can be used to define value of assets in the absence of cash flows;

- Prospect Theory helps to model the loss-averse nature of navies through riskadjustment of the value of naval options;

- Game Theory is used to model the interdependencies of naval options and the resulting feedback to the operating environment.

The following sections outline ongoing work on how the use of PB-ROA in concept naval ship design and architectural modelling of the concept ship design could be 
brought together to address TLS aspects, such as operational and through-life adaptability.

\subsection{CASE STUDY: MISSION BAY ADAPTABILITY}

As explained in Section 3.1, through-life supportability performance and architectural decisions are tightly linked together. This simplified example shows how the PB-ROA framework might be used to introduce some degree of quantification of adaptability when comparing the extent of adaptability features for a set of ship design variants.

This example considers a blue-water navy frigate sized combatant with a wide operational profile, including wartime and peacetime operations as well as anti-piracy and policing activities. Being able to deliver satisfactory performance across such a wide operational profile requires quick adaptation to meet new requirements and roles. The concept of introducing a large mission bay in modern naval combatants is seen as a capability enabler, able to significantly improve the adaptability performance of the vessel, both operational and through-life.

In this example, the naval option available to the ship operator is to reconfigure the mission bay when new operational tasks arise. Applying Knight and Singer's approach, outlined in [21] and [22], two design variants have been investigated. For simplicity, the adaptability performance of both designs has been measured in terms of how many Twenty-Foot Equivalent Unit (TEU) containers can be fitted into the mission bay after its reconfiguration. This approach is considered reasonable as containerised mission support equipment has already been employed in both the USN LCS variants [20].

The mission bay in the proposed baseline design variant has a fixed area. The other variant also has the same initial mission bay size, but it can provide the ship operator with the capability to enlarge the mission bay by 'consuming' the 'soft areas' (e.g. accommodation spaces, dining halls and stores) located adjacent to the mission bay. The use of 'soft areas' for rapid conversion of spaces was proposed by Gates [25] to implement the 1980's UK modular proposal known as Cellularity.

\section{3 (a) PB-ROA Implementation Procedure}

In using the PB-ROA framework to help decide which design variant should be chosen, the relevant set of risk factors that a particular naval option is exposed to need to be identified first.

In this example, the relevant risk factor is taken as the number of vessels within the fleet, denoted as $x$, to undergo the mission bay reconfiguration. From [21] and [22], if the initial fleet size is $n$, and the number of new vessels to be acquired is $m$, then the binomial distribution may be used to model the probability density function associated with the risk factor:- 


$$
p(x, \beta)=\left(\begin{array}{c}
n+m \\
x
\end{array}\right) \beta^{x}(1-\beta)^{n+m-x}
$$

Where $\beta$ is the probability for any one vessel within the fleet being considered for the mission bay reconfiguration. Knight and Singer [21] refer to the probability density function, $\mathrm{p}(\mathrm{x}, \beta)$ simply as the real probability measure and it can be calculated for all values of $\beta \in[0,1]$.

The next step in deciding whether to choose the baseline or the cellular design variant as a new vessel, is to measure the value of the naval option using utility scores through the following equations [23]:-

$$
\begin{aligned}
& u_{1}(c(x))=\frac{1}{\xi_{1}}-e^{-a c_{1} x} \\
& u_{2}(c(x))=\frac{c_{2}}{c_{1}}\left(\frac{1}{\xi_{2}}-e^{-a c_{2} x}\right)
\end{aligned}
$$

Where subscripts 1 and 2 denote the baseline and cellular design variants respectively. The symbols are defined as:-

- $u-$ Value of naval option to be measured

- $c$ - Design capability (in this case is the number of TEUs the mission bay can accommodate)

- $\quad \xi$ - Design complexity metric

- $a$-Steepness of the utility curves

Knight and Singer [21] define the design complexity metric as a known quantity, a direct result from the design features, and an important measure for differentiating between design variants with similar capabilities.

The steepness of the utility curves is an important measure as its value indicates the attitude of the designers towards risk. In short, it is interpreted as:-

- $a>0$ for risk-aversion

- $a=0$ for risk-neutrality

- $a<0$ for risk-seeking

Traditional expected utility hypothesis assumes all risk-aversion is captured by the shape of the utility curves (i.e. the value of a) and ignores human attitudes towards risk [24]. This approach employs the real probability measure (Equation 1) when evaluating options (Equations 2 and 3), naval or otherwise.

However, the reliability of the results obtained through expected utility hypothesis is questionable as ship designers, especially in the naval domain, always have a riskaverse attitude due to the high value of assets like human life and naval ships. PB- 
ROA accounts for this through Prospect Theory and using the risk-adjusted probability measure when evaluating naval options.

The risk-adjusted probability measure is defined as the product of the real probability measure and the marginal utility [21]:-

$$
q(x) \propto p(x) \times u^{\prime}(c(x))
$$

Where $\mathrm{q}(\mathrm{x})$ is the risk-adjusted probability measure for the event $\mathrm{x}$ (i.e. mission bay reconfiguration), $\mathrm{p}(\mathrm{x})$ is the real probability measure for the event $\mathrm{x}$, and $\mathrm{u}^{\prime}$ is the marginal utility for the event $\mathrm{x}$ defined as:-

$$
u^{\prime}(c(x))=\frac{\partial u}{\partial c} \mid c=c(x)
$$

Hence the risk-adjusted probability measure for each design variant is defined as [21]:-

$$
\begin{aligned}
& q_{1}(x) \propto\left(\begin{array}{c}
n+m \\
x
\end{array}\right) \beta^{x}(1-\beta)^{n+m-x} \times a e^{-a c_{1} x} \\
& q_{2}(x) \propto\left(\begin{array}{c}
n+m \\
x
\end{array}\right) \beta^{x}(1-\beta)^{n+m-x} \times a \frac{c_{2}}{c_{1}} e^{-a c_{2} x}
\end{aligned}
$$

To calculate the risk-adjusted probability measure for each variant, the following intermediate variables for utilisation and de-utilisation of the option need to be found first [21]:-

$$
\begin{aligned}
& t_{1}^{u}=p \times a e^{-a c_{1} x} \\
& t_{1}^{d}=(1-p) \times a e^{-a c_{1}(n+m-x)} \\
& t_{2}^{u}=p \times a \frac{c_{2}}{c_{1}} e^{-a c_{2} x} \\
& t_{2}^{d}=(1-p) \times a \frac{c_{2}}{c_{1}} e^{-a c_{2}(n+m-x)}
\end{aligned}
$$

The real probability measure, $p$, is found using Equation (1) for all of values of $\beta \in[0,1]$. Hence the intermediate variables resulting from Equations (8), (9), (10) and (11) correspond to the same range $\beta \in[0,1]$. The superscripts $u$ and denote the utilisation and de-utilisation, respectively, of the option to reconfigure the mission bay.

Probability theory principles make it necessary for all risk-adjusted probability measures to be re-normalised such that they integrate to one for each design variant [21]. This is carried out through the following equations:- 


$$
\begin{aligned}
& q_{1}=\frac{t_{1}^{u}}{t_{1}^{u}+t_{1}^{d}} \\
& q_{2}=\frac{t_{2}^{u}}{t_{2}^{u}+t_{2}^{d}}
\end{aligned}
$$

The results from Equations (12) and (13) can then be used to calculate the risk-adjusted value of the design variants through Equations (2) and (3).

\section{3 (b) Case Study Results}

The following arbitrary assumptions were made in order to carry out the case study:-

- The steepness of the exponential utility curves was assumed to be, $a=0.002$.

- The baseline design variant has a fixed mission bay area of $300 \mathrm{~m}^{2}$. Considering a standard TEU container and allowing 10\% extra space for accessibility, the mission bay has the capability to accommodate 18 TEUs. Hence $c_{1}=18$

- The baseline design variant was assumed to have a fixed design complexity metric, $\xi_{1}=1$

- Three cases were tested for the increased TEU capacity of the cellular design. The mission bay was enlarged by $1 / 3,1 / 2$, and $2 / 3$. Space requirement per TEU was unchanged.

- The design complexity metric was also varied in steps of 0.01 over the arbitrary range between 1.01 and 1.03 for the cellular design.

For the case of $n=0$ and $m=1$ (i.e. initial fleet size is zero and acquiring one new vessel, see Section 5.3(a)), the risk-adjusted value of both design variants was measured with the inputs listed above. The crossover point between the risk-adjusted utility curves could help answer the important question about which design variant should be chosen. Figure 8 illustrates how the risk-adjusted utility varied with the probability of the mission bay being reconfigured to host containerised mission support equipment. The Inputs for Figure 8 were $c_{2}=27$ TEUs (i.e. mission bay is $50 \%$ larger than the baseline design variant) and $\xi_{2}=1.02$ (i.e. design complexity metric is $2 \%$ larger than the baseline design variant). 


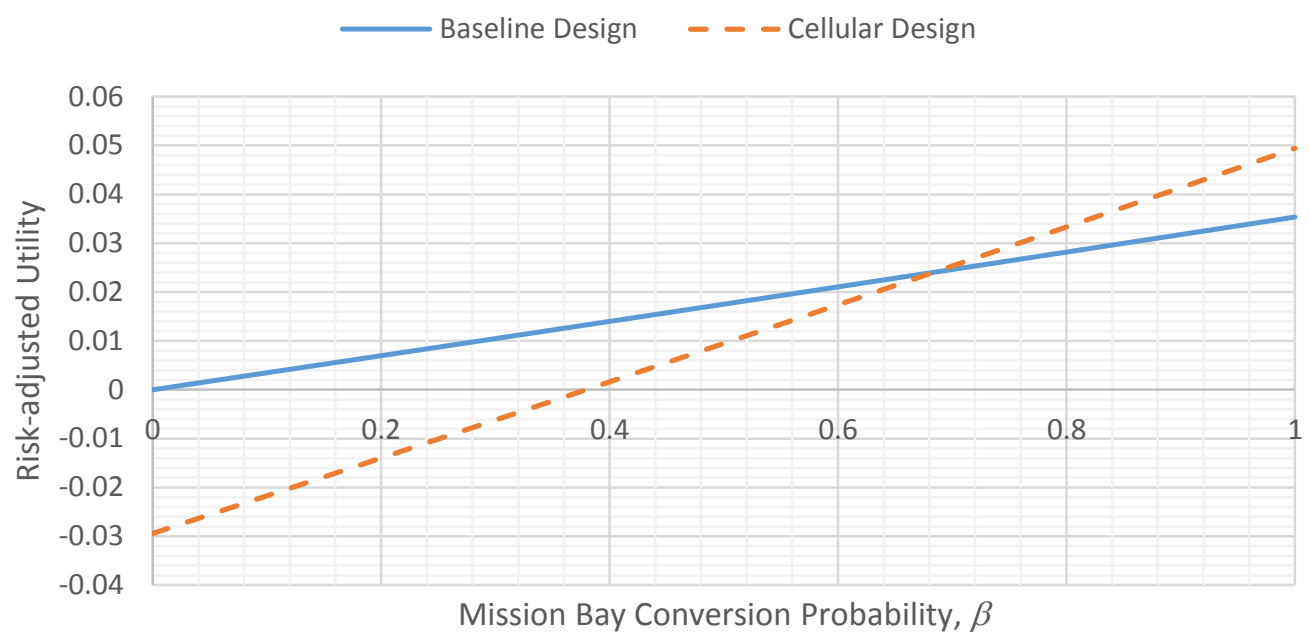

Figure 8: Variation of risk-adjusted utility with mission bay conversion probability

In this case, the probability threshold at which it would be more desirable to use the cellular design is approximately $70 \%$.

Figure 9 demonstrates how the probability decision threshold varied with the factor of mission bay enlargement for each of the design complexity metric values.

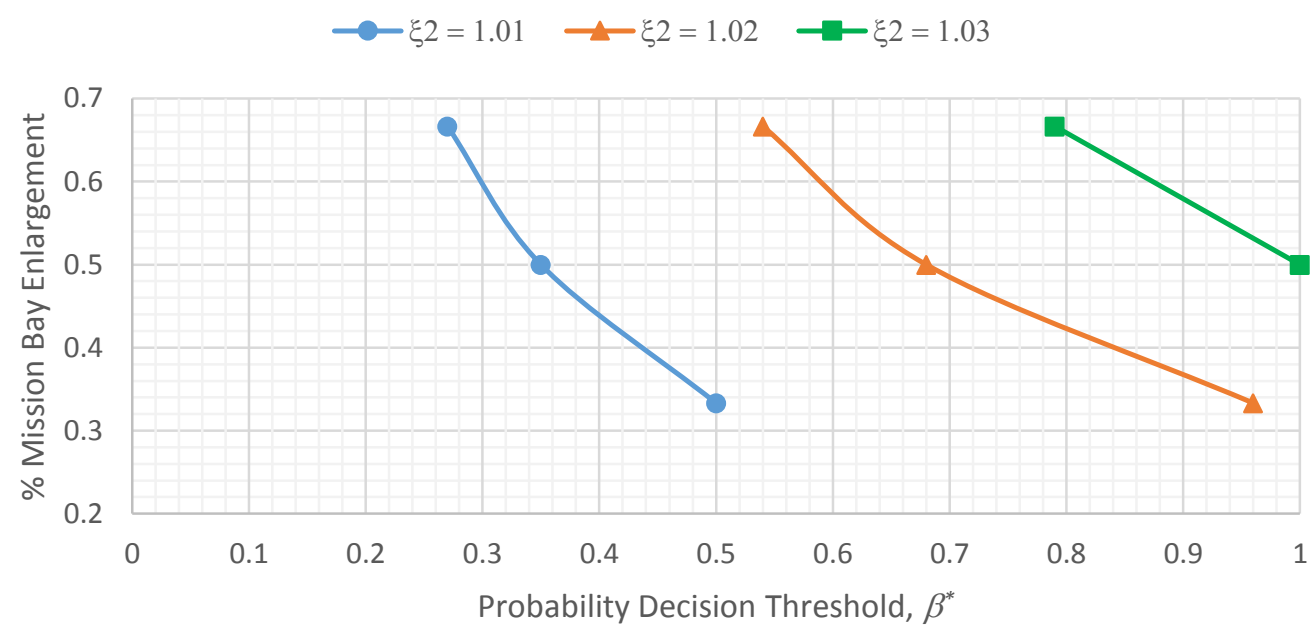

Figure 9: Variation of mission bay enlargement with mission bay conversion probability

Note that the curve showing the results for the highest design complexity metric, $\xi_{2}=$ 1.03 , does not include the case in which the mission bay was enlarged by $1 / 3$ of the original area. This is because with the highest design complexity metric, enlarging the mission bay by $1 / 3$ was not sufficient for the risk-adjusted utility of the cellular design to overtake that of the baseline design. This is illustrated in Figure 10. 


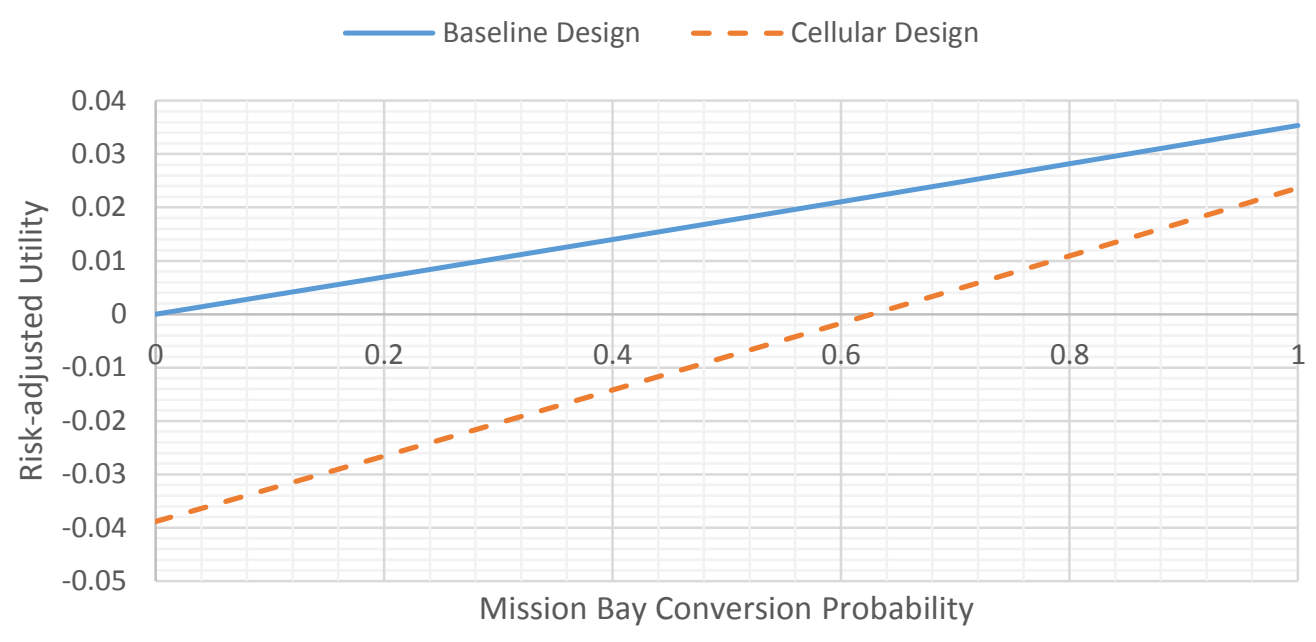

Figure 10: Illustration of no crossover point for the case $\xi_{2}=1.03, c_{2}=24$

\section{3 (c) Discussion of Case Study Results}

From Figure 8, the cellular design is seen to have negative utility up until the mission bay conversion probability $(\beta)$ is approximately $40 \%$, and the largest negative utility occurs when the ship is not used at all (i.e. $\beta=0 \%$ ). In all the cases tested, the cellular design was observed to have varying degrees of negative utility up to certain $\beta$ values. Based on the assumption that the cellular design always has a higher design complexity metric than the baseline design, the negative utility may be interpreted as being due to the larger purchase and through-life maintenance costs most likely associated with a more complex design. However, this assumption is subject to verification.

The probability decision threshold is also an important observation. From Figure 8, it means that if the ship operator believes there is a $70 \%$ or more probability that the mission bay will be reconfigured to host containerised mission support equipment, the cellular design should be chosen. For smaller probability values the baseline design will be better value.

The probability decision threshold value also depends on the assumed steepness of the utility curves. This parameter (Section 5.3(a)) indicates the attitude of the designers towards risk. Due to the risk-averse nature of naval ship design, a positive value of 0.002 was arbitrarily chosen. Changing this value to 0.0015 (i.e. less risk-averse) while keeping $\xi_{2}$ and $c_{2}$ as they were, will shift the probability decision threshold from $70 \%$ to $90 \%$. Similarly, if increased to 0.0025 (more risk-averse) the probability decision threshold will be lowered to approximately $55 \%$. Note that these steepness values are by no means representative of any real navy's utility curves.

From Figure 9, it can be seen for constant values of $\xi_{2}$, the probability decision threshold is smallest for the largest enlargement of the mission bay. In other words, choosing the cellular design will be increasingly the more sensible option the more the 
mission bay is enlarged, as one might expect. However, a great deal of uncertainty is associated with the underlying assumption. In this example, it was assumed that as the mission bay is enlarged in the cellular design, the design complexity metric could be kept constant, as illustrated by the three separate plots in Figure 9. In reality however, aspects such as Vertical Centre of Gravity (VCG) and topside arrangement of the ship will be increasingly affected the larger the mission bay becomes, with possible effects on the design complexity metric.

In addition to the above, perhaps more obvious aspects, other knock-on effects could result from having a reconfigured mission bay. Examples include but are not limited to the following:-

- Will more crew be required on-board to operate the containerised mission support equipment?

- How will requirements for hotel systems such as power generation be affected?

- Will the need for command and control be affected by hosting containerised mission support equipment?

$\bullet$

\section{BASELINE SHIP DESIGN FOR THE RESEARCH PROJECT}

The main merit of the research reported is seen to be the proposal of a supportability evaluation approach to assist naval ship designers during ESSD. Thus the baseline design and other design variants can be used to demonstrate the robustness of the approach and to provide a stimulus to the discussions needed to develop the approach. Hence, ongoing work on the current baseline design, to which this approach will be applied, is briefly outlined in this section.

Having entered the Demonstration Phase in February 2015 [26], the Type 26 GCS will form the backbone of the Royal Navy for the next 40 years. The programme consists of the design, plan for manufacture and support solution of eight Anti-Submarine (ASW) and five general purpose frigates [27]. While not using or planning to use any current official design solutions, the specification for the general purpose frigates has been drawn on to form the baseline design broad requirements set for these DfS studies, and to demonstrate the new UCL ship concept design toolset (Section 4).

Major ship characteristics of the research project baseline design, at the time of writing, are given in Table 1.

\begin{tabular}{|l|r|l|}
\hline Total Enclosed Volume & 21500 & $\mathrm{~m}^{3}$ \\
\hline Deep Displacement & 5565 & $\mathrm{te}$ \\
\hline Waterline Length & 126.5 & $\mathrm{~m}$ \\
\hline Overall Length & 134.5 & $\mathrm{~m}$ \\
\hline Waterline Beam & 18 & $\mathrm{~m}$ \\
\hline Amidships Upper Deck Beam & 20.5 & $\mathrm{~m}$ \\
\hline Amidships Draught & 5.5 & $\mathrm{~m}$ \\
\hline
\end{tabular}

Table 1: Baseline ship major design characteristics 
Major payload equipment:-

- $1 \times$ BAE Systems $127 \mathrm{~mm}$ naval gun;

- $2 \times$ Quad MK 141 Harpoon launchers;

- $6 \times 8$ cell MK 41 VLS;

- $2 \times$ Phalanx CIWS;

- $64 \times$ Quad packed MBDA Sea Ceptor SAMs;

- $1 \times$ Hangared Merlin HM.2;

- $1 \times$ Medium range 3-D BAE Systems 997 Artisan radar.

- $1 \times$ Medium range Thales type 2050 bow sonar

\section{CONCLUSIONS AND FURTHER WORK}

This paper presents ongoing work at UCL to develop a new ship concept design software tool. It outlines the disadvantages of using sophisticated (fully 3D) CAD models in ESSD and how they could be tackled by the new developmental tool.

The analytical framework proposed by Knight [23] was used to evaluate the adaptability of a blue-water navy frigate sized combatant. In the absence of a market and cash flows, the PB-ROA framework allows the evaluation of naval options in nonmonetary terms through Utility Theory. The framework also considers the risk-averse nature of naval domains through Prospect Theory.

Seeing the concept of a large mission bay as a capability enabler, two design variants were investigated; the baseline design with a fixed mission bay area, and the cellular design with the option to enlarge the original mission bay. For simplicity of the analysis, design capability was defined as the number of TEUs that the mission bay can accommodate.

The results from this simple case study demonstrate how PB-ROA can be used to compare the value of design variants in terms of their adaptability performance. The probability decision threshold and negative utility values were significant observations from the case study.

Further work will include a more detailed and multidimensional consideration of the risk factors and the capability parameters used in the example analysis, as well as incorporation of game theoretic interdependencies (the third aspect of the PB-ROA framework in addition to Utility and Prospect theories), which were not considered in this case study.

Parallel to developing the proposed approach, ongoing work on the baseline design will continue. To discern any distinction in applying DfS metrics across ship roles and types, it is intended that the investigation will extend from the baseline design to include studies of smaller, larger and multihull combatant designs meeting different specifications. 


\section{ACKNOWLEDGEMENTS}

Financial support for the first author's PhD studentship has been provided through an EPSRC industrial CASE award with BAE Systems sponsorship and is greatly acknowledged, as is the latter's ongoing input to the research project.

\section{REFERENCES}

1. ANDREWS, D.J., 'A Creative Approach to Ship Architecture', Transactions of RINA, Vol. 145, 2003.

2. UK MOD., 'The Defence Logistic Support Chain Manual - Integrated Logistic Support', Integrated Logistic Support Policy JSP 886, 2013.

3. BROWN, D.K., and ANDREWS, D.J., 'The Design of Cheap Warships', Proceedings of the International Naval Technology Expo 1980, Rotterdam, Netherlands, February 1980.

4. PAWLING, R.J., ANDREWS, D.J., PIKS, R., SINGER, D., DUCHATEAU, E., HOPMAN, H., 'An Integrated Approach to Style Definition in Early Stage Design', Proceedings of COMPIT 2013, Cortona, Italy, May 2013.

5. ANDREWS, D.J., 'Creative Ship Design', Transactions of RINA, Vol. 123, 1981.

6. BROWN, D.K., 'Naval Architecture', US Naval Engineers Journal, Vol. 105 (1), 1993.

7. ANDREWS, D.J., 'An Integrated Approach to Ship Synthesis', Transactions of RINA, Vol. 128, 1986.

8. ANDREWS, D.J., DICKS, C.A., 'The Building Block Design Methodology Applied to Advanced Naval Ship Design', Proceedings of IMDC 1997, Newcastle, UK, May 1997.

9. TUPPER, E.C., 'Introduction to Naval Architecture, Fifth Edition', Butterworth-Heinemann, UK, 2013.

10. ANDREWS, D.J., 'Marine Requirements Elucidation and the Nature of Preliminary Ship Design’, IJME Vol. 153, Part A1, 2011.

11. ANDREWS, D.J., PAWLING, R.J., 'The Impact of Simulation on Preliminary Ship Design', Proceedings of IMDC 2009, Trondheim, Norway, May 2009.

12. MUNOZ, J.A., FORREST, C.J.M., 'Advantages of Software Integration from Initial Design Through to Production Design', Proceedings of ICCAS 2002, Malmö, Sweden, September 2002. 
13. ANDREWS, D.J., PAWLING, R.J., 'SURFCON - A 21st Century Ship Design Tool', Proceedings of IMDC 2003, Athens, Greece, May 2003.

14. PAWLING, R.J., PIPERAKIS, A.S., ANDREWS, D.J., 'Developing Architecturally Oriented Concept Ship Design Tools For Research and Education', Proceedings of IMDC 2015, Tokyo, Japan, May 2015.

15. PAWLING, R.J., ANDREWS, D.J., 'Large Unmanned Vehicles and the Minor War Vessel', Proceedings of Warships 2013, Bath, UK, June 2013.

16. ANDREWS, D.J., CASAROSA, L., PAWLING, R.J., GALEA, E., DEERE, S., LAWRENCE, S., 'Integrating Personnel Movement Simulation into Preliminary Ship Design', IJME Vol. 150 Part A1, Discussion IJME, Vol. 150, Part A3, 2008.

17. PAWLING, R.J., GRANDISON, A., LOHRMANN, P., MERMIRIS, G., PEREIRA DIAS, C., 'Methods and Tools for Risk-Based Approach to Fire Safety in Ship Design', Ship Technology Research/Schiffstechnik, Vol. 59(3) pp. 38-49, 2012.

18. PIPERAKIS, A.S., ANDREWS, D.J., 'A Comprehensive Approach to Survivability Assessment in Naval Ship Concept Design', IJME Vol. 156, Part A4, 2014.

19. ANDREWS, D.J., BURGER, D., ZHANG, J., 'Design for Production Using the Design Building Block Approach', Transactions of RINA, 2005.

20. DOERRY N. H., 'Institutionalising Modular Adaptable Ship Technologies', Journal of Ship Production and Design Vol. 30(16) pp. 126-141, 2012.

21. KNIGHT J. T., SINGER D. J., 'Prospect Theory-based Real Options Analysis for Non-commercial Assets', ASCE-ASME Journal of Risk and Uncertainty in Engineering Systems, 2015.

22. KNIGHT J. T., SINGER D. J., 'Applying Real Options Analysis to Naval Ship Design', ASNE, 2014.

23. KNIGHT J. T., 'A Prospect Theory-Based Real Option Analogy for Evaluating Flexible Systems and Architectures in Naval Ship Design', PhD Thesis, University of Michigan, 2014.

24. GISIGER N., 'Risk-Neutral Probabilities Explained', Journal of Economic Literature, 2010.

25. GATES P. J., 'Cellularity: An Advanced Weapon Electronics Integration Technique', Transactions of RINA, 2012.

26. FALLON, M., 'Type 26 Global Combat Ship', House of Commons: Written Statement (HCWS289), 2015. 
27. PRICE, G., WILSON D., ROTTIER, P., KYTE, P., 'UK Type 26 Global Combat Ship Support Solution Decision Point Project', Proceedings of INEC 2014, Amsterdam, Netherlands, May 2014.

\section{AUTHORS BIOGRAPHY}

Syavash Esbati completed his MEng in Naval Architecture and Marine Engineering at UCL in 2013. He is currently a PhD student at UCL DRC investigating naval ship through-life support in preliminary ship design.

Dr Alexander S. Piperakis, MEng, PhD, AMRINA, completed the MEng in Mechanical Engineering at the University of Bath in 2008. He then joined the UCL DRC as a research student investigating naval ship survivability in preliminary ship design. Gaining his PhD in 2013, he has continued his research in the DRC in projects involving the incorporation of human factors into Risk-Based Design of ships and preliminary ship design methodology.

Dr Rachel J. Pawling, MEng, PhD, AMRINA, is the BMT DSL Research Fellow for Naval Ship Design at UCL. She completed the MEng in Naval Architecture and Marine Engineering at University College London in 2001 and then joined the UCL DRC as a research student investigating the application of the Design Building Block approach to innovative ship design. Gaining her PhD in 2007, she has continued her research both in the DRC and via a secondment in industry. She is the recipient of the 2008 RINA Samuel Baxter Prize, 2009 RINA WHC Nicholas Prize, and 2012 COMPIT GL Award for papers describing her research.

Professor David J. Andrews, FREng, FRINA, FIMechE, RCNC, was appointed Professor of Engineering Design at UCL in September 2000 following an extensive career in ship and submarine design and acquisition management in the UK Defence Procurement Agency. He leads UCL's design research in computer aided ship design, design methodology and design practice. He is a Fellow of RINA, Fellow of IMechE and was elected to the Royal Academy of Engineering in 2000 and as a Vice President of RINA in 2006. He is the International Committee Chairman of the IMDC. 


\section{Appendix 5 Ship Design Case Studies}

\section{Baseline Frigate Design}

\section{Margins and Equipment Mountings}

The ability to alter or enhance the weapons fit and capabilities of a warship during its lifetime is entirely dependent on the adopted margin policy (Brown and Andrews, 1980). Hence considering margins as future investment opportunities (Brown, 1987) that can have significant effects on the ship's size, cost and performance, a clear margin policy should be developed prior to starting a ship design (Heather, 1990). Such policy should consider margins of weight, space, Vertical Centre of Gravity (VCG), stability, strength, propulsion power, electric loads, and ship services (Brown, 1987) (Heather, 1990). Margins are either applied across the traditional weight groups (Gale, 1975) or rather consciously allocated to specific spaces and compartments where the requirement is more likely to develop (Andrews, 1985).

The adopted margin policy should allow for the uncertainty in the design of ships, weapons and systems, the planned upgrades and addition of new equipment, and the unplanned through-life growth. The three types of margins generally considered are:

- Design and Construction Margins are weight and space margins (UCL, 2013a), allocated either to the weight or functional groups, that help handle the uncertainties associated with developing and constructing the ship (Andrews and Brown, 1982), allow for errors and omissions (Heather, 1990), and account for the anticipated relatively minor changes in the top level requirements as the design is developed (Gale, 1975);

- Board Margins are weight and space margins (UCL, 2013a) that allow for future requirement additions to the ship (Andrews and Pawling, 2007) by enabling the upgrade of existing ship equipment and the insertion of new ones (Andrews and Brown, 1982) (Heather, 1990). Andrews (1987) suggested that the current practice of applying Board Margins at the whole ship level should be replaced with an architecturally-based approach to better reflect the configurational and associated ships services demands of future changes. Applying Board Margins at specific locations is considered to deliver genuine 
adaptability by enabling specific capability enhancements without significantly affecting the overall ship size;

- Growth Margins are weight margins (UCL, 2013a) applied at the whole ship level so that the unplanned, inevitable accumulation of stuff does not have disproportionate effects on the safety or performance of the ship (Andrews and Brown, 1982) (Heather, 1990).

Table A5. 1 shows the margins used in the development of the Baseline Frigate Design as instructed by the guidelines provided for the UCL MSc course ship design exercise (UCL, 2013a).

\begin{tabular}{|l|l|r|}
\hline Design and Construction Margins - Weight Group 1 & Space & $0 \%$ \\
\cline { 2 - 3 } (Hull) & Weight & $+15 \%$ \\
\hline Design and Construction Margins - Weight Group 2 & Space & $+5 \%$ \\
\cline { 2 - 3 } (Personnel) & Weight & $+5 \%$ \\
\hline $\begin{array}{l}\text { Design and Construction Margins - Weight Group 3 } \\
\text { (Ship Systems) }\end{array}$ & Space & $+2 \%$ \\
\cline { 2 - 3 } $\begin{array}{l}\text { Design and Construction Margins - Weight Group 4 } \\
\text { (Main Propulsion) }\end{array}$ & Weight & $+5 \%$ \\
\cline { 2 - 3 } $\begin{array}{l}\text { Design and Construction Margins - Weight Group 5 } \\
\text { (Electrical Power) }\end{array}$ & Weight & Space \\
\cline { 2 - 3 } $\begin{array}{l}\text { Design and Construction Margins - Weight Group 6 } \\
\text { (Payload) }\end{array}$ & Weight & Space \\
\cline { 2 - 3 } & Weight & $+5 \%$ \\
\hline $\begin{array}{l}\text { Design and Construction Margins - Weight Group 7 } \\
\text { (Variables) }\end{array}$ & Space \\
\cline { 2 - 3 } & Weight & $+10 \%$ \\
\hline \multirow{2}{*}{ Board Margins } & Space & $+4 \%$ \\
\cline { 2 - 3 } & Weight & $+5 \%$ \\
\hline \multirow{2}{*}{ Growth Margin } & Space & $+5 \%$ \\
\cline { 2 - 3 } & Weight & $+7.5 \%$ \\
\hline
\end{tabular}

Table A5. 1: Weight and space margins used in the development of the Baseline Frigate Design (UCL, 2013a)

The following were taken into consideration when applying the margins:

- Design and Constructions Margins apply only if there are uncertainties regarding the weight and space information of individual equipment and spaces, hence do not apply if the information is directly obtainable from genuine manufacturers' data sheets and brochures;

- Board Margins only apply to the Lightship weight and not the variables; 
- Growth Margins should take into consideration the issue of short or long life ship. The Type 26 has been described by the (UK MoD, 2015a) as the future backbone of the Royal Navy for at least 40 years. Hence the Growth Margins, set to be $0.5 \%$ per year, were applied with the assumption that the accumulated growth will be removed during a major refit after 15 years.

The weight of equipment mountings required for noise and vibration reduction purposes vary depending on the weight group. Table A5. 2 shows the advisory values provided for the UCL MSc course ship design exercise (UCL, 2013a).

\begin{tabular}{|l|l|r|}
\hline Weight Group 2 (Personnel) & Weight & $+2.5 \%$ \\
\hline Weight Group 3 (Ship Systems) & Weight & $+5 \%$ \\
\hline Weight Group 4 (Main Propulsion) & Weight & $+30 \%$ \\
\hline Weight Group 5 (Electrical Power) & Wight & $+10 \%$ \\
\hline Weight Group 6 (Payload) & Wight & $+10 \%$ \\
\hline
\end{tabular}

Table A5. 2: Weight of equipment mountings used in the development of the Baseline Frigate Design for noise and vibration attenuation purposes (UCL, 2013a)

\section{Hullform Geometry Coefficients}

According to Griethuysen (1993), ship designers are generally inconsiderate or unaware of the underlying reasons that cause form parameters to vary from one type of warship to another. As a general rule, each warship type is associated with a collection of typical values and working within the small range of parameters, the ship designer attempts to satisfy the often conflicting operational and performance requirements and improve the ship design. Griethuysen (1992) listed some, though by no means all of the significant constraints and performance aspects that drive the process of selecting the form parameters for different types of warships as:

- Absolute dimensional constraints;

- Layout considerations;

- Resistance, propulsion, range and fuel cost;

- Seakeeping;

- Manoeuvring;

- Structural considerations;

- Survivability;

- UPC and TLC. 
In the first iteration of the design process, the initial sizing has classically relied heavily on extensive amounts of existing ship data and scaling ratios to produce the first estimates of weight and space requirements and costs (Brown and Andrews, 1980). As part of the work aimed at creating a framework to help understand and improve the process of selecting form parameters, Griethuysen (1992) listed some of the geometrical characteristics and form parameters associated with different types of warships. Since the baseline design for this research is representative of a blue-water navy frigate-sized combatant, the advisory values typical of frigates provided by Griethuysen (1992, 1993) and UCL (2013a) were used to begin the initial sizing process. These values are shown in Table A5. 3 and Table A5. 4.

\begin{tabular}{|l|r|}
\hline Payload Volume Fraction (PVF) & $0.16-0.22$ \\
\hline Total enclosed volume & $5,000-40,000 \mathrm{~m}^{3}$ \\
\hline Overall density & $0.27-0.33 \mathrm{te} / \mathrm{m}^{3}$ \\
\hline
\end{tabular}

Table A5. 3: Typical frigate characteristics (UCL, 2013a)

\begin{tabular}{|l|r|}
\hline Volume of displacement $(\nabla)$ & $3,000-5,000 \mathrm{~m}^{3}$ \\
\hline Waterline length to waterline beam ratio & $8-9.5$ \\
\hline Overall length to amidships hull depth ratio & $<12$ for concept design \\
\hline Waterline beam to amidships hull depth ratio & $1.4-1.6$ \\
\hline $\begin{array}{l}\text { Waterline beam to amidships Deep draught } \\
\text { ratio }\end{array}$ & $2.8-4$ \\
\hline Circular M & $7-8.5$ \\
\hline$C_{B}$ & $0.4-0.56$ \\
\hline$C_{M}$ & $0.7-0.85$ \\
\hline$C_{P}$ & $0.57-0.66$ \\
\hline
\end{tabular}

Table A5. 4: Typical frigate characteristics (Griethuysen, 1992, 1993)

\section{Ship Resistance and Propulsion}

The widely applied Holtrop and Mennon method was used to estimate the total ship resistance and the required propulsion power of the Baseline Frigate Design. Like all resistance prediction methods, Holtrop and Mennon is also subject to applicability limitations in terms of certain hullform parameters, as shown in Figure A5. 1. 


\begin{tabular}{|l|c|c|c|c|c|c|c|}
\hline \multicolumn{1}{|c|}{ Ship type } & Max & \multicolumn{2}{|c|}{ Cp } & \multicolumn{2}{|c|}{ L/B } & \multicolumn{2}{|c|}{ B/T } \\
\cline { 4 - 9 } & Froude & Min & Max & Min & Max & Min & Max \\
& no. & & & & & & \\
\hline Tankers, bulk carries & 0.24 & 0.73 & 0.85 & 5.1 & 7.1 & 2.4 & 3.2 \\
\hline Trawlers, coasters, tugs & 0.38 & 0.55 & 0.65 & 3.9 & 6.3 & 2.1 & 3.0 \\
\hline Containerships, destroyer types & 0.45 & 0.55 & 0.67 & 6.0 & 9.5 & 3.0 & 4.0 \\
\hline Cargo liners & 0.30 & 0.56 & 0.75 & 5.3 & 8.0 & 2.4 & 4.0 \\
\hline RORO ships, car ferries & 0.35 & 0.55 & 0.67 & 5.3 & 8.0 & 3.2 & 4.0 \\
\hline
\end{tabular}

The method subdivides the estimation of total ship resistance into:

$$
R_{\text {Total }}=R_{F}\left(1+k_{1}\right)+R_{A P P}+R_{W}+R_{B}+R_{T R}+R_{A}
$$

Where:

- $\mathrm{R}_{\text {Total }}$ is the total ship resistance;

- $\mathrm{R}_{\mathrm{F}}$ is the frictional resistance according to ITTC-1957 correlation line;

- $1+\mathrm{k}_{1}$ is the hull form factor;

- $\mathrm{R}_{\mathrm{APP}}$ is the appendage resistance;

- $\mathrm{R}_{\mathrm{W}}$ is the wave-making resistance;

- $\mathrm{R}_{\mathrm{B}}$ is the additional pressure resistance of the bulbous bow near the water surface;

- $\mathrm{R}_{\mathrm{TR}}$ is additional pressure resistance of the immersed transform stern;

- $\mathrm{R}_{\mathrm{A}}$ is the model-ship correlation resistance.

Holtrop (1984) provided empirical equations to measure each of the above components of total resistance. Once the total resistance of a ship is estimated, the process shown in Figure A5. 2 is used to estimate the required ship propulsion power, taking into account various efficiency losses, margins and correction factors. 


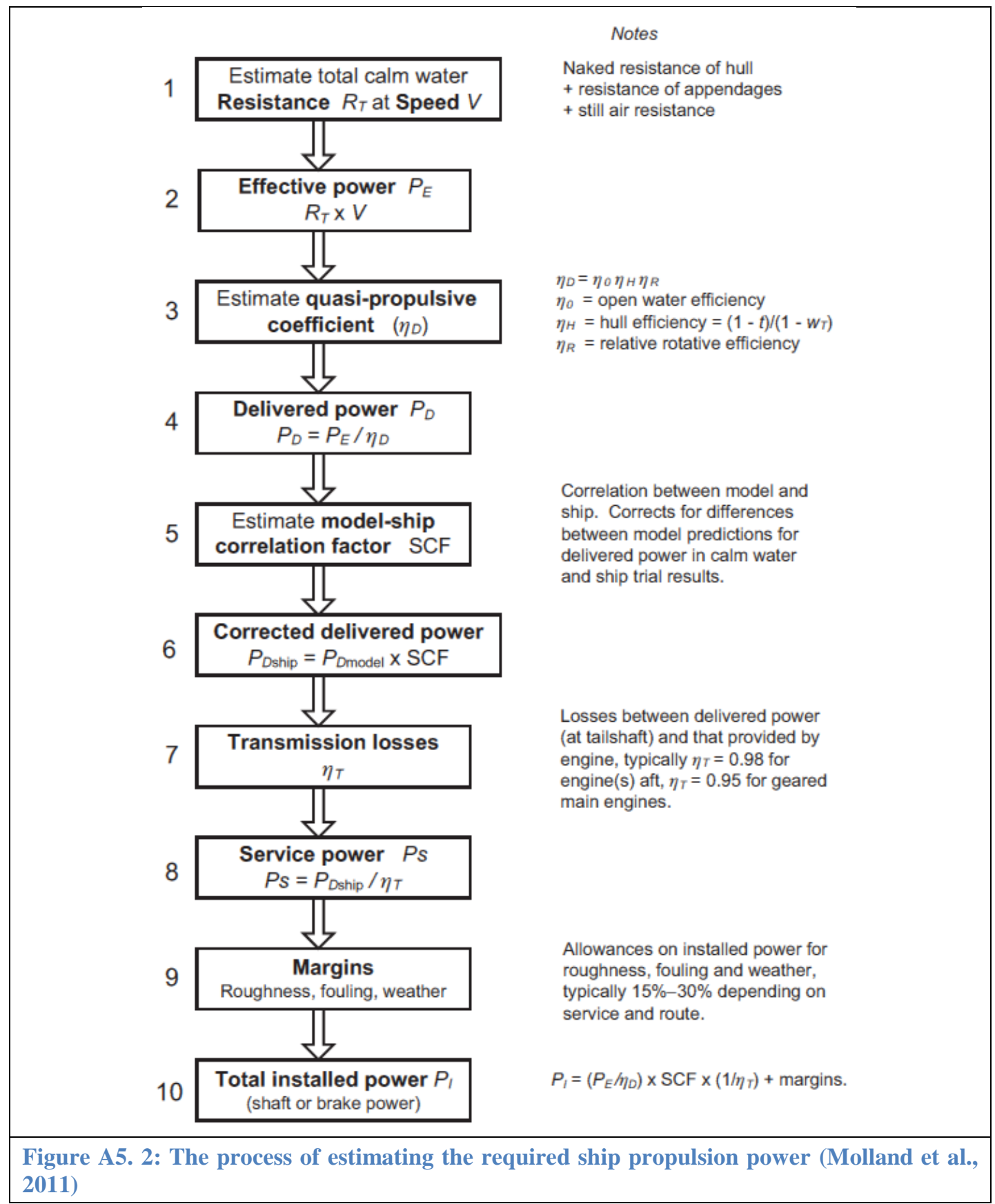

\section{Ship Costing}

Figure A5. 3, produced by NATO (2009), shows the various cost estimation methods and their applicability at each stage of a defence programme. The typical methods used for developing a cost estimate at the Concept Phase are Bayesian, parametric, analogous, expert opinion or rule of thumb techniques, with parametric methods being the most widely used. The parametric method 'estimates costs based upon various characteristics or measurable attributes of the system, hardware and software being estimated. It depends upon the existence of a causal relationship between system costs and these parameters. Such relationships, known as Cost Estimating Relationships 
(CER), are typically estimated from historical data using statistical techniques. If such a relationship can be established, the CER will capture the relationship in mathematical terms relating cost as the dependent variable to one or more independent variables. Examples would be estimating costs as a function of such parameters as equipment weight, vehicle payload or maximum speed, number of units to be produced or number of software lines of code to be written. The CER describes how a product's physical, performance and programmatic characteristics affect its cost and schedule' (NATO, 2007). The advantages of parametric cost estimation include the consistent, quantitative, and non-subjective nature of its inputs, its ability to readily perform what-if sensitivity analyses, and the validity of the output estimates despite the lack of sufficient information at the Concept Phase. Its disadvantages are the large amounts of historical data required by the statistical techniques used to develop the relationships, that the system being designed has to be similar to the systems in the underlying database, and its limited ability to provide low level visibility and reflect on subtle changes (NATO, 2007; UCL, 2013b).

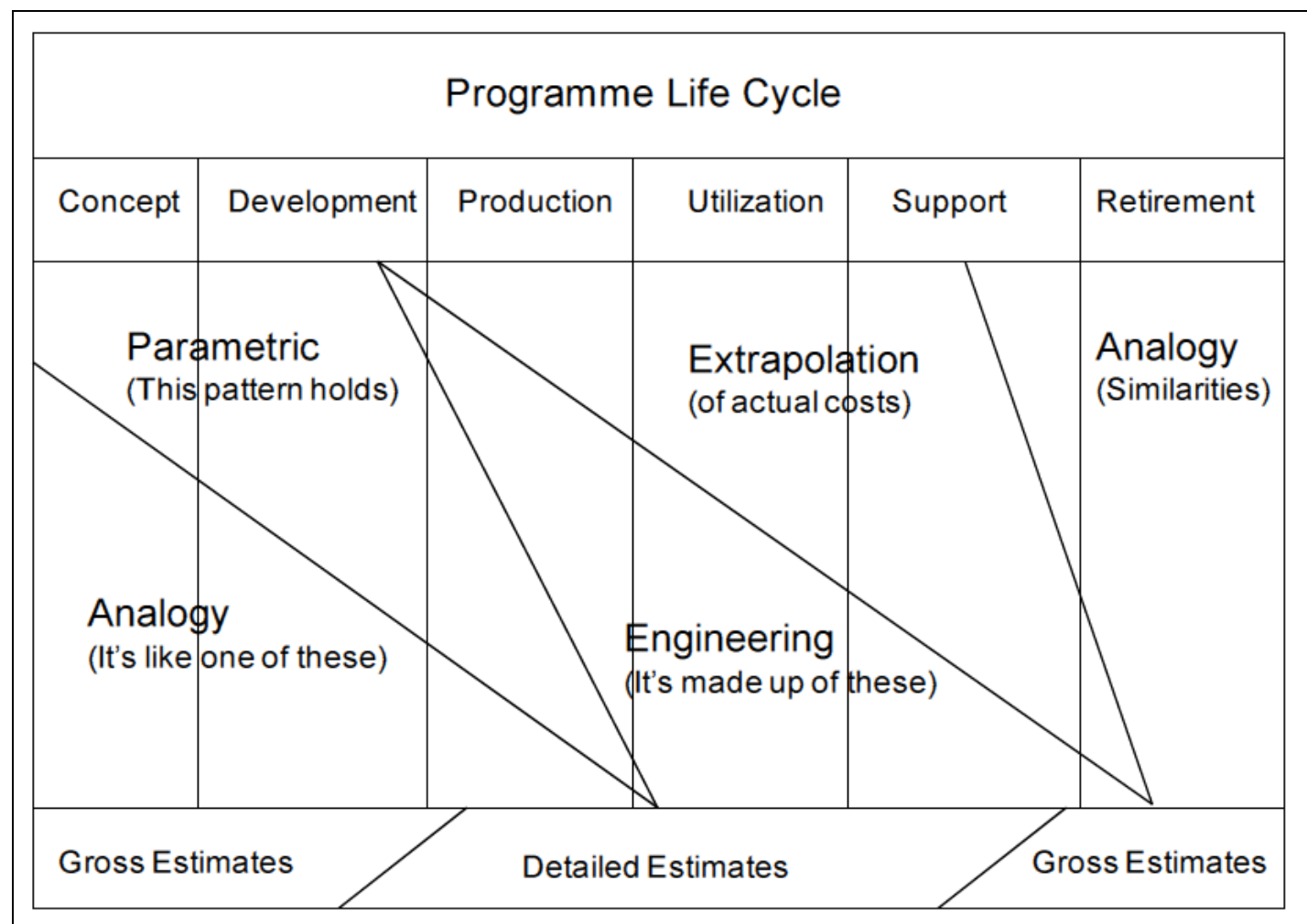

Figure A5. 3: Various cost estimation methods and their applicability at each stage of a defence programme (NATO, 2009) 
Ship costing is a highly data driven process and the quality of the available data determines the costing method to be used and accuracy of the resultant cost estimates (NATO, 2009). Given the limited availability of data at the Concept Phase, parametric ship costing analysis inevitably relies on multiple assumptions. Dirksen (1996) and UCL (2013a, 2013b) listed these as:

- FOC costs as a percentage of UPC;

- The selected shipyard and the associated purchasing overheads;

- Material and labour costs, knowing that shipbuilding is still a labour intensive industry comparing to some other industries (e.g. automotive) (Kålås, 2015);

- Degree of modularity;

- The extent of adopting COTS equipment;

- Repetition effects and learning curves, considering that conditions change over long periods of naval shipbuilding and two warships of the same class are rarely exactly the same (Usher and Dorey, 1982);

- Inflation, interest rates, relative strength of different currencies, and variance of wage rates, influenced by factors not controlled by the ship designers, builders, and operators (Usher and Dorey, 1982);

- Frequency of dockings and refits;

- Degree of external and sub-contractor involvement.

In addition to the above uncertainties, parametric ship costing during ESSD is made more difficult by the reluctance of the involved parties to release costing data on the grounds of confidentiality (Carreyette, 1978).

The parametric method used at UCL (2013b) is mainly applicable to naval ships and largely based on the work by Dirksen (1996), with updates to reflect the Smart Acquisition process and the current UK MoD practice (UK MoD, 2002). For the seven individual weight groups (excluding individual items of equipment), UPC is estimated through cost/tonne relationships for both material and labour costs. The actual cost/tonne values are ship type dependent (i.e. naval or commercial structural standards) and the construction location (i.e. naval yard). For individual items of equipment, UPC is dominated by the cost of discrete items (i.e. gas turbines, diesel engines and missile systems) plus an additional allowance for installation costs. For 
such items of equipment, the UCL (2013b) parametric method provides representative UPC values and the installation costs are estimated in terms of cost/tonne values.

\section{Ship Design Variants}

Baseline Frigate Design Configurational Variant

The complete internal arrangement of the configurational variants of the Baseline Frigate Design (see Table 4.11) and breakdown of the estimated UPC values are provided in the following figures.

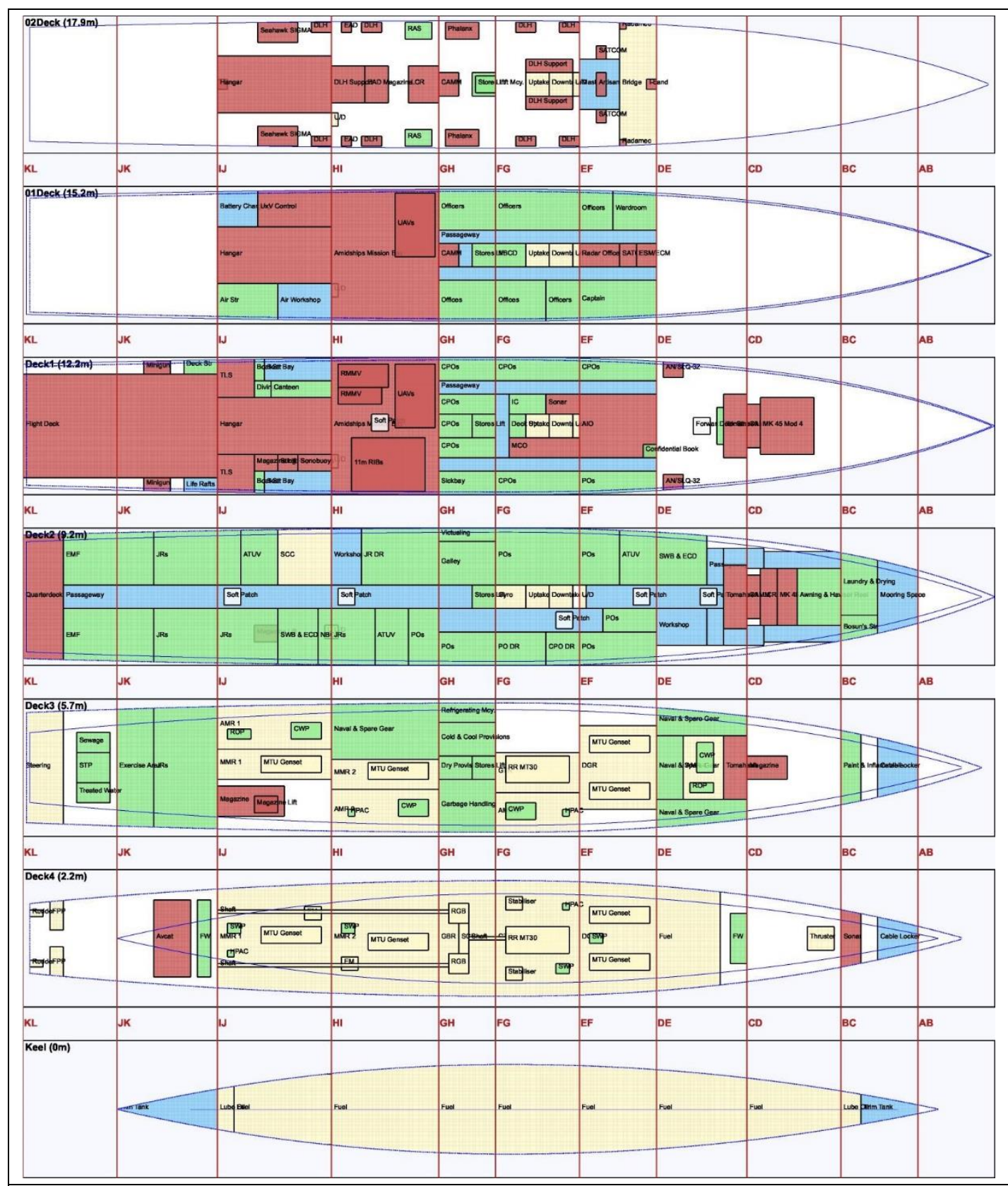

Figure A5. 4: Ship design Variant 1 (two workshops and two Naval and Spare Gear Stores) internal arrangement, produced by the UCL JavaScript ship design tool 


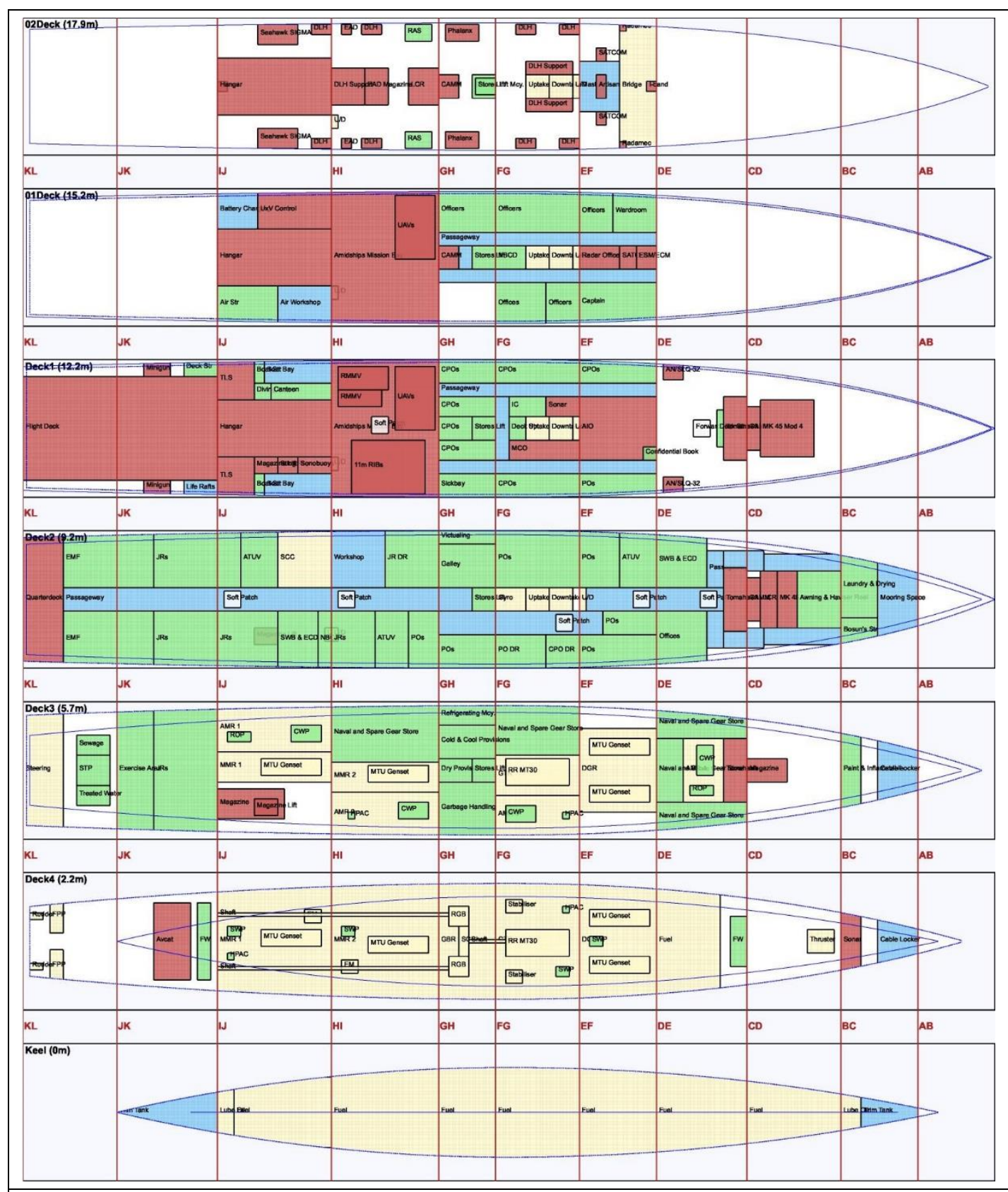

Figure A5. 5: Ship design Variant 2 (one workshop and three Naval and Spare Gear Stores) internal arrangement, produced by the UCL JavaScript ship design tool 


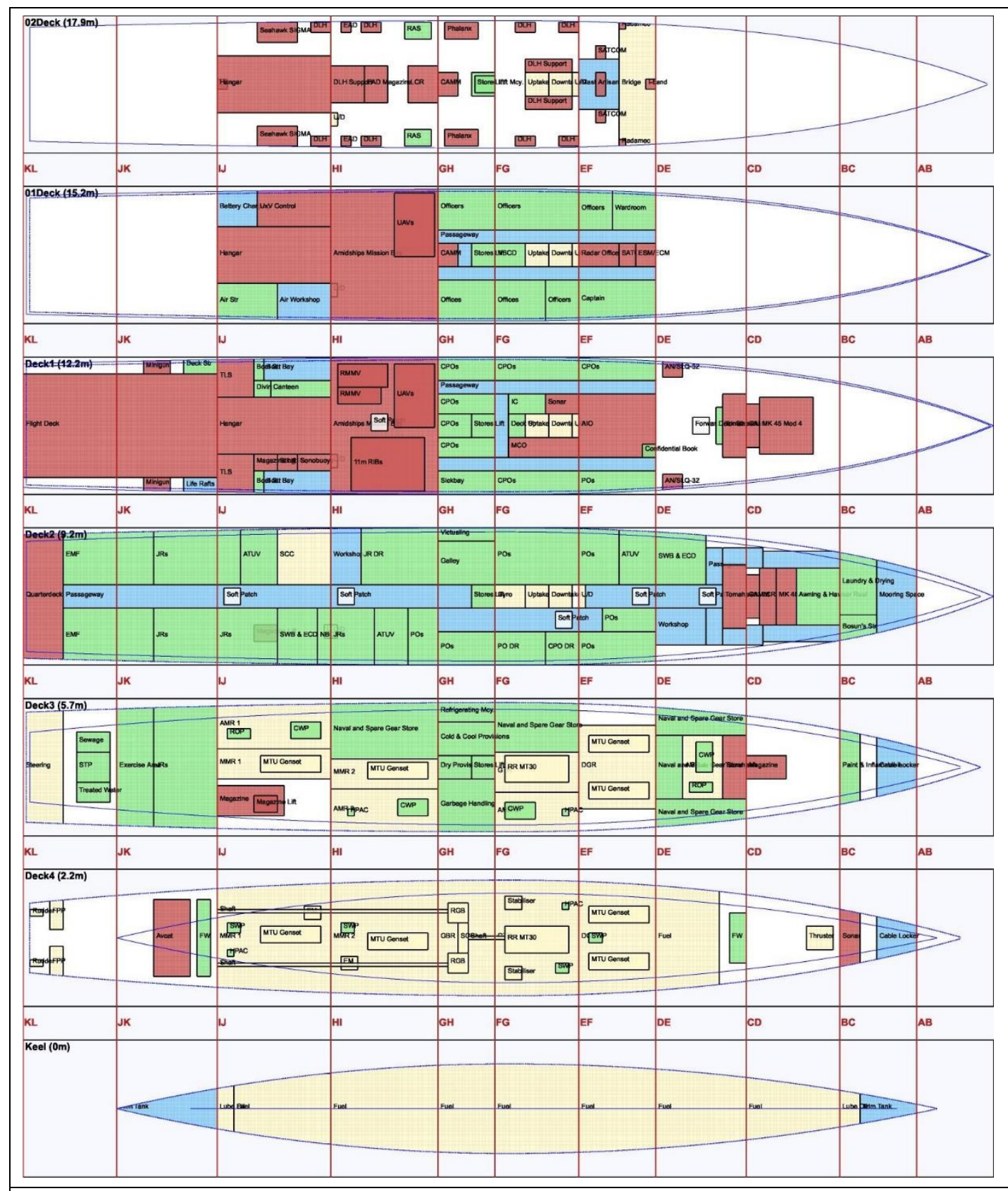

Figure A5. 6: Ship design Variant 3 (two workshops and three Naval and Spare Gear Stores) internal arrangement, produced by the UCL JavaScript ship design tool 

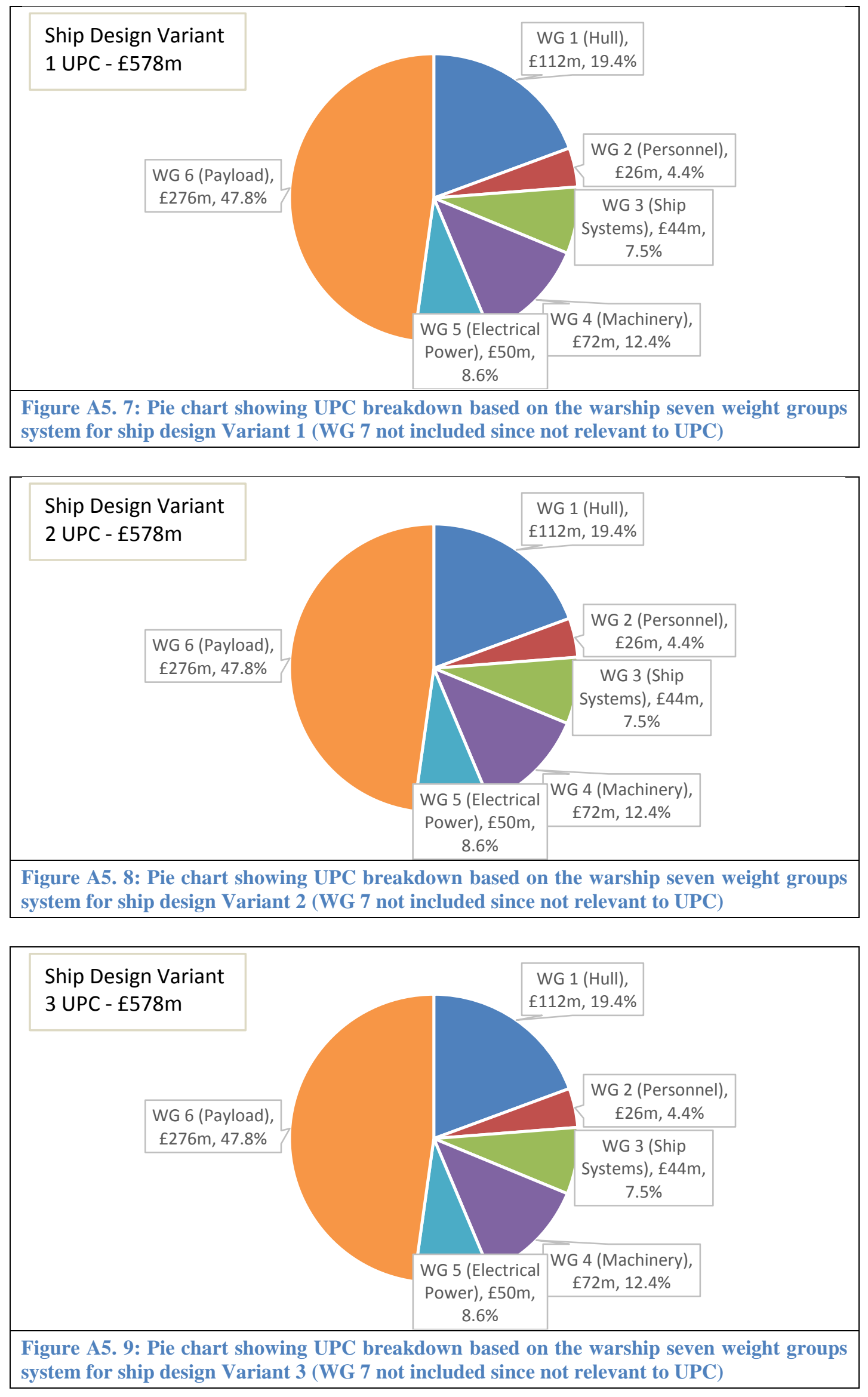
Enhanced Supportability Features

The complete internal arrangement of the ship design Variants 1.1 and 1.2 (see

Table 4.13) and breakdown of the estimated UPC values are provided in the following figures.

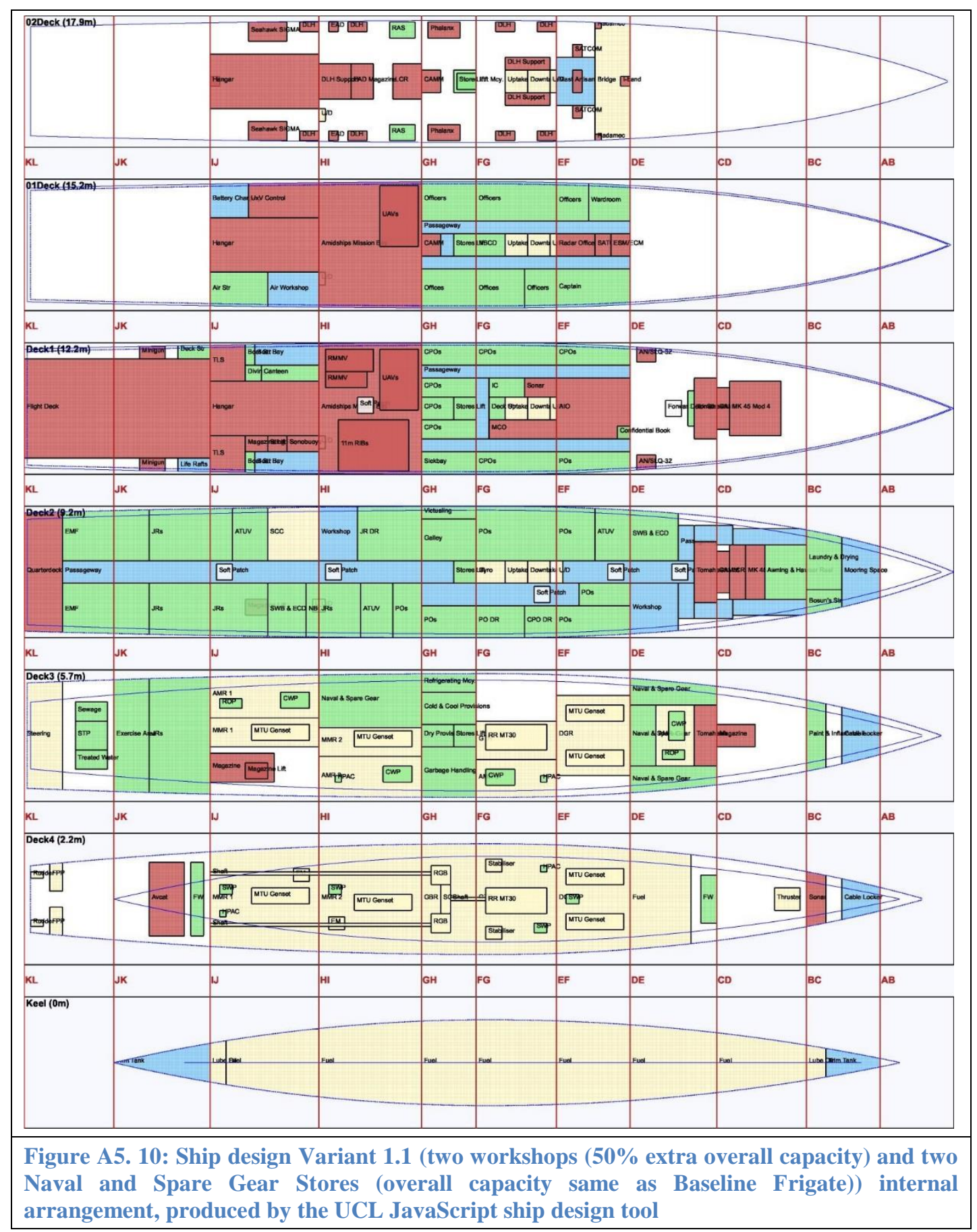




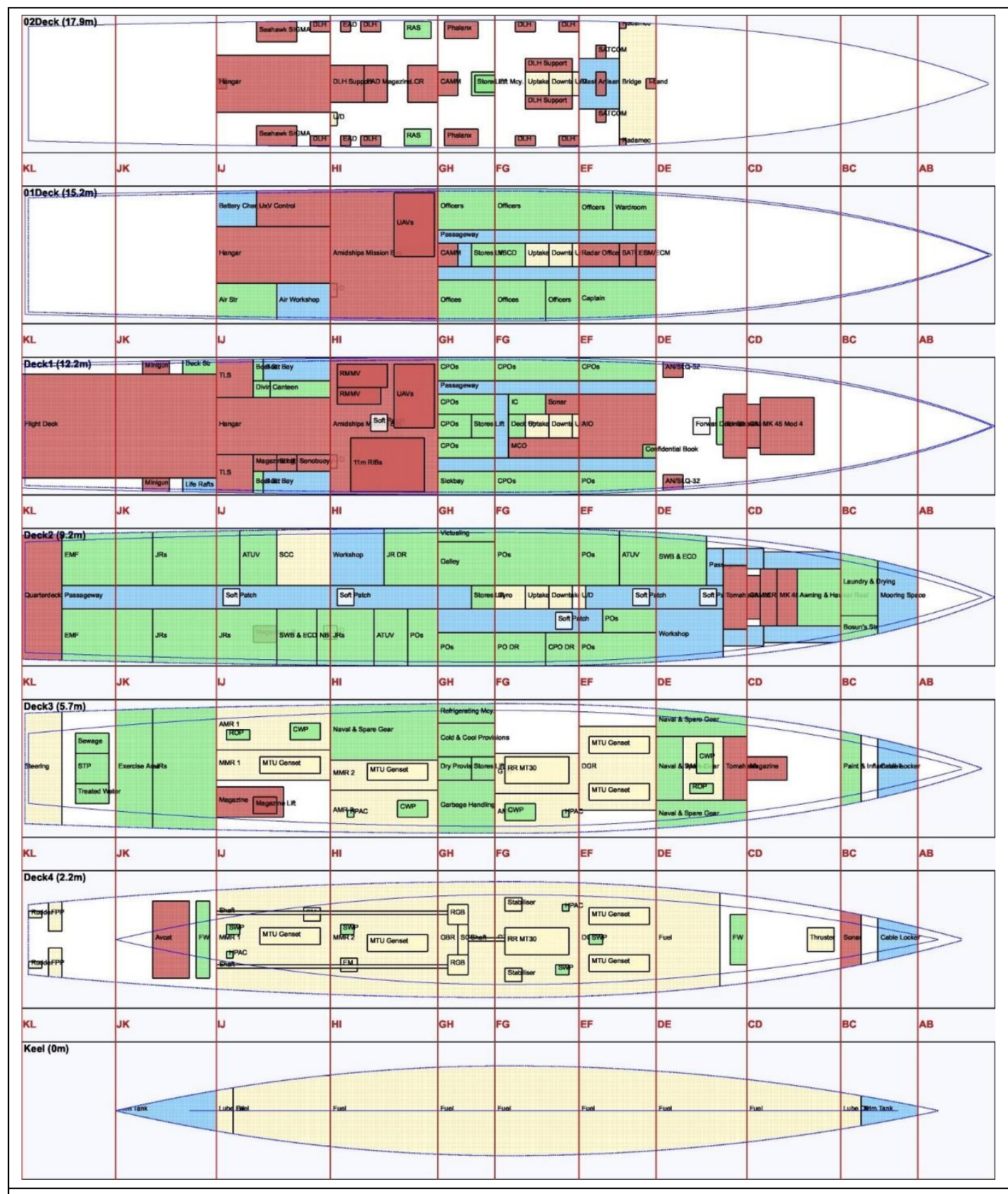

Figure A5. 11: Ship design Variant 1.2 (two workshops (100\% extra overall capacity) and two Naval and Spare Gear Stores (overall capacity same as Baseline Frigate)) internal arrangement, produced by the UCL JavaScript ship design tool 

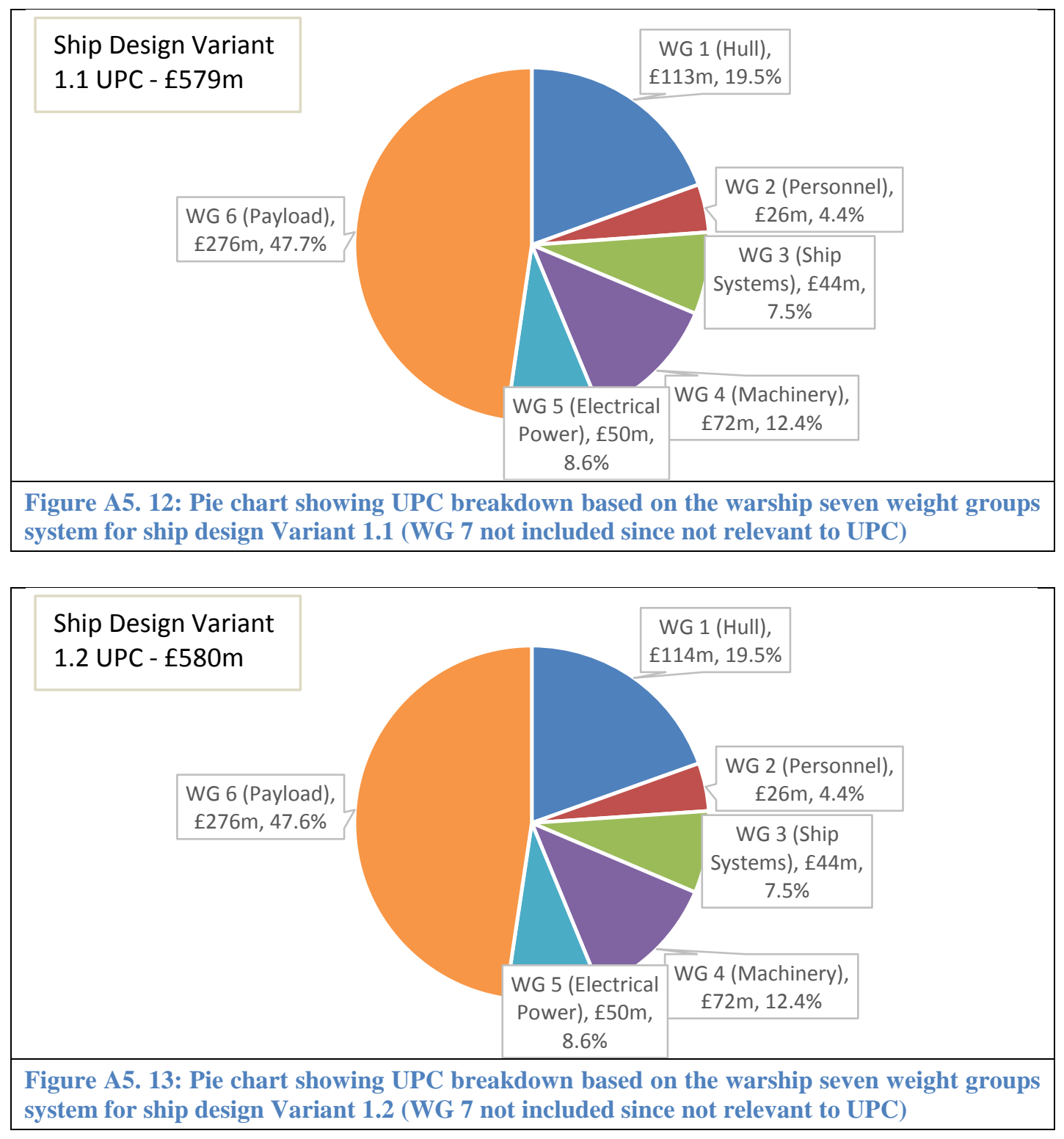
The complete internal arrangement of the ship design Variants 2.1 and 2.2 (see

Table 4.13) and breakdown of the estimated UPC values are provided in the following figures.

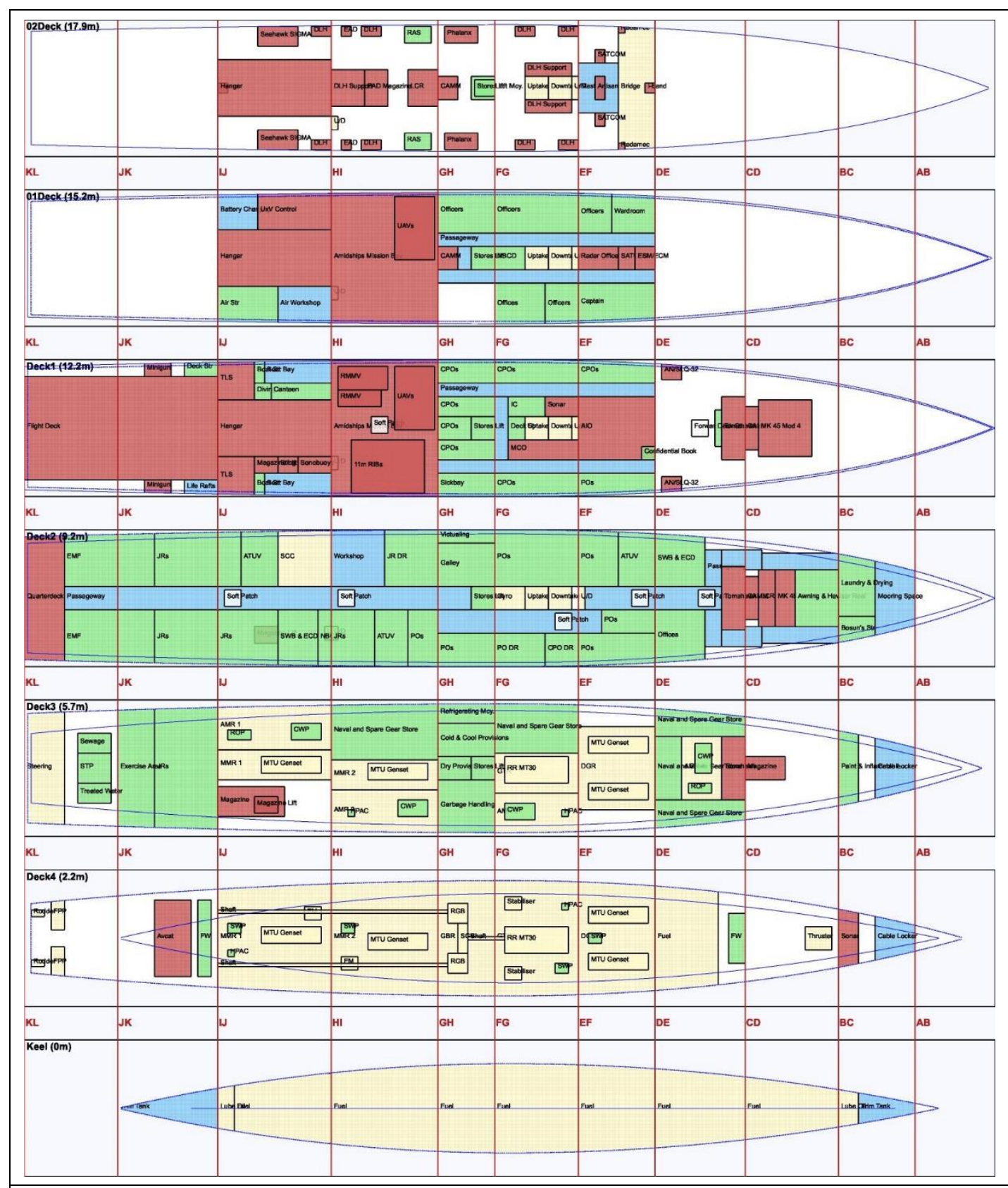

Figure A5. 14: Ship design Variant 2.1 (one workshop (overall capacity same as Baseline Frigate) and three Naval and Spare Gear Stores (50\% extra overall capacity)) internal arrangement, produced by the UCL JavaScript ship design tool 


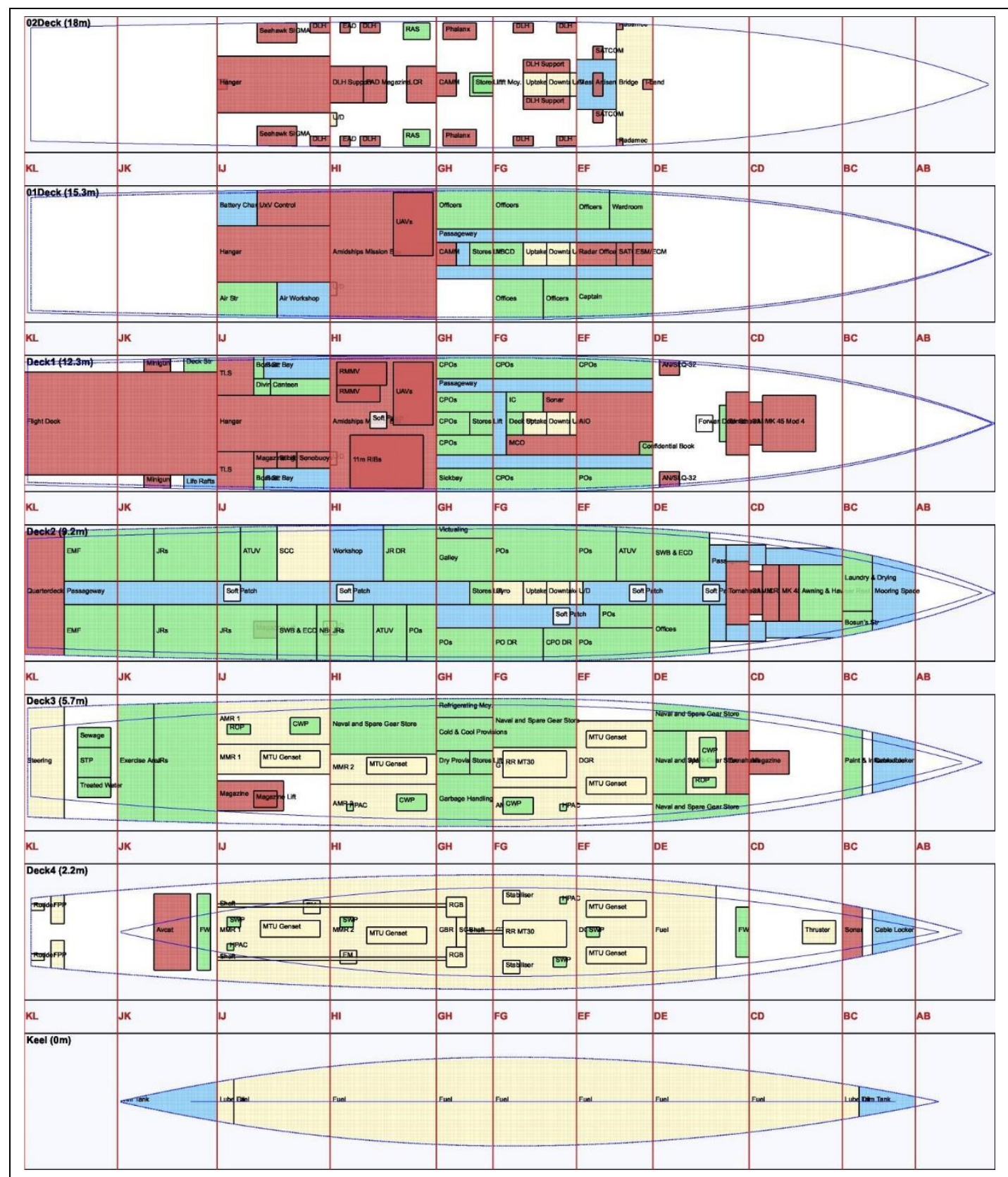

Figure A5. 15: Ship design Variant 2.2 (one workshop (overall capacity same as Baseline Frigate) and three Naval and Spare Gear Stores (100\% extra overall capacity)) internal arrangement, produced by the UCL JavaScript ship design tool 

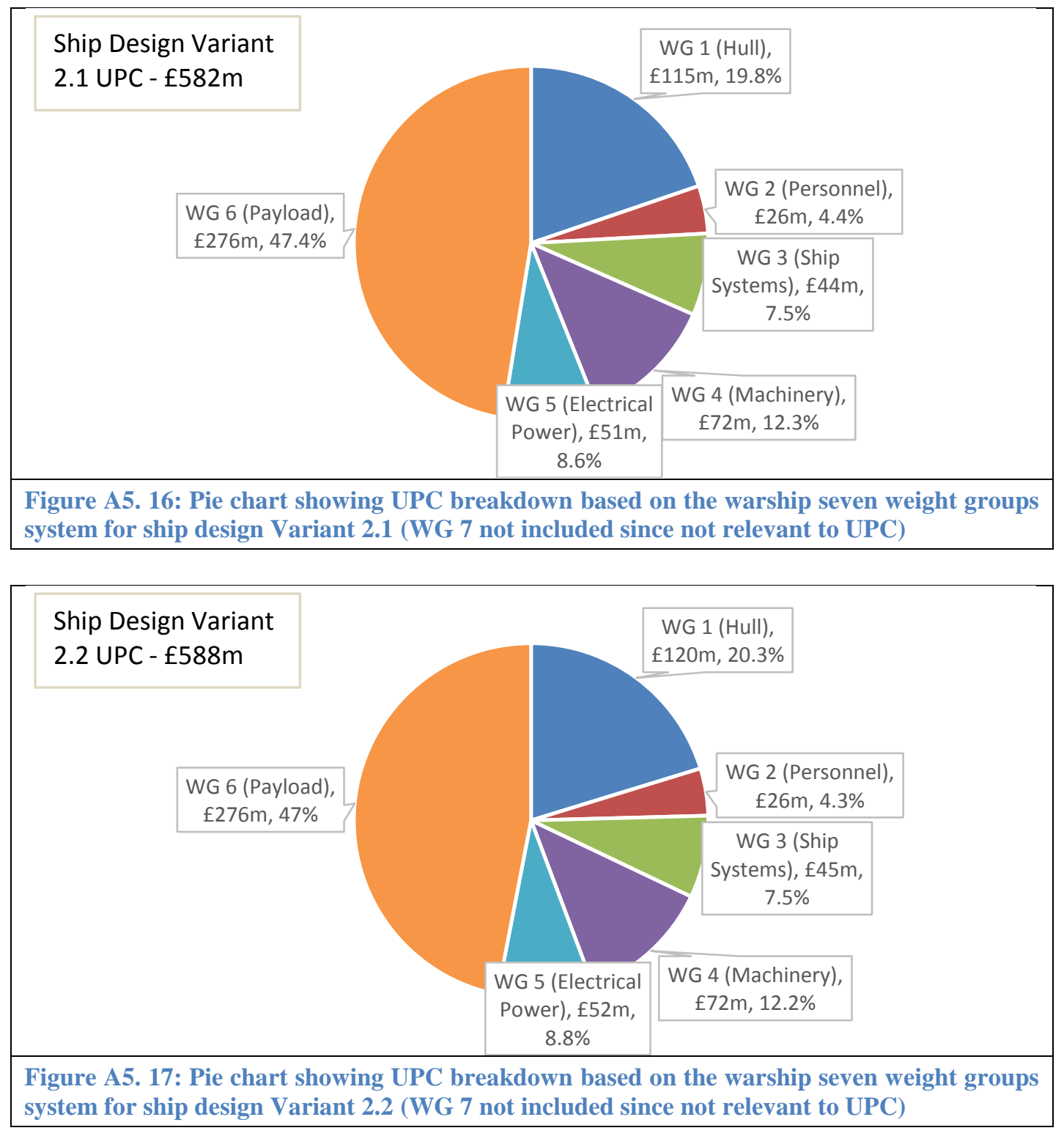
The complete internal arrangement of the ship design Variants 3.1 and 3.2 (see

Table 4.13) and breakdown of the estimated UPC values are provided in the following figures.

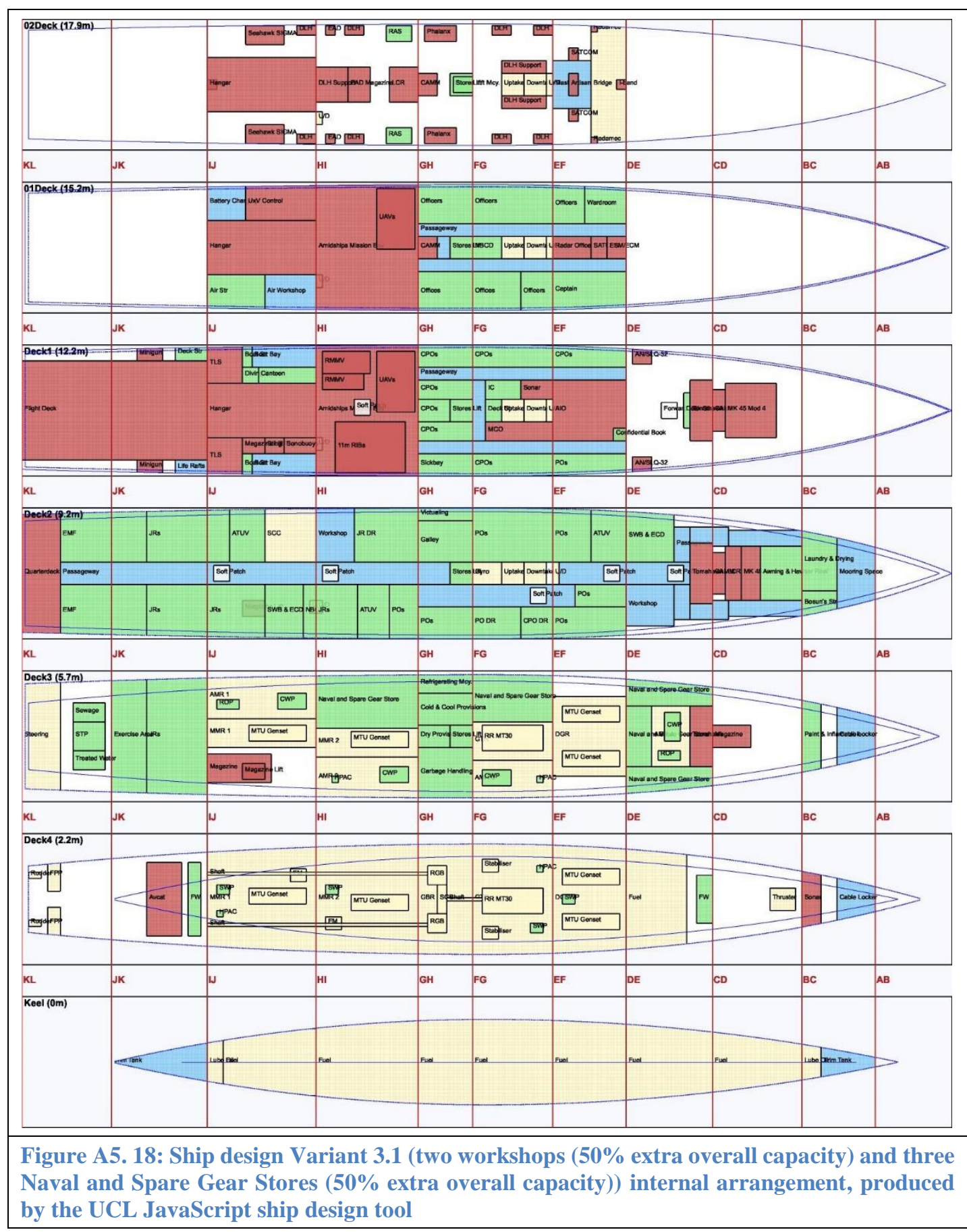




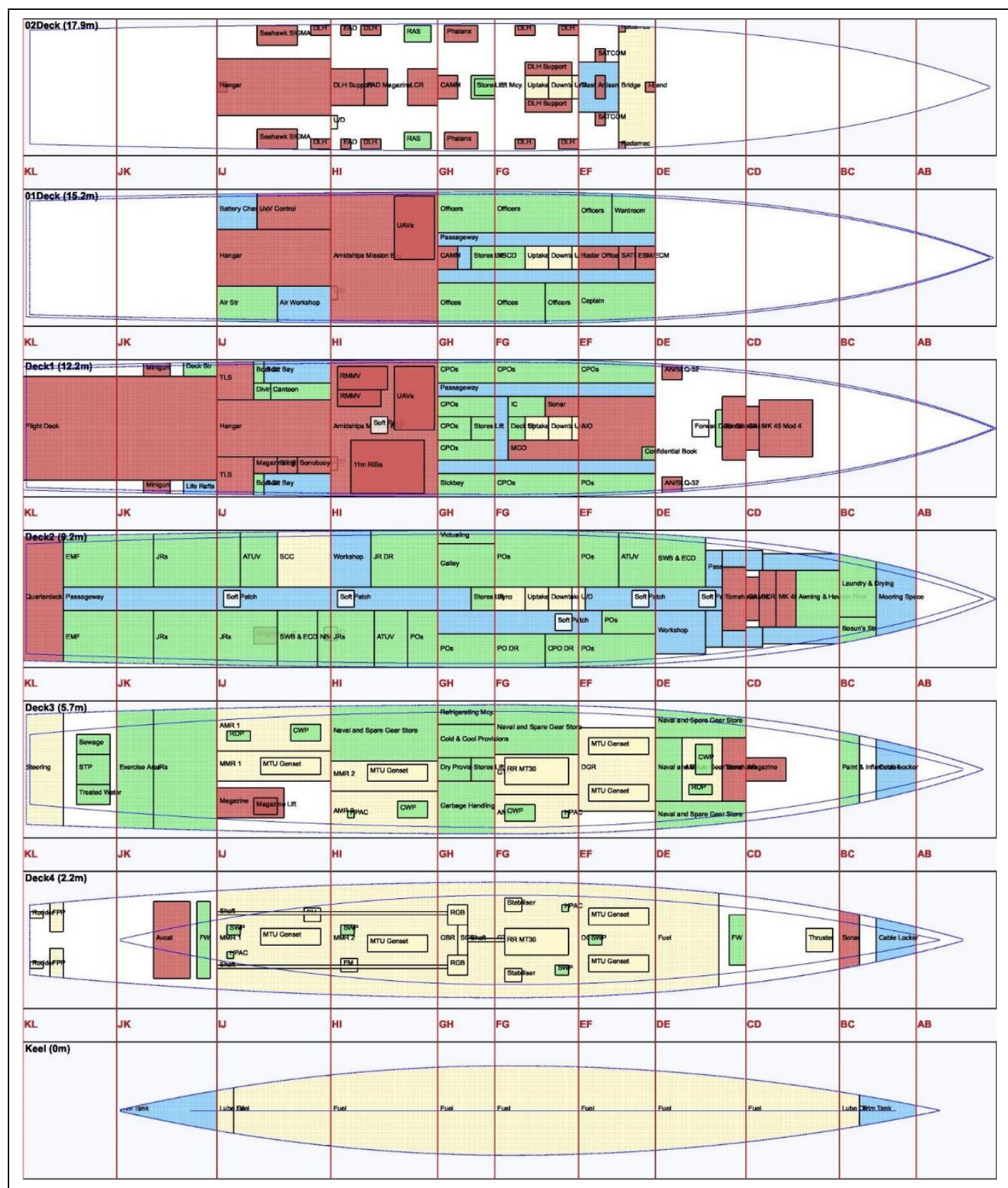

Figure A5. 19: Ship design Variant 3.2 (two workshops (100\% extra overall capacity) and three Naval and Spare Gear Stores (100\% extra overall capacity)) internal arrangement, produced by the UCL JavaScript ship design tool 

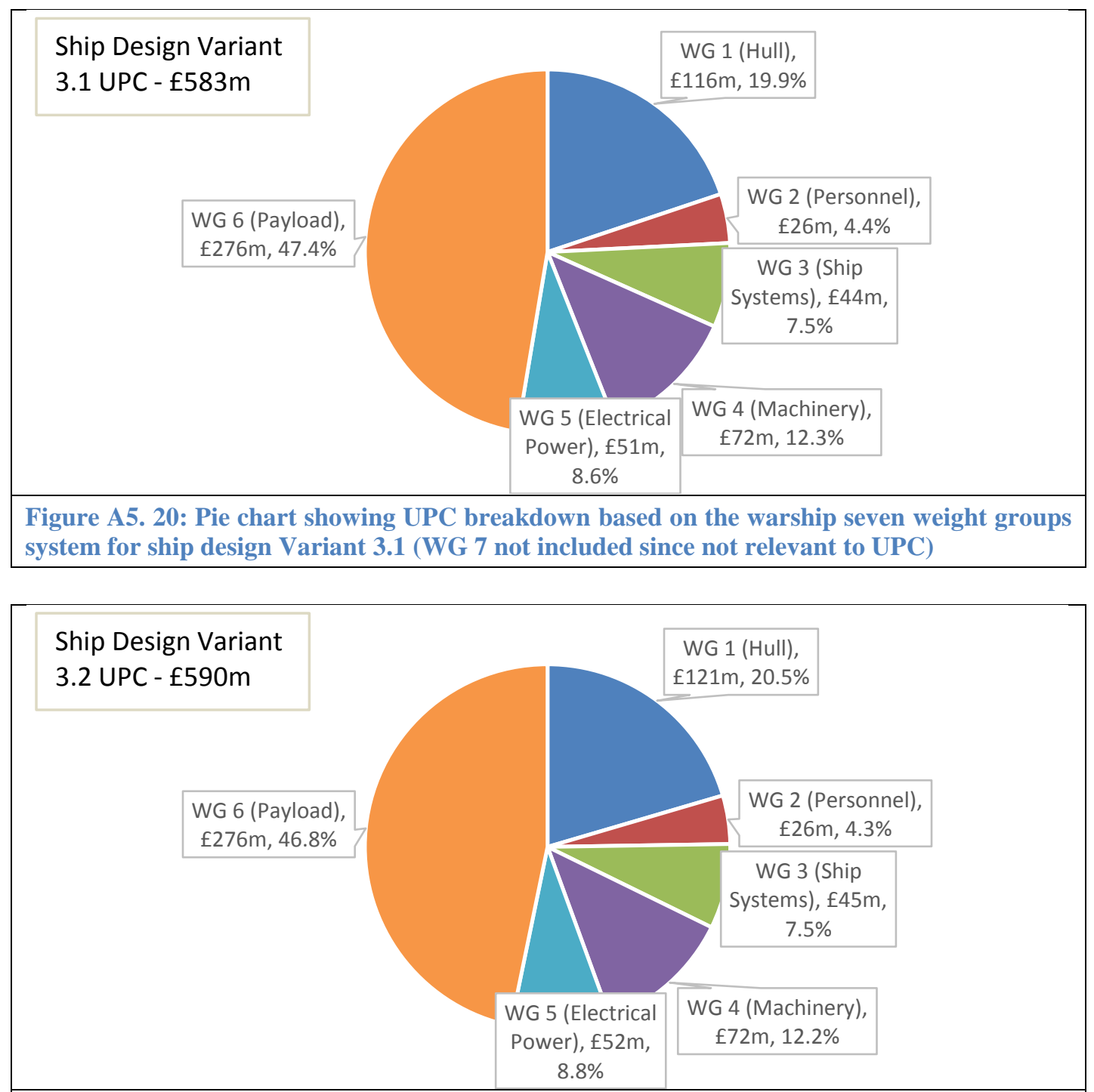

Figure A5. 21: Pie chart showing UPC breakdown based on the warship seven weight groups system for ship design Variant 3.2 (WG 7 not included since not relevant to UPC) 
Alternative Ship Design Style

The complete internal arrangement of the ship design Variants 1.2.1, 2.2.1, 3.2.1 (see

Table 4.17) and breakdown of the estimated UPC values are provided in the following figures.

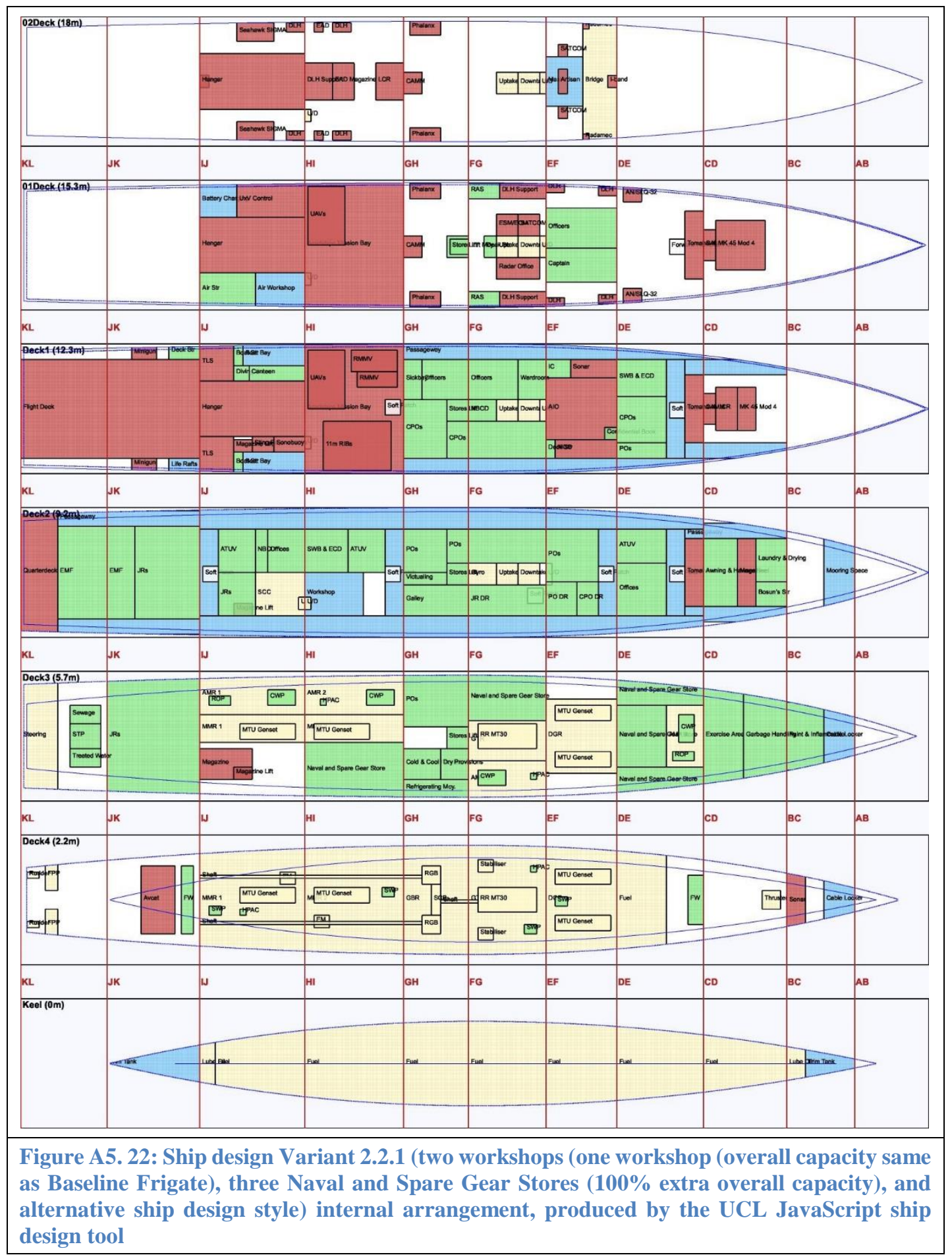




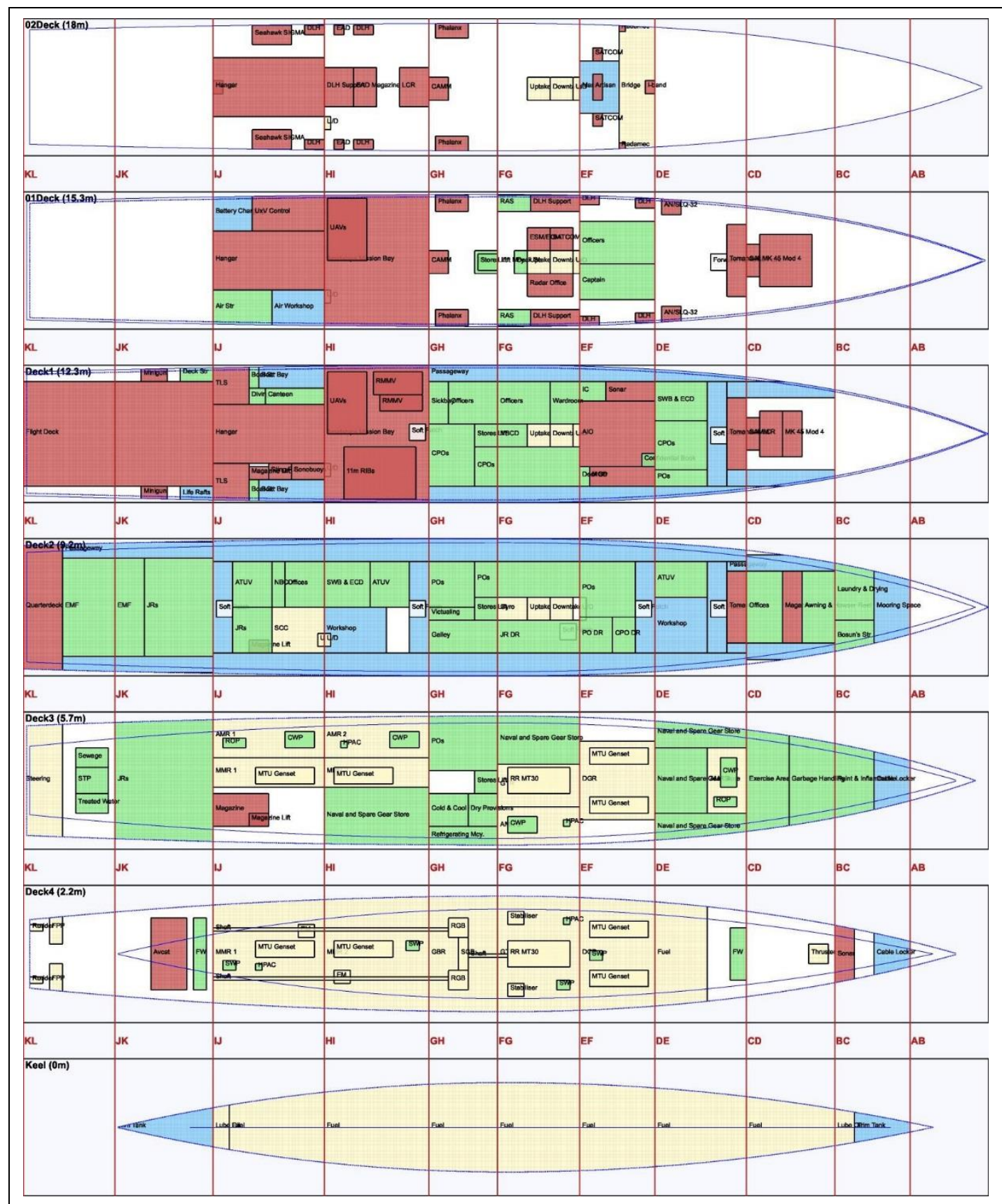

Figure A5. 23: Ship design Variant 3.2.1 (two workshops (100\% extra overall capacity), three Naval and Spare Gear Stores (100\% extra overall capacity), and alternative ship design style) internal arrangement, produced by the UCL JavaScript ship design tool 

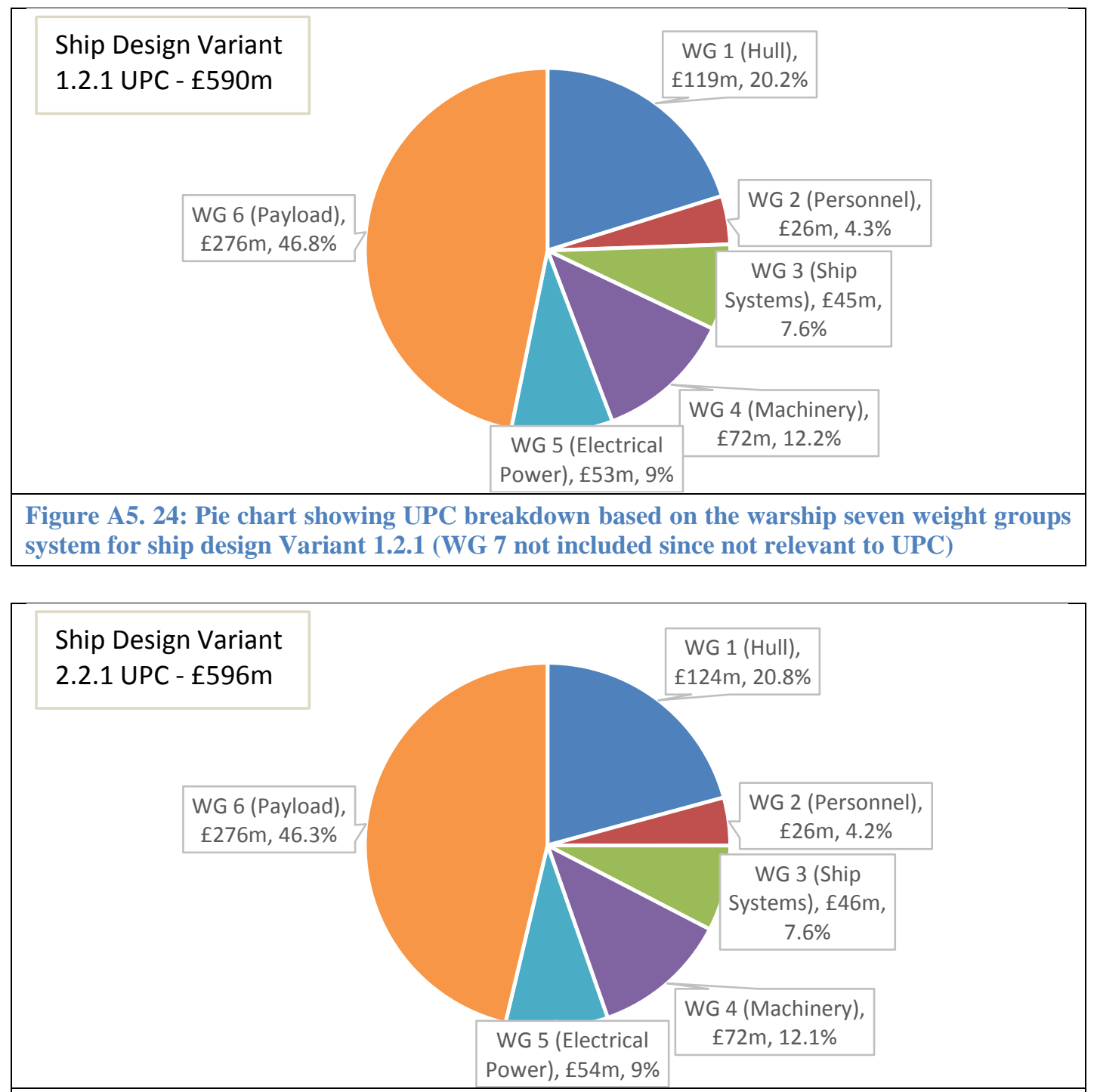

Figure A5. 25: Pie chart showing UPC breakdown based on the warship seven weight groups system for ship design Variant 2.2.1 (WG 7 not included since not relevant to UPC)

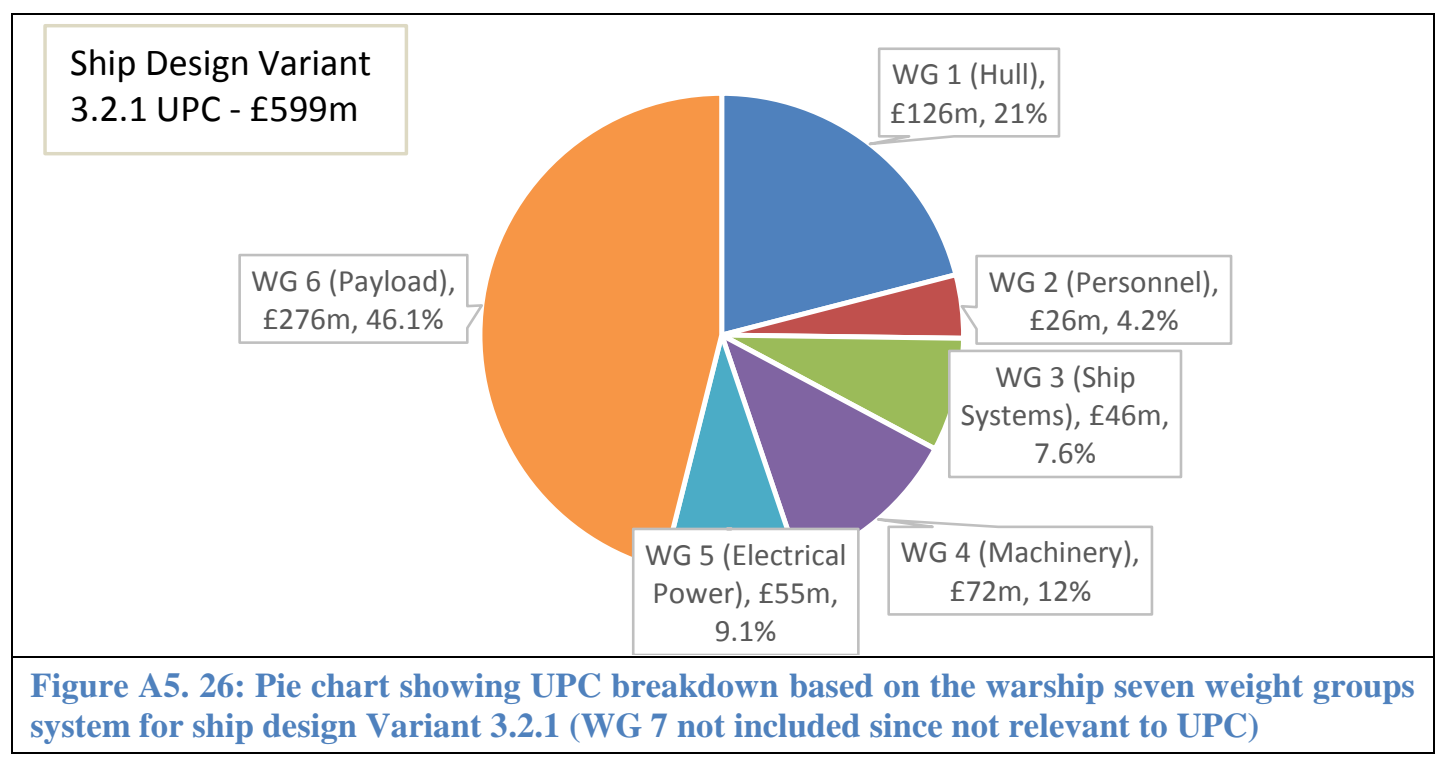

